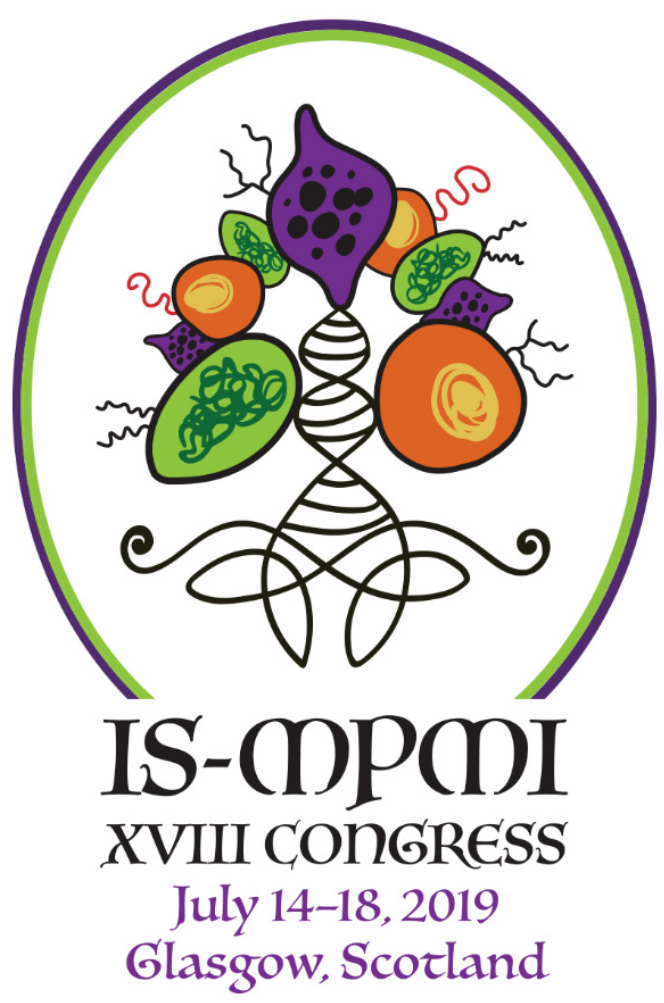

\title{
IS-MPMI XVIII Congress Abstracts of Poster Presentations
}

\begin{abstract}
IS-MPMI XVIII Congress in Glasgow, Scotland, July 14-18, 2019. The recommended format for citing congress abstracts, using the first abstract below as an example, is as follows:

Li, F., Upadhyaya, N., Schwessinger, B., Sperschneider, J., Matny, O., Raley, C., Miller, M. E., Silverstein, K., Nguyen-Phuc, H., Hirsch, C. D., Visser, B., Pretorius, Z. A., Steffenson, B., Dodds, P. N., and Figueroa, M. 2019. Contribution of a somatic hybridization event to the emergence of the Ug99 lineage of the wheat stem rust pathogen. (Abstr.) Molecular Plant-Microbe Interactions 32:S1.1. https://doi.org/10.1094/MPMI-32$10-\mathrm{S} 1.1$
\end{abstract}

The abstracts are published as a supplement to $M P M I$ for citation purposes. They were not reviewed by the MPMI Editorial Board and were not edited by the APS editorial staff.

https://doi.org/10.1094/MPMI-32-10-S1.1

(C) 2019 International Society for Molecular Plant-Microbe Interactions

Contribution of a somatic hybridization event to the emergence of the Ug99 lineage of the wheat stem rust pathogen F. LI (1), N. Upadhyaya (2), B. Schwessinger (3), J. Sperschneider (2), O. Matny (4), C. Raley (5), M. E. Miller (6), K. Silverstein (1), H. Nguyen-Phuc (1), C. D. Hirsch (1), B. Visser (7), Z. A. Pretorius (7), B. Steffenson (4), P. N. Dodds (2), M. Figueroa (1), (1) University of Minnesota, St. Paul, MN, U.S.A.; (2) CSIRO Agriculture and Food, Canberra, ACT, Australia; (3) Research School of Biology, The Australian National University, Canberra, ACT, Australia; (4) University of Minnesota, Saint Paul, MN, U.S.A.; (5) The George Washington University, Washington, DC, DC, U.S.A.; (6) Pairwise, Durham, NC, U.S.A.; (7) University of the Free State, Bloemfontein, South Africa

Stem rust, caused by Puccinia graminis f. sp. tritici (Pgt), is a devastating disease of wheat and barley. The recognition of Pgt as a global threat to food security was substantiated by the emergence of races in the Ug99 lineage from Africa, which are virulent to most wheat cultivars in the world. Similar to other rust fungi, Pgt is a dikaryotic organism, with two haploid nuclei (karyons) during the infection process on wheat. We developed a new strategy to generate haplotype-phased genome assemblies of Pgt using PacBio long reads, to better understand the genomic architecture and evolution of this pathogen. Comparison of a fully haplotype-resolved assembly of Ug99 to two Pgt isolates of the race 21 group found in South Africa and Australia provides evidence that Pgt Ug99 arose by a somatic hybridization event caused by nuclear exchange during the vegetative phase. Inter-isolate comparisons indicated that one complete haplotype of the race 21 isolates is shared with Pgt Ug99. This is 
supported by whole-genome sequence alignments, structural variation analysis, read cross mapping, and phylogenetic analyses. DNA proximity analysis from Hi-C data supports the physical separation of the two predicted haplotypes into separate nuclei. Based on these findings, we propose a model that ascribes the origin of the Pgt $\mathrm{Ug} 99$ lineage to a somatic hybridization event that occurred in Africa.

Understanding the molecular intricacies of rice-Rhizoctonia solani interactions S. GHOSH, P. Kanwar, G. Jha, National Institute of Plant Genome Research, India

Sheath blight disease is a devastating disease in rice that causes huge crop losses worldwide. It is caused by a necrotrophic fungus Rhizoctonia solani. In spite of global efforts till now there are no reports of complete resistance against this pathogen. We observed that $R$. solani has a brief biotrophic phase wherein mycelial grows parallel to rice veins without showing any morphological and anatomical changes. However, at later stage, i.e. necrotrophic phase, infection cushions along with anatomical changes are observed at the site of disease symptoms. Through transcriptome and metabolome studies we have identified several candidate host susceptibility factors and pathogenicity determinants that might play an important role during pathogenesis of $R$. solani in rice. Furthermore, using whole genome studies, we have identified several gene/gene families that might contribute to the aggressiveness of the $R$. solani strains. Although gene manipulation studies in $R$. solani is difficult we have devised ways wherein we can functionally characterize these pathogenicity determinants. Overall the present study will help in better understanding of the rice- $R$. solani pathosystem which would eventually be helpful in developing strategies for durable sheath blight disease tolerance in rice.

Decoding the myrtle rust genome, the largest fungal genome (1 Gbp) sequenced to date P. A. TOBIAS (1), D. Chagné (2), G. Smith (2), B. Schwessinger (3), C. M. Dong (4), J. Tibbits (5), C. Deng (2), R. F. Park (4), (1) University of Sydney, Eveleigh, NSW, Australia; (2) The New Zealand Institute for Plant \& Food Research Ltd., New Zealand; (3) Research School of Biology, The Australian National University, Canberra, ACT, Australia; (4) Plant Breeding Institute, University of Sydney, Narellan, NSW, Australia; (5) AgriBio, Agriculture Victoria, Melbourne, VIC, Australia

Austropuccinia psidii, originating from South America, is a globally invasive plant pathogen causing myrtle rust. Several biotypes are recognised, the most widely distributed one known as Pandemic. Unlike many other rust fungi, A. psidii has a very broad host range (currently $>445$ myrtaceous species), making it a particularly dangerous plant pathogen. In only 8 years since the Pandemic biotype was first found in Australia, the rust has caused near extinction of at least 3 species (Lenwebbia sp. Main Range, Rhodamnia rubescens, Rhodomyrtus psidioides), caused the decline of at least one keystone species, Melaleuca quinquenervia, and impacted commercial production of Myrtaceae such as the tea-tree (Melaleuca alternifolia) and lemon myrtle (Backhousia citriodora). Critical to combatting this pathogen is understanding the mechanisms it uses to parasitize its many hosts. To enable genomic studies of pathogenicity in $\mathrm{A}$. psidii, we assembled a highly contiguous haplo-phased genome for the pandemic biotype based on PacBio sequence data and scaffolding with Hi-C technology. With a haploid size of just over 1 Gbp, it is the largest assembled fungal genome to date and one of the largest rust genomes known. Annotations based on repeat analysis and post-inoculation mRNA sequence capture from a susceptible host have begun to provide insight into potential infection strategies.

Time-series transcriptome analysis of Brachypodium distachyon in response to infection by Rhizoctonia solani Y. KOUZAI (1), K. Inoue (1), M. Shimizu (1), Y. Uehara (1), K. Takahagi (1), Y. Noutoshi (2), K. Mochida (1), (1) RIKEN, Yokohama, Japan; (2) Okayama Univ, Okayama, Japan

Rhizoctonia solani AG-1, 1A is the causal agent of sheath blight which severely reduces the yield of cereals such as rice. Because there are no useful genetic resources for resistance against $R$. solani in both cultivated and wild rice, the molecular mechanisms underlying plant native sheath blight resistance are mostly unknown. In this study, we report the identification of five natural accessions of Brachypodium distachyon, which showed much fewer symptoms upon $R$. solani infection. Our time-series comparative transcriptome analysis during $R$. solani infection illustrated that these resistant accessions commonly induced defense-associated genes potentially regulated by WRKY transcription factors at $8-16$ hours postinoculation (hpi), while the susceptible accession Bd21 induced the similar set of genes at 16-24 hpi. We next inferred gene regulatory networks (GRNs) that include WRKYs based on the time-series transcriptome dataset and found the WRKYs constituting hubs in the GRNs of both susceptible and resistant accessions. For example, the hubs of the GRN from Bd3-1, one of the resistant accessions, contained the salicylic acid (SA)-inducible WRKYs, which may suggest the contribution of SA in its $R$. solani resistance. All our findings suggest that rapid activation of WRKY-dependent defense genes is important to the $R$. solani resistance in $B$. distachyon. We are now investigating the WRKYs to verify their roles in the $R$. solani resistance by RNAi-based silencing.

Functional and genomic characterization of a Pseudomonas syringae strain isolated from natural populations of Arabidopsis plants D. Zavala (1), I. Fuenzalida (1), A. HERRERA-VÁSQUEZ (1,2), M. V. Gangas (1), C. Meneses (1), F. Roux (3), M. F. Blanco (1,2,4), (1) Universidad Andres Bello, Chile; (2) Fundación Instituto de Biología Integrativa, iBio, Chile; (3) LIPM, Université de Toulouse, INRA, CNRS, INPT, Castanet-Tolosan, France; (4) FONDECYT regular $\mathrm{N}^{\circ} 1170259$, Chile

$P$. syringae is a phytopathogenic bacterium used to study the plant-pathogen interaction. In this work, we evaluate the infective process of the isolate $P$. syringae RAYR-BL (Psy RAYR-BL) described as highly infective in natural accessions of Arabidopsis. Despite the $P s y R A Y R-B L$ grows faster on different culture media compared to other Pseudomonas strains, when Arabidopsis plants (WT Col0 ecotype) were infected with a virulent (Pst DC3000), avirulent (Pst AvrRPMI), and a non-host (Pst hrcC) P. syringae strains, the growth kinetics of Psy RAYR-BL was similar to the Pst hrc $C^{-}$strain. Consequently, the markers of disease progression evaluated were almost absent in the plants inoculated with $P s y R A Y R$ $B L$. To assess if WT Col0 plants can induce a defense mechanism more efficiently in response to $P S y R A Y R-B L$, we evaluate molecular markers associated with PAMP- and Effector-trigger immunity (PTI and ETI). Psy RAYR-BL displays a slight accumulation of the ETI marker, PR-1, and induce a PTI response similar to the $P s t h r c C^{-}$strain suggesting that a less effective virulence mechanism could explain the low proliferation of Psy RAYR-BL in plant tissue. To asses this hypothesis, the genomic DNA of Psy RAYR-BL was sequenced and analyzed. Despite the presence of 
the complete repertoire of genes coding for the T3SS components, the strain has a small collection of genes coding for effector proteins and lacks other virulence factors described as necessary in the infective process of $P$. syringae.

Phytoplasma SAP11 effector homologs have evolved to differentially interact with plant TCP transcription factor subclasses S. CAPDEVIELLE (1), P. Pecher (2), G. Moro (1), A. Al-Subhi (3), T. C. Mathers (1), W. Huang (1), A. M. Al-Sadi (3), S. A. Hogenhout (1), (1) John Innes Centre, Norwich, U.K.; (2) Leibniz Inst of Plant Biochemistry, Halle Saxony-Anhalt, Germany; (3) College of Agricultural and Marine Sciences, Sultan Qaboos University, Seeb, Oman

Phytoplasmas are bacterial plant pathogens vectored by sap-feeding leafhoppers and secrete effectors to modulate processes in their plant hosts. The Aster Yellows phytoplasma Witches' Broom (AY-WB) effector Secreted AY-WB Protein 11 (SAP11) and SAP11 effector homologs of other phytoplasmas bind and destabilize plant TCP (TEOSINTE BRANCHED1, CYCLOIDEA, PROLIFERATING CELL FACTORS 1 and 2) transcription factors, which play key-roles in regulating plant development and defense. AY-WB is a generalist pathogen that infects diverse plant species, including Arabidopsis thaliana, while Maize bushy stunt phytoplasma (MBSP) is a specialist that infects maize (Zea mays L.). Stable production of SAP $11_{\mathrm{AYWB}}$ and SAP1 $11_{\mathrm{MBSP}}$ in $A$. thaliana and maize leads to the induction of specific developmental phenotypes consistent with destabilization of Class II CYC/TB1 and CIN-TCPs. We expanded the analysis to different SAP11 effector homologs from various phytoplasma groups and showed that they group in different clades that each interact with different TCP (sub)classes. We found that all SAP11 homologs bind Class II CYC/TB1 TCPs, but differentially interact with Class I PCF TCPs and class II CIN-TCPs. We identified the SAP11 region involved in the binding specificities toward class I PCF and II CIN-TCPs. These results provide a reliable predictive model to determine the binding specificities of SAP11 toward TCP classes.

An emerging new model system: The smut fungus Thecaphora thlaspeos infects Brassicaceae S. Gul (1), K. Bösch (1), L. Plücker (2), K. J. Courville (1), L. Frantzeskakis (1), M. Feldbrügge (3), V. GOEHRE (1), (1) Heinrich-Heine University, Duesseldorf, Germany; (2) Heinrich-Heine University, Universitaetsstraße 1, Building 26.12.01.31, Duesseldorf, Germany; (3) Heinrich-Heine-Universität, Düsseldorf, Germany

Smut fungi, such as Ustilago maydis in maize or Thecaphora solani in potato, are globally distributed plant pathogens that infect agriculturally important crop plants. During the major part of their life cycle, these pathogens grow as endophytes in their host plant to finally produce large amounts of fungal spores in reproductive organs. To date, the lack of genetically tractable host plants limits investigations of plant responses, and several attempts were made to infect Arabidopsis thaliana with $U$. maydis. In a reverse approach, we establish the Brassicaceae smut fungus $T$. thlaspeos as a pathogen of model plants.

To this end, we follow three approaches. 1. Establishing the fungus as a model organism comparable to U. maydis. This included cultivation, genetic manipulation, and sequencing the genome, which is a typical smut genome (Courville 2019 New Phytologist). 2. Characterization of the infection biology, which has already revealed several interesting differences, e.g. root colonization previously unknown for the grass smut fungi (Frantzeskakis 2017 MPMI). 3. Characterization of protein and RNA virulence factors to identify conserved and specific virulence factors. Interestingly, our current top candidate effector links infection to plant cold acclimation. Here, I will report on our latest findings on the interaction between $T$. thlaspeos with its host plants in this novel model pathosystem.

Good fungus, bad fungus: Interactions between barley and Ramularia collo-cygni

C. BURRELL, University of Edinburgh and Scotland's Rural College, Edinburgh, U.K.

Barley yield is reduced by Ramularia Leaf Spot, caused by the fungus Ramularia collo-cygni (Rcc). Rcc can be present throughout the life of the plant, but disease symptoms don't occur in the field until after flowering. Although there have been significant advances in our understanding of the barley $-R c c$ relationship in recent years, the nature of the interactions between plant and fungus during the period before disease symptoms appear is still not well understood. Specifically it is not known to what extent photosynthesis and plant growth is restricted during the asymptomatic phase of pathogen growth. My project aims to answer whether infection with Rcc affects barley leaf physiology during the asymptomatic period, and if so whether there is any indication that effects from this stage of infection could contribute to yield loss. I have used techniques such as qPCR, infra-red gas analysis, and chlorophyll fluorescence imaging to determine the relationship between the amount of pathogen infection, the severity of visible symptoms, and the rates of photosynthesis and net carbon assimilation in both the asymptomatic and symptomatic phases of pathogen growth. My results so far show no indication of significant damage to the photosynthetic apparatus before symptom appearance, making it unlikely that the asymptomatic stage of infection is a major contributor to yield loss, however, once symptoms appear effects on photosynthetic performance extend beyond the lesion area.

Prevalence of mixed infection and putative facilitative interactions between blueberry viruses

M. L. FALL, P. L. Lemoyne, C. L. Vincent, C. L. Michel, Agriculture and AgriFood Canada, Saint-Jean-sur-Richelieu, QC, Canada

Most of the knowledge of plant virus diversity is biased heavily toward viruses that cause visible symptoms, yet many of the known blueberry viruses are latent or asymptomatic. Many questions, including what is driving symptom expression during mixed virus infection in blueberry, remain unanswered. Large-scale molecular screening of blueberry viruses in 111 leaf samples was carried out. The prevalence, coinfection, and interaction of major blueberry viruses were then specifically studied by PCR-mediated detection. In 7\% of the samples, mixed infections with at least two viruses were observed. Tomato ringspot virus (ToRSV), blueberry mosaic virus (BIMV), and blueberry shock virus (BIShV) were present in $50 \%$ of the mixed infections, suggesting the absence of antagonistic interactions between those viruses. All leaf samples infected with multiple viruses expressed symptom of reddish whereas the majority of the samples infected with ToRSV or BIShV did not express leaf symptoms. However, all samples infected with BIMV expressed symptoms, suggesting that BIMV is driving symptom expression. The seedborne virus, $\mathrm{BlShV}$, was the most prevalent virus, infecting up to $15 \%$ of the samples. The prevalence of aphid-borne viruses and viruses with unknown vectors was very low, $1.1 \%$ and $2.3 \%$, respectively. This study will facilitate the surveillance of emerging viruses and the management of viral diseases in blueberry. 
Characterization of expression profiles of effector genes of Rhizoctonia solani on Brachypodium distachyon

S. S. H. ABDELSALAM (1), S. Isoya (2), M. Watanabe (3), Y. Kouzai (4), H. Matsui (5), M. Yamamoto (3), Y. Ichinose (5), K. Toyoda (3), S. Tsuge (6), Y. Noutoshi (7), (1) Okayama Uni, Okayama, Japan; (2) Okayama Uni, Japan; (3) Okayama University, Japan; (4) RIKEN, Yokohama, Japan; (5) Okayama University, Okayama, Japan; (6) Kyoto Prefectural Univ, Kyoto, Japan; (7) Okayama Univ, Okayama, Japan

Rhizoctonia solani is a necrotrophic fungal pathogen with a broad host range worldwide. It is known as a causal agent of rice sheath blight. Recently, we reported that pretreatment of salicylic acid conferred resistance to $R$. solani in both Brachypodium distachyon and rice. These results suggest that $R$. solani should undergo biotrophic phase in its infection. To execute this process, the pathogen would use effector proteins to suppress host immunity. Therefore, we are now trying to identify effector proteins of $R$. solani. Using several bioinformatics tools we could predict 88 genes in the $R$. solani genome which encode small secreted proteins with no apparent enzymatic motifs and transmembrane domains. Then we developed an inoculation method using Brachypodium detached leaves to collect much amount of synchronized infection hyphae for detection of fungal RNAs in host plant by RT-pPCR. In our inoculation system, symptoms appeared at 24 hours postinoculation (hpi) and fungal biomass was detected from $6 \mathrm{hpi}$ with a primer set for 18S rRNA. Leaves were sampled at 6,10,16,24, and 32 hpi and expression profiles of the effector candidate genes were examined. The transcripts of 68 genes could be detected at any time points during the infection and they were classified into 7 clusters based on their expression patterns. Nine genes were found as early induced genes with highest expression peak at 6 hpi. These identified genes are candidates for further functional analysis.

OchraVine Control - Implementation of integrated and innovative management strategies to reduce the occurrence of ochratoxins along the vine value chain products: Grapes, raisins/currants and wine

D. I. TSITSIGIANNIS (1), C. S. Lagogianni (1), G. Xanthopoulos (1), S. Fountas (1), P. Battilani (2), S. Giannoukos (3), S. Taylor (3), Z. Tsiropoulos (4), C. Drosou (5), F. Chatzipapadopoulos (6), N. Marianos (6), B. Tisseyre (7), C. Moszkowicz (8), (1) Agricultural University of Athens, Athens, Greece; (2) Università Cattolica del S. Cuore di Piacenza, Piacenza, Italy; (3) Q-Technologies Ltd, U.K.; (4) Agricultural \& Environmental Solutions-AGENSO, Athens, Greece; (5) KPAD Ltd, London, U.K.; (6) NEUROPUBLIC, Athens, Greece; (7) Institut National D’ Etudes Superieures Agronomiques De Montpellier-SUPAGRO, Montpellier, France; (8) ENEO, Montpellier, France

Ochratoxin A (OTA) is a very toxic mycotoxin that constitutes a severe problem for viticulture and taking into account the extreme climatic events that are frequently faced in recent years, the OTA problem is arising in wine and raisins/currants. OchraVine Control project offers an inexistent innovative, sustainable and integrated smart ICT solution (OchraVine Control DSS) considering fungal, host and environmental indicators that affect OTA contamination along the vine grape-wine value chain. The OchraVine Control DSS will allow prediction and monitoring at pre- and post-harvest level to control Aspergillus infection and OTA contamination in vine cultivation by combining epidemiological data, biological and chemical management strategies, post-havrest technologies and precision agriculture tools. OchraVine Control DSS solution will pursue a field-to-fork approach and will link and translate the information derived from the OchraRisk and OchraDetect predictive map tools and real data obtained during the monitoring controls by the OchraSensor. OchraVine Control DSS tool in combination with data from the OchraRed Integrated Management Strategy will provide risk prediction information (i.e. geographic OTA vine alerts), practical recommended solutions for OTA management and will verify the compliance with legislation requirements. This project has received funding from the European Union's Horizon 2020 Research and Innovation Program under grant agreement No 778219.

Comparative genomics screen identifies MAMPs from Candidatus Liberibacter species that trigger immunity in citrus Y. Chen (1), C. Bendix (1), J. D. LEWIS (1,2), (1) University of California, Berkeley, Albany, CA, U.S.A.; (2) USDA ARS, Plant Gene Expression Center, Albany, CA, U.S.A.

Citrus greening or Huanglongbing (HLB) is a serious threat to the worldwide citrus industry. HLB is caused by three uncultureable bacteria, Candidatus Liberibacter asiaticus (CLas), Ca. L. americanus (CLam) and Ca. L. africanus (CLaf), and is vectored by the psyllid Diaphorina citri. Control of the disease is hampered by the inability to manipulate the pathogens, a lack of resistance in hosts and the disease's long latency period. Evolutionary approaches have identified microbe-associated molecular patterns (MAMPs) from different bacteria that elicit pattern-triggered immunity (PTI) in host plants. We carried out a bioinformatic-based comparative genomics screen to identify putative MAMPs that could elicit immune responses in citrus. We established a 96-well plate assay to measure reactive oxygen species (ROS) production as a measure of PTI in citrus, and demonstrated that flg22, a well-characterized PAMP, elicits ROS production. We identified two new MAMPs that elicit PTI, as evidenced by ROS production, and enhanced defense gene expression. This work identifies new targets to boost the host immune system and potentially help control HLB.

The Small RNA Chaperone Hfq Regulates Quorum Sensing and Pathogenicity of Pantoea ananatis B. KANG (1), O. Choi (2), Y. Lee (3), S. Kim (3), J. Kim (3), (1) Division of Applied Life Science (BK21 Plus), Gyeongsang National University, Jinju, Korea, Republic of (South); (2) Institute of Agriculture \& Life Science, Gyeongsang National University, Jinju, Korea, Republic of (South); (3) Department of Plant Medicine, Gyeongsang National University, Jinju, Korea, Republic of (South)

Hfq is an abundant bacterial RNA binding protein which has many important physiological roles that are usually mediated by interacting with Hfq binding small RNA (sRNA). Pantoea ananatis PA13 causes bacterial rice sheath rot and onion center rot diseases. $\Delta h f q$ mutant exhibited the deficiency in carotenoid pigmentation, swimming motility, EPS production, HR in tobacco leaves and virulence in rice and onion. All phenotype deficiencies of $\Delta h f q$ mutant were recovered by genetic complementation with intact $h f q$ (pCOK335; pLAFR3::hfq). A small regulatory RNA (sRNA) SraH is known to bind Hfq and increase translation of sigma factor RpoS. $\triangle$ sraH mutant of P. ananatis exhibited deficiencies in EPS production, $\mathrm{HR}$ and virulence in rice and onion. All phenotypes deficiencies of $\triangle \mathrm{sraH}$ mutant were recovered by genetic complementation with intact $s r a H$ (pBS28; pLAFR3::sraH), indicating SraH controls pathogenicity of PA13. In the present study, we found an additional regulatory circuit that Hfq regulates quorum sensing (QS) of Pantoea ananatis. We showed that Hfq regulates the transcription of eanI (QS signal synthetic gene) and QS signal production. 
Understanding the Physiological and Molecular Components of Elicitor-induced Resistance in Tomato against Fusarium Wilt S. L. CARMONA JR., M. Gomez, D. Burbano, M. Soto-Suárez, Corporación Colombiana de Investigación Agropecuaria AGROSAVIA, Mosquera, Colombia

Fusarium oxysporum f. sp. lycopersici ( $F o l)$ is a major pathogen causing Fusarium wilt disease, an economically important disease of tomato crops (Solanum lycopersicum L.). In Colombia, there is a lack of knowledge about the Fusarium wilt, farmers use agrochemicals to control the disease and, in most cases, without knowledge. Plant resistance induction by treatment with elicitors such as Chitosan and Trichoderma spp., that improve the plant physiology, is a promising management strategy. The aim of this work was to evaluate the physiological and molecular response of elicitor-induced resistance in tomato against Fusarium wilt. A highly virulent Colombian isolate of Fol (pathogenic race 2) was obtained, morphologically and molecularly characterized. The effectiveness to control Fol of chitosan and Trichoderma spp. was evaluated using both in vitro and in planta approaches. Chitosan was effective controlling Fol, by inhibiting mycelial growth (in vitro) and also controlled $70 \%$ of the damage caused by the race 2 isolate (in planta). In addition, chitosan protected photosynthetic components such as Fv/Fm, Y(II) and pigments against the damage caused by Fol and induced resistance through PRla gene expression. This is the first study to describe Colombian Fol races, and the understanding of plant physiological and molecular alterations induced by fungal elicitors during pathogen attack might be helpful to develop better alternatives to control the Fusarium wilt disease in tomato.

Pseudomonas syringae evades phagocytosis by animalcells via type III effector-mediated regulation of actinfilament plasticity S. J. Yoon (1), Y. J. Park (1), J. S. Kim (1), S. Lee (1), C. M. RYU (1,2), (1) KRIBB, Daejeon, Korea, Republic of (South); (2) University of Science and Technology, Daejeon, Korea, Republic of (South)

Certain animal and plant pathogenic bacteria have developed virulence factors including effector proteins that enable them to overcome host immunity. A plant pathogen, Pseudomonas syringae pv. tomato (Pto) secretes a large repertoire of effectors via a type III secretory apparatus, thereby suppressing plant immunity. Here, we show that Pto causes sepsis in mice. Surprisingly, the effector HopQ1 disrupted animal

phagocytosis by inhibiting actin rearrangement via direct interaction with the LIMdomain of the animal target protein LIM kinase, a key regulator of actin polymerization. The results provide novel insight into animal host-plant pathogen interactions. In addition, the current study firstly demonstrates that certain plant pathogenic bacteria such as Pto evade phagocytosis by animal cells due to crosskingdom suppression of host immunity.

Uncovering the secrets of success of Phakopsora spp. on crop plants

M. LOEHRER (1), I. V. Primiano (2), L. Amorim (2), U. Schaffrath (1), (1) RWTH Aachen University, Aachen, Germany; (2) University of São Paulo (ESALQ), Piracicaba, Brazil

Asian soybean rust (Phakopsora pachyrhizi) is known as an aggressive plant pathogen on soybean and is responsible for considerable economic damage. Similar to Asian soybean rust, Asian grapevine leaf rust (Phakopsora meliosmae-myrianthae = Phakopsora euvitis) recently spread in South America and is able to cause severe rust epidemics on the perennial plant grapevine. Histopathological examinations and comparative growth kinetics on their respective hosts revealed the formation of satellite uredinia within lesions as an important source for secondary inoculum and illuminated the high risk of epidemic spreading for both pathogens. While for P. pachyrhizi, analysis of the genome and the effector repertoire already led to deeper insights into its infection strategy, there is not much known about the biology of $P$. euvitis. Our detailed knowledge of the Asian soybean rust fungus was used as a base for studying similarities and differences to the Asian grapevine leaf rust. Comparative genome analysis between both Phakopsora species and the bean rust fungus Uromyces appendiculatus uncovered genome size and degree of heterozygosity in the dikaryotic stage of these rusts. We present novel data on Asian soybean rust effectors and how this knowledge can be used to increase our understanding of the infection strategy of $P$. euvitis.

Genome analysis and effector repertoire of emerging Fusarium oxysporum pathogens J. P. CLARKSON (1), A. D. Armitage (2), S. Jenkins (1), H. J. Bates (2), A. C. Jackson (1), M. K. Sobczyk (2), L. Baxter (1), B. P. Greenfield (3), A. Taylor (1), F. M. Wilson (4), S. Ott (1), R. J. Harrison (2), (1) University of Warwick, U.K.; (2) NIAB EMR, East Malling, U.K.; (3) Swansea University, U.K.; (4) NIAB-EMR, East Malling, U.K.

Fusarium oxysporum $(\mathrm{Fo})$ is a soilborne pathogen of global significance that affects many horticultural crops. Host adaptation has resulted in a species complex of $>120$ 'formae speciales' (f. spp.). Furthermore, new races of $F o$ f. spp. continue to emerge in response to the deployment of single major gene plant resistance. We carried out comparative genomics of several $F o$ f. spp. including those affecting onion (f. sp. cepae), Narcissus (f. sp. narcissi; FON) and column stocks (f. sp. matthiolae) as well as different races affecting pea (f. sp. pisi; FOP) and lettuce (f. sp. lactucae; FOL). This allowed discrimination of highly conserved 'core' and asyntenous 'pathogen/lineage (LS) specific' regions of the genome while bioinformatic analysis identified distinct effector repertoires between the different $F o$ f. spp. and between races within FOP and FOL. Variation in effector complement was also apparent within FON although there is no known race structure. Putative novel effectors and homologs of secreted in xylem (SIX) genes previously described in other Fo f. spp. were also identified. Transcriptome sequencing and RT-PCR for selected $F o$ f. spp confirmed expression of effector candidates and SIX genes, allowing prioritisation for functional validation. Transposon content of LS regions provides a putative mechanism for evolution of new Fo f. spp. and races. This approach is particularly relevant to FOL race 4 which has recently emerged in The Netherlands, Belgium and the U.K.

Unraveling the genetic determinism of barley resistance to root lesion nematodes

H. PIDON (1), S. Shah (2), G. Capistrano-Goßmann (2), E. Laubach (3), N. Stein (1,4), C. Jung (2), (1) Leibniz Institute of Plant Genetics and Crop Plant Research (IPK), Gatersleben, Germany; (2) Univ of Kiel, Kiel, Germany; (3) Nordsaat Saatzucht GmbH, Langenstein, Germany; (4) Georg-August-Universität, Göttingen, Germany 
Root lesion nematodes (RLN) are important pests worldwide but, as underground pests, they are often neglected as causes of yield loss. However, there is increasing evidence that the species of the genus Pratylenchus, and in particular P. neglectus, are causing severe damage in cereal production. Amplified by early sowing dates and mild winters, those damages are expected to increase in the context of climate change. So far very few is known about barley resistance to RLN and the resolution of previous QTL studies is low. We are using two complementary approaches and state-of-the-art genotyping methods to identify the genetic determinism of $P$. neglectus resistance in barley. The first approach is a QTL mapping in a biparental population of 500 DH-lines between the susceptible accession Valentina and the resistant accession Beysehir. By genotyping the population by GBS, we retrieved a high density of markers that will allow us to achieve a very precise localization of the resistance QTLs of Beysehir. In a second approach, we performed a GWAS on a collection of 425 diverse barley accessions phenotyped for $P$. neglectus resistance. Using 44,441 high-quality SNPs and imputation of missing data, we identified three main QTLs that colocalize with QTLs identified in biparental populations. Detail analysis of the QTLs identified by those two approaches will soon give us candidates genes and allow us to unravel mechanisms of RLN resistance in barley.

Lipopolysaccharide-induced plant defense priming against Xylella fastidiosa in Vitis vinifera grapevines C. A. CASTRO (1), M. C. Roper (2), (1) University of California, Riverside, Riverside, CA, U.S.A.; (2) University of California, Riverside, CA, U.S.A.

Plants have developed complex mechanisms to defend themselves from constant biotic and abiotic challenges presented by a fluctuating environment. One of these mechanisms, called plant defense priming, is a tool that exploits plant 'memory' to counteract pathogens and abiotic stress. Microorganisms have signature molecules called microbe-associated molecular patterns (MAMPs) or pathogen-associated molecular patterns (PAMPs) that can act as stimuli to induce the primed state. This 'memory' allows plants to quickly recognize pathogens and activate strong immune responses that result in disease resistance or tolerance. Xylella fastidiosa $(X f)$ is a Gram-negative, xylem-limited bacterium that causes severe diseases in many economically important plants including grape, olive, and citrus. Lipopolysaccharide (LPS) covers most of the cell surface in Gram-negative bacteria and is a well-described PAMP that elicits defense responses in plants. In grapevines, $X f$ LPS-mediated elicitation of plant immunity leads to systemic and prolonged activation of defense pathways related to $X f$ recognition. The goal of this project was to explore the persistence and molecular mechanisms underlying the LPS-mediated plant defense priming phenomenon against $X f$. We demonstrate $X f$ LPS treatment primes the immune system and results in reduced symptom development and lower bacterial titer in grapevines inoculated with $X f$. Interestingly, an additional LPS booster dose enhances this reduced symptom phenotype.

Variation of barriers to geminivirus movement within vector body affects virus transmission

S. S. LIU, Institute of Insect Sciences, Zhejiang University, Hangzhou, China

Begomoviruses (genus Begomovirus; family Geminiviridae) are a large group of viruses with about 400 species recorded, many of which are major viral agents of crop diseases. These viruses are transmitted by whiteflies in a circulative manner. Following oral acquisition from virusinfected plants by whiteflies, the virus has to move along food canal, reach the esophagus/midgut, cross the midgut wall to reach the haemolymph, and then translocate into the primary salivary glands, from where the virus is egested with saliva into new plants for virus inoculation. The efficiency of a begomovirus to cross the barriers will determine the feasibility and speed of the virus being transmitted. In this presentation, case studies are analyzed to illustrate the complexity and variation of the virus' journey. Several scenarios occur: (1) a virus can be intercepted by the midgut wall or the salivary glands; (2) partially intercepted by midgut wall and partially by the salivary glands; (3) mainly intercepted by the midgut wall; (4) mainly intercepted by the salivary glands; and (5) moving smoothly all the way along the path to be eventually transmitted to a plant. These scenarios imply that potential receptors are located in the midgut wall or the salivary glands, the receptors may differ between species of whitefly, and even between tissues within the same whitefly. Understanding these receptors will likely offer new visions for the development of novel methods to block virus transmission.

Cladosporium fulvum Avr2 effector inhibits cysteine proteases secreted from its mycoparasite Dicyma pulvinata H. Sushida, Y. Higashi, Y. IIDA, National Agriculture and Food Research Organization, Japan

Most fungal pathogens secrete effector molecules that function as virulence factors to facilitate disease on host plants, but they are also recognized by cognate plant resistance proteins to arrest fungal growth. Avr2 effector of leaf mold pathogen Cladosporium fulvum encodes cysteine protease inhibitor and inhibits cysteine proteases Rcr3 and Pip1 of tomato during infection. The fungal mycoparasite Dicyma puvinata is known as a biocontrol agent that parasitizes $C$. fulvum. The draft genome of $D$. pulvinata revealed 2 cysteine proteases which have the signal peptides for secretion at the N-terminus. These cysteine proteases were strongly up-regulated when it is co-cultured with C. fulvum, and enzymatic activities were high at basic $\mathrm{pH}$ and very low at acidic $\mathrm{pH}$. Although Avr2 inhibits the activity of Rcr3 and Pip1 only at acidic pH, cysteine proteases from D. pulvinata were inhibited by Avr2 at basic $\mathrm{pH}$, suggesting that Avr2 is able to function at a wide $\mathrm{pH}$ range. These results suggest that fungal effector Avr2 is important not only in plant-pathogen interactions but also in fungal-fungal interactions. In other words, C. fulvum deploys the same effector to protect itself against both resistance response of plants and mycoparasitic invasion.

First report of Phomopsis fukushii causing stem cankers and sudden death of young apple trees in Canada P. A. ABBASI, S. Ali, W. Renderos, Agriculture and Agri-Food Canada, Kentville, NS, Canada

Apple nurseries in Annapolis Valley, Nova Scotia reported severe necrotic, stem canker symptoms and death of young apple trees. Those trees had been grafted on four different rootstocks EMLA 9, Supporter 4, M9-T337, and Pajam 2. To determine the causes of stem canker and death of young apple trees, pathogen isolations from the diseased tissue obtained from the infected rootstocks showing visible necrotic and canker symptoms were made on potato dextrose agar (PDA). Almost all isolations on PDA yielded a similar type of fungal colony. Based on the colony and spore morphology and presence of both $\alpha$ and $\beta$ spores, Phomopsis was identified as a potential pathogen. The identity of the pathogen was then confirmed as P. fukushii, based on sequence analysis of the ITS-5.8S region of rDNA and the translation elongation factor 1 alpha (EF1- $\alpha$ ) gene region of the representative fungal isolate. The pathogenicity of the $P$. fukushii isolate was confirmed on 1-year-old young apple trees 
grafted on four rootstocks in the greenhouse tests. Necrotic stem canker symptoms like those observed on the nursery-grown young apple rootstocks appeared on stems of all inoculated plants. The necrotic and canker lesions were shown within 3 days of inoculation, and death of the young trees was observed after 4 weeks. This is the first report of Phompsis causing stem canker and sudden death of young apple trees in Canada and also first report of $P$. fukushii occurring as a pathogen on any host in Canada.

Detection and identification of a Candidatus Liberibacter species from ash tree infesting psyllids

F. O. WAMONJE (1), T. Wist (2), S. Banniza (1), S. M. Prager (1), (1) Department of Plant Sciences, University of Saskatchewan, Saskatoon, SK, Canada; (2) Saskatoon Research and Development Centre, Agriculture and Agri-Food Canada, Saskatoon, SK, Canada

Candidatus Liberibacter species are best known for causing severe economically important diseases of Citrus and Potato. These pathogens are transmitted by sucking insects known as psyllids. Its detection in plants is complicated by its uneven distribution in host plants and largely unculturable nature. The death of black (Fraxinus nigra) and mancana (F. mandshurica) ash trees in parts of western Canada, including the provinces of Alberta and Saskatchewan, has been associated with infestation with the cottony ash psyllid (Psyllopsis discrepans). This insect is native to southern Europe and in recent times has spread to most of Europe and the United Kingdom, Canada and the United States. The psyllids cause dieback over time due to stunted growth of branches and a thinning canopy. However, how the insect actually kills the trees remained a mystery. We hypothesized that the death could be due to a psyllid-transmitted Liberibacter. We used a combination of conventional and quantitative TaqMan PCR using 16S rDNA-based TaqMan primer-probes specific for Liberibacter and Sanger sequencing to determine the presence of Liberibacter from psyllids collected from Ash trees in Saskatoon. Sanger sequencing generated two sequences (1058 and $1085 \mathrm{bp}$ long). A BLAST search showed 99-100\% sequence similarity to $C$. Lib. solanacearum (Genbank: KX197200) isolated from Nearctic psyllids (Bactericera maculipennis) of United States provenance. These results provide first evidence on the likely cause of Ash dieback in Saskatchewan.

Gene duplication and mutation in the emergence of a novel aggressive allele of the AVR-Pik effector in the rice blast fungus A. LONGYA (1), C. Chaipanya (1), M. Franceschetti (2), J. H. Maidment (2), M. J. Banfield (3), C. Jantasuriyarat (1), (1) Kasetsart University, Bangkok, Thailand; (2) John Innes Centre, Norwich, U.K.; (3) John Innes Centre, Norwich Research Park, Norwich, U.K.

Higher yield potential and greater yield stability are common targets for crop breeding programs, including those in rice. Despite these efforts, biotic and abiotic stresses continue to impact rice production. Rice blast disease, caused by Magnaporthe oryzae, is the most devastating disease affecting rice worldwide. In the field, resistant varieties are unstable and can become susceptible to disease within a few years of release due to the adaptive potential of the blast fungus, specifically in the effector (avirulence or $A V R$ ) gene pool. Here, we analyzed genetic variation of the effector gene $A V R-P i k$ in 58 rice blast isolates from Thailand and examined the interaction between AVR-Pik and the cognate rice resistance gene Pik. Our results reveal that Thai rice blast isolates are very diverse. We observe four $A V R-P i k$ variants in the population, including three previously identified variants, $A V R-P i k A, A V R-P i k D$, and $A V R-P i k E$, and one novel variant which we named $A V R-P i k F$. Interestingly, 28 of the isolates contained two copies of $A V R$-Pik, always in the combination of $A V R-P i k D$ and $A V R-P i k F$. Blast isolates expressing only AVR-PikF show high virulence to rice cultivars encoding allelic Pik resistance genes, and the AVR-PikF protein does not interact with the integrated HMA of the Pik resistance protein in vitro, suggesting a mechanism for immune evasion.

Host Genotype-dependent Interactions among Pathogenic and Commensal Pseudomonas Strains in A. thaliana O. SHALEV SKRIPTCHAK, T. Karasov, D. S. Lundberg, H. Ashkenazy, D. Weigel, Max Planck Institute for Developmental Biology, Tuebingen, Germany

Members of the genus Pseudomonas often dominate the phyllosphere of A. thaliana in the wild. A multi-year survey of Pseudomonas in local $A$. thaliana populations revealed co-colonization of numerous strains in the same population, and even within the same plant (Karasov et al., 2018). We are examining the interactions of such strains both with each other and with a panel of local $A$. thaliana genotypes, by infecting soil-grown $A$. thaliana accessions with three synthetic communities: pathogens only, commensals only (both defined based on single strain-plant interactions in vitro), and a mixture of both. Strains were genetically barcoded, allowing precise quantification of all strains. While infections with the pathogen community reduced the weight of all plant genotypes, infections with the mixture of pathogens and commensals gave a host genotype-dependent result; there was no weight reduction in any genotype but one. Pseudomonas strain abundance data implied that commensals suppressed all pathogenic strains except one. By associating bacterial composition with the plant weight, we managed to find that genotype-dependent control of the unsuppressed strain could explain differential commensal protection. Collectively, our results demonstrate the complexity of interactions among local Pseudomonas strains that co-occur in nature as well as the effect of the host genotype on these interactions. We are now investigating how which genes are responsible for host and bacterial effects.

Identification of candidate genes for resistance to lettuce downy mildew using Renseq k-mer association studies L. B. PARRA (1), M. J. Truco (1), K. Fletcher (1), R. W. Michelmore (2), (1) UC Davis- Genome center, Davis, CA, U.S.A.; (2) University of California, Davis, CA, U.S.A.

Downy mildew (DM), caused by Bremia lactucae, is the most important foliar disease of lettuce worldwide. The use of resistant varieties carrying dominant genes ( $D m$ genes) is the most effective method for controlling this disease; however, pathogen variability has led to the rapid defeat of individual resistance genes. Many resistances have been introgressed from wild species by repeated backcrosses to cultivated lettuce resulting in numerous near-isogenic lines (NILs). Segregating populations derived from these NILs were used to genetically map resistance genes against several Bremia isolates. Most of the resistances were conferred by single dominant genes that were mapped to individual loci in the lettuce reference genome. Resistance gene enrichment sequencing (RenSeq) allowed the identification of numerous NBS-LRR sequences from these NILs and from multiple lettuce cultivars carrying known $D m$ genes. These, in combination with phenotypic data from these lines was used for Kmer-association mapping, which allowed the identification of several candidate genes for known and new Dm genes. Knowledge of resistance genes at the sequence level provides the foundation for engineering resistance cultivars carrying multiple $D m$ genes by using genome editing leading to more durable resistance to multiple isolates of lettuce DM. 
Phase variation in rice pathogen Xanthomonas oryzae pv. oryzae

V. N. MADHAVAN (1), H. K. Patel (1), P. B. Patil (2), R. V. Sonti (3), (1) CSIR-Centre for Cellular and Molecular Biology (CSIR-CCMB), Hyderabad, India; (2) CSIR - Institute of Microbial Technology (CSIR-IMTECH), Chandigarh, India; (3) National Institute of Plant Genome Research (NIPGR), New Delhi, India

Xanthomonas oryzae pv. oryzae (Xoo) is the causal pathogen of bacterial blight of rice. Diverse factors contribute to its virulence, e.g., secreted enzymes, protein involved in adhesion, gene regulation. Two such studied virulence factors are Exopolysaccharide (EPS) and Lipopolysaccharide (LPS). LPS is a structural component of the outer membrane of all gram-negative bacteria. LPS is necessary for the function of many outer membrane proteins and protect the bacteria from antimicrobial plant products. EPS is a complex polymer secreted by Xanthomonas genus and the known functions of EPS include biofilm formation, and suppression of the plant immune responses. In this study, we isolated non-mucoid and virulence compromised Xoo colonies from stationary phase cultures. These spontaneous mutants are called as stationary phase variants (SPV). Our data show that, SPVs arises due to insertion of endogenous Insertion Sequence (IS) elements in EPS or LPS O-antigen biosynthetic clusters or by slipped-strand mispairing (SSM) in wxoA gene of LPS O-antigen biosynthetic cluster. The SPVs reverts to wild-type colony morphology and showed true reversion, i.e. restoration of wild-type genotype. The results suggest that we are observing phase variation in $X o o$. We hypothesize that similar phase variation may be a part of Xoo's life cycle. Which may help the bacteria survive in nutrient limited conditions during late stages of infection and on rice seeds and plant debris.

External RNA uptake by Cercospora zeina and Exserohilum turcicum and the possible development of RNA fungicides against maize yield limiting pathogens

I. MARAIS (1), J. Theron (2), B. G. Crampton (3), D. K. Berger (4), (1) Dept. Plant \& Soil Sciences, FABI, University of Pretoria, South Africa; (2) Dept. Biochemistry, Genetics \& Microbiology, University of Pretoria, South Africa; (3) Forestry \& Agricultural Biotechnology Institute (FABI), University of Pretoria, South Africa; (4) Dept. Plant \& Soil Sciences, FABI, University of Pretoria, Pretoria, South Africa

External RNA uptake and silencing has previously been shown against Botrytis cinerea and Fusarium graminearum. We aimed to test this novel approach in two foliar pathogens Cercospora zeina and Exserohilum turcicum, that cause the maize foliar diseases grey leaf spot and northern corn leaf blight, respectively. The uptake of RNA and the knockdown of complementary GFP sequences in the $C$. zeina fungus were evaluated, as well as RNA uptake by E. turcicum. Fluorescently labelled dsRNA was created which was complementary to GFP gene regions in a GFPtransformed $C$. zeina isolate. Confocal microscopy was used to observe the uptake of externally applied fluorescently labelled RNAs into nontransgenic $C$. zeina mycelia, conidia and protoplasts, as well as E. turcicum mycelia. RNA uptake was observed in all the fungal growth forms. Transgenic $C$. zeina mycelia, conidia and protoplasts expressing the GFP gene were inoculated with non-fluorescently labelled RNAs, and GFP fluorescence was observed using confocal microscopy. GFP fluorescence in RNA treated samples was reduced by $35 \%$ on average as quantified by confocal imaging and fluorescence assays. The results obtained from this study form the basis to further develop novel RNA-based fungicides.

Generation and characterization of tomato lines with CRISPR/Cas9-mediated mutations in 150 immunity-associated genes N. ZHANG (1), H. M. Roberts (1), Y. Zheng (1), Z. Fei (1), G. B. Martin (1,2), (1) Boyce Thompson Institute, Ithaca, NY, U.S.A.; (2) School of Integrative Plant Science, Cornell University, Ithaca, NY, U.S.A.

CRISPR/Cas9 is a powerful tool for making precise gene knockouts in plants. To better understand the mechanisms involved in plant-pathogen interactions, we are developing 150 tomato lines with CRISPR-mediated mutations in immunity-associated genes and characterizing them for their responses to the bacterial pathogen Pseudomonas syringae pv. tomato. To increase the mutation efficiency and minimize transformation attempts, we evaluated guide-RNA efficiency by Agrobacterium-mediated transient expression in tomato leaves and conducted tomato transformation with Agrobacterium pools containing various gRNA constructs targeting different genes. Currently, more than 103 independent tomato mutant lines have been developed, most of which have one mutated gene while others have mutations in two or three targeted genes. Several mutants showed enhanced or reduced disease symptoms compared to wildtype plants when inoculated with various $P$. syringae strains, indicating the mutated gene(s) play a positive or negative role in tomato immunity. Additionally, we have developed a Plant Genome Editing Database (PGED; http://plantcrispr.org/), a central repository for managing plant mutant data and a platform for sharing mutant lines with the research community. Taken together, the generation and characterization of CRIPSR-mediated tomato mutants provides a powerful platform for understanding the molecular basis of the plant immune system and for improving disease resistance in plants.

Legume nodules: Are they new niches or/and traps for pathogens?

C. BENEZECH (1), A. Le scornet (1), J. George (2), F. Berrabah (2), T. Badet (1), M. F. Jardinaud (1), P. Ratet (2), F. Vailleau (1), S. Raffaele (1), B. Gourion (1), (1) Laboratory of Plant-Microbe Interactions, Toulouse, France; (2) IPS2, Paris, France

Thanks to the nitrogen they supply to the plants, rhizobium-legume interactions are often placed on a pedestal. However, the plant tolerance to an enormous rhizobium colonization raises the question of potential negative consequences associated with these symbiosis. We initiated experiments to evaluate whether nodules could represent infection sites for pathogens. We set up two tripartite interaction systems involving Medicago truncatula nodulated by Sinorhizobium medicae and two distinct pathogens: the bacterium Ralstonia solanacaearum or the fungus Sclerotinia sclerotiorum. Our studies indicate that $M$. truncatula, pea and soybean nodules are vulnerable to pathogens. We observed that $S$. sclerotiorum development is more important on functional nodules than on root segments and on nonfunctional nodules. Nodules also serve as gate for R. solanacearum to enter into the plant. Nevertheless, dissemination outside the nodules is hampered suggesting the existence of a diffusion barrier separating symbiotic and non-symbiotic organs or/and a particular tropism for the nodules. Our transcriptomic analyses showed that, when facing pathogens, both nodules and roots can develop responses. However, these responses are organ specific. Finally, we are currently estimating whether symbiosis has associated drawbacks at the plant scale: we are using our systems to assess the influence of nodulation and of nitrogen fixation on the sensitivity to pathogens. 
Induced and primed defence responses of Fragaria vesca against grey mold

R. BADMI (1), Y. Zhang (1), T. Tengs (1), M. B. Brurberg (1), P. Krokene (1), C. G. Fossdal (1), T. Hytonen (2), T. Thorstensen (1), (1) Biotechnology and Plant Health, Norwegian Institute of Bioeconomy Research, Ås, Norway; (2) Department of Agricultural Sciences, University of Helsinki, Helsinki, Finland

Plants are exposed to various pathogens in their environment and have developed immune systems with multiple defense layers to prevent infections. However, often pathogens overcome these resistance barriers, infect plants and cause disease. Pathogens that cause diseases on economically important crop plants incur huge losses to the agriculture industry. For example, the 2016 outbreak of strawberry grey mold (Botrytis cinerea) in Norway caused up to $95 \%$ crop losses. Such outbreaks underline the importance of developing novel and sustainable tools to combat plant diseases, for example by increasing the plants' natural disease resistance. Priming plant defenses using chemical elicitors may enhance resistance against multiple pathogens. Such an approach may reduce the use of chemical fungicides and pesticides that often select for resistant strains of pests and pathogens. My presentation will focus on the effectiveness of different chemical agents to prime woodland strawberry (Fragaria vesca) defenses against the necrotroph B. cinerea. We have identified several genes that seem to play a role in disease resistance in strawberry and associated epigenetic memory mechanisms. Our results point out new management avenues for more sustainable crop protection schemes.

Engineering synthetic plant-microbe signal communication in the rhizosphere

P. PARAMASIVAN (1), B. Geddes (2), P. S. Poole (2), G. Oldroyd (1), (1) Sainsbury Laboratory, Cambridge, U.K.; (2) University of Oxford, U.K.

The rhizosphere microbiome influences host plant growth and development in the natural environment. Plants can shape their root microbiomes through root exudates and by trans-kingdom signaling. Three types of signaling mechanisms are reported in the plant rhizosphere: inter and intraspecies signaling among microbes, signaling from host plant to microbes and signaling from microbes to host plant. We aim to engineer synthetic plant-microbe signaling in the rhizosphere to control gene expression in rhizobacteria for delivery of beneficial traits to plants. I will be presenting the plans for testing biosynthetic pathway of a synthetic molecule in transient systems as well as engineering biosensor strains to test cross-kingdom signaling in transient root transformation systems for establishing synthetic trans-kingdom signaling in the cereal rhizosphere.

Deciphering Arabidopsis thaliana responses to bacterial virulence factors of Ralstonia solanacearum through the study of natural variation of both biotic partners

N. S. RAZAVI EBRAHIMI, F. Lonjon, B. Gomes Ribeiro, H. Desaint, M. Turner, S. Genin, F. Roux, R. Berthomé, F. Vailleau, LIPM, Université de Toulouse, INRA, CNRS, INPT, Castanet-Tolosan, France

Ralstonia solanacearum species complex (RSSC), the causal agent of bacterial wilt, is one of the most harmful bacterial diseases, attacking more than 200 plant species. The main pathogenicity determinant of the RSSC is its type III secretion system, a molecular syringe that injects more than 70 bacterial type III effectors (T3Es) directly into the plant cell. These T3Es will target plant proteins to suppress plant innate immunity, or to directly provide nutrients to the bacteria. In this context, my thesis project aims at identifying Arabidopsis thaliana plant proteins targeted, directly or indirectly, by T3Es using Genome-wide association (GWA) mapping. GWA is a powerful method to map genomic regions associated to a phenotypic natural variation with a high mapping resolution. First, we have looked for association peaks for the plant responses to T3Es through the analysis of A. thaliana accessions after R. solanacearum mutant inoculation. Several plant candidate genes have been identified and are under functional characterization. Secondly, we will study the natural diversity of plant responses to one T3E coming from different bacterial backgrounds. With this project, we want to bring information on the evolving interacting genetic determinants of both organisms, i.e. TEs and the plant proteins they target, to try to identify novel resistance mechanisms, potentially more sustainable.

\section{Understanding microbial life in total controlled environment agriculture: A knowledge transfer partnership} E. ERSKINE, N. Holden ${ }^{1}$. F. Greig James Hutton Institute, U.K.

The total controlled environment agriculture (TCEA) developed by Intelligent Growth Solutions is a specialised form of indoor vertical horticulture, enabling real-time sensing and modification of the plant growth environment through LED light cycling, nutrient delivery and climate control. The TCEA system maximises production and minimises waste, growing crops year-round in optimised conditions, with a closed irrigation system to save water and internet-of-things communication to decrease labour costs.

We are currently working to understand microbial life within TCEA; identifying and tracking pathogens, developing risk management strategies, and characterising the plant-associated microbiome. The primary concern is human health, so we are undertaking hazard identification via molecular detection of pathogens such as E. coli and Salmonella for use in risk analyses. We are also interested in the utilisation of plant-growth promoting rhizobacteria and the use of biocontrol in plant pathogen competition in the TCEA context.

The goal of the project is the transfer of academic knowledge at the James Hutton Institute to the industrial partner (Intelligent Growth Solutions). This partnership encourages collaboration and presents unique opportunities to pursue questions in food safety, water systems, plant production, and microbial community analysis.

Genetic bases of natural variation of plant response to Ralstonia solanacearum under elevated temperature conditions N. AOUN (1), L. Tauleigne (2), H. Desaint (3), F. Vailleau (4), L. Deslandes (5), F. Roux (3), R. Berthomé (3), (1) INRA Occitanie-Toulouse, Toulouse, France; (2) LIPM, Institut National de la Recherche Agronomique, Castanet-Tolosan, France; (3) LIPM, Université de Toulouse, INRA, CNRS, INPT, Castanet-Tolosan, France; (4) Laboratory of Plant-Microbe Interactions, Toulouse, France; (5) Université de Toulouse, LIPM, CNRS-INRA, Toulouse, France

In the context of climate warming, plants are threatened by an increased risk of epidemics and the emergence of highly aggressive pathogen species. Studies showed that an increase of temperature alters most plant resistances. However, the underlying molecular mechanisms are poorly 
understood. Our project aims to identify and characterize the genetic bases of resistance mechanisms remaining efficient at elevated temperature to the phytopathogenic bacterium Ralstonia solanacearum. To uncover quantitative trait loci (QTLs) associated with the natural variation of response to $R$. solanacearum at $30^{\circ} \mathrm{C}$ and to identify resistance mechanisms remaining efficient at elevated temperatures, we adopted a genome wide association (GWA) mapping approach using worldwide and local natural collections of Arabidopsis thaliana. From the worldwide collection, we identified a totally resistant accession to $R$. solanacearum GMI1000 reference strain and the genetic bases of this resistance are under investigation. Also, GWA mapping revealed a polygenic architecture involving several QTLs underlying the response to GMI1000. One of these QTLs involves two genes encoding for strictosidine synthase-like proteins (SSL) in early resistance to $R$. solanacearum at $30^{\circ} \mathrm{C}$. For the local collection, playful dynamics of several QTLs were monitored during disease progress. The functional validation of several candidates known to be involved in plant defense responses such as JAZ-9 or a NLR protein is in progress.

Burkholderia gladioli strain NGJ1 deploys a prophage tail-like protein to feed on fungi

D. M. SWAIN (1), S. Yadav (1), I. Tyagi (2), R. Kumar (2), R. Kumar (2), S. Ghosh (2), J. Das (2), G. Jha (3), (1) NATIONAL INSTITUTE OF PLANT GENOME RESEARCH, NEW DELHI, India; (2) NATIONAL INSTITUTE OF PLANT GENOME RESEARCH, India; (3) National Institute of Plant Genome Research, India

Fungal pathogens are responsible for approximately two third of the infectious plant diseases. Due to extensive usages, pathogens have evolved resistance against most of the commonly used fungicides. Rice sheath blight disease caused by Rhizoctonia solani remains a serious threat to global sustainable agriculture. The pathogen has a complex biology and has been reported occurring world-wide causing necrosis and dampingoff on numerous host plant species. The pathogen is polymorphic and despite extensive efforts, till today no source of complete disease resistance has been identified against this pathogen. We have recently discovered a novel bacterium Burkholderia gladioli strain NGJ1 which has broad spectrum mycophagus ability. We also demonstrated that the bacteria NGJ1 deploys a prophage tail like protein (Bg_9562) to feed over fungi in a T3SS dependant manner. Moreover we observed that Bg_9562 protein has a broad spectrum antifungal activity on Rhizoctonia solani as well as several other phytopathogenic fungi. Furthermore, being equipped to kill and feed upon fungi, the mycophagous bacteria can serve as an experimental tool box to facilitate discovery of novel anti-fungal molecules. This opens up a new biotechnological application of this prophage tail like protein in controlling fungal diseases in rice as well as in other plants.

\section{Terpene synthases in Trichoderma gamsii T6085}

I. V. MUÑOZ, University of Pisa, Pisa, Italy

Secondary metabolites (SM) play a crucial role in the interaction between fungi and the environment, sometimes acting as signals inducing physiological responses in plants, or as antibiotics and mycotoxins to constrain the growth of their competitors. The beneficial isolate T6085 of Trichoderma gamsii is an effective biocontrol agent, with demonstrated activity against Fusarium graminearum, one of the most aggressive causal agents of Fusarium Head Blight on wheat. The genome sequence of 31 Trichoderma strains were analysed and screened with antiSMASH 3.0, which predicts gene clusters involved in SM biosynthesis. The genome of T. gamsii T6085 harbours 11 putative terpene synthase-encoding genes, seven of which have been predicted to belong to SM clusters. Interestingly, one of these was predicted to encode a trichodiene synthase (TRI5), which has only been described in the trichotecene-producer species of the Trichoderma brevicompactum clade. A comparative genomic approach has been carried out to characterize terpene synthase genes. In addition, expression patterns of T6085 TS encoding genes have been assessed in several induction media and during T6085/wheat, and T6085/wheat/F. graminearum interactions, in order to decipher their putative function.

Analysis of small RNA silencing in the interactions between wheat and an exclusively apoplastic fungal pathogen Zymoseptoria tritici G. J. Kettles (1), B. J. Hofinger (1), P. Hu (2), C. Bayon (1), J. Rudd (1), D. Balmer (3), M. Courbot (3), K. Hammond-Kosack (1), G. Scalliet (3), K. KANYUKA (4), (1) Rothamsted Research, Harpenden, U.K.; (2) Syngenta Biotechnology Inc., Research Triangle Park, NC, U.S.A.; (3) Syngenta Crop Protection AG, Stein, Switzerland; (4) Department of Biointeractions and Crop Protection, Rothamsted Research, Harpenden, U.K.

Cross-kingdom small RNA (sRNA) mediated silencing (or RNAi) has recently been shown to occur in several plant-pathogen interactions. However, it remains unknown how universal this phenomenon is, and in particular whether RNAi facilitates colonization of plants by fungal pathogens that invade extracellular spaces and do not form specialized feeding structures or penetrate host cells either during entire life cycle or during prolonged initial phases of infection. One such pathogen is Zymoseptoria tritici, which causes Septoria tritici blotch - a serious foliar disease of wheat. In this study we defined the global sRNA populations in Z. tritici and computationally predicted their potential wheat mRNA targets. However, molecular approaches failed to validate targeting of selected wheat mRNAs by fungal sRNAs, and bioassays using newly generated $Z$. tritici mutant strains carrying $D C L$ and $A G O$ gene deletions suggested that these key components of RNAi are dispensable for full infection of wheat. Moreover, we demonstrated that $Z$. tritici is incapable of environmental dsRNA uptake, and neither in vitro nor in planta application of dsRNAs in a virus-mediated Host-induced gene silencing procedure was effective in preventing fungal growth or disease. Collectively, our data suggest that RNAi approaches for gene function analyses in Z. tritici and potentially also as a disease control measure may not be as effective as has been demonstrated for some other plant pathogenic fungi.

Mutual potentiation of immune mechanisms triggered by cell surface and intracellular receptors

P. M. NGOU, P. Ding, J. D. Jones, The Sainsbury Laboratory, University of East Anglia, Norwich Research Park, Norwich, U.K.

Pathogens secrete effectors to facilitate infection in plants. Intracellular NLR immune receptor proteins enable plants to detect effectors and activate an immune response known as the effector-triggered immunity (ETI). The hypersensitive response (HR), accompanied by cell death and tissue collapse, is one of the hallmarks of ETI. Estradiol-induced expression of AvrRps4 and AvrRPP4, recognised by the TIR-NLRs (NLRs with Toll/Interleukin-1 receptor/Resistance domain) RPS4/RRS1 and RPP4, does not elicit HR in Arabidopsis. Whereas, ETI induced by the CCNLRs (NLRs with coiled-coil domain), such as RPM1, RPS2 and RPS5, can directly trigger HR. We observed a potentiation of ETI-induced HR 
through activation of cell surface pattern recognition receptors (PRRs) by either Pseudomonas or pathogen-associated molecular patterns (PAMPs). Potentiation of ETI-induced HR by pattern-triggered immunity (PTI) requires activation of mitogen-activated protein kinases (MAPKs). On the other hand, we observed elevated transcript and protein accumulations of multiple PTI signaling components during ETI. In addition, ETI activation leads to more robust PTI responses such as stronger ROS burst and prolonged MAPKs activation. Thus, the robustness of plant innate immunity involves a mechanism of mutual potentiation between two distinct immune systems.

Searching for host resistance: Studying early potato stem response to Dickeya inoculation via RNA-seq Y. LIU (1), K. Bao (2), Z. Fei (3), M. J. Filiatrault (4), (1) Cornell University, U.S.A.; (2) Boyce Thompson Institute, U.S.A.; (3) Boyce Thompson Institute, Ithaca, NY, U.S.A.; (4) USDA ARS, Ithaca, NY, U.S.A.

Dickeya spp. are necrotrophic bacterial pathogens that can cause blackleg disease on potatoes. The blackleg disease has resulted in significant economic losses in the United States and continues to devastate the potato industry. Breeding for resistance to blackleg in commercial potato cultivars is difficult due to the limited knowledge of the host-microbe interactions in this pathosystem. We conducted global transcriptomic analysis to understand the molecular interactions between Dickeya dadantii 3937 and susceptible or tolerant diploid potatoes. One-month old potato stems were inoculated with $D$. dadantii suspension or mock buffer and stem tissues were harvested at 0 and 12 -hour postinoculation (hpi). RNAs from two biological replicates per potato line per time point were subjected to RNA sequencing and subsequent computational analyses. Overall, 1328 differentially expressed genes (DEGs) were detected at 12 hpi when comparing susceptible and tolerant plants. Of those 1328 genes, 512 DEGs were unique to infected plants vs mock inoculated. Gene ontology and cluster analyses revealed different enriched terms and different gene expression patterns when comparing the expression profiles between tolerant and susceptible potatoes. Our study of potato global gene expression patterns will provide critical information to facilitate the development of novel disease management strategies and accelerate disease-resistance potato breeding processes.

Suppression of bacterial biofilm formation contributes to PAMP-triggered immunity in the Arabidopsis-Pseudomonas syringae pv. tomato interaction

R. K. CAMERON, A. B. Fufeng, N. W. Xiao, G. M. Nunn, A. Halim, McMaster University, Hamilton, ON, Canada

Biofilms consist of bacterial cells and associated extracellular poylsaccharides (EPS), DNA, lipids and proteins. Biofilms are thought to protect bacterial pathogens from plant defense responses. Using Pseudomonas syringae pv. tomato (Pst) and Arabidopsis, we investigated the role of biofilms in virulence by asking: Does biofilm formation contribute to Pst success and does the PAMP-Triggered Immunity (PTI) response limit biofilm formation in an SA-dependent manner? PTI was induced by flg22 treatment in wild-type Col-0, fls2 (PTI mutant) and sid2-2 (SA biosynthesis mutant). In vivo bacterial aggregate formation was monitored in leaves using Pst DC3000 PDSK-GFPuv and epifluorescence microscopy. Pseudomonas aggregate occurrence was positively correlated with bacterial success in $f l s 2$ and sid2-2, while fewer and smaller aggregates were observed during PTI providing evidence that Pst biofilm formation is suppressed in an SA-dependent manner during PTI. To determine if bacterial aggregates are biofilms, the importance of two common biofilm components was examined. Aggregate formation was similar in alginate-deficient and wild-type Pst-GFP suggesting alginate (EPS) is not a major component of Pst biofilms. Extracellular (e)DNA was observed at sites of Pst-GFP aggregate formation suggesting that eDNA is a component of Pst biofilms. These studies indicate that biofilm formation is important for Pst virulence and suppression of biofilm formation is a key component of PTI.

\section{The Rice CYP78A Gene BSR2 Confers Resistance to Rhizoctonia solani and Affects Seed Size and Growth Speed in Arabidopsis and Rice \\ S. Maeda (1), J. G. Dubouzet (1), Y. Kondou (2), K. Oda (3), M. Matsui (2), H. Hirochika (1), M. MORI (1), (1) NIAS, Tsukuba, Japan; (2) RIKEN Yokohama, Japan; (3) RIBS Okayama, Japan}

The fungal pathogen Rhizoctonia solani causes devastating diseases in hundreds of plant species. Among these, R. solani causes sheath blight, one of the three major diseases in rice. To date, few genes have been reported that confer resistance to $R$. solani. Here, rice-FOX Arabidopsis lines identified as having resistance to a bacterial pathogen, Pseudomonas syringae pv. tomato DC3000, and a fungal pathogen, Colletotrichum higginsianum were screened for disease resistance to R. solani. BROAD-SPECTRUM RESISTANCE2 (BSR2), a gene encoding an uncharacterized cytochrome P450 protein belonging to the CYP78A family, conferred strong resistance to R. solani in Arabidopsis. When overexpressed in rice, $B S R 2$ also conferred resistance to two $R$. solani anastomosis groups. Both Arabidopsis and rice plants overexpressing $B S R 2$ had slower growth and produced longer seeds than wild-type control plants. In contrast, $B S R 2$-knockdown rice plants were more susceptible to $R$. solani and displayed faster growth and shorter seeds in comparison with the wild-type control. These results indicate that BSR2 is associated with disease resistance, growth rate (plastochron) and seed size in rice and suggest that its function is evolutionarily conserved in both monocots and dicots.

Decipher regulation mechanism and evolutionary process of a pair of $R$ proteins Pit-1 and Pit-2 Y. LI, Y. Kawano, Shanghai Center for Plant Stress Biology, Chinese Academy of Sciences, China

Plants have evolved resistance (R) proteins to recognize cognate avirulence (Avr) proteins (effectors) that are secreted into plant cells from phytopathogens. However, exact mechanisms of how R proteins execute their functions through detecting pathogen effectors and triggering downstream immune responses still remain poorly understood. Previously, we have found that small GTPase OsRac1 acts as a molecular switch, working downstream of an R protein for rice blast fungus, Pit-1. Here, we found that although Pit-1 induced cell death, Pit-2 does not have this activity but it suppresses Pit-1-induced cell death and immunity. Pit-1 and Pit-2 have ability to form homocomplexes by themselves but Pit-2 suppressed the homocomplex formation of Pit-1, resulting in promoting the heterocomplex between Pit-1 and Pit-2. Pit-2 prevented the plasma membrane localization of Pit-1 and captured it in the cytosol to suppress Pit-1-induced immunity. We tried to identify important residues to produce the functional differences between Pit-1 and Pit-2, and found three residues which are important for Pit-1 activities. Interestingly, only these three amino acids exchange can convert functions between Pit-1 and Pit-2. We also solved the evolutionary process of a pair of $R$ genes Pit- 
1 and Pit-2. Taken together, these findings suggest that Pit-1 works with Pit-2 by forming the hetero-oligomer to trigger disease resistance in rice, and we shed light on the evolutionary process of pairs of R proteins.

Combinatorial biosynthesis of desmethyl-lasiodiplodin and its derivatives for new nematicide development Q. Yue (1), R. Zhang (1), I. Molnár (2), Y. XU (1), (1) Biotechnology Research Institute, Chinese Academy of Agricultural Sciences, Beijing, China; (2) Southwest Center for Natural Products Research, University of Arizona, Tucson, AZ, U.S.A.

Nematode infections of plants seriously endanger agricultural and forestry productivity. However, only a few chemical nematicides gained market authorization, and these still show liabilities in animal and environmental toxicity. Demand for new green nematicides is increasing. Desmethyl-lasiodiplodin (DLD) displayed significant nematocidal activity in our previous study, but its poor water solubility limits its application in nematode control. Glycosylation is a prominent strategy to modulate the solubility, stability, and bioactivity of compounds. Here, we reconstituted the production of DLD by expression of Lasiodiplodia theobromae PKSs (LtLasS1 and LtLasS2) in heterologous host Saccharomyces cerevisiae. Methylglucosylated and glucosylated DLD were produced by S. cerevisiae cotransformed with LtLasS1, LtLasS2, $B b G T 86$ and BbMT85. BbGT86 and BbMT85 are glycosyltransferase and methyltransferase, respectively, and form a methylglucosylation functional module in Beauveria bassiana. The nematocidal activity of the compounds was tested against second instar larvae of Caenorhabditis elegans. After treatment with $0.5 \mathrm{mg} / \mathrm{mL}$ of DLD, DLD 3-O-glucoside and DLD 4'-O-methyl-3-O-glucopyranoside for 24 hours, the corrected mortality rate of C. elegans $(\%)$ were $27.47 \%, 17.09$ and 13.65 , and the $\mathrm{EC}_{50}$ were $1.10 \mathrm{mg} / \mathrm{mL}, 2.89 \mathrm{mg} / \mathrm{mL}, 6.20 \mathrm{mg} / \mathrm{mL}$, respectively. Thus, improved solubility does not correlate to increased bioactivity, providing an important lesson for new nematicide development.

Broad-spectrum fungal protection in transgenic Arabidopsis by the overexpression of an acyl-CoA-binding protein S. P. Narayanan (1), P. Liao (1), P. W. J. Taylor (2), C. Lo (1), M. L. CHYE (1), (1) School of Biological Sciences, University of Hong Kong, Pokfulam, Hong Kong; (2) Faculty of Veterinary and Agricultural Sciences, University of Melbourne, Australia

Lipids have many diverse roles. They are involved in the formation of cellular membranes, storage compounds, signaling molecules and defense components. Acyl-CoA-binding proteins (ACBPs) contain an acyl-CoA-binding domain which facilitates binding to lipids such as acyl-CoA esters. The ACBPs from plants, mammals and the nematode, Caenorhabditis elegans, have been reported to participate in plant development and stress responses. $A C B P s$ such as the Arabidopsis $A C B P 3$ (AtACBP3) and rice $A C B P 5$ (OsACBP5) were induced by pathogen infection. AtACBP3 was induced by both bacterial (Pseudomonas syringae pv tomato DC3000) and fungal (Botrytis cinerea) pathogens and transgenic Arabidopsis AtACBP3-overexpressors (OEs) were protected against $P$. syringae but not $B$. cinerea. OsACBP5, was upregulated upon infection by the rice fungal pathogen Magnaporthe grisea. In this study, transgenic Arabidopsis plants overexpressing OsACBP5 (OsACBP5-OEs) showed protection against necrotrophic, hemibiotrophic and biotrophic phytopathogens. Proteomic analysis of the changes in protein expression in OsACBP5-OEs infected with the fungal necrotroph Rhizoctonia solani will be presented. A better understanding on the role of OsACBP5 in plant-microbe interactions is expected to lead to its application in the genetic engineering of crop plants in agriculture.

Funded by the Research Grants Council of Hong Kong [HKU17109917M]

Regulation of fungal effector gene expression during infection through chromatin dynamics M. KRAMER (1), D. E. Cook (2), M. F. Seidl (1), B. P. H. J. Thomma (1), (1) Wageningen University, Wageningen, Netherlands; (2) Kansas State University, Manhattan, KS, U.S.A.

Fungal plant pathogens require tight control over the expression of their effector genes, which encode secreted proteins that facilitate host invasion. Effector genes are generally induced upon infection and failure to express these genes at the appropriate time or location may result in failure of the infection. Epigenetic mechanisms may regulate the expression of effector genes that reside in secluded genomic regions through the dynamic folding of DNA from restrictive heterochromatin into permissive euchromatin. Besides induction of effector genes upon infection, differential growth conditions differentially induce effector gene expression, which is likely similarly regulated through dynamic chromatin folding. We use ChIP-sequencing to isolate restrictive and permissive chromatin of the broad host-range fungus Verticillium dahliae grown under various conditions. We show that effector gene-containing regions are enriched for the heterochromatin-associated tri-methylation of lysine 27 on histone 3 (H3K27me3) under all tested conditions, and that deletion of the H3K27me3-deploying enzyme leads to the induction of a subset of effector genes. However, no obvious differential H3K27me3 patterns are observed upon growth of the fungus under different conditions. This indicates that, although $\mathrm{H} 3 \mathrm{~K} 27 \mathrm{me} 3$ is involved in repressing transcription of effectors, dynamics in H3K27me3 levels does not appear to drive effector gene expression under the conditions tested using $V$. dahliae.

\section{Involvement of jasmonates signaling pathway in watermelon-Fusarium oxysporum f. sp. niveum interactions}

P. F. L. CHANG (1), H. T. Lin (2), T. H. Chang (3), Y. H. Lin (4), K. S. Chen (5), J. W. Huang (1), (1) IDCSA Center and Department of Plant Pathology, National Chung Hsing University, Taiwan; (2) Department of Plant Pathology, National Chung Hsing University, Taiwan; (3) IDCSA Center and Department of Plant Pathology, National Chung Hsing University, Taichung, Taiwan; (4) Dept. of Plant Medicine, National Pingtung University of Science and Technology, Pingtung, Taiwan; (5) FTHEB, Taiwan Agricultural Research Institute, COA, Kaohsiung, Taiwan

Fusarium oxysporum f. sp. niveum (Fon) is one of the limiting factors for watermelon production. Uncovering the defense mechanisms provides new insight in controlling watermelon $F u$ sarium wilt. Differentially expressed genes (DEGs) related to watermelon hormone pathways were obtained from transcriptome profiling of watermelon-Fon interaction. The expression of hormone pathway-related genes was analyzed by quantitative real time polymerase chain reaction (qRT-PCR) to understand hormone regulation in the shoot base of resistant (JSB) and susceptible (Sugar Baby, SB) watermelon lines during early infection with Fon. The results suggest that salicylic acid and ethylene pathways might not involve in the defense response in JSB shoot base during early infection of Fon, whereas jasmonate (JA) biosynthesis pathway was significantly activated in the Fon-susceptible SB. Activating JA signaling pathway triggers JA-dependent defense responses. However, over-activated defense responses may consume excessive energy and break the hormone homeostasis thus promoting the susceptibility of watermelon to Fon. Furthermore, in early watermelon-Fon interaction, the expression two pathogenesis-related protein 5 (PR5) genes, the thaumatin-like protein 
genes (TLPS), was significantly higher in JSB than in SB. It suggested that induction of watermelon TLPs might involve in JSB resistance against Fon. The work was funded in part by MOST, Executive Yuan, Taiwan, R.O.C. and IDCSA, NCHU, Taiwan, R.O.C.

The surfactin and fengycin played an important role in biological ability of Bacillus subtilis NCD-2 against cotton damping-off P. MA, Q. Guo, L. Dong, S. Li, Plant Protection Institute, Hebei Academy of Agricultural \& Forestry Sciences, Baoding, China

Surfactin and fengycin are the main lipopeptides produced by Bacillus subtilis and play an important role in suppressing plant diseases. B. subtilis NCD-2 is an excellent biocontrol agent against plant soil-borne diseases, early study showed this bacterial isolate could produce surfactin and fengycin. In this study, the surfactin deficient mutant (MS), fengycin deficient mutant (MF) and surfactin/fengycin double deficient mutant (MSF) of strain NCD-2 were obtained by deleting the synthetase genes, respectively. Compared to strain NCD-2, the mutant MF decreased the antifungal ability, but the mutant MS decreased the swimming ability and biofilm formation ability, adding surfactin standard could restore the biofilm formation ability of MS to the wild type level. Comparatively, the mutant MSF decreased the antifungal activity, the swimming ability and the biofilm formation ability at more obvious level. The colonization ability on cotton root was compared between strain NCD-2 and mutant strains, results showed that when compared to strain NCD-2, the mutant MF had a light decrease of population in the rhizosphere, but the mutant MS and MSF had significantly decreased the root colonization abilities. Finally, the suppression against cotton damping-off was evaluated between strain NCD-2 and mutant strains, results indicated all the mutant strains decreased the biocontrol abilities, and the mutant MSF had the worst biocontrol efficiency, when compared to wild type strain.

Dual resistance of transgenic plants against Cymbidium mosaic virus and Odontoglossum ringspot virus T. Y. CHEN (1), P. Hsuan (2), C. C. Hao (1), T. T. Lin (1), Y. H. Hsu (3), N. S. Lin (2), (1) Academia Sinica, Taipei, Taiwan; (2) Institute of Plant and Microbial Biology, Academia Sinica, Taipei, Taiwan; (3) Graduate Institute of Biotechnology, National Chung Hsing University, Taichung, Taiwan

Cymbidium mosaic virus (CymMV) and Odontoglossum ringspot virus (ORSV) are two of the most prevalent viruses worldwide. When coinfecting orchids, they cause synergistic symptoms. Because of the huge economic loss in the orchid industry with virus-infected orchids, virusresistant orchids are urgently needed. Until now, no transgenic resistant lines against these two viruses have been reported. In this study, we generated transgenic Nicotiana benthamiana, expressing various constructs of partial CymMV and ORSV genomes and assayed them for viral resistance. Most transgenic lines grew normally and remained symptomless after mixed inoculation with CymMV and ORSV. The replication of CymMV and ORSV was approximately $70 \%$ to $90 \%$ lower in protoplasts of transgenic lines than wild type (WT) plants. Of note, we detected extremely low or no viral RNA or capsid protein of CymMV and ORSV in systemic leaves of transgenic lines, despite a moderate virus level accumulated in inoculated leaves after co-infection. Grafting experiments further revealed that CymMV and ORSV were unable to traffic from co-infected WT stocks to transgenic scions, which implies that novel blocking of long-distance movements of CymMV and ORSV co-contributes to the viral resistance in the transgenic lines. This study describes the first successful creation of dual resistant transgenic lines against CymMV and ORSV. Our studies shed light on the development of transgenic orchid to combat the global viral threat.

Phylogenetic and Phylogenomic Approaches to Decipher the Evolution of the Nitrogen Fixing Root Nodule Symbiosis J. KELLER (1), T. Vernié (1), H. San Clemente (1), L. Cottret (2), C. Girou (1), P. M. Delaux (1), (1) LRSV UMR5546 CNRS/Université de Toulouse III, Castanet-Tolosan, France; (2) LIPM (INRA-CNRS), Castanet-Tolosan, France

Root-nodule nitrogen-fixing symbiosis (RNS) is a key component of many natural and agronomic ecosystems. RNS is present in four orders (Fabales, Fagales, Cucurbitales and Rosales) that form the NFN clade and share a common ancestor. Only few families, spread among the NFN clade, can form RNS. A long-lasting question was whether RNS has been lost or gained multiple times. Recently, using phylogenomics analysis of nodulating and non-nodulating species we found two genes, NIN and RPG that are lost in non-nodulating species and conserved in the nodulating ones, suggesting that RNS has been acquire once and then lost multiple times. However, these two genes are also present outside the NFN clade. Thus, we now aim at identifying the molecular changes that drove the evolution of RNS in the NFN clade. We developed SymDB, a centralized plant genomes database gathering more than 100 species and including large-scale orthology relationships. Using this database, we are now investigating whether changes in cis-regulatory elements are linked to the evolution of RNS. Preliminary analyses identified such a motif in the promoter of NIN.

The RNA-binding protein MOB7 is a novel negative regulator of plant immunity in Arabidopsis thaliana

J. GEORGE (1,2), M. G. Stegmann (3,4), J. Monaghan (5,6), C. Zipfel (6,7), (1) The Sainsbury Laboratory, University of East Anglia, Norwich, U.K.; (2) Inst. of Plant and Microbial Biology, University of Zürich, Zürich, Switzerland; (3) Phytopathology Department, Technical University of Munich, Freising, Germany; (4) The Sainsbury Laboratory, Norwich, U.K.; (5) Queen's University, Kingston, ON, Canada; (6) The Sainsbury Laboratory, University of East Anglia, Norwich Research Park, Norwich, U.K.; (7) Inst. of Plant and Microbial Biology, University of Zürich, Zurich, Switzerland

Cell-surface pattern recognition receptors sense invading pathogens by perceiving microbial patterns, to activate innate immunity. These responses are often under tight control to avoid excessive or untimely activation of cellular responses, which may otherwise be detrimental to the homeostasis of host cells. How plant innate immune signaling is fine-tuned remains largely unknown. A forward-genetics suppressor screen was performed to identify Arabidopsis thaliana mutants that regained immune signaling in the otherwise immunodeficient genetic background bakl5. This screen led to the identification of 10 modifier of bakl-5 $(\mathrm{mob})$ mutants, which regained immune signaling and resistance to the bacterial pathogen Pseudomonas syringae (Monaghan et al., 2014; Stegmann et al., 2017). Here, we report the characterization of MOB7. MOB7 acts as a negative regulator of immunity, as measured by reactive oxygen species production and resistance to $P$. syringae. Interestingly, MOB7 is a RNAbinding protein conserved in land plants, and we could show that it co-localises with several proteins involved in mRNA stability. Ongoing work aims to identify MOB7-associated RNAs and proteins in order to elucidate how MOB7 regulates immune signaling. 
A genome editing approach to establish de novo blast disease resistance in wheat

S. PODDAR (1,2), J. Tanaka (2), M. J. Cho (2), B. J. Staskawicz (3), (1) Department of Molecular and Cell Biology, University of California, Berkeley, CA, U.S.A.; (2) Innovative Genomics Institute, University of California, Berkeley, CA, U.S.A.; (3) Department of Plant and Microbial Biology, University of California, Berkeley, Berkeley, CA, U.S.A.

Blast, caused by the fungus Magnaporthe oryzae, is a destructive disease that leads to substantial loss of annual rice and wheat harvests. Wheat blast emerged in South America and recently spread to Asia, with no known wheat cultivars resistant to the disease. We are deploying gene editing in wheat to knock out genes of rice orthologs already characterized to control resistance. Loss of function of these negative regulators of resistance lead to broad spectrum resistance that is more durable than that conferred by effector specific resistance genes. Given the family level phylogenetic relationship between rice and wheat, we reason that homologous genes may retain similar function. We have computationally identified these genes in wheat and designed Cas 9 based editing to target these genes for knockout in wheat immature embryos. By optimizing Cas9 guide RNAs and developing high frequency transformation by microparticle bombardment, we have identified homozygous susceptibility gene knockouts across all three genomes of a wheat plant in the first generation. These plants will be characterized for their response to blast and bacterial pathogen challenges to potentially demonstrate establishment of de novo resistance in wheat.

Nicotiana benthamiana Argonaute10 plays a proviral role in Bamboo mosaic virus infection

Y. H. HSU (1), Y. W. Huang (1), C. C. Hu (1), C. H. Tsai (1), N. S. Lin (2), (1) Graduate Institute of Biotechnology, National Chung Hsing University, Taichung, Taiwan; (2) Institute of Plant and Microbial Biology, Academia Sinica, Taipei, Taiwan

In plants, RNA silencing plays a major defense mechanism against invading viruses. Argonaute proteins (AGOs) are the key players in RNA silencing. The number of AGO family members varies depending on the plant species and they play distinct or sometimes redundant roles in antiviral defense. Here, we found Nicotiana benthamiana AGO1 restricted Bamboo mosaic virus (BaMV) accumulation, however, NbAGO10, the closest paralog of NbAGO1, positively regulated BaMV accumulation. AtAGO10 has been reported to act as a miRNA locker and prevent it from associating with AtAGO1. In this study, BaMV vsiRNA was found in the NbAGO10 complexes. Transient overexpression of NbAGO10 increased BaMV RNA accumulation, while co-expression of NbAGO1, BaMV RNA accumulation was reduced, suggesting that NbAGO10 sequestered BaMV vsiRNA from NbAGO1 RISC formation. In addition, overexpression of NbAGO10 decreased BaMV vsiRNA accumulation. A small RNA degrading nuclease 1 (SDN1) was demonstrated to interact with NbAGO10 by the in vivo pull-down assay. Silencing of SDN1 resulted in elevated BaMV vsiRNA level and decreased BaMV RNA accumulation in $N$. benthamiana. These data indicated that NbAGO10 might recruit SDN1 for the BaMV vsiRNA degradation. All together, our study suggests that NbAGO10 plays a proviral role in a way of the BaMV vsiRNA sequestration and degradation.

Reconstitution of TIR-domain NLR cell death in tobacco by coevolved EDS1 family proteins with helper NLR NRG1 from Arabidopsis D. LAPIN (1), X. Sun (1), V. Kovacova (2), J. A. Dongus (1), J. Rzemieniewski (1), D. D. Bhandari (1), J. Stuttmann (3), A. Beyer (2), J. E. Parker (1), (1) Max Planck Institute for Plant Breeding Research, Cologne, Germany; (2) University of Cologne, Cologne, Germany; (3) Martin Luther University, Halle (Saale), Germany

Pathogen effector proteins are detected by diversified plant immune receptors which share a central nucleotide-binding (NBARC) domain and Cterminal leucine-rich repeats (NLRs). Effector recognition by NLR receptors can induce plant defense and cell death pathways by poorly understood mechanisms. NLR resistance, however, requires a number of conserved plant signaling proteins including helper NLRs and three nonNLR, lipase-like proteins of the Enhanced Disease Susceptibility1 (EDS1) family. Here, we report an assay to reconstitute bacterial effectordependent cell death from a TIR-domain NLR (TNL) immune receptor Roq1 in Nicotiana benthamiana plants without functional EDS1-family genes. This was achieved by co-expressing a module of three proteins from Arabidopsis: two EDS1 family proteins and a helper NLR, NRG1. No pairwise combination was sufficient to mediate TNL cell death, indicating that cell death signaling and/or execution requires TNL Roq1 and EDS1-family proteins with a coevolved helper NLR. Using this reconstitution assay powered by EDS1-family protein structural information, we have identified molecular features within this module required for cell death signaling from the activated TNL. We are using this tobacco reconstitution system to study molecular interactions and co-functions between a receptor TNL, EDS1-family proteins and a helper NLR in cell death signaling and bacterial immunity.

\author{
ORA59 Transcription Factor Positively Regulates Resistance to Necrotrophic Pathogens through Binding to Two Distinct cis-Acting \\ Elements in Arabidopsis \\ Y. N. YANG, H. Kim, D. S. Lee, Y. S. Kim, O. K. Park, Department of Life Sciences, Korea University, Seoul, Korea, Republic of (South)
}

Plant defense against pathogens depends on the action of several phytohormones, including jasmonic acid (JA) and ethylene (ET).

Phytohormones activate a network of transcription factors that regulate the expression of defense genes. Notably, ET response factors (ERFs) are responsible for the transcriptional regulation of JA/ET-responsive defense genes. Members of ERF family transcription factors typically bind to GCC-box, a conserved motif found in the promoters of JA/ET-responsive genes. In this study, we report that a previously undefined cis-element, so called ET-responsive element (ERE), is critical for ET-induced transcription and frequently present in the promoters of ET-responsive genes. In yeast one-hybrid screening, ORA59 was identified as a transcription factor that binds to the ERE element. Our results give an insight into how ORA59 can generate specific patterns of gene expression dynamics through JA and ET hormone pathways.

Transcriptomic impact of the nitrogen fixing root symbiotic signaling pathway in the green lineage T. VERNIÉ, J. Keller, C. Girou, C. Langlet, P. M. Delaux, LRSV UMR5546 CNRS/Université de Toulouse III, Castanet-Tolosan, France

Nitrogen is a life component available in the food chain thanks to plants. Air is full of dinitrogen, but this form is not accessible to plants. The Haber Bosch process uses fossil energy to fix dinitrogen in ammoniac, the nitrogen active form present in chemical fertilizers. This process has 
huge environmental and economical costs. One alternative is the biological nitrogen fixation by nitrogen fixing bacteria. Few plant species are able to establish symbioses with these bacteria. However this nitrogen-fixing root nodule symbiosis (RNS) is limited to four plant orders that belong to a single clade. Recent phylogenomics studies have suggested that RNS has been acquired once and lost multiple times. Only two genes have been shown to be lost in non-nodulating species and conserved in the nodulating ones. Evolution of these 2 genes does not correlate with the evolution of RNS. We hypothesize that the evolution of RNS is due to neofunctionalization and/or redirected gene expression. We are currently working on this second hypothesis by searching for cis element specific to RNS. Our global approach is to genetically activate RNS signaling in nodulating and non nodulating plant species to identify downstream genes expressed only in nodulating species and then look for conserved cis elements in their promoters. In parallel we have identified conserved cis elements in known RNS-related genes. Validation of these cis elements in the legume Medicago truncatula is ongoing.

Bacterial outer membrane vesicles: A new player in plant-microbe interactions G. Mordukhovich, L. Katsir, N. Sela, O. BAHAR, ARO - Volcani Center, Rishon LeZion, Israel

Outer membrane vesicles (OMVs), pinching off the cell-wall of Gram-negative bacteria, are involved in multiple processes including response to stresses, delivery of virulence factors, host immune modulation and more. Nevertheless, very few reports studied the role OMVs play during plant-microbe interactions. We recently demonstrated that Arabidopsis up-regulates specific immune markers in response to OMVs purified from Xanthomonas campestris pv. campestris $(X c c)$ and other bacterial pathogens. To better understand the broader response of Arabidopsis to OMV challenge, we conducted RNA-seq analysis and plant pretreatment assays. Arabidopsis seedlings challenged with Xcc OMVs induced the expression of a large suite of immune-related genes including surface-bound and intracellular immune receptors. This transcriptional shift towards immune activation, primed Arabidopsis plants, rendering them more resistant to subsequent infection. OMVs proteomic analyses of four different plant-associated bacteria revealed a variety of potential MAMPs, virulence factors and secreted enzymes that may play a role during plant colonization. Interestingly, grouping OMV proteins by their predicted cell compartment localizations revealed variations among the different bacteria, hinting on a slightly different secretion mechanism or perhaps a different protein sorting process. Further research is underway to explore the full spectrum of OMV functions during plant colonization.

Sex mutants reveal components of Bs3-triggered immunity

D. R. HOLMES, C. Kroenauer, R. Morbitzer, T. Lahaye, ZMBP, Tübingen, Germany

Plant resistance $(R)$ genes mediate recognition of microbial effector proteins and in turn initiate a defense reaction. Executors are a unique class of plant $R$ genes that are transcriptionally activated by matching Xanthomonas transcription activator-like effectors (TALEs). In pepper, one of these executor $R$ genes is $B s 3$, which mediates recognition of and is activated by the TALE AvrBs3. Upon transcriptional activation of $B s 3$, a plant immune response is triggered, culminating in cell death. Up until now the mechanism that Bs3 employs to activate an immune response has been elusive. We discovered that Bs3 expression causes growth arrest in yeast. Bs3-triggered growth arrest in yeast facilitated a rapid forward genetic approach by which we identified a handful of SEX (suppressor of executor) genes. For one of the identified yeast SEX genes, the corresponding knockout of the Arabidopsis homologue was previously shown to compromise functionality of NLR type plant $R$ proteins. This observation suggests that executor- and NLR-type plant R proteins employ common immune signaling components. In support of this notion, we also found that the Bs3-triggered immune response correlates with an increase in salicylic acid, which is a well-known hallmark of NLRtriggered immunity. In summary, these observations suggest that Bs3 is hijacking well established immune pathways to execute its own distinct immune response.

Uncoupling Growth Inhibition from Plant Immunity in the Hyperresistant dmr6 dlo1 Mutant

T. VAN BUTSELAAR (1), G. Van Den Ackerveken (2), (1) Utrecht University, Utrecht, Netherlands; (2) Plant-Microbe Interactions, Dept of Biology, Utrecht University, Utrecht, Netherlands

Plants have evolved an elaborate immune system to combat microbial pathogens. Resistance to biotrophic pathogens, which thrive on living host tissue, is predominantly mediated by the phytohormone salicylic acid (SA). Although SA stimulates immunity, it actively suppresses growth. This growth-immunity tradeoff exerts itself in SA-accumulating mutants, like the dmr6 dlol double mutant. Arabidopsis plants mutated in DMR6 and $D L O 1$, which encode 2-oxoglutarate iron-dependent SA-oxygenases, are hyperresistant to biotrophs but show growth defects. Here we describe our forward genetics (EMS-mutagenesis) screen for restored growth of the $d m r 6$ dlo1 mutant. We identified 104 mutants with growth phenotypes similar to Col-0. Moreover, half of these restored growth mutants retained high resistance levels to the downy mildew Hyaloperonospora arabidopsidis. These modifier of $\mathrm{dmr6/dlo1-mediated} \mathrm{immunity} \mathrm{(mdi)} \mathrm{mutants} \mathrm{are} \mathrm{being} \mathrm{backcrossed} \mathrm{and} \mathrm{homozygous}$ progeny is being genome-sequenced to map the causal $M D I$ genes. We will present the phenotypic analysis of our $m d i$ mutants and provide an update on the genetic analysis of the underlying $M D I$ genes. Understanding the mode of action of the $M D I$ genes in mediating growth suppression during plant immunity will greatly aid to our understanding of the plant immune network.

Harnessing the diversity of biocontrol-related traits in plant-beneficial phenazine-producing Pseudomonas spp. to control potato diseases.

M. FILION, A. Biessy, A. Novinscak, G. Léger, Université de Moncton, Moncton, NB, Canada

Some plant-beneficial rhizosphere-associated Pseudomonas spp. strains produce phenazine antibiotics, which are not only inhibitory to numerous soil-dwelling plant pathogens but also promote survival and persistence of bacterial cells in the rhizosphere. We assembled a collection encompassing 63 phenazine-producing Pseudomonas spp. strains isolated from a wide diversity of host plants on four continents. The strains greatly vary in their ability to inhibit the growth of three major potato pathogens, Phytophthora infestans, Verticillium dahliae and Streptomyces scabies. Their capacity to produce different phenazine antibiotics also significantly impact growth inhibition and is pathogen-specific. Genome sequencing and comparative genomics unveiled an important genomic diversity, as indicated by the size of the pangenome, which accounts for more than 25,000 protein-coding genes. This diversity is also reflected by the myriad of phytobeneficial traits encoded in the genomes, which 
includes antibiotics, cyclic lipopeptides and siderophores. Using a reverse genetic approach, we are investigating the roles played by the different phenazine antibiotics and some biocontrol-related traits in the pathogen suppression activity towards the three potato pathogens.

Sustained crop protection by surface functionalization

C. LANGENBACH (1), P. Schwinges (1), F. Jakob (2), S. Pariyar (3), G. Noga (3), A. Pich (4), U. Schwaneberg (5), U. Conrath (1), (1) RWTH Aachen University, Plant Physiology Department, Aachen, Germany; (2) RWTH Aachen University, Biotechnology Department, Aachen, Germany; (3) Department of Horticultural Science, University of Bonn, Germany; (4) RWTH Aachen University, Institute of Technical and Macromolecular Chemistry, Germany; (5) Department of Biotechnology, RWTH Aachen University, Germany

To reduce pesticide use while preserving crop productivity alternative pest and disease control measures are needed. Although safer than ever, pesticides are still subject to wash-off by rain. We developed a technology platform that functionalizes the crop surface for sustained disease protection. The technology uses the immobilization of so-called anchor peptides on crop leaves. We show that anchor peptides are retained on the epicuticular wax layer of barley, corn, and soybean leaves even when treated leaves or plants are subjected to simulated rain or natural rainfall in ambient field conditions. Dipeptides consisting of an N-terminal antifungal peptide fused to a C-terminal anchor peptide were not phytotoxic at all but effectively inhibited the germination of spores of the Asian soybean rust-causing fungus Phakopsora pachyrhizi in vitro. On planta, they reduced rust disease severity in a rainfast manner. Furthermore, we provide evidence that anchor peptides can be employed to immobilize microgel containers on the surface of multiple crops. Controlled release of compounds from anchor peptide-microgel hybrids may provide prolonged disease protection by shielding pesticides from environmental degradation and wash off by rain.

Secondary metabolism pathway engineering for crop protection

P. SCHWINGES (1), D. Spencer (1), S. Dreischoff (1), J. Weber Böhlen (1), A. Beesley (1), S. F. Beyer (1), S. Holger (2), U. Conrath (1), C. Langenbach (1), (1) RWTH Aachen University, Plant Physiology Department, Aachen, Germany; (2) BASF Plant Science, Limbugerhof, Germany

Engineering crops for the enhanced accumulation of antimicrobial secondary metabolites is a promising means for sustainable disease management. Here, we show that stress-induced enzymes can be used to optimise the production of coumarins in planta. As a first step, we heterologously expressed an $O$-methyltransferase from sunflower (Helianthus annuиs) in Escherichia coli. Substrate feeding assays revealed its activity towards different hydroxylated coumarins leading to biosynthesis of the antifungal methoxycoumarins scopoletin, isoscopoletin, and scoparone. Transient expression of the $O$-methyltransferase in Nicotiana benthamiana led to the accumulation of high amounts of the dimethoxylated scoparone. The latter coumarin was absent from non-transformed plants. Our results indicate that fine-tuning the secondary metabolism of crops by exploiting biosynthetic enzymes from different origins in the plant kingdom is a promising strategy for sustainable disease management.

RNAi-based silencing of Citrus sinensis callose synthase 7, important gene to defense response to Candidatus Liberibacter asiaticus L. M. GRANATO (1), N. D. R. D’Alessandre (1,2), R. L. Boscariol Camargo (1), M. A. Machado (1), (1) Centro de Citricultura Sylvio Moreira/Instituto Agronômico, Cordeirópolis, Brazil; (2) UFSCAR, Araras, Brazil

Huanglongbing (HLB) is a destructive disease of citrus caused by the phloem-limited proteobacterium Candidatus Liberibacter asiaticus (CaLas). Symptoms of HLB including blotchy chlorosis have been related by the callose accumulation in the phloem sieve plates. Callose synthase genes are involved in callose synthesis and its expression is modulated in response to biotic stresses. In this study, nine Citrus sinensis calS genes (CscalS) were identified, and the expression patterns were analyzed in CaLas-inoculated and healthy plants. CscalS7 was the only gene differentially expressed in both evaluated times after $\mathrm{CaLas}$ infection (120 and 360 days after infection), which was correlated with increased callose deposition in the phloem sieve tubes and the accumulation of starch in the leaves. We hypothesized that the up-regulation of CscalS is a citrus defense response to increase the callose deposition in the phloem, plugging the sieve plates to reduce bacterial colonization by decrease of the transport of photoassimilates. To confirm this hypothesis, we have tested the use of RNA interference (RNAi) technology in C. sinensis plants to knockdown the CscalS7 expression. To induce silencing, we used two methods: infiltration of leaves with Agrobacterium tumefaciens carrying the hpRNAi-transgene and the establishment of transgenic plants using Agrobacterium-mediated transformation of epicotyls. RT-qPCR analysis showed reduction of CscalS7 expression which indicated the gene silencing.

Rhizosphere colonization by phytobeneficial phenazine-producing Pseudomonas spp.: A metabolic and genomic approach A. ZBORALSKI, M. C. Savoie, A. Biessy, A. Novinscak, M. Filion, Université de Moncton, Moncton, NB, Canada

Phenazine-producing Pseudomonas spp. are mutualistic bacteria able to colonize the plant rhizosphere and inhibit several soil-dwelling plant pathogens. Rhizosphere colonization relies on multiple genetic and metabolic factors driving plant-bacteria interactions. To decipher these factors, a collection of 60 phenazine-producing Pseudomonas strains representative of the worldwide known diversity was assembled and their genomes were sequenced and annotated. These strains were individually inoculated in the rhizosphere of Solanum tuberosum (potato) and Arabidopsis thaliana. Rhizosphere colonization was assessed by quantitative PCR using a newly developed TaqMan probe/primer set targeting the phenazine biosynthetic operon. In parallel, metabolic abilities of the strains were assessed using the Biolog Phenotype Microarray technology with a total of 758 tested substrates, including sugars, amines, organic acids and amino acids. Rhizosphere colonization levels were related to taxonomic subgroups in both plant species. Combining rhizosphere colonization results and the tested metabolic abilities of the 60 strains lead to the identification of multiple substrates associated with high or low colonization levels in both plant species, such as nitrogenous substrates and sugars, respectively. Biosynthetic genes and clusters of genes associated with higher colonization levels, especially related to antibiotic and antibacterial proteins, were also identified. 
The emerging case for epigenetic regulation of plant immunity

J. Stassen (1), L. Furci (1), S. W. Wilkinson (1), A. Lopez Sanchez (2), R. Jain (3), L. M. Smith (1), J. TON (1), (1) The University of Sheffield, Sheffield, U.K.; (2) National Centre of Biotechnology, Madrid, Spain; (3) La trobe University, Melbourne, Australia

Plants can prime their immune system after perception of hostile environmental signals, causing acquired resistance. In Arabidopsis, priming of jasmonic acid (JA)- and salicylic acid (SA)-dependent defences lasts weeks and involves regulation by DNA methylation and histone modifications. While long-term priming of the JA pathway specifically targets MYC2-dependent defences against herbivores, long-term priming of the SA pathway requires the regulatory protein NPR1 and chromatin remodeler KYP. Transcriptome analysis of mutants in DNA (de)methylation revealed that half of the defence-related transcriptome is influenced by DNA methylation. Further evidence for epigenetic control of plant immunity came from our finding that progeny from heavily diseased Arabidopsis express transgenerational acquired resistance (TAR). TAR can be elicited by both biotrophic and necrotrophic pathogens, and its durability and intensity are determined by the level of parental disease stress. Bisulfite-sequencing of TAR-expressing progeny revealed changes in gene body methylation, although it remains uncertain whether this causes TAR. To identify TAR-regulating epigenetic loci, we screened epigenetic recombinant inbred lines (epiRILs) for downy mildew resistance, and identified four hypo-methylated pericentromeric loci that provide heritable resistance. Transcriptome analysis of selected epiRILs suggested that methylation of transposable elements at these loci trans-regulates defence gene priming.

\section{CRISPR/Cas9-mediated targeting of Cacao swollen shoot virus DNA}

M. A. GOMEZ (1), N. Chingandu (2), J. Pham (1), J. Jones (1), E. Zhang (1), M. J. Cho (1), J. K. Brown (2), B. J. Staskawicz (3), (1) Innovative Genomics Institute, University of California, Berkeley, CA, U.S.A.; (2) School of Plant Sciences, University of Arizona, Tucson, AZ, U.S.A.; (3) Department of Plant and Microbial Biology, University of California, Berkeley, Berkeley, CA, U.S.A.

Theobroma cacao is a tree crop and the source of chocolate. Cacao production is distributed across the tropics with over $70 \%$ of cacao beans coming from West Africa. Here, Cacao swollen shoot virus (CSSV, family Caulimoviridae, genus Badnavirus) threatens cacao production. In this study, we investigate whether CRISPR/Cas9-mediated viral interference can be applied to confer resistance against this DNA virus. This strategy targets the CSSV genome for degradation and/or mutagenesis via the CRISPR/Cas9 system. We identified conserved sequences across multiple CSSV isolates to design guide RNAs, which exhibited functional CRISPR/Cas9 targeting of CSSV DNA in vitro. We assembled the guide RNAs into a CRISPR/Cas9 plant expression vector for multiplex targeting of multiple CSSV isolates. The targeted region within the CSSV isolates were cloned into surrogate gemini-vectors that facilitate delivery and amplification of DNA fragments of interest in planta. Co-expression of the CRISPR/Cas9- and gemini-vectors in the model plant Nicotiana benthamiana enabled rapid evaluation of targeting CSSV DNA. Analysis of the targeted sites show CRISPR/Cas9 induced mutagenesis of the CSSV DNA. Stable N. benthamiana and T. cacao transgenic lines bearing this CRISPR/Cas9 expression system will be generated and evaluated for their ability to interfere with CSSV infection.

Preliminary investigations of extracellular vesicles from the wheat pathogen Zymoseptoria tritici E. H. HILL, P. S. Solomon, The Australian National University, Canberra, Australia

The fungal wheat pathogen Zymoseptoria tritici resides exclusively in the apoplast during the extended latent phase of the foliar infection. Consequently, the apoplast is likely the site of significant molecular events mediating interactions between pathogen and host. The mechanisms used by $Z$. tritici to secrete disease-associated molecules into the harsh apoplastic environment and/or deliver them to host cells is currently unknown and represents a critical gap in our understanding of pathogen infections. While many molecules are likely secreted by classical pathways, non-classical mechanisms may also be used. Extracellular vesicles (EVs) have emerged as a potential mechanism of secretion and cross-kingdom transport. We hypothesise that EVs may play a role in pathogen-host communication during $Z$. tritici infection of wheat. We have made preliminary investigations of putative $Z$. tritici EVs isolated from in vitro culture filtrates. Ultracentrifugation-based techniques, transmission electron microscopy and single-particle analysis were used to purify and characterise putative EVs; these have been optimised to allow for proteomic profiling to begin dissecting vesicle cargoes. The establishment of these techniques in vitro will allow us to expand our investigation to in planta contexts and examine if $Z$. tritici EVs are deployed during infection of wheat. We anticipate this study will begin to address unanswered questions about how fungal pathogens communicate with their hosts.

\section{Surcrose non-fermenting-1 related protein kinase 1 (GmSnRK1) play positive role in regulating the resistance to Phytophthora sojae in} soybean

X. ZHU, D. Fang, S. He, X. Zhou, P. Zhang, C. Chen, Y. Qiao, Shanghai Normal University, China

Phytophthora root and stem rot (PRSR) is one of the most devastating diseases in world soybean production, therefore, exploring new Phytophthora sojae resistance genes and developing new resistant germplasm is vital important. The surcrose non-fermenting-1 related protein kinase 1 (SnRK1) subfamily plays a central role in metabolic responses to nutritional and environmental stresses in plants. However, the role of SnRK1 in the regulation of biotic stress remain largely unexplored. In this study, we showed that two SnRK1 genes (GmSnRK1-1 and GmSnRK12) in soybean belong to the conserved family that were up-regulated during the late infection stage of Phytophthora sojae by analyzing soybean RNA-seq and qRT-PCR data. The Phytophthora inoculation assays showed that GmSnRK1-1/2 genes are pathogenesis-related genes and play a positive role in resistance to Phytophthora sojae and Phytophthora capsici by over-expression and silencing (VIGS or CRISPR/cas9 assays) of GmSnRK1-1/2 in Nicotiana benthamiana leaves and soybean hairy roots. A two-hybrid screen in yeast with GmSnRK1-1/2 as bait together with bimolecular fluorescence complementation (BiFC) assay in planta suggested that GmSnRK1-1/2 interact with GmTPS6 (Trehalose phosphate synthase 6) in the nucleus and cytosol, which involved in sugar signaling pathway. Over all, these data suggest the GmSnRK1 regulate innate immunity in plants which is associated with sugar related signal pathway and plant- Phytophthora interactions.

The WY domain in the Phytophthora effector PSR1 is required for virulence and RNA silencing suppression activity Y. QIAO, P. Zhang, Y. Jia, J. Shi, C. Chen, Shanghai Normal University, China 
Phytophthora pathogens manipulate host innate immunity by secreting numerous RxLR effectors, thereby facilitating pathogen colonization. Predicted single and tandem repeats of WY domains are the most prominent C-terminal motifs conserved across the Phytophthora RxLR superfamily. However, the functions of individual WY domains in effectors remain poorly understood. The Phytophthora sojae effector PSR1 promotes infection by suppressing small RNA biogenesis in plant hosts. We identified one single WY domain following the RxLR motif in PSR1. This domain was required for RNA silencing suppression and virulence activity in Nicotiana benthamiana, Arabidopsis, and soybean. Mutations of the conserved residues in the WY domain did not affect the subcellular localization of PSR1 but abolished its effect on plant development and resistance to viral and Phytophthora pathogens. This is at least in part due to decreased protein stability of the PSR1 mutants in planta. The identification of the WY domain in PSR1 allows the prediction of a family of PSR1-like effectors also possess RNA silencing suppression activity. Mutation of the conserved residues in two members of this family, PpPSR1L from P. parasitica and PcPSR1L from $P$. capsici, perturbed their biological functions, indicating that the WY domain is critical in Phytophthora PSR1 and PSR1-like effectors.

Phytophthora sojae effector suppresses RNA silencing and plant immunity by activating GmDCP2 mediated mRNA decay in soybean J. SHI, H. Liu, X. Ji, R. Jia, Y. Zhu, Y. Qiao, Shanghai Normal University, China

Phytophthora sojae is a soilborne pathogen, which causes a serious Phytophthora root and stem rot in the soybean product region. RNA silencing suppressor proteins are pathogenicity determinants widely expressed by plant and animal viruses that have recently also been identified amongst P. sojae effectors. However, the mechanism of how Phytophthora effectors suppress host RNA silencing remain unclear. In this study, we identified a novel RNA silencing suppressor, PSR3, in $P$. sojae using a stable transgenic system by checking the suppression activity of $G U S$ gene silencing in $A$. thaliana L1 plants instead of traditional RNA suppressor screening system in $N$. benthamiana $16 \mathrm{C}$ plants. Further studies showed that the expression of PSR3 is highly up-regulated in the early infection period and PSR3 can enhance the plant susceptibility to both PVX and Phytophthora parasitica in N. benthamiana leaves and to P. sojae in soybean hair root. Y2H screening showed that PSR3 directly interacts with soybean ASYMMETRIC LEAVES like proteins GmASL2-1/2 and greatly induce those genes expression. Consistent with these findings, GmASL2-1/2 transient expression in 16C plants suppressing GFP expression also confirmed GmASL2-1/2 are involved in the RNA silencing pathway. GmASL2-1/2 were proven to interact with soybean RNA decapping protein GmDCP2b. Our results suggests that PSR3 may activate RNA degradation to suppress Post transcription of gene silencing (PTGS) and immunity in soybean.

Induced proximity of a signaling domain on a plant-mammalian NLR chimera triggers defense activation in plants. Z. DUXBURY (1), J. D. Jones (2), (1) Gregor Mendel Institute, Vienna, Austria; (2) The Sainsbury Laboratory, University of East Anglia, Norwich Research Park, Norwich, U.K.

Plant and animal intracellular nucleotide-binding, leucine-rich repeat (NLR) immune receptors detect pathogen-derived molecules and activate defense. Upon ligand detection, mammalian NAIP and NLRC4 NLRs oligomerize, forming an inflammasome that induces proximity of its Nterminal signaling domains. To investigate plant NLR signaling mechanisms, we fused the N-terminal TIR (Toll-like, interleukin-1 receptor, resistance protein) domain of several plant NLRs to the N-terminus of NLRC4. Inflammasome-dependent induced proximity of the TIR domain in planta initiated defense signaling. We provide proof-of-concept that TIR-NLRC4/NAIP inflammasomes can provide an additional layer of intracellular surveillance of phytopathogenic bacteria. Thus, induced proximity of a plant TIR domain imposed by oligomerization of a mammalian inflammasome is sufficient to activate authentic plant defense.

Ribonuclease-type effectors from Colletotrichum orbiculare potentiate host immune responses in a catalytic residue-dependent manner N. KUMAKURA (1), S. S. Ogawa (2), P. Gan (3), A. Tsushima (1,4), M. Narusaka (5), Y. Narusaka (5), Y. Takano (2), K. Shirasu (3,6), (1) RIKEN Center for Sustainable Resource Science, Plant Immunity Research Group, Yokohama, Japan; (2) Graduate School of Agriculture, Kyoto University, Kyoto, Japan; (3) RIKEN Center for Sustainable Resource Science, Yokohama, Japan; (4) The University of Tokyo, Japan; (5) Res Inst for Biological Sciences, Kaga-gun Okayama, Japan; (6) The University of Tokyo, Tokyo, Japan

Members of the genus Colletotrichum infect many commercially important crops. The genome of the hemibiotrophic anthracnose fungus, Colletotrichum orbiculare, encodes a large set of effector candidates, but the vast majority of their roles in plant-microbe interactions are still obscure. Here, we show that secreted ribonuclease-type effectors from C. orbiculare, named SRN1 and SRN2, potentiate host immune responses in a catalytic residue-dependent manner. By comparing different Colletotrichum species genomes, SRNs were found to be conserved effectors in the genus Colletotrichum. We established a transient gene expression system in Cucumis sativus, the host plant from which C. orbiculare was isolated, and showed that the $C$. orbiculare SRNs potentiate pattern triggered immunity (PTI) responses, including chitin-triggered reactive oxygen species (ROS) bursts, chitin-triggered MPK phosphorylation, and PTI marker gene expression. Consistent with this, C. orbiculare SRN1 and SRN2 deletion mutants showed increased penetration ratios and fungal biomass compared to that of wild-type C. orbiculare. Importantly, the potentiation of $C$. sativus PTI responses by SRN2 depends on its ribonuclease catalytic residues and signal peptide. We hypothesize that $C$. sativus is able to perceive the pathogen through the catalytic activities of SRNs.

Silencing genes in Arabidopsis downy mildew using exogenously applied small RNAs

M. TOR (1), O. Bilir (2,3), O. Telli (1), S. John (2), C. Norman (2), H. Budak (2,4), Y. Hong (2,5), (1) School of Science and the Environment, Worcester, U.K.; (2) University of Worcester, Worcester, U.K.; (3) Directorate of Trakya Agricultural Research Institute, Edirne, Turkey; (4) Montana State University, U.S.A.; (5) Hangzhou Normal University, Hangzhou, China

The interaction between Arabidopsis thaliana and diploid biotrophic oomycete Hyaloperonospora arabidopsidis (Hpa) has been a model for investigating plant-pathogen systems. Several $R$-genes conferring resistance to $H p a$ and signaling genes playing a role in the immune system have been cloned from Arabidopsis. Similarly, several effectors from Hpa that are recognized by the cloned $R$-genes have been identified and investigated. Although a vast amount of resources including mutant lines for Arabidopsis exist, lack of genetic manipulation methods for Hpa has hampered studies on pathogenicity related genes. This, in turn, has hindered the discovery of Hpa genes that are involved in spore germination, appressorium, haustorium and conidiophore development. We investigated whether the exogenous application of small RNAs, known as spray- 
induced gene silencing (SIGS), could silence selected target genes in Hpa. We showed that it is possible to use single or double stranded sRNAs to silence these targeted genes. The latest findings will be presented.

From male-killers to plant pathogens? Investigating the capacity of insect-associated Arsenophonus symbionts to colonize plants J. DITTMER (1), X. Foissac (2), F. Faoro (1), (1) Dipartimento di Scienze agrarie e ambientali (DISAA), University of Milan, Italy; (2) INRA Bordeaux, UMR 1332, France

Bacteria of the genus Arsenophonus represent one of the most widespread clades of insect endosymbionts and establish diverse symbiotic interactions with their insect hosts, ranging from the initially discovered male-killing strain of parasitoid wasps to obligate mutualists of several blood-feeding insects. Within this group of insect symbionts, two strains have been identified as insect-vectored plant pathogens, causing diseases in strawberry and sugar beet. In both cases, the bacteria are vectored by planthoppers and accumulate in the plant phloem, ultimately causing yellows and plant death. Hence, the switch from an ancestral purely insect-associated to a multi-host lifestyle occurred independently multiple times within this genus. These symbionts therefore represent outstanding model systems to investigate bacterial adaptations at the early stages of transition towards insect-vectored phytopathogens. Here, we used comparative genomics to investigate whether the ability to infect plants was achieved via genomic adaptations compared to other Arsenophonus strains or whether the genetic repertoire within this clade is sufficiently versatile to allow rapid adaptations to plant host environments, should the ecological opportunity arise. To this end, we searched the available Arsenophonus genomes for orthologs of known effector molecules mediating plant pathogenicity and discuss whether numerous insects carrying Arsenophonus might be reservoirs of potential plant pathogens.

Enhancement of growth and salt tolerance of rice by ACC deaminase-producing endophytic streptomycetes A. THAMCHAIPENET, W. Kruasuwan, S. Yoolong, R. Jaemsaeng, Kasetsart University, Bangkok, Thailand

1-Aminocyclopropane-1-carboxylate (ACC) deaminase is one of the plant growth promoting (PGP) traits found in beneficial bacteria including streptomycetes and responsible for growth and stress tolerance. In this work, ACC deaminase-producing endophytic streptomycetes have been evidently demonstrated to increase salt tolerance of rice by reduction of ethylene via the action of ACC deaminase and further assist rice to scavenge ROS, balance ion content and osmotic pressure. Expression profiles of stress responsive genes in rice in association with the endophytes were correlated to the physiological characteristics. Furthermore, acdS encoding ACC deaminase of streptomycetes was highly expressed in vivo. In contrast, $a c d S$ mutant showed no such beneficial effect to rice. However, the ACC deaminase-overexpressing mutant did boost more growth and significantly enhanced more salt tolerance in rice. Transcriptomic profiling revealed up-regulation of transcription factors involved in growth and development, plant hormones, ion translocation, ROS scavenging, and compatible solutes of rice. Moreover, up-regulation of plant-microbe interacting genes were observed. Whereas, genes involved in ethylene biosynthesis were down-regulated. The results strongly validated that ACC deaminase-producing endophytic streptomycetes enhance growth and alleviate salt tolerance of rice.

The impacts of quantitative disease resistance on downy mildew epidemiology in mixed host populations E. K. MOFFAT, M. Yassin, S. Rolfe, J. Stassen, J. Ton, The University of Sheffield, Sheffield, U.K.

Quantitative disease resistance (QNDR) has great potential to provide durable disease protection in plants. Whilst there are ample studies about QNDR in individual plants, little is known about the effects of QNDR on disease spread within closely grouped plants, such as those found in agricultural/horticultural settings. Here, we characterise the patterns by which downy mildew (Hyaloperonospora arabidopsidis; Hpa) spreads across Arabidopsis microcosms of varying composition and canopy density, using hyperspectral imaging (HSI). These microcosms consisted of combinations of high-QNDR edr1-1 plants, medium-QNDR wild-type plants (Col-0), and low-QNDR NahG plants. HSI maps the reflectance of light at 480 different wavelengths, providing high-throughput disease assessment at high spatial resolution. Since HSI is non-destructive, it allows us to track the infection spread through time and space. We created disease indices that accurately indicate the presence and Hpa disease severity by correlating HSI data to conventional Trypan-blue scoring. We will discuss how canopy density and different combinations of QNDR-host genotypes affect the dynamics of disease spread. Our aim is to translate these findings from the Arabidopsis-Hpa interaction to the lettuce-Bremia interaction, which remains a global threat to lettuce cultivation.

Symbiotic nodules, the Achilles heel of legumes?

C. Benezech (1), F. Berrabah (2), J. George (2,3,4), T. Badet (1), A. Le scornet (1), M. F. Jardinaud (1), F. Vailleau (1), P. Ratet (2), S. Raffaele (1), B. GOURION (1), (1) Laboratory of Plant-Microbe Interactions, Toulouse, France; (2) IPS2, Paris, France; (3) Inst. of Plant and Microbial Biology, University of Zürich, Zürich, Switzerland; (4) The Sainsbury Laboratory, University of East Anglia, Norwich, U.K.

In the diversity of multicellular organisms, there are many examples where tolerance of a massive microbial density can be beneficial for the host. This tolerance generally requires local adaptations of the immune system to prevent inappropriate defenses that would defeat or reduce the benefit provided by the mutualistic microbes. On the other hand, to avoid pathogen exploitation, immunity should not be totally suppressed in the tolerant organs. Due to the tremendous rhizobial population size they host, legume nodules are good models to study this trade-off. We investigate the influence of symbiosis on plant vulnerability and defenses and vice versa notably through tripartite systems involving the Medicago-Sinorhizobium couple. Using two pathogens, we showed that nodules represent a risk of infection by the bacterium Ralstonia solanacearum and offer favorable proliferation sites for the fungus Sclerotinia sclerotiorum. Transcriptome analysis indicate that Medicago nodules can develop specific defenses. Interestingly, those responses are much less intense in the nodule than in roots. This might contribute to the niche exploitation by the pathogens. We are now using genetics to characterize the systems. Interestingly, the two pathogens display opposite behaviors when facing fix- nodules. Furthermore, they have contrasted impacts on symbiosis. Our data provide keys to better understand the molecular basis ruling the trade-off between tolerance and vulnerability. 
FtsH membrane protease genes of Flavescence dorée phytoplasma exhibit host-dependent differential expression in both natural and experimental pathosystems

C. Jollard (1), X. Foissac (2), D. Desque (2), F. Razan (2), C. Garcion (2), L. Beven (2), S. EVEILLARD (2), (1) INRA, France; (2) INRA

Bordeaux, UMR 1332, France

Flavescence Doré (FD) is a severe disease of grapevine caused by $16 \mathrm{SrV}-\mathrm{C}$ and $16 \mathrm{SrV}-\mathrm{D}$ phytoplasmas (FDp), transmitted by the leafhopper Scaphoideus titanus. Differences in virulence among 'Candidatus Phytoplasma mali' isolates have previously been associated to sequence variations in FtsH and membrane AAA+ ATPases. Interestingly, the FDp genome encodes for an unusual number of genes encoding ATP dependent zinc-binding membrane proteases FtsH. Eight $f t s \mathrm{H}$ genes were identified along the FDp chromosome and encoded all FtsH functional domains. Genetic environments of $f t s \mathrm{H} 1$ to $f t s \mathrm{H} 8$ and phylogenetic analysis of FtsH proteins both pointed out FtsH6 as the original ortholog of the conserved cellular FtsH. The other seven gene copies clustered on a distinct monophyletic branch suggesting possible paralogous duplications. All fts $\mathrm{H}$ genes but one were expressed in S. titanus, grapevine natural hosts and in Euscelidius variegatus and broad bean experimental hosts. Relative Gene Expression showed a host-dependent differential expression. Gene copies fts $\mathrm{H} 3$, fts $\mathrm{H} 4$ and $f t s \mathrm{H} 8$ were overexpressed in FDp infecting grapevine as compared to their expression in S. titanus. The FtsH C-terminal proteolytic domain was predicted to be intracellular, except for FtsH6 and FtsH7 that could be extracellular. The soluble part of three FtsH expressed in Escherichia coli exhibited an ATPAse activity in vitro. The involvement of some of the $\mathrm{FtsH}$ in of host protein degradation remains to be investigated.

A Marchantia transcription factor involved in both plant-pathogen interactions and development

D. J. HOEY, P. Carella, S. Schornack, Sainsbury Laboratory, University of Cambridge, Cambridge, U.K.

Studies in vascular plants have revealed examples where families of transcription factors control pathogenic and symbiotic interactions as well as plant development. Recently established non-vascular model systems have reduced numbers of transcription factors, and family sizes are often also smaller. In order to uncover transcription factors involved in plant-pathogen interactions and plant development, we are exploiting a pathogenic interaction between the oomycete Phytophthora palmivora and the liverwort Marchantia polymorpha. Here, we report on a member of a liverwort and angiosperm transcription factor family which is transcriptionally responsive to $P$. palmivora attack. In unchallenged plants, its expression is spatially restricted to reproductive organs. This suggests that it contributes developmental and biotic stress-related functions. Using genetics, transcriptomics and cell biology, we plan to dissect how a single gene integrates both functions and whether these are conserved in angiosperms.

Interplay of carbonic anhydrase activity and virulence in the plant pathogenic bacterium Pseudomonas syringae pv. tomato M. Fishman (1), W. ZHANG (1), M. J. Filiatrault (2), (1) Cornell University, Ithaca, NY, U.S.A.; (2) USDA ARS, Ithaca, NY, U.S.A.

Bacterial carbonic anhydrases (CAs) are a class of metalloenzymes that catalyze the physiologically process of carbon dioxide hydration to bicarbonate and protons. Recently they have received attention because of described roles in survival, invasion and pathogenicity of microorganisms. Information regarding what role CAs play in bacterial plant pathogens is lacking. We previously identified a CA produced by the plant pathogenic bacterium Pseudomonas syringae that is regulated by a calcium responsive two-component system CvsSR. Deletion of CA impacted virulence. Here we performed functional and phenotypic assays and show CA activity affected calcium dissolution by $P$. syringae and production of the extracellular molecule cellulose. Cellulose is associated with various aspects of the lifecycles of bacteria and functions in competitive interactions with microbes. Deletion of the CA resulted in reduced competition of $P$. syringae with bacteria and influenced expression of genes involved in Type VI secretion, such as $h c p-2$ and the GacA-regulated small RNA $r s m Y$. The gene encoding CA is conserved among Pseudomonas, however discrepancies were discovered with predictions of the size of the protein produced. A model depicting the regulatory circuit and roles of CA activity in $P$. syringae are presented. Studies are underway to evaluate global impacts on virulence gene expression and in vivo inhibition of the CA to investigate impacts on pathogenicity and growth $P$. syringae in planta.

High throughput phenotyping to optimize CRISPR/Cas9 mutation efficiency in cereal crops M. LENK (1), M. Wenig (1), C. Knappe (1), S. Dey (1), J. Stuttmann (2), D. Lang (3), I. Fischer (3), J. Imani (4), K. H. Kogel (4), K. F. X. Mayer (3), C. Vlot (1), (1) Institute of Biochemical Plant Pathology, Helmholtz Zentrum Muenchen, Germany; (2) Martin Luther University, Halle (Saale), Germany; (3) Institute of Plant Genome and Systems Biology, Helmholtz Zentrum Muenchen, Germany; (4) Institute of Phytopathology, Justus-Liebig-Universität Gießen, Germany

CRISPR/Cas9 is increasingly used for gene editing applications in different plant species. Research on polyploid species such as hexaploid wheat will benefit in particular from the use of this simple and versatile technique, because it potentially allows for disruption of all gene copies in a genome via multiplexing. We want to use CRISPR/Cas9 to knockout barley and wheat genes associated with systemic acquired resistance (SAR), a process in which systemic plant parts are protected from infection after application of a local stimulant. Also, we aim to facilitate the process of mutant generation in cereals using CRISPR/Cas9. To this end, we will establish a high throughput pipeline for target gene selection and guide RNA design using a fluorescent reporter system in protoplasts combined with an automated microscopy phenotyping platform. For selected gRNAs, we will also sequence target sites and the whole genome to assess gRNA efficiency and off-target mutations. We aim to analyze a large number of gRNAs that are randomly distributed across various regions of barley and wheat chromosomes (e.g. euchromatin or heterochromatin). Comparing the associated mutagenesis efficiencies will allow the deduction of gRNA design rules that are tailored to the structure of cereal chromosomes and allow e.g. specificity among the subgenomes of wheat. Using this knowledge, we will target potential SAR-associated genes in barley and wheat to phenotype the resulting mutants' defense responses.

Identification of Sex (Suppressor of Executor) genes suggests shared immune signaling components of classical Bs1- and executor-type plant resistance proteins

R. MORBITZER, A. Dressel, M. Wunderlich, T. Lahaye, ZMBP, Tübingen, Germany 
Executor plant resistance $(R)$ genes are transcriptionally activated by Xanthomonas TAL effectors (TALEs) that bind to the executor $R$ promoter. In turn, TALE-induced expression of executor R proteins triggers plant immunity. We study the pepper (Capsicum annuum) executor $R$ genes $B s 3$ and Bs4C, that mediate recognition of the Xanthomonas TALEs AvrBs3 and AvrBs4, respectively (Römer et al.,2007 and Strauß et al.,2012). To identify suppressor of executors (SEX) genes we initiated a forward genetic screen in the pepper genotype ECW123 that contains functional copies of the pepper executor genes $B s 3$ and $B s 4 C$. In addition, the chosen pepper genotype mediates recognition of the Xanthomonas non-TALE proteins AvrBs1 and AvrBs2 that are recognized by the pepper $B s 1$ and $B s 2$ genes, respectively. So far 1.500 EMS-mutagenized M2 families have been screened resulting in identification of two independent M2 families in which AvrBs4 recognition was no longer observed and the Bs1 reaction was strongly reduced suggesting that the executor R protein Bs $4 \mathrm{C}$ and the Bs1 protein share common immune signaling components. By contrast, the Bs3 reaction was not affected, suggesting that the identified signaling component is not common to Bs3-and Bs4C-triggered immune reactions. In summary the identification of SEX genes provides the very first insights into executor R protein triggered plant immune reactions. Recent progress on the cloning and the molecular characterization of SEX genes will be presented.

A molecular toolkit for gene function studies using CRISPR/Cas in monocots and dicots

F. Hahn (1), A. Korolev (1,2), C. Sparks (1), K. Kanyuka (3), V. NEKRASOV (1), (1) Rothamsted Research, Harpenden, U.K.; (2) John Innes Centre, Norwich, U.K.; (3) Department of Biointeractions and Crop Protection, Rothamsted Research, Harpenden, U.K.

Genome editing technologies, such as CRISPR/Cas, have recently become available as tools for plant reverse genetics as well as crop improvement, including enhancement of disease resistance. Targeting susceptibility (S) genes by genome editing has proven to be a viable strategy for generating resistance to bacterial, fungal and viral pathogens in various crops. In this study we generated a set of Golden Gate compatible plasmid modules that enable rapid assembly and deployment of CRISPR/Cas-based molecular tools for genome editing applications in both monocot and dicot plant species. As a proof of concept, we targeted a few positive regulators of plant immunity as well as putative Sgenes in bread wheat (Triticum aestivum). We managed to generate homozygous/biallelic mutant lines as primary transformants suggesting a high efficiency of the CRISPR/Cas-mediated mutagenesis in wheat. CRISPR/Cas is therefore a valuable tool for gene function studies, and potentially breeding for enhanced disease resistance, in a polyploid crop species like wheat.

Engineering disease resistance in crops by enhancing scopoletin production A. BEESLEY (1), S. F. Beyer (1), P. F. W. Rohmann (1), V. Wanders (1), S. Holger (2), U. Conrath (1), C. Langenbach (1), (1) RWTH Aachen University, Plant Physiology Department, Aachen, Germany; (2) BASF Plant Science, Limbugerhof, Germany

The plant secondary metabolite scopoletin has multiple properties that promote its use in agriculture. In plants, for example, scopoletin supports the defense response to biotic and abiotic stress. Through genetic engineering we aim to generate crops with high amounts of scopoletin, thus enhancing stress tolerance. Feruloyl-CoA 6'-hydroxylase 1 (AtF6'H1), an enzyme in the phenylpropanoid pathway, has an important role in scopoletin biosynthesis. When constitutively overexpressed, $A t F 6^{\prime} H 1$ enables the production of scopoletin and its glycoside scopolin in transgenic Arabidopsis, tobacco and soybean plants as well as in tobacco BY-2 culture cells. Feeding AtF6'H1-overexpressing BY-2 cells with the scopoletin-precursor ferulic acid further increased scopoletin accumulation. This finding indicates a metabolic bottleneck upstream of F6' $\mathrm{H} 1$ in scopoletin biosynthesis. To overcome AtF6'H1 substrate limitation we co-expressed a transcription factor that globally triggers the expression of secondary metabolism-associated genes. By doing so we enormously increased the accumulation of scopoletin and scopolin in transgenic plants. We are currently investigating the applicability of different promoter/transporter combinations for regulating the temporal and spatial accumulation of scopoletin thus avoiding detrimental effects of its hyperaccumulation in transgenic plants. Crops engineered this way will be tested for their tolerance to biotic and abiotic stress in the field.

Arabidopsis nonhost resistance-associated coumarin scopoletin provides plant protection

S. F. BEYER (1), A. Beesley (1), P. F. W. Rohmann (1), S. Holger (2), U. Conrath (1), C. Langenbach (1), (1) RWTH Aachen University, Plant Physiology Department, Aachen, Germany; (2) BASF Plant Science, Limbugerhof, Germany

Scopoletin supports the response to biotic and abiotic stress. However, the potential of scopoletin for applied plant protection has remained unknown. Here, we show that mRNA transcript for the scopoletin-biosynthesis enzyme Feruloyl-CoA 6'-hydroxylase 1 (AtF6'H1) and scopoletin both accumulate in Arabidopsis leaves during postinvasion defense to Phakopsora pachyrhizi ( $P p)$. Pp is a fungus that causes Asian soybean rust, and thus threatens global soybean production. Different from Arabidopsis, scopoletin is absent from soybean leaves. In-vitro experiments disclosed fungistatic activity of scopoletin against $P p$ associated with reduced accumulation of reactive oxygen species (ROS) in fungal preinfection structures. Non-antioxidant and antioxidant molecules with a similar structure to scopoletin were inactive or much less effective at inhibiting fungal accumulation of ROS and germination of $P p$ spores. When sprayed onto Arabidopsis leaves, scopoletin also suppressed the formation of $P p$ preinfection structures and fungal penetration. However, scopoletin neither directly activated plant defense nor did it prime Arabidopsis for enhanced defense, thus indicating direct fungistatic activity as the exclusive mode of action of scopoletin in inhibiting $P p$. Because spray application of scopoletin also protected soybean from $P p$ infection the coumarin may serve as a natural fungicide, or as lead for developing near-to-nature fungicides that enable Asian soybean rust control.

Uncovering novel peptide signaling molecules intrinsic to plant-microbe interactions in Populus by leveraging high-performance mass spectrometry, neural networks, and deep learning.

P. E. ABRAHAM (1), S. Poudel (2), M. I. Villalobos Solis (2,3), H. K. Shrestha (2,3), R. J. Giannone (2), A. Matthiadis (2), U. Kalluri (2), R. L. Hettich (1), (1) Oak Ridge National Laboratory, Oak Ridge, TN, U.S.A.; (2) Oak Ridge National Laboratory, U.S.A.; (3) Department of Genome Science and Technology, University of Tennessee, U.S.A.

A major research goal is to characterize and interpret the physical, molecular, and chemical interfaces between the plant and microbes and determine their functional roles in biological and environmental systems. Small polypeptides are emerging as key signaling molecules that 
mediate cell-cell communication and biological processes that occur within and between plants and microbes. However, discovery and characterization of these polypeptides is not trivial because their post-translational processing is not well understood nor predictable. Therefore, we aimed to develop experimental and bioinformatic workflow designed to characterize unknown polypeptide molecules at the plant-microbe interface. For this study, an artificial peptide mixture was used as a benchmark to demonstrate the ability to comprehensively and accurately identify and quantify small polypeptides. From the data collected, several de novo sequencing algorithms, including DeepNovo which recently introduced neural networks and deep learning to de novo peptide sequencing, were qualitatively evaluated. Following the successful testing of this approach, small polypeptides were measured across a 2-week colonization period of Laccaria S238N or Pantoea YR343 on 5-week old Populus roots. Preliminary results suggest there are a substantial number of differentiable small polypeptides that respond across the colonization period and we are currently working to quantitatively map the de novo peptide annotations.

Ebb and flow of vascular pathogenesis through repeated gain and loss of a molecular switch

E. GLUCK-THALER (1), A. Cerutti (2), A. L. Perez Quintero (3), C. Pesce (4), A. Jauneau (2), T. Vancheva (5), J. M. Lang (3), C. Allen (6), V. V. Verdier-Michel (7), L. Gagnevin (7), B. Szurek (7), S. Cunnac (7), C. Bragard (5), J. E. Leach (3), L. D. Noel (8), J. Slot (1), R. Koebnik (7), J. M. Jacobs (1), (1) The Ohio State University, Columbus, OH, U.S.A.; (2) INRA-CNRS - LIPM, Toulouse, France; (3) Colorado State University, CO, U.S.A.; (4) University of New Hampshire, Durham, NH, U.S.A.; (5) Université Catholique Louvain-la-Neuve, Louvain-laNeuve, Belgium; (6) University of Wisconsin-Madison, Madison, WI, U.S.A.; (7) IRD UMR IPME, Montpellier, France; (8) Université de Toulouse, LIPM, CNRS-INRA, Toulouse, France

Microbes cause disease in plants through two distinct processes. Vascular pathogens move through host veins leading to widespread infection, while non-vascular pathogens remain restricted to the site of infection. The systemic nature of vascular pathogenicity poses a much greater risk to host health; yet the mechanisms underpinning this lifestyle are unknown. Here, we examine the molecular and evolutionary basis for vascular pathogenicity in Xanthomonas, a diverse genus of plant-associated bacteria that cause vascular and non-vascular diseases. As opposed to equally complex traits controlled by multiple loci, we identified a single gene, celA (encoding a cellobiosidase), that acts as a switch for vascular pathogenesis. Heterologous expression of celA in non-vascular Xanthomonas species resulted in pathotype conversion allowing for xylem colonization, while its deletion in vascular species resulted in the loss of xylem colonization, demonstrating that celA is both sufficient and necessary for vascular pathogenesis. We inferred several gains and losses of celA in Xanthomonas, where celA was acquired through horizontal transfer in what are now vascular lineages, and alternatively lost through transposon-mediated insertion in non-vascular lineages. The dynamic evolution of celA suggests that rather than representing evolutionary endpoints, vascular and non-vascular modes of infection exist on a continuum, and populations can easily flow from one end to another.

The design of immune receptors RRS1/RPS4 with diverse integrated decoys to extend recognition capacity S. WANG (1), Z. Duxbury (2), Y. Ma (3), J. D. Jones (3), (1) The Sainsbury Laboratory, Norwich, U.K.; (2) Gregor Mendel Institute, Vienna, Austria; (3) The Sainsbury Laboratory, University of East Anglia, Norwich Research Park, Norwich, U.K.

Plants have evolved an effective immune system to detect and respond to pathogens, while pathogens are highly divergent and evolve rapidly to evade plant surveillance. The ability to design immunity receptors to provide new recognition capacities against pathogens is highly desirable for crop disease control. Arabidopsis RRS1, a nucleotide-binding, leucine-rich repeat (NLR) immune receptor, paired with RPS4, responds to diverse effectors from different pathogens. The perception of effectors is through the integrated WRKY domain of RRS1. WRKY transcription factors have been reported to be the target of pathogen effectors. We set out to test whether if we swap the WRKY domain of RRS1 with diverse WRKY domains, the engineered RRS1-WRKY might be able to recognize new effectors, and thus provide new resistance resources. Like many other NB-LRR immune receptors, the structure of RRS1 is tightly fine-tuned. Many WRKY domain swaps display effectorindependent, RPS4-dependent, defense activation. We were able to engineer non-autoactive RRS1-WRKY chimeras. However, the WRKY41 and WRKY70 chimeras did not show immune response to effectors. Our work showed that the interaction of integrated domain and effector is not sufficient to activate immunity. This provides potential molecular mechanistic insights into paired immune receptors. Future work will focus on screening more WRKY domain diversity, aiming at engineering novel non-autoactive RRS1-WRKY alleles which respond to effectors.

Host-induced silencing of Aspergillus flavus genes to control preharvest aflatoxin contamination in maize K. RAJASEKARAN, J. W. Cary, M. K. Gilbert, M. D. Lebar, R. Majumdar, C. M. Sickler, Q. Wei, USDA, ARS, SRRC, New Orleans, LA, U.S.A.

RNA interference (RNAi)-mediated host-induced gene silencing (HIGS) of key Aspergillus flavus genes to reduce fungal virulence, growth and/or toxin production by the fungus in susceptible crops such as maize is a promising and consumer-friendly approach to control dangerous levels of carcinogenic aflatoxins (AF). This RNAi-mediated technology does not require production of a foreign protein in the host plant; as such, food and feed produced from these resistant lines of maize should be more acceptable to regulatory agencies and consumers. For prevention of AF contamination in maize, we targeted A. flavus genes that are critical to its growth, development, and AF biosynthesis. These include the veA and $n s d C$ genes, both global regulators required for normal $A$. flavus development and AF production and the $\alpha$-amylase gene, required for starch degradation by the invading fungus, among others. Fungal growth was monitored in transgenic kernels using a green fluorescent protein (GFP)expressing A. flavus and AF measurement was carried out by UPLC. Results from transgenic kernel infection studies demonstrated significant reductions in fungal growth, invasion, and AF production in transgenic maize RNAi lines that targeted fungal genes critical for growth and/or $\mathrm{AF}$ biosynthesis, either alone or in combination. Further details on effective reduction in aflatoxin contamination of maize kernels from several transgenic lines will be presented.

Near-saturation efficiency and novel counter-selection strategies for genome editing in Arabidopsis and Nicotiana benthamiana R. Gruetzner (1), J. Ordon (2), P. MARTIN (2), J. Gantner (2), S. Marillonnet (1), J. Stuttmann (2), (1) Leibniz Institute of Plant Biochemistry, Halle, Germany; (2) Martin Luther University, Halle (Saale), Germany 
RNA-guided nucleases based on Streptococcus pyogenes Cas9 (SpCas9) are most commonly employed for genome editing in different plant species. We mainly used transgene-encoded SpCas9 in Arabidopsis and Nicotiana benthamiana, and encountered two major bottlenecks: Efficiencies were low in Arabidopsis and separation of desired genome modifications from the $S p C$ as 9 -encoding transgene required screening of large populations in $N$. benthamiana due to multiple transgene copies. Therefore, nuclease system components were systematically evaluated for their performance in Arabidopsis, and novel counter-selection markers for use in $N$. benthamiana developed. Resulting improved systems achieve near-saturation efficiency in both model species we use, and allow the isolation of transgene-free, genome-edited individuals from PCR-screening of just a few plants. Development and benchmarking of novel genome editing vector systems, together with their use for isolation of multiple immune-compromised Arabidopsis and N. benthamiana lines, will be presented.

Dissecting the role of RNA-binding proteins in plant immunity

M. BACH-PAGES (1), F. Kaschani (2), R. A. L. Van Der Hoorn (3), A. Castello (4), G. M. Preston (5), (1) University of Oxford, U.K.; (2)

University of Duisburg-Essen, Germany; (3) Department of Plant Sciences, University of Oxford, Oxford, U.K.; (4) Department of Biochemistry, University of Oxford, U.K.; (5) University of Oxford, Oxford, U.K.

RNA-Binding Proteins (RBPs) are master regulators of gene expression and are involved in virtually all steps of RNA life from synthesis to decay. RBPs can modulate the cellular transcriptome in response to developmental, physiological or environmental cues. Importantly, recent studies have pinpointed the crucial role of RBPs in plant immunity, although the identification of the RBPs involved in this process has been collected stepwise and comprehensive approaches are missing.

We have successfully applied the cutting-edge technique 'RNA interactome capture' (RNA-IC) to mature Arabidopsis thaliana plants to determine the dynamic responses of the RNA-Binding Proteome (RBPome) to the bacterial elicitor flg22. We have been able to use RNA-IC to identify more than five hundred RBPs in Arabidopsis leaves, many of which were previously unknown, of which about $10 \%$ are differentially regulated in response to flg22, indicating that they have potential roles in plant immunity.

We have obtained mutant lines for selected RBPs and screened them for resistance to the bacterial pathogen Pseudomonas syringae pv. tomato DC3000. The results from this screen have allowed us to identify RBP mutant lines that show differential resistance to $P$. syringae pv. tomato DC3000. We are currently performing targeted functional analyses to elucidate the specific role of these RBPs in plant immunity.

Novel sensor technology using capacitive micromachined ultrasonic transducers (CMUT) for rapid detection and interrogation of hostpathogen interactions

N. Constantino, M. Mahmud, E. Sennik, C. Seok, Y. Oh, F. Yamaner, O. Oralkan, R. A. DEAN, NC State University, U.S.A.

During times of stress, plants rapidly release a wide variety of volatile organic compounds (VOCs). Each type of stress, be it abiotic or biotic, induces plants to emit unique volatile patterns, much like a fingerprint, which can activate their own defenses, attract beneficial insects, and warn neighboring plants of an impending attack. In addition, microbes including pathogens also produce specific VOCs. These unique volatile fingerprints provide an opportunity to develop low-cost, non-invasive, real-time sensor monitoring technology, which can be used to facilitate earlier and more targeted intervention of management strategies. We have designed, developed, and fabricated a new sensor technology based on capacitive micromachined ultrasonic transducers (CMUT) functionalized with selective polymers, which has shown high sensitivity for detecting VOCs produced by systemically wounded, herbivore challenged, and infected plants as well as microbial pathogens. To characterize each unique VOC fingerprint, GC-MS analysis was performed in tandem with the CMUT analysis. By identifying which VOCs are emitted during different types of stress, the CMUT sensor can be made more selective and sensitive by selecting polymers that interact more exclusively with the VOCs emitted by distressed plants. Examples of the success of using CMUT sensors for early detection as well as specific VOC species associated with abiotic or biotic challenge and from microbial pathogens will be presented.

Regulation of a geminivirus late gene promoter by PRC2

G. SUNTER (1), E. P. Regedanz (2), M. Berger (3), D. M. Bisaro (2), (1) University of Texas at San Antonio, San Antonio, TX, U.S.A.; (2) The Ohio State University, Columbus, OH, U.S.A.; (3) Texas Woman's University, U.S.A.

Geminiviruses are small ssDNA viruses causing significant yield loss in agriculturally important crops. Upon entering the nucleus, the viral genome is converted by host enzymes into a dsDNA replicative form, which associates with histones to form non-integrating episomes that facilitate virus replication and transcription and serve as targets of host defense pathways. As viral chromatin is formed de novo in infected cells, geminiviruses are unique models for examining mechanisms that target and establish epigenetic modifications. Polycomb Repressive Complex 2 (PRC2) is an important repressive regulator of developmental processes via its ability to deposit H3K27me3, but how PRC2 activity is regulated at target genes is unclear. We propose that geminivirus gene expression is a model for PRC2 control, as we have found that $\mathrm{H} 3 \mathrm{~K} 27 \mathrm{me} 3$ is localized to the viral coat protein (CP) promoter, which is repressed early in infection. The CP promoter is bound by TCP24, a plant-specific transcription factor, which has been implicated in PRC2 recruitment. Importantly, TCP24 mRNA levels decrease in virus-infected cells. Thus, we hypothesize that TCP24 recruits PRC2 to inhibit premature CP expression and allow viral genome amplification. Once a threshold of genomes is produced, reduced TCP24 levels allow CP expression, promoting virion assembly. Our studies offer insight into the temporal control of the geminivirus infection cycle, as well as principles underlying developmental gene regulation.

Non-integrative viral-delivery system for plant genome editing by CRISPR/Cas9

J. KIM (1,2), J. Yu (3), J. Woo (1,2), S. Bae (3), S. In (1,2), E. Park (1,2,4), D. Choi (1,5), (1) Department of Plant Science, Seoul National University, Seoul, Korea, Republic of (South); (2) Plant Immunity Research Center, Seoul, Korea, Republic of (South); (3) Department of Chemistry, Hanyang University, Seoul, Korea, Republic of (South); (4) Department of Molecular Biology, University of Wyoming, Laramie, WY, U.S.A.; (5) Plant immunity research center, Seoul National University, Seoul, Korea, Republic of (South)

The CRISPR/Cas (clustered regularly interspaced short palindromic repeats/CRISPR-associated proteins) system has been tremendously advanced in a short period due to its usability to manipulate a specific position on genome. However, compared with its application for other 
species, plant genome editing by the CRISPR/Cas system is less effective and limit on specific plants. The current CRISPR/Cas methods in plants are necessarily accompanied by the regeneration of plants, causing somaclonal variations for genome-edited transgenic plant. Our goal is to develop an optimized CRISPR/Cas tool for plant genome editing in progeny via viral delivery system without the plant regeneration process. Most virus systemically infect in plants without integration in genome. We utilized Tobacco rattle virus system which have wide host range as a shuttle, with several modifications on SpCas9 for the efficiency of genome editing and viral sub-promoter for powerful release of gRNA.

Plant Extracellular Vesicles Interact with Pathogenic Fungi and Can Alter their Morphology and Gene Expression B. D. RUTTER, H. Zand Karimi, R. W. Innes, Indiana University, Bloomington, IN, U.S.A.

During fungal infections, plant cells secrete small ( 120 nm in diameter), membranous compartments known as extracellular vesicles (EVs). Plant EVs are enriched for stress-related proteins and small RNAs. They accumulate underneath sites of fungal penetration and around intruding haustoria. While EVs are though to contribute to defense, little is known about their functions. To better understand the interactions between plant EVs and fungal pathogens, we incubated EVs isolated from Arabidopsis leaves with the hemibiotrophic fungus, Colletotrichum higginsianum. Plant EVs labeled with a fusion marker protein, GFP-PENETRATION1 (PEN1), accumulated on fungal secondary germ tubes. GFP-PEN1 EVs did not accumulate on formaldehyde-fixed fungi, suggesting that the interaction requires an active process. Fungus-associated EVs were also protected from detergent treatment, suggesting that they may have been taken up. Fungal spores germinated in the presence of EVs, though still viable, had an altered morphology. Finally, Arabidopsis EVs were able to deliver a transgenic small RNA into C. higginsianum, resulting in the down regulation of important fungal genes. Combined, our results suggest that plant EVs have specific interactions with pathogenic fungi that can alter fungal morphology and gene expression.

Plant A20/AN1 proteins play important roles in the SA-mediated antiviral immunity L. CHANG (1,2,3), H. H. Chang (1), Y. S. Chiu (1), J. C. Chang (1), D. W. Hsu (4), Y. Tzean (1), H. H. Yeh (1,5), (1) Agricultural Biotechnology Research Center, Academia Sinica, Taiwan; (2) Molecular and Biological Agricultural Sciences Program, Academia Sinica, Taiwan; (3) Graduate Institute of Biotechnology, National Chung-Hsng University, Taiwan; (4) Department of Biotechnology, National Kaohsiung Normal University, Taiwan; (5) Department of Plant Pathology and Microbiology, National Taiwan University, Taiwan

Salicylic acid (SA) plays important roles in antiviral immunity; however, the detailed mechanism of SA mediated-antiviral immunity remains elusive. We previously identified an A20/AN1 zinc-finger domain-containing protein, Pha13, which serves as a hub to regulate SA-mediated immune response for antiviral immunity (Chang et al., 2018). In addition to Pha13, we identified Pha21 that shares $69.5 \%$ amino acid sequence identity to Pha13 and is also involved in SA-mediated antiviral immunity. Similar to Pha13, Pha21 is early-induced by SA treatment and virus infection, localize in nucleus, exhibits self-E3 ligase and ubiquitin binding activity mainly through the A20 domain, and is involved in the expression of orchid homolog of NPRI (a master regulator in SA-mediated plant immunity), PhaNPR1. Transgenic orchid and Arabidopsis overexpressing Pha21 is similar to Pha13 in enhanced antiviral immunity. However, in contrast to Pha13, Pha21 negatively regulates two Pha13 positively regulated genes involved in SA-mediated antiviral immunity, PhaRdRI (orchid homolog of RNA-dependent RNA polymerase 1) and PhaGRXC9 (orchid homolog of Glutaredoxin-C9). Because Pha13 expression is 15 times higher than Pha21, Pha13 regulated PhaRdR1 and PhaGRXC9 still play important roles in SA-mediated antiviral immunity. Together, our results suggest that although both Pha13 and Pha21 are involved in SA-mediated plant immunity, Pha13 and Pha21 function through distinctive manner.

Two LSD1-like Histone Demethylases Suppress Immune Response in Arabidopsis.

S. W. Noh, H. W. JUNG, Dept. of Applied Bioscience, Dong-A University, Busan, Korea, Republic of (South)

Plants need to build up diverse defense barriers against unpredictable pathogen infection, but also to reduce an energy consumption in the absence of pathogen infection. Recent lots of studies propose that epigenetic modifications including histone modification are responsible for maintaining the balance between growth and immunity, as well as priming syndrome. Methylation at lysine 4 residue of histone $\mathrm{H} 3$ is regarded as an active mark for transcription. Here we addressed roles of two different LSD1-like genes encoding lysine-specific demethylase, LDL1 and LDL2 in immune response against Pseudomonas infection. $l d l l$, $l d l 2$ and $l d l l l d l 2$ mutants exhibits enhanced disease resistance to the infection with virulent Pseudomonas strains, and resistance response shown in double mutant are more stable and stronger than that in single mutants. Two different mutants corresponding to LSD1-like genes, including FLD, show wild type-like response to virulent Pseudomonas infection. Furthermore, primary immunization by avirulent strain of Pseudomonas carrying AvrRpt 2 confers systemic resistance in distal leaves of ldll and $l d l 2$ to secondary virulent Pseudomonas infection, whereas $l d l 1$ ldl2 double mutant is hyper-resistance regardless of the primary immunization. Levels of di- and tri-methylation of histone $\mathrm{H} 3 \mathrm{~K} 4$ are increased in $l d l l l d l 2$ mutants, compared with those in wild-type plants. We present several pathophysiological changes in the single and double mutants during immune response.

\author{
Mycoviruses infecting Fusarium species isolated from Ethiopian wheat fields and their potential association with hypovirulece of a \\ Fusarium Head Bright fungus \\ Y. MIZUTANI (1), A. Adane (2), K. Uesaka (3), H. Kondo (2), H. Suga (4), N. Suzuki (2), S. Chiba (1), (1) Nagoya Univ., Japan; (2) Okayama \\ Univ., Japan; (3) Nagoya University, Japan; (4) Gifu Univ., Japan
}

Diverse mycoviruses have been found in a wide range of fungal species during the past several decades, including increasing number of hypovirulence-conferring viruses. In this study, a hypovirulent strain of the Fusarium Head Bright fungus, Fusarium boothii, was isolated from Ethiopian wheat samples. The strain Ep-BL13 was infected by a unique virus with $12.8 \mathrm{~kb}(+)$ ssRNA genome that resembles to the order Tymovirales but dissimilar to some extent with the order members. Interestingly, very close strains of a novel mitovirus $(2.8 \mathrm{~kb}(+) \mathrm{ssRNA}$ genome) were commonly infected to virulent $F$. boothii strains Ep-BL14 and Ep-N28, as well as the hypovirulent Ep-BL13, suggesting a possible etiology of hypovirulence by the tymo-like virus. Although virulence to wheat plants is unknown, several Fusarium strains were isolated as mycovirus-infected isolates. Viruses identified involve a hypovirus, a polymycovirus, an alternavirus and the most prevalent mitoviruses. 
Together with previously isolated mycoviruses, taxonomic consideration of obtained mycoviruses and possible their behavior in wheat fields will be discussed.

Three novel deoxynivalenol-degrading bacteria isolated from Poaceae planted soils in Japan. H. MORIMURA, S. Chiba, D. Takemoto, K. Kawakita, I. Sato, Nagoya Univ., Japan

Deoxynivalenol (DON) is a hazardous and globally prevalent phytotoxin and mycotoxin in cereals. DON commonly accumulates in the grains of wheat, barley and other cereals affected by Fusarium head blight which was caused by Fusarium graminearum species complex. The cereals which are accumulated DON by $F$. graminearum can contaminate agricultural systems, resulting in harm to both humans and livestock, and leading to large economic losses. Since production and storage are threatened by DON contamination, a more effective approach for the degradation of DON is required. A biological approach for the degradation of DON using DON-degrading bacteria appears to be promising, although information about DON-degrading bacteria is limited. In this study, we isolated three novel DON-degrading bacteria from Poaceae planted soils. The obtained bacteria were classified into genera Sphingomonas, Nocardioides and Marmoricola and they were named strain IMS, HEM and KOM respectively. DON-assimilating activities of the bacteria were tested and the result showed that only strain HEM had the capacity. We conducted experiment on DON-degrading activities used by three different types of liquid media containing DON. Strain KOM had obviously DON-degrading activity when the bacterium was cultured in 3-fold diluted R2A medium including DON. We carried out examination on nivalenol (NIV)-degrading activity using the bacteria. As a result, it was found that strain HEM had an ability to degrade NIV.

Host-induced hairpin RNA-mediated gene silencing of Foc SGE1 for development of resistance against Fusarium wilt in banana R. F. LETCHUMANN, J. Y. Paul, J. Kleidon, R. M. Harding, J. L. Dale, U. K. S. Shekhawat, Queensland University of Technology, Brisbane, QLD, Australia

Banana is one of the most important fruit crops in the world. Particularly export bananas are grown as monocultures which are incredibly vulnerable to disease outbreaks. Fusarium wilt caused by Fusarium oxysporum f. sp. cubense (Foc) is considered one of the most economically damaging fungal disease of banana. Genetic modification provides the opportunity to introduce resistant traits to commercial cultivars as opposed to developing new cultivars by conventional breeding. We are investigating the use of host-induced gene silencing as a strategy to mediate resistance in transgenic bananas against Fusarium wilt. A small group of cysteine-rich secreted proteins, called "Secreted In Xylem" (SIX), coded on the mobile pathogenicity chromosome of Fusarium oxysporum f. sp. lycopersici (Fol), are known to function as important effectors in causing Fusarium wilt of tomato. Both Foc race 1 and Foc Tropical Race 4 (TR4) possess sequences encoding close homologs of these Fol SIX proteins. A master regulator known as SIX Gene Expression 1 (SGE1) has been shown to control transcription of these SIX genes in Fol. We postulate that targeting the $S G E 1$ homolog of $F O C$ and by implication SIX proteins can dramatically reduce the infection capacity of $F o c$. We have generated transgenic banana plants to express small interfering RNAs targeted towards the Foc SGE1 gene sequence as a strategy to produce effective resistance against $F O C$.

Plant genome editing using the CRISPR-Cas9 system to establish bs5 resistance gene in tomato A. ORTEGA (1), J. Pham (2), M. J. Cho (2), B. J. Staskawicz (3), (1) University of California Berkeley, Berkeley, CA, U.S.A.; (2) Innovative Genomics Institute, University of California, Berkeley, CA, U.S.A.; (3) Department of Plant and Microbial Biology, University of California, Berkeley, Berkeley, CA, U.S.A.

Agricultural productivity is severely affected by plant pathogens. Sustaining efficient productivity is essential for maintaining long-term food security. Bacterial spot disease is globally spread and has caused major productivity losses in commercial crops. A recessive allele of the $B s 5$ gene, $b s 5$, from pepper confers disease resistance against Xanthomonas euvesicatoria, the causative agent of bacterial spot of pepper and tomato. The causative mutation of $b s 5$ is an in-frame six nucleotide deletion in the third and last exon. The Bs 5 gene is conserved among Solanaceae and encodes a protein with a different structure to that of nucleotide binding leucine-rich repeat proteins (NLRs). Our objective is to study the underlying mechanisms of $b s 5$ resistance using tomato as a model system. Pepper and tomato are not sexually compatible, thus classical plant breeding strategies cannot be employed to establish bs 5 resistance. Using Cas 9 gene editing tools, we have introduced knockout mutations in the endogenous $B s 5$ gene in tomato while simultaneously introducing the pepper $b s 5$ genomic region. Preliminary experiments suggest $b s 5$ confers disease resistance against bacterial spot in these transgenic tomatoes. Using Cas9-mediated homology directed repair, we will introduce the $b s 5$ allele in the endogenous tomato $B s 5$ gene and evaluate disease resistance. Studying $b s 5$ resistance will demonstrate the feasibility of the $b s 5$ allele in tomatoes and other commercial Solanaceous crops.

flg22-induced root growth-immunity trade-offs can be overcome by de-repression of cell cycle arrest R. EICHMANN, J. Finch, P. Schafer, The University of Warwick, U.K.

Plants use plasma membrane receptors to detect conserved molecules of potentially harmful microbes, and thereupon activate pattern triggered immunity (PTI) to protect plants against pathogen invasion. However, PTI activation appears to have some trade-offs and is well known to inhibit plant growth and yield. These growth-immunity trade-offs may impair efforts to breed for plants with high resistance and full growth and yield levels. In Arabidopsis, flg22 (active flagellin epitope) detection by the receptor FLAGELLIN-SENSING 2 has been key to uncovering PTI regulation and we scrutinised the effects of flg22 on root growth inhibition. We found that flg22 inhibits cell division as a key growth determinant in the root meristem but does not affect stem cell niche function. Moreover, flg22-induced PTI affects the G2-mitosis pathway and specific stimulation of this pathway by over-expressing CYCLIN-DEPENDENT PROTEIN KINASE B1;1 resulted in regular growth upon full PTI activation. Our findings demonstrate the feasibility of uncoupling negative growth-immunity trade-offs. Considering the conservation of cell cycle regulation, it may inform breeding strategies for crops with high resistance traits without impairing growth. 
Establishment of anthracnose disease resistance of maize by Cas endonuclease-mediated mutagenesis and host-induced gene silencing K. M. PATHI (1), N. Budhagatapalli (1), M. Groß (2), H. B. Deising II (3), J. Kumlehn (4), (1) Leibniz Institute of Plant Genetics and Crop Plant Research (IPK), 06466 Gatersleben, Germany; (2) Phytopathology and Plant Protection, Martin Luther University, Halle, Germany; (3) MartinLuther-Universitat Halle-Wittenberg, Halle (Saale), Germany; (4) Leibniz Inst of Plant Genetics \& Crop Plant Research, Gatersleben, Germany

Maize anthracnose is caused by the devastating hemibiotrophic fungal pathogen Colletotrichum graminicola which accounts for up to $80 \%$ of the yield loss in maize production worldwide. During the infection process, C. graminicola takes the advantage of maize oxylipins (products of the 9lipoxygenase pathway) for its spore production and successful colonization, which renders maize LIPOXYGENASE 3 (ZmLOX3) a host susceptibility gene. Therefore, we are aiming to generate loss-of-function mutations of $Z m L O X 3$ using Cas endonuclease technology to establish durable resistance of maize to infection by $C$. graminicola. The regenerated $\mathrm{T}_{0}$ plants were efficiently mutated at the target sites with predominantly homozygous and bi-allelic mutations. The majority of mutations detected in $\mathrm{T}_{0}$ plants were stably transmitted to the $\mathrm{T}_{1}$ generation, plants with mutations are currently being subjected for the infection assays. Besides this approach, we are pursuing a host-induced gene silencing (HIGS) strategy to control the anthracnose disease. HIGS has emerged as an effective principle for the improvement of plant resistance by silencing genes that are essential for the pathogens. In our approach, fungicide target genes (i.e. $\beta$-TUBULIN and SUCCINATE DEHYDROGENASE) were used as potential HIGS targets, since these genes are well established as being indispensable for fungal growth and pathogenicity. Infection assays will be carried out once we obtained homozygous transgenic maize plants.

Secondary metabolite expression could shape the biocontrol efficiency of Pseudomonas bacteria against plant parasitic nematodes S. CLOUGH (1), P. E. Urwin (2), V. Friman (3), (1) University of York, York, U.K.; (2) University of Leeds, Leeds, U.K.; (3) University of York, U.K.

Soil-borne pathogens, such as plant parasitic nematodes, are a significant threat to crop production. One way to control these nematodes in a more environmentally friendly manner is to use naturally occurring plant growth promoting bacteria that can suppress nematodes via production of secondary metabolites including 2,4-diacetylphloroglucinol (DAPG) and cyclic lipopeptides. DAPG is a universal antimicrobial produced by fluorescent Pseudomonas bacteria and is widely studied. However, the production and the role of other metabolites, such as cyclic lipopeptides, are less well understood. We investigated this using 8 rhizospheric Pseudomonas strains and C. elegans nematode as our model system. By using feeding and nematode survival experiments on bacterial lawns, we found clear Pseudomonas strain-dependent inhibition effects. Specifically, two strains, CHA0 and Pf-5, were really effective causing $100 \%$ nematode death within $3 \mathrm{~h}$ of bacterial exposure. Based on initial genome analysis we identified potentially important metabolic clusters of a subgroup of cyclic lipopeptides called "orfamides" in the genomes of these two strains. Together these results suggest that orfamides produced by Pseudomonas bacteria could contribute to the killing of C. elegans. Ongoing work to investigate the effects of these strains on plant parasitic nematodes through in vivo and in vitro experiments will also be discussed.

Exploring the sound-modulated delay in tomato ripening through expression analysis of coding and non-coding RNAs S. K. Kim (1), J. Jung (1,2), C. M. RYU (1,3), (1) KRIBB, Daejeon, Korea, Republic of (South); (2) KAIST, Daejeon, Korea, Republic of (South); (3) University of Science and Technology, Daejeon, Korea, Republic of (South)

Sound is omnipresent in nature. Recent evidence supports the notion that naturally occurring and artificially generated sound waves induce interand intracellular changes in plants. These changes, in turn, lead to diverse physiological changes, such as enhanced biotic and abiotic stress responses, in both crops and model plants. We previously observed delayed ripening in tomato fruits exposed to $1 \mathrm{kHz}$ sound vibrations for $6 \mathrm{~h}$. Here, we evaluated the molecular mechanism underlying this delaying fruit ripening by performing RNAsequencing analysis of tomato fruits at 6 $\mathrm{h}, 2 \mathrm{~d}, 5 \mathrm{~d}$ and $7 \mathrm{~d}$ after $1 \mathrm{kHz}$ sound vibration treatment. Bioinformatic analysis of differentially expressed genes and non-coding small RNAs revealed that some of these genes are involved in plant hormone and cell wall modification processes. Ethylene and cytokinin biosynthesis and signaling-related genes were downregulated by sound vibration treatment, whereas genes involved in flavonoid, phenylpropanoid and glucan biosynthesis were upregulated. Furthermore, we identified two soundspecific microRNAs and validated the expression of the pre-microRNAs and the mRNAs of their target genes. Our results indicate that sound vibration helps to delay fruit ripening through the sophisticated regulation of coding and non-coding RNAs and transcription factor genes.

\section{Phenomics for plant quantitative disease resistance}

J. STASSEN, F. Chatziavgerinos, J. Ton, C. Voigt, S. Rolfe, The University of Sheffield, Sheffield, U.K.

Quantitative disease resistance (QNDR) is a broad range resistance phenotype with the potential to revolutionise crop protection. QNDR protects plants against a broad-spectrum of taxonomically unrelated diseases and is based on multiple resistance genes, each contributing to different defence mechanisms that act additively. Accordingly, QNDR provides 'strength-in-depth' crop protection with increased durability and, hence, sustainability. We are using phenomics techniques to characterise QNDR in plant pathogen-interactions. Using wheat and Zymoseptoria tritici as a case study we will discuss how imaging methods can quantify the dynamic and heterogeneous effects of disease progression and host response. Multispectral imaging (MSI) can quantify lesion development, underlying processes (e.g. chlorosis and necrosis) and host response (stress associated pigments). Chlorophyll fluorescence imaging can quantify disease development, but also host physiology (e.g. increases in photosynthetic assimilation rate outside of lesions). Phenomics analysis of individual plants can be scaled to field use, or inform the development of low cost phenotyping systems for adoption by the wider scientific and agricultural communities. Alternatively, microscopy provides information concerning mechanisms underpinning resistance. Ultimately, the concerted efforts by biologists, computer scientists and engineers in developing phenomics applications can greatly enhance approaches to breeding for QNDR.

Functional analysis of Argonaute3 in Phytophthora parasitica

J. XU (1), Y. Li (2), Q. Zhang (1), G. Huang (3), X. Qiang (2), W. X. Shan (2), (1) College of Plant Protection, Northwest A\&F University, Yangling, China; (2) College of Agronomy, Northwest A\&F University, Yangling, China; (3) College of Life Sciences, Northwest A\&F University, Yangling, China 
Oomycetes, particularly Phytophthora species, infect more than one hundred kinds of plants and cause devastating crop diseases, such as potato late blight. It is of importance to understand the mode of action of key pathogenic factors. Argonaute, named after the Argonauta argo, was firstly found in Arabidopsis. The Argonaute protein is highly conserved in both prokaryotes and eukaryotes. It is thought to regulate gene expression by binding DNA or RNA. Further studies demonstrate that it plays an important role in the sRNA biogenesis. Nevertheless, the function of Argonaute proteins, especially their potential function in plant-microbe interaction is largely unknown. Using the Phytophthora parasitica, a model oomycete pathogen, we have got eleven $P p A G O 3$ knock-out mutants through CRISPR-Cas9 system. Compared with wildtype strain, the $P p A G O 3$ mutants exhibit much vigorous pathogenicity and insensitivity to abiotic stress. Combining with small RNA sequencing and RNA transcription sequencing of $P p A G O 3$ mutant, we found specific small RNA species change dramatically, accompanied with the dramatic alternation of target genes. Taken together, we speculate that $P p A G O 3$ plays a key role in regulating the pathogenicity of Phytophthora. Results are presented to decipher the regulating mechanism as well as its biological significance for novel crop disease resistance.

\section{Odontoglossum ringspot virus replicase uncouples RNA silencing suppressor and replication activities in Cymbidium mosaic virus mixed-infection synergism}

S. C. LEE (1), H. Pai (1), M. H. He (1), Y. H. Hsu (2), N. S. Lin (1), (1) Institute of Plant and Microbial Biology, Academia Sinica, Taipei, Taiwan; (2) Graduate Institute of Biotechnology, National Chung Hsing University, Taichung, Taiwan

Plant RNA viruses encode RNA silencing suppressors (VSRs) to counteract with the host RNA silencing system for successful infection. Synergism in virus-virus and virus-host interactions during mixed infection might be contributed by VSRs, with an unclear mechanism. Mixed infection with two important orchid viruses, Cymbidium mosaic virus (CymMV) and Odontoglossum ringspot virus (ORSV), produced synergistic effects in symptom intensification. In this study, co-infection of CymMV and ORSV infectious cDNA clones showed similar synergistic effects as isolated wild-type viruses and were beneficial to enhanced accumulation and systemic movement of CymMV but not ORSV in both orchid and tobacco. Among CymMV- and ORSV-encoded proteins, the ORSV replicase P126 showed strong VSR activity. Accumulation of CymMV could be enhanced when inoculated plants expressed P126, so P126 is the key determinate of synergistic effects in CymMV. Analysis of P126 mutants showed VSR activity attenuated and protein stability decreased in mutants with one amino acid substitution, Met to Arg (P126$\mathrm{R}$ ), in the helicase domain but not in Met to Ala (P126-A) or Ser (P126-S) mutants. However, all of these ORSV P126 mutants showed abolished replication. Our results provide evidence to support that ORSV P126, a viral VSR, could be a key determinate in CymMV and ORSV synergism, and the function of P126 in virus replication is distinct for its VSR activity.

\section{Revealing How SA187 Induces Salt Tolerance in the Model Organism Arabidopsis thaliana: Functional Characterization of GWAS} Candidate.

H. ALZUBAIDY (1), A. De Zélicourt (2), K. Mariappan (3), S. B. Satbhai (4), W. Busch (4), H. Hirt (3), (1) King Abdullah University of Science and Technology, Thuwal, Saudi Arabia; (2) Institute of Plant Sciences Paris Saclay, France; (3) King Abdullah University of Science and Technology, Saudi Arabia; (4) Salk Institute for Biological Studies, U.S.A.

In a world with a rapidly growing population, clear-cut solutions should be found to increase crop yield in order to provide enough food for everyone. However, abiotic stresses are among the most limiting factors to achieve this goal. A possible solution might come from studying the plant microbiota communities and their effects on plants under stress. Indeed, rhizophere and endosphere microbial communities are distinct from the surrounding soil, and these specific communities highly contribute to the health and growth of plants. We identified a beneficial plant microbe Enterobacter sp. SA187 that induces plant tolerance to abiotic stresses. However, the key genes for this beneficial interaction between the plant and the bacterium are unknown. Here we show that Ethylene Receptor Sensor 2 (ERS2) acts as a positive regulator of plant-bacterial interaction. We identified the ERS2 gene from a Genome-Wide Association study. When colonized with SA187, we found that mutant ers 2 plants are compromised in fresh weight and beneficial index under salt stress conditions. Our results confirm the important role of ethylene signaling in this beneficial interaction to enhance plant stress tolerance of the model plant Arabidopsis in vitro and in open field agriculture of crops. Taken together, our work shows that ethylene is a key signal from beneficial bacteria to the host plant to enhance tolerance to abiotic stress thereby increasing yield and biomass.

Novel antibiotic producting bacteria from the phyllosphere of Arabidopsis thaliana

S. QI, Gent university, Gent, Belgium

Recent studies have found that cryptic antibiotic biosynthetic clusters compounds in soil and phyllosphere microbiota could be induced by coculture with microbes from the same niche. In this study, we compiled a library of 198 genetically distinct strains isolated from the phyllosphere and rhizosphere of Arabidopsis thaliana, enriched for the known antibiotic-producing groups Actinobacteria, Bacillales, Burkholderiales and Pseudomonads. We tested these isolates in co-culture in more than 10000 pairwise combinations for antibiotic activity against two human pathogens of the ESKAPE group: Acinetobacter baumannii and Staphylococcus aureus. Our results show that the phyllosphere is enriched in antibiotic-producing strains, including some new taxa, with the most productive combinations resulting from the interactions between Proteobacteria and Bacteroidetes. Overall, although the results revealed that the number of antibiotic-producing strains was much higher in cocultures than monocultures, only around $10 \%$ of all combinations were positive, indicating that induction of cryptic phenotypes is highly specific Together, our research shows that the phyllosphere can be a promising environment for the isolation of novel antibiotic-producing microorganisms.

Double trouble, co-inoculation of potato with both the early and late blight pathogens S. M. BROUWER (1), C. Million (1), A. Andriani (2), P. J. Wolters (2), E. Andreasson (3), V. G. A. A. Vleeshouwers (4), L. J. Grenville-Briggs (3), (1) Swedish University of Agricultural Sciences (SLU), Alnarp, Sweden; (2) Wageningen University and Research (WUR), Wageningen, Netherlands; (3) Swedish University of Agricultural Sciences, Alnarp, Sweden; (4) Plant Breeding, Wageningen University and Research, Wageningen, Netherlands 
Simultaneous multispecies diseases are understudied in phytopathology but studies in animal and human pathology have shown that the presence of multiple pathogens can alter the virulence, and course of diseases, and are important drivers of epidemiological dynamics. Potato production worldwide is plagued by multiple pathogens, amongst which are Phytophthora infestans and Alternaria solani, the causal agents of potato late and early blight respectively. While these pathogens are successful on their own, observations of both pathogens present in the same field at the same time, led us to wonder about their effect when present together. Hence, we investigate the effects of co-inoculation and sequential inoculation of both $P$. infestans and A. solani on disease development in potato. The infection process is followed using confocal microscopy, measurements of lesion development, and time-resolved transcriptomics of all three organisms during early infection. We found that leaves infected with both pathogens simultaneously, developed larger necrotic lesions morphologically resembling early blight lesions, but with both pathogens present and sporulating within the lesion. Thus, we hypothesize that the necrotroph $A$. solani benefits from the presence of the hemibiotroph $P$. infestans. Transient expression studies with effectors of $P$. infestans and $A$. solani, followed by disease tests further substantiate our observed findings and indicate altered virulence phenotypes.

Ecological implications of microscopic leaf wetness on the phyllosphere M. Grinberg (1), T. OREVI (1), S. Steinberg (1), N. Kashtan (2), (1) The Hebrew University of Jerusalem, Israel; (2) The Hebrew University of Jerusalem, Rehovot, Israel

Microscopic wetness occurs in many microbial habitats including the phyllosphere - the above ground parts of plants - which is a huge microbial habitat of global importance. There is increasing evidence that during daytime, microscopic wetness in the form of thin films and droplets, invisible to the naked eye, persist on leaf surfaces worldwide. This wetness results from deliquescence of hygroscopic aerosols that are ubiquitous on leaves. Little is known on the impact of such microscopic wetness on the ecology of leaf bacteria. Here we study how such microscopic wetness affects central features of microbial life, including cells' survival. We developed an experimental system that create microscopic wetness on artificial surfaces. Using advanced microscopy and image processing we analyzed microscopic wetness, bacterial self-organization and survival. We revealed that stable microscopic droplets are formed around bacterial aggregates on a drying surface under moderate relative humidity, due to pinning and deliquescence. Notably, droplet size correlated with aggregate size, and cell survival was higher within larger droplets. Similar results were observed for $>15$ species, two of them - Pseudomonas fluorescens and P. putida - studied in depth here. The formation and retention of microscopic droplets around bacterial aggregates during daytime are likely central features of the leaf surface as a microbial habitat and key for bacterial survival on plant leaf surfaces.

Development of a flavonoid-responsive transcriptional biosensor

I. Bervoets (1), L. H. CHAPPELL (2), B. De Paepe (3), R. Soldan (2), V. K. Ramachandran (2), G. M. Preston (2), (1) Vrije Universiteit Brussel, Belgium; (2) University of Oxford, U.K.; (3) University of Ghent, Belgium

Flavonoids are an extensive group of secondary plant metabolites that exhibit a broad spectrum of biological activities, including acting as physiological regulators, chemical messengers and signals in the legume-Rhizobium symbiosis. Flavonoids also display antimicrobial and allelopathic activity. One metabolite central in the biosynthesis of flavonoids is naringenin, which is noted, amongst others, for its antiinflammatory, antioxidant and DNA protective qualities. Given the importance of this metabolite, the development of an accurate and rapid analytical tool to detect the presence of naringenin is necessary. Previous work led to the development of naringenin-responsive transcriptional biosensors that were characterised in Escherichia coli. We have built on this to transfer the biosensors to broad-range vectors for use in a wide range of gram-negative bacteria. In addition to the fluorescent proteins originally used as outputs, we have developed naringenin-inducible reporter constructs incorporating luminescent lux and ilux operons. We have validated the activity of these biosensors in Pseudomonas fluorescens and Pseudomonas syringae and demonstrated increased sensitivity to naringenin using the luminescent lux/ilux operons. We are currently using these biosensors to study the spatiotemporal biosynthesis of naringenin in planta with Phaseolus vulgaris as a model plant and testing their activity in Rhizobium strains.

Verticillium longisporum induces SA-responsive gene expression in roots of Arabidopsis thaliana in the absence of SA L. ULRICH, J. Schmitz, C. Thurow, C. Gatz, Georg-August-University Göttingen, Göttingen, Germany

Infection of $A$. thaliana with the soil-borne vascular pathogen $V$. longisporum leads to the induction of 329 genes in roots 10 days after infection. 96 of these genes are known to be regulated by salicylic acid (SA) in the shoot. Infections in the SA-deficient sid2 mutant reveal the induction of these 96 SA-responsive genes in roots at 10dpi in the confirmed absence of SA. Among these genes is SARD1, a master regulator of SA biosynthesis in shoots. Many of the $96 \mathrm{~V}$. longisporum-induced genes contain SARDI binding sites in their promoters and induction of a number of them in roots is abolished in sard1xcbp60g mutants. We hypothesise that SARD1 directly controls defence genes in the absence of SA in roots, while in shoots it regulates the expression of the same set of genes indirectly through induction of the SA biosynthesis gene isochorismate synthase1 (ICS1). The contribution of other signaling components known to be involved in SA-related defence responses (NPR1, FMO1) to the induction of $S A R D 1$ and its target genes in roots is under investigation. Taken together, this study reveals that signaling networks leading to expression of defined target genes are constructed in a different way in roots as compared to shoots.

Influence of T6SS in temporal and spatial colonization of Agrobacterium tumefaciens during infection and tumorigenesis processes C. F. CHIEN (1), E. M. Lai (2), (1) Institute of Plant-Microbe Biology (IPMB), Academia Sinica, Taipei, Taiwan; (2) Academia Sinica, Taipei, Taiwan

Agroabcterium tumefacienscauses crown gall disease by delivering transferred (T)-DNA to plant cell via type IV secretion system (T4SS). Type VI secretion system (T6SS), which is found in nearly $25 \%$ of the Gram(-) bacteria, is also widespread in A. tumefaciensgenomospecies. We previously showed that $A$. tumefaciensexhibits T6SS-mediated antibacterial activity to enhance competitive growthin planta. However, whether or when T6SS impacts Agrobacterium competitive colonization in its ecological niche remains unexplored. Here, we aim to analyze the influence 
of T6SS in temporal and spatial colonization of A. tumefaciens during infection and tumorigenesis processes. We engineered both wild type C58 and T6SS mutant with each harboring different fluorescent proteins but conferring same antibiotic resistance gene. Each pair of four combinations was infected on stem of tomato seedlings and wounded sites were collected for analysis with a time course experiment up to 35 days. To this end, we observed modest but significant higher cell numbers of wild type than T6SS mutant one or two weeks after infection. All four combinations induced similar size of tumors. This result suggested that T6SS plays a role in colonization efficiency, which however does not impact tumorigenesis. We are currently in the process to visualize the spatial localization of colonized strains in tumors and analyze the relative expression levels of T6SS and T4SS genes during infection processes.

Origin, expansion, and diversity of the NLR integrated domains in the grasses

J. Russell (1), S. Holden (1), H. J. Brabham (1), M. Barrett (2), M. J. MOSCOU (1), (1) The Sainsbury Laboratory, University of East Anglia, Norwich Research Park, Norwich, U.K.; (2) Botanic Gardens and Parks Authority, West Perth, Australia

Membrane and intracellular immune receptors recognize conserved and variable components of plant pathogens or the modification of host proteins. Characterization of nucleotide-binding, leucine-rich repeat (NLR) family recently identified prolific chimeric fusions with domains from diverse protein families. These integrated domains (ID) are non-random and derive from host proteins that are common targets of pathogen effectors. In previous work, the grass-specific major integrated clade (MIC1) was found to be enriched with NLRs with diverse ID. MIC1 members include the rice genes Pi-ta (NLR-TRX) and RGA5 (NLR-HMA), and barley Rpg 5 (NLR-Kinase). We set out to investigate the evolutionary origin, expansion, and diversity of NLRs within MIC1 in the grasses (Poaceae). Using the genomes of Streptochaeta angustifolia (Poaceae) and Ecdeiocolea monostachya, a grass-like species, we found that integration in MIC1 clade members emerged after speciation of $S$. angustifolia but prior to the radiation of BEP and PACMAD grasses. We developed a bioinformatic pipeline to use publicly available RNAseq data to assemble and annotate NLRs with ID. Investigation of NLR diversity in 269 accession from 115 species in the Poales identified 62,366 full length NLRs with 4,455 NLR-ID. ID diversity spans $>40$ domains with the most prominent belonging to protein kinases (44\%). NLR-ID in MIC1 represent a genetic innovation developed during the radiation of the grasses and encompasses extensive ID diversity.

The role of viral and oomycete suppressors of silencing in modulating plant defense

R. R. VETUKURI (1), P. B. Kalyandurg (2), D. Sen (1), S. Kushwaha (1), M. Brus-Szkalej (1), P. Boevink (3), S. C. Whisson (4), E. I. Savenkov (2), L. J. Grenville-Briggs (1), (1) Swedish University of Agricultural Sciences, Alnarp, Sweden; (2) Swedish University of Agricultural Sciences, Uppsala, Sweden; (3) Cell and Molecular Sciences, James Hutton Institute, Dundee, U.K.; (4) The James Hutton Institute, Dundee, U.K.

Plants deploy RNA silencing as first line of defense against invading viral pathogens. However, as a counter defense, successful viruses have consequently evolved diverse mechanisms to avoid silencing, most notably through the expression of suppressors of RNA silencing and cause devastating plant diseases. Similar suppressors have recently been discovered in oomycete plant pathogens. Both the Oomycete, Phytopthora infestans and potato mop-top virus (PMTV) causes significant economic losses to potato production worldwide and both deploy silencing suppressors to disable plant-silencing pathways, suggesting that disruption of host plant silencing is a general virulence strategy across several kingdoms of plant pathogens. Here, we have characterized RNA silencing suppression activity of AVR1-like in P. infestans, an RXLR effector, and the $8 \mathrm{~K}$ protein from PMTV. Using mutagenesis analysis, we found that a GW motif in Avr1-like and the SWIM zinc finger motif in PMTV play a major role in their suppression activity. To provide insights into the mechanisms of RNA silencing suppression by viral (8k, HC-Pro, P12) and oomycete (Avr1-like) proteins, we have sequenced small RNAs resulting from the sense mediated PTGS. Understanding how the hostsilencing pathways are modified in response to pathogen attack and the ways in which the pathogens avoid or manipulate these pathways to ultimately cause disease can help design better disease control strategies.

Unveiling the role of LysM-RLKs in priming Arabidopsis defence responses against fungal pathogens M. GIOVANNONI (1), D. Lironi (2), C. Paparella (1), L. Marti (3), A. Gust (4), T. Nurnberger (5), G. De Lorenzo (1), S. Ferrari (6), (1) Sapienza University of Rome, Rome, Italy; (2) University of Aarhus, Denmark; (3) Sapienza, University of Rome, Rome, Italy; (4) Eberhard Karls Univ, Tubingen, Germany; (5) University of Tübingen, Germany; (6) Sapienza University Of Rome, Rome, Italy Plant receptor-like kinases (RLKs) characterized by the presence of one or more lysin motifs (LysM-RLKs, or LYKs) are responsible for the recognition of chito-oligosaccharide signals released from microbial symbionts and pathogens. The Arabidopsis thaliana genome encodes five LYKs (AtCERK1/AtLYK1 and AtLYK2 to 5) and among them, AtCERK1, AtLYK4 and AtLYK5 are important for perception and signaling of fungal chitin, whereas AtLYK3 negatively regulates defense responses. Insertional mutants for AtCERK1, AtLYK2, AtLYK4 and AtLYK5 show wild-type susceptibility to the fungal pathogen Botrytis cinerea. However, pre-treatment with different elicitors significantly increases resistance in WT plants and in atcerk1, atlyk4 and atlyk 5 mutants, but not in atlyk2 mutants although the lack of AtLYK2 affects chitin responsiveness only to a limited extent. Notably, AtCERK1, AtLYK2, AtLYK4 and AtLYK5 are all necessary to enhance chitin-dependent gene expression after elicitor pre-treatments, indicating that the specific role of AtLYK2 in priming of resistance might be more complex than anticipated.

Pathways involved in the Arabidopsis growth-defense trade off mediated by conditionally altered levels of cell wall-derived damage associated molecular patterns (DAMPs)

L. MARTI (1), F. Locci (1), S. Ferrari (2), S. Raggi (3), F. Cervone (4), G. De Lorenzo (4), (1) Sapienza, University of Rome, Rome, Italy; (2) Sapienza University Of Rome, Rome, Italy; (3) Umea Plant Science Centre | UPSC, Umea, Sweden; (4) Sapienza University of Rome, Rome, Italy

Oligogalacturonides (OGs) are damage-associated molecular patterns (DAMPs) that are recognized by plant receptors as signals of an altered-self and activate the plant immune response. The accumulation of OGs in vivo is favored by the interaction of microbial polygalacturonases (PGs) with plant-derived inhibitors (PGIPs). Transgenic Arabidopsis plants conditionally expressing a chimeric protein made by a fusion between a PG of Fusarium phyllophilum and a plant-derived PGIP, named "OG machine" (OGM), have been generated, allowing on command release of OGs in planta and the consequent activation of defense-related responses. If the command persists, plant growth is reduced or completely arrested; this 
effect reflects the natural trade-off existing between growth potential and plant defense capacity. Notably, a very high level of OGM expression triggers cell death. To better understand key aspects of the trade-off between development and defense that takes place in response to the OGM action, we generated plant lines expressing the OGM in genetic backgrounds defective in key elements involved in immunity (ndr $1-1, e d s 1-2$, $m p k 6, r b o h D, \mathrm{NahG})$. Our results suggest the existence of both SA-dependent and SA-independent pathways leading to inhibition of root growth in the presence of the OGs.

Assessing the role of small RNAs in inter-kingdom communication during plant-bacterial interactions M. SINGLA RASTOGI (1), M. Charvin (1), O. Thiebeauld (2), A. E. Fortunato (3), A. Ravet (3), V. Mendu (3), L. Navarro (3), (1) IBENSCNRS UMR8197, Paris, France; (2) IBENS-ImmunRise, Paris, France; (3) IBENS, Paris, France

During the last decade, small RNAs have emerged as critical fine-tuners of plant immune responses. For example, we and others have shown that a subset of Arabidopsis microRNAs (miRNAs) are differentially expressed upon bacterial PAMP detection to orchestrate antibacterial immunity. In addition, we have reported a major role of the Arabidopsis miRNA pathway in PAMP-Triggered Immunity (PTI) and, as a corollary, have identified bacterial effectors from Pseudomonas syringae pv. tomato strain DC3000 (Pst DC3000) that suppress this small RNA pathway to cause disease. In the present work, we wanted to extend these findings by investigating whether host-encoded small RNAs could not only fine-tune plant immunity but also reprogram Pst DC3000 gene expression as part of an antibacterial defence response. This intriguing hypothesis would suggest that plant small RNAs must be capable of passing through both the host plasma membrane and the double membrane of the bacterial cell envelope, and subsequently trigger gene silencing in this bacterium, which does not possess canonical eukaryotic-like RNA silencing factors. Here, I will provide experimental evidence for such trans-kingdom regulatory process. I will additionally report on the ongoing approaches that are being used to investigate whether extracellular vesicles could contribute to this Antibacterial Gene Silencing (AGS) phenomenon in the context of infection.

Potato virus $Y$ infection alters small RNA metabolism and immune responses in tomato F. CILLO, CNR - IPSP, Bari, Italy

Potato virus $Y(\mathrm{PVY})$ is an important pathogen of solanaceous crops. The $\mathrm{PVY}^{\mathrm{C}}$-to isolate induces stunting and foliar symptoms on tomato, but new vegetation displays symptom recovery at a later stage. In order to investigate the role of micro (mi)RNA-regulated mechanisms in tomato defenses against PVY, we performed small RNA sequencing from healthy and PVYC ${ }^{\mathrm{C}}$-to infected plants at 21 and 30 days-post-inoculation (dpi). A total of 756 unique miRNA sequences were obtained, among which 118 canonical miRNA sequences, many isomiR variants and 22 novel miRNAs. 46 mature miRNAs were overexpressed in infected vs. healthy plants, and showed a consistent alteration at both 21 and 30 dpi, whereas only a few miRNAs were underexpressed. Increased accumulation of isomiRs was correlated with viral infection. Among miRNA-targets, enriched gene ontology categories included resistance (R) gene families such as NBS-LRR receptors, RLPs and RLKs that are posttranscriptionally regulated by sly-miR482, sly-miR6023, sly-miR6024, sly-miR6027. These 22-nt miRNAs were shown to target R genes and trigger the production of 21-nt secondary phased sRNAs (phasiRNAs). 500 phasiRNA-generating loci were identified, mostly active in PVYinfected tissues. Our data demonstrate that sRNA-regulated host responses, encompassing quantitative miRNA alteration, diversification within miRNA families and miRNA-driven phasiRNA accumulation, regulate R genes, suggesting a functional role in the recovery phenotype.

S-acylation: A key regulator of the plant immune system

C. H. HURST (1), D. Turnbull (1), P. A. Hemsley (1,2), (1) University of Dundee, Dundee, U.K.; (2) The James Hutton Institute, Dundee, U.K.

Receptor-Like Kinases (RLK) conduct extracellular signals across the plasma membrane into cells, allowing cells to respond and adapt to environmental changes. We have recently identified a novel fatty acid based post-translational modification of RLKs called S-acylation. Using FLS2 as a model we show how S-acylation affects RLK function.

Levels of S-acylated FLS2 increase within minutes of bacterial flagellin perception. To identify when FLS2 S-acylation is occurring, mutants in FLS2 signaling pathway components were tested for flagellin-mediated increases in FLS2 S-acylation. Loss of components required for activation or ubiquitination prevented the flagellin-mediated increase in FLS2 S-acylation. However, mutants in endocytic processes displayed a wild-type increase in FLS2 S-acylation. This indicates that S-acylation occurs after ubiquitination but before endocytosis of activated FLS2. We subsequently demonstrated that FLS2 S-acylation is required for endocytosis of activated FLS2. Interestingly FLS2 mutants that cannot undergo flagellin-mediated S-acylation show reduced acute immune responses, suggesting a role in initial signal amplification, but abolished sustained responses such as immunity against P. syringae, consistent with the requirement of FLS2 endocytosis for sustained signaling. This suggests that a single S-acylation event plays two distinct roles in RLK function. We will also discuss our recent finding on the underlying enzymology of RLK S-acylation.

Establishing a system to study cross-kingdom communication via extracellular vesicles between the crop plant Hordeum vulgare and the biotrophic fungus Blumeria graminis f. sp. hordei

H. S. THIERON (1), R. Panstruga (1), P. D. Spanu (2), (1) RWTH Aachen University, Aachen, Germany; (2) Imperial College London, London, U.K.

The intercellular space is a major battlefield for plants and their pathogens. In recent years, it has become clear that plants and pathogens exchange a plethora of molecules that influence the outcome of their interaction, significantly via small extracellular vesicles (EVs). In this study, we describe the development of a procedure that allows the analysis of intercellular fluid (IF) of barley (Hordeum vulgare) plants infected with its powdery mildew pathogen, Blumeria graminis f. sp. hordei $(B g h)$. For IF extraction, a protocol following the infiltration-centrifugation technique was established and the fluid subsequently analysed using immunoblotting. We show that a) cytosolic contamination, tested by detection of a component of photosystem II (PsbA/D1), is present in the IF below detection limits, b) secreted proteins, exemplarily tested for PR-1, accumulate in the extracted IF, and c) pathogen-induced changes can be detected by PR-1 signal increase after Bgh infection. Furthermore, we observed the accumulation of ROR2, whose ortholog PEN1 was shown to be an EV marker in Arabidopsis. Together, these data suggest that the method is 
appropriate to isolate enriched IF with low cytosolic contamination and that it is sensitive enough to detect pathogen-induced changes. The enrichment of ROR2 in the IF indicates that this protein might qualify as an EV marker in Hordeum vulgare.

A microbial fermentation-based elicitor primes wheat plants against powdery mildew

A. TWAMLEY (1), M. Gaffney (2), A. Feechan (3), (1) UCD; School of Agriculture and Food Science, UCD Earth institute, Ireland; (2) Alltech European Bioscience Centre, Dunboyne, Co. Meath, Ireland; (3) UCD, School of Agriculture and Food Science, UCD Earth institute, Dublin, Ireland

Fungal pathogens alone are responsible for losses of $15-20 \%$ of wheat production annually, costing billions of dollars to the global economy. With changing consumer demand and increasing regulatory pressures, new strategies are required to help improve overall plant fitness and reduce the burden of biotic stress. One such strategy is the use of elicitors to induce the plant's own defence mechanisms. In this project, the ability of a microbial fermentation-based product to elicit defence priming was investigated. Wheat seedlings were foliar-treated with the elicitor and pathogenesis related $(P R)$ gene expression was measured. $P R$ genes involved with the salicylic acid dependent signaling pathway were found to be significantly upregulated within $24 \mathrm{hrs}$ of treatment compared to controls $(\mathrm{p} \leq 0.05)$. In order to assess if the elicitor had a priming effect, plants were inoculated with powdery mildew eight days post elicitor treatment. Quantitative real time PCR studies indicated a significant upregulation of $P R 1, P R 4, P R 5$ and $P R 9$ genes, in the immediate hours post challenge. Disease scoring showed significantly reduced powdery mildew pustule incidence in treated wheat plants $(\mathrm{p} \leq 0.05)$. These findings suggest that a microbial fermentation-based elicitor can prime the upregulation of specific $P R$ genes in wheat for protection against powdery mildew.

\section{Phenotypic heterogeneity in Pseudomonas syringae}

N. LOPEZ-PAGAN (1), M. A. Sánchez-Romero (2), J. S. Rufian Plaza (3), L. Aussel (4), F. Govantes (5), J. Ruiz-Albert (6), C. R. Beuzon Lopez (6), (1) University of Malaga, Spain; (2) University of Sevilla, Spain; (3) Shanghai Center for Plant Stress Biology, China; (4) Aix Marseille Université, CNRS, France; (5) Universidad Pablo de Olavide, Spain; (6) Univ of Malaga, Malaga, Spain

The notion of isogenic bacterial populations displaying phenotypic differences is widely accepted today. A particular case of phenotypic heterogeneity is bistability. Bistability occurs when bacterial population splits into two subpopulations showing distinct phenotypes. Phenotypic heterogeneity can allow some individuals to survive sudden environmental changes (risk-spreading) and can also lead to the cooperation (division of labour) between individuals. The relevance of this process has been highlighted in some animal pathogens, nevertheless, little is known about the occurrence or impact of these processes in the adaptation of bacteria to non-animal hosts. Pseudomonas syringae is a plant-pathogenic bacterium whose virulence depend of the T3SS expression. We have reported that T3SS expression is bistable in hrp-induction medium. This bistability generates two subpopulations, that show differences in virulence. Flagella is also an important virulence determinant for Pseudomonas syringae colonization. Here, we show how flagella expression also displays markedly phenotypic heterogeneity during growth within the plant. Although subpopulations displaying flagONT3SSON and flagOFFT3SSOFF can be identified within the plant, we provide evidence of cross regulation between T3SS and flagella expression at the individual cell level and propose phenotypic heterogeneity as an adaptative value for Pseudomonas adaptation to the plant host.

Identification and characterization of a dysbiosis mutant in Arabidopsis Y. T. CHENG (1,2), C. A. Thireault (1,2), S. Y. He (1,3,4,5), (1) MSU-DOE Plant Research Laboratory, U.S.A.; (2) Howard Hughes Medical Institute, U.S.A.; (3) Howard Hughes Medical Institute, East Lansing, MI, U.S.A.; (4) Plant Resilience Institute, U.S.A.; (5) Department of Plant Biology, Michigan State University, U.S.A.

In nature, plants co-exist with diverse microbes, consist of bacteria, fungi, viruses, and protists collectively called the plant microbiota. Using Arabidopsis as a model, many important plant immunity players that protect plants against pathogenic microbes have been revealed in the past several decades. However, little is known about how healthy plants select and maintain a homeostatic microbiota. By carrying out a forward genetic screen in the Arabidopsis bakl bkkl cerkl $(b b c)$ triple mutant that is defective in pattern-triggered immunity, we identified several dysbiosis mutants that we named gardiens of normal microbiota (germs). One of the germ mutants, germ2, exhibits interesting phenotypes. The germ 2 mutant cannot control microbiome homeostasis and harbors a greatly increased level of endogenous microbiota in leaves. By identifying and characterizing germ 2 and other germ mutants, we hope to shed light on the uncharted puzzle of host control of microbiota dynamics and answer the fundamental question of how plants select and maintain health-promoting microbiota in the phyllosphere.

\footnotetext{
Applying recent developments in our understanding of Lr67 function to identify new sources of broad-spectrum disease resistance in cereals

K. DIBLEY (1), R. Milne (1), S. Belide (1), W. Schnippenkoetter (1), J. Bose (2), A. Ashton (1), P. Ryan (1), D. Odeny (3), Z. Nikkiema (4), B. Zhou (5), D. Jordan (6), T. Mengiste (7), S. Tyerman (2), E. Lagudah (1), (1) CSIRO Agriculture and Food, Canberra, ACT, Australia; (2) School of Agriculture, Food and Wine, The University of Adelaide, Adelaide, SA, Australia; (3) International Crops Research Institute for the Semi-Arid Tropics, Nairobi, Kenya; (4) Institut de l'Environnement et Recherches Agricoles (INERA), Ouagadougou, Burkina Faso; (5) 7Regional Office for Asia and Pacific, Bangkok, Thailand, Thailand; (6) Queensland Alliance for Agriculture \& Food Innovation, University of Queensland, Warwick, QLD, Australia; (7) Purdue University, West Lafayette, IN, U.S.A.

Lr67 confers partial, durable, broad-spectrum disease resistance in wheat against stem, leaf and yellow rusts and powdery mildew. When transformed into other cereal crops Lr67 confers resistance to fungal pathogens adapted to the recipient crop species. Lr67 is encoded by a hexose transporter gene from the STP13 family, with two amino acid substitutions differentiating the susceptible and resistant alleles. As STP13s are highly conserved in plants, Lr67 was identified as a potential target to deliver improved fungal disease resistance in subsistence crops. Screening of STP13 orthologs in diversity panels of sorghum (Sorghum bicolor) and rice (Oryza glaberrima and O. rufipogon) failed to identify any mutations corresponding to those of Lr67. However our recent increased understanding of Lr67 molecular function, when expressed of $L r 67$ in yeast and Xenopus laevis oocytes, has led to the development of an in vitro screening system to identify STP13 variants likely to confer
} 
resistance. This screen is now being deployed to help identify sources of "Lr67-like" resistance in mutagenised sorghum and rice populations. The implications for delivery of germplasm with resistance against diseases such as sorghum anthracnose and rice blast, for inclusion in regional breeding programs, is discussed.

Assessing the costs and benefits of chemical defence priming agents using emerging hyperspectral imaging technologies M. YASSIN (1), E. K. Moffat (1), J. Stassen (1), S. Rolfe (1), M. Cromey (2), N. Holden (3), T. A. Valentine (3), A. C. Newton (3), J. Ton (1), (1) The University of Sheffield, Sheffield, U.K.; (2) Royal Horticultural Society, Wisley, U.K.; (3) The James Hutton Institute, Dundee, U.K.

Priming agents (PAs) hold promise as an alternative to pesticides in crop protection strategies. They are molecules of varied origins which sensitise plant immune systems and enable faster and/or stronger deployment of inducible defences upon pathogen exposure. The protection conferred by PAs is broad-spectrum, and as it bolsters the effects of multiple defence genes/pathways, is more durable than pesticides.

Furthermore, PAs generally do not have direct biocidal activity and are therefore considered less damaging for the environment. However, the exploitation of PAs has been hampered by the fact that they are rarely fully effective and that they can cause non-target effects on growth and yield. While the effectiveness of disease protection by PAs increases with dose, over-stimulation often leads to phytotoxic effects. We have used hyperspectral imaging to assess the costs benefits of combinations of PAs that act on different pathways, with the aim to find optimal combinations through additive or synergistic interactions. Using beta-aminobutyric acid, R-beta-homoserine, jasmonic acid and chitosan in the tomato-Botrytis and lettuce-Bremia pathosystems, we show that the protective and phytotoxic interactions of various PAs can be disentangled. Our results show that hyperspectral imaging provides high-throughput non-destructive tracking of disease progression and plant growth to assess the effectiveness of combinations of PAs in disease control.

Integrated electrochemical Chip-on-Plant Biosensor for monitoring biotic and abiotic stress A. AVNI (1), R. Pandey (1), A. Jog (1), O. Teig-Sussholz (1), I. Marsh (2), M. Bar (2), Y. Shacham-Diamand (1), (1) Tel Aviv University, Tel Aviv, Israel; (2) ARO, The Volcani Center, Rishon LeZion, Israel

In vivo monitoring of plant physiological parameters will be a key enabler of precision farming. The in vivo, in loco and real-time detection of qualitative and quantitative changes to a plant's physiological state in response to biotic or abiotic stress is of great relevance for the success of precision farming, crop management and plant phenotyping. Currently available platforms designed for the study of plant physiology are not compatible with in vivo monitoring. There are currently no biosensors detecting stress in plant combining in vivo sensing with electronic devices. Integrating micro-electro-mechanical systems, with bio electrochemical sensing and the associated electronics will allow sensing in real time biotic and abiotic stress. Recognizing that the advancement of precision agriculture requires amalgamated technologies aimed at direct on-plant sensing, we developed an electrochemical approach to monitor the expression of the $\beta$-glucuronidase (GUS) reporter gene. We have designed an electrode microchip able to transduce GUS expression, by chronoamperometry, to a measurable electrical current. Here, we demonstrate the possibility of real time monitoring of GUS enzyme expression in transgenic cell cultures and plants, using GUS activity as a biomarker for functional sensing of drought and pathogenic processes.

Structure vs. Chemistry: Alternate mechanisms for controlling leaf microbiomes

K. J. X. LAU (1), P. Balakrishnan (1), V. Vettath (1), A. Wong (1), A. Uchida (1), T. Y. Poh (2), D. D. I. Moses (1), A. C. Junqueira (3), S. C. Schuster (1), (1) Nanyang Technological University/Singapore Ctr for Env Life Sci Eng, Singapore, Singapore; (2) Nanyang Technological University/Lee Kong Chian School of Medicine, Singapore, Singapore; (3) Instituto de Biologia, Universidade Federal do Rio de Janeiro, Rio de Janeiro, Brazil

The analysis of phytomicrobiome communities residing on plant leaves traditionally relies on sonicated, filtered washes collected from foliage before DNA extraction. This process aggregates the total microbial communities found on both top (adaxial) and bottom (abaxial) leaf surfaces. To segregate microbial communities from both surfaces, we conducted a whole genome shotgun metagenomics approach of swabs of two plant species, Rhapis excelsa and Cordyline fruticosa. Our results show significant differences in microbial community composition and diversity between the adaxial and abaxial surfaces in both plants. The abaxial surface of $R$. excelsa was found to have approximately $98 \%$ reduction in bacterial load and only $10 \%$ reduction in fungal load, while C. fruticosa showed $95 \%$ decrease in bacterial load and $45 \%$ decrease in fungal load when compared to their adaxial counterpart. It has been well described in plant ecology literature about the two broad categories of plant defence mechanisms, namely chemical and mechanical defence. Plants synthesize either toxic chemicals or physical structures such as trichomes, spines and sticky surfaces to protect themselves from herbivories. In this study, we show that these protective mechanisms exist even at the microscopic level on the abaxial leaf surface. $R$. excelsa deploys a chemical defence approach while $C$. fruticosa uses physical structures that resemble like labyrinth walls and trichomes to deter pathogens on their abaxial surface.

A pair of Receptor-Like Proteins fine-tunes growth and immune signaling in Arabidopsis

H. S. LEE (1), J. Kong (2), S. B. Satbhai (3), W. Busch (3), Y. Belkhadir (4), (1) Gregor mendel insititute, Vienna, Austria; (2) GMI, Vienna, Austria; (3) Salk Institute for Biological Studies, U.S.A.; (4) Gregor Mendel Institute of Molecular Plant Biology (GMI), Vienna, Austria

Plants should orchestrate the balance between pathogen defense and growth because both are metabolically high-cost processes. To obtain a finetuned decision between defense and growth, plants sense their constantly changing environments and make crosstalk between them. Plants exploit Receptor Kinase (RKs) or Receptor-Like Proteins (RLPs) to relay environmental changes to the cell. These phylogenetically related protein families are involved in defense and multiple aspects of plant development such as root growth and stomata development. One of the well-known studies on RK pathways showed that RK pathway regulates defense and growth depending on their environment through Brassinosteroid (BR) signaling. BR perceived from the cell surface which is exactly the same place for the beginning of pathogen attack. We used large-scale phenotyping method, genome-wide association (GWA) studies, to find novel genes which can modulate the sensitivity to BR in Arabidopsis. We obtained candidate genomic regions that encode two uncharacterized RLP genes, which we named BR-REGULATOR1 (BRR1) and BR-REGULATOR2 (BRR2). We found that $b r r 1$ and $b r r 2$ are involved in the BR signaling, damage associated molecular patterns (DAMPs) 
and pathogen associated molecular patterns (PAMPs) triggered pathways. BRR1 and BRR2 interact with BAK1 and are involved in BAK1regulated developmental pathways. We propose that a pair of Receptor-like proteins fine-tunes growth and immune signaling in Arabidopsis.

Effect of a fresh water algae, Chlorella fusca as a Bio-stimulant on Growth promoting and Improving Qualities of Corn and Leek in Organic Farming

S. S. KIM (1), M. J. Kim (2), C. K. Shim (3), (1) 1Wonju Agricultural Technology and Extension Center, Wonju, Korea, Republic of (South); (2) 1Oragnic Agricultural Division, National Institute of Agricultural Sciences, Wanju, Korea, Republic of (South); (3) Organic Agricultural Division, National Institute of Agricultural Sciences, Wanju, Korea, Republic of (South)

Chlorella, widely known as a functional food material, has been studied as an organic agricultural material and has various growth promoting and disease suppressing effects for each crops in Korea. This study was conducted to investigate the effects of freshwater green alga Chlorella fusca on the growth and quality of corn and leek. The germination rate of corn ('Mibaek 2 ho') was improved to $6.7 \%$ and $4.8 \%$ in $0.2 \%$ and $0.4 \%$ chlorella treatments, respectively, as compared with the control, after 2 treatments of chlorella (Chlorella fusca) after corn sowing. As a result of 20 days after corn seeding, the growth of corn seedlings by chlorella treatment was accelerated by $15 \%$ and $26.3 \%$ in $0.2 \%$ and $0.4 \%$ chlorella treatments, respectively, as compared with the control. After 65 days of formal application, the characteristics of harvest treated with chlorella were examined, and weight and quantity of the corns were increased in $1 \%$ chlorella treatment. Chlorella was treated at once intervals per week during leek cultivation and gray matter fungus development and fresh weight were investigated. As a result, gray mold disease decreased by $22.5 \%$ and fresh weight was increased by $9.0 \%$ in chlorella treatment compared to the control. Further studies on the growth promotion factors and the metabolites produced during chlorella culture will be needed in the future.

Assessment of 12 Domestic Potato Varieties Resistant Against Common Scab and Late Blight at Five Organic Potato Fields in Korea M. J. KIM (1), C. K. Shim (1), B. G. Ko (1), J. Kim (1), S. S. Km (2), J. H. Park (1), J. Y. Yoon (1), J. Seo (1), E. W. Lee (1), (1) Organic Agricultural Division, National Institute of Agricultural Sciences, Wanju, Korea, Republic of (South); (2) Wonju Agricultural Technology and Extension Center, Wonju, Korea, Republic of (South)

Potato scab caused by Streptomyces scabies is known to be important factors causing degradation of quality and reduction of yield in organic potato cultivation. The purpose of this study was to assess the suitablility of potato varieties for organic farming. The influences of growing conditions and variety were significant on potato yields. Among the 12 potato varieties, in the trial for evaluating disease resistance against late blight, the disease was occurred in only one variety, called 'Sumi' potato, in Wonju. Among the tested five areas, there was no the incidence of scab disease on 12 tested potato varieties at Hamyang. However, Jeju and Wonju were showed the most severe infection of common scab caused by S. scabies ranged with 2.0 to $28.0 \%$. Among the tested 12 potato varieties, four varieties had shown the slightly common scab symptoms with less than $6.3 \%$ disease severity. As a result, among the 12 domestic potatoes varieties, four varieties, 'Daese', 'Sumi; 'Jopung', and 'Hongyoung' hade drameticlely shown the resistance against common scab in five major organic potato production areas. Further studies on the analysis of resistant genes related with common scab and molecular diversity of Streptomyces species are needed in the future.

Characterization of Septoria leaf spot and stem canker of lowbush blueberry

S. ALI (1), P. A. Abbasi (1), P. Hildebrand (2), W. Renderos (1), (1) Agriculture and Agri-Food Canada, Kentville, NS, Canada; (2) Agriculture and Agri-Food Canada, Kentville, Canada

In Canada, wild lowbush blueberry is a valuable horticultural crop that is managed on a 2-year production cycle with the harvesting of fruits, which occurs every other year, follows pruning of the stems. Several fungal diseases cause premature defoliation in fruiting fields and negatively affect fruit quality and yields. A Septoria-like leaf spot and stem canker have been described from both sprout and fruiting fields as one of the major causes of leaf and fruit drop. The pathogen was isolated from leaf and stem tissues of plants naturally infected in the field and based on morphology, the causal agent was described as Septoria-like pathogen. The pathogen was further characterized based on molecular analysis of several gene sequences. Analysis of the ITS, TEF-1 $\alpha, \beta$-tubulin, Calmodulin and LSU genes of the fungal isolates from lowbush blueberry revealed its identity as Sphaerulina amelanchier. The pathogenicity of the representative leaf and stem isolates was confirmed on lowbush blueberry clones, Vaccinium angustifolium and V. myrtilloides, in a growth chamber. Symptoms such as minute water-soaked specks to irregular reddish purple spots like those observed in the field appeared on leaves and stem followed by defoliation. In highbush blueberry, Septoria leaf spot and stem canker is caused by Septoria albopunctata that is very different from S. amelanchier based on both spore morphology and sequences of several genes. Two pathogens also produce different symptoms on two hosts.

Engineering bacteriocin-mediated resistance against plant pathogenic bacteria in plants W. M. ROONEY (1), R. W. Grinter (2), J. J. Milner (3), D. C. Walker (1), (1) University of Glasgow, Glasgow, U.K.; (2) Monash University, Clayton,, Clayton, VIC, Australia; (3) Glasgow University, Glasgow, U.K.

Pseudomonas syringae $(P s)$ and related plant pathogenic bacteria are responsible for damaging losses in crops as diverse as tomato, kiwifruit, peppers, olives, soybean etc. Current solutions, involving the use of chemicals and the introduction of resistance genes, have enjoyed only limited success, encourage the evolution of resistance among the bacterial populations and may have adverse environmental impacts. There is, therefore, a pressing need to develop alternative technologies to address the problem of bacterial disease in crops. Bacteriocins are naturally occurring antibiotic proteins. They are produced by nearly all genera of bacteria, including Pseudomonas, and offer the potential to provide robust disease resistance against $P$ s. Here we demonstrate that putidacin L1 (PL1), a bacteriocin from the non-pathogenic $P$. putida, selectively kills a range of Ps pathovars. We have expressed PL1 transiently in Nicotiana benthamiana and by stable transformation in Arabidopsis and have obtained effective resistance against PL1-susceptible pathovars of $P$ s. Our results provide proof-of-principle that transgene-mediated expression of a bacteriocin in planta is an effective strategy for providing disease resistance against bacterial pathogens. 
Co infection of OMMV and OLV-1 enhances symptoms and increases both viruses accumulation and viral derived siRNAs in plants C. M. Varanda (1), P. MATERATSKI (1), M. D. Campos (1), M. I. Clara (1), N. Marques (2), G. Nolasco (3), M. R. Felix (1), (1) ICAAM, University of Évora, Évora, Portugal; (2) University of Algarve, Faro, Portugal; (3) MeditBio, University of Algarve, Faro, Portugal

Previous extensive field surveys in olive orchards have revealed high levels of Olive mild mosaic virus (OMMV) and Olive latent virus 1 (OLV1), frequently appearing in mixed infections. These viruses belong to genus Alphanecrovirus and their RNA dependent RNA polymerase (RdRp), as well as their p6 and p8 amino acid sequences share over $87 \%$ identity. Preliminary studies have shown that co infection of OMMV and OLV-1 is associated to an intensification of symptoms, as well to an increase in transmission efficiency, suggesting a synergistic effect. Single and double infections of OMMV and OLV-1 were obtained through mechanical inoculation of Nicotiana benthamiana plants and the second upper leaf from each inoculated plant was collected at different stages and used for quantitative PCR. In this study we found that the co infection of OMMV and OLV-1 causes an exacerbation of symptoms and increases the accumulation of both viruses in N. benthamiana plants. Highthroughput sequencing of siRNAs from both viruses in singly and co infected plants showed that OMMV and OLV-1 co infection increased the accumulation of siRNAs, mainly of 21 and $22 \mathrm{nt}$ in length, with most non distinguishable between OMMV and OLV-1 siRNAs. Our findings suggest that siRNAs of both viruses have possible roles in the synergistic interaction between OLV-1 and OMMV in N. benthamiana plants. Whether a similar situation occurs in olive fields is not yet known and studies are being pursued.

Isolate specific responses to the fungal pathogen Zymoseptoria tritici in wheat and Brachypodium distachyon

A. Reilly (1), S. J. Karki (2), M. Mascarello (3), A. Twamley (1), A. M. M. Tiley (1), S. Kildea (4), A. FEECHAN (5), (1) UCD; School of Agriculture and Food Science, UCD Earth institute, Ireland; (2) UCD; School of Agriculture and Food Science, UCD Earth institute, Dublin, Ireland; (3) University College Dublin, Ireland; (4) Teagasc Crops Environment and Land Use Programme, Teagasc, Ireland; (5) UCD, School of Agriculture and Food Science, UCD Earth institute, Dublin, Ireland

Zymoseptoria tritici is the causal agent of Septoria tritici Blotch in wheat which threatens wheat yields. Disease assays using the wheat cultivar Longbow revealed that an Irish field isolate displays disease symptoms including chlorosis and cell death, prior to the occurrence of these symptoms with the Dutch reference isolate IPO323. Increased pycnidia formation was also observed at 21 days postinfection (dpi) with the Irish isolate. RNAseq was carried out to compare gene expression between isolates and revealed a subset of differentially expressed small secreted proteins (SSPs). Similar isolate specific responses were found in the symptoms induced when the non-host grass $B$. distachyon was challenged, including cell death, hydrogen peroxide production and defence gene induction. The Irish isolate was found to be more aggressive on both wheat and $B$. distachyon and formed hyphae within $B$. distachyon. Therefore $B$. distachyon may serve as a useful model to study $Z$. tritici.

Host induced gene silencing in $P$. pachyrhizi effectors interfere in virulence of soybean attenuating fungal pathogenicity K. Carvalho (1), V. Yukari Abe (1), L. M. Darben (1), M. P. Rincão (1), M. Qi (2), A. S. Utiyama (1), M. C. C. G. Carvalho (3), I. O. N. Lopes (1), R. V. Abdelnoor (1), S. A. Whitham (2), F. C. MARCELINO-GUIMARAES (1), (1) Embrapa Soybean, Brazil; (2) Iowa State University, Ames, IA, U.S.A.; (3) State University of North of Paraná, Brazil

Asian soybean rust, caused by Phakopsora pachyrhizi (Pp), is currently the main disease affecting the crop in Brazil, with an estimated cost for its control of U\$ 2.8 billion per crop season. In rusts and other filamentous plant pathogens, haustoria have been shown to secrete effector proteins into their hosts to permit successful completion of their life cycle. The identification and characterization of Pp effectors are expected to provide insight into the mechanisms by which this fungus manipulates soybean to promote infection and elicit Rpp-mediated defense. Pp families of effector have been predicted based on $P$. pachyrhizi transcriptome expressed in planta, and its ability to suppress plant immunity was previously demonstrated. For the functional characterization, effectors candidates able to suppress PAMP and effector-triggered immunity were evaluated by host induced gene silencing (HIGS) bioassays. Fifteen effectors candidates were first tested independently, and five that showed significant reductions in different phenotypic parameters analyzed were also tested combined. Silencing was confirmed by RT-qPCR and effectors acting at initial times after infection (6/12hai) showed a reduction in three of the four phenotypic parameters evaluated when they were tested alone. When combined, four combinations showed reduced infection.

PGPR-mediated modulation of the wheat microbiome

P. CARRIL (1), J. Cruz (2), J. Melo (1), R. Tenreiro (2), C. Cruz (1), (1) Center for Ecology, Evolution and Environmental Changes, University of Lisbon, Lisbon, Portugal; (2) BioISI-Biosystems and Integrative Sciences Institute, University of Lisbon, Lisbon, Portugal

Plant defenses can be primed upon local contact of naïve plants with plant growth promoting rhizobacteria (PGPR), leading to enhanced resistance against pathogens. Upon germination, however, plant tissues are quickly colonized by seed-borne bacteria. The presence of such microbiome led us to hypothesize that, in addition to the direct PGPR recognition, PGPR-mediated priming may occur through indirect mechanisms, via modulation of the seed-borne microbiome. To test this hypothesis, characterization of the cultivable microbiome in wheat seedlings inoculated with PGPR Herbaspirillum seropedicae (RAM10) was carried out. The results showed a significant decrease of the seedborne bacterial load in the roots and in the medium surrounding the roots of RAM10-inoculated wheat seedlings, when compared to noninoculated seedlings. Furthermore, bacterial diversity was reduced in RAM-inoculated seedlings, suggesting that that RAM10 modulates the composition of the plant microbiome by favoring specific bacteria. Currently we are involved in 1) characterizing the plant growth promoting traits of the identified seed-borne isolates; and 2) determining the potential of RAM10 as an effective priming agent against the wheat pathogen Pseudomonas syringae pv. atrofaciens. These results will allow us to establish the priming effect as the result of a direct PGPR recognition by the plant, a PGPR-mediated modulation of the plant microbiome, or as the combination of both strategies.

Bacterial vesicles: Double agents for plant defense

H. M. MCMILLAN (1), S. G. Zebell (1), X. Dong (2), M. J. Kuehn (1), (1) Duke University, Durham, NC, U.S.A.; (2) HHMI/Duke University, Durham, NC, U.S.A. 
Bacteria constantly face a wide array of stress. In the context of host-pathogen interactions this stress includes low pH, oxidative stress, and antimicrobial compounds. To survive in these harsh conditions, bacteria have developed many ways to adapt including through the production of vesicles. Vesicles are selectively packaged with molecules that the cell needs to eliminate, such as toxic compounds, misfolded proteins, hydrophobic molecules, and lipids that aid in membrane remodeling; however, they also play a much larger role in pathogenic interactions by delivering virulence factors to host cells. While studies have revealed many ways in which vesicles interact with mammalian systems, very little is known about how vesicles play a role in the natural environment. One way to shed light on this interaction is by studying vesicles in the context of plant-pathogen interactions. My work shows that bacterial vesicles elicit many plant immune responses, including ICS1 expression and salicylic acid accumulation. Furthermore, these responses protect the plant and lead to better outcomes upon future bacterial and fungal pathogen challenge. Uncovering mechanisms through which vesicles mediate plant immune responses will add to the growing body of research on hostpathogen interactions generally and will directly lead to better control of a wide array of agricultural pathogens, thus reducing crop loss and ensuring food security worldwide.

A DCL1-associated tRNA-derived small RNA is a potential positive regulator in Arabidopsis immunity H. G. KANG (1), S. I. Kim (1), D. S. Pujara (1), Y. Bordiya (1), J. Kim (2), (1) Texas State University, San Marcos, TX, U.S.A.; (2) University of Texas, Austin, TX, U.S.A.

Plants respond to stress by massive transcriptional reprogramming process. Small RNAs (sRNAs), generally known as an epigenetic inhibitor, are important in this reprogramming. To characterize the role of an sRNA in plant immunity, the Dicer enzyme which produces a majority of sRNAs were assessed in defense responses in Arabidopsis. To this end, all the mutants for Arabidopsis Dicers were analyzed in resistance against a bacterial pathogen, Pseudomonas syringae. Out of all the Dicers, a mutant in Dicerl (DCL1) displayed significantly compromised resistance to avirulent $P$. syringae. Moreover, the induction kinetics of defense genes was notably delayed/dampened in a $d c l 1$ mutant relative to wild type, when checked with targeted transcriptome analysis, suggesting that a DCL1-generated sRNA is a potential positive regulator for the defense genes. To further characterize the regulatory role of sRNAs for defense genes, sRNA transcriptomes were analyzed in the nucleus. Interestingly, select tRNA-derived sRNAs were highly induced in response to the bacterial infection. Furthermore, AGO2, an sRNA-loading protein, which was rapidly translocated to the nucleus in response to bacterial infection, physically interacted with this stress-inducible sRNAs. We are currently testing the hypothesis that the tRNA-derived sRNAs are involved in altering chromatin conformation to facilitate transcriptional reprogramming.

Rapid evolutionary adaptation of phytopathogenic powdery mildew fungi to highly selective plant environments S. KUSCH (1), L. Frantzeskakis (2), L. Pesch (3), R. Panstruga (1), (1) RWTH Aachen University, Aachen, Germany; (2) DOE Joint Genome Institute, Walnut Creek, CA, U.S.A.; (3) University of Cologne, Cologne, Germany

The obligate biotrophic ascomycete fungus Blumeria graminis causes the powdery mildew disease on grasses including wheat and barley. Different formae speciales of $B$. graminis exhibit strict host specificity, e.g. the barley powdery mildew (B. graminis f. sp. hordei, Bgh) can only complete its pathogenic life cycle on barley. We hypothesize that the evolution of the fungus is fast enough to detect changes in its virulence spectrum in real time. To identify the nature of genomic alterations underlying such putative rapid evolutionary adaptations, we perform experimental evolution experiments with $B g h$ by exposing the fungal pathogen to normally inaccessible host plant environments. We found that three experimentally evolved Bgh isolate K1 derivatives (named SK1-SK3) display variable levels of virulence on otherwise fully resistant barley mlo (Mildew locus $O$ ) mutant plants. By RNA-Seq we identified 123 genes that are differentially expressed between SK1 and K1 at 18 hours postinoculation (the time of host cell penetration), suggesting that $m l o$ virulence is polygenic and quantitative. Furthermore, we discovered that a gene coding for a transcription factor known to affect virulence and hyphal development is part of a $\sim 40 \mathrm{~kb}$ genomic deletion. By contrast, we observed no deleterious genomic alterations in genes encoding candidate secreted effectors. Nonetheless, we noted copy number variation of candidate secreted effectors between $B$. graminis formae speciales as well as a recent lineage-specific expansion of transposable elements in our near-chromosome level genome assembly of $B g h$, which together with the evidence from SK1 indicates that the fungus is capable of rapid evolution and fast adaption towards new host environments.

Role of genetic and epigenetic modifications in the bacterial plant pathogen Ralstonia solanacearum during adaptation to the resistant tomato Hawaii 7996 plant

R. GOPALAN NAIR (1), L. Legrand (2), X. Barlet (3), S. Genin (4), A. Guidot (3), (1) INRA, Université Toulouse III - Paul Sabatier, Castanet Tolosan, France; (2) INRA, Castanet Tolosan, France; (3) INRA, CNRS, Castanet Tolosan, France; (4) LIPM, Université de Toulouse, UMR INRA-CNRS 441-2594, Castanet Tolosan, France

Ralstonia solanacearum, the causal agent of bacterial wilt disease infects more than 250 plant species. The ability of this pathogen to adapt to many host plants and counteract plant resistance is supported by field observations reporting strains more aggressive and able to colonize novel hosts. The global objective is to investigate the genetic/epigenetic bases of host adaptation in $R$. solanacearum. Experimental evolution was conducted with GMI1000 strain on tomato Hawaii 7996 plant, a reference cultivar resistant to bacterial wilt, by serial passage experiments during 300 bacterial generations. The experimentally evolved clones are better adapted to tomato Hawaii and the hypotheses is that the genetic and epigenetic modifications (DNA methylation) could explain fitness gain. The fitness gain of these clones was measured by competitive experiment in tomato Hawaii; genome, methylome and transcriptome of these clones were compared to the ancestral GMI1000; and the modifications in genomic/methylome profile will be characterized. Genomic sequence analysis was performed by Illumina sequencing; methylome analysis by PacBio SMRT sequencing and bisulfite treatment/lllumina sequencing; transcriptome analysis by RNA-seq. The 25 tested evolved clones had increased in planta fitness compared to the ancestral clone. Whole genome sequencing of evolved clones showed between 0 and 2 polymorphism per clone. Methylome and transcriptome analyses of these clones are being currently run.

The impact of deployed resistances in agriculture on pathogen effector diversity M. R. Armstrong (1), E. M. Gilroy (2), P. R. J. Birch (3), J. H. Vossen (4), I. HEIN (5), (1) The James Hutton Institute, Invergowrie, Dundee, U.K.; (2) The James Hutton Institute, Dundee, U.K.; (3) Cell and Molecular Sciences, James Hutton Institute, Dundee, U.K.; (4) Plant Breeding, 
Wageningen University and Research, Wageningen, Netherlands; (5) The James Hutton Institute/University of Dundee, Invergowrie, Dundee, U.K.

The fundamental question of how resistances in agriculture are shaping pathogen populations has been the subject of scientific fascination for many years. Until recently, such important studies lacked efficient tools to asses deployment patterns of functional NLRs in crop varieties and, in parallel, to track all known cognate avirulence genes from the pathogens. Diagnostic resistance gene enrichment sequencing (dRenSeq) enables the high-confidence identification and sequence validation of functional NLRs in crops. Our study reveals a very limited base of utilised NLRs in all major potato cultivars but has identified additional and currently very effective NLRs in potato varieties that could be combined with the help of dRenSeq. Further input for a rationale to combine and deploy disease resistances comes from the diversity within pathogen populations which can be monitored through PenSeq, an enrichment-sequencing approach that targets critical pathogen genes including effectors. The combination of PenSeq and dRenSeq enables, for the first time, comprehensive Pathogen/Crop coevolution studies on local and global scales. Such studies are critical for the durable deployment of natural resistances in agriculture. Here, an example is presented for Phytophthora infestans, causing the major problem of potato late blight.

Phytoanticipin tolerance is critical for Pantoea ananatis virulence on onion S. P. STICE (1), B. H. Kvitko (1), B. Dutta (2), (1) University of Georgia, Athens, GA, U.S.A.; (2) University of Georgia, Tifton, GA, U.S.A.

Pantoea ananatis is a Gram-negative phytopathogenic bacterium of significant importance to Vidalia onion production. Pathogenic strains cause leaf blights and associated bulb rots that lead to significant post-harvest losses. Comparative genomic analysis confirmed that pathogenic strains lack typical virulence-associated protein secretion systems and instead correlated bacterial virulence with four mega-plasmid borne genetic loci we termed the Onion Virulence Regions (OVR A-D). Using mutation and complementation analysis in P. ananatis strain PNA 97-1 and other natural variants of Pantoea sp. with/without these gene clusters, we identified a $10 \mathrm{kbp}$ sub-region of OVR A that contributes to bacterial proliferation in onion bulbs. We found that the OVR A1 sub-region carries genes that confer tolerance to the reactive sulfur antimicrobial phytoanticipins of onion. The OVR A1 sub-region encodes genes annotated as sulfur redox proteins, two thioredoxins, a cystine transporter family member and a carbon-sulfur lyase. Additionally, a sequenced strain of onion pathogenic Enterobacter cloacae carries a locus of plasmidborne genes similar to OVR A1. We currently hypothesize that $P$. ananatis expresses an additive cohort of OVR A1 genes to confer to tolerance to reactive sulfur species produced in onion bulbs. Together, our data is consistent with a coevolutionary model whereby this non-soft rot necrotrophic pathogen evolved chemical tolerance to phytoanticipins produced by the host.

A genetically unlinked NLR network that modulates plant immunity against diverse pathogens originated from an ancestral gene cluster C. H. WU (1), S. Kamoun (2), (1) Institute of Plant and Microbial Biology, Academia Sinica, Taipei, Taiwan; (2) The Sainsbury Laboratory, University of East Anglia, Norwich Research Park, Norwich, U.K.

NLR immune receptors in the NRC superclade form an intricate network that confers resistance to a multitude of plant pathogens. This network is comprised of sensor NLRs, specialized in detection of pathogen effectors, that require helper NLRs, known as NRCs, to initiate immune responses. The network originated from a linked NLR pair that evolved prior to the divergence of asterids and caryophyllales over 100 million years ago, and expanded to half of all NLRs in some asterid species. We used phylogenomics methods to reconstruct the evolutionary history of the NRC family. One of the NRCs, we termed NRC0, is the only family member shared among different asterid species, leading us to investigate its evolutionary history and genetic organization. In tomato, carrot, coffee and morning glory, NRC0 is closely linked to NLRs that are phylogenetically related to NRC-dependent sensors. This prompted us to hypothesize that the ancestral state of the NRC network is an NLR helper-sensor gene cluster that was present in early asterids. We validated this hypothesis by demonstrating that tomato NRC0 is essential for the hypersensitive cell death induced by its genetically linked NLR. We are testing the degree to which the functional dependency between NRC0 and the linked NLRs is conserved across asterids. These results revealed the evolutionary dynamics of NLR receptor networks and how coevolution with pathogens drives the expansion and diversification of NLRs.

Virulence variation of Acidovorax citrulli isolates depending on inoculation sites in watermelon Y. SONG, I. S. Hwang, C. S. Oh, Department of Horticultural Biotechnology, Kyung Hee University, Yongin, Korea, Republic of (South)

Acidovorax citrulli causes bacterial fruit blotch (BFB) in Cucurbitaceae, including watermelon. Although A. citrulli is a seed-borne pathogen, it can cause BFB diverse symptoms in other plant organs like leaves, stems and fruits. To determine the infection routes of $A$. citrulli and their relationship with virulence, we examined the virulence of six isolates on watermelon, using several inoculation methods. Among the six isolates, only three, regardless of Clonal Complex (CC) groups of A. citrulli, efficiently infected seeds during germination in soil, and two of these also infected the roots of watermelon seedlings wounded prior to inoculation. Infection through leaves was successful only by three isolates belonging to CC group II, and two of these efficiently infected the mature watermelon fruits. One isolate infected seeds during germination as well as mature fruits. There may be a direct correlation between profiles of 16 putative type III effector genes and virulence through leaves. Overall, our results indicate that $A$. citrulli can infect host plants through various routes and organs and that there is natural variation in virulence of $A$. citrulli isolates, depending on infection routes. Moreover, the CC grouping of A. citrulli was only correlated with virulence in leaf infection assays.

Differential analysis of secondary metabolites of upland cotton line 91079-80 under the stress of Verticillium dahliae S. LI (1), B. Li (2), H. Zhou (3), L. Niu (4), X. Lu (1), Q. Guo (1), P. Ma (1), Z. Ma (5), (1) Plant Protection Institute, Hebei Academy of Agricultural \& Forestry Sciences, Baoding, China; (2) Department of Bioengineering, University of California at San Diego, San Diego, CA, U.S.A.; (3) Baoding Academy of Agricultural Sciences, Baoding, China; (4) Hebei University of Engineering, Handan, China; (5) Hebei Agricultural University, Baoding, China

Cotton verticillium wilt caused by Verticillium dahliae is one of the most serious cotton diseases in China. So far, the changes of cotton secondary metabolites are not well-known presented in the interaction between pathogen $V$. dahliae and cotton. In this study, secondary 
metabolite profiles of 6 groups of cotton seedling tissue samples were detected by UPLC-Q/TOF-MS, and the difference between treatments were analyzed. Results showed there were $4744 \mathrm{ESI}+(\mathrm{m} / \mathrm{z}$ from 101.022312 to 1484.517108$)$ and $3370 \mathrm{ESI}$ ( $\mathrm{m} / \mathrm{z}$ from 100.040801 to 1483.382150 ) characteristic ions of cotton metabolites presented in the interaction. Compared to healthy control, 1578 metabolites (933 ESI+ and 645 ESI-) significantly altered with a VIP threshold (VIP $>1$ ) from the OPLS-DA model as well as $p$ values calculated from Student's t-test $(\mathrm{p}<0.05)$. Among them, the peak area of 538 metabolites (398 ESI+ and 140 ESI- metabolites) presenting in pathogen-treated cotton tissues changed more than one fold. These included 64 increased metabolites (47 ESI+ and 17 ESI-) and 61 decreased ones (59 ESI+ and 2 ESI+) presenting in root, 82 increased (66 ESI+ and 16 ESI-) and 49 decreased ones (37 ESI+ and 12 ESI-) in stem, and 141 increased (98 ESI+ and 43 ESI-) and 185 decreased ones (131 ESI+ and 54 ESI-) in leaf. These results will not only enrich the data of cotton metabolomics, but also provide a scientific basis for further understanding the interaction between pathogen and cotton.

Profiles and characterization of Clavibacter capsici natural variants isolated from pepper plants during infection I. S. HWANG, H. M. Lee, E. J. Oh, C. S. Oh, Department of Horticultural Biotechnology, Kyung Hee University, Yongin, Korea, Republic of (South)

Gram-positive plant pathogenic bacterium Clavibacter capsici type strain PF008 causing canker disease in pepper carries one chromosome and two plasmids, pCM1 and pCM2. Our previous report showed that C. capsici lacks a chromosomal pathogenicity island (PAI) and celA, whereas this pathogen carries most putative virulence genes in both plasmids that directly contribute to its pathogenicity. Using over 50 natural isolates of C. capsici isolated from infected pepper in Korea, we investigated the pathogenicity of these strains. Disease symptoms generated by these natural isolates displayed dramatically different in pepper plants. The most notable finding was that natural isolates lacking pCM1 were commonly classified as low virulent. The disease-causing ability of these pCM1-lacking strains in host plants was successfully increased by overexpressing plasmid-borne virulence genes, $\operatorname{ch} p E$ or $\operatorname{ch} p \mathrm{G}$. Interestingly, the strains, 1106 and 1207, showing low virulence even with the presence of the $\operatorname{ch} p G$ gene were also increased by overexpression of $\operatorname{ch} p$. The whole genome analysis of 1101 and 1106 showed that indeed, both strains lack pCM1 and that the $\operatorname{ch} p G$ gene in 1106 is located in pCM2. In conclusion, our results demonstrated that virulence of natural isolates was also determined by the presence of $\mathrm{pCM} 1$, particularly the $\operatorname{ch} p G$ gene in $\mathrm{pCM} 1$.

Identification of Clavibacter michiganensis virulence genes via transposon mutagenesis I. S. HWANG, E. Song, E. J. Oh, C. S. Oh, Department of Horticultural Biotechnology, Kyung Hee University, Yongin, Korea, Republic of (South)

Gram-positive bacterium Clavibacter michiganensis is considered an economically important pathogen that causes bacterial canker in tomato. So far, a few virulence factors have been functionally characterized in detail in this bacterium. To fully understand the virulence mechanisms of $C$. michiganensis, it is necessary to find more virulence factors. For this purpose, transposon mutants of type strain LMG7333 were generated by insertion of transposon $\mathrm{Tn} 1409 \mathrm{C} \beta$ and were screened in terms of virulence and induction of hypersensitive responses (HR), compared to the wild type strain in tomato and on Nicotiana benthamiana, respectively. As the results from these assays, 23 mutants showing reduced virulence and/or HR were selected. To determine the mutated genes, TAIL PCR was performed. Among 23 mutants, 5 mutants had a transposon in celA encoding cellulase and one mutant had in pat- 1 encoding serine protease, confirming that these genes are very important virulence genes, as previously reported. Other 17 mutants had a transposon in different sites of $C$. michiganensis pathogenicitiy island (PAI) region, indicating that there are more uncovered virulence genes in PAI region of this pathogen. Overall, these results will help to understand virulence mechanisms of $C$. michiganensis in host plants.

Effector adaptation in a host-specialized lineage of Phytophthora

E. ZESS (1), Y. F. Dagdas (2), A. Maqbool (1), T. O. Bozkurt (3), M. J. Banfield (4), S. Kamoun (1), (1) The Sainsbury Laboratory, University of East Anglia, Norwich Research Park, Norwich, U.K.; (2) Gregor Mendel Institute of Molecular Plant Biology, Vienna, Austria; (3) Imperial College London, U.K.; (4) John Innes Centre, Norwich Research Park, Norwich, U.K.

There are very few examples detailing how plant pathogen proteins have adapted to their host environments at the resolution of single amino acids, obscuring the molecular evolutionary steps that shape the process of host specialization. Here, we use closely related Phytophthoraspecies - Phytophthora infestans, a pathogen of Solanum plants, and Phytophthora mirabilis, a pathogen of four-o'-clock flower (Mirabilis jalapa) — as a molecular platform to study the specialization of a well-characterized secreted effector, PexRD54. Previously, it was shown that the $P$. infestansPexRD54 (PiPexRD54) targets the potato host selective autophagy protein ATG8CL during infection via a C-terminal ATG8-interacting motif (AIM), counteracting host defences and re-routing autophagosome traffic. The P. mirabilisPexRD54 ortholog (PmPexRD54) has a fixed amino-acid polymorphism in its AIM which significantly reduces binding to the M. jalapahost ATG8s (MjATG8s), as tested in both in planta co-immunoprecipitation and isothermal titration calorimetry experiments. We propose that the interaction between PmPexRD54 and the M. jalapahost ATG8s is deleterious for P. mirabilisinfection. Ongoing experiments will determine whether PmPexRD54 retains the capacity to modulate selective autophagy via other mechanisms, such as through association with host vesicle trafficking proteins.

Genomic mechanisms of pathogenic plasticity in Phytophthora sojae B. M. TYLER (1), R. G. Anderson (2), F. D. Arredondo (1), A. L. Co (1), M. C. Duquette (1), Y. M. Fang (1), K. Fedkenheuer (3), M. Fedkenheuer (3), B. A. Kronmiller (1), C. Mahadevan (4), J. M. McDowell (3), A. Robertson (5), M. A. Saghai-Maroof (3), H. A. Wise (1), (1) Oregon State University, Corvallis, OR, U.S.A.; (2) University of North Carolina at Chapel Hill, Chapel Hill, NC, U.S.A.; (3) Virginia Tech, Blacksburg, VA, U.S.A.; (4) Rajiv Gandhi Centre for Biotechnology, Trivandrum, India; (5) Iowa State University, Ames, IA, U.S.A.

Oomycete pathogens cause destructive diseases on a huge range of plants of importance to agriculture, horticulture and natural ecosystems. Many of these pathogens are highly adaptable, readily overcoming chemical and genetic control measures, and jumping to new host species. Genomic studies have implicated thousands of genes as potentially contributing to the virulence of oomycete pathogens. The products encoded by these genes include toxins, secreted effector proteins, and hydrolytic enzymes. In the soybean pathogen, Phytophthora sojae, the genome contains 
nearly 400 genes that encode RxLR effectors that can enter plant cells to target specific components of the plant signaling and defense machinery. $P$. sojae strains differ in their repertoires of effector genes, in the DNA sequences of their effector genes, and to a surprisingly large extent, by which effector genes are transcribed. Using gene silencing, and more recently CRISPR/Cas9-mediated gene knockouts, we have shown that the pathogen relies on a relatively small subset of "elite" effectors that seem essential for full virulence. We have successfully targeted these genes for find new soybean resistance genes. Genetic selection for strains that come overcome the loss of essential effector genes has however revealed that the pathogen is extraordinarily effective at recovering from losses of essential effectors through epigenetic changes that affect the expression of other genes in the genome.

Identification and characterization of revertants of the dde2/ein2/pad4/sid2-quadruple mutant, which exhibit disease resistance S. ASAI (1), Y. Ayukawa (2), K. Shirasu (1), (1) RIKEN Center for Sustainable Resource Science, Yokohama, Japan; (2) RIKEN, Japan

Susceptible genes are host genes that are necessary for pathogen infection. As defects in host susceptible genes lead to resistance to pathogens, these genes are important targets for development of durable resistance. To hunt such susceptible genes for crops, we have employed a forward genetics approach using the $d d e 2 /$ ein2/pad4/sid2-quadruple mutants in Arabidopsis. The dde2/ein2/pad4/sid2-quadruple mutation largely abolishes the two major surveillance systems that recognize Microbe-Associated Molecular Patterns (MAMPs) and pathogen effectors, leading to Pattern-Triggered Immunity (PTI) and Effector-Triggered Immunity (ETI), respectively, due to defects in the jasmonic acid, ethylene, salicylic acid, and PAD4 signaling networks (Tsuda et al. 2009 PLoS Genet. e1000772). Here, we report identification and characterization of revertants of the quadruple mutant, which exhibit disease resistance to Hyaloperonospora arabidopsidis, Colletotrichum higginsianum, and Pseudomonas syringae.

Recessive Resistance to Potyviruses in Cucumber is Determined by a Single Mutation in the Vps4 Gene Y. IMURA (1), M. Amano (2), N. Kita (1), (1) Nihon university, Fujisawa, Japan; (2) Saitama Gensyu Ikuseikai co,. Itd., Kuki, Japan

Cucumber inbred line A192-18 shows strong resistance to Zucchini yellow mosaic virus (ZYMV) and other potyviruses. We have already confirmed that ZYMV resistance is conferred by a single recessive locus, which is different from the gene encoding eukaryote translation initiation factor (eIF) 4E or iso4E. By high-resolution mapping, it is revealed that the gene encoding a vacuolar protein sorting-associated protein (Vps) 4 is a candidate for the recessive resistance gene. The coexpression analysis of an infectious ZYMV cDNA with the Vps4 cDNA from A192-18, in which double-mutations have naturally occurred in the microtubule interacting and trafficking (MIT) domain, showed drastically reduced virus accumulation in the susceptible cucumber leaves. In addition, the expression of Vps4 originated from the susceptible cucumber allowed ZYMV to replicate in A192-18 leaves. Additional analyses indicated that only a single nucleotide difference in Vps4 MIT domain determined resistance to the virus. The genes encoding ZYMV proteins which interact with Vps4 protein from the susceptible cucumber (SVps4), but not from A192-18 (A-Vps4), were identified by using yeast two-hybrid system. Each of the genome-linked viral protein Vpg and HCpro interacted with S-Vps4, but not with A-Vps4. Furthermore, some host proteins specifically interacted with S-Vps4. The complex formation of Vps4 with viral proteins might determine whether potyvirus could replicate and infect in the cucumber plants.

Defining the putative minimal gene set required for host colonization by a fungal phytopathogen with an obligate biotrophic lifestyle Blumeria graminis f. sp. hordei M. BARSOUM (1), S. Kusch (1), L. Frantzeskakis (2), R. Panstruga (1), (1) RWTH Aachen University, Aachen, Germany; (2) DOE Joint Genome Institute, U.S.A.

The barley powdery mildew, Blumeria graminis f. sp. hordei $(B g h)$, is a fungal phytopathogen with an obligate biotrophic lifestyle. The unfeasibility to culture it on artificial media renders it inaccessible to conventional functional genomics approaches. Here, we propose a novel, ultraviolet (UV) radiation-based mutagenesis experimental approach for functional profiling of its genome. We first determined a sub-lethal dose for UV-C radiation $(254 \mathrm{~nm})$. To avoid the possible repair of the potential UV-mutations by the photolyase repair system, a sub-lethal dose was also determined when the UV treatment was followed by a dark incubation overnight. To this end, we performed several iterative rounds of UV radiation on sporulating $B g h$ colonies on detached barley leaves. Whole genome resequencing will be initially carried out for two isolates (one with and one without dark incubation) to validate the successful UV mutagenesis of $B g h$ conidiospores and the ability to obtain mutants with detectable loss-of-function mutations (e.g. stop codons, frame shifts and altered splice sites). Following this proof of concept, a pool of $B g h$ isolates that have undergone saturating UV mutagenesis will be created. These isolates will be subjected to deep genome and transcriptome resequencing. The analyses of multiple mutant strains will provide a global overview about which genes are "dispensable" and which are seemingly "indispensable" for Bgh pathogenesis.

Characterizing serine protease effectors from a vascular Gram-positive tomato pathogen, Clavibacter michiganensis D. M. STEVENS, Q. Lyu, S. P. Thapa, G. L. Coaker, University of California, Davis, CA, U.S.A.

Pathogen secreted effectors shape plant-pathogen interactions by shifting host susceptibility, facilitating pathogen colonization, and promoting disease. The causal agent of bacterial wilt and canker of tomato, Clavibacter michiganensis $(\mathrm{Cm})$, is an economically important GC-rich Grampositive actinobacteria. Core effectors secreted by $\mathrm{Cm}$ are encoded on the $\mathrm{Chp} / \mathrm{TomA}$ pathogenicity island (PAI), which, with virulence genes celA and pat-1, are hypothesized to drive a pathogenic relationship with its plant host. Genes in the Chp/TomA PAI are necessary for virulence since deletion of the entire region render the bacteria nonpathogenic on tomato. Within the PAI, $\mathrm{Cm}$ carries a variety of putative serine protease effectors from the Chp, Ppa, and Sbt family whose plant targets remain elusive. Other pathogenic Clavibacter within the genus cause equivalent wilting diseases in different hosts despite lacking many of the serine proteases found in $\mathrm{Cm}$. My research focuses on characterizing the role of serine proteases from $\mathrm{Cm}$ for disease development. Thus far, we have identified a group of serine proteases core to $\mathrm{Cm}$, verified protein accumulation and enzymatic activity of serine proteases in xylem mimicking conditions and found some to be functionally redundant since single gene deletions do not significantly change pathogenicity with it host. We are now working towards developing knockouts of genetically linked serine proteases to identify suites of effectors necessary to cause disease. 
Holistic investigation of the rice sheath blight disease

E. G. M. OREIRO (1), I. L. Quibod (2), E. K. Grimares (1,3), G. A. Grande (1,3), V. Roman-Reyna (1,4), R. Oliva (1), (1) International Rice Research Institute, Los Banos, Philippines; (2) International Rice Research Institute, Philippines; (3) University of the Philippines Los Banos, Philippines; (4) Ohio State University, U.S.A.

Rice sheath blight caused by the necrotrophic fungus Rhizoctonia solani AG-1 IA continues to be challenging disease in areas with intensive rice cultivation. Here we reported genome-wide association study that explored high density rice array to detect genomic regions linked to sheath blight resistance. We identified multiple regions with interesting signals in chromosomes 1,4 and 11. In particular, we investigated $q L N 11^{28}$, a quantitative trait loci enriched with defense related genes. RNA profiling of near-isogenic line carrying $q L N 11^{28}$ showed a number of differentially expressed genes related to ROS-redox pathway. Histochemical staining revealed less ROS accumulation on resistant line suggesting efficient ROS deregulation that halts the pathogen's colonization. In addition, we identified two candidate genes within the $q L N 11^{28}$ that might contribute to pathogen recognition. To contrast the host, we sequenced the genome of $R$. solani LR-1 using Nanopore and Illumina technology and developed a pipeline to identify a repertoire of secreted protein, which might facilitate its necrotrophic lifestyle. Functional characterization of $R$. solani effectors will be performed on heterologous system. Overall, this study provides an insight in multigenic resistance and novel pathogenicity mechanisms in rice sheath blight.

Role of soil $\mathrm{pH}$ in selection of root-nodule microsymbionts by nodulating legumes

N. TAK, S. Rathi, G. Bissa, B. Chouhan, P. Choudhary, H. S. Gehlot, Jai Narain Vyas University, Jodhpur, India

The specificity in legume-rhizobia symbiosis is based on Nod factors which play crucial role in molecular discourse between compatible symbiotic partners. Besides these signaling molecules the environmental factors such as soil moisture regime, $\mathrm{pH}$ of soil and temperature plays a significant role in determining the rhizobial diversity associated with legumes. We studied the effect of soil $\mathrm{pH}$ on selection of root-nodule microsymbiont(s) by native legume Chamaecrista pumila and species of Tephrosia grown in wet-acidic soils of Shillong, Meghalaya and in dryalkaline soils of Indian Thar Desert. These promiscuous host species are nodulated in both soil conditions. However, the ecological variables have resulted in niche-specific evolution of microsymbiont(s). In Thar Desert these host legumes are nodulated by novel strains of Ensifer and Bradyrhizobium (specifically by strains diversified from B. yuanmingense). In contrast to this the acidic soils enriched diversity of Bradyrhizobium microsymbiont(s) harbouring novel and various types of symbiotic (nodA and $n i f H$ ) genes. To conclude the acidic soils are more favorable for speciation and evolution of Bradyrhizobium strains when compared with alkaline-dry areas. Nevertheless, the Ensifer strains have evolved as dominant root-nodule microsymbionts of different native legumes growing in alkaline soils and hot-arid climate of Thar Desert.

Bacterial community composition shaped by variety and geographic distance in rice seeds

X. X. ZHANG (1), H. B. Guo (2), K. K. Thin (2), S. W. He (2), X. Wang (2), (1) Institute of Agricultural Resources and Regional Planning, CAAS, China; (2) Institute of Agricultural Resources and Regional Planning, CAAS, China

To compare the diversity and composition of endophytic bacteria of rice seeds, Japonica PA64 and Indica Dular were selected and planted in four main rice production regions of China, Hainan, Yunnan, Jiangxi and Hebei. Culture-dependent method and Illumina high-throughput sequencing were used to investigate the seed microbiome. A total of 788 endophytic bacteria isolated from parental and filial rice seeds, were assigned to Proteobacteria, Actinobacteria, Firmicutes according to 16S RNA gene sequence analysis. The shared genus in all samples was Xanthomonas, which was further identified as Xanthomonas sacchari, but Pantoea was the dominate group in most seeds. According to highthroughput sequencing analysis, rice seed endophytic bacteria belonged to Proteobacteria, Actinobacteria, Firmicutes and Bacteroidetes. At the genus level, Xanthomonas and Pantoea were the shared OTUs in all samples. We also found that the composition of endophytic bacterial communities of two varieties in the same region under the same planting conditions was similar. In contrast, the same varieties in different regions had obvious differences. The results from both methods above revealed that rice seeds existed conservative bacteria (Xanthomonas and Pantoea), which might be transmitted vertically from parental seeds. Geographic distance accounted for rice seed bacterial community more than rice varieties did.

Adaptive evolution at a pathogen effector-host target binding interface is associated with host specificity.

A. R. BENTHAM (1), Y. Petit (2,3), S. Kamoun (3), M. J. Banfield (1), T. Langner (3), (1) John Innes Centre, Norwich Research Park, Norwich, U.K.; (2) UMR BIOGER, INRA, AgroParisTech, Université Paris-Saclay, Thiverval-Grignon, France; (3) The Sainsbury Laboratory, University of East Anglia, Norwich Research Park, Norwich, U.K.

Targeting of host proteins by effectors is essential for pathogen infection. The AVR-Pik effector from the blast fungus Magnaporthe oryzae can interact with host small Heavy Metal Associated (sHMA) proteins. Here, we analysed genomes of M. oryzae and identified an AVR-Pik-like (AVR-PikL) family of effectors with sequence similarity to AVR-Pik. We predicted a conserved HMA binding site with only few, lineagespecific polymorphisms. We hypothesise these AVR-PikL family polymorphisms reflect adaptation to different sHMA proteins across grass hosts. To test our hypothesis, we focussed on AVR-PikL2 which is conserved in all host specific lineages of M. oryzae. A yeast 2-hybrid screen of AVR-PikL2/sHMA interactions revealed a broader sHMA binding spectrum for AVR-PikL2_Ta (from wheat-infecting M. oryzae isolates), compared to the AVR-PikL2_Os allele (from rice-infecting isolates). A crystal structure of AVR-PikL2 bound to an sHMA protein suggested an Asp to Asn mutation may underpin the increased binding spectrum of AVR-PikL2_Ta over AVR-PikL2_Os. To assess the importance of this residue, we generated point mutations in AVR-PikL2_Os and AVR-PikL2_Ta and will determine how these impact affinity for sHMA proteins using structural and biophysical analyses. Polymorphisms at the HMA-binding interface of AVR-PikL2 may reflect evolutionary adaptions in the effector, which emerged after host jumps and specialisation to different effector targets across new host species. 
Identification and Characterization of Novel Plant Immune Components Using the Arabidopsis-Powdery mildew Pathosystem Q. ZHANG (1,2), R. Berkey (2), J. J. Blakeslee (3), J. Lin (3), X. Ma (2,4), H. King (2), Y. Wu (2), W. Wang (2), A. Liddle (2), L. Guo (5), T. Munnik (6), X. Wang (7), S. Xiao (2), (1) University of California, Berkeley, CA, U.S.A.; (2) University of Maryland, Rockville, MD, U.S.A.; (3) The Ohio State University/OARDC, Wooster, OH, U.S.A.; (4) Hunan Agricultural University, Changsha, MD, China; (5) Huazhong Agricultural University, Wuhan, China; (6) University of Amsterdam, Amsterdam, Netherlands; (7) University of Missouri, St. Louis, MO, U.S.A.

Despite tremendous progress made in plant immunity in the past two decades, how plants mount spatiotemporally appropriate defenses against pathogens is still elusive. Here we use forward and reverse genetic approaches to uncover novel mechanisms used by plants to fight against fungal pathogens, or exploited by fungi for host adaptation using the Arabidopsis-powdery mildew pathosystem. Through a reverse genetics approach, we found that two phospholipase D (PLD) genes $P L D \alpha 1$ and $P L D \delta$ play opposing roles in modulating basal, post-penetration resistance against mildew through a yet-to-be characterized mechanism that is independent of EDS1/PAD4, salicylic acid (SA), and jasmonic acid. Inspired by this, we designed and performed a forward genetic screen in the background of a super-susceptible Arabidopsis eds $1 p a d 4 s i d 2$ (eps) triple mutant. By screening EMS-mutagenized eps plants using powdery mildew species with different levels of adaptation on Arabidopsis, 5 susceptible to non-adapted PM (snap) and 18 compromised immunity yet poor infection (cipi) mutants have been identified. So far this has revealed that loss of the MAP KINASE PHOSPHATASE1 gene, or the MILDEW RESISTANCE LOCUS 2 gene results in powdery mildew resistance independent of EDS1/PAD4 and SA. Characterization of additional causal cipi or snap mutations is in progress. Together, our results may contribute to a better understanding of the multi-layered plant immune system and powdery mildew host adaptation mechanisms.

Diversity of TAL effectors from Xanthomonas axonopodis pv. glycines isolated in Korea

S. HEU (1), I. J. Kang (2), J. W. Yang (2), (1) Natl Inst of Crop Sci, RDA, Suwon, Korea, Republic of (South); (2) National Institute of Crop Science, RDA, Suwon, Korea, Republic of (South)

Bacterial pustule disease is one the most important bacterial diseases in Korean soybean cultivation. The incidence of bacterial pustule had been increased from $3.8 \%$ to $8.8 \%$ during last three years. Increasing disease incidence may suggest the diversification of pathogen, Xanthomonas axonopodis pv. glycines. Pathogen produces effectors that transfer into plant cells and support bacterial virulence, proliferation, and dissemination. The unique effector family found in Xanthomonas spp. is the TAL (transcription activator-like) family. TAL effectors act as transcriptional activators in the plant nucleus and involved in the host specificity. In order to investigate the diversity of recent isolates of $X$. axonopodis pv. glycines, southern blotting analyses of 108 X. axonopodis pv. glycines isolated within 10 years had been conducted using avrBs 3 as a probe. Recent isolates are classified into 14 types than 6 types published 2002. It suggests that X. axonopodis pv. glycines isolates from Korea may be more diversified to adapt newly developed resistant soybean cultivar. Whole genome of 6 types strains had been characterizaed and the response of soybean cultivar to type strains had been investigated. The relations between TAL effectors and the response to soybean cultivar will be discussed.

Genetic basis of host specificity to fungal rust pathogens in barley

Y. WANG (1,2), S. Subedi (2), P. Doornenbal (2), H. D. Vries (2), G. Hensel (3), J. Kumlehn (3), P. A. Johnston (4), X. Qi (5), I. Blilou (1), R. E. Niks (2), S. G. Krattinger (1), (1) King Abdullah University of Science and Technology, Saudi Arabia; (2) Wageningen University \& Research, Netherlands; (3) Leibniz Inst of Plant Genetics \& Crop Plant Research, Gatersleben, Germany; (4) The New Zealand Institue for Plant and Food Research Limited, New Zealand; (5) Institute of Botany, Chinese Academy of Sciences, China

Most cereal species are attacked by rust fungi, many of which cause diseases of global significance for cereal production. Cereal rusts display a narrow host specificity and most cereal rust species can only colonize one or a few closely related grass species. Cultivated barley (Hordeum vulgare) and wild bulbous barley (H. bulbosum) are two closely related grass species that diverged about six million years ago. The two species are colonized by separate rust pathogens that show a strict host specificity. The leaf rust pathogen of cultivated barely (Puccinia hordei) can only infect cultivated barley but not wild bulbous barley, while the opposite is true for $P$. hordei-bulbosi. We used this barley-rust pathosystem to study the genetic and molecular basis of host specificity to rusts. We identified two orthologous lectin receptor-like kinases as quantitative determinants of host specificity. Both genes conferred resistance to adapted as well as to non-adapted rusts, but the effect against non-adapted rusts was much stronger. Our results show that quantitative host and non-host resistance can be conferred by the same genes.

BcCPDH1 is involved in the detoxification of sesquiterpenoid phytoalexin capsidiol, and specifically required for the pathogenicity of Botrytis cinerea on Nicotiana species

T. Kuroyanagi (1), M. Ojika (1), T. Suzuki (2), I. Sato (1), S. Chiba (1), K. Kawakita (1), D. TAKEMOTO (1), (1) Nagoya Univ., Japan; (2) Chubu Univ., Japan

The gray mold pathogen Botrytis cinerea have a broad host range, causing diseases in over 200 plant species, however how this pathogen obtained polyxenous nature is largely unknown. B. cinerea can detoxify a wide range of phytoalexins, including resveratrol from grape (stilbenoid), capsidiol from tobacco and rishitin from potato (sesquiterpenoids). In this study, we first analyzed metabolism of sesquiterpenoid phytoalexins by $B$. cinerea. Capsidiol was dehydrogenized to capsenone which was then further oxygenized, while rishitin was directly oxygenized to epoxy- or hydroxyrishitin, indicated $B$. cinerea has separate mechanisms to detoxify these phytoalexins. RNAseq analysis revealed that distinctive set of genes were induced in $B$. cinerea treated with capsidiol and rishitin, indicating $B$. cinerea distinguish these structurally similar phytoalexins to activate appropriate detoxification mechanisms. Most highly upregulated gene by capsidiol treatment was a dehydrogenase gene, named $B C C P D H 1$. Capsidiol-sensitive fungi transformed with $B C C P D H 1$ became resistant to capsidiol by detoxifying capsidiol. bccpdh1 KO showed reduced pathogenicity to Nicotiana benthamiana but remained pathogenic to potato. Homologues of $B c C P D H 1$ were found in taxonomically distant Ascomycota fungi pathogenic to insects and animals, suggesting $B$. cinerea obtained ancestral $B c C P D H 1$ by horizontal gene transfer, which is probably a critical event for pathogenicity of this pathogen to capsidiol-producing plants. 
The evolution of effectome-based strategies to establish beneficial symbiosis

R. OSBORNE (1), S. Lehmann (1), J. Roberts (1), T. Nussbaumer (2), P. Falter-Braun (2), P. Schäfer (1), (1) The University of Warwick, U.K.; (2) Helmholtz-Zentrum München, Germany

Beneficial plant symbionts enabled plants to colonise land in the harsh environment of the Mid-Palaeozoic era and have been essential for stress protection of plants ever since. Cultivation practice (e.g. tillage, pesticides) in modern cropping systems often prevent the establishment of symbiosis. Therefore, understanding how symbionts activate stress protection in plants can equip us with novel and sustainable crop protection strategies. Irrespective of lifestyle (pathogenic or mutualistic), plant colonising microbes secrete modular proteins called effectors to colonise their hosts. We identified 150 effectors released by the plant symbiotic fungus Serendipita indica and analysed their ability to activate beneficial pathways in colonised host plants. We applied three complimentary screens to identify effectors with growth-promoting and stress-protecting activities. First, we identified the plant proteins targeted by these 150 effectors in a yeast-two-hybrid screen against $12 \mathrm{~K}$ Arabidopsis genes. Next, we determined the role of all effectors in modulating different growth and stress pathways in a high-throughput protoplast screen. Finally, we overexpressed all 150 effectors in Arabidopsis and phenotyped these plants for improved growth and stress tolerance. Here, we present the outcome of this global screen together with biochemical and cell biology analyses that have been applied to reveal beneficial plant effects activated by these effectors.

Excision of genomic island PPHGI-1 leads to broad changes in expression of island genes.

J. D. PAYNE (1), H. C. Neale (1), R. Laister (1), G. M. Preston (2), R. W. Jackson (3), D. L. Arnold (1), (1) Univ of the West of England, Bristol, U.K.; (2) University of Oxford, Oxford, U.K.; (3) University of Reading, Reading, U.K.

The bean pathogen Pseudomonas syringae pv. phaseolicola (Pph) carries a genomic island (PPHGI-1) encoding the type III effector avrPphB, which triggers the hypersensitive response (HR) in a resistant bean cultivar. However, the pathogen evolves to avoid host resistance by losing the PPHGI-1 from the cells, but this does not lead to island extinction because a sub-population of cells carrying PPHGI-1 remain. We have shown that when PPHGI-1 excises from the chromosome to become a supercoiled episome, avrPphB is downregulated. Our current aim is to assess how the expression of genes on PPHGI-1 are regulated when PPHGI-1 is supercoiled after excision from the chromosome. RNAseq of the bacterium indicated genes on PPHGI-1 are downregulated after excision and supercoiling. Analysis showed that 53\% of genes on excised PPHGI-1 are downregulated compared to PPHGI-1 in the chromosome and that in general down regulation is greater than upregulation, with an average downregulation of -5.914 ( 2 fold $\mathrm{log}$ ) compared to only an increase on average of 0.804 for the upregulated genes. We have also shown that sections of PPHGI-1 cloned into cosmids are lost from the bacterial cells at different rates when exposed to host resistance. Therefore, we will use qRT-PCR to investigate the gene expression of selected genes on these sections of PPHGI-1 using cosmids mapped to the sections of PPHGI1.

Highly dynamic effector clusters are involved in host adaptation of cereal powdery mildew M. C. MÜLLER, C. R. Praz, T. Wicker, B. Keller, University of Zurich, Zurich, Switzerland

Plant pathogenic fungi display a remarkable capacity to adapt to new environments and host. This is thought to be due to their extremely modular set of effectors, small secreted genes that are involved in host manipulation during infection. The biotrophic fungus Blumeria graminis causes powdery mildew disease on many important cereal crops and exist in several highly host specialized subgroups. We recently created a chromosome-scale assembly of the wheat powdery mildew revealing that the fungus' effector complement possesses large standing genetic variation, characterized by a high frequency of deletions and duplications as well as transcriptional plasticity. B. graminis has undergone several host jumps and host expansion during its evolution. For instance powdery mildew recently emerged on triticale, the hybrid between rye and wheat, through a hybridization of wheat and rye powdery mildew. Here we use a QTL mapping approach in powdery mildew to depict the genetic basis of its host adaptation on the new host triticale. We show that host adaptation is controlled by multiple loci contributed by both the rye and wheat powdery mildew parent of the new hybrid triticale fungus. In addition, one of the major host adaptation locus encodes for a highly dynamic effector cluster suggesting a role for effector copy number variation in host adaptation.

A single cellobiosidase is required for barley hydathode and xylem colonization by Xanthomonas translucens A. Cerutti (1), A. Jauneau (1), M. C. Auriac (2), J. Butchacas (3), E. Gluck-Thaler (3), J. Slot (3), A. L. Perez Quintero (4), R. Koebnik (5), C. Bragard (6), L. D. Noel (7), J. M. JACOBS (3), (1) INRA-CNRS - LIPM, Toulouse, France; (2) INRA CNRS LIPM, Castanet-Tolosan, France; (3) The Ohio State University, Columbus, OH, U.S.A.; (4) Colorado State University, Fort Collins, CO, U.S.A.; (5) IRD UMR IPME, Montpellier, France; (6) Université Catholique Louvain-la-Neuve, Louvain-la-Neuve, Belgium; (7) Université de Toulouse, LIPM, CNRS-INRA, Toulouse, France

Many vascular pathogenic bacteria in the genus Xanthomonas enter host hydathode pores before reaching the water transporting xylem for longdistance disease development. The biological basis for hydathode entry and vascular pathogenesis in bacteria such as Xanthomonas translucens remains poorly understood. Here we describe the role of cellobiosidase, CelA, during $X$. translucens hydathode colonization. Live imaging revealed that $X$. translucens pv. translucens colonized the hydathodes pores and epithem. After epithem colonization, $X$. translucens pv. translucens crossed the hydathode-xylem barrier for long-distance vascular movement. Related X. translucens pv. undulosa, a barley non-vascular pathogen, moderately colonized hydathodes, entered the xylem but could not move past the initial vessels. We identified that CelA, a xylem pathogen-conserved protein, was present in X. translucens pv. translucens but inactivated by a transposon in X. translucens pv. undulosa. Notably, heterelogous expression of CelA in X. translucens pv. undulosa promoted barley hydathode colonization, vascular entry and long-distant movement for pathogenesis. Phylogenomic analysis also revealed that celA was lost from multiple $X$. translucens pv. undulosa and thus suggests that hydathode infection was an early behavior in X. translucens host colonization. We overall posit that cellobiosidase activity promotes both successful barley hydathode colonization and vascular pathogenesis. 
DNA double-strand breaks-mediated ectopic recombination between solo-long terminal repeats triggered pathogenic changes and genome rearrangement in the rice blast fungus

A. TAKAYUKI (1), D. Hiraoka (2), S. Murochi (1), K. Kiguchi (3), T. Tanaka (3), T. Sakuma (4), T. Yamamoto (4), T. Arie (5), S. Kuwata (3), I. Chuma (6), S. Ohsato (3), Y. Tosa (2), T. Kamakura (1), (1) Tokyo University of Science, Japan; (2) Kobe University, Japan; (3) Meiji University, Japan; (4) Hiroshima University, Japan; (5) Tokyo University of Agriculture and Technology, Japan; (6) Obihiro University of Agriculture and Veterinary Medicine, Japan

The rice blast fungus, Pyricularia oryzae, rapidly adapts to newly developed resistant cultivars through frequent loss of the avirulence (Avr) gene. In addition, chromosomal polymorphism, including genome-wide multiple translocation of $A v r$-Pita (deletion, duplication, and translocation), are detected in various field isolates (Chuma et al., 2011). Considering the DNA repair properties and genome structure of $P$. oryzae, ectopic recombination between solo-long terminal repeats (solo-LTRs) may cause pathogenic and genomic evolution. To confirm this, using engineered nuclease TALEN, we introduced a specific DNA double-strand break (DSB) into the closed-to-solo-LTR, flanking Avr-Pita, in the O29-J isolate. Southern blot analysis showed that TALEN-mediated DSB triggered the deletion and translocation of $A v r-P i t a$, which enabled it to infect the resistant cultivar Yashiro-mochi. Sequencing analysis revealed that the recombination between the tandem solo-LTRs flanking Avr-Pita resulted in the loss of Avr-Pita. The loss of Avr-Pita was also observed at the subtelomeric region of the supernumerary chromosome in the O23IN isolate. In this case, telomere capping and duplication/translocation of the other subtelomeric region via ectopic solo-LTR recombination led to the loss of Avr-Pita and genome rearrangement. Using a marker system to detect ectopic solo-LTR-like recombination showed that various stress conditions dramatically accelerated the recombination in the genome of $P$. oryzae.

The relationships between G. ambrosiae, G. circumfusus and G. spadiceus and their hosts S. LIU, Jilin Agricultural University, Changchun, China Jilin Agricultural University, Changchun, China

Golovinomyces on host plants belonging to the Heliantheae formed a single lineage in previous phylogenetic analyses based on ITS and 28S sequence data, comprised of a morphologically differentiated complex of species, which included G. ambrosiae, G. circumfusus, and G. spadiceus. However, the lineage also encompassed sequences retrieved from Golovinomyces specimens on composite hosts belonging to other tribes and other plant families, suggesting the involvement of a plurivorous species. A multilocus phylogenetic examination of this complex, using ITS, 28S, IGS (intergenic spacer), TUB2 (beta-tubulin), and CHS1 (chitin synthase I) sequence data was carried out to clarify the discrepancies between ITS and 28S sequence data and morphological differences. The phylogenetic and morphological analyses conducted in this study revealed three distinct species named, viz., (1) G. ambrosiae emend. (including G. spadiceus), a plurivorous species that occurs on a multitude of hosts including, Ambrosia spp., multiple species of the Heliantheae and plant species of other composite tribes including the Asian species of Eupatorium, (2) G. latisporus comb. nov. ( ${ }^{\circ}$ Oidium latisporum), the closely related, but morphologically distinct species confined to hosts of the Heliantheae genera Helianthus, Zinnia, and most likely Rudbeckia, and (3) G. circumfusus, the unrelated species confined to Eupatorium cannabinum in Europe.

Plant responses involved in cold temperature suppression of Xylella fastidiosa in grapevine L. BURBANK, USDA-ARS, Parlier, CA, U.S.A.

Xylella fastidiosa $(X f)$ colonizes a wide range of host plants and has emerged as a threat to agricultural crops worldwide. Climate factors impact both the pathogen and its insect vectors, limiting diseases caused by $X f$ to warmer regions. In the case of Pierce's disease of grapevine, $X f$ can be eliminated from infected plants over the winter, a phenomenon that has been associated with reduced disease severity in colder regions. The molecular basis for reduced pathogen colonization following cold stress is poorly understood but is likely a combination of bacterial vulnerability to cold shock, host plant physiological response, and degree of pathogen colonization prior to winter temperature exposure. The cellular response of $X f$ to cold stress appears to be limited. Two cold shock protein homologs have been identified in a grape strain of $X f$, but both are expressed independent of temperature. A peptide derived from the $X f$ cold shock protein Csp1 however, induces production of reactive oxygen species in grape tissue from a susceptible variety of Vitis vinifera suggesting it can act as an elicitor of plant innate defense response. Cultivar dependent differences in $X f$ survival and disease re-occurrence also were identified by artificial inoculations followed by cold treatment. Further understanding of how plant physiological response contributes to temperature-dependent elimination of $X f$ infections is important as it relates to climate factors associated with disease spread.

Successive subculture induced the degeneration of virulence and development on Fusarium oxysporum f. sp. niveum T. H. CHANG (1), Y. L. Wan (2), Y. H. Lin (3), K. S. Chen (4), J. W. Huang (5), P. F. L. Chang (5), (1) IDCSA Center and Department of Plant Pathology, National Chung Hsing University, Taichung, Taiwan; (2) Deparment of Plant Pathology, National Chung Hsing University, Taichung, Taiwan; (3) Dept. of Plant Medicine, National Pingtung University of Science and Technology, Pingtung, Taiwan; (4) FTHEB, Taiwan Agricultural Research Institute, COA, Kaohsiung, Taiwan; (5) IDCSA Center and Department of Plant Pathology, National Chung Hsing University, Taiwan

Successive cultivation of fungus has been reported to cause the sectorization which leads to degeneration of phenotypes and virulence. This event was found in Fusarium oxysporum f. sp. niveum (Fon), a limiting factor in watermelon production. Two types of Fon degenerated variants, mycelial type (MT) and pionnotal type (PT), were identified. Phenotype analysis showed PT variants produced more conidia but formed fewer chlamydospores than Fon wild type (sporodochial type, ST). In contrast, the MT variant produced fewer conidia and formed more chlamydospores than ST. Furthermore, virulence of different variants on susceptible watermelon Grand Baby was determined by two inoculation systems. In root dipping method, Fon variants showed increased disease severity than ST. Conversely, Fon variants showed a significant decrease in disease progression compared with ST by infested soil inoculation. The contrary results of two methods suggest that the cultural degeneration may curb the early stage of Fon infection process. Interestingly, Fon variants showed significantly decreased CWDEs (cell wall-degrading enzymes) activities compared with ST. Meanwhile, results of random amplified polymorphic DNA (RAPD) suggest that genomics of Fon variants and ST are highly similar. Our results reveal that successive cultural degeneration reduces CWDEs activities which affects the virulence of Fon. More details on cultural degeneration remain uncovering. This work was funded in part by IDCSA, NCHU. 
Resilience and sensitivity of pattern triggered immunity signaling pathways at elevated temperature in Arabidopsis thaliana A. T. SEROKA (1), R. Hilleary (1), C. D. M. Castroverde (1), S. Y. He (2), (1) Michigan State Unviersity, East Lansing, MI, U.S.A.; (2) Michigan State University, U.S.A.

Plants face a variety of environmental and biotic stressors that challenge their survival, such as warmer growing seasons and emergence of novel pathogens. Despite substantial knowledge on the molecular mechanisms controlling plant's response to temperature or disease in isolation, research on how these pathways interact to affect disease progression is gaining greater attention. Our previous research showed that Arabidopsis thaliana is more susceptible to the pathogen Pseudomons syringae at elevated temperature. We are conducting experiments to identify temperature-sensitive components of the plant immune system. Pattern-triggered immunity represents a major form of plant immunity and was previously shown to be temperature-resilient based on MAPK-regulated phenotypes (Cheng et al. 2014). Surprisingly, we found that flg22primed plants displayed weakened protection against $P$. syringae at elevated temperature. We saw that flg22-triggered MAPK signaling responses remained temperature-resilient, but we identified calcium-regulated responses were diminished at elevated temperature. Direct measurement of calcium confirmed a negative impact of elevated temperature on cytosolic calcium. Identification of calcium signaling as a temperature-sensitive component of the plant immune system provides a framework for understanding the molecular interplay between temperature and immunity and offers opportunities to safeguard plant productivity in the face of climate change.

Cytokinin-Mediated Processes Promote Heat-Induced Disease Susceptibility of Arabidopsis to Pseudomonas syringae pv. tomato DC3000 A. M. SHIGENAGA, S. Johns, D. R. Bush, C. T. Argueso, Colorado State University, Fort Collins, CO, U.S.A.

Under increased temperatures, such as those predicted as a result of global climate change, plant defense responses are attenuated leading to a process known as heat-induced disease susceptibility (HIS). The plant growth hormone cytokinin is known to regulate responses to both biotic and abiotic pressures. To address the role of cytokinin in HIS of Arabidopsis to Pseudomonas syringae pv. tomato DC3000 (Pst), wild-type plants and a cytokinin receptor mutant (ahk2,3 mutated on ARABIDOPSIS HISTIDINE KINASE 2 and 3) were exposed to Pst at two different temperatures, normal $\left(22^{\circ} \mathrm{C}\right)$ and high $\left(28^{\circ} \mathrm{C}\right)$. Pst populations were measured to assess pathogen fitness and host susceptibility. Stomatal conductance, fluorescent microscopy, and gene expression were measured to evaluate how cytokinin signaling impacts HIS. Results show that $a h k 2,3$ plants are less susceptible at $28^{\circ} \mathrm{C}$, with $P$ st populations plateauing 36 hours postinoculation. Moreover, pathogen-induced stomatal closure and expression of defense-marker genes were impaired under heat stress. However, a synthetic cytokinin reporter showed that high temperature increased cytokinin signaling. These results suggest that apart from its role in defense under normal temperature conditions, under high temperature cytokinin promotes physiological conditions that contribute to pathogen proliferation and highlight the value of cytokinin-based approaches to understand plant susceptibility and improve crop protection under increased temperatures.

The Nature of Drought Stress Drives Differential Metabolomic and Endophytic Responses in Leaves of Populus deltoides T. TSCHAPLINSKI (1), B. Garcia (1), J. Labbe (1), P. E. Abraham (1), P. Jones (1), I. Hodge (2), S. Climer (3), S. Jawdy (1), L. Gunter (1), M. Martin (1), N. Engle (1), X. Yang (1), G. A. Tuskan (1), D. Jacobson (1), (1) Oak Ridge National Laboratory, Oak Ridge, TN, U.S.A.; (2) Stanford University, Stanford, CA, U.S.A.; (3) University of Missouri, St. Louis, MO, U.S.A.

Some Populus sp. genotypes have the capacity to increase dehydration tolerance by lowering the osmotic potential via osmotic adjustment. Few studies have contrasted the degree and nature of solute accumulation if the nature of drought stress progression is varied. Multi-'omic analyses were conducted on $P$. deltoides 'WV94' subjected to cyclic mild (-0.5 MPa predawn water potential) drought vs cyclic severe (-1.26 MPa) drought after 2 or 4 drought cycles in contrast to well-watered controls $(-0.1 \mathrm{MPa})$, and in contrast with plants subjected to acute drought, where plants were allowed to dessicate for up to 8 days. The nature of drought onset, frequency and severity of drought interacted to dictate the degree of osmotic adjustment, the nature of the organic solutes that accumulated, and surprisingly, the nature of the microbial endophytes retained within leaves, as determined from cryptic signatures within the leaf transcriptome. Acute onset of prolonged, severe drought induced the greatest osmotic adjustment after withholding water for 7 days $(1.42 \times)$ with the greatest accumulations in the large, complex higher-order salicylate conjugates, and hydroxycinnamic acid conjugates of salicin; the populosides, particularly elevated. It will be discussed how the nature of drought can drive higher-order salicylate accumulation and an associated shift in the leaf microbiome away from beneficial endophytes to favor fungal pathogens that have consequences for plant-microbe interactions.

Defense responses against Botrytis cinerea are dampened by photoreceptor-controlled transcriptome changes in tomato. S. COURBIER (1), B. L. B. Snoek (2), K. Kajala (1), S. C. Van Wees (3), R. Pierik (1), (1) Plant Ecophysiology, Dept. of Biology, Utrecht University, Utrecht, Netherlands; (2) Bioinformatics, Dept. of Biology, Utrecht University, Utrecht, Netherlands; (3) Plant-Microbe Interactions, Department of Biology, Utrecht University, Utrecht, Netherlands

Plants growing in dense vegetation experience severe pathogen and plant competition pressures. Plants detect neighbors through reflection of farred (FR) light and respond through so-called shade avoidance responses to reach for light. Importantly, FR also increases susceptibility to several pathogens and herbivores in various plant species. In this study, we aimed to decrypt this phenomenon in the high impact crop species tomato. We investigated the effect of additional FR radiation on lesion development and defense responses against Botrytis cinerea, using tomato RNA sequencing. Our time series experiment unravels the chronology of the infection process and shows that FR perceived prior to inoculation strongly dampens jasmonic acid-mediated defenses leading to a higher susceptibility. We also show that FR modulates the tomato soluble sugar pool such that it promotes pathogen development within plant tissue. These findings will advance our understanding of FR-induced susceptibility in tomato, and will help optimize plant resistance in greenhouse by implementing LED lighting.

Disease incidence, severity and molecular characterization among field isolates of Sphaceloma manihoticola infecting cassava. A. T. ALLEYNE, S. Mason, R. Crichlow, The University of the West Indies, Cave Hill Campus, Bridgetown, Barbados 
Fungal diseases of cassava present a critical risk to the growing cassava industry in the Caribbean. Sphaceloma manihoticola, (Bitancourt \& Jenkins) causal agent of Superelongation disease (SED) of cassava Manihot esculenta (Crantz) is one such pathogen. It produces increased quantities of gibberellin $\mathrm{GA}_{4}$ in late stages of infection by over-expression of Smp 450 genes in-planta. From 2015 to 2017, field experiments were conducted in three rainfall zones in Barbados: low; intermediate and high; to quantify incidence and severity of SED. A disease rating scale and digital assessment of disease severity were used to measure SED leaf symptoms, while cassava tuber size and mass were also measured, after production. Results showed high disease incidence (100\%) in each zone with significant differences in disease severity between rainfall zones. No statistically significant differences were seen in yields of tuber mass, while tuber sizes were significantly different. DNA was then extracted from selected diseased leaf tissues which was then sequenced using ITS 1 and 4 PCR primers. This study therefore sought to characterize SED using field studies and provide a molecular characterization of S. manihoticola in diseased cassava using ITS sequencing. Varying patterns of disease severity with rainfall and molecular characterization provide new insights on S. manihoticola isolates for improved management of SED in the cassava industry in the Caribbean.

Gene expression dynamics of the obligate filamentous pathogen Blumeria graminis and its host plant under the field environments K. YOSHIDA (1,2), N. Mizoo (1), K. Wakamori (3), K. Oikawa (4), H. Yaegashi (4), S. Natsume (4), A. Uemura (4), A. Abe (4), R. Terauchi $(4,5)$, H. Mineno (2,3), S. Takumi (1), (1) Graduate School of Agricultural Science, Kobe University, Kobe, Japan; (2) Japan Science and Technology Agency, Presto, Japan; (3) Graduate School of Integrated Science and Technology, Shizuoka University, Hamamatsu, Japan; (4) Iwate Biotechnology Research Center, Kitakami, Japan; (5) Graduate School of Agriculture, Kyoto University, Kyoto, Japan

Molecular interaction between obligate filamentous pathogen and its host plant has not been well studied under field environments. Unstable temperature and humidity outsides potentially influence disease resistance of the host plant and virulence of pathogen. There may be unknown mechanisms of plant-pathogen interaction that have not been clarified under the laboratory conditions. To reveal plant-obligate filamentous pathogen interaction at molecular level under the field environments, we studied seasonal gene expression dynamics of both the powdery mildew Blumeria graminis and its host plant Aegilops umbellulata Zhuk., a diploid wild wheat relative, by using RNA-sequencing for naturally infected leaves in the field trails during three years from 2016 to 2018. We found that infection level of the powdery mildew mostly contributed to alteration in gene expression of the host plant and the pathogen compared to the other environmental factors. Clustering analyses based on the gene expression clearly showed that co-expressed genes of the host plant were categorized into the same clusters between years, even though it was difficult to find common time series dynamics of their gene expression between the years. This result suggests that under the field environments, most of the host plant genes are activated/repressed in response to temporary biotic and abiotic stimuli on the infected leaves rather than in response to seasonal environmental changes.

Dissection of molecular events involved in signal perception and root infection by Fusarium graminearum

Y. DING (1), M. Colgrave (1), D. M. Gardiner (2), K. Kazan (1), (1) Commonwealth Scientific and Industrial Research Organization, St Lucia, QLD, Australia; (2) CSIRO Agriculture and Food, St Lucia, QLD, Australia

How soil-borne fungal pathogens and roots sense each other remains elusive. Fusarium graminearum causes diseases of important cereal plants and is a major soil-borne pathogen. Here, we asked how $F$. graminearum initiates root recognition to then go on to cause root disease. We performed transcriptomics during the precontact stage of infection of $F$. graminearum on the model plant Brachypodium distachyon. Significant changes were found in multiple processes such as reduced pathogenesis, transport and induced ribosome biogenesis. Clustering analyses grouped a large number of differentially regulated genes encoding putative signaling proteins. From this set an ankyrin-domain containing protein encoded by $F g A N K 1$ was identified and was mutated in F. graminearum. The mutant exhibited strong defects in growth and reduced pathogenicity. In the knockout mutant, expression of several other precontact differentially expressed genes were also affected. FgANK1 was nuclear and partially cytosolic localized during precontact and may physically interact with a $\mathrm{Zn}_{2} \mathrm{Cys}_{6}$ transcription factor with predicted binding motifs in promoters of a number of the precontact-inducible genes. We also performed untargeted metabolomics and found significant changes for precontact metabolites. Together, we propose that $F$. graminearum is able to sense roots before physical contact and FgANK1 may play an important role in the sensing process.

Dickeya dadantii in poorly drained soil causes sudden death of fruit trees.

T. FUJIKAWA, N. Ota, T. Iwanami, National Agriculture and Food Research Organization, Tsukuba, Japan

Dickeya dadantii is a well-known plant pathogen that causes soft-rot disease to a wide range of plants, especially to economic vegetables, such as potato, tomato, and cabbage. Many crops are damaged by this pathogen all over the world. Surprisingly, Dickeya dadantii afflicts not only vegetables but also fruit trees in Japan. Peach, Japanese pear, mango, and apple trees have been quickly declined by this pathogen, resulting in sudden death. This may be due to the environmental conditions peculiar to Japan. Because fruit trees are grown in a temperate humid climate and there is a warm rainy season, soil often becomes hot and humid. Furthermore, in recent years, localized heavy rains and rising soil temperatures due to global warming have been frequently observed. In addition, because some orchards are sloped or transformed from paddy fields, the low part of orchard tends to be poorly drained. Then the soil of this field becomes often anaerobic. Thus, it seems that these environments are very suitable for the growth and pathogenicity of Dickeya dadantii. Regarding the virulence of Dickeya dadantii, we focus on various molecular factors not as the soft-rot pathogen but as the quick decline pathogen of fruit trees.

Rain Evokes a Primitive Plant Immune Response Via Trichome Hair-like Cell M. MATSUMURA (1), M. Nomoto (2), T. Itaya (1), Y. Aratani (3), M. Iwamoto (4), T. Suzuki (5), M. Toyota (3), S. Betsuyaku (4,6), Y. Tada $(1,2),(1)$ Division of Biological Science, Graduate School of Science, Nagoya University, Nagoya, Japan; (2) Center for Gene Research, Nagoya University, Nagoya, Japan; (3) Graduate School of Science and Engineering, Saitama University, Saitama, Japan; (4) Graduate school of Life and Environmental Sciences, Tsukuba, Japan; (5) Chubu Univ., Japan; (6) Fac. of Life and Environ. Sci. MiCS, Tsukuba, Japan

Rain is an essential element for every life form but is also an environmental stress. When it rains, pathogenic spores travel in water droplets that splash off an infected plant, causing diseases in neighbouring healthy plants. In addition, because raindrops are known to contain bacteria, fungi 
and virus, rain may directly mediate pathogen infection. Therefore, we hypothesized that plants may perceive raindrops as the emergency signal. However, if and how plants respond to raindrops is totally unknown. Transcriptome analysis of Arabidopsis leaves demonstrated that artificial raindrops unambiguously express genes related to disease resistance via mechanical stress. Genetic and bioimaging studies revealed that the mechanical stress induces $\mathrm{Ca}^{2+}$ wave and the expression of immune gene concentrically around trichomes. Furthermore, our results indicate that a member of the calmodulin binding transcription activator (CAMTA) family is involved in the transcriptional changes induced by raindrops. Finally, we confirmed that trichome-mediated immunity significantly reduces the growth of virulent Pseudomonas syringae. Here we discuss about a role of trichome-mediated immunity evoked prior to the known immune systems such as pattern- and effector-triggered immunity.

Plant growth promoting under iron-deficiency mediated by the Arabidopsis thaliana bacterial root microbiota H. INOUE (1,2), M. Hashimoto (3), Y. Bai (4,5,6), A. D. Rombolà (7), P. Schulze-Lefert (8), (1) NARO Institute of Agrobiological Sciences (NIAS), Tsukuba, Japan; (2) JST, PRESTO, Kawaguchi, Saitama, Japan; (3) The University of Tokyo, Tokyo, Japan; (4) State Key Laboratory of Plant Genomics, Chinese Academy of Sciences, Beijing, China; (5) CEPAMS, Chinese Academy of Sciences \& John Innes Centre, Beijing, China; (6) University of Chinese Academy of Sciences, Beijing, China; (7) University of Bologna, Department of Agricultural and Food Sciences, Bologna, Italy; (8) Max Planck Institute for Plant Breeding Research, Cologne, Germany

Although abundant in mineral soils, Iron (Fe) is sparingly soluble under aerobic conditions in high pH soils, especially in calcareous soil. Consequently, plants grown in this soil type often exhibit severe chlorosis owing to an Fe deficiency. In nature, the rhizosphere and plant roots are colonized by an increasingly differentiated community of soil-derived bacteria, designated the root-associated microbiota. Root-associated bacterial communities are a likely natural source for the isolation of rhizobacteria that might play an important role in Fe uptake by plant roots. Thus, we hypothesize that the root microbiota of plants grown in natural calcareous soils is enriched for bacteria that increase Fe bioavailability for plant growth. To test this hypothesis, we collected natural calcareous soil from an organic vineyard in Italy. To purify rhizobacteria, we prepared a bacterial library from $A$. thaliana roots of plants grown in the calcareous soil with a limited dilution approach. In parallel work, we have established a gnotobiotic $A$. thaliana microcosm that rescues plant growth under defined Fe-deficiency in the presence of individual microbiota. Several rhizobacteria were found to rescue plant growth under severe Fe-deficiency. Our protocol is suitable for the screening of comprehensive rhizobacterial libraries and will provide insights in the taxonomic diversity of root microbiota members that increase Fe bioavailability for plant growth.

The role of circadian entrainment in the outcome of rice blast infection

C. D. GRIFFIN (1), A. Foster (2), D. Soanes (3), A. Plessis (1), M. E. Hanley (1), N. J. Talbot (4), G. Littlejohn (1), (1) University of Plymouth, Plymouth, U.K.; (2) The Sainsbury Laboratory, Norwich, U.K.; (3) University of Exeter, Exeter, U.K.; (4) The Sainsbury Laboratory, University of East Anglia, Norwich Research Park, Norwich, U.K.

Rice blast is a fungal disease, which kills enough rice to feed 60 million people annually. Magnaporthe oryzae, the hemibiotrophic ascomycete and causative agent of rice blast disease, exhibits diurnal conidiation, where spores are produced each night. Magnaporthe colonies entrained in a $12 \mathrm{~h}$ light-dark photoperiod prior to transfer to free-running, constant light conditions exhibit a conidiation periodicity of approximately $24 \mathrm{~h}$, indicating circadian control. Rice plants inoculated with Magnaporthe spores either at dawn or dusk were scored for lesion number and severity after six days. Plants inoculated at dawn exhibit more than three times as many lesions than dusk inoculated plants. In addition, dawn inoculation gives rise to lesions approximately $70 \%$ larger than those formed in dusk-inoculated rice. These results suggest the timing of inoculation during the day is important in terms of determining disease outcome and have led us to investigate the role of circadian machinery in both partners of the rice blast pathosystem. We have identified putative core clock genes and photoreceptors present in Magnaporthe by homology to genes of known circadian function in Neurospora crassa. We are generating circadian mutants in Magnaporthe genes using CRISPR-mediated gene editing and investigate the mutants to elucidate the role of the Magnaporthe circadian clock in pathogenicity.

Stress, resistance and virulence of pectobacteria

O. PETROVA (1), V. Gorshkov (1,2), H. Osipova (1), A. Daminova (1), L. Moleleki (3), Y. V. Gogolev (1), (1) Kazan Institute of Biochemistry and Biophysics FRC "KSC of RAS", Kazan, Russian Federation; (2) Kazan Federal University, Kazan, Russian Federation; (3) Univrsity of Pretoria, Pretoria, South Africa

The formation and functioning of plant-microbial systems largely depend on environmental conditions. Under the influence of stress factors, microorganisms realize a specific reaction - a stress response, as a result of which bacteria acquire resistance to stressors. Phytopathogenic bacterium Pectobacterium atrosepticum under carbon starvation formed multiple resistance to repeated stress effects: heat shock, osmotic and oxidative stresses, antibiotics. In addition, starving bacteria were more virulent in tobacco and potato plants than bacteria growing in LB broth. Transcriptome profiling of starving cells revealed a repression of genes encoding the main virulence factors of pectobacteria: the enzymes for plant cell wall degradation, cfa-cluster, components of type III secretion system. At the same time, an induction of genes and gene clusters encoding factors of invasiveness, i.e. recognition, attachment and penetration into the host plant organism: capsular polysaccharides, bacterial biofilms, motility and chemotaxis, certain proteins of type III secretion system. Thus, starvation is a trigger for the "searching behavior" of bacteria that determines their readiness to interact with host plant and induce pathogenesis. This study was supported by RSF (19-14-00170).

\section{Cold priming memory reduces susceptibility to virulent pathogens and requires a functional plastid peroxidase system in Arabidopsis} thaliana

T. GRIEBEL, A. Ebert, H. H. Nguyen, M. Baier, Dahlem Centre of Plant Science, Plant Physiology Group, Freie Universität, Berlin, Germany

Plants are able to benefit from the experience and memorization of an environmental stress phase, a phenomenon referred to as priming. Priming and subsequent stress events can be of same nature (cis-priming) or different types (trans-priming). This brings up questions about specificity, duration, and cross signaling of plant stress memorization. We study if plant responses to bacterial pathogens are altered after the experience of an abiotic stress treatment. Our data show that a 24-hour cold priming phase $\left(4^{\circ} \mathrm{C}\right)$ increases plant resistance to a subsequent infection with 
virulent Pseudomonas syringae pv. tomato DC3000 (Pst) when the bacterial inoculation was done immediately after the cold treatment or after a recovery phase of 5 days. Hyper-susceptible null mutants of the plant immune regulator EDS1 (Enhanced Disease Susceptibility1) are like the Arabidopsis accession Col-0 immune-responsive to cold stress priming and memory. In contrast to basal immunity, resistance against avirulent Pst strains inducing effector-triggered immunity is not affected by cold priming. Recently, the plastid peroxidase system was described as signaling hub in cold cis priming. We found that interference of the plastid peroxidase system leads to perturbation of the memory effect and does not reduce susceptibility upon cold stress priming.

\section{Investigating the effect of temperature on bacterial infections of bean}

J. I. O. PIAT (1), D. Fourie (2), N. J. Kruger (3), G. M. Preston (4), (1) Doctoral Training Centre \& Department of Plant Sciences, Oxford University, Oxford, U.K.; (2) Agricultural Research Council, Pretoria, South Africa; (3) Department of Plant Sciences, Oxford University, Oxford, U.K.; (4) University of Oxford, U.K.

Three bacterial pathogens of bean (Phaseolus vulgaris) have a substantial impact on bean production worldwide: halo blight (Pseudomonas syringae pv phaseolicola), common blight (Xanthomonas axonopodis pv phaseoli) and brown spot (P. syringae pv syringae). Despite being capable of co-occurring on the same leaf, their relative impact on bean crops differs through the season: halo blight symptoms appear earlier, at daytime temperatures of $23^{\circ} \mathrm{C}$ and above, while the other two mostly appear above $27^{\circ} \mathrm{C}$. A number of factors could be responsible for this seasonal pattern, but the best documented one is the temperature regulation of phaseolotoxin production by P. syringae pv. phaseolicola: maximal at $18^{\circ} \mathrm{C}$, it is repressed at $28^{\circ} \mathrm{C}$. We are currently comparing various bacterial traits at low and high temperatures in vitro, including phaseolotoxin production and resistance, oxidative stress tolerance, metabolism and mobility. Interestingly, we find that the pathogens' tolerance to hydrogen peroxide differs between temperature treatments, suggesting that temperature could affect the ability of bacteria to tolerate stress encountered in plant tissue. We plan to extend this work to in planta assays and to identify key genes responsible for the temperature dependence of these pathogens. In the face of climate change, understanding temperature effects on the outcome of plant disease will be instrumental to outbreak prediction and control.

Geographic dependence of the genetic architecture of resistance to leaf rust (Puccinia novopanici) in locally adapted ecotypes of the biofuel crop switchgrass (Panicum virgatum)

A. VANWALLENDAEL, Michigan State University, East Lansing, MI, U.S.A.

Pathogens play an important role in the evolution of plant populations, but the genetic mechanisms of resistance vary greatly. This study uses locally adapted ecotypes of switchgrass (Panicum virgatum) to examine the temporal and spatial variation in the genetic architecture of resistance to a fungal pathogen, switchgrass leaf rust (Puccinia novopanici). We planted clones of an outcrossed mapping population at eight locations in the United States from Texas to Michigan. We measured rust progression at these sites for three years, then mapped quantitative trait loci (QTLs) using function-valued transformations of rust progression curves. We mapped fifty-one total QTLs over the three-year period. Two large-effect loci were associated with resistance across multiple sites and years, but were almost exclusively expressed in northern sites, raising questions about the geographic dependence of resistance. There is no difference in rust species composition between the north and south, so QTL differences may be due to rust strain variation or environmental influence on the underlying resistance genes. The beneficial alleles at the focal loci confer a $33 \%$ decrease in rust, and a $26 \%$ increase in biomass, indicating that these loci have high adaptive importance. Our results show an role for rust in the local adaptation of switchgrass and illustrate the variation in the genetic architecture of disease resistance.

Trehalose synthesis contributes to Ralstonia solanacearum fitness during bacterial wilt disease and outside the plant A. MACINTYRE, J. Barth, C. Allen, University of Wisconsin-Madison, Madison, WI, U.S.A.

The economically devastating, xylem-dwelling pathogen Ralstonia solanacearum (Rs) changes host tomato xylem sap composition during bacterial wilt disease. The disaccharide trehalose, implicated in stress tolerance across all kingdoms of life, is enriched in sap from infected tomato plants. To investigate the role(s) of trehalose metabolism during disease, our first goal was to determine if the host, the pathogen, or both produce the trehalose detected in xylem sap. To explore bacterial trehalose contribution, we evaluated the phenotypes of targeted $R s$ mutants lacking one or more of the bacterium's three bacterial trehalose synthesis pathways: TreYZ, TreS, and OstAB. Following naturalistic soil soak infection of tomato plants, the otsA single mutant and the treY/treS/ots $A$ triple mutant both had defects in virulence and in tomato stem colonization compared to wild-type $R s$. Further, both these mutants had reduced competitive fitness in planta when they were co-inoculated with wild-type, suggesting that $R s$ needs trehalose to overcome stresses during the plant infection process. This is consistent with our finding that in vitro these mutants were less tolerant of osmotic and desiccation stresses, conditions the bacteria likely confront in planta. The ots $A$ single mutant, which lacks trehalose-6-phosphate synthase, behaved indistinguishably from the triple mutant in all experiments, indicating that the OtsAB pathway is the dominant trehalose synthesis pathway in $R s$.

Disease progression in a field trial of transgenic bananas modified for resistance to banana bunchy top virus in Malawi J. L. DALE (1), B. Dugdale (1), L. Tripathi (2), R. M. Harding (1), M. Soko (3), (1) Queensland University of Technology, Brisbane, QLD, Australia; (2) International Institute of Tropical Agriculture (IITA), Nairobi, Kenya; (3) Malawi |Dept of Agricultural Research Services, Bvumbwe, Malawi

Banana bunchy top virus (BBTV) causes the most serious virus disease of bananas. The disease is causing major production losses particularly in Africa where it continues to regularly move into countries where it has not been previously reported. Bananas are an extremely important component of diets in many African countries and the major types of bananas grown there, the dessert bananas such as Cavendish, and the cooking types, East African Highland bananas and plantains, are all susceptible to BBTV. Genetic modification is the most appropriate strategy to introduce resistance into these bananas as suitable sources of conventional resistance have not been identified in banana germplasm. We have generated transgenic Cavendish bananas and plantains using a range of different potential resistance strategies. The most advanced of these are transgenic lines transformed to trigger an RNAi response targeting three different BBTV genes. A number of lines appeared to be immune in 
glasshouse trials. These and additional lines were progressed through to a field trial in Malawi. We have followed the progression of the disease over more than two years. In contrast to the glasshouse trials, we have identified transgenic lines with resistance but not immunity. Importantly, we have gained valuable insights into the factors that affect the rates and patterns of disease spread within a field.

An ABC transporter-two component regulatory system locus is required for host metabolite-induced type III secretion by Pseudomonas syringae pv. tomato DC3000

Q. Yan, C. J. Rogan, Y. Y. Pang, J. C. ANDERSON, Department of Botany and Plant Pathology, Oregon State University, Corvallis, OR, U.S.A.

Pseudomonas syringae is a plant bacterial pathogen that uses a type III secretion system (T3SS) to suppress host immunity. Plant-derived organic acids induce the expression of T3SS-encoding genes in P. syringae pv. tomato DC3000 through unknown mechanisms. Here we report that the acidic amino acid-transport (aat) and-utilization (aau) locus in DC3000 positively regulates T3SS gene expression in response to bioactive plant metabolites. The aat/aau locus is predicted to encode an ABC transporter (AatMQP), periplasmic solute-binding protein (AatJ), and a twocomponent system (AauS/R). DC3000 mutants lacking aatJ, aauS and aauR have decreased expression of T3SS-encoding genes and decreased virulence during infection of Arabidopsis, as well as delayed growth in a glutamate-only medium, indicating that these genes have dual roles in T3SS regulation and metabolite uptake. In contrast, a DC3000 mutant lacking aatP, encoding an ATPase required for transporter activity, has delayed growth in glutamate-only medium but no defect in T3SS deployment or virulence, suggesting T3SS regulation and transporter functions of this locus are genetically separable. The predicted DNA motif bound by AauR is present in only two promoters in the DC3000 genome: upstream of the aat/aau operon and upstream of T3SS regulators $h r p R / S$. We hypothesize that AatJ-AauS-AauR signaling directly regulates $h r p R / S$ expression in response to host metabolite signals. Our progress in testing this model will be presented.

Plant-pathogen interactions in the halophytic crop species Chenopodium quinoa

F. V. SCRAFTON, G. M. Preston, J. A. C. Smith, University of Oxford, Oxford, U.K.

Climatic change and the utilisation of new land for cultivation introduces novel environmental pressures for crops. Chenopodium quinoa (quinoa) is a highly nutritious crop native to the Andes that is adapted to marginal climates and is cultivated in over 100 countries. It is a halophytic crop able to prosper on nutrient-poor and highly saline soils, across altitudinal gradients of $4000 \mathrm{~m}$ and temperature fluctuations of up to $40{ }^{\circ} \mathrm{C}$. Immune mechanisms in quinoa have yet to be fully characterised, and neither have potential synergies or trade-offs between salt tolerance and biotic stress responses been studied in depth. We have established a model pathogen system with Pseudomonas syringae that can be used to study disease resistance in quinoa, and a hydroponics system for the controlled exposure of plants to salt. Preliminary results show that quinoa plants exposed to high salt concentrations maintain comparable physical parameters (biomass, growth rate) and show significantly higher tolerance to infection. We have also shown that quinoa is capable of exhibiting a range of well-characterised PAMP-triggered immunity (PTI) responses, including the ROS burst, MAPK signaling and upregulation of peroxidase activity. We are using this combinatorial stress system to investigate how quinoa's halophytic adaptations crosstalk with immunity at multiple levels: morphologically, and via cellular and molecular components.

In search of genes required for Paraburkholderia phymatum's high competitiveness in legume root infection S. Hug, P. Bellés Sancho, M. Lardi, L. Paganini, S. B. de Campos, L. Eberl, Y. Liu, G. PESSI, Department of Plant and Microbial Biology/ UZH Zurich, Switzerland

Most legume species can fix atmospheric nitrogen via symbiotic bacteria called rhizobia. This gives the legumes a pronounced growth advantage in nitrogen $(\mathrm{N})$ deprived soils. In exchange for reduced $\mathrm{N}$, plants provide rhizobia with nutrients and highly specialized root structures, called nodules. In addition to the well-known alpha-proteobacteria, several beta-proteobacteria of the genera Cupriavidus and Paraburkholderia have also been shown to be able to establish a nitrogen-fixing symbiosis with legumes (beta-rhizobia). In our laboratory, we use Paraburkholderia phymatum, a strain isolated from several Mimosa spp. and from papillionoid legumes as a model system to elucidate the molecular mechanisms underlying beta-rhizobial symbiosis. We recently showed that $P$. phymatum is highly competitive against other beta-rhizobial strains for nodulating several papilionoid legumes of major agricultural importance including the common bean. Mutations in the two P. phymatum clusters coding for type VI secretion systems (T6SS) caused a reduced fitness in interbacterial competition, suggesting that a functional T6SS is one of the traits that renders this strain competitive. In this study we present the further characterization of $P$. phymatum T6SSs, the construction of strains mutated in both T6SSs as well as our first attempts to identify additional genetic factors responsible for the success of $P$. phymatum in nodulating the roots of agriculturally important grain and forage legumes.

\section{Hormetic pulsed UV light treatment induces plant defences and reduces Botrytis cinerea disease progression on tomato (Solanum} lycopersicum)

M. PAPP-RUPAR (1,2), G. Scott (2), A. Fletcher (2), G. Shama (3), M. J. Dickinson (2), (1) NIAB-EMR, East Malling, U.K.; (2) University of Nottingham, Nottingham, U.K.; (3) Loughborough University, U.K.

Botrytis cinerea is one of the most important fungal pathogens causing losses estimated to over $£ 88$ million per year in U.K. tomato and cut flower industries alone. Quiescent life cycle and resistance to fungicides are main challenges of Botrytis control. In this work we have developed non-chemical method to control Botrytis in pre-harvest environments. The method consisted of short pulses of extremely high intensity broad spectrum light (UV-VIS-IR) applied to vegetative part of tomato. Light treatment elicited defense-like responses leading to reduction of $B$. cinerea disease progression by $25-40 \%$. Optimized dose of pulsed light induced maximum resistance with minimum or no damage to the plants in terms of growth, visual damage or chlorophyll fluorescence $(\mathrm{Fv} / \mathrm{Fm})$. Effects of induced resistance were detectable up to 12 days post treatment and were observed on three different tomato cultivars. To shed light on the molecular basis of pulsed light induced resistance we have performed transcriptome analysis of light treated and untreated plants before and after $B$. cinerea inoculation. Involvement of reactive oxygen species (ROS) signaling, ethylene signaling and WKRY transcription factors were highlighted in pulsed light induced resistance. This method illustrates that transient changes to the light conditions can contribute to disease control in growth environment. 
One of the best-known ecological consequences of climate change is the advancement of spring phenology. Yet, we lack insights into how changes in climate interact with genetic variation in shaping tree phenology, and how such changes in phenology will translate in the seasonal dynamics of tree attackers. To elucidate the impact of warming and tree genotype on phenology, as well as the consequences for the population dynamics of a fungal pathogen (Erysiphe alphitoides) and plant-feeding insect (Tuberculatus annulatus), we conducted an active field heating experiment with grafted oak trees. We found that experimental warming generally advanced oak bud burst in spring and delayed leaf senescence in autumn, but that reaction norms differed strongly among tree genotypes. Warming or tree genotype did not affect disease levels or herbivore abundance at the beginning of the season, but shaped both disease levels and aphid abundance during the latter part of the season. Our findings demonstrate that elevated temperatures and genetic variation each affect spring and autumn phenology, as well as the seasonal dynamics of higher trophic levels. To achieve a predictive understanding of the ecological responses, and potential evolutionary changes, of natural food webs in response to climate warming, we should thus merge the frameworks of global warming and community genetics.

Investigating the circadian clock of Verticillium dahliae and its influence on pathogenicity E. CASCANT-LOPEZ, H. J. Bates, S. Crosthwaite, R. J. Harrison, NIAB EMR, East Malling, U.K.

The circadian clock allows organisms to anticipate and synchronise to daily environmental changes. Little is known about circadian clocks in other fungi than Neurospora crassa (Nc). We sought to characterise the presence of a circadian clock in Verticillium dahliae (Vd) and its influence on pathogenicity. We identified homologs of all the $N c$ circadian clock genes in $V d$. No evidence for an entrainable, free-running rhythm was observed at the morphological level. Moreover, temporal gene expression profiling using RNA-Seq showed a lack of rhythmic gene expression. Deleting the core clock gene frq did not have an effect on fungal morphology. However, the deletion of the TF and photoreceptor $w c-$ 1 resulted in the abolishment of microsclerotia production, and the light-regulated spore production, confirming that $w c-1$ is involved in the production of the daily developmental rhythm by mediating transcriptional responses to white light. RNA-Seq carried out in $w c-1$ knockout mutants demonstrated a widespread effect of $w c-1$ on metabolism, redox processes and pathogenicity. Finally, frq and $w c-1$ were found to be required for full pathogenicity of $V d$ on Arabidopsis thaliana and strawberry plants under light/dark conditions. In conclusion, $V d$ presents all the necessary genetic loci for a functional clock, but there is no evidence of rhythmicity. However, putative clock genes play an important role in the overall fitness of the fungus, suggesting that they have additional non-circadian roles.

Salmonella persistence in agricultural soil and ability to colonize plants vary in different soils and plant species S. Jechalke (1), J. Schierstaedt (2), A. Zarkani (1), B. Flemer (2), R. Grosch (2), K. Smalla (1), A. SCHIKORA (1), (1) Julius Kühn-Institut, Institute for Epidemiology and Pathogen Diagnostics, Braunschweig, Germany; (2) Leibniz Institute of Vegetable and Ornamental Crops, Dep. Plant-Microbe Systems, Großbeeren, Germany

Salmonella and other human pathogenic bacteria may use plant as alternative hosts. Until now, little is known about factors influencing this colonization. This knowledge, however, is essential for efficient strategies aiming at safe produce. In this study, we investigated the interplay between different factors which might influence the persistence of Salmonella in agricultural soils and its ability to colonize plants. Different soil type, organic fertilisation, plant species and the means of Salmonella entry into the plant production system were amongst them. We selected $S$. Typhimurium strain 14028s and LT2 and S. Senftenberg. Our results revealed that all strains are able to persist in soil during the vegetation period. Loamy soil supported Salmonella better than sandy soil, also an application of fertilizers had a positive effect. All tested plants: lettuce, tomato and corn salad were colonised, providing evidence for internalisation from the soil via the root. Transcriptome analysis assessed during Salmonella response to plants or root exudates revealed an upregulation of genes associated with biofilm formation and virulence. Plants inoculated with Salmonella showed a strong up-regulation of genes associated with immune and stress responses. In summary, we showed that environmental factors influence the persistence and interactions between human pathogens and plants. This understanding is therefore a starting point for new strategies to provide safe food for the consumer.

Establishment of a high throughput system for screening bacterial quorum sensing inhibitors J. W. Zhang, C. G. Xuan, W. J. Jiang, L. Q. ZHANG, Department of Plant Pathology, China Agricultural University, Beijing, China

Quorum Sensing (QS) is a mechanism by which bacteria coordinate gene expression in response to fluctuations of population density. In many bacterial pathogens, QS is a central regulator for virulence expression and thus offers an attractive target for novel antibacterial drugs. We constructed a QS reporter containing a beta-lactamase gene fused with the traG gene promoter fragment of Agrobacterium tumefaciens, which expressed in the presence of TraR and signal molecules $N$-acyl homoserine lactones (AHLs) and used this reporter to establish a high-throughput system for screening QS inhibitors. From nearly 5,000 tested compounds, at least 20 chemicals were identified to be able to inactivate the betalactamase activities of the QS reporter. Four representative inhibitors effectively suppressed QS-dependent phenotypes in different pathogenic bacteria, including the production of pyocyanin and proteases in Pseudomonas aeruginosa, conjugational transfer of Ti plasmid in $A$. tumefaciens, and the swimming motility and production of cell wall degrading enzymes in Pectobacterium carotovorum. In the meantime, a modified protocol was applied to screen quorum quenching genes from cosmid libraries of bacterial genomic DNA or metagenomic DNA. Four AHL-degrading genes were identified from over 80,000 cosmid transformants. Together, our high throughput method established in this study is suitable for screening both small molecular QS inhibitors and genetic elements for AHL inactivation.

Effects of fomesafen on soil enzyme activities, microbial population and its degradation in the rhizosphere soil of soybean H. HU, Institute of Agricultural Resources and Regional Planning, CAAS, Beijing, China 
Fomesafen is used widely in soybean fields in northern China. However, the impact of fomesafen on microbial communities in rhizosphere soils is unknown. In this research. We investgated fomesafen degradation and the impact of herbicide on soybean rhizosphere soil in rhizoboxes in a greenhouse. Fomesafen had shorter half-life in rhizosphere soil than previously reported for bulk soil from the same location (87 days vs 120$)$. Although not immediately apparent, urease and invertase activities in the rhizosphere soil were decreased significantly by both the high ( $37.5 \mathrm{mg}$ $\left.\mathrm{kg}^{-1}\right)$ and low $\left(18.75 \mathrm{mg} \mathrm{kg}^{-1}\right)$ doses of fomesafen from days 30 and 45 respectively until the end of the experiment ( 90 days). The $16 \mathrm{~S}$ amplicons analysis demonstrated that fomesafen had a dose dependent effect, reducing alpha diversity and altering beta diversity. Significant phylum level was decreased in five of the ten phyla that were most abundant in the control. Proteobacteria was the only phylum whose relative abundance increased in the presence of fomesafen, driven by increases in the Methylophilacaea, Dyella, and Sphingomonas genera. Our data suggest that, despite being degraded more rapidly in the rhizosphere than in bulk soil, fomesafen had long-lasting functional impacts on the soil microbial community.

Plant nitrogen supply affects Arabidopsis thaliana susceptibility to Botrytis cinerea and reveals novel virulence functions M. C. Soulie (1,2), A. Davière (2), V. Brunaud (3), L. Soubigou-Taconnat (3), M. FAGARD (2), (1) Sorbonne Universités, UPMC Université Paris 06, UFR 927, Paris, France; (2) Institut Jean-Pierre Bourgin, INRA, AgroParisTech, CNRS, Université Paris-Saclay, Versailles, France; (3) IPS2, CNRS, INRA, Université Paris-Sud, Université Evry, Université Paris-Saclay, Orsay, France

Botrytis cinerea is a nectrotrophic plant pathogen that leads to important economic losses in many crops. $B$. cinerea has a large arsenal of virulence, including the production of cell wall degrading enzymes, reactive oxygen species and toxins. $B$. cinerea has a wide host range and plant resistance to this pathogen is quantitative. Nitrogen $(\mathrm{N})$ is a major limiting factor of plant growth and soils frequently lack sufficient $\mathrm{N}$. Large quantities of $\mathrm{N}$ fertilizers are used worldwide and have an impact on crop disease. However, the variety of effects observed indicate that there is a complex relationship linking $\mathrm{N}$ availability to plant disease. We showed previously that high $\mathrm{N}$ supply increases the susceptibility of $A$. thaliana to $B$. cinerea. A transcriptomic approach of $B$. cinerea-infected $A$. thaliana plants grown in low or high $\mathrm{N}$ has enabled us to identify both fungal and plant genes for which expression is affected by the plant's N status. Characterization of corresponding fungal knock-out mutants led to the identification of novel B. cinerea virulence genes, including a GST. On the plant side, we identified the jasmonate signaling pathway as a key player in the modulation of $A$. thaliana susceptibility by $\mathrm{N}$ supply. Our current working model is that $\mathrm{N}$ availability for plants primarily affects the outcome of plant-pathogen interactions through its effect on plant defense and pathogen virulence gene expression.

Investigating the control of clock genes in Verticillium dahliae

E. Cascant-Lopez, H. J. BATES, S. Crosthwaite, R. J. Harrison, NIAB EMR, East Malling, U.K.

Verticillium dahliae $(V d)$ is a soil-borne fungal plant pathogen of many crop species causing Verticillium wilt. Timing of the events during the life cycle and in response to environmental conditions is important for successful infection and disease progression. In the related soil dwelling saprotrophic fungus, Neurospora crassa (Nc), the molecular mechanisms of the circadian clock have been well characterised. The central components of the clock, WCC and FRQ produce transcription-translation feedback loops that generate pools of mRNA and protein leading to rhythmic physiological changes including daily formation of conidia. Analysis of the $V d$ genome reveals homologues to all of the core clock components. Despite this we have found no evidence of a circadian clock but there is evidence of involvement of WC1 in daily spore and conidial formation. Gene expression profiling showed a lack of rhythmic frq expression which, in contrast to Nc frq, appears to be highly constitutively expressed. There is slight induction of $f r q$ in response to light in WT $V d$ whereas a $w c l$ deletion mutant maintains high constitutive levels of $f r q$ with no light induction. ONT dRNAseq shows differences in the transcripts originating from the frq locus in $V d$ compared to $N c$. Transcription at $f r q$ in $N c$ is subject to tight control involving antisense $q r f$ and an upstream lncRNA, both of which are absent in $V d$. Work is ongoing to elucidate the roles of WC1 and FRQ in the control of light regulated genes in $V d$.

The effects of immune activation on clock and defence gene expression

S. J. CARGILL, S. H. Spoel, G. van Ooijen, School of Biological Sciences, University of Edinburgh, Edinburgh, U.K.

Organismal behaviour and biological processes are often coordinated by molecular circadian clocks to anticipate predictable daily changes in the environment. The expression of approximately a third of all genes in Arabidopsis are clock-regulated, including many genes involved in immunity. In plants, the circadian clock primes immune responses when pathogen attack is most likely to occur, resulting in temporal differences in susceptibility to pathogens over the course of a day. The mechanisms underpinning clock-gated immune responses, however, are poorly understood, hampering the agricultural exploitation of this system to boost plant immunity. Here we provide evidence that pathogen-associated molecular patterns (e.g. bacterial flagellin, EF-Tu) and the immune hormone salicylic acid alter the oscillating transcription of core clock genes. Conversely, we show that immune gene activation is differentially regulated during subjective dawn and dusk. We propose that reciprocal interaction between the circadian clock and different layers of the immune response shape the outcome of plant-pathogen interactions.

Characterizing the role Ralstonia solanacearum's catabolic pathways play in pathogenesis in the xylem \& root tomato environment C. HAMILTON, O. Steidl, A. MacIntyre, C. Allen, University of Wisconsin-Madison, Madison, WI, U.S.A.

The soilborne pathogen Ralstonia solanacearum (Rs) causes bacterial wilt disease by colonizing plant xylem vessels, triggering wilt and death. $R s$ affects over 200 species, including tomato. Although xylem sap has been considered nutrient poor, $R s$ populations in wilting plants surpass $10^{9}$ $\mathrm{CFU} / \mathrm{g}$ of stem and ex vivo sap from infected plants supports bacterial growth. Previous genomic, transcriptomic, and metabolomic analyses offered clues about the pathogen's carbon sources during infection. These datasets suggested $R s$ can use dozens of carbon sources at low cell densities, as in soil or very early disease. However, once the bacterium is established in host xylem, a major regulatory switch limits its available carbon sources to some present in xylem sap like sucrose, glucose, fructose, and myo-inositol. We tested the hypothesis that $R s$ has adapted to use a narrow set of carbon sources abundant in host xylem sap during disease. Using bacterial mutants lacking specific catabolic pathways, plant virulence assays, and quantitative metabolomics of xylem sap, we defined the link between xylem sap composition and virulence in the $R s$ tomato pathosystem. These studies showed that sucrose is the most important carbon source for the pathogen when it is growing in tomato stems. 
Overall, the sugars that were important in xylem colonization were not needed for successful root colonization and vice versa. The contribution of each carbon source to $R s$ fitness aligned with infection stage.

Virulence characters of Erwinia amylovora and their variation under plant abiotic stress

R. JERIDI (1), M. Farjad (2), M. A. Mekni (3), A. Landoulsi (4), M. Fagard (5), (1) Institut JeanPierre Bourgin, INRA, AgroParisTech, CNRS, Université Paris-Saclay, Versailles, France; (2) Institut JeanPierre Bourgin, INRA, AgroParisTech, CNRS, Université Paris-Saclay, France; (3) Centre National de Greffe de Moelle osseuse (C.N.G.M.O.) Tunisie., Tunisia; (4) Laboratoire de biochimie Biologie moléculaire, FSB, U Carthage, BizerteTunisie, Tunisia; (5) Institut Jean-Pierre Bourgin, INRA, AgroParisTech, CNRS, Université Paris-Saclay, Versailles, France

Plant diseases can be caused by adverse environmental conditions related to climate, nutrition and pollution or by phytopathogenic agents such as bacteria. In this context Erwinia amylovora, the causal agent of the fire blight disease is considered an invasive disease that threatens several rosaceous plants distributed worldwide and threatens their commercialization. During this work, 20 strains of E. amylovora were isolated from different regions of North Tunisia and identified by biochemical and molecular methods. Then, we studied virulence characters such as biofilm formation, motility and pathogenicity in pear and found a variation in these traits between the strains. These strains also showed a variation in virulence on the non-host plant Arabidopsis thaliana. On the other hand, we showed that nitrogen supply to plants affects the susceptibility of $A$. thaliana to E. amylovora CFBP1430. We also found that increased susceptibility was correlated to an increase or decrease of specific metabolites in response to bacteria. To understand the natural variability of this process, we will study in planta virulence gene expression of the Tunisian strains in plants grown under different $\mathrm{N}$ regimes. Identification of apoplastic metabolite and protein accumulation in plants grown under different $\mathrm{N}$ regimes could help understand the variations in plant susceptibility observed.

Dissecting the Disease Triangle: Hosts, pathogens and the environment R. BART, Donald Danforth Plant Science Center, St. Louis, MO, U.S.A.

Xanthomonas is a known pathogen of most crop plants. As a culturable, genetically tractable organism with a small genome, Xanthomonas is also an excellent model for studying the disease triangle - host, pathogen and environment. Distinct yet complementary projects of diverse hosts will be presented with an emphasis on our use of transcriptomics to simultaneously monitor molecular changes in host and pathogen. These data are being combined with phenomics analyses to elucidate the role of the environment on disease progression.

The White-Collar Complex and FREQUENCY drive pathogenesis in Cercospora zeae-maydis J. B. Ridenour, B. H. BLUHM, University of Arkansas, Fayetteville, AR, U.S.A.

Cercospora zeae-maydis is an important foliar pathogen of maize. In C. zeae-maydis, pathogenesis requires light and Crp1, an ortholog of the White-Collar Complex (WCC) subunit WC-1 of Neurospora crassa. Here, Crp2, an ortholog of the WCC subunit WC-2, was found to be required for infection. The absence of light or disruption of Crp1 or Crp2 impairs stomatal tropism and appressorium formation, suggesting that light coordinates pathogenesis through the WCC. However, the genetic network regulated by the WCC in C. zeae-maydis is not known. In N. crassa, the WCC regulates the circadian clock component FREQUENCY. An ortholog of FREQUENCY, designated Frq1, was identified in $C$. zeae-maydis. Expression of FRQ1 was induced by light in a Crp1-dependent manner and showed circadian oscillation in the absence of environmental input, demonstrating that $C$. zeae-maydis has a circadian clock. Deletion of $F R Q 1$ impaired aspects of pathogenesis, including stomatal tropism and appressorium formation. Overexpression of frequency in $N$. crassa impairs clock function. Overexpression of $F R Q 1$ in the C. zeae-maydis wild type did not impact virulence, but overexpression of FRQ1 in a Crp1-deficient strain restored pathogenesis. These findings suggest that a Crp1-mediated light response regulates pathogenesis by driving expression of $F R Q 1$ and Frq1 may have a clock-independent role in the process, a phenomenon that has not been described previously.

Evaluation and characterization of systemic resistance in forest trees: Eucalyptus grandis as a case study. S. K. FLEMINGTON (1), L. Zwart (2), L. Shuey (3), S. Naidoo (4), (1) Forest Molecular Genetics Program, FABI, University of Pretoria., Pretoria, South Africa; (2) Chameleon Communications International, U.K.; (3) Department of Agriculture and Fisheries, Queensland Government, Brisbane, Australia; (4) Forest Molecular Genetics Program, FABI, University of Pretoria, Pretoria, South Africa

In plants, systemic acquired resistance (SAR) and induced systemic resistance (ISR), elicited by non-lethal infection, provides distal tissues with long-lasting, broad spectrum resistance to biotic stress. These phenomena have been poorly studied in woody-hosts but are particularly relevant for these species as they are exposed to multiple biotic stresses for an extended period of time. RNA-sequencing revealed dramatic phytohormone related transcriptional responses in systemic tissue of a resistant Eucalyptus grandis clone upon biotic challenge with the fungal pathogen Chrysoporthe austroafricana. We aimed to (i) validate the systemic responses at the molecular level, (ii) determine how long the systemic responses were sustained and (iii) determine whether this induction is accompanied by phytohormone changes. We analyzed systemic tissue (leaves) at 3,7,14, 21- and 28-days postinoculation (dpi) of E. grandis stems using RT-qPCR and metabolite quantification. From our preliminary analysis, we suspect that significant changes at key signaling points in the Salicylic acid (SA) and Jasmonic acid (JA) pathways promote resistance in distal tissue rather than leading to induction of SAR or ISR. We hypothesize that the phytohormone related genes involved in these key points become primed at a transcriptional level once the initial infection is contained. Our inquiry will characterize and evaluate the relevance of systemic defence responses to secondary biotic challenges in trees.

BABA and ABA: The relation between a novel (signaling?) molecule in plant defense and a classic plant hormone. F. STEFANELLI (1), I. Baccelli (2), B. Mauch-Mani (3), (1) University of Neuchatel, Neuchatel, Switzerland; (2) National Research Council, Sesto Fiorentino, Italy; (3) University of Neuchâtel, Neuchatel, Switzerland 
Beta aminobutyric acid (BABA) is a well-known priming agent, recently shown to be produced by plants. The relation between exogenously applied $\mathrm{BABA}$ and $\mathrm{ABA}$ is visible in the $\mathrm{BABA}$-insensitive mutant $i b s 3$ that does not synthetize $\mathrm{ABA}$ and is impaired in priming. Furthermore, BABA-induced resistance against biotrophic and necrotrophic pathogens is based on ABA-dependent callose deposition. However, it is not known if there is an interaction between plant-produced BABA and ABA. This gap in knowledge is due to a lack of information on the mechanisms of biosynthesis and signaling of endogenous BABA. Here, we show a direct connection between ABA and endogenous BABA. Treatment of Arabidopsis plants with ABA leads to an accumulation of BABA. We will present our results concerning BABA accumulation in various $\mathrm{ABA}$-biosynthetic and $\mathrm{ABA}$-signaling mutants during pathogen infection, senescence, and salt stress. Moreover, to investigate the biosynthetic pathway of BABA, we have performed an RNA-seq analysis to identify common genes expressed during various BABA-inducing stresses (i.e. salt stress, submergence, infection with avirulent Pseudomonas syringae and Plectosphaerella cucumerina) and in the mutant cpr $5-2$ (a constitutive producer of high levels of BABA). We are currently screening T-DNA mutants for candidate genes presumably involved in BABA biosynthesis and will present these results.

Mutation of repressive NAC transcription factors as lead to pathogen resistance

M. PEREIRA MENDES, R. Hickman, C. M. J. Pieterse, S. C. Van Wees, Plant-Microbe Interactions, Department of Biology, Utrecht University, Utrecht, Netherlands

The phytohormone, salicylic acid (SA), is a critical regulator of the immune system that protects plants from infection. To advance our understanding of the transcriptional reprograming controlled by SA and underlying gene regulatory, we performed a high-density time course RNA-Seq experiment (14 time points), upon treatment of Arabidopsis leaves with exogenous salicylic acid (SA). Subsequent analysis of these expression data led to the discovery of two homologous NAC transcription factors (TFs) from, $A N A C A$ and $A N A C B$, that are coordinately upregulated by SA. The double mutant anacA anacB displayed enhanced resistance to the biotrophic pathogen Hyaloperonospora arabidopsidis (Hpa) and the hemi-biotrophic pathogen Pseudomonas syringae pv tomato (Pto), to a level that was comparable (Hpa) or even higher (Pto) than that of $d m r 6$, a mutant that was previously shown to contain increased SA levels. Interestingly, compared to the dmr 6 mutant, the anac double mutant was less severely compromised in plant growth. Altogether, this shows the great potential of these specific NACs for breeding of biotroph-resistant crops. Collectively, our work pinpoints and validates new ANACs TFs in plant immunity and provides a valuable resource for functional studies on SA signaling components in plant defense and development.

Rhamnolipids trigger induced-systemic resistance against Botrytis cinerea in Arabidopsis thaliana. M. TOUCHARD, R. Schellenberger, M. Cordier-Demissy, E. Huby, F. Mazeyrat-Gourbeyre, C. Clément, F. Baillieul, S. Dhondt-Cordelier, J. Crouzet, S. Cordelier, S. Dorey, Reims Champagne-Ardenne University, Reims, France

To combat diseases, plants possess an arsenal of defence mechanisms activated following microorganism perception. We have previously shown that a rhamnolipid secretome (RLsec) secreted by bacterial Pseudomonas species is highly effective on Arabidopsis thaliana leaves to induce local resistance against phytopathogenic microorganisms. The aim of this study is to determine the ability of Arabidopsis to perceive RLsec at the root level, and to investigate whether this perception result in an induced systemic resistance (ISR). Classical plant immunity markers such as reactive oxygen species (ROS) production and mitogen-activated protein kinases (MAPK) activation were investigated in roots after RLsec challenge. Surprisingly, whereas both markers are induced in leaves, RLsec perception by roots only triggered MAPK activation. In addition, early differentially expressed genes in roots were identified through a transcriptomic approach. Resistance of Arabidopsis plants against $B$. cinerea was investigated by applying RLsec at the root level followed by fungal inoculation on leaves. Our data show that RLsec triggers a systemic immune response in Arabidopsis resulting in an effective ISR against the necrotrophic fungus. Using a genetic approach, we investigated the signaling pathways involved in RLse ISR.

Systemic acquired susceptibility caused by the fungal wheat pathogen Zymoseptoria tritici H. SEYBOLD (1,2), T. Demetrowitsch (3), M. A. Hassani (1,2), S. Szymczak (4), K. Schwarz (3), E. H. Stukenbrock (1,5), (1) Max Planck Institute for Evolutionary Biology, Plön, Germany; (2) Environmental Genomics, Botanical Institute, University of Kiel, Germany; (3) Institute of Human Nutrition and Food Science, University of Kiel, Germany; (4) Institute of Medical Informatics and Statistics, University of Kiel, Germany; (5) Environmental Genomics, Botanical Institute, University of Kiel, Kiel, Germany

The fungal wheat pathogen Zymoseptoria tritici is the causal agent of Septoria tritici Blotch (STB), a major threat to wheat production worldwide. Infection begins with a symptom-free and presumably biotrophic phase of fungal growth followed by a lifestyle switch to necrotrophic growth. We aimed to elucidate the nature of the symptom-free infection phase and the putative immune suppression during biotrophic fungal growth. In addition to wheat cultivars of varying STB susceptibility, we used different pathovars of Pseudomonas syringae bacteria to study the physiological responses and the extent of immune suppression in wheat caused by $Z$. tritici. During the biotrophic infection, we observed that non-adapted $P$. syringae strains were able to co-infect wheat. This effect was not limited to the fungal infection site but extends to adjacent and systemic leaf areas. In contrast, after $Z$. tritici infection of wheat cultivars with STB resistance genes, we observed systemic acquired resistance also towards adapted $P$. syringae strains. Using a plant metabolomics approach, we could confirm the fungus-mediated local and systemic resistance suppression during colonization of susceptible wheat. Our findings suggest that (I) virulent $Z$. tritici infections of wheat cause systemic acquired susceptibility allowing colonization by non-adapted phytopathogens and (II) the fungal infection impacts leaf microbiome dynamics.

Identification and characterization of small-molecular compounds that inhibit salicylic acid-mediated signaling pathway in Arabidopsis N. ISHIHAMA (1), Y. Noutoshi (2), S. Choi (1,3), I. Saska (1), S. Asai (1), K. Takizawa (1), Y. Kondoh (4), Y. Nomura (1), H. Nakagami (5,6), H. Osada (4), K. Shirasu (1), (1) RIKEN Center for Sustainable Resource Science, Yokohama, Japan; (2) Okayama Univ, Okayama, Japan; (3) Department of Life Science, International Christian University, Mitaka, Japan; (4) RIKEN Center for Sustainable Resource Science, Wako, Japan; (5) RIKEN Center for Sustainable Resource Science, Japan; (6) Max Planck Institute for Plant Breeding Research, Germany 
Chemical biology is an expanding discipline that utilizes bioactive small molecules to elucidate biological mechanisms. We applied a chemical biology approach to investigate Arabidopsis immune responses to Pseudomonas syringae pv. tomato (Pst) DC3000 avrRpm1. Using 2,768 compounds from natural and synthetic compound libraries, we identified three structurally related inhibitors of plant immune responses. Interestingly, Arabidopsis plants treated with these inhibitors, designated as P7, P8 and P9, significantly increased susceptibility to Pst DC3000. These compounds suppressed the expression of salicylic acid (SA) marker gene, PR1, in response to SA treatment, with P7 showing the greatest effect. Transcriptome analysis revealed that P7 widely affects the expression of SA-responsive genes. Moreover, we found that P7 prevented an increase of cellular GSH/GSSG ratio and suppressed the nuclear accumulation of NPR1, which is a redox-sensitive protein and plays a central role in SA signaling pathway, upon SA treatment. These results suggest that P7 targets component(s) of SA-mediated signaling pathway, upstream of NPR1, or component(s) of another pathway that converges and impinges on the SA-mediated signaling pathway. Furthermore, we have identified a cupin domain containing protein, CUPIN1, as a target of P7. The possible roles of CUPIN1 in plant immune signaling will be discussed.

Long-distance transport factors of cucurbit-infected potyvirus W. C. HU (1), H. W. Cheng (1), S. D. Yeh (2), (1) NCHU, Taichung, Taiwan; (2) Natl Chung Hsing Univ, Taichung, Taiwan

Plant viruses systemically infect plant hosts by hijacking the traffic machinery though phloem, a conducting pathway for long-distance translocation of informational macromolecules within plants. However, the details of plant virus long-distance factors and mechanisms remain to be demonstrated. Here, we established a delicate system for collecting phloem sap from cucumber plants to analyze the possible viral complex involved in systemic translocation. Zucchini yellow mosaic virus (ZYMV, genus Potyvirus) is an important cucurbit-infecting virus. A novel nsstag system was used to label individual potyviral genes for long-distance trafficking study. The nss monoclonal antibody combined with specific antisera to viral proteins revealed that, ZYMV HC-Pro, CI, NIb, and CP proteins are present as viral phloem-rich proteins from infected cucumber plants. The phloem sap also containing infectious viral RNA, but the virions were not observed under TEM, suggesting that the ZYMV translocation is through a form of ribonucleic protein complex. Moreover, the long-distance movement complex, which comprises of the aforementioned viral proteins and other host factors, in the phloem sap was also identified by co-immunoprecipitation with our antibodies. Several candidate host proteins including phloem proteins and RNA-binding protein have been identified by LC/MS/MS method. The interaction and functional analysis between viral and host factors are being investigated.

Root-to-shoot signaling in mycorrhizal plants upon Botrytis cinerea infection.

N. SANMARTín MARTÍNEZ (1), J. Pastor Fernández (1), P. Sánchez Bel (1), V. Pastor Fuentes (1), M. J. Pozo (2), V. Flors (3), (1) Universitat Jaume I, Castellón, Spain; (2) EEZ-CSIC, Granada, Spain; (3) Universidad Jaume I, Castellón, Spain

Arbuscular mycorrhiza fungi (AMF) establish a symbiosis with plant roots to exchange nutrients and water. Besides the nutrient improvement, AMF enhance plant defences upon pathogen attack, so called Mycorrhiza Induced Resistance (MIR), which is under the hormonal control of JA pathway. Plants have to keep a balance between growth and defence, thus when a stress is present, they readjust their metabolism to deal with it. Mycorrhizal plants change their metabolism and hormone levels, not only in roots, but also in the shoots. This rearrangement prepares mycorrhizal plants to defend themselves better against pathogens. In this study, we aim to decipher metabolic changes in mycorrhizal roots during a shoot Botrytis cinerea infection and to identify those metabolites transported from roots to shoots. Some pathways of secondary metabolism, such as lignans and other pathways related to defence, present priming profile. Then, some of these lignans were identified. Since AMF symbiosis takes place in roots, some of the compounds responsible of the shoot protection could be transported from roots to shoots. Root efflux was analysed and some compounds were identified, such as some lignans. In conclusion, mycorrhizal roots change their metabolism and manipulates the plant shoot defence mobilizing some compounds such as lignans, which may have relevance in MIR due to their priming profile.

Salicylic acid-driven association of LENRV and NIMIN1/NIMIN2 binding domain regions in the C-terminus of tobacco NPR1 transduces SAR signal

D. Neeley, E. Konopka, A. Straub, F. Maier, U. M. PFITZNER, Universitaet Hohenheim, Germany

NONEXPRESSOR OF PATHOGENESIS-RELATED (PR) GENES1 (NPR1) is the central regulator of salicylic acid (SA)-induced $P R$ lgeneexpression and systemic acquired resistance (SAR). The mechanism how SA is transduced through NPR1 is discussed controversially. Previously, we showed that Arabidopsis and tobacco (Nt) NPR1 contain two domains in their C-terminal thirds with relevance to SA signaling.SA sensitivity of NPR1 relies on the arginine residue in the LENRV motif, and SA-induced NIM1-INTERACTING (NIMIN, N) proteins bind to a highly conserved sequence termed N1/N2 binding domain (BD). We demonstrate that LENRV and N1/N2BD regions of tobacco NPR1, separated from each other, interact in yeast, in vitro, in plant and in animal cells. Physical association of LENRV and N1/N2BD parts is enhanced considerably by SA and functional analogs, but not by a non-functional analog. Furthermore, physicalassociation requires R431 and is most effective with intact LENRV and N1/N2BD interfaces.Association of separated LENRV and N1/N2BD parts by SA reconstitutes a functional NtNPR1 C-terminus, displaying transcription activity and able to interact with TGA transcription factors at two distinct sites. Tobacco NIMIN proteins can assemble LENRV and N1/N2BD parts into ternary complexes suggesting that NIMINs shape the NPR1 C-terminus to modulate SA signaling.

A look on the wild side of tomato plants to tackles biotic stresses

A. K. JAISWAL (1), T. Mengiste (1), J. Myers (2), L. Hoagland (1), (1) Purdue University, West Lafayette, IN, U.S.A.; (2) Oregon State University, OR, U.S.A.

Crop domestication events followed by targeted breeding practices across plant taxa was a pivotal achievement for human civilization to introduce superior agronomic traits needed to meet human needs, and adapt varieties to local agronomic environments. However, domestication also caused a strong reduction in the genetic diversity of modern cultivars compared to their wild relatives. It remains undetermined how plant domestication may have influenced plant interaction with aboveground pathogens and belowground beneficial microbes. We examined the levels 
of induced resistance and changes in hormonal signaling pathways by Trichoderma harzianum, a beneficial soil microbe, against two foliar pathogens in 30 diverse tomato genotypes across a domestication gradient, including wild relatives, landraces and modern commercial cultivars. Our results have demonstrated that Trichoderma enhanced both aboveground canopy and belowground root biomass in all tested genotypes. Wild relatives and landraces tomato were more responsive to Trichoderma against foliar pathogens in comparison to early modern cultivars. In addition to these finding, transcriptional profiling of responsive and non-responsive genotypes will be discussed. These findings have important implications for understanding the mechanisms behind differences in the induction of resistance among genotypes and may someday be used to identify molecular markers for efficient selection of responsive cultivars.

Chlorella fusca primes systemic immunity against Pseudomonas syringae in Arabidopsis S. M. LEE (1,2), C. M. Ryu (1,2), (1) KRIBB, Daejeon, Korea, Republic of (South); (2) University of Science and Technology, Daejeon, Korea, Republic of (South)

Biological control agents including beneficial bacteria and fungi has been well studied as plant protection strategy. Previously, as novel biological control agents, microalgae Chlorella-mediated plant protection against pathogenic fungi and nematode has been reported on different crop species. However, the underlying mechanism and defense signaling is largely unknown. To establish the system of Chlorella-elicited immunity in model plant, C. fusca was sprayed on Arabidopsis thaliana leaves to protect against Pseudomonase syringae pv. tomato DC3000 (Pto DC3000). Chlorella-elicited immune activation can be confirmed by the lack of direct antagonism between Chlorella and Pto DC3000. Transcriptome analysis to understand Chlorella-mediated defense priming reveled that Chlorella activates multi-layers of defense signal cascades from cysteinerich receptor kinase to $W R K Y$ transcription factors only after pathogen challenge. Furthermore, defense priming-derived foliar Chlorella treatment leads flg22- and Pto DC3000-triggered PAMP-triggered immunity including oxidative busting and callose deposition. The attempts to find Chlorella determinants allow us to identify the secreted metabolites including D-lactic acid. Our study first demonstrates the molecular mechanisms of Chlorella-elicited plant immunity and its bioactive determiant.

\section{Alternatation of WRKY62 and WRKY76 Expression Reprograms Rice Metabolism for Defense} X. Chen, J. Liu, X. Liang, Z. GUO, China Agricultural University, Beijing, China

WRKY transcription factors are involved in plant defense signaling pathway. Here we report WRKY62 and WRKY76, two WRKY IIa subfamily genes in rice, which were induced by chitin and flg22 treatments. They interacted with each other physically through N-terminal leucine-zipper motif and regulated rice defense responses. The RNA interference of WRKY62/WRKY76 (dsOW62/76), knockout lines of WRKY62 and WRKY76, enhanced resistance to the blast fungus Magnaporthe oryzae and the leaf blight bacterium Xanthomonas oryzae pv oryzae, with greatly increased expression of defense-related genes and the accumulation of phytoalexins. Further, metabolomic approaches were used to dissect the enhanced disease resistance in dsOW62/76 transgenic rice plants. A large number of phenolamides, conjugates of the phenolic acids with amines, were elevated, most of which were at higher levels in dsOW62/76 compared with wild type (ZH17) plants; however, most of the free pools of flavonoids were at lower levels in dsOW62/76. Contents of salicylic acid (SA) and jasmonic acid (JA)/JA-Ile were increased in dsOW62/76 and knockout lines of individual WRKY62 and WRKY76 genes. These results indicate that WRKY62 and WRKY76 may form a highly complex interacting regulatory network and function as negative regulators of biosynthetic defense-related metabolites in rice.

Bacterial diversity associated with the rhizosphere and endosphere of two halophytes: Glaux maritima and Salicornia europaea K. YAMAMOTO (1), Y. Shiwa (1), T. Ishige (2), H. Sakamoto (3), K. Tanaka (2), M. Uchino (1), N. Tanaka (1), S. Oguri (3), H. Saitoh (4), S. Tsushima (1), (1) Department of Molecular Microbiology, Tokyo University of Agriculture, Japan; (2) NODAI Genome Research Center, Tokyo University of Agriculture, Japan; (3) Department of Northern Biosphere Agriculture, Tokyo University of Agriculture, Japan; (4) Department of Molecular Microbiology, Tokyo University of Agriculture, Tokyo, Japan

Root-associated microbial communities are important in the adaptation of halophytes to coastal environments. Here, we studied the diversity and community structure of both rhizosphere and root endosphere bacteria in two halophytic plants: Glaux maritima and Salicornia europaea. We sampled the rhizosphere $(\mathrm{Rh})$, the root endosphere (Re), and bulk control soil (Bl) samples, and performed bacterial 16S rRNA sequencing. Among the G. maritima samples, the richness and diversity of bacteria in the $\mathrm{Rh}$ were higher than those in the Re but were lower than those of the Bl. In contrast for S. europaea, the Bl, the Rh, and the Re all had similar bacterial richness and diversity. The number of unique operational taxonomic units within the Re, the Rh, and the Bl were 181, 366, and 924 in G. maritima and 126, 416, and 596 in S. europaea, respectively. The dominant phyla across all samples were Proteobacteria and Bacteroidetes. Actinobacteria was abundant in the Re from G. maritima. A principal coordinate analysis demonstrated significant differences in the microbiota composition associated with the plant species and type of sample. These results indicate that there are clear differences in bacterial community structure and diversity between G. maritima and S. europaea. This is the first report to characterize the root microbiome of G. maritima, and to compare the diversity and community structure of Rh and Re bacteria between G. maritima and S. europaea.

Distinguishing Nutrient-Dependent Plant Driven Bacterial Colonization Patterns in Alfalfa K. M. MOCCIA, A. Willems, S. Lebeis, University of Tennessee, Knoxville, TN, U.S.A.

Recent studies from the Earth Microbiome Project have shown that in moving from the rhizosphere to the surface or inside of the plant, the microbial diversity plummets (Thompson et al., 2017). While few in number the microbes that are able to live in close association with the plant, endophytes or strongly adherent epiphytes, have been shown to be widely culturable (Bai et al., 2015). Small synthetic communities in an environment that is both highly selective and easily cultured can then provide scientists a way to study the interactions between a host and it's colonizers. We created a synthetic community from isolates of the agriculturally relevant plant Medicago sativa and screened for physiological traits relating to plant growth promotion such as phosphate and potassium solubilization. Using drop out communities, where each community member is removed one at a time to determine its role in community structure, we identify two members who have large roles in overall 
community structure; Pantoea sp. R4 and Williamsia sp. R60. Within the drop out communities, we modulate nitrogen levels and uncover microbial structure changes that only occur within the presence of the plant and ones that are dependent on changes in nitrogen.

The characteristics of microbiota cultivated potato (Solanum tuberosum) cv. Sumi in South Korea

G. BAK, Rural Development Administration, PyeongChang, Korea, Republic of (South)

The relationship between plant and microbes is highly complicate. Although many researchers have tried to elucidate that to apply crop production in agriculture, it is very difficult. However, supported by researcher's efforts, it is generally known that microorganisms and microbiota could influence on crop growth and productivity. Potato (Solanum tuberosum) is the four major food crops in worldwide. Various cultivars are cultivated depending on climate and environment each country. In South Korea, cultivar Sumi which was originated cultivar Superior, is most widely cultivated. While there are some potato microbiota research works, there is no study on cv. Sumi in South Korea. Here, we tried to identify microbiota cultivated potato cv. Sumi, and environmental factors which could affect microbiota on three different regions through 16S rDNA and ITS1 targeted NGS (next generation sequencing) analysis. As a result, there are some distinct OTUs were detected while some universal OTUs which already reported by previous potato research works, were detected showing clear distribution on beta diversity analysis. The analysis of correlation with environmental factor using soil chemical properties, climate factor and potato yield showed significance with organic matter, available phosphate, respiration, temperature and yield on fungal microbiota, while calcium, magnesium, available phosphate, $\mathrm{pH}$, solar radiation and temperature showed significance with bacterial microbiota.

Deciphering the influence of arbuscular mycorrhiza on the root exudate and microbiome of Lotus japonicus G. STABL (1), S. Schalk (2), R. Siani (3), M. Schloter (3), C. Dawid (2), C. Gutjahr (1), (1) Technical University of Munich (TUM) - Plant Genetics, Freising, Germany; (2) Technical University of Munich (TUM) - Food Chemistry, Freising, Germany; (3) Helmholtz Center Munich (HZM), Neuherberg, Germany

Plant roots are associated with a multitude of microorganisms, collectively called microbiota, which play symbiotic, pathogenic and commensal roles. Of great prominence in and around plant roots are arbuscular mycorrhiza fungi (AMF), which form symbiotic relationships with plants since 450 million years. In addition, the soil layer in direct contact with the root (rhizosphere) and the internal root tissues (endosphere) are colonized by numerous bacteria. Both the rhizosphere and endosphere represent very selective environments and the composition of the bacterial community inhabiting them differs from open soil. One strong determinant of the bacterial community composition in the rhizosphere is the chemical cocktail of the root exudate. In addition, it can be assumed, that the microbes, affect each other either directly or indirectly via the plant and this may contribute to a specific niche formation in the rhizosphere. It has been previously observed that AMF affect the metabolite composition of the root and the root exudates. However, it is unknown how this affects the microbial communities in the rhizosphere. To understand whether AMF influence a bacterial rhizosphere community directly or indirectly we study the impact of root colonization by AMF on the chemical composition of the root exudate of Lotus japonicus and how in turn this affects a synthetic bacterial community.

Inoculation of a microbial consortium for enhanced nutritional quality of maize (Z. mays) and potato (S. tuberosum) grown under field conditions

W. OVERBEEK (1), P. Page (2), S. Arunachalam (1), D. L. Smith (1), (1) McGill University, Department of Plant Science, Sainte Anne de Bellevue, QC, Canada; (2) SynAgri Company, Saint-Hyacinthe, QC, Canada

An intense interest in "biologicals", biological inputs for crop production, has developed over the last several years with microbial inoculums being some of the most promising. While most of the research done on inoculants have looked at enhanced plant growth promotion, one of the major benefits of those inoculants is their ability to enhance nutritional value of crops' harvest. Maize and potato are two staple food consumed throughout the world. However, there is no knowledge on increasing the nutritional content of those two crops through microbial inoculation. To answer this question, corn and potato were grown under field conditions where two different microbial consortiums were applied. For both consortiums, different concentrations, either the commercial rate or twice the commercial rate and timings of application, either at seeding, during side-dressing or at both times. were established. At the end of the growing season, cobs and tubers were harvested and dried. The total starch and protein content of cobs and tubers were quantified, additionally the total phenolic content of tuber was also quantified. Preliminary results show a yield increase of $11 \%$ and $4.65 \%$ for potato and corn respectively, with an increase of the starch content of $3.6 \%$, enhancing the nutritional value of those staple crops. One of the two microbial consortia is currently being investigated for its metabolome production to identify its possible mode of action as a plant growth promoter.

Rhizosphere community selection reveals bacteria associated with reduced root disease

C. YIN (1), J. M. Casa Vargas (1), D. C. Schlatter (1), C. Hagerty (2), S. Hulbert (1), T. Paulitz (3), (1) Washington State University, Pullman, WA, U.S.A.; (2) Oregon State University, Pendelton, OR, U.S.A.; (3) USDA-ARS, Pullman, WA, U.S.A.

Microbes benefit plants by increasing nutrient availability, producing growth hormones, and protecting against pathogens. However, it is largely unknown how plants shape root microbial communities. Here, we used a multi-cycling selection system with Rhizoctonia solani AG8 (AG8) inoculum and an AG8-susceptible wheat cultivar Alpowa to select for soil microbiomes that improve or reduce wheat susceptibility to AG8. Analysis of the soil microbial community using deep sequencing of the $16 \mathrm{~S}$ rDNA revealed distinct bacterial community profiles assemble over multiple plant cycles and in response to pathogen inoculum. After nine cycles of wheat, the bacterial community of plants was different between the wheat more tolerant to AG8 and the wheat less tolerant to AG8. Taxa in the phyla Acidobacteria, Actinobacteria and Candidate division WPS-1 were found at higher abundance in plants grown without AG8 inoculum than with AG8 inoculum, while the phylum Bacteroidetes showed the opposite trend. Interestingly, all taxa levels for Gemmatimonas, including phylum, class, order, family, genus, and OTU were consistently more abundant in wheat less tolerant to AG8, compared to the wheat more tolerant to AG8. Furthermore, a species of Janthinobaterium was isolated from the rhizosphere soil of wheat more tolerant to AG8 and exhibited broad antagonism against Pythium, AG8, and $R$. oryzae. 
Comparisons of Bacterial Community between Rhizosphere of Potato in Fields and the Surface of Potato Tuber in Storage S. JEE, G. Bak, G. Lee, RDA, Pyeongchang, Korea, Republic of (South)

Potato (Solanum tuberosum L.) is one of the most important crops in the world and cultivated as cash crops in highland agriculture in South Korea. The rhizosphere and the surface of potato tubers are considered as a reservoir for bacteria that might be interact with host and the environment. With 16S rRNA gene, molecular techniques such as Next Generation Sequencing are able to provide relatively rapid insight for bacterial diversity and abundance. The objectives of this study are to trace the changes of bacterial community on stage of tuber maturation, harvest, storage of potato, and to understand relationship of bacterial community and stage of potato tuber in field and storage. Rhizosphere soil and surface of tuber of Cv. Sumi and Cv. Jayoung were collected as the sample and total DNA were extracted. In regions of 16S rRNA gene, amplicons were amplified and sequenced on a Miseq sequencer. The meta data was analyzed with SILVA database (release 123) and Mothur software. Samples were compared between time, stage, and cultivar. The results show that there is not significantly different between time and cultivar. But, bacterial community in fields was different from it of storage condition.

A citrus huanglongbing-associated microbiota has the capacity to metabolize sugars in the sieve elements K. FUJIWARA (1), T. Fujikawa (2), (1) National Agriculture and Food Research Organization, Koshi, Japan; (2) National Agriculture and Food Research Organization, Tsukuba, Japan

Candidatus Liberibacter asiaticus (CLas) infects citrus plants and survives in the phloem, causing citrus huanglongbing (greening disease). It has become apparent that CLas needs CLas-associated microbiota for disease development. However, the functional relationships between CLas and other microbes in the community have not yet been clarified. We conducted metagenomics to investigate CLas-associated microbiota in leaf samples from rough lemon (Citrus jambhiri Lush.) with CLas infection, finding some bacterial species corresponding to the growth of CLas in contrast to healthy leaf samples. Then, we examined the enzymatic properties of CLas-associated microbiota between the infected and healthy leaves. Some enzymatic activities including alkaline phosphatase, alpha-glucosidase, beta-glucosidase, and N-acetyl-beta-D-glucosaminidase were relatively higher than those in the microbiota of healthy samples. Understanding associations between CLas-associated microbiota and its functional property may help us find the microbial factors influencing CLas survivals in citrus plants.

Species Specificity of Bacterial Colonization and Maintenance in the Rhizosphere of Arabidopsis thaliana S. L. HARRIS (1), C. A. Pelaez (2), E. A. Shank (3), (1) University of North Carolina, Chapel Hill, Chapel Hill, NC, U.S.A.; (2) University of North Carolina at Chapel Hill, Chapel Hill, NC, U.S.A.; (3) The University of North Carolina at Chapel Hill, Chapel Hill, NC, U.S.A. Bacteria living on plant roots form complex multispecies microbiomes in which microbes interact with each other and the abiotic environment. Root colonization by plant-growth-promoting bacteria (PGPB) may increase the health or development of host plants under diverse stressors; however, these bacterial treatments often do not provide substantial benefits to crops in field settings. Interactions with endogenous soil bacteria may affect behavior of PGPB, but few studies focus on the maintenance of rhizosphere communities. We hypothesized that bacterial maintenance in the rhizosphere microenvironment depended on the species of bacteria present. Using of a hydroponic plant growth assay, we quantified bacterial presence and visualized their spatial distributions on seedlings of Arabidopsis thaliana roots after transfer into different growth environments. We then investigated how the presence of multiple bacterial species affected colonization and maintenance of each bacterium, focusing primarily on species originally isolated from the $A$. thaliana rhizosphere. Future studies of multispecies plant microbiomes in changing environmental conditions hold the potential to improve crop productivity.

Temporal dynamics of the sap microbiome of grapevine under high Pierce's Disease pressure E. A. DEYETT (1), P. E. Rolshausen (2), (1) University of California Riverside, Riverside, CA, U.S.A.; (2) University of California, Riverside, CA, U.S.A.

In California, grapevine is valued at $\$ 5$ billion and is threatened by Pierce's Disease (PD), caused by X. fastidiosa. X. fastidiosa resides in the xylem vessels and infections lead to occlusions eventually leading to vine death. The negative pressure and low nutrient content of the xylem sap make it a unique unexplored microbial environment. Disease severity and sap samples were collected from 10 grapevines at bloom (spring), veraison (summer) and post-harvest (fall). Culture-based and amplicon metagenomics was used to profile the bacterial (16S -V4) and fungal (ITS) communities of the sap. A core microbiome was identified in response to season and vine condition. Season significantly affected fungal populations whereas bacteria showed a stronger community divergence in relation to condition. The number of unique bacterial and fungal families increased over seasons and years, suggesting a gain in microbial richness as canes mature and plants age. We were able to reveal potential biomarkers to seasons and show microbial cycling associated with major phenological stages. Interestingly, the closely related genus to $X$. fastidiosa, Stenotrophomonas, was found in higher abundance to mildly symptomatic plants, making it a potential biocontrol to the pathogen. Taken together, we explored a novel ecological niche in phytobiomes to create a better understanding in grapevine-microbiome-Xylella interactions that can help improve management practices to limit disease.

Accumulation of agrocinopine-like substrate in Ipomoea batatas

A. TANAKA (1), D. Takemoto (2), T. Suzuki (3), K. Uesaka (1), N. Yamaguchi (4), M. Otani (5), O. Nakayachi (5), N. Tanaka (4), (1) Nagoya University, Japan; (2) Nagoya Univ., Japan; (3) Chubu Univ., Japan; (4) Hiroshima University, Japan; (5) Ishikawa Prefectural University, Japan

Previously T-DNA sequences originated from Agrobacterium spp. were discovered in the genome of cultivated sweet potato (Ipomoea batatas) (Kyndt et al., 2015). One of the genes in the Agrobacterium-derived regions is agrocinopine synthetase gene, Acs. Agrocinopine is known to be produced in tumors induced by pathogenic Agrobacterium strains. To validate that the product synthesized by I. batatas Acs, IbAcs, is agrocinopines or its related substrate, we introduced IbAcs under constitutive promoter into Nicotiana tabacum. Crude extracts obtained from IbAcs-expressing tobacco leaves were separated by high voltage paper electrophoresis (HVPE). Agrociopines were extracted from tomato tumors 
induced by pathogenic A. tumefaciens. HVPE analysis showed that the extract from IbAcs-expressing tobacco leaves and that from tomato tumors had alkaline silver nitrate-positive spots migrated similar distance whereas no positive spot detected at similar distance in the extract from the transgenic leaves with empty vector. Crude extracts were also obtained from storage roots of I. batatas and alkaline silver nitrate-positive spots were detected by HVPE analysis. The substrates from the spot of IbAcs-expressing tobacco leaves were analyzed by Orbitrap LC-MS and MALDI-QIT-TOF-MS. Both analyses showed that the molecular mass of this substrate was identical to that of agrocinopine A suggesting that the substrate detected in storage roots of sweet potatoes is also agrocinopine A produced by IbAcs.

Understanding the mechanisms by which root hair mutations alter the barley rhizosphere bacterial microbiota.

S. ROBERTSON (1), T. S. George (2), N. Koebernick (3), J. Abbott (4), D. Bulgarelli (1), (1) Plant Sciences, School of Life Sciences, University of Dundee, Dundee, U.K.; (2) James Hutton Institute, U.K.; (3) University of Southampton, U.K.; (4) University of Dundee, U.K.

Deciphering the molecular mechanisms regulating plant-microbiota interactions in the rhizosphere will be key to exploiting soil microbes for agriculture. Using barley (Hordeum vulgare) as a model and a 16S RNA gene Illumina sequencing approach I demonstrated that root hair mutants (RHMs) impact on microbiota recruitment. Specifically, the significant enrichment of Actinomycetales, Burkholderiales, Rhizobiales, Sphingomonadales and Xanthamonadales discriminates between RHM and wild type (wt) microbiota. To investigate the molecular basis of this observation I took a two-pronged approach. As the absence of root hairs increases rhizosphere soil density, I performed a comparative analysis of the microbial communities from soil with differing compaction. Interestingly, I revealed that soil density is a determinant of the microbiota but is not sufficient to explain the taxonomic diversification observed. In parallel, the analysis of exudation profiles indicated that although exudation patterns are mainly conserved between genotypes the presence of Phenylalanine and organic acids can differentiate between wt and RHMs. This suggests that the barley microbiota is shaped by physical and chemical cues modulated by the presence and development of root hairs. To understand the specific role played by differentially enriched rhizosphere bacteria, I established a genome-annotated, reduced complexity, synthetic community recapitulating the major differentiating orders which I will present.

Healthy wheat spikes microbiome contains fungi with biological control potential against Fusarium head blight E. C. ROJAS, H. J. Lyngs Jorgensen, B. Jensen, D. B. Collinge, University of Copenhagen, Frederiksberg C, Denmark

The study of plant microbiome is opening new doors to the use of microbes in plant disease control. Fungal endophytes have evolved to exploit the same resources as plant pathogens. They can be considered as potential biological competitors and have the potential to become biological alternatives to chemical disease control. We have collected visually healthy wheat spikes from heavily Fusarium head blight (FHB) infested fields. We hypothesise that these spikes harbour naturally occurring fungi with biological control potential against FHB in their microbiome. Using community meta-barcoding, we have identified several fungal species that negatively correlate with Fusarium pathogens abundance and appear to be enriched in healthy compared to symptomatic spikes, specifically among Tremellomycetes yeasts. Moreover, we have recovered 163 fungal endophyte isolates from such healthy spikes. A greenhouse test using complete spikes showed that four fungal isolates reduced FHB severity by $50 \%$ and significantly reduced pathogen growth inside the endophyte-treated compared to non-treated spikes. Differentially expressed genes, enzyme activity and classical confrontation assays suggest that the biocontrol effect exerted by these isolates is mediated by the plant through activation of defence when inoculated onto the spikes before pathogen arrival. Our results confirm the potential of using plant microbiome as a reservoir for environmentally friendly disease control mechanism in agriculture.

Pathogen detection and beyond: Development and application of new sequence-based methods for characterisation of real-world microbial communities

Y. HU (1), G. Green (1), A. Milgate (2), L. Irinyi (3,4), T. Eenjes (1), W. Meyer (3,4,5), E. Stone (1,6), J. Rathjen (1), B. Schwessinger (1), (1) Research School of Biology, The Australian National University, Canberra, ACT, Australia; (2) New South Wales Department of Primary Industries, Wagga Wagga, NSW, Australia; (3) Westmead Institute for Medical Research, Sydney, NSW, Australia; (4) Westmead Clinical School, The University of Sydney, Sydney, NSW, Australia; (5) Westmead Hospital (Research and Education Network), Sydney, NSW, Australia; (6) ANU-CSIRO Centre for Genomics, Metabolomics and Bioinformatics, Canberra, ACT, Australia

Fungal diseases of plants are responsible for major losses in agriculture, highlighting the need for rapid and accurate identification of fungal plant pathogens. Disease outcomes are defined not only by main pathogens but are influenced by diverse microbial communities known as microbiomes at sites of infection. We conducted the first use of whole community DNA sequencing with a portable sequencer as a method for detecting fungal pathogens from wheat (Triticum aestivum) in a standard molecular biology laboratory. The data revealed that our method is robust and capable of diagnosing stripe rust (caused by Puccinia striiformis f. sp. tritici), septoria blotch (caused by Zymoseptoria tritici) and yellow spot (caused by Pyrenophora tritici repentis). We also identified the bacterial genus Pseudomonas co-present with Puccinia and Zymoseptoria but not Pyrenophora infections. One limitation of the method is the over-representation of redundant wheat genome sequences from samples. This could be addressed by long-range amplicon-based meta-barcode sequencing approaches and host DNA depletion during or after DNA extraction. Here we will present recent progress in our method development and the biological insight gained during this process. Our work outlines a new approach for detection of a broad range of plant pathogens and associated microbes using a portable sequencer, providing the basis for the development of a high-throughput, large scale and on-site disease monitoring system.

Plant-microbiome interaction in tomato for disease response under defined soil condition K. CHOI, P. A. Lee, J. Choi, N. Roy, R. Khan, S. Y. Lee, R. Thapa Magar, S. W. Lee, Dong-A University, Busan, Korea, Republic of (South)

Plant-microbiome interaction for plant disease or resistance has been poorly understood. To investigate the role of bacterial community for disease development in tomato rhizosphere, we established a system that we can treat various microbiome under defined soil condition and disease pressure. Our investigation showed that the system can maintain consistent soil physicochemical properties but carry various microbiome to study plant-microbiome interaction for plant disease. Microbial fractions which were isolated from various natural or cultivated soils including paddy, alluvial, upland, and forest soil were treated to tomato seedlings, and bacterial pathogen such as Ralstonia solanacearum causing bacterial 
wilt (BW) was inoculated to the grown plants under different microbiome. A specific upland microbiome conferred the BW-resistant cultivar Hawaii 7996 with the significant suppression of BW progress whereas a specific forest one caused Hawaii 7996 more conducive to BW occurrence than non-microbiome treated control. Analysis of bacterial communities of tomato rhizosphere treated with different microbiomes displayed distinct community structure. More intensive analysis of bacterial community is conducting to identify the core microbiome involved in the disease response. Taken together, the treated microbiomes isolated from natural field soils influenced BW progress positively or negatively in a resistant tomato cultivar.

\section{Investigating the structure of the leaf and root microbiome of the wild strawberry $F$. vesca}

J. MITTELSTRASS, Universität Zürich, Zürich, Switzerland

Because plants are anchored in place, they rely on their ability to adapt and respond to their surrounding environment through changes in metabolism, root exudates, and their physiology or phenotypic diversity. At the same time, plants host a variety of microbes that may also contribute to this environmental adaptation and therefore provide an important means to extend the host plant's functionality. Here, the bacterial and fungal leaf and root microbiome of the wild strawberry Fragaria vesca was analyzed regarding community diversity, richness and taxonomic composition. Communities of plants sampled across different sites in the European Alps and the United States were sequenced using a phylogenetic approach. By including climatic and geochemical factors, we are able to gain additional insights about the main factors that structure the microbiome and the microbiome metrics that best describe the natural variation of the microbiome in $F$. vesca.

Investigating the virome of crops and trees using RNA-sequencing

S. JONES (1), S. MacFarlane (1), G. Cowan (1), J. Beaton (1), B. Mukoye (2), B. C. Mangeni (2), G. Iason (1), H. Were (2), L. Torrance (1), (1) The James Hutton Institute, U.K.; (2) Masinde Muliro University of Science and Technology, Kenya

Virus infection is of importance in plants in the natural environment and those cultivated for food and fuel. RNA-sequencing of plant material allows for hypothesis-free detection of multiple viruses simultaneously, as the majority of plant viruses have RNA as their genetic material and those that have DNA genomes produce RNA transcripts. In this work we report on the use of RNA-sequencing of cultivated groundnuts (Arachis hypogaea) from Kenya and Scots Pine trees (Pinus Sylvestris) from the U.K., for the detection of plant viruses. For both plants, we have mapped raw RNA-sequence reads to host reference genomes (or close relatives), to remove host reads, assembled the non-host reads into contigs and then aligned the contigs to known plant virus genomes in the GenBank database. This work revealed multiple plant viruses present in the groundnut samples exhibiting virus-like symptoms, including groundnut rosette umbravirus, cowpea polerovirus 2 and a potentially novel Caulimovirus. The number of nonhost reads achieved from the RNA-sequencing of Scots Pine samples was more limited than the groundnut samples, which led to contig assembly being very restricted. However, contigs have been assembled that include virus-like sequences, and these are currently being investigated further.

Comparison of endophytes associated with the invasive weed, medusahead (Taeniatherum caput-medusae): Native range vs. introduced range.

C. DUNLAP (1), B. Rector (2), (1) Crop Bioprotection Research Unit, Peoria, IL, U.S.A.; (2) Great Basin Rangelands Research, NV, U.S.A.

Medusahead is an invasive weed impacting the Great Basin region of the western United States with damage to the natural and agricultural ecosystems. The native range of the annual grass is the northern Mediterranean basin of Europe. It is well understood that microbial endophytes play an important role in a plant's ability to adapt to abiotic and biotic stresses. However, there is currently little knowledge on how endophytes enhance or alter the phenotype of invasive weeds. The goal of the current study is to compare the endophytes of medusahead in the native range to endophytes of medusahead in the introduced range. The results show the introduced plants generally had a lower biodiversity of endophytes in all plant parts tested. In addition, there were significant differences in the types of microorganisms that colonized the plants. The endophytes found in the seeds of the plants also demonstrated significant differences between the ranges, which suggests the plants vertically transmit different microorganisms in a range dependent manner. Culture isolations were made to begin to evaluate the impact of these endophytes on the plant phenotype.

The International Alliance for Phytobiomes Research: Advancing international phytobiomes research for food security T. P. Alliance (1), N. W. BREAKFIELD (2), J. E. Leach (3), G. A. Beattie (4), K. Eversole (5), M. Guilhabert (6), M. J. Ryan (7), A. Sessitsch (8), (1) Phytobiomes Alliance, Lee's Summit, MO, U.S.A.; (2) NewLeaf Symbiotics, Saint Louis, MO, U.S.A.; (3) Colorado State University, CO, U.S.A.; (4) Iowa State University, Ames, IA, U.S.A.; (5) Eversole Associates, Bethesda, MD, U.S.A.; (6) Bayer, West sacramento, CA, U.S.A.; (7) CABI, Egham, U.K.; (8) AIT Austrian Institute of Technology GmbH, Vienna, Austria

A major paradigm shift in agricultural production is required to meet the demands of a growing world population projected to reach 9.8 billion in 2050. We need to sustainably increase crop productivity while preserving biodiversity and natural resources, particularly in the face of climate change. To optimize sustainable productivity and profitability on farms, grasslands, and forests, scientists must embrace a holistic, systems-level approach and focus on the complexity within phytobiomes. The term "phytobiome" refers to a plant growing within a specific environment, or biome, and all of the micro- and macro-organisms living in, on, or around it—such as microbes, animals, insects, and other plants—as well as the environment, which includes soil, air, water, weather, and climate. By establishing a foundation of knowledge on how phytobiome components interact and affect each other, the International Alliance for Phytobiomes Research, a nonprofit alliance of industry, academic, and governmental partners, aims to address today's agricultural challenges. The Alliance, established in 2016, facilitates and coordinates international efforts toward expanding phytobiomes research to accelerate the sustainable production of food, feed, and fiber for food security.

Biocontrol activity of Pantoea dispersa against black rot disease of sweetpotato caused by Ceratocystis fimbriata J. LEE, Korea Reserach Institute of Bioscience and Biotechnology, Jeongeup, Korea, Republic of (South) 
Biocontrol has been developing as a promising alternative to synthetic fungicides to control a variety of pre-or postharvest disease of crops. Black rot is the most destructive postharvest disease of sweetpotato caused by pathogenic fungus Ceratocytis fimbriata. However, little is known about the biocontrol agent of this fungus. In this study, we isolated culturable microorganisms from tuberous roots and shoots of field-grown sweetpotato plants. Analysis of the rRNA sequences from isolated microorganisms was revealed that genus Pantoea is one of a major portion of the microbes, residing within a sweetpotato plant tissue. To identify the role of genus Pantoea in sweetpotato plants, we found that four Pantoea dispersa strains RO-18, RO-20, RO-21, and SO-13, strongly inhibits mycelium growth, spore germination of $C$. fimbriata, and alter the morphology of fungal hyphae, while not significantly changed by other Pantoea strains. Evans blue staining to detect the dead cell of $C$. fimbriata after exposure to $P$. dispersa revealed that $P$. dispersa strains have a fungicidal activity rather than fungistatic activity. Finally, $P$. dispersa strains significantly suppress the $C$. fimbriata growth on leaves and tuberous roots of sweetpotato. Our finding suggests that $P$. dispers $a$ strains, an associating with sweetpotato tissue, could strongly inhibit $C$. fimbriata growth. $P$. dispersa is highly worthwhile for biocontrol agent of sweetpotato

Unveiling seed microbiotas as an archive of evolution of Oryza

H. KIM (1), K. Lee (1), J. Jeon (2), W. Harris (1), Y. H. Lee (1,2,3,4,5), (1) Department of Agricultural Biotechnology, Seoul National University, Seoul, Korea, Republic of (South); (2) Interdisciplinary Program in Agricultural Genomics, Seoul National University, Seoul, Korea, Republic of (South); (3) Research Institute of Agriculture and Life Sciences, Seoul National University, Seoul, Korea, Republic of (South); (4) Center for Fungal Genetic Resources, Seoul National University, Seoul, Korea, Republic of (South); (5) Plant Immunity Research Center, Seoul National University, Seoul, Korea, Republic of (South)

Despite the close physiological and ecological relationships between host and host-associated microbiomes, effects of domestication on structure and functions of microbiomes are debatable. Here, we show the evidence that evolutionary factors shape seed bacterial and fungal community structures using 17 wild and 26 domesticated rice accessions. Enrichment and switching of dominant genera in bacterial and fungal biotas are predicted as key events during domestication with deepening inequality and simplifying network structures. Furthermore, in spite of compositional variations, conserved microbiotas which can be vertically transmitted exist regardless of host genetic variations and geographical distance. Composition of bacterial biota also shows a significant eco-evolutionary relationship with rice phylogeny. Our findings show that evolutionary factors affect overall composition of microbial communities in stochastic ways whereas dramatic changes in environments during domestication contribute to assembly of microbiotas in deterministic ways.

Impact of Arabidopsis immunity sectors on accommodation of a beneficial root endophyte in a community context C. UHLMANN (1), K. Raj (1), A. S. Piro (1), A. Zuccaro (2), J. E. Parker (1), (1) Max Planck Institute for Plant Breeding Research, Cologne, Germany; (2) University of Cologne, Germany

In natural environments, plant hosts interact with phylogenetically diverse microorganisms. Central to these interactions is the plant innate immune system because it is involved in microbe perception as well as in activation or repression of defence pathways against pathogens or beneficial symbionts, respectively. Although accommodation of microbes in plant tissues is a common feature of both disease and symbiosis, these were classically separate area of studies. Serendipita vermifera $(\mathrm{Sv})$ is a widespread soil borne filamentous fungus that has been shown to colonize plant roots of many species, including Arabidopsis. We are examining the Arabidopsis - Sv interaction in a set of immunity mutants representing different sectors of plant immunity. Comparing mutant responses with those of wild type will highlight which defence sectors are important for restriction or accommodation of Sv. Plant genotype-Sv combinations that display the most interesting phenotypes will be selected for the second phase, in which we will test whether selected root-associated bacterial communities (SynComs) modify the plant immune response and/or Sv colonisation phenotypes. We expect that SynComs might change Sv accommodation by plants, revealing how immunity functions in a community context. Finally, specific plant genotype-Sv-SynComs will be selected for physiological, metabolic phenotyping and RNA-seq analyses, to build and test functional gene networks for plant host-root microbe associations.

Investigation of endosphere and rhizosphere microbial communities in kiwifruits plant.

G. CHO, Y. S. Kwak, Gyeongsang National University, Korea, Republic of (South)

Plants interacts with their environment, micro and macro organism is defined in a term, phytobiome. Knowledge of phytobiome can be develop new concepts and applicate tools for future sustainable agriculture. Psudomonas syringeae pv. actinidiae (Psa), caused bacterial canker disease in kiwifruit, leads to the great economic losses in kiwifruit production. To understand kiwifruit's microbiota ecology including Psa, 16S rRNA and ITS library sequencing were performed at three kiwifruit's sap and rhizosphere using Illumina Miseq platform. Their raw sequences were filtered and clustered to OTUs by DADA2. Bacterial OTU reads were normalized once again based on known 16S rRNA multiple copies by PICRUSt2. Interestingly, variations in fungal and bacterial community composition were very large in the sap samples but the rhizosphere samples showed relatively similar structure. Genus Psudomonas composition ratio varied from $0.3 \%$ to $95 \%$ for each sap. The largest variation in fungal genus composition was Didymella at $2 \%$ to $34 \%$ for each sap. In DESeq2 results, Family Deinococcaceae, Propionibacteriaceae, Pseudomonadaceae, Enterobacteriaceae and so on were revealed as dominant bacterial members in the saps compared to the rhizospheres. Dominant fungal members in the sap were Family Sirobasidiceae, Tubeufiaceae, Leptosphaeriaceae and Coniothyriaceae in order. Co-occurrence analysis was conducted to understand the interactions between the fungi and the bacteria by FlashWeave.

Investigating the mycoparasitic interaction of Pythium oligandrum with a major phytopathogenic fungus, Fusarium graminearum C. FAURE (1), T. Rey (2), E. Gaulin (1), B. Dumas (1), (1) LRSV UMR5546 CNRS Université Toulouse III, Castanet-Tolosan, France; (2) De Sangosse, Pont-du-Casse, France

Pythium oligandrum is a soilborn mycoparasite oomycete, with a wide host range ranging from fungi to oomycetes. When confronted with the fungus Fusarium graminearum, we observed that $F$. graminearum growth was stopped after the first contact with $P$. oligandrum. Six days after 
contact, all remaining fungal structures were dead, while oospores of the oomycete were produced. Microscopical observations revealed the necrotrophic behavior of $P$. oligandrum and differentiation of specialized cellular structures. Notably, specialized hyphae of $P$. oligandrum have been observed which are able to enter fungal cells causing host cell death. To identify the genetic determinants of mycoparasitism, whole genome PacBio sequencing of the $P$. oligandrum strain in combination with RNAseq analyses were performed. Gene annotation revealed 15,007 genes encoding proteins. Comparative genomics with several Pythiales led to the identification of a set of 2,222 genes specific to mycoparasite species. In addition, the transcriptomic data indicate that among them 323 genes are highly induced in confrontation with $F$. graminearum. Interestingly 123 genes encode putative secreted proteins such as chitinases, peptidases and elicitins. These results show that, following the perception of $F$. graminearum mycelium, $P$. oligandrum engages a specific cellular and genetic program, leading to host death. Further studies will aim to functionally characterize the genes and molecular signals involved in mycoparasitism.

Bacterial Diversity in the Rhizosphere of Rice Plant in Response to Nitrogen Induced Susceptibility to Rice Blast Disease M. ROY, G. Shanmugam, J. Jeon, Yeungnam University, Gyeongsan, Gyeongbuk, Korea, Republic of (South)

The interaction between plant roots and their rhizospheric microbiome plays an important role in plant fitness and in modulating the outcomes of interactions with pathogens. In order to identify the culturable bacterial community associated with the rhizosphere of rice plants under low and high nitrogen regime in presence of fungal pathogen M. oryzae, we sequenced the V3 and V4 region of the 16S rRNA of 100 isolates cultured on two different media: TSA and R2A. Based on 16S rRNA gene sequencing, the overall bacterial diversity found in the rhizosphere of rice plants showed an abundance of four phyla: Proteobacteria, followed by Actinobacteria, Firmicutes and Bacteroidetes, making up most of the bacterial community of root microbiota. Among 100 isolates, 32 different bacterial strains were identified as Gryllotalpicola genus and found to be higher in both regimes. On the contrary, Ochrobactrum genus and Herbaspirillum genus is specific to high and low nitrogen regime, respectively. Thus, the root microbiome enriched with these bacterial species might potentially help the rice plant to combat $M$. oryzae infection. Exploiting the interspecies interactions of this rhizosphere bacterial community could provide a sustainable solution towards devastating crop loss due to rice blast disease.

Microbiome-guided selection to identify plant growth-promoting bacteria that enhance juvenile maize growth under cold stress S. GOORMACHTIG (1), S. Beirinckx (2), T. Viaene (3), A. Haegemen (4), J. De bode (4), F. Amery (4), S. Vandenabeele (3), H. Nelissen (5), D. Inzé (6), R. Tito (7), J. Raes (7), C. De Tender (8), (1) Ghent University - VIB, Gent, Belgium; (2) Ghent Univerity - VIB, Zwijnaarde, Belgium; (3) Aphea.Bio, Zwijnaarde, Belgium; (4) ILVO, Merelbeke, Belgium; (5) VIB, Zwijnaarde, Belgium; (6) VIB-Ghent University, Zwijnaarde, Belgium; (7) VIB-KULeuven, Leuven, Belgium; (8) ILVO-UGent, Merelbeke, Belgium

When maize (Zea mays L.) is grown in the Northern hemisphere, its development is heavily arrested by cold temperatures, especially at the juvenile phase. It is well-known that plant organs, including leaves and roots are occupied by a vast majority of microbes that are expected to support plant fitness in changing biotic and abiotic environments. Here we investigated how root endophytes contribute to the growth of juvenile maize plants in cold conditions. The root endomicrobiome mainly consisted of bacteria recruited from the soil and seed-derived endophytes were only lowly represented. Additionally, over two independent repeats, cold caused significant changes in the root endomicrobiome composition causing a depletion of the Actinobacteria and an enrichment of several families of which the Comamonadaceae and the Xanthomonadaceae were the most abundant. Based on these microbiome experiments, we executed a screen for growth-promoting bacteria on juvenile maize and identified two new strains, a Pseudoduganella sp. and a Bosea sp., that could increase juvenile maize growth when grown in the cold. This screen demonstrates that selecting bacterial representatives from families enriched in the root compared to the soil, but not necessarily enriched in the root upon cold, is a successful parameter to find new plant growth promoting rhizobacteria.

Bacterial endophytes to protect plants against cold temperatures

S. GARCIA MENDEZ (1,2), S. Goormachtig (3), A. Willems (4), (1) Laboratory of Microbiology University of Ghent, Gent, Belgium; (2) VIB Plants Systems Biology, Gent, Belgium; (3) Ghent University - VIB, Gent, Belgium; (4) Ghent University, Gent, Belgium

Environmental factors have a great impact on crop production due to their effect on plant growth. Finding an environmentally friendly way to overcome adverse environmental conditions has gained great importance during the past years. One solution is the use of Plant Growth Promoting Rhizobacteria (PGPR), free living bacteria that have beneficial effects on plant health. In this study we want to determine the importance and nature of the contributions of bacterial endophytes to the survival and growth of plants under cold temperatures. For that purpose, the microbiome of Arabidopsis thaliana, Valerianella locusta and Poа annua grown under normal and cold conditions is being studied. Through the comparison of the results from the 16S rRNA amplicon sequencing that are being obtained for Valerianella locusta plants, and that will be acquired in a nearby future for the other two pants, we want to identify which bacterial populations are being favored in cold conditions. We already conducted a bacterial isolation campaign to cultivate endophytes from these plants so that representative strains can be tested for their ability to promote plant growth under cold conditions. The use of MALDI TOF mass spectrometry and 16S rRNA sequencing has provided insights in the taxonomic diversity of the cultivable endophytes isolated from the three plants and showed they belong to different phyla which are common in soil, such as Firmicutes, Actinobacteria, Proteobacteria and Bacteroidetes.

Deciphering the modulation of compartment-specific microbiome during vascular wilt interaction by Fusarium oxysporum in Tomato M. SABALE (1), A. Redkar (1), S. Hacquard (2), A. Di Pietro (1), (1) University of Cordoba, Cordoba, Spain; (2) Max Planck Inst for Plant Breeding Res, Cologne, Germany

Plant roots are colonized by diverse microbes that affect plant health either in beneficial or harmful ways. These include benign rhizosphere inhabitants, as well as endophytes, some of which contribute to plant health. Alternatively, root infecting pathogens have evolved to outcompete their microbial competitors and cause disease. Fusarium oxysporum is a soil-borne fungal pathogen, that causes vascular wilt disease in broad range of crops including both monocots and dicots. The fungus grows asymptomatically in the root cortex and then colonizes the vasculature. The chemical control methods are difficult to apply, as the low visibility of fungus in early stages. Biological control of disease by bacteria is 
emerging as a promising method, however the functional relevance of the root microbiome during the pathogen-host interaction is largely unknown. The present work aims to understand the host compartment-specific native tomato microbiome and its role during Fusariuminfection. Soils with diverse characteristics collected across tomato fields in Southern Spain, were used in culture dependent and independent approaches to monitor the changing microbiome under Fusarium infection by Amplicon Sequencing. Moreover, the isolated bacterial communities are classified based on compartments such as soil, rhizosphere, root, endophytic root and xylem. The final goal of the study is to understand the interplay and the modulation of microbiome assembly during pathogen-plant interaction.

\section{Phytobeneficial Traits under the Control of Multiple Crosstalks' Belowground}

W. ACHOUAK (1), A. Laveilhé (2), S. Fochesato (3), J. Tulumello (3), M. Huleux (3), M. Barakat (3), T. Heulin (4), (1) BIAM/LEMiRE UMR 7265 CNRS/CEA/Aix Marseille University, Saint-Paul-Lez-Durance, France; (2) Syngenta, France; (3) BIAM/LEMiRE UMR 7265 CNRSCEA-Aix Marseille University, France; (4) BIAM/LEMiRE UMR 7265 CNRS-CEA-Aix Marseille University, Saint-Paul Lez Durance, France

Pseudomonads play crucial roles in plant growth promotion and control of plant diseases. We hypothesized that failure of bioncontrol agents in suppressing plant disease, under natural conditions, could be linked to a negative control of their phytobeneficial traits by others microroganisms competing for the same nutritive resources in the rhizosphere. In this work, we assessed the expression of phytobeneficial genes, phlD and $h c n A$, in Pseudomonas brassicacearum co-cultivated with three phytopathogenic fungi, Fusarium culmorum, Fusarium graminearum and Microdochium nivale and two other rhizobacteria, Kosakonia sacchari NO9 and Rhizobium alamii YAS34 in the presence or absence of plant, as well as in relation to iron availability. We also investigated the impact of the introduction of these bacteria on the assembly of the plant rootsassociated microbiota in the natural soil. We found that the antifungal activity of $P$. brassicacearum depends on the production of DAPG and not on $\mathrm{HCN}$ whose production is suppressed by fungi. We have also shown that the two rhizobacteria NO9 and YAS34 strains modulate the antifungal activity of $P$. brassicacearum by modifying the expression of phlD and hcnA in relation to iron availability. Inoculation of plant with these bacteria led to a shift in microbiota assemblage and ecological network.

Targeting the phyllosphere microbiome to combat downy mildew disease P. GOOSSENS (1), K. Baremans (1), G. Vismans (1), R. L. Berendsen (2), G. Van Den Ackerveken (3), (1) Utrecht University, Utrecht, Netherlands; (2) Plant-Microbe Interactions, Department of Biology, Utrecht University, Utrecht, Netherlands; (3) Plant-Microbe Interactions, Dept of Biology, Utrecht University, Utrecht, Netherlands

Downy mildews are host-specific obligate biotrophic pathogens belonging to the Oomycetes that cause major crop loss worldwide. Damage by downy mildews is reduced by breeding for disease resistance. Although this is a highly effective strategy, turnover rate of resistance genes is high due to the rapid evolution of pathogen isolates. As an alternative approach, we are exploring downy mildew interactions with phyllosphere microbes and ultimately aim to exploit these interactions to combat downy mildew disease. We have generated gnotobiotic cultures of the downy mildew Hyaloperonospora arabidopsidis ( $\mathrm{Hpa}$ ) on the model plant Arabidopsis thaliana. Preliminary data show that the Hpa-associated microbial community influences host infection. By comparing the phyllosphere microbial communities of susceptible and resistant plants, in the presence and absence of $H p a$, we were able to identify bacterial taxa that are consistently enriched in the phyllosphere of $H p a$-infected leaves. These bacteria were subsequently isolated from $\mathrm{Hpa}$-infected leaves and we are currently assessing co-inoculation with gnotobiotic $\mathrm{Hpa}$ cultures to test for pathogen-helper functions or antagonistic activities.

The role of secreted effectors in complex phyllosphere microbial communities

K. EITZEN (1,2), S. Kroll (2), E. Kemen (2,3), G. Doehlemann (1), (1) University of Cologne, Cologne, Germany; (2) Max Planck Institute for Plant Breeding Research, Cologne, Germany; (3) University of Tübingen - ZMBP, Tübingen, Germany

As the phyllosphere is a resource-limited niche, microbes have evolved different survival strategies by collaborating or competing with other organisms in network structures. To establish such stable microbial networks so-called microbial hubs, like the oomycete pathogen Albugo laibachii in the Arabidopsis phyllosphere, are essential factors. In addition, recent findings suggest that particularly negative interactions of antagonistic microbes are important to stabilize microbial communities. The basidiomycete yeast Moesziomyces was isolated from wild Arabidopsis plants showing white rust symptoms. This yeast, which is closely related to plant pathogenic smut fungi, is a strong microbial hub which both suppresses the infection of $A$. laibachii and inhibits growth of several bacterial phyllosphere members. To functionally investigate the antimicrobial interactions of Moesziomyces, we established a high-quality annotated genome sequence and an efficient transformation system. RNA-sequencing identified several secondary metabolite clusters of Moesziomyces being upregulated in microbe-microbe interactions. Interestingly, several genes encoding secretory proteins, mostly glycoside hydrolases and peptidases, are particularly induced upon interaction with A. laibachii. Those candidate genes are tested in a reverse genomics approach which aims to identify crucial factors that determine microbial antagonism of Moesziomyces and thereby shape complex phyllosphere microbial communities.

Combined approaches to identify the incidence of Zymoseptoria tritici on microbial community in the wheat residues ecosystem. L. Kerdraon (1), M. H. Balesdent (1), M. Barret (2), F. Suffert (1), V. LAVAL (1), (1) INRA BIOGER, Thiverval-Grignon, France; (2) INRA IRHS, Beaucouzé, France

The wheat pathogen Zymoseptoria tritici reproduces sexually during the intercropping season on crop residues, which act as primary inoculum source. Few studies have examined the impact of the whole microbial community on the saprophyte/sexual stage of such residue-borne pathogens. We characterized the interactions between $Z$. tritici and the micro-organisms present on residues and assessed the impact of some factors (soil contact, seasonality). To this end, residues of wheat plants preliminary inoculated with $Z$. tritici in greenhouse were left outdoor either with or without contact with soil. Non-inoculated residues were left in the same conditions, as control. Residue sampling was performed in July (before contact with ground), October, December and February. The experiment was repeated a second year. Following a metagenomic description of fungal and bacterial communities, linear discriminant analyses and ecological network analyses were combined to investigate how microbial communities were impacted by the infection. Foremost, we showed the influence of the infection, even after Z.tritici disappearance, on 
the structure and composition of the microbial communities of wheat residues. Interactions appeared to be mainly indirect, despite Z.tritici position in networks as key taxa. The presence of $Z$. tritici also impacted fungal and bacterial species previously reported as beneficial. Describing interactions within residues ecosystem may lead to new disease-management solutions.

Fungal endophytes from wild plants induce resistance in commercial tomato plants against Pseudomonas syringae E. LLORENS, L. Scalschi, A. I. Gonzalez-Hernandez, E. Fernandez-Crespo, G. Camañes, B. Vicedo, P. Garcia-Agustin, UNIVERSITAT JAUME I, CASTELLON, Spain

Fungal endophytes are able to improve the performance of plants under stress situations due to a mutualistic relationship. However, it has been demonstrated that the presence and richness of fungal endophytes is highly dependent on the host plant and the environment. Moreover, it has been hypothesized that this diversity in crop plants has been reduced due to the domestication and the continuous treatment with pesticides, and therefore endophytes communities might be less diverse compared with wild plants. In this work, we tested whether the ability of fungal endophytes isolated from Solanum nigrum to induce resistance in tomato plants (Solanum lycopersicum cv. Ailsa Craig) against Pseudomonas syringae. Fungal endophytes isolated from S. nigrum were inoculated on seeds of tomato plants. Four-week-old tomato seedlings were infected with the pathogenic bacterium $P$. syringae. The plants inoculated with endophytes showed significantly lower symptoms of bacterial infection as well as lower levels of colony forming units per leaf compared with control plants. Moreover, the presence of the endophytes induces an altered balance of hormones as well as a reduction of abscisic acid that may be related with the colonization of the endophyte. In conclusion, our results show that solanaceae wild plants as $S$. nigrum are promising sources of endophytes able to induce resistance in tomato against the biotrophic bacterium $P$. syringae, which were associated with changes in phytohormones levels.

Comparative metagenomic analysis of highly abundant Pseudomonas within the maize rhizosphere J. L. SUTTER, N. Youngblut, W. A. Walters, R. Ley, Max Planck Institute for Developmental Biology, Tuebingen, Germany

Plant health is known to be significantly influenced by the microbial structure of the rhizosphere. Despite no formal terminology, a "bloom" can refer to an event where an individual rhizosphere taxon substantially increases in relative abundance within a defined period. Studies of pathogenic or growth-promoting rhizobacteria often place blooms in the context of increased root colonization and a subsequent plant phenotype. Less is understood concerning non-pathogenic blooms, especially among competing strains within the same genus. A non-pathogenic bloom of the genus Pseudomonas was observed by our group during a field study of the maize rhizosphere. To investigate the cause of this phenomenon, rhizosphere Pseudomonas genomes were assembled via shotgun metagenomics to assess their relative abundance at strain level. We identified blooming species as $P$. brassicacearum, P. chlororaphis, and P. fluorescens. We will match these strains to genomes within our collection of 500 Pseudomonas isolated from the same maize field soil. A comprehensive pan-genome analysis will compare phylogenies, gene content, SNP variation, and growth rates between blooming and static genomes. Through this approach, we aim to identify genetic and molecular mechanisms that underlie differences in competitive fitness between individual Pseudomonas strains when colonizing the rhizosphere of maize.

The inconspicuous gatekeeper: Endophytic Serendipita vermifera acts as extended plant protection barrier in the rhizosphere L. K. MAHDI (1), D. Sarkar (1), H. Rövenich (2), A. Tissier (3), G. U. Balcke (3), G. Langen (1), A. Zuccaro (1), (1) University of Cologne, Germany; (2) University of Cologne, Cologne, Germany; (3) IPB halle, Halle, Germany In nature, beneficial and pathogenic fungi simultaneously colonize plants. Despite substantial efforts to understand the composition of natural plant-microbe communities, the mechanisms driving such multipartite interactions remain largely unknown. Here we address how interaction between the beneficial root endophyte Serendipita vermifera (S.v) and the pathogen Bipolaris sorokiniana (B.s) affects fungal behavior and barley host responses. Fungal confrontation in soil resulted in induction of $B . s$ genes involved in secondary metabolism and repression of genes encoding putative effectors. In S.v, genes encoding hydrolytic enzymes were induced. These antagonistic responses were not activated during tripartite interaction. Instead S.v genes involved in detoxification and redox homeostasis were induced. Pathogen infection but not endophyte colonization resulted in host transcriptional reprogramming and defense activation. S. $v$ colonization significantly reduced pathogen infection and disease symptoms despite no marked alterations of the plant transcriptional response. Activation of stress response and repression of putative effectors in B.s during fungal-fungal confrontation suggest a reduction of the pathogen's virulence potential prior to host plant infection. Molecular mechanisms underlying the tripartite system will be further analyzed by proteomics, metabolomics and reciprocal transcriptional responses of barley WT and mutants to fungal colonization and bacterial accommodation.

Microbial Community Dynamics Associated with Host Phenological Stages of Citrus sinensis N. A. GINNAN, P. E. Rolshausen, M. C. Roper, University of California, Riverside, CA, U.S.A.

Citrus trees are a woody perennial crop that can be in production for decades. Each year the host undergoes a progressive series of phenological stages that culminates in fruit maturity and, ultimately, fruit harvest. We characterized the leaf/root microbiomes of field grown citrus over seven major phenological stages (from leaf flush to fruit maturity) to test our hypothesis that significant shifts in microbial community composition occur in relation to host phenology. Beta diversity analyses indicated significant clustering of bacterial and fungal communities residing in leaves/roots by phenological stage $\left(p \leq 0.003, R^{2} \geq 0.1\right)$. This supports our hypothesis that phenology is a driver of structural shifts of the microbiome. Furthermore, we have identified 66 bacterial/fungal genera that are significantly enriched during specific host stages. A subset of the genera significantly enriched during flowering are known bee gut microbes. An additional subset are known to produce phytohormones, such as Acinetobacter spp. This suggests that plant microbiota may play a role in co-regulating host phenological phases that are driven by phytohormone balances. Knowledge of how the architecture of the microbiome relates to host phenology provides an avenue for testing the role of the microbiome in modulating plant development. Phenological microbiome information could potentially be integrated into phenology models to aid in predicting the timing of annual phenological events in citrus.

Absolute counts versus relative abundances in A. thaliana leaf bacterial networks 
H. WHITEHURST (1), B. Brachi (2), R. Leff (1), T. Karasov (3), S. Holm (4), J. Bergelson (1), (1) University of Chicago, Chicago, IL, U.S.A.; (2) French National Institute for Agricultural Research, U.S.A.; (3) Max Planck Institute for Developmental Biology, Tuebingen, Germany; (4) Mid Sweden University, Sundsvall, Sweden

Plant microbial communities, called microbiomes, affect host productivity, defense responses, and fitness. As a consequence, researchers aim to tease apart interactions among hosts, microbes and the abiotic environment to develop means of using microbiomes to improve crop productivity in the field. For these experiments, we need effective methods of characterizing the microbiome within plant leaves, as well as effects on host health. Canonical methods for characterizing the microbiome use relative abundances of sequenced marker genes to generate community profiles, but we can increase our statistical power by using absolute counts of microbes. Deriving absolute counts requires extensive sequencing because there is currently no effective microbial DNA enrichment step, and therefore host DNA fragments inundate the reads.

Here we use deep sequencing to derive absolute microbial counts from the leaves of $A$. thaliana grown in the presence of microbes in a field site in Sweden. We also test the efficacy of two potential methods of microbial enrichment for future research and examine the introduced biases in the characterization of the microbial community. We use these results to generate networks of inferred microbe-microbe interactions and compare these networks to previous networks derived from relative abundance sequencing. Finally, we briefly describe testing the importance of key members of the community in $A$. thaliana greenhouse experiments.

Selecting soybean-specific beneficial microbes for sustainable yield improvement

S. VAN BENTUM (1), H. A. van Pelt (2), P. A. H. M. Bakker (1), C. M. J. Pieterse (1), R. L. Berendsen (1), (1) Plant-Microbe Interactions, Department of Biology, Utrecht University, Utrecht, Netherlands; (2) Utrecht University, Utrecht, Netherlands

The demand for higher crop yields and sustainable agricultural practices calls for innovative solutions. In this endeavor, the use of plantbeneficial microbes that sustain plant health is a viable alternative. It was recently demonstrated that the model plant species Arabidopsis thaliana can adjust its root microbiome upon pathogen infection and specifically recruits a group of disease resistance-inducing microbes. In this project, we will study such microbiome-shaping capacities in soybean and aim to select soybean-specific consortia of beneficial microbes. The ability of soybean to recruit specific microbes upon infection with different pathogens will be investigated both under controlled and field conditions. The microbes that are recruited by diseased soybean plants will be isolated and assessed for their ability to protect soybean plants from diverse attackers. To explore the mechanisms of beneficial microbe recruitment, metabolite profiles in soybean roots and root exudates of control and infected plants will be analyzed. Combining metabolite profiling and microbiome data is expected to lead to effective industrialization of soybean-specific microbes, thereby providing soybean growers with a novel, stable biocontrol product applicable in the field.

The Role of Agricultural Soil Management Practices in Shaping the Microbiome of Sorghum bicolor H. M. L. WIPF (1), J. Mitchell (2), D. Coleman-Derr (3), (1) University of California, Berkeley, Berkeley, CA, U.S.A.; (2) Kearney Agricultural Research \& Extension Center, Parlier, CA, U.S.A.; (3) USDA-ARS, Albany, CA, U.S.A.

Soils are a living and finite resource that play vital roles in sustaining biological productivity, impeding crop disease and maintaining air and water quality. Recent work has demonstrated the major role microbes play in shaping soil health, yet it is largely unknown how agricultural soil management regimes shape crop microbiomes. Using a field site managed under conservation till (CT), standard till (ST), and cover-cropping (CC), we employed 16S rDNA and ITS2 amplicon sequencing and meta-transcriptomics to determine how soil management impacts microbial composition and function in sorghum's microbiome. Preliminary results show that tillage type and CC both impact microbial profiles, with significant shifts in the relative abundance of bacterial and fungal lineages across treatments. We observed that fungal communities appear more dissimilar between CT and ST treatments than bacterial ones, and that changes in alpha diversity across treatments are also greatest for fungi in the rhizosphere and soil. Across treatments, we identified significantly enriched Clusters of Orthologous Groups (COG) categories that highlight the role of lipid and carbohydrate transport and metabolism. Determining the impact of agronomic practices on the soil environment and crop microbiomes not only informs efforts to configure trait-associated microbiomes through traditional agricultural techniques, but also allows for the development of a beneficial microbiome component to crop production systems.

Genomic and Phenotypic Characterization of Novel Rhizoplane and Endophytic Verrucomicrobia Reveal Features for Plant Association B. REINHOLD-HUREK, Univerity of Bremen, BREMEN, Germany

Although Verrucomicrobia are widely distributed and cosmopolitan in the plant rhizosphere, only few strains are cultivated. Thus little is known about their potential environmental role and capacities, especially in microbe-plant interactions. We report four new Verrucomicrobia, representing two novel species, one novel genus, and a new subdivision. The divergent strains were isolated from roots or rhizomes of Oryza sativa and O. longistaminata. Opitutus oryzae ER46 and Albicoccus flocculans EW11, or Spartobacter rhizophilum LR76, belonged in subdivision 4 or 2, respectively, while we propose a new diverse subdivision for Ardumicrobium roseum LW23. Bioinformatic analysis of the draft genomes and phenotypic tests revealed potential features for plant association and plant growth promotion, like phytohormone production, phosphate solubilisation and siderophore production. Remarkably, two strains colonized rice roots endophytically in gnotobiotic culture, representing the first endophytes in Verrucomicrobia. Comparison of protein domains encoded in the genomes of novel Verrucomicrobia and reference endophytes, rhizoplane and soil bacteria allowed us to postulate genomic features for association with plants across phyla. Thus, characterization of these four novel isolates increased knowledge on plant-related capacities of Verrucomicrobia and provided a diverse range of novel strains that interact with plant roots for studies on causal relationships using synthetic communities.

Field analyses for structural dynamics of rice associated microbiome

Y. D. UTAMI (1), M. Fuji (1), Y. Shimizu (1), Y. Hongoh (2), Y. Sato (3), Y. Saijo (4), (1) NARA Institute of Science and Technology, Ikoma, Japan; (2) Tokyo Institute of Technology, Tokyo, Japan; (3) National Institute of Genetics, Mishima, Japan; (4) Nara Institute of Science and Technology, Japan 
Plants associate with diverse microorganisms, which dynamically change in the community structure throughout the host life cycle and influence plant adaptation under fluctuating environments. However, the driving force and functional significance for environmental modulation of plantassociated microbiomes remain poorly understood, in particular under field conditions. To grasp field dynamics of microbiome assemblies in rice (Oryza sativa), we performed a DNA amplicon sequencing analysis for bacterial and archaeal 16S rRNA gene, fungal ITS region, and metagenomic analyses, in five cultivars from 5 different regions across Japan over the growing season of 2018. Our current results reveal dynamic changes in the microbial community structure of rice roots, which may be attributable to the environmental cues including soil conditions and plant developmental stages and/or genotypes. Of particular note, direct comparison between neighboring fertilized and nonfertilized fields points to overrepresentation of specific bacterial taxa in the latter, which are expected to involve plant growth-promoting (PGP) species that help host nutrition. In addition, we have initiated culture isolation and characterization of PGP bacterium candidates with inoculation onto rice seedlings. Based on these studies, we will discuss the microbial characteristics that may influence the growth and yield of rice under different field conditions, with attention to soil nutrients.

Exploring the effects of soybean genotype and environment on oomycete populations in Ohio using a microbiome approach K. A. NAVARRO, S. Wijeratne, A. Dorrance, The Ohio State Univ, Wooster, OH, U.S.A.

Pythium and Phytophthora spp. are a major concern in soybean production due to losses in stand and yield when susceptible cultivars are planted. Among the different management strategies, the use of resistant cultivars has shown to be the most effective method to reduce yield losses. However, plant genotype, soil edaphic factors and temperature are known to play a critical role in the community composition. This study investigated the effects of three soybean cultivars on the oomycete community composition across 11 different environments using a microbiome approach paired with direct isolation methods. Lower yields were obtained in the susceptible cultivar Sloan compared to cultivars Kottman with high partial resistance to $P$. sojae and Lorain with high partial resistance to both Pythium spp. and $P$. sojae. Based on the Shannon's diversity index, across environments different levels of diversity were present, which correlated with the number of isolates retrieved from seedlings when using direct isolation methods. Community composition using non- metric multidimensional scaling with Bray-Curtis dissimilar distances indicated that each environment differed significantly from one another. Cultivar also exhibited an effect on community composition but to a lesser degree than that contributed by the environment. This study provides insights on how environments and cultivars play a key role on the diversity and abundance of Pythium and Phytophthora species.

The genes that shape the plant microbiome

M. HORTON (1), J. Mittelstrass (2), (1) University of Zurich, Zurich, Switzerland; (2) Universität Zürich, Zürich, Switzerland

Roots provide plants mineral nutrients and stability in soil; while doing so, they come into contact with diverse soil microbes that affect plant health and productivity. However, despite their ecological and economic relevance, the factors that shape the root microbiome remain poorly understood. Here, we characterize the bacteria and fungi that colonize the roots (the rhizoplane and endosphere) of Arabidopsis thaliana. In addition to bacteria, frequently overlooked fungi shape the structure of the root microbiome. Characterizing both bacteria and fungi helped when identifying candidate genes using genome-wide association studies (GWAS). These genes have diverse roles in immunity, root development, and cell-wall integrity. Our results suggest that future analyses of the root microbiome should focus on multiple kingdoms, and that the root microbiome is shaped not only by genes involved in defense, but also by genes involved in plant form and physiology.

New Plant-Specific Transcript Factors Interact with NLRs and Regulates Broad-Spectrum Blast Resistance in Rice K. Zhai, Y. Dong, Z. HE, Institute of Plant Physiology \& Ecology, SIBS, CAS, Shanghai, China

Plant NLR receptors (NLR) family plays a major role in plant immunity. We previously reported that an antagonistic NLR pair, PigmR and PigmS, confers durable and broad-spectrum resistance to rice blast through epigenetic regulation and heterodimerization. However, the molecular mechanism underlying Pigm-mediated broad-spectrum resistance remains unclear. Here, we show that PigmR protein interacts with the PigmInteracting and Blast Resistance Protein 1 (PIBP1) through its CC domain. PIBP1 belongs to a new plant-specific protein family, and functions as a positive regulator of PigmR-mediated immune responses to blast fungus. PigmR-dependent nuclear localization of PIBP is critical to its role in blast resistance. Our extensive experiments demonstrate that the PIBP1 and its homologs can directly bind to consensus DNA sequences in vitro and in vivo. Furthermore, PIBP1 binds to the promoter of $O s W A K 14$ and $O S P A L$ to activate their expression, resulting in defense responses. Collectively, our study discovers new transcription factors that directly interacts with NLRs to activate plant defense, establishing a direct link between transcriptional activation of immune responses with NLR-mediated pathogen perception.

Pathogen effector triggered plant immunity is modulated by light rhythm

C. GAO (1), J. Huang (1), B. Sun (1), F. Zhang (1), C. H. Wu (2), Y. Wang (1), V. G. A. A. Vleeshouwers (3), T. O. Bozkurt (4), S. Kamoun (2), S. Dong (1), (1) Nanjing Agricultural University, China; (2) The Sainsbury Laboratory, University of East Anglia, Norwich Research Park, Norwich, U.K.; (3) Plant Breeding, Wageningen University and Research, Wageningen, Netherlands; (4) Imperial College London, U.K.

Plant responses to biotic stress are affected by light rhythm, however how light rhythm regulates the pathogen effector triggered plant immunity (ETI) remains largely unknown. Here, we showed that light rhythm specifically determined the activation of potato resistant gene Rpi-vnt1 against Phytophthora infestans carrying cognate effector gene Avrvnt1. A further investigation indicates that Avrvnt1 triggered ETI response required its associated plant protein glycerate kinase (GLYK). To adapt to light changes, natural plant will induce alternative promoter selection (APS) on a set of genes including GLYK. Interestingly, we found Avrvnt1 physically binds to a longer isoform of GLYK which is generated under normal light cycle (LD) and consequently triggers ETI. However, this AVR effector fails to bind the shorter isoform produced by APS under constant dark (DD) and therefore fails to induce ETI response. This study highlights an appealing molecular basis of how plant immune response is determined by light rhythm. 
Chitin elicitor receptor kinase 1 (CERK1) signaling and sugar transporter (SWEET) gene expression in leaves and roots of Brassica rapa infected with Plasmodiophora brassicae

M. H. OH, Chungnam National University, Daejeon, Korea, Republic of (South)

Two cabbage (Brassica rapa) cultivars 'M7' and 'M8' were identified to be resistant and susceptible to several pathotypes of Plasmodiophora brassicae respectively. In plants, sugar plays an important role in the plant growth and development, and also serves as a carbon source for surrounding microbes. In this study, we investigated how BrSWEET genes of both M7 and M8 cultivars respond to P. brassicae infection. Quantitative reverse transcription-PCR (qRT-PCR) analysis revealed that the expression levels of six BrSWEET genes were up-regulated in roots of the resistant cultivar at 72 hours after inoculation with $P$. brassicae compared with uninoculated plants, suggesting that these transporters may support or be involved in transporting sugars into sink roots associated with $P$. brassicae colonization. These findings provide new insights into the complex transcriptional regulation of BrSWEET genes, as well as potential candidate BrSWEET genes that promote sugar transport to enhance clubroot disease resistance in cabbage. CERK1 is essential for perception of the fungal cell wall component chitin and for resistance to fungal pathogens. Our data shown that in Brassica, CERK1 is a major chitin signaling depends on CERK1 post-translational modification and kinase activity and interact with diverse proteins according to Yeast Two Hybrid.

The inactivation of MdDIPM4 by CRISPR/Cas9 editing reduces the susceptibility of apple to fire blight disease V. POMPILI, S. Piazza, L. Dalla Costa, M. Pindo, M. Malnoy, Fondazione Edmund Mach, San Michele a/Adige, Italy

Fire blight, caused by the bacterium Erwinia amylovora (E.a.), is one of the most economically important and invasive diseases affecting apple (Malus $\times$ domestica). The molecular mechanisms of E.a.-apple interaction are widely elucidated but the management of the disease remains still arduous. Apple protein MdDIPM4 interacts with the DspA/E effector, mandatory for the pathogenesis of Erwinia amylovora, but its biological function is still unknown. In this work, the knock-out of MdDIPM4 has been produced in two Malus $\times$ domestica susceptible varieties, by using the CRISPR/Cas9 system delivered via Agrobacterium tumefaciens. Fifty-seven transgenic lines were analyzed using a Next Generation Sequencing to identify the CRISPR/Cas9-induced mutations. Some edited plants with a loss of function mutation were selected and inoculated with the pathogen. An increased resistance was observed, demonstrating that MdDIPM4 is involved in plant susceptibility to fire blight. Moreover, with the aim of producing plants 'clean' from exogenous DNA, we used a heat shock-inducible FLP-FRT recombination system designed specifically to remove the entire T-DNA in those plants with an increased pathogen resistance. Our data demonstrated the possibility to produce apple varieties more resistant to fire blight by using the CRISPR/Cas9 technology and containing a minimal trace of exogenous DNA. These plants may be used in further analysis to elucidate how MdDIPM4 is involved in the onset of the disease.

A receptor-like protein kinase in maize involved in jasmonic acid-mediated phytoalexin production in response to fungal pathogens A. K. BLOCK, USDA-ARS-CMAVE, Gainesville, FL, U.S.A.

Maize (Zea mays) responds to infection by fungal pathogens using jasmonic acid regulated defenses that include the production of anti-microbial compounds termed phytoalexins. The specific mechanisms maize uses to regulate these responses are not well understood. In this study we identify a receptor-like protein kinase in maize whose expression is strongly upregulated in response to infection with the fungal pathogen Cochliobolus heterostrophus. Loss-of-function mutants in this gene display enhanced susceptibility to C. heterostropus, indicating its important role in defending maize against this pathogen. Metabolite analysis revealed that mutant plants had reduced production of both jasmonic acid and the phytoalexins, zealexins and kauralexins in response to $C$. heterostropus infection when compared to wild type plants. These data suggest that this receptor-like protein kinase may be involved in the recognition of $C$. heterostropus and mediate the activation of jasmonic acid induced defenses

Multiple deletion mutation analysis of T3E effectors in Ralstonia solanacearum N. LEI, L. Chen, A. Kiba, Y. Hikichi, K. Ohnishi, Kochi Univ, Japan

A Japanese Ralstonia solanacearum strain OE1-1 infects host plants through the secretion of type III effectors (T3Es) by the type III secretion system (T3SS). There are almost 70 putative T3Es have been identified in OE1-1. It contains four (GALA, SKWP, HLK, and AWR) effector families, which are composed of multiple homologous genes. In addition, several T3Es are conserved in almost all $R$. solanacearum strains and called as "core" and "next core" T3Es, which seem to be important for pathogenicity. When all the effector family genes including 21 T3Es genes were deleted, the resulting strain RK7113 still retained a substantial pathogenicity to Nicotiana benthamiana and eggplant. The mutant strain RK7147, which deleted all the "core" T3Es from RK7113, strongly reduced the pathogenicity. When all the "next core" T3Es were deleted from RK7147, the resulting strain RK7161 completely lost pathogenicity when inoculated from root or directly to xylem. While the mutant cells could not multiply in xylem, RK7161-infiltrated $N$. benthamiana leaves still showed necrotic lesions, and the mutant cells could proliferate in the intercellular spaces of the infiltrated leaves. RK7161 also lost pathogenicity against eggplant and could not multiply in both intercellular spaces and xylem. These results demonstrate that the "core" and "next core" T3Es are definitely needed for proliferation in xylem, but the necessary T3Es for proliferation in the intercellular spaces are diverse between host plants.

Better Bait and Faster Traps: Optimizing the RPS5/PBS1 Decoy System to Enhance Immune Responses to Turnip Mosaic Virus S. E. POTTINGER (1), A. Bak (2), M. Helm (1), C. Casteel (3), R. W. Innes (1), (1) Indiana University, Bloomington, IN, U.S.A.; (2) INRA, Montpellier Cedex, France; (3) University of California, Davis, CA, U.S.A.

RPS5 is activated via cleavage of PBS1 by the Pseudomonas syringae effector AvrPphB. We have shown replacement of PBS1's cleavage sequence with a motif cleaved by Turnip Mosaic Virus (TuMV) NIa protease enabled RPS5 activation upon TuMV infection. However this produced a trailing necrosis phenotype indicative of a cell death response too slow to contain the virus. We theorized this resulted from a mismatch in sub-cellular localization of PBS1 ${ }^{\text {TuMV }} /$ RPS5 and the NIa protease, to test this we transiently re-localized PBS1 ${ }^{\text {TuMV }}$ and RPS5 to sites of NIa accumulation. These experiments revealed location doesn't affect PBS1 ${ }^{\text {TuMV }}$ cleavage, but relocation eliminated RPS5's ability to activate 
cell death. We also created Arabidopsis lines overexpressing PBS1 ${ }^{\mathrm{TuMV}}$ that displayed resistance to TuMV infection initiated by both Agrobacterium and by aphids. Thus the PBS1/RPS5 decoy system can be improved by overexpressing the decoy protein and RPS5, which normally confers resistance to a bacterium, can confer resistance to a virus. Finally, the decoy system may be translated to crops as soybean, barley and wheat respond to AvrPphB. Insertion of an NIa cleavage site in GmPBS1 orthologs enables their cleavage by Soybean Mosaic Virus NIa protease. Significantly co-expression of $G m \mathrm{PBS}^{\mathrm{SMV}}$ with SMV NIa triggers cell death in soybean protoplasts. This establishes PBS1 decoy engineering in crop plants using endogenous proteins could be used to generate resistance to agriculturally important pathogens.

PUB4, a novel CERK1 interactor, positively regulates chitin-induced immune signaling

Y. DESAKI (1), S. Takahashi (2), S. Matsui (2), I. Yoshimi (2), M. Kohari (2), E. Yumoto (3), K. Miyamoto (4), N. Shibuya (2), H. Kaku (2), (1) Dept. Biological Science and Technology, Tokyo University of Science, Katsushika-ku, Tokyo, Japan; (2) Dept. Life Sciences, Sch. Agriculture, Meiji University, Tama-ku, Kawasaki, Kanagawa, Japan; (3) Advanced Instrumental Analysis Center, Teikyo University, Toyosatodai, Utsunomiya, Tochigi, Japan; (4) Dept. Biosciences, Fac. Science and engineering, Teikyo University, Toyosatodai, Utsunomiya, Tochigi, Japan

The LysM-RLK CERK1 is essential for chitin-induced signaling in Arabidopsis and rice. At/OsCERK1 is also known to be important for some other carbohydrate MAMPs signaling. CERK1 kinase activates these immune signaling cascades through the interacting proteins. Arabidopsis PBL27 was identified as a CERK1 interacting RLCK and shown to be activated by CERK1 kinase. Activated PBL27 directly phosphorylates MAPKKK3/5 and positively regulates downstream signaling. Moreover, other RLCK VII members are also involved in the chitin signaling.We here report PUB4, an E3 ubiquitin ligase, is a novel CERK1 interactor and contributes to chitin signaling. Knockout of PUB4 resulted in the alteration in the chitin-induced defense responses, indicating that PUB4 positively regulates the ROS generation and callose deposition but negatively regulates the MAPK activation and defense gene expression. On the other hand, pub4 had enhanced SA accumulation and expression of $P R$ genes in the basal state, indicating spontaneous activation of SA-triggered immunity. To evaluate the effect of this abnormal SA accumulation, we established the double knockout mutant of pub4 and sid2, a mutant of salicylic acid synthesis pathway. Characterization of this double knockout mutant showed that the contradictory phenotype of the pub4 mutant was actually caused by the abnormal accumulation of SA. Therefore, PUB4 is a novel positive regulator of immune responses and seems to be "guarded" in the host plant itself.

\section{Maize lectin receptor-like kinase associated with QTL for resistance to grey leaf spot disease identifed by de novo assembly of transcriptomes}

D. K. BERGER (1), T. Welgemoed (2), R. Pierneef (3), L. Sterck (4), Y. Van de Peer (4), (1) Dept. Plant \& Soil Sciences, FABI, University of Pretoria, Pretoria, South Africa; (2) Dept. Biochemistry, Genetics \& Microbiology, FABI, University of Pretoria, Pretoria, South Africa; (3) Biotechnology Platform, Agricultural Research Council, South Africa; (4) VIB - UGent Center for Plant Systems Biology, Belgium

Gray leaf spot disease of maize, caused by the fungus Cercospora zeina is a threat to maize production in Africa, the Americas, and Asia. Understanding the molecular basis for quantitative resistance to GLS is therefore important for food security. We developed a de novo assembly pipeline to identify candidate maize resistance genes. Near isogenic lines of maize inbred B73 - with and without the QTL for GLS resistance were challenged with C. zeina in the field and samples collected for RNAseq. The reads that did not map to the B73 or C. zeina genomes were expected to contain the novel defence genes and were de novo assembled. A total of 95 protein coding sequences with plant annotations were identified from the B73-QTL plants exposed to C. zeina. To determine if candidate gene expression was induced by C. zeina, a parallel analysis was conducted. The RNAseq reads from each replicate were mapped to a master assembly of all the B73-QTL RNAseq reads, and differential gene expression analysis was carried out. Combining results from both bioinformatics approaches led to identification of seven candidate genes encoding two receptor-like kinases, a LRR-receptor, a chitinase, a thaumatin, a nuclear pore protein and a transcription factor. The most promising candidate was a novel allele of a lectin receptor-like kinase that (i) was induced by C. zeina, and (ii) mapped to the QTL region using PCR-RFLP. Defining the role of this candidate in quantitative resistance to GLS is ongoing.

\section{Differential regulation of TNL-mediated immune signaling by redundant helper CNLs}

\section{Z. WU, University of British Columbia, Vancouver, BC, Canada}

Higher plants utilize nucleotide-binding leucine-rich repeat domain proteins (NLRs) as intracellular receptors to recognize effectors and trigger a robust defense. Although the first NLR-encoding genes were cloned about 25 years ago, the mechanisms of their activation and downstream signaling remains poorly understood. The ADR1 family of coiled-coil NLRs (CNLs) have evolved as helper NLRs that function downstream of many TIR-type NLRs (TNLs). Close homologs of ADR1s form the NRG1 family in Arabidopsis, the function of which is unclear. Through CRISPR/Cas9 gene editing methods, we discovered that the tandemly repeated NRG1A and NRG1B are functionally redundant and operate downstream of TNLs. Interestingly, ADR1s and NRG1s function in two distinct parallel pathways contributing to TNL-specific immunity. Synergistic effects on basal and TNL-mediated defense were detected among ADR1s and NRG1s. An intact P-loop of NRG1s is not required for mediating signals from sensor TNLs, while auto-active NRG1A exhibits autoimmunity. Importantly, NRG1s localize to the cytosol and endomembrane network regardless of the presence of effectors, suggesting a cytosolic activation mechanism. Taken together, different sensor TNLs differentially use two ancient helper NLRs, ADR1s and NRG1s, to transduce downstream defense signals. My current work is focusing on addressing how NRG1s transduces signals from the sensor NLRs through mutagenesis and biochemistry, which will be presented at the conference.

\section{Effectors from Alternaria solani and evidence for a resistance gene in wild potato}

P. J. WOLTERS (1), D. Wouters (2), G. Bijsterbosch (3), R. G. F. Visser (2), M. F. Seidl (3), V. G. A. A. Vleeshouwers (2), (1) Wageningen University and Research (WUR), Wageningen, Netherlands; (2) Plant Breeding, Wageningen University and Research, Wageningen, Netherlands; (3) Wageningen University, Wageningen, Netherlands

The necrotroph Alternaria solani causes early blight on several Solanaceous crops. Potato cultivars show variation in their level of susceptibility to the disease, but no fully resistant cultivars exist. To help understand the infection strategy of $A$. solani and aiming to speed up the identification 
of resistance genes, we have initiated an effectoromics approach. We have sequenced the genome of $A$. solani using PacBio technology and obtained a gapless genome assembly. Various effector candidates were identified, of which some showed to trigger a cell death response in potato. We are now studying the role of these effectors in pathogenicity of $A$. solani through gene-knockouts and transient expression assays. In parallel, we have explored wild relatives of potato for resistance to early blight. Various genotypes with high levels of resistance were detected. Populations derived from some of these genotypes show a 1:1 segregation of resistance, which suggests that the resistance is caused by a single dominant gene. We have started genetic mapping and found markers that are closely linked to resistance. The effectoromics approach should help us to quickly characterise candidate genes. This research is an important step towards developing a potato cultivar that is resistant to early blight.

Multiple MAMPs-triggered immune responses are mediated by BSR1, a rice RLCK, and enhanced by its overexpression, resulting in broad-spectrum disease resistance

Y. KANDA (1,2), Y. Nishizawa (2), T. Kamakura (3), M. Mori (1,4), (1) Tokyo Univ of Science, Noda, Chiba, Japan; (2) NIAS, Tsukuba, Ibaraki, Japan; (3) Tokyo University of Science, Japan; (4) NIAS, Tsukuba, Japan

Recently, receptor-like cytoplasmic kinases (RLCKs) have been found to play roles in intracellular signaling pathways for MTI. BROADSPECTRUM RESISTANCE 1 (BSR1), a rice RLCK, is unique because its overexpression confers the resistance to fungal and bacterial pathogens. The mechanisms underlying the broad-spectrum disease resistance remain unclear. Here, we first validated the contribution of BSR1 to MTI using knockout plants. BSR1 was disrupted by CRISPR/Cas9 system-mediated mutagenesis. The amount of $\mathrm{H}_{2} \mathrm{O}_{2}$ produced in oxidative bursts and the transcript levels of defense-related genes were measured in rice suspension-cultured cells. Knocking out of BSRI suppressed the responses induced by various MAMPs (chitin, peptidoglycan, and lipopolysaccharide), suggesting that BSR1 should function downstream of OsCERK1, a receptor-like kinase corresponding to these MAMPs. Furthermore, whether overexpression of BSRl enhances defense responses was investigated. In response to MAMPs, cells and leaves overexpressing $B S R I$ displayed increased $\mathrm{H}_{2} \mathrm{O}_{2}$ production. The co-cultivation of leaves with living conidia of the blast fungus revealed that BSRI's overexpression allowed host plants to cause detectable oxidative bursts against compatible pathogens. The competition between host-derived ROS production and degradation by pathogens is crucial for host-microbe interactions. Thus, we concluded that the $\mathrm{H}_{2} \mathrm{O}_{2}$ overproduced in planta confers the robust resistance to broad-spectrum pathogens.

Application of plant-immune biosensors for screening and discovery of Actinobacteria biocontrol candidates against necrotrophic fungal pathogens

K. BELT, CSIRO Agriculture and Food, Floreat, WA, Australia

Actinobacteria present a phylum of gram-positive bacteria, which can be terrestrial or aquatic. They are of great interest in agriculture for their abilities as plant growth promoting agents or production of pathogen inhibiting compounds. Plant beneficial isolates are able to inhabit the plant root system without causing a disease. We have a collection of Actinobacteria isolated from south-west Western Australia. Our goal is to identify isolates that can prime a plants' immune response with potential to provide protection against a broad range of phytopathogens, but with a focus on protection against economically destructive necrotrophic fungal pathogens. This is being achieved through a novel approach, non-destructively capturing visible plant immune-biosensor output (gene-based reporter systems) in real-time following inoculation with potential Actinobacteria biocontrol agents to identify isolates with novel plant immunity inducing properties. This way, plant microbe interactions can be monitored and involvement of Actinobacteria in plant defense signaling pathways can be determined. In combination, the Actinobacteria isolates are assessed for production of biocontrol compounds (e.g. antifungals) via routine in vitro pathogen growth inhibition assays. We aim to identify, extract and purify these compounds, and develop a biocontrol fungicide for agricultural disease control.

The involvement of pattern recognition receptors in Caulobacter sp. RHG1 induced growth promotion in Arabidopsis thaliana A. LAMPENS, VIB-UGent Center for Plant Systems Biology, Ghent, Belgium

During the last years, plant growth promoting rhizobacteria (PGPR) are emerging as a promising alternative fertilizer for application on the field as part of a sustainable agricultural system. To date, many phenotypic data on PGPR induced effects on a whole spectrum of crops are available. However, the gathering and reporting of molecular data on these intriguing plant-microbe interactions is still lagging behind. Therefore, my $\mathrm{PhD}$ project aims at unravelling the molecular networks, and in particular the plant receptors, at work during the interaction between Caulobacter species RHG1 and Arabidopsis thaliana. Caulobacter species RHG1 has been isolated from maize roots grown in Belgian field soil and showed growth promoting effects both on maize and Arabidopsis. Mutant and GUS marker line testing for the involvement of the phytohormones auxin, cytokinin and ethylene didn't result in a significant response, whereas the brassinosteroid biosynthesis pathway seems to be necessary for the growth promoting effect. Mining of the bacterial genome also didn't provide a clear clue towards the molecular mechanisms that give rise to the observed phenotype, since no phytohormone biosynthesis genes, biofertilization or biocontrol involved genes could be detected. By combining transcriptomic, proteomic and phosphoproteomic data of inoculated and control treated root samples, we are digging into this plant-microbe interaction until it's true essence will become clear.

Mitogen-Activated Protein Kinase Phosphatase 1 (MKP1) dependent changes of plasma membrane proteome during PAMP elicitation G. RUPP, L. A. Greeley, S. Peck, Univ of Missouri, Columbia, MO, U.S.A.

By 2050, a 70 percent increase in food production is needed to continue to feed the worlds growing population. Pathogen infection of crops plays a major role in the disruption of food production. Thus, understanding how plants defend themselves is an important step towards developing plants with improved resistance. In Arabidopsis thaliana, loss of Mitogen-Activated Protein Kinase Phosphatase 1 (MKP1), a negative regulator of plant defense, results in plants which have enhanced resistance to bacterial infection by Pseudomonas syringae pv. tomato DC3000. In addition, this resistance in $m k p l$ is due to a decrease in the extracellular accumulation of bioactive metabolites that induce the bacteria's type III secretion system, a critical component of bacterial infection. Whether this reduction in $m k p 1$ metabolites is due to an overall change in plant metabolism or due to a reduction of plasma membrane transporters has yet to be determined. In this project, we examine differences in plasma membrane proteome of elicited and unelicited $m k p 1$ plants as compared to wildtype to determine if differential accumulation of transporters for 
the bioactive metabolites may explain the phenotype observed in $m k p 1$. Ultimately, identification of these key plasma membrane proteins will increase the understanding of how resistance in $m p k l$ is controlled.

Animal TIR domains inform the molecular and structural basis of signaling by plant NLR TIR domains B. KOBE (1), H. Burdett (1), X. Zhang (2), J. Chen (3), M. X. Rank (1), S. Horsefield (1), M. K. Manik (1), Y. Shi (4), T. Qi (5), I. B. Dry (6), S. J. Williams (7), B. J. Staskawicz (8), T. Ve (4), P. N. Dodds (9), (1) University of Queensland, Brisbane, QLD, Australia; (2) Commonwealth Scientific \& Industrial Research Organization, Canberra, ACT, Australia; (3) Commonwealth Scientific and Industrial Research Organisation, Canberra, ACT, Australia; (4) Griffith University, Southport, QLD, Australia; (5) University of California, Berkeley, Berkeley, CA, U.S.A.; (6) CSIRO Plant Industry, Urrbrae, SA, Australia; (7) Australian National University, Canberra, ACT, Australia; (8) Department of Plant and Microbial Biology, University of California, Berkeley, Berkeley, CA, U.S.A.; (9) CSIRO Agriculture and Food, Canberra, ACT, Australia

A large group of plant NLR immune receptors contain TIR (Toll/interleukin-1 receptor, resistance) domains involved in signaling at their Ntermini. However, the signaling mechanism has remained unclear. TIR domains are also present in proteins involved in innate immunity and celldeath signaling pathways in animals. We have therefore been able to learn about the molecular mechanisms of signaling by TIR domains by complementary studies of both animal and plant systems. We reconstituted large assemblies of the TLR (Toll-like receptor) adaptor TIR domains and determined the structure of the filamentous assembly of the TLR adaptor MAL by cryo-electron microscopy. We further determined the crystal structure of the TIR domain from the protein SARM1, involved in axon degeneration, a cell-death process operating in neurons that involves cleavage of the dinucleotide $\mathrm{NAD}^{+}$. These studies have informed on the function of plant TIR domains. We have evidence that plant TIR domains can also self-associate though more than one interface. Structural similarities between SARM1 and plant TIR domains led us to demonstrate that plant TIR domains can cleave $\mathrm{NAD}^{+}$, and this activity likely plays a role in their HR function. Our studies unify the mechanism of function of TIR domains as "signaling by cooperative assembly formation (SCAF)" with prion-like features that leads to the activation of effector enzymes and show that some TIR domains can themselves function as effector enzymes.

RNA-Seq transcriptome analysis reveals candidate genes involved in crown-rot (Phytophthora cactorum) resistance in strawberry (Fragaria vesca)

A. GOGOI (1), E. Lysøe (2), J. Davik (2), H. Eikemo (2), A. Stensvand (1), M. B. Brurberg (1), (1) Department of Plant Sciences, Norwegian University of Life Sciences (NMBU), Ås, Norway; (2) Div. of Biotech. and Plant Health, Norwegian Institute of Bioeconomy Research, Ås, Norway

The phytopathogenic oomycete Phytophthora cactorum is a devastating pathogen of strawberry (Fragaria spp.). Genotypes of wild strawberry (Fragaria vesca) vary in resistance to crown-rot caused by the pathogen. To gain insights into the molecular mechanisms underlying the defence response, the whole transcriptome of a susceptible (CFRA1218) and two resistant (CFRA1603 and Bukammen) strawberry genotypes were studied at an early infection stage (48 hours postinoculation). The genes involved in flavonoid and isoprenoid biosynthesis, as well as auxin, ethylene, jasmonic acid, and salicylic acid pathways, were more expressed in the susceptible genotype than the resistant ones. This was also the case for pathogenesis-related proteins. On the other hand, several potential resistance gene-related transcripts, including transcripts encoding receptor-like kinases, nucleotide-binding leucine-rich repeat (NB-LRR) containing proteins, and toll/interleukin-1 receptor domain-containing proteins, were highly expressed in the resistant genotypes compared to the susceptible one. One NB-LRR protein transcript, which is contained within the RPc-1 (Resistance to Phytophthora cactorum 1) locus, is uniquely upregulated in the resistant genotypes upon P. cactorum infection.

The Arabidopsis receptor-like kinase FERE is a crucial component of pattern-triggered immunity responses to Fusarium species A. D. COLEMAN, L. Raasch, J. Maroschek, S. Ranf, R. Hueckelhoven, Technical University of Munich, Freising, Germany

Fusarium is a large genus of fungi causing severe economic damage in many crop species. Molecular patterns from diverse origins (collectively termed elicitors) can be perceived by plants resulting in quantitative disease-resistance via the activation of pattern-triggered immunity (PTI). Several studies have described elicitors present in Fusarium species which elicit PTI in various plant systems. However, precise knowledge of these elicitors or corresponding plant immunity components is often lacking. Our aim is to improve our mechanistic understanding of Fusariumtriggered PTI. We employed a forward-genetics screen in Arabidopsis to isolate mutants strongly impaired in calcium influx upon treatment with an elicitor fraction derived from either $F$. graminearum or F. oxysporum. We obtained several independent 'Fusarium elicitor reduced elicitation' mutants and successfully mapped the causal mutation of ferel to a receptor-like kinase with an undescribed role in fungal elicitor responses. PTI loss-of-function in ferel was fully complemented with the full-length recombinant FERE protein. FERE contributes significantly towards canonical PTI-responses and basal resistance to F. oxysporum. Genetic studies suggest that FERE shares some common PTI-signaling partners but also shows unique features. Our approach demonstrates the feasibility to identify novel PTI players relevant for Fusarium resistance in Arabidopsis which may be of value to breed or engineer Fusarium-resistant crops.

\section{Characterization and functional analysis of the Md-miR285N gene promoter in transgenic apple (Malus $\times$ domestica) and Arabidopsis thaliana \\ V. POMPILI, S. Piazza, M. Li, C. Varotto, M. Malnoy, Fondazione Edmund Mach, San Michele a/Adige, Italy}

MicroRNAs are key regulators of plant physiological activities by functioning in RNA silencing and post-transcriptional regulation of gene expression. In apple, the uncharacterized Md-miR285N is predicted to target 35 RNA transcripts, mostly coding for TMV, SCN1 and CDPK proteins involved in plant defense to pathogens. In this work, ca. $2 \mathrm{~Kb}$ of the $M d-m i R 285 \mathrm{~N}$ promoter were isolated and cloned into an expression vector with its 3'-end fused to the $\beta$-glucoronidase (GUS) reporter gene. The expression vector was used for Agrobacterium-mediated transformation of both Malus $\times$ domestica (cv. 'Gala') and Arabidopsis thaliana (ecotype Col-0). In transgenic apple plantlets a strong GUS activity was detected in stems, leaves and veins. In Arabidopsis seedlings the promoter was shown to be highly expressed in roots, shoot apex meristem, primary and secondary leaves and veins. To understand the functional response of $M d-m i R 285 \mathrm{~N}$ against pathogen infections, apple and Arabidopsis plantlets were inoculated, respectively, with Erwinia amylovora strain Ea273 and Pseudomonas syringae pv. tomato DC3000. The 
GUS activity was qualitatively and quantitatively evaluated in treated plants at 12, 24, 36, and 48 hours postinoculation. A differential regulation of promoter expression was detected compared to untreated plants. These data suggest that Md-miR285N may act as important regulator of plant defense after bacterial infections.

The Citrus reticulata CrRAP2.2 transcriptional factor shares similar functions to the Arabidopsis homologue and increases resistance to Xylella fastidiosa

W. E. L. Pereira (1), M. A. Takita (1), M. Melotto (2), A. A. DE SOUZA (1), (1) Centro de Citricultura "Sylvio Moreira" - Agronomic Institute (IAC), Cordeiropolis, Brazil; (2) University of California, Davis, CA, U.S.A.

Xylella fastidiosa is a multi-host pathogen that affects many different crops worldwide. In Brazil it causes Citrus Variegated Chlorosis (CVC) in Citrus sinensis, but not in Citrus reticulata. Many genes have been associated with C. reticulata resistance to X. fastidiosa, but without functional validation. Among them is a transcriptional factor of the AP2/ERF family that is homologous to the Arabidopsis thaliana AtRAP2.2.

Bioinformatics analysis together with subcellular localization and auto-activation assays indicate that $R A P 2.2$ from $C$. reticulata (CrRAP2.2) is a transcriptional factor ortholog to AtRAP2.2. Thus, we used Arabidopsis rap2.2 mutant to better understand defense mechanisms in response to $X$. fastidiosa. Bacterial inoculation in rap2.2 mutant resulted in a larger population compared to wild type, which was complemented by $C r R A P 2.2$. In addition, symptoms of anthocyanin accumulation in consequence of bacterial infection were higher in the mutant and restored by $C r R A P 2.2$, indicating they have conserved functions in plant defense response to X. fastidiosa. We therefore transformed C. sinensis with CrRAP2.2 and verified a positive correlation between $\mathrm{CVC}$ resistance and gene expression in transgenic lines. In our knowledge this is the first work to characterize the function of a gene related with $X$. fastidiosa defense response and its application in generating transgenic resistant lines with a gene from a closely related species.

The receptor-like cytoplasmic kinase BSK5 associates with immune receptors and is required for PTI B. B. Majhi, S. Sreeramulu, G. SESSA, Tel Aviv University, Tel Aviv, Israel

We investigated the role of Arabidopsis brassinosteroid-signaling kinase 5 (BSK5), a receptor-like cytoplasmic kinase of subfamily XII, in pattern-triggered immunity (PTI). BSK5 localized to the plant cell periphery, interacted in yeast and in planta with several immune receptors, including EFR and PEPR1, and it was phosphorylated in vitro by PEPR1 and EFR. Remarkably, bsk5 T-DNA insertion mutants displayed enhanced susceptibility to the fungal pathogen Botrytis cinerea and to Pseudomonas syringae bacteria. Moreover, bsk5 mutant plants were impaired in PAMP/DAMP-induced generation of reactive oxygen species and callose deposition at the cell wall but were not affected in MAP kinase signaling. BSK5 forms mutated in the BSK5 myristoylation site, ATP binding site, and kinase activation loop were not able to complement defective PTI responses of bsk5 mutant plants, suggesting that localization to the cell periphery, kinase activity, and phosphorylation by immune receptors are important for the function of BSK5 in PTI. Together, these results suggest that BSK5 associates with immune receptors and plays a central role in PTI. Further studies are in process to assess the involvement of additional BSK family members in immunity, their functional redundancy, and whether they are targeted by effector proteins of plant pathogens.

Characterization of the molecular mechanism of action of the AsES elicitor protein in plant immunity

M. D. P. CARO (1), N. Holton (2), G. Conti (3,4), A. L. Venturuzzi (3,4), C. Zipfel (2,5), S. Asurmendi (3,4), J. C. Díaz-Ricci (1), (1) INSIBIO, CONICET-UNT, San Miguel de Tucumán, Argentina; (2) The Sainsbury Laboratory, University of East Anglia, Norwich Research Park, Norwich, U.K.; (3) Instituto de Biotecnología, CICVyA, INTA Castelar, Buenos Aires, Argentina; (4) IABIMO, INTA-CONICET, Buenos Aires, Argentina; (5) Inst. of Plant and Microbial Biology, University of Zürich, Zurich, Switzerland

AsES (Acremonium strictum Elicitor Subtilisin) is a subtilase purified from the supernatant of the fungus $A$. strictum that is capable of inducing a defence response in strawberry and Arabidopsis plants. The aim of this work was to obtain AsES protein by recombinant expression in order to determine the role of the enzymatic activity in the induction of the defence response and to study the mechanism of action by which AsES is perceived by the plant. Notably, AsES induces a PTI-like response as observed by seedling growth inhibition and induction of PTI-marker genes. Interestingly, AsES proteolytic activity is not required for defence induction since catalytically inactive AsES mutants were still able to induce defence-related genes. In addition, we found that the common co-receptor BAK1 is involved in AsES perception, suggesting that AsES is perceived as a PAMP by a leucine-rich repeat receptor. Pre-treatment of Arabidopsis plants with AsES resulted in a significant reduction of TMV-Cg viral titre as well as increased resistance to the fungus Botrytis cinerea in Arabidopsis. Similar results with TMV-Cg were obtained in Nicotiana benthamiana plants, which additionally showed a reduction in cell to cell movement and a delay in the systemic movement. Our results contribute to the understanding of the mechanism of action of AsES and can be exploited for the development of strategies to confer durable resistance in plants.

Coat protein of conserved structure from multiple potyviruses triggers Rysto-mediated immunity M. GRECH-BARAN (1), K. Witek (2), J. D. Jones (2), J. Hennig (1), (1) Institute of Biochemistry and Biophysics, PAS, Warsaw, Poland; (2) The Sainsbury Laboratory, University of East Anglia, Norwich Research Park, Norwich, U.K.

$R y_{\text {sto }}$ from the wild relative of potato, Solanum stoloniferum, confers extreme resistance to potato virus Y (PVY) that causes severe losses in potato production. Using SMRT RenSeq, we cloned $R y_{\text {sto }}$ and showed that it encodes a TNL-class nucleotide-binding, leucine rich repeat immune receptor. To identify the elicitor of the $R y_{\text {sto }}$-mediated immune response, the ORFs encoding putative viral proteins were cloned and transiently expressed in transgenic $R y_{\text {sto }}$ tobacco plants. Among all tested proteins, only expression of PVY coat protein (CP) resulted in strong HR, showing that viral CP is an elicitor of $R y_{s t o}$-triggered immunity. To further understand the mechanism of $R y_{s t o}$-mediated resistance, we showed that $R y_{s t o}$ targets a conserved element of the $\mathrm{CP}$ structure, rather than a linear amino acid motif. Consistent with this hypothesis, we showed that Ry $\mathrm{sto}^{-}$ mediated resistance can be triggered by CPs of similar structure from multiple potyviruses, but not by the ones lacking it. This allows to predict the range of viral pathogens against which $\mathrm{Ry}_{\text {sto }}$ could confer resistance. 
A cysteine-rich receptor-like protein kinase is involved in CERK1-4 dependent cell death responses in Arabidopsis

C. TRIPPEL, E. K. Petutschnig, V. Lipka, Georg-August-University, Goettingen, Germany

Chitin Elicitor Receptor Kinase 1 (CERK1) is a major component of the Arabidopsis chitin receptor. Accordingly, CERK1 knockout plants are defective in chitin signaling and induction of chitin-triggered immunity. A CERK1 mutant line (cerk1-4) with a point mutation in the ectodomain exhibits normal chitin signaling but shows a deregulated salicylic acid-dependent cell death reaction upon infection with the powdery mildew fungus Erysiphe cruciferarum. The cerk1-4 phenotype is coupled with altered CERK1 processing and increased PRI induction upon pathogen challenge (Petutschnig et al., 2014). In a forward genetic suppressor screen, mutants were identified that showed WT-like cell death and PRIexpression upon infection with E. cruciferarum. One mutant was examined in more detail in this study. The cell death suppressor phenotype of this mutant is semi-dominant and early PAMP responses are comparable to wild type. Mapping by new generation sequencing identified a single amino acid exchange in a cysteine-rich receptor-like protein kinase (CRK) as the causative suppressor mutation. The mutation affects a highly conserved cysteine in one of the extracellular DUF26 domains, likely disrupting CRK ligand binding or interaction with other proteins.

Dual functionality of a fungal LysM effector: Substrate sequestration and receptor complex perturbation? H. TIAN (1), H. Rövenich (2), B. P. H. J. Thomma (3), (1) Wageningen University \& Research, Wageningen, Netherlands; (2) University of Cologne, Cologne, Germany; (3) Wageningen University, Wageningen, Netherlands

Chitin is a structural component of fungal cell walls that is recognized by LysM domain-containing cell surface immune receptors in many plant species. One of the strategies that fungal plant pathogens evolved to perturb chitin-triggered recognition is through the secretion of LysMcontaining effector proteins that bind chitin. The effector protein Ecp6 of the tomato leaf mould fungus Cladosporium fulvum sequesters chitin with ultra-high $(\mathrm{pM})$ affinity because two of its three LysM domains undergo ligand-induced intramolecular dimerization. The remaining LysM domain can also bind chitin, albeit with significantly lower $(\mu \mathrm{M})$ affinity. Until now, it has remained unclear whether this low-affinity binding site contributes to the virulence function of Ecp6. Through the analysis of C. fulvum mutants that express Ecp6 mutants in which the chitinbinding ability of individual LysM domain is compromised, we show that the low-affinity chitin binding site contributes to fungal virulence which involves the suppression of chitin responsive tomato genes, including candidate chitin receptor genes. Potentially, Ecp6 not only outcompetes plant LysM receptors for chitin binding, but also interferes in the receptor complex formation that is crucial for activation of chitininduced immunity.

LysM-mediated elicitor recognition in the liverwort Marchantia polymorpha

H. Iwakawa (1), I. Yotsui (2), K. Melkonian (1), H. Matsui (2), R. Nishihama (3), T. Kohchi (3), H. NAKAGAMI (1), (1) Max Planck Institute for Plant Breeding Research, Germany; (2) RIKEN CSRS, Japan; (3) Kyoto University, Japan

Plasma membrane localized LysM proteins recognize microbe-derived molecules and thereby mediate plant immune activation or beneficial symbiosis establishment. Extensive studies in angiosperms revealed that the LysM proteins perceive fungal chitin and bacterial peptidoglycan (PGN) as pathogen-associated molecular patterns with different modes of action. The genome of Marchantia polymorpha, a species in a basal land plant lineage, encodes four LysM domain-containing receptor-like proteins, MpLYK1, MpLYK2, MpLYR, and MpLYP, anticipating the existence of a LysM-mediated immune system in Marchantia. We confirmed that M. polymorpha recognizes chitin and PGN and exerts early immune responses. Analysis of loss-of-function mutants suggested that MpLYK1 and MpLYR are involved in the recognition of chitin and PGN. To evaluate the function of genes during the plant-microbe interaction, we established an Agrobacterium-mediated transient transformation system. With the use of a GUS reporter gene, we found that Agrobacterium preferentially transferred T-DNA into rhizoids and assimilatory filaments, suggesting that pathogenic bacterial species preferentially target these cell types for infection. Interestingly, we found that $\mathrm{Mp} L Y K 1$ and $\mathrm{Mp} L Y R$ genes are primarily expressed in rhizoids and assimilatory filaments. These results suggest that Marchantia has established a MpLYK1/MpLYR-mediated immune system in these specific cell types to effectively protect themselves against pathogenic microbes.

Plant autoimmunity activated by oligomerization of the atypical resistance protein RPW8/HR4 and NLR RPP7

L. LI, D. Weigel, Max Planck Institute for Developmental Biology, Tuebingen, Germany

Immune responses that occur without an external trigger provide an effective platform for understanding the mechanisms underlying activation of immune signaling. In certain intra- and interspecific plant hybrids, autoimmunity can be triggered when immune system components meet that have not been co-adapted. A particularly interesting case of hybrid necrosis in Arabidopsis thaliana is that of the RPP7 and RPW8 loci. RPP7, which confers race-specific resistance against oomycetes, encodes members of canonical coiled-coil, nucleotide-binding and leucine rich repeat (CC-NLR) proteins. NLR proteins are the most common class of plant immune receptors. RPW8, which confers broad-spectrum resistance to biotrophic pathogens, belongs to the small family of RPW8/HR (Homologous to RPW8) coiled-coil-only domain proteins. Here, we will describe the mechanisms by which the HR4 $4^{\mathrm{Fei}-0}$ and RPP7b $\mathrm{b}^{\mathrm{Lerik1-3}}$ pair activates immune responses. Based on our findings, we propose a model that extends previous scenarios of NLR signaling based on ON/OFF equilibrium of closed, inactive and open, active NLR states.

Putting yellow rust to BED: Elucidating the relationship between Yr7, Yr5 and YrSP

C. MARCHAL (1), J. Zhang (2), P. Zhang (2), P. Fenwick (3), B. Steuernagel (1), N. Adamski (4), L. Boyd (5), R. McIntosh (6), B. B. Wulff (1), S. Berry (3), E. Lagudah (7), C. Uauy (4), (1) John Innes Centre, Norwich, U.K.; (2) Commonwealth Scientific and Industrial Research Organisation (CSRIO), Australia; (3) Limagrain UK Ltd, U.K.; (4) John Innes Centre, U.K.; (5) National Institute of Agricultural Botany (NIAB), U.K.; (6) University of Sydney, Australia; (7) CSIRO Agriculture and Food, Canberra, ACT, Australia

Crop diseases reduce wheat yields by $\sim 25 \%$ globally and thus pose a major threat to global food security. Yellow (stripe) rust caused by Puccinia striiformis f. sp. tritici, is distributed worldwide and is currently the most globally damaging cereal rust. Despite over 70 designated yellow rust resistance genes $(Y r)$ in wheat, few have been cloned. Using mutational resistance gene enrichment sequencing (MutRenSeq) we successfully 
cloned three non-canonical BED containing Nucleotide-binding and Leucine-rich Repeat proteins (BED-NLRs) in wheat that confer different resistance spectra to yellow rust: $Y r 7, \operatorname{Yr} 5$ and $Y r S P$. We showed that all three genes are genetically linked and $Y r 5$ is distinct from $Y r 7$, whereas $\operatorname{YrSP}$ is a truncated allele of $Y r 5$ with $99.8 \%$ sequence identity. We showed that a single amino-acid change in the BED domain of $Y r 7$ was sufficient to lead to a loss of resistance. Additionally, Yr5 and YrSP BED domains are identical and there is only one amino-acid polymorphism between Yr7 and Yr5/YrSP BED domains. We thus hypothesize that recognition specificity is not solely governed by the BED domain. We are currently setting-up Agrobacterium-mediated expression of $Y r 7$ in Nicotiana benthamiana to test the effect of full-length and different truncations on defence response signaling. These results will provide more insights into the mode of action of BED-NLRs in disease resistance in crops.

Deciphering the sensing of rhamnolipid secretome by Arabidopsis thaliana

R. Schellenberger, M. Touchard, E. Huby, F. Mazeyrat-Gourbeyre, C. Clément, F. Baillieul, S. Dhondt-Cordelier, S. Cordelier, J. Crouzet, S. DOREY, Reims Champagne-Ardenne University, Reims, France

Plant innate immunity is mediated by the perception of invasion patterns (IPs), originating from the pathogen or the plant, by plant IP receptors. Rhamnolipids are amphiphilic molecules secreted by bacteria, including Pseudomonas and Burkholderia species. In the last few years, we have shown that rhamnolipid secretome (RLsec) from Pseudomonas aeruginosa is perceived by plants and stimulates an immune response. RLsec induces early immunity-related signaling events such as calcium influx, extracellular reactive oxygen species (ROS) production and mitogenassociated protein kinase (MAPK) phosphorylation followed by late defense responses including defense gene activation. RLsec also triggers local resistance against necrotrophic and (hemi)biotrophic pathogens. Although RLsec-triggered immune responses have been characterized in several plant species, its perception mechanism by plant cells remains largely unknown. Here, we investigated how RLsec is sensed at the plasma membrane level in Arabidopsis. Our results demonstrate that RLsec is recognized by Arabidopsis cells through two distinct mechanisms, one involving a proteinaceous IP receptor and another which requires a lipid-driven process. This work therefore highlights the singular molecular mechanisms involved in the perception of RLsec by plants.

OsWAKL21, a putative receptor of rice cell wall damage activates alternate signaling in rice and Arabidopsis to induce immunity K. K. MALUKANI (1), A. Ranjan (2), S. J. Hota (1), H. K. Patel (1), R. V. Sonti (3), (1) CSIR-Centre for Cellular and Molecular Biology (CSIR-CCMB), Hyderabad, India; (2) University of Wisconsin, Madison, WI, U.S.A.; (3) National Institute of Plant Genome Research (NIPGR), New Delhi, India

Xanthomonas oryzae pv. oryzae (Xoo) causes the serious bacterial blight disease of rice. As part of its virulence repertoire, Xoo secretes various cell wall degrading enzymes (CWDEs) such as cellulases, xylanases and a Lipase/esterase (LipA). Conversely, treatment of rice tissues with any of these purified enzymes activates immune responses. Plants sense this cell wall damage as a mark of infection and induce immune responses. Very little information is available about the plant functions that are involved in the elaboration of cell wall damage induced immune responses. Transcriptome analyses revealed a rice cell wall-associated receptor kinase, OsWAKL21 that is upregulated following treatment with either LipA or Xoo. VIGS mediated downregulation of OsWAKL21 attenuates LipA induced immune responses. Overexpression of OsWAKL21 in rice mimics LipA treatment in induction of immune responses, activation of JA pathway and enhanced expression of defence related genes, indicating that it plays an important role in elaboration of LipA induced immune responses. Ectopic expression of OsWAKL21 in Arabidopsis also activates plant immune responses. OsWAKL21 is a moonlighting kinase having in vitro kinase and guanylate cyclase activities. Interestingly, OsWAKL21 needs kinase activity to activate immune responses in rice while in Arabidopsis it needs the guanylate cyclase activity. Thus OsWAKL21 is activating similar immune responses in two different species but via different mechanisms.

Effector-dependent interactions between RRS1 N- and C- terminal domains are required for RPS4/RRS1 derepression H. GUO, H. K. Ahn, J. Sklenar, Y. Ma, F. L. H. Menke, J. D. Jones, The Sainsbury Laboratory, University of East Anglia, Norwich Research Park, Norwich, U.K.

Plant intracellular nucleotide-binding leucine-rich repeat (NLR) immune receptors are encoded by Resistance (R) genes and confer specific resistance to pathogen races carrying the corresponding recognized effectors. Arabidopsis RRS1 and RPS4 are a pair of NLR proteins that function together to recognize two bacterial effectors, PopP2 and AvrRps4. The RRS1-R allele in accessions Nd-1 and Ws-2 confers AvrRps4 and PopP2 recognition, whereas the Col-0 allele of RRS1 (RRS1-S) confers only AvrRps4 recognition. Here we show that de-repression of RRS1-R/S involves effector-triggered proximity between the TIR domain and the C-terminal WRKY transcription factor domain. The TIR domain of RRS1-S interacts with its C-terminus only upon AvrRps4 perception, while TIR domain of RRS1-R associates with C-terminus constitutively but in an AvrRps4- and PopP2-enhanced manner. We propose that the effector-promoted interaction between TIR ${ }^{R R S 1}$ and the Cterminus is required to relieve the inhibitory effect of $\mathrm{TIR}^{\mathrm{RRS} 1}$ on $\mathrm{TIR}^{\mathrm{RPS} 4}$. Intriguingly, the C-terminus of RRS1-R, but not RRS1-S, is phosphorylated. Mutational analysis reveals that phosphorylation at Thr1214 is required for maintaining RRS1-R inactive state. Phosphorylation at 5 Ser sites is required for PopP2, but not AvrRps4, responsiveness. Our findings collectively uncover an effector-triggered and phosphorylation-regulated conformational difference within RRS1 that results in distinct modes of derepression of the complex by PopP2 and AvrRps4.

Structural and functional analysis of LORE-dependent 3-hydroxy fatty acid immune sensing in Brassicaceae L. J. SHU, M. Schäffer, S. Ranf, Technical University of Munich, Freising, Germany

Plants are constantly exposed to pathogens and pests, and thus evolved a sophisticated immune system to monitor and resist biotic threats in their environment. Plants utilize cell-surface pattern recognition receptors (PRR) to detect signs of danger - microbe-associated molecular patterns (MAMPs) - in the apoplast and trigger local and systemic defense responses. Medium chain 3-hydroxy fatty acids (3-OH-FAs), particularly 3hydroxy decanoic acid, released from Gram-negative bacteria, are sensed as MAMPs by the bulb-type lectin receptor kinase LORE (also known as SD1-29) in Arabidopsis thaliana, and LORE senses 3-OH-FAs in a carbon chain length-specific manner. How LORE is activated by 3-OHFAs at the molecular level is still unknown. Therefore, we assess binding of 3-OH-FAs to the LORE ectodomain by different methods and apply 
homology modelling to predict the protein structure of the LORE ectodomain and potential binding sites of 3-OH-FAs. Candidate sites are currently being validated by mutational studies. Additionally, we functionally explore the natural diversity of LORE orthologs within the Brassicaceae family. Overall, our findings reveal the mechanism of LORE-mediated 3-OH-FA sensing in Brassicaceae.

The Ptr1 locus in Solanum lycopersicoides confers resistance to race 1 strains of Pseudomonas syringae pv. tomato and to Ralstonia pseudosolanacearum by recognizing type III effectors AvrRpt2/RipBN

D. C. MAZO-MOLINA (1), S. Mainiero (2), S. R. Hind (2), C. M. Kraus (2), M. Vachev (2), F. Maviane-Macia (3), M. Lindeberg (1), S. Saha (2), S. R. Strickler (2), A. Feder (2), J. J. Giovannoni (2), C. D. Smart (4), N. Peeters (5), G. B. Martin (1,2), (1) School of Integrative Plant Science, Cornell University, Ithaca, NY, U.S.A.; (2) Boyce Thompson Institute, Ithaca, NY, U.S.A.; (3) Laboratory of Plant-Microbe Interactions, France; (4) Cornell University, Geneva, NY, U.S.A.; (5) LIPM, Université de Toulouse, INRA, CNRS, INPT, Castanet-Tolosan, France

Race 1 strains of Pseudomonas syringae pv. tomato (Pst), which cause bacterial speck disease of tomato, are becoming increasingly common and no simply-inherited genetic resistance to such strains is known. We discovered that a locus in Solanum lycopersicoides, termed Pseudomonas tomato race 1 (Ptr1), confers resistance to race 1 Pst strains by recognizing the type III effector AvrRpt2. In Arabidopsis and apple, strains of Pst and Erwinia amylovora expressing AvrRpt2 degrade the RIN4 protein thereby activating RPS2 or Mr5-mediated immunity, respectively. Ptr 1 also recognized homologs of AvrRpt2 from diverse bacteria including one in Ralstonia pseudosolanacearum and this correlated with the ability of AvrRpt2 to degrade RIN4. Using site-directed mutagenesis of AvrRpt2 we found that, like RPS2, activation of Ptr1 requires AvrRpt2 proteolytic activity. Ptr1 recognition of AvrRpt2 suggests it likely encodes an NLR protein or possibly a guardee such as Rin4. Based on genetic mapping we currently have a list of nine Ptrl candidate genes. Interestingly, none of them encode proteins with similarity to RPS2 or Mr5. Each candidate gene is being tested using Agrobacterium-mediated transient expression for its ability to recognize AvrRpt2 in the presence of tomato Rin4. Ptr 1 could play an important role in controlling bacterial speck disease, and its future cloning may shed light on an example of convergent evolution for recognition of a widespread type III effector.

Trans-(auto)phosphorylation events between SOBIR1 and BAK1 are required for immune signaling

M. H. JOOSTEN (1), J. Postma (2), S. Robatzek (3), J. Wu (1), A. M. van der Burgh (1), (1) Wageningen University, Wageningen, Netherlands; (2) Heinrich Heine University, Center for Advanced Imaging, Dusseldorf, Germany; (3) Ludwig-Maximilians-Universität München, Genetics, Martinsried, Germany

Receptor-like proteins (RLPs) and receptor-like kinases (RLKs) trigger immune signaling of plants against pathogens. RLPs lack an intracellular kinase domain (KD), and they constitutively interact with the RLK SOBIR1 to form signaling-competent receptor complexes. Ligand perception recruits the co-receptor BAK1/SERK3 to the RLP/SOBIR1 complex, thereby activating RLP-mediated immunity. Arabidopsis thaliana (At) SOBIR1, constitutively activating immune responses upon its overexpression in Nicotiana benthamiana, is highly phosphorylated. In addition to kinase activity of SOBIR1, kinase-active BAK1 is essential for AtSOBIR1-induced constitutive immunity and for the phosphorylation of AtSOBIR1. Furthermore, defence initiation upon perception of Avr4 from the extracellular pathogenic fungus Cladosporium fulvum by the tomato RLP Cf-4, depends on kinase-active SOBIR1 and BAK1. Likely, SOBIR1 and BAK1 trans-phosphorylate, thereby activating the Cf4/Avr4-containing receptor complex. AtSOBIR1 T519, T523, and T529 are all probable phosphorylation sites, similar to T522, Y532, and Y538. Constitutively active AtSOBIR1 is phosphorylated on several additional Thr and Ser residues and specific phosphorylation of the KD of SOBIR1 likely enables this regulatory RLK to initiate immune signaling downstream of RLPs. Interestingly, the bacterial effector HopD1 suppresses AtSOBIR1-triggered constitutive immunity and the Cf-4/Avr4-triggered signaling pathway, similar to the effector AvrPto.

Using natural variation in tomato to understand and improve the plant immune system

S. MAINIERO (1), R. Roberts (1), A. F. Powell (1), S. R. Strickler (1), A. Collmer (2), G. B. Martin (1,2), (1) Boyce Thompson Institute, Ithaca, NY, U.S.A.; (2) School of Integrative Plant Science, Cornell University, Ithaca, NY, U.S.A.

The interaction between tomato and Pseudomonas syringae pv. tomato (Pst) is a well-developed model for investigating the molecular basis of the plant immune system. There is extensive natural variation in tomato but it has not been fully leveraged to enhance our understanding of the tomato-Pst pathosystem. Using available whole-genome resequencing data, we chose a set of 216 genetically diverse accessions. Plants of each accession were inoculated by spraying Pst mutant strains that lack different effectors and/or flagellin to help elucidate whether the observed host responses involved NLR-or PRR-triggered immunity. The screen discovered a broad range of previously unseen host symptoms in response to Pst and subsequent analyses indicate some of these phenotypes are simply inherited. Reactive oxygen species assays, reporter genes, and bacterial population measurements were also performed to investigate host responses and identify candidate accessions for genetic analysis. We will present our results of mapping-by-sequencing efforts to identify loci involved in both resistance and novel host responses to Pst strains. This project opens up new avenues for investigating the molecular mechanisms underlying the plant immune system and augments plant breeding efforts to develop disease-resistant tomato varieties.

An acetyltransferase effector HopZ5 activates RPM1 (Resistance to Pseudomonas syringae pv. maculicola 1)-mediated defence responses in Arabidopsis thaliana.

S. CHOI (1), M. Prokchorchik (1), J. Jayaraman (2), K. H. Sohn (3), (1) POSTECH, POHANG, Korea, Republic of (South); (2) The New Zealand Institute for Plant and Food Research Ltd, Auckland, New Zealand; (3) Pohang University of Science and Technology, Pohang, Korea, Republic of (South)

Plants resistance $(\mathrm{R})$ proteins have evolved to recognize corresponding pathogen effectors and activate effector-triggered immunity (ETI). Plant innate immunity is highly dependent on repertoire of $R$ genes, therefore, it is not surprising that, in some cases, an $\mathrm{R}$ protein can recognize multiple effectors from distinct pathogens with diverse biochemical activities. However, detailed mechanism of how an R protein can differentially recognize biochemically unrelated effectors is unclear. RPM1 (Resistance to Pseudomonas syringae pv. maculicola 1) -mediated 
defence responses are known to be activated by two different effectors from Pseudomonas, AvrRpm1 and AvrB. Both AvrRpm1 and AvrB activate RPM1 by inducing RIPK-dependent phosphorylation of a gaurdee protein, RIN4 (RPM1-interacting protein 4. Here, we found that an acetyltransferase effector HopZ5 from a kiwifruit pathogen Pseudomonas syringae pv. actinidiae can trigger RPM1-mediated defence responses also in RIN4-dependent manner. We hypothesize that HopZ5 acetylates RIN4 and activate RPM1-dependent signaling pathway in Arabidopsis.

Molecular basis of effector recognition by independently evolved NLRs in distantly related plant species M. PROKCHORCHIK (1), S. Choi (2), E. H. Chung (3), K. Won (4), J. L. Dangl (5), K. H. Sohn (1), (1) Pohang University of Science and Technology, Pohang, Korea, Republic of (South); (2) POSTECH, POHANG, Korea, Republic of (South); (3) University of North Carolina, Chapel Hill, NC, U.S.A.; (4) National Institute of Horticultural and Herbal Science, Naju, Korea, Republic of (South); (5) Department of Biology, University of North Carolina at Chapel Hill, Chapel Hill, NC, U.S.A.

Plant pathogenic bacteria secrete effector proteins to the plant cell to promote virulence. On the other hand, plants developed mechanisms to recognize these effectors and respond with elevated immunity often concluding into hypersensitive response (HR). Effector recognition is often mediated by the intercellular NOD-like receptors (NLRs) directly by binding effectors or indirectly by detecting effector-mediated modifications on plant 'guardee' proteins. RIN4 protein (RPM1-interacting protein 4) is conserved in a wide range of plant species and targeted by several bacterial effector proteins including a cysteine protease AvrRpt2. AvrRpt2 homologs from P. syringae and E. amylovora are recognized in Arabidopsis and apple by RPS2 and MR5 NLRs respectively. In Arabidopsis rin4 knock-out plants, RPS2 leads to autoimmunity and consequent plant lethality. Interestingly RPS2 and MR5 share very little protein sequence homology implying they adapted convergently to recognize the same bacterial effector. We found that apple RIN4 (MdRIN4) is specifically required for MR5-mediated AvrRpt2 recognition. We further found that MdRIN4 cleavage generating a C-terminal cleavage product is essential for MR5-activation. Finally, we identified two polymorphic residues in AtRIN4 and MdRIN4 that are crucial in the regulation of and physical association with NLRs. We propose that diversification of RIN4 allows it to remain an effector target while maintaining compatibility with multiple NLRs.

Lineage-specific Pepper NRCs Have Roles in Recognizing of Multiple Effectors of Non-adapted Pathogen P. infestans S. OH (1), S. Kim (1), H. Park (1), D. Choi (2), (1) Plant Immunity Research Center, Seoul National University, Seoul, Korea, Republic of (South); (2) Plant immunity research center, Seoul National University, Seoul, Korea, Republic of (South)

NHR (Non-host resistance) is a robust form of plant immune response associated with complex molecular mechanisms including ETI (effectortriggered immunity). In a recent report, multiple recognition of Phytophthora infestans effectors is suggested as a molecular mechanism of NHR in pepper (Lee et al., 2014). To identify pepper NLRs which trigger ETI by recognizing $P$. infestans effectors, we selected three among fiftyseven $P$. infestans core effectors from HR (hypersensitive response) phenotype screening on pepper and co-expressed with 415 pepper NLRs in Nicotiana benthamiana. But none of the pepper NLRs induced HR when co-expressed with candidate effectors in 1:1 manner. In this context, to investigate whether additional factors could be required for effector-triggered HR on pepper, we characterized fourteen NRC (NLR-required for cell death)-type helper NLRs of pepper from phylogenetic analysis with a whole set of NLRs of Solanaceae plants, and tested whether these NRC candidates of pepper could function downstream of recognizing $P$. infestans effectors. As a result, we confirmed that pepper-specifically expanded group of NRCs are function downstream of several known R/Avr gene pairs including Avr-blb2 and Rpi-blb2. These results indicate that complex NLR network of pepper underlies NHR by recognizing effectors of non-adapted pathogen, $P$. infestans.

Global identification and transcriptional co-regulation analysis of receptor-like protein genes in pepper W. H. KANG (1), N. Koo (2), Y. M. Kim (2), S. I. Yeom (1), (1) Gyeongsang National Univeristy, Jinju, Korea, Republic of (South); (2) Korea Research Institute of Bioscience and Biotechnology, Daejeon, Korea, Republic of (South)

Receptor-like proteins (RLPs) are cell surface receptors, and it is well known to be involved in plant development and defense response. Despite this, there have been limited reports on genome-wide identification and comprehensive evolutionary study of the $R L P$ gene family in plants. To study the characterization of RLPs in pepper, we identified and analyzed RLPs using pepper genome and transcriptome. A total of 438 RLP genes were identified in the pepper genome. CaRLPs were divided into 11 subgroups showing differential expansion. Phylogenetic comparison with Arabidopsis RLPs revealed that pepper and Arabidopsis RLP families evolved independently. Furthermore, transcriptome of RLP genes showed dynamics of global gene expression changes during either plant growth stages or pepper-pathogen interactions. We constructed multidimensional co-expression network for predicting RLP gene functions by functional modules. Several pathogen-responsive regulatory modules of CaRLPs were identified through integrating the co-expression network and function enrichment tools, which covered variable function such as immune responses, metabolism, transcriptional regulation. Taken together, our results would be useful keys for functional analysis of RLPs in pepper.

\section{OsAPXs regulate the response to Xanthamonas (Xoo) infection in rice}

Yanwen Wang, Joanne Teo, Dongsheng Tian and Zhongchao Yin. Temasek Life Sciences Laboratory, 1 Research Link, National University of Singapore, 117604, Singapore

Bacterial blight caused by Xanthomonas oryzae pv. oryzae (Xoo), is one of the most destructive diseases in rice plantation worldwide. Plant generates reactive oxygen species (ROS) during plant immune response against pathogen infection. However, excessive ROS may cause significant cellular damage. Thus, regulating ROS at a proper level is significant important for plant. Ascorbate peroxidase (APX) is a key enzyme regulating ROS levels acting in different subcellular compartments. Rice has eight $A P X$ genes. To reveal the biological functions of these $A P X$ genes involved in ROS regulation during Xoo infection, we have successfully created APX mutants by using CRISPR/Cas9 genome editing system and studied subcellular localization of OsAPX proteins. Using these gene-edited mutants, the biological functions of $A P X$ genes would be investigated by Xoo infection. Furthermore, the $\mathrm{H}_{2} \mathrm{O}_{2}$ content in those mutants would be measured to understand their roles in ROS regulation. 
A Rice OsAGO18-interacted Helicase OsHRQ1 Promotes Virus Infection In Plants

K. XU, Peking University, Beijing, China

RNA silencing is a vital antiviral mechanism in plants, but was not well studied in monocotyledon grain crops, like rice. OsAGO18 is the first identified Argonaute protein which plays a crucial role in rice antiviral resistance. Previous studies show that OsAGO18 is induced after Rice stripe virus (RSV) infection and competes with OsAGO1 for the binding of miR168, resulting in the release of OsAGO1 and enhanced rice antiviral immunity. We found that DEAD-box helicase OsHRQ1 associated with OsAGO18 directly in nuclei. We firstly explored the function and characteristics of its homolog in Arabidopsis, AtHRQ1. Compared with Col-0, Athrq1 plants were more efficient in reproductive growth, earlier bolting time, longer flower stems and larger capsules. Virus infection assay using Cucumber mosaic virus (CMV) showed that CMV RNAs appeared later in new leaves of Athrql than Col-0. Athrql developed larger rosette leaves and stronger flower stem than Col-0 after CMV infection. Interestingly, more CMV RNAs were accumulated in Athrq1 than Col-0 two weeks postinfection. We also find that Oshrq1 plants had more branches and less effective seeds. Virus infection assay using RSV infection showed that Oshrql were more resistant and less virus accumulation than Nipponbare (NPB). On the contrary, OsHRQ1 over-expression transgenic rice showed a more susceptible phenotype and more virus accumulation than NPB. Taken together, these results suggest that HRQ1 promotes virus infection in plants.

The type III effectors of Ralstonia solanacearum interact with tobacco chloroplastic proteins K. OHNISHI, A. Dahal, L. Kharel, A. Kiba, Y. Hikichi, Kochi Univ, Japan

Ralstonia solanacearum type III effectors are known to cause disease in several host plants. A well-known family of type III effectors called RipG possesses a group of seven genes. RipG effectors contain an F-box domain and leucine-rich repeats (LRR). The F-box domain resembles eukaryotic F-box proteins, which in combination with SKP1 and Cullin1 forms SCF ubiquitin ligase complex and controls specific protein ubiquitinylation. RipG effectors interact with Skp1-like protein in Arabidopsis thaliana through their F-box domain and act by hijacking host SCF ubiquitin ligase complex. In this study, yeast two-hybrid screening of Nicotiana benthamiana and Nicotiana tabacum cDNA library was conducted to screen proteins that interact with the RipG effectors of $R$. solanacearum OE1-1 strain. In addition to the previously reported Skp1, several chloroplastic proteins were found to interact with the RipG effectors, especially RipG2 and RipG7. While Skp1 bound to RipGs through the F-box domain, these chloroplastic proteins did not bind to the F-box domain but bound to either LRR or the N-terminal region of RipGs. The simultaneous binding of RipG7 with Skp1 and chloroplast chaperonin-like RbcX was confirmed by yeast three-hybrid assay. From these results, we hypothesize that chloroplastic proteins could be the targets for ubiquitination via RipG effectors. The core effector RipAJ was also found to interact with several tobacco chloroplastic proteins.

The LRR-RLK REAL1 is a novel component of PRR complex that negatively regulates PAMP-triggered immunity in Arabidopsis Y. GOTO (1,2), Y. Kadota (1,3), H. Matsui (4), J. Sklenar (3), P. Derbyshire (3), F. L. H. Menke (3), H. Nakagami (5), C. Zipfel (3,6), K. Shirasu $(7,8)$, (1) RIKEN Center for Sustainable Resource Science, Plant Immunity Research Group, Yokohama, Japan; (2) The University of Tokyo, Hongo, Japan; (3) The Sainsbury Laboratory, University of East Anglia, Norwich Research Park, Norwich, U.K.; (4) Okayama University, Okayama, Japan; (5) Max Planck Institute for Plant Breeding Research, Germany; (6) Inst. of Plant and Microbial Biology, University of Zürich, Zurich, Switzerland; (7) RIKEN Center for Sustainable Resource Science, Yokohama, Japan; (8) The University of Tokyo, Tokyo, Japan

The production of reactive oxygen species (ROS) is a conserved signaling output in immunity across kingdoms. It has to be tightly controlled to avoid detrimental effects on host cells and to induce appropriate immune responses. In plants, the perception of pathogen-associated molecular patterns (PAMPs) by pattern recognition receptors (PRRs) induces PAMP-triggered immunity (PTI). Upon PRR activation, receptor-like cytoplasmic kinases, such as BIK1, directly phosphorylate and activate the NADPH oxidase RBOHD to activate ROS production. RBOHD exists in complexes with the leucine-rich repeat receptor kinases (LRR-RKs) EFR and FLS2, which are the PRRs for bacterial EF-Tu and flagellin, respectively. To understand the regulatory mechanism of PAMP-induced ROS production, we employed co-immunoprecipitation with EFR and RBOHD coupled with mass spectrometry analyses to identify novel components of PRR-RBOHD complex(es). We identified an LRR-RLK, which we named RBOHD/EFR-associated LRR-RLK1 (REAL1), as a novel component of PRR-RBOHD complex. Arabidopsis reall knockout mutant plants showed enhanced PTI outputs, such as ROS production, MAP kinase activation and disease resistance against bacterial pathogens. In contrast, REAL1 over-expressers showed reduced PTI. Notably, FLS2 protein levels were lowered in REAL1 over-expressers, suggesting that REAL1 negatively controls PRR protein levels at the plasma membrane. Possible roles of REAL1 in PTI and FLS2 protein control will be discussed.

Structural and Biochemical Study of DANGEROUS MIX pairs in Arabidopsis thaliana

N. CHAROENNIT (1), G. Kim (2), W. L. Wan (1), Y. Y. Liew (1), J. Wang (1), R. Lee (1), Y. Zhang (1), J. J. Song (2), E. Chae (1), (1) National University of Singapore, Singapore; (2) Korea Advanced Institute of Science and Technology, Daejeon, Korea, Republic of (South)

In the innate immune system in plants, nucleotide-binding domain leucine-rich repeat (NLR) receptors function to perceive non-self invasion and activate immune signaling. Reflecting co-evolutionary arms race, certain genomic loci encoding NLRs are highly variable in natural populations and carry potential risk of causing autoimmunity in hybrid plants. The Dangerous Mix (DM) interactions for autoimmunity involve NLRs from multi-gene clusters in Arabidopsis thaliana and provides the pair information for such NLRs. To elucidate molecular mechanisms underlying NLR activation, we exploit these NLR partnerships, DM1/DM2, DM2/DM3 and DM6/DM7 to structurally determine the modes of NLR activation. We established an in vivo and in vitro protein expression of such pair, consisting of an NLR and its partner, to characterize the complex and identify signaling partners. This study is likely to provide a structural insight on how NLR signaling complex assembles and activates immune signaling in plants. 
Effectors-assisted breeding approach for resistance to Ralstonia solanacearum in potato

B. KIM (1), H. Moon (2), K. S. Cho (3), K. H. Sohn (2), S. Cécile (1), (1) Seoul National University, Seoul, Korea, Republic of (South); (2) Pohang University of Science and Technology, Pohang, Korea, Republic of (South); (3) Highland Agriculture Research Institute, National Institute of Crop Science, Pyeongchang, Korea, Republic of (South)

Ralstonia solanacearum (Rso) is a soil-borne bacterium causing severe disease in Solanaceae crops, including potato. The most effective way to manage this disease is to breed resistant potato hybrids. Conventional approaches to identify genetic source of resistance are limited by environmental effect on disease development, host genome complexity, and diversity among Rso species complex. We are developing an effector-assisted breeding approach, using the hypersensitive response triggered upon recognition of pathogenic effector by resistance (R) protein as a proxy indicating disease resistance to enhance accuracy of phenotyping. First, we have selected and cloned 9 effectors from Rso isolates that cause disease on potato or wild relative species. Next, we tested three delivery systems to express or transfer Rso effector individually within the host cells: Agrobacterium tumefaciens-mediated transient transformation, secretion by non-pathogenic Pseudomonas fluorescens (Pf0-1), and Pseudomonas syringae pv. tomato DC3000 polymutant lacking all 36 type III secretion effectors (DC3000D36E). Our results indicate that we can use DC3000D36E as a delivery system in potato leaves. This method will contribute to identify Rso effectors that are recognized in potato or wild-relative species and to map $R$ genes in segregating populations.

Colonisation of Arabidopsis leaves by a Streptomyces sp. results in a protection against fungal diseases relying on plant immunity D. GAYRARD (1), S. Vergnes (2), M. Veyssière (2), J. Toulotte (2), Y. Martinez (2), V. Dumont (3), O. Bouchez (4), T. Rey (5), B. Dumas (2), (1) CNRS / De Sangosse, Castanet-Tolosan, France; (2) CNRS, Castanet-Tolosan, France; (3) CRITT BIO-INDUSTRIES, Toulouse, France; (4) Genotoul Genome \& Transcriptome, INRA, Toulouse, France; (5) De Sangosse, Pont-du-Casse, France

A Streptomyces strain was isolated from vine rhizosphere and screened among other soil bacteria for secreting potent activators of SA signaling. Multilocus Sequence Typing coupled to morphological characteristics assigned it to the S. violaceusniger clade. Microscopic examination of treated plant tissues revealed that the strain is able to transiently colonise leaf surface. Arabidopsis thaliana plants treated with the bacterial suspensions or culture medium showed intense defence responses such as SID2 dependent necrotic lesions. These treatments resulted in significant reduction of symptoms when plants were challenged with fungal pathogens. Arabidopsis transcriptome following treatment with bacteria culture medium was then examined by RNA Sequencing. Multiple markers of salicylic acid and jasmonate/ethylene as well as secondary metabolism pathways such as camalexin were upregulated by the treatment. Partial protection of sid2, jar1 and ein 2 immune-deficient Arabidopsis mutants inoculated with A. brassicicola conidies suggests that defence responses are responsible for plant protection. Together, this work shows that Streptomyces bacteria produce compounds harbouring strong elicitor activity and could be used as foliar treatment to efficiently protect plants against pathogen attacks.

Wheat pattern-triggered immunity against bacteria

S. REN, Y. Qin, C. Pedersen, H. Thordal-Christensen, University of Copenhagen, Frederiksberg C, Denmark

Bacterial pathogens cause serious diseases on plants that use plasma membrane receptor-like kinases (RLKs) to recognise conserved pathogen molecules, so-called pathogen-associated molecular patterns (PAMPs). FLS2 and EFR are examples of such receptors, which recognize the abundant bacterial PAMP proteins, flagellin and elongation factor Tu (EF-Tu). Upon binding of these molecule, FLS2 and EFR dimerize with the same co-receptor-like kinase (co-RLK), BAK1, which activates cross-phosphorylation of the kinases and activation of pattern-triggered immunity (PTI). We recently discovered a novel PAMP, potentially broadly present in pathogenic bacteria, to be recognised by 144 of the 440 wheat lines in the Wagtail population, and by the help of a genome-wide association study (GWAS) and subsequent fine-mapping using a MAGIC population, a narrow genome interval of the wheat genome was found to be responsible for the recognition of the novel PAMP. In the interval, a gene encoding a co-RLK has been identified, which has a two amino-acid difference co-segregating with the 144 sensitive vs. the 296 insensitive lines. Knocking out of the presumed active wheat allele is ongoing, aiming at confirming the involvement of the candidate receptor in the recognition of the novel PAMP. Precise identification of the PAMP is in progress involved tests of a number of candidate proteins.

Investigation of the physical interaction of Arabidopsis thaliana CERK1 and Pathogen and Circadian Controlled 1 (PCC1) B. SABELLECK, R. Panstruga, RWTH Aachen University, Aachen, Germany

MAMP-triggered immunity relies on the perception of microbe-associated molecular patterns (MAMP) by receptor-like kinases (RLKs) to detect microbes that can act as symbionts or pathogens. RLKs bind MAMPs on the cell surface and transmit the signal intracellularly by phosphorylation reactions. In the past, an increasing number of proteins, participating in different signaling networks, were identified to participate in protein-protein interactions with RLKs and were extensively characterized, but the interacting protein network is presumably not complete. In Arabidopsis thaliana, the chitin elicitor receptor kinase 1 (CERK1) is involved in the perception of the fungal cell wall component chitin and the bacterial cell wall unit peptidoglycan. We identified potential interaction partners of CERK1 in a split-ubiquitin yeast two-hybrid cDNA library screen and validated them in planta by split-luciferase assays. One candidate, Pathogen and Circadian Controlled 1 (PCC1), belongs to a small protein family involved in biotic and abiotic stress responses. We tested truncated versions of PCC1, together with other PCC1 family members, for association with CERK1 and additional RLKs to explore the molecular basis and specificity of this interaction. The cellular functions of PCC1 and the biological significance of the PCC1-CERK1 interaction need to be further investigated. Our analysis improves the understanding of the protein-protein interaction network in plant immunity.

Investigating the mechanistic differences underlying the divergent immune response readouts of the FLAGELLIN SENSING 2 and FLAGELLIN SENSING 3 receptors in tomato

R. ROBERTS (1), G. B. Martin (1,2), (1) Boyce Thompson Institute, Ithaca, NY, U.S.A.; (2) School of Integrative Plant Science, Cornell University, Ithaca, NY, U.S.A. 
Plant pattern recognition receptors (PRRs) recognize a variety of conserved, diverse pathogen-associated molecular patterns to effectively activate basal immune responses and repel most potential pathogens. Two PRRs, FLAGELLIN SENSING 2 (FLS2) and FLAGELLIN SENSING 3 (FLS3), both recognize bacterial flagellin (epitopes flg22 and flgII-28, respectively), although their output differs: activation of FLS3, for example, results in ROS production over a much longer time period than does FLS2 activation. To investigate possible mechanistic differences between these two PRRs, we generated chimeric constructs by swapping the leucine rich repeat (LRR), transmembrane (TM), and kinase (KD) domains between FLS2 and FLS3 and tested their functions using ROS assays. We found that the LRR domain was sufficient for the specific recognition of flg22 or flgII-28. However, to activate a ROS response, there was a requirement that the TM and KD domains originate from the same PRR. Both tomato FLS2 and FLS3 have strong kinase activity in an in vitro assay, and this activity requires the presence of the cognate inner juxtamembrane domain. We are currently making mutations in conserved motifs to investigate whether there are mechanistic differences in kinase activation between the two PRRs. Collectively, our data indicate that FLS2 and FLS3, though they both recognize flagellin-derived PAMPs, have unique mechanisms underlying their signaling activities.

A conserved peptide pattern from fungal endopolygalacturonase triggers plant immunity in Arabidopsis

L. ZHANG, T. Nuernberger, Centre for Plant Molecular Biology, Eberhard Karls University of Tübingen, Tübingen, Germany Plant plasma membrane pattern recognition receptors are key to microbe sensing and activation of immunity to microbial invasion. The Arabidopsis leucine-rich repeat receptor-like protein (LRR-RP) RLP42 (also known as Responsiveness to Botrytis PolyGalacturonase 1, RBPG1) mediates plant immunity by perceiving fungal endopolygalacturonases (PGs). RLP42 recognizes several PGs from the plant pathogen Botrytis cinerea and from the saprotroph Aspergillus niger. In this study, we showed that RLP42 recognizes a conserved 9-amino-acid peptide from fungal PGs (pg9), but not from plant PGs, thereby triggering plant immune responses, including ROS burst, MAPK activation, ethylene production, and defense-related gene expression. Mutagenesis analysis of pg9 revealed that 3 residues conversed among fungal PGs (including an aspartic acid residue required for enzymatic activity) are crucial for RLP42 recognition. RLP42 forms a ligand-independent complex with LRR receptor kinase (LRR-RK) SOBIR1 (Suppressor of Brassinosteroid insensitive 1 (BRI1)-associated kinase (BAK1)-interacting receptor kinase 1). In addition, we show that RLP42 recruits LRR-RKs SERK proteins into a tripartite complex upon ligand perception. Expression of RLP42 in Arabidopsis $\mathrm{Br}-0$ (an accession that is non-responsive to fungal PGs) confers pg9 pattern recognition and enhances immunity against bacterial pathogen Pseudomonas syringae pv. tomato after pg9-priming.

Arabidopsis PAD4 lipase-like domain is sufficient for limiting green peach aphid infestation

J. A. DONGUS (1), L. Dijkgraaf (1), M. Patel (2), D. D. Bhandari (1), J. Shah (3), J. E. Parker (1), (1) Max Planck Institute for Plant Breeding Research, Cologne, Germany; (2) University of North Texas, Denton, TX, Lewisville, TX, U.S.A.; (3) University of North Texas, Denton, TX, U.S.A.

PHYTOALEXIN DEFICIENT4 (PAD4) is a central regulator of plant anti-microbial and anti-aphid responses. As a heterodimer with EDS1, PAD4 promotes basal and effector triggered immunity (ETI), thereby limiting (hemi)-biotrophic pathogen growth. Independently of EDS1, PAD4 limits aphid infestation. The molecular function(s) of PAD4 in these two plant responses remains elusive. PAD4 consists of an N-terminal lipase-like domain and a unique C-terminal EDS1-PAD4 (EP) domain. To gain an insight to the molecular function of PAD4, we investigated whether PAD4 has domain-specific activities. Using information from a PAD4 protein structure model we generated Arabidopsis plants expressing solely the PAD4 lipase-like domain. We found that the Arabidopsis PAD4 lipase-like domain, without its EP domain, is sufficient to limit infestation of the green peach aphid (Myzus persicae). In contrast, the PAD4 lipase-like domain is insufficient for triggering ETI and, without its EP domain, was unable to stabley bind EDS1. These results show that PAD4 has domain-specific functions and could function as a bipartite protein to defend plants against different stresses.

Connecting structure to function: How NRG1 helps signal in immunity

J. M. FEEHAN (1), A. R. Bentham (2), H. K. Ahn (1), B. Castel (1), M. J. Banfield (2), J. D. Jones (1), (1) The Sainsbury Laboratory, University of East Anglia, Norwich Research Park, Norwich, U.K.; (2) John Innes Centre, Norwich Research Park, Norwich, U.K.

Plants encode intracellular immune receptors to aid in the detection and defense against pathogen threats. These so-called NBD-LRR (NLR) receptor proteins may directly bind pathogen molecules, as sensor NLRs, or act as a coreceptors in immune networks as helper NLRs. NRG1 is broadly required as the helper NLR to a distinct class of sensor NLRs with amino-terminal TIR (toll/interleukin1 receptor/resistance protein)domains. Enhanced disease susceptibility 1 (EDS1), a non-NLR immune protein, is also required by TIR-NLRs for immune activation and we have some evidence that it directly interacts with NRG1 in an immune activation-dependent way. The mechanisms by which NLRs mediate immune responses are not well understood, further complicated by our limited understanding of their structures. We want to determine the structure of the helper NLR, N-requirement gene 1 (NRG1) in an effort to better elucidate its role in immune signaling networks. Here, we produce high amounts of very pure NRG1 protein by Agro-infiltration and transient expression in N. benthamiana for structural investigations. Determination of NRG1 structure will guide new investigations for functional studies, help resolve the specificities of the NRG1/EDS1 interaction and supply a model for future NLR structural investigations. Our studies utilizing a novel expression technique will greatly contribute to our understanding of how these receptors function in immunity.

1001 Ways of detecting Danger-Activation of a secreted Decoy Signal by a bacterial Protease L. P. MAIER (1), V. Fallahzadeh-Mamaghani (2), A. Schaller (3), G. Felix (1), (1) Center for Plant Molecular Biology (ZMBP), Tübingen University, Germany; (2) Plant Protection Department, Azarbaijan Shahid Madani University, Azerbaijan; (3) Institute of Plant Physiology and Biotechnology, Hohenheim University, Germany

In order to mount active defense responses against potentially harmful microorganisms, plants employ plasma membrane-localized patternrecognition receptors (PRRs), which perceive the presence of microbes through danger signals present in the apoplast. These signals are either derived from the microbes themselves or are released by action of the pathogen from the plant tissue. We identified a conserved protease that is secreted by pathogenic Xanthomonas bacteria and elicits typical immune responses in non-host plant Arabidopsis thaliana. Biochemical evidence indicates an apoplastic plant protein, which, when digested by the Xanthomonas protease, is converted into an active peptide signal that gets 
perceived by the plant. Our current work aims at identification of this plant protein and its active epitope. Taking advantage of natural variation in Arabidopsis thaliana we are also aiming towards the identification of the corresponding PRR. Orthologs of the protease have been implicated in the extracellular activation of bacterial effector proteins. Thus, we propose a model, in which a secreted plant protein acts as a decoy to detect activity of the bacterial protease as a proxy for the presence of Xanthomonas bacteria.

\section{An over-excited FLS2}

Y. WANG (1), D. Chinchilla (2), P. Bittel (3), A. Jehle (1), K. Mueller (1), G. Felix (4), (1) Center for Plant Molecular Biology (ZMBP), Tuebingen University, Germany; (2) University of Freiburg, Switzerland; (3) University of Basel, Switzerland; (4) Center for Plant Molecular Biology (ZMBP), Tübingen University, Germany

The flagellin receptor FLS2 is one of the best studied LRR (Leucine-Rich-Repeat) receptors in plants. While At-FLS2 from Arabidopsis and S1FLS2 from tomato both recognize the flagellin epitope flg22, they show characteristic differences with respect to the affinity and specificity for flg22-variants. To map these differences on the LRR binding domain we constructed chimeric receptors with swaps of various parts of their LRR domains (Mueller et al. 2012). Here, we report on an additional chimeric construct with the LRRs 15-24 of At-FLS2 replaced by the respective LRRs of S1-FLS2. This hybrid exhibits all the characteristics of a constitutively active receptor when expressed in Arabidopsis. None of the other replacements, including the ones with the LRRs 19-24 or 11-24, respectively, show activation in the absence of ligand, indicating that the activated state depends on the particular combination of LRRs from S1-FLS2 embedded in the LRRs of Arabidopsis. More interestingly, ligandindependent activation did not occur in the absence of functional AtBAK1/SERK3, indicating that BAK1/SERK3 from Arabidopsis plays a crucial role for the activated state of this receptor. Currently, we are trying to narrow down the LRR-regions of At-FLS2 and S1-FLS2 that cause the autoimmune. The self-activating hybrid provides a model case for studying how FLS2 interacts with its co-receptor BAK1/SERK3 and triggers downstream signaling.

Structure-function analysis of ZAR1 immune receptor reveals key molecular interactions for activity M. BAUDIN (1), K. J. Schreiber (2), E. C. Martin (3), A. J. Petrescu (4), J. D. Lewis (1), (1) University of California, Berkeley, Albany, CA, U.S.A.; (2) University of California, Berkeley, Berkeley, CA, U.S.A.; (3) Institute of Biochemistry of the Romanian Academy,, Bucharest, Romania; (4) Institute of Biochemistry of the Romanian Academy, Bucharest, Romania

Pathogenic bacteria such as Pseudomonas syringae can inject effector proteins into host cells to promote disease. Plants have evolved genes encoding NLRs (Nucleotide-binding [NB] Leucine-rich repeat [LRR] Receptor) that can detect the activity or presence of the pathogenic effector and induce strong immune responses that can lead to the hypersensitive response. Therefore, NLRs must be tightly regulated to prevent inappropropriate activation in the absence of a pathogen. The ZAR1 NLR recognizes diverse effector proteins from $P$. syringae, including HopZ1a, and Xanthomonas species. Specificity for different effector proteins, such as HopZ1a, is determined by receptor-like cytoplasmic kinases such as ZED1, which interact with ZAR1. We previously developed a transient expression system in Nicotiana benthamiana, that allowed us to demonstrate ZAR1 function is conserved from brassica species to solanaceous species. Here, we combined structural modeling of ZAR1, with molecular and functional assays in our transient system, to show that multiple intramolecular and intermolecular interactions regulate ZAR1 activity. We found that the coiled-coil (CC) domain dimerized in a MLA10-like fashion, and induced immunity. The CC and LRR interact with the central NB domain of ZAR1. Finally, we took advantage of multiple loss-of-function mutations in ZAR1 to evaluate their impact on intramolecular interactions. This work identifies molecular constraints on immune receptor function and activation.

Roles of the protein domains of the B-lectin S-Domain receptor kinase LORE in oligomerization and immune signaling S. ESCHRIG, M. Schäffer, S. Ranf, Technical University of Munich, Freising, Germany

Plants employ a complex, multi-layered innate immune system to detect and fight off invading pathogens. Sensing of highly conserved microbeassociated molecular patterns (MAMPs) by pattern recognition receptors (PRRs) at the plant plasma membrane activates pattern-triggered immunity (PTI) and confers resistance to a broad spectrum of pathogens. We identified the B-type lectin S-domain receptor kinase LORE (LIPOOLIGOSACCHARIDE-SPECIFIC REDUCED ELICITATION or SD1-29) (Ranf et al. 2015) as a PRR which senses 3-hydroxylated fatty acid (3-OH-FA) metabolites in Brassicaceae. The ectodomain of the S-locus receptor kinase 9 (SKR9) was shown to form homodimers in a ligand- and receptor-mediated manner (Ma et al. 2016). Structural modelling suggests a similar structure of the LORE ectodomain and accordingly a similar complex formation may be assumed. Hence, we are currently investigating homo-dimerization of LORE by different methods and its role in signaling activation upon 3-OH-FA sensing. Further work aims to analyse the contribution of the different LORE protein domains to homo-dimerization and regulation of LORE signaling. Cross-family transfer of PRRs and engineering of PRR signaling in crops are promising tools for development of sustainable disease management strategies. Therefore, we are currently generating LORE-transgenic tomato to investigate whether 3-OH-FA sensing can be transferred to a crop plant.

Blast resistance gene Pita-2 is allelic to Pi19 but not to Pita

A. TAKAHASHI, N. Hayashi, Institute of Agrobiological Sciences, NARO, Tsukuba, Ibaraki, Japan

Several rice blast resistance genes, including Pita, Pita-2 and Pi19, to M. oryzae have been mapped on the short arm of chromosome 12. Among them, Pita gene has been isolated, however other genes have not been, yet. We screened Nipponbare mutants which showed loss of Pi19-

dependent resistance against a rice blast isolate containing AvrPi19 from about 10,000 lines and identified two independent mutants. Map-based cloning and complementation analysis revealed that an amino-acid substitution or deletion mutation of Pi19 caused the loss-of-function phenotypes in these mutants. Isolation of Pi19 revealed that it was not allelic gene to Pita. Previously, it was reported Pi19 was allelic or closely linked to another resistance gene Pita-2. The deduced amino acid sequences encoding in Pil19 locus of Pi No.4 which has Pita-2 is highly similar and the identity to that of Nipponbare is $98 \%$. These data raised a possibility that Pi19 is allelic to Pita-2. To confirm this hypothesis, we analyzed the transgenic lines which contained the sequence of Pi19 locus of Pi No.4 including promoter region. The complementation analysis 
indicated that Pi19 locus of Pi No.4 encodes Pita-2. Pi19 and Pita-2 encode non-NBS-LRR type proteins and the polymorphisms in these proteins are only detected at their C-terminal end. This is the first report of making possible to distinguish Pita from Pita-2 and Pi19 genes.

Investigation of Regulating Mechanism of the Novel Susceptibility Factor RTP1 in Plant Resistance to Phytophthora

X. Qiang (1), X. LIU (1), Q. Zheng (2), L. Kang (1), H. Zhao (2), W. X. Shan (1), (1) College of Agronomy, Northwest A\&F University, Yangling, China; (2) College of Plant Protection, Northwest A\&F University, Yangling, China

Oomycetes, particularly Phytophthora species, cause serious crop diseases, such as potato late blight, and threaten the sustainable crop production worldwide. Due to the prominent problem of virulence variation and loss of genotype-specific resistance, understanding and utilization of novel factors that confer broad-spectrum and durable resistance have been a central focus in the field of plant-oomycete interaction. Based on the compatible interaction between Phytophthora parasitica, a model oomycete pathogen, and the model plant Arabidopsis thaliana, we have identified a novel susceptibility factor, namely RTP1 (Resistance to P. parasitica 1), which encodes an endoplasmic reticulum (ER) localized protein. The loss-of-function $r t p 1$ mutant showed resistance against $P$. parasitica and several other biotrophic pathogens. Based on recent genetic, molecular, biochemical and cytological studies, we speculate that RTP1 might play an important role in sensing ER stress and regulating ER stress-mediated plant immunity upon infection by Phytophthora. We will summarize and discuss the data that address this hypothesis, which is expected to facilitate development of novel strategies for the utilization and molecular design of genetic resources required for crop disease resistance breeding.

Characterization of autoimmunity triggered by DANGEROUS MIX pairs in Arabidopsis thaliana W. L. WAN, Y. Y. Liew, N. Charoennit, J. Wang, R. Lee, Y. Zhang, E. Chae, National University of Singapore, Singapore

The plant innate immune system employs receptors and other proteins to build up complex signaling networks. Numerous nucleotide-binding and leucine-rich repeat domain (NLR) proteins function as intracellular receptors that can recognize molecular signatures of pathogenic threats and activate immune responses in plants. Hybrid necrosis studies have shown that unique allelic interactions between NLRs or between NLRs and other proteins can trigger autoimmunity in multiple plant species. We take such Dangerous Mix (DM) pairs in A. thaliana to investigate the molecular mechanism underlying NLR receptor activation and signaling. Our primary focus lies on two NLR and non-NLR pairs, DM2h (RPP1) /DM3(alpha/beta hydrolase) and DM6 (RPP7)/DM7 (RPW8/HR), as to explore how the natural variants of these host factors are being recognized as equivalent to non-self. Proximity dependent biotin identification (BioID) has emerged as a powerful tool for studies of proteomics, therefore we established BioID2 system, which is based on a substantially smaller promiscuous biotin ligase, in A. thaliana for identifying interacting partners of DM pairs of which autoimmunity is engineered to be inducible. In our BioID2 DM pair system, the necrosis causing pairs and normal pairs will be compared to identify specific factors tethered to active signaling NLR complexes in vivo.

-coil domain of a subgroup of plant NLR immune receptor induces cell death

H. Y. LEE (1), E. Choi (2), S. B. Kim (3), Y. E. Seo (4), H. E. Kim (4), E. Park (5), H. G. Mang (1), D. Choi (1,4), (1) Plant immunity research center, Seoul National University, Seoul, Korea, Republic of (South); (2) LG Chem, Seoul, Korea, Republic of (South); (3) North Carolina State University, Raleigh, NC, U.S.A.; (4) Department of Plant Science, Seoul National University, Seoul, Korea, Republic of (South); (5) Department of Molecular Biology, University of Wyoming, Laramie, WY, U.S.A.

Plants possess hundreds of intracellular immune receptors encoding nucleotide-binding domain and leucine-rich repeat (NLR) proteins that can recognize pathogen effectors. After infection, activated NLRs confer disease resistance that is often accompanied by localized cell death termed by hypersensitive response. N-terminal domains (NTDs) of NLRs are known to function as a signal inducer and remain in an auto-inhibited state through intramolecular interaction with other domains in absence of pathogen infection. In this study, cell death-inducing activity of pepper fulllength NLRs and their NTDs was analyzed by transient overexpression in Nicotiana benthamiana. The screening assay revealed that the NTDs, not full length NLRs, of specific group-NLRs trigger cell death. These NTDs-mediated cell deaths appear to mimic the HR cell death triggered by activation of NLR protein, as evident by upregulated defense-related genes and requirement of molecular chaperone complex for resistance. Here, we will discuss the detailed molecular mechanisms of NTDs-mediated cell death as well as the distinct function of the specific group of NLRs in terms of plant immunity.

Functional analysis of RIN4 natural variants reveals the essential properties for compatibility with NLRs H. Kim, M. Prokchorchik, K. H. SOHN, Pohang University of Science and Technology, Pohang, Korea, Republic of (South)

RIN4 (RPM1-interacting protein 4) is targeted by multiple bacterial type III effectors. In some cases, effector-mediated modifications of RIN4 is monitored by NLR (nucleotide-binding and leucine repeat R) proteins and activate plant immunity. For instance, Pseudomonas syringae effectors AvrRpt2 or AvrRpm1 cleaves or induces phosphorylation of RIN4 that triggers RPS2 or RPM1 dependent immunity in Arabidopsis. Do RIN4 natural variants that are present in diverse plant species carry similar function? In order to address this question, we have analyzed multiple RIN4 natural variants that have not been characterized previously for their ability to regulate several known NLRs that monitor effector-driven RIN4 modifications. Our results indicate that RIN4 is a highly adaptive protein that has ability to be compatible with multiple NLRs from distantly related plant species.

Identification and analysis of RTP5, a susceptibility factor of Arabidopsis thaliana to Phytophthora W. LI (1), W. X. Shan (2), (1) College of Plant Protection, Northwest A \& F University, Yangling, China; (2) College of Agronomy, Northwest A\&F University, Yangling, China

Oomycetes include many plant pathogens that cause disastrous diseases of many ornamental and food crops. Phytophthora pathogens deliver highly polymorphic effectors to facilitate successful infection. While plant disease resistance mediated by recognition of pathogen effectors is 
well studied, little is known about how plant susceptibility is established. Here we show identification of a susceptibility factor of Arabidopsis thaliana-RTP5, which is obtained by screening T-DNA insertion mutants for enhanced resistance against $P$. parasitica. Leaf and root inoculations consistently showed that RTP5 acts as a negative regulator of plant immunity. Molecular and genomic analyses suggest that $R T P 5$ mediates plant immunity by being involved in regulating plant hormone signaling pathways, which play vital roles both in growth and in plant immunity.

Screening wild tomato germplasm for resistance to race 2 of Verticillium dahliae

J. VERMEULEN, K. Hanika, B. P. H. J. Thomma, H. Schouten, Y. Bai, Wageningen University, Wageningen, Netherlands

Verticillium dahliae is a fungal pathogen that causes vascular wilt disease on a wide range of crop species. Most fungicides are ineffective once $V$. dahliae has entered the plant. Host resistance therefore plays an important role in the management of $V$. dahliae. Currently, only one resistance gene against $V$. dahliae, $V e 1$, has been identified. As race 2 strains of $V$. dahliae are not recognized by Ve1, additional resistance sources are required to control these strains. We have screened a wide collection of tomato germplasm for enhanced resistance to race 2 strains and identified several promising accessions. We further assessed the broadness of the resistance spectrum of these accessions using a phylogenetically diverse set of $V$. dahliae strains. The accessions showed reduced Verticillium wilt symptoms upon inoculation with most of the race 2 strains. Intriguingly, some of the accessions did show not a clear reduction in symptoms upon inoculation with most race 1 strains. To further characterize the resistance of these accessions, segregating recombinant inbred line populations have been developed. These populations are currently being screened to identify the chromosomal regions and the underlying genes associated with race 2 resistance.

Exploring the Role of RanGAP2 in Recognition by the Potato CC-NB-LRR Immune Receptors Rx1 and Gpa2

O. SUKARTA (1), A. Diaz Granados (2), E. Slootweg (1), J. Bakker (1), G. Smant (1), A. Goverse (1), (1) Laboratory of Nematology, Wageningen University and Research, Wageningen, Netherlands; (2) Wageningen University, Wageningen, Netherlands

The Ran GTPase activating protein 2 (RanGAP2) is a co-factor of the potato CC-NB-LRR immune receptor Rx1. However, its role in defence remains to be fully elucidated. Artificial tethering of RanGAP2 to the nematode effector GpRbp-1 was shown to enhance the cell death response of Gpa2, a closely related immune receptor of Rx1 that also interacts with RanGAP2 (Sacco et al. 2009). This suggests that RanGAP2 may contribute to immunity by facilitating effector recognition. Here, we expand on this model using a combination of structure-informed approaches, including co-immunoprecipitation and cellular imaging studies. We show that RanGAP2 can in fact form complexes in planta with the cognate effectors of Rx1 and Gpa2, namely the PVX coat protein (CP) and GpRbp-1. Interestingly, this was noted for both the eliciting and non-eliciting effector variants, suggesting that the RanGAP2/effector interaction may not be sufficient to confer recognition. This is in line with existing data demonstrating that the C-terminal region of the Rx1 and Gpa2 LRR domains is required for successful pathogen perception (Slootweg et al 2013). Currently, we are performing structural and biochemical analysis to discern whether RanGAP2 acts as a bait that facilitates specific effector recognition either by bridging or enhancing the effector/cognate LRR interaction. This model may explain the bifurcation of pathogen recognition specificities of these two closely related immune receptors during evolution.

Investigating the function of the tomato receptor-like cytoplasmic kinase BSK830 in PTI signaling G. SOBOL (1), B. B. Majhi (1), N. Zhang (2), H. M. Roberts (2), G. B. Martin (2), G. Sessa (1), (1) Tel Aviv University, Tel Aviv, Israel; (2) Boyce Thompson Institute, Ithaca, NY, U.S.A.

Arabidopsis brassinosteroid signaling kinases (BSKs) are receptor-like cytoplasmic kinases (RLCKs) with an established function in both growth and immunity. To investigate a possible role for the tomato BSK830 in pattern-triggered immunity (PTI), we analyzed its physical association with pattern recognition receptors (PRRs). BSK830 interacted in yeast with the FLS2, FLS3, and Bti9 PRRs. Split luciferase complementation assays validated these interactions in planta and revealed that plant treatment with the PAMP flgII-28 reduce the BSK830-FLS3 interaction. Consistent with a function in PTI, preliminary analysis of CRISPR/Cas9-generated $b s k 830$ mutant plants revealed a lower production of reactive oxygen species, but unaltered MAPK activation, upon treatment with the flg22 and flgII-28 PAMPs. Moreover, bsk830 mutant plants displayed enhanced susceptibility to Botrytis cinerea. Finally, to start investigating whether BSK 830 is targeted by bacterial type III effectors, we analyzed in planta the interaction of BSK 830 with 35 Xanthomonas euvesicatoria effectors. This analysis identified seven effectors that interacted with BSK830. Together, the results obtained so far support the hypothesis that BSK830 plays a role in PTI and may be a target of bacterial effectors.

Genome-wide investigation for 2,4-diacetylphloroclucinol responsible genes in Arabidopsis thaliana D. CHAE, Y. S. Kwak, Gyeongsang National University, Korea, Republic of (South)

2,4-diacetylphloroglucinol (DAPG) is a known secondary metabolite produced by a biocontrol agent Pseudomonas spp. DAPG display remarkably broad spectrum of antibiotic activity against various of pathogens. However, high concentrations of DAPG also has been known to have negative effect on plants, such as inhibition seed germination and growth of plants. In previous studies, toxicity of DAPG in plants has been hypothesized to be linked to inhibition of proton secretion across the plasma membrane, but the mechanisms underlying these effects are not understood clearly. Dependent effects of DAPG on the plant suggest that its' action mechanisms may involve multiple molecular targets. In this presentation Arabidopsis thaliana, a powerful material for genomic research, was employed to reveal plant responsible genes to DAPG. The approach used genome wide activation tagged population of $A$. thaliana (pSKI015). Among 63,000 T-DNA insertion lines, 16 relatively tolerance lines to DAPG were obtained. TAIL - PCR was performed to determine the loci of T-DNA integration in the genome. O-transferase or hydrolase enzyme (TBL27, SBH1, GLX2-5, WSD-1, BRIZ1, GDSL), transmembrane protein (MCA1), phylloquinone biosynthesis (ICS2), golgi organization (DUF833), reduction oxidase (FRO5) were confirmed as related DAPG tolerance Arabidopsis lines. The selected 16 genes will contribute to understand plant physiological response to DAPG and develop hypothesis of DAPG in plant ISR. 
Fusarium and PRSV resistance genes in melon: Protein interactions and functional validation by CRISPR-Cas9 S. Nizan, K. Pashkovsky, A. Amitzur, M. Normantovich, G. Miller, A. Bar-Ziv, R. PERL-TREVES, Bar Ilan University, Ramat Gan, Israel

Fom-1 and Prv genes reside in a head-to head orientation in a single locus, and control melon resistance to Fusarium races 0 and 2, and to Papaya ring spot virus, respectively. They encode TIR-nucleotide binding-leucine rich repeat (NBL) proteins. To confirm Prv function, we used CRISPCas9 mutagenesis. Transgenic melons from the appropriate resistant genotypes were regenerated, with high frequency of bi-allelic mutations in the target gene. We observed deletions of the region between two targets, and even beyond that area. To our best knowledge, this is a first report of CRISPR mutants in melons. Plants were fertile, and their progeny is being tested for breaking resistance. Since a few paired R genes reportedly form functional units, we explore the possible interaction between Fom-1 and Prv. In a previous proteomic study, we identified in the xylem a candidate $\mathrm{Avr}^{2}$ for another melon $R$-gene, Fom-2, and studied their protein interactions. We constructed a yeast-expressed cDNA library from Fusarium infected melon roots and screened it with an $\mathrm{Avr}^{2}$ bait; we also isolated interactors by co-immunoprecipitation. Several putative interactors were identified that could mediate the defense response initiated by Fom-2, and these, as well as plant xylem proteins induced upon infection, serve as a starting point to study Fusarium-melon recognition at the protein level.

\section{Activation of Heterotrimeric G Proteins in Arabidopsis Immune Signaling}

M. MA (1), X. Liang (1), J. M. Zhou (2), (1) Chinese Academy of Science, IGDB, Beijing, China; (2) Institute of Genetics and Developmental Biology, Chinese Academy of Sciences, Beijing, China

$\mathrm{G}$ proteins are universal signaling modules in eukaryotic organisms. Unlike animal $\mathrm{G}$ proteins that are activated by the ligand-induced guanine nucleotide exchange activity of seven-transmembrane G protein-coupled receptors (GPCRs), plant G proteins are self-activating and couple to single transmembrane receptors. Our previous study showed that G proteins are directly coupled to FLS2, a receptor kinase that perceives bacterial flagellin epitope flg22 to activate immunity in Arabidopsis. How plant receptors regulate G proteins and how plant G proteins regulate downstream signaling remain poorly understood. Here we show that RGS1, a GTPase accelerating protein (GAP), maintains the G proteins in an inactive state in complex with FLS2 by its GAP activity. Activation of FLS2 by flg22 induces BIK1/PBLs-mediated phosphorylation of RGS1, which promotes RGS1 dissociation from FLS2-G proteins complex, relieves the G proteins from the RGS1-mediated repression, and enables positive regulation of immune signaling. Our data provided a paradigm for plant receptor-mediated regulation of heterotrimeric $\mathrm{G}$ proteins that is distinct from animals. We additionally found that RGS1 is similarly regulated by multiple immune receptors. Finally, we have identified new $\mathrm{G}$ protein targets that negatively regulate plant immune responses. We will discuss how $\mathrm{G}$ proteins regulate these newly identified proteins during immune activation.

Interaction and structural study between plant TIR-containing NLRs and EDS1 immune regulatory complex

M. X. RANK (1), B. Kobe (1), H. Burdett (1), S. Horsefield (1), M. K. Manik (1), X. Zhang (2), Y. Shi (3), (1) University of Queensland, Brisbane, QLD, Australia; (2) Commonwealth Scientific \& Industrial Research Organization, Canberra, ACT, Australia; (3) Griffith University, Southport, QLD, Australia

An important category of innate immune plant NLRs carry a TIR (Toll/interleukin-1 receptor, resistance) signaling domain at their N-termini. The molecular mechanism of plant TIRs once activated is unknown. Much of what we have learnt about plant TIRs has come from our studies of animal TIR-containing proteins. Assemblies of TLR (Toll-like receptor) adaptor TIR domains can be produced in vitro and the structure of the MAL (MyD88 adaptor-like protein) filamentous assembly has been determined by cryo-EM. Plant TIR domains can also self-associate through more than one interface. The crystal structure of the TIR domain from the catalytically active protein SARM1 (sterile alpha and armadillo-motif containing protein), involved in a cell death pathway operating within neurons, has also been determined. Structural similarities between SARM1 and plant TIR domains has led our lab to demonstrate that plant TIR domains can cleave NAD+, and this activity likely plays a role in their hypersensitive response function. However, it is unclear how self-association and the cleavage event of NAD+ relates the TIR-containing NLRs to their dependence on the downstream signaling nucleo-cytoplasmic complex, EDS1 (enhanced disease suseptability 1) and its interactive partners, SAG101 (Senescence associated gene 101) and PAD4 (phytoalexin deficient 4). Through structural and molecular biological techniques I seek to understand and link plant TIR function to the EDS1 immune regulatory complex.

Elucidating the role of SOBIR1 and its signaling partners in plant immunity to microbial pathogens W. HUANG, A. M. van der Burgh, M. H. Joosten, Wageningen University, Wageningen, Netherlands

Plants are continuously challenged by pathogens. To defend themselves against pathogenic microorganisms, plants possess an innate immune system. Receptor-like proteins (RLPs) and receptor-like kinases (RLKs), localized at the plasma membrane, are essential for the first line of defence against pathogens. The tomato RLP Cf-4, which recognises the effector Avr4 secreted by the apoplastic fungal pathogen Cladosporium fulvum, lacks an intracellular kinase domain, and the RLK SUPPRESSOR OF BIR1-1 (SOBIR1) constitutively interacts with Cf-4, thereby providing Cf-4 with a downstream cytoplasmic signaling domain. The bimolecular complex Cf-4/SOBIR1 recruits BRI1-ASSOCIATED

KINASE 1 (BAK1) to trigger downstream signaling upon Avr4 perception. We propose that trans-phosphorylation events take place between the kinase domains of SOBIR1 and BAK1, leading to the activation of the receptor complex

(https://onlinelibrary.wiley.com/doi/epdf/10.1111/mpp.12767). For subsequent signaling into the cytoplasm, it is hypothesized that this tripartite complex recruits receptor-like cytoplasmic kinases (RLCKs). In this study, we are interested in determining what kind of phosphorylation events happen between the kinase domains of SOBIR1 and BAK1, and in addition we aim to decipher which particular RLCKs are essential in the Cf-4mediated defence signaling pathway and how these RLCKs eventually activate immunity.

Partners and signaling in SOBIR1-related immune complexes

S. LANDEO, N. Pelzers, M. H. Joosten, Wageningen University, Wageningen, Netherlands 
Detrimental plant pathogens infect plants, thereby causing poverty and food shortage. Plants rely on their innate immunity to control pathogen invasions and they either perceive pathogens by extracellular plasma-membrane-localized receptors or by cytoplasmic receptors. Extracellular receptors mediate the first line of defence and are classified into receptor-like kinases (RLKs), containing an extracellular domain for ligand recognition as well as an intracellular kinase domain for downstream signaling, and receptor-like proteins (RLPs) that lack the intracellular signaling domain. The RLK SOBIR1 is an essential co-receptor that associates with RLPs triggering immune responses (thereby providing a kinase domain), and allowing the key central regulator of plant immunity, BAK1, to be recruited to the RLP/SOBIR1 complex upon ligand recognition by the RLP. Very little is known about how this RLP/SOBIR1/BAK1 complex functions in immunity. In this study, we aim to decipher the defence signaling pathway that is activated by SOBIR1-containing complexes by identifying SOBIR1 downstream interactors, determining their biological relevance, and eventually testing whether we can exploit biologically relevant interactors to improve plant resistance. Candidates, obtained from a network-assisted analysis of expression data, are evaluated in Nicotiana benthamiana by means of co-immunoprecipitations and measurements of immune output after silencing of the encoding genes.

Analysis of downstream signaling components of LORE-mediated immune sensing in Arabidopsis L. RAASCH, M. Schäffer, S. Ranf, Technical University of Munich, Freising, Germany

Plants are constantly exposed to a multitude of pathogens. Their innate immune system employs specific pattern recognition receptors (PRRs) to recognise conserved microbe-associated molecular patterns (MAMPs). Translation of this signal into a pattern-triggered immunity response is achieved via a conserved and converging signal transduction network. We are working with the PRR LORE (LipoOligosaccharide-specific Reduced Elicitation), a crucial component for detection of 3-OH fatty acids of Gram-negative bacteria. Currently, we are searching for downstream signaling components during LORE-mediated immune sensing in Arabidopsis thaliana. For this, we performed an untargeted LORE-immunoprecipitation experiment in $A$. thaliana coupled with LC-MS/MS analysis to identify LORE-interacting proteins. We identified several putative LORE interaction partners, including members of diverse receptor-like kinase and receptor-like cytoplasmic kinase families. Currently, we are further investigating promising candidates on the genetic level with regard to changes in early immune responses and resistance to Pseudomonas syringae infection. In parallel, we are confirming direct interaction of these candidates with LORE via co-immunoprecipitation and FRET-FLIM measurements in vivo. Overall these analyses will reveal common but also specific signaling components of different receptor classes in immune sensing in Arabidopsis.

\section{Signalling dynamics of the RRS1-RPS4 NLR immune complex at the chromatin during ETI}

E. GRUND (1), B. Zhou (2), P. Dabos (1), X. Sun (3), S. Carrere (4), D. Rengel (5), J. E. Parker (3), D. Tremousaygue (1), L. Deslandes (6), (1) Laboratory of Plant-Microbe Interactions, INRA/CNRS, Castanet-Tolosan, France; (2) UCD Centre for Plant Science, Dublin, Ireland; (3) Max Planck Institute for Plant Breeding Research, Cologne, Germany; (4) Université de Toulouse, INRA, CNRS, Toulouse, France; (5) Université de Toulouse, Toulouse, France; (6) Université de Toulouse, LIPM, CNRS-INRA, Toulouse, France

Pathogenic microorganisms deliver virulence factors (effectors) into host cells, in order to oppress the plant immune response and to facilitate the infection. Effectors interfere with PTI (pattern-triggered immunity) basal resistance pathways, causing effector triggered susceptibility (ETS). Intracellular plant nucleotide-binding/leucin rich repeat receptors (NLRs) can detect effector activities and induce effector triggered immunity (ETI). The Ralstonia solanacearum PopP2 effector triggers ETS in Arabidopsis by inhibiting defensive WRKY transcription factors through acetylation. The TIR (Toll-interleukin-1 receptor) - NLR pair RRS1-R/RPS4 confers resistance to this effector. PopP2 acetylates a key lysine within the C-terminal WRKY DNA-binding domain of RRS1-R, causing dislodging of the RRS1-R/RPS4 complex from chromatin sites and resulting in the activation of RPS4-dependent immunity (Le Roux et al., 2015, Cell, 161; Sarris et al., 2015, Cell, 161). To date, the mechanisms by which NLRs activate plant defense reprogramming are still poorly understood. By using DNA adenine methylation IDentification (DamID) coupled with Illumina DNA sequencing (Dam-Seq), we are currently studying at which chromatin sites pre- and post-activated RRS1-R/RPS4 complex binds and evaluating its potential involvement in the regulation of plant defence processes.

Grapevine (Vitis vinifera) receptor-like cytoplasmic kinases VvPBL27 and VvPBS1 connect chitin perception to MAPK activation R. ZHANG (1), P. D. Spanu (2), L. Davies (3), I. B. Dry (4), (1) Imperial College London, U.K.; (2) Imperial College London, London, U.K.; (3) CSIRO, Urrbrae, Australia; (4) CSIRO Plant Industry, Urrbrae, SA, Australia

Perception of microbe-associated molecular patterns by pattern recognition receptors (PRRs) leads to activation of mitogen-activated protein kinase (MAPK) cascades, which induce robust immune responses. VvLYK1-1, a V. vinifera PRR, participates in chitin-triggered immunity of grapevine and is required for resistance against non-host powdery mildew (PM) species. However, the downstream components by which VvLYK1-1 transmits immune signals to the MAPK cascade are not known. The grapevine family of RLCKs and MAPKKKs were annotated to select potential candidates which act downstream of VvLYK1-1. Here, we show that in yeast two-hybrid assays all three VvRLCKs interacted with VvLYK1-1, but only VvPBL27 and VvPBS1 interacted with VvMAPKKK7/16. The protein interactions were validated by coimmunoprecipitation and bimolecular fluorescence complementation assays. To further investigate the role of RLCKs and MAPKK proteins in non-host PM resistance, the Arabidopsis mutants AtPBL27 and AtMAPKKK5 were challenged with grapevine and barley PM. Both mutants showed a significant increase in penetration of these two non-adapted PM species. Overall, we demonstrate that components of chitin-mediated MAPK activation pathway play an important role in resistance against non-adapted powdery mildew pathogens. Based on our results, we propose a signaling transduction pathway from PRR-mediated chitin recognition to MAPK activation in $V$. vinifera involving of VvLYK1-1, VvPBL27/VvPBS1 and VvMAPKKK6/17.

\footnotetext{
A helper and sensor NLR pair focally accumulate at the host-pathogen interface to provide resistance to the Irish potato famine pathogen

C. DUGGAN (1), C. H. Wu (2), Z. Savage (1), E. Moratto (1), K. Prabhu (1), S. Nohut (1), L. Derevnina (2), S. Kamoun (2), T. O. Bozkurt (1),

(1) Imperial College London, U.K.; (2) The Sainsbury Laboratory, University of East Anglia, Norwich Research Park, Norwich, U.K.
} 
In asterids, a complex signaling network of nucleotide-binding leucine-rich repeat (NLR) proteins mediates resistance to viruses, nematodes, bacteria, aphids and oomycetes. In this network, the helper NLR NRC4 pairs with many sensor NLRs, including agronomically important Rpiblb2, Mi-1.2 and Rx, mediating resistance against Phytophthora infestans, nematodes/aphids, and Potato virus X (PVX), respectively. Rpi-blb2 provides broad-spectrum resistance to $P$. infestans in both potato and Nicotiana benthamiana by detecting the core pathogen effector protein AVRblb2. However, the molecular basis of resistance mediated through the NRC network is unknown. Moreover, due to the strong cell death activated following pathogen recognition, the cellular processes triggered upon NLR activation during infection remain cryptic. Because $N$. benthamiana naturally lacks sensor NLRs that respond to P. infestans, we were able to study the subcellular trafficking of NRC4 during infection. By coexpressing a functional NRC4-GFP construct with a variety of established subcellular markers, we discovered that NRC4 accumulates at the host membrane which engulfs haustoria. Interestingly, NRC4 localized to microdomains that are also positively labelled by AVRblb2 and Rpi-blb2. Remarkably, Rpi-blb2 accumulation around haustoria is dependent on NRC4, indicating that NRC4 functions include the recruitment of sensor NLR to effector delivery sites, possibly to maximise immune recognition of its cognate effector.

The Recognition of Conserved RxLR Effectors of Phytopthora Species Might Help to Defeat Multiple Oomycete Diseases X. LIN (1), K. Witek (1), A. Witek (1), H. McLellan (2), C. F. Nellist (3), A. D. Armitage (3), R. J. Harrison (3), P. R. J. Birch (4), V. G. A. A. Vleeshouwers (5), J. D. Jones (1), (1) The Sainsbury Laboratory, University of East Anglia, Norwich Research Park, Norwich, U.K.; (2) Cell and Molecular Sciences, the James Hutton Institute, Dundee, U.K.; (3) NIAB EMR, East Malling, U.K.; (4) Cell and Molecular Sciences, James Hutton Institute, Dundee, U.K.; (5) Plant Breeding, Wageningen University and Research, Wageningen, Netherlands

Oomycetes, especially Phytophthora species, cause diseases of many economically important crops. For example, potato late blight, tobacco black shank disease and strawberry crown rot are caused by $P$. infestans, $P$. parasitica and $P$. cactorum respectively. So far, many $R$ genes against potato late blight have been cloned from wild Solanum species. However, little is known about resistance against these other Phytophthora species. In 2016, Rpi-amr3 was cloned from Solanum americanum. Here we report a newly identified Avr gene Avr-amr3 from $P$. infestans, which was identified by effectoromics. Unlike other RxLR effectors, orthologs of Avr-amr3 are present in many other Phytophthora species. Surprisingly, we found homologs of Avr-amr3 from P. parasitica, P. cactorum, P. palmivora, P. sojae, P. megakarya, P. litchii and $P$. pluvialis can be recognized by the same R protein after co-expression in $N$. benthamiana. To further test if Rpi-amr 3 confers resistance to other Phytophthora species carrying the Avr-amr3 homolog, we used N. benthamiana - P. parasitica as a model system. We found that Rpi-amr3 transgenic $N$. benthamiana is resistant to $P$. parasitica Race 0 and Race 1 , but the wild type $N$. benthamiana is highly susceptible. This is the first report of a conserved RxLR effector from different Phytophthora species that can be recognized by the same $R$ gene, and has the potential to enable broad spectrum resistance against many oomycete diseases.

Identification of Arabidopsis LysM-type receptors involved in immune response induced by polymeric chitin H. KAMINAKA, M. Yoshioka, K. Naito, S. Matsukawa, R. Y. Parada, M. Egusa, S. Ifuku, Tottori University, Japan

Chitin, an $N$-acetyl D-glucosamine polymer, is known as one of microbe-associated molecular patterns and recognized by specific pattern recognition receptors in plants. In Arabidopsis, the immune response induced by chitins occurs mainly via the LysM-type receptor CERK1. Using chitin nanofiber $(\mathrm{CNF})$, which can be produced by nanofibrillation of chitin, we have studied the disease resistance induced by polymeric chitin in plants. We found that CNF systemically induces disease resistance against necrotrophic fungal pathogen Alternaria brassicicola even in Arabidopsis cerk1-2, supposing the involvement of the receptor(s) different from CERK1 in response to polymeric chitin. Here, we examined CNF-induced immune response in loss-of-function Arabidopsis mutants of LysM receptor-Like Kinases (LYKs) including CERK1. Quantitative analysis of the time course of reactive oxygen species (ROS) production revealed that ROS production induced by CNF was abolished in cerk1-2, but also suppressed in $l y k 2-1$ and $l y k 3-1$. The disease resistance against $A$. brassicicola systemically induced by CNF was impaired in $l y k 3-1$ and lyk5-2. Taken together, LYK2 and LYK3 are involved in local disease resistance induced by polymeric chitin, whereas LYK3 and LYK5 are required for chitin-induced systemic disease resistance against $A$. brassicicola. It's suggested that the receptor complex consisting of LYK3 and other LysM-type receptors is involved in response to polymeric chitin in Arabidopsis.

From crystals to crops: Using effector targets to engineer novel immune receptors to combat rice blast disease

J. H. MAIDMENT (1), M. Franceschetti (1), C. Jantasuriyarat (2), A. Maqbool (1), A. Bialas (3), S. Vera (1), H. Saitoh (4), R. Terauchi (5,6), S. Kamoun (3), M. J. Banfield (7), (1) John Innes Centre, Norwich, U.K.; (2) Kasetsart University, Bangkok, Thailand; (3) The Sainsbury Laboratory, University of East Anglia, Norwich Research Park, Norwich, U.K.; (4) Department of Molecular Microbiology, Tokyo University of Agriculture, Tokyo, Japan; (5) Graduate School of Agriculture, Kyoto University, Kyoto, Japan; (6) Iwate Biotechnology Research Center, Kitakami, Japan; (7) John Innes Centre, Norwich Research Park, Norwich, U.K.

The rice blast fungus Magnaporthe oryzae threatens global rice production. During infection, M. oryzae secretes effector proteins which manipulate host targets to promote virulence. Some effectors are recognised by specific nucleotide-binding leucine-rich repeat (NLR) proteins in rice to activate plant defences. Recognition of the effector AVR-Pik is mediated by the paired NLR proteins Pik-1/Pik-2. Pik-1 contains an integrated heavy metal associated (HMA) domain, to which certain alleles of AVR-Pik directly bind to trigger resistance. Multiple Pik-1 alleles have evolved in rice and exhibit differential responses to different AVR-Pik alleles. AVR-PikC and AVR-PikF do not interact with any Pik-1 alleles identified to date, evading plant defences. A family of small HMA domain-containing (sHMA) proteins have been identified as host targets of AVR-Pik. Biochemical/biophysical techniques revealed AVR-PikC/F bind to sHMA1 in vitro. We hypothesised that modifying the HMA domain of Pik-1 to resemble sHMA1 would extend the response of the NLR to AVR-PikC/F. To guide engineering, we solved the crystal structure of AVR-PikF in complex with sHMA1. Using this, we produced a Pik-1 variant which triggered an immune response to AVR-PikC/F in Nicotiana benthamiana. This engineered NLR protein is currently being incorporated into transgenic rice and barley plants. This study reveals a novel approach to engineer NLR proteins capable of triggering immunity to previously unrecognised effectors.

Identification of new Cuscuta derived factors

M. KÖRNER, Eberhard Karls Untiversity Tübingen, Tübingen, Germany 
Parasitic plants are a constraint on agriculture worldwide. Plants of the genus Cuscuta spp. are obligate holoparasites with a broad host spectrum for nearly all dicotyledonous plants. As leaf- and rootless plants, Cuscuta spp. wind around stems of host plants and penetrate host tissue with haustoria. They directly connect to the vasculature and withdraw water, nutrients and carbohydrates. Thus, the haustorium development and the establishment of a connection to the host represent essential steps in the parasite's life cycle.

Little is known concerning the development of such host-parasite connections on molecular level. In this project we want to gain knowledge about specific molecular signals of Cuscuta spp. that get sensed by host plants and manipulate them towards susceptibility or resistance, respectively. On the host plant side, we are interested in identifying receptors that recognize parasitic molecules and further induce cellular signaling programs related to susceptibility or development. To identify novel Cuscuta factors that reprogram the host cellular signaling, we cloned the promoters of host genes that are upregulated at Cuscuta infection sites and fused them to the luciferase reporter gene. In a promotor::luciferase based bio-assay, we now screen different haustorial Cuscuta-extract preparations for bioactive Cuscuta factors.

Molecular cues from Cuscuta their perception and effects on host plants

P. SLABY, Eberhard Karls University Tuebingen, Tuebingen, Germany

Parasitic flowering plants occupy a specialized niche in the plant kingdom. They obtain inorganic and organic nutrients from their hosts. The parasite Cuscuta reflexa belongs to the genus Cuscuta which comprises about 100-150 species. C. reflexa is a holoparasite consisting of threadlike vines which coil around the shoots of potential host plants. Upon infection, the parasite produces specialized haustoria which penetrate the aboveground parts of plants. Therefore, the host cell wall needs to be overcome followed by the fusion with the host's xylem and phloem. Receptors, molecular cues as well as susceptibility-related signaling of this plant-plant dialogue remain elusive. To get any idea about the susceptibility related signaling mainstream, we investigate the role of phytohormones in hosts during the infection. With the help of the COLORFUL biosensor system we aim to monitor the diverse phytohormone activities while Cuscuta haustorium penetration. Additionally, we examine the role of yet unknown parasitic molecular triggers and their perception. In the resistant species Solanum lycopersicum, one resistance receptor was already identified which recognizes a parasite-associated molecular pattern and induces defense-related responses. In contrast, Solanum pennellii a susceptible tomato species does not display defense responses upon treatments. By comparison of sequenced genome parts in recombinant inbred lines, we are now able to identify the corresponding resistance genes.

Contrasting potato defense responses and Phytophthora infestans virulence between leaves and tubers A. LACAZE, D. L. Joly, Université de Moncton, Moncton, NB, Canada

Organ-specificity of plant defense is an under-represented axis of research. However, recent publications point out the importance of this concept in plant-microbe interactions. Indeed, blindly assuming the resistance status of a whole plant against a given pathogen following studies conducted only on leaves appears inappropriate. This study aims at understanding the extent of potato (Solanum tuberosum) defense organspecific mechanisms. Our results show that potato leaves and tubers activate contrasted defense responses, in terms of production of reactive oxygen species (ROS) and the activation of PTI-associated genes, following treatments with the oomycete PAMP Pep-25 or the bacterial PAMP flg22. Transcriptional reprograming following flg22 induction differed between leaves and tubers, while ROS production was similar, showing that PTI-associated genes responses do not necessarily overlap. Following Pep-25 treatment, ROS production was induced in leaves but not in tubers. Also, StPotlx-3, StPR-1, StRbohB and StCAST exhibited organ-specific expression patterns. Since PTI appears to act differently in leaves and tubers, we also sought to identify organ-specific effectors in Phytophthora infestans. We thus investigated the expression level of $P$. infestans effectors (RXLR and CRN) at three time points and identified six organ-specific effectors overexpressed at all time points in tubers compared to leaves.

A widely conserved N-terminal motif in the coiled-coil domain of NLR immune receptors is required for activation of hypersensitive cell death

H. ADACHI, A. Harant, L. Derevnina, C. H. Wu, J. Win, S. Kamoun, The Sainsbury Laboratory, University of East Anglia, Norwich Research Park, Norwich, U.K.

The majority of plant NLR immune receptors carry an N-terminal coiled-coil domain, which mediates self-association and is required or, in some cases, sufficient for activation of hypersensitive cell death. However, our understanding of how CC-NLRs trigger cell death remains limited. Here, we used the in vitro Mu transposition system to generate a random truncation library and identify the minimal region required for CC-NLRmediated cell death. We applied this method to NRC4 - a helper NLR that functions with a multitude of sensor NLRs within a complex receptor network. This revealed that the N-terminal 29 amino acids of NRC4 are sufficient to induce cell death. Remarkably, this region is defined by a motif that follows the consensus MADAxVSFxVxKLxxLL and is conserved not only in NRC proteins but also in $20 \%$ of all CC-NLRs of dicot and monocot species. Motif swapping experiments revealed that this sequence is functionally conserved between NLRs from distantly related plant species. Interestingly, NRC-dependent NLRs and many other sensor NLRs lack the N-terminal motif which may have become nonfunctional over evolutionary time. We conclude that the evolutionarily constrained "MADA motif" is critical for the cell death inducing activity of CC domains from a significant fraction of plant NLR proteins.

Widening the bottleneck: Strategies to enhance protein expression and purification of NLRs for structural studies. H. Brown (1), N. MUKHI (2), H. Guo (1), Z. Ma (1), J. M. Feehan (1), R. K. Hughes (2), J. D. Jones (1), M. J. Banfield (2), (1) The Sainsbury Laboratory, University of East Anglia, Norwich Research Park, Norwich, U.K.; (2) John Innes Centre, Norwich Research Park, Norwich, U.K.

A highly evolved surveillance mechanism in plants detects diverse pathogen-derived, disease-promoting effector proteins to activate defence. Plants use intracellular innate immune receptors known as NLRs (nucleotide-binding leucine-rich repeat proteins) to detect and respond to pathogen effectors. In the $>25$ years since the discovery of NLRs as molecular determinants of plant immunity, an increasing number of these receptors have been defined. However, our understanding of the biochemical and structural mechanisms underpinning effector recognition and 
activation of downstream defence signaling pathways by NLRs has lagged behind. The difficulties in recombinant expression of NLR proteins has been a major stumbling block to determining their in vitro activities, and their structures. In recent years, advances have been made in understanding the structure of individual domains of NLRs, but full-length structures remain a major challenge. There is no one-size-fits-all approach to the process of purifying proteins, and there remains a need to develop additional methods for generating high yields of recombinant proteins amenable for structural studies. Here, we summarize multiple approaches to high throughput expression and purification of NLRs, using the Arabidopsis paired NLRs RRS1/RPS4 as an example, in the context of both individual domains and full-length proteins.

A pair of rice NLR immune receptors work together via cooperation rather than negative regulation to initiate cell death.

R. ZDRZALEK (1), H. Saitoh (2), R. Terauchi (3,4), S. Kamoun (5), M. J. Banfield (1), (1) John Innes Centre, Norwich Research Park, Norwich, U.K.; (2) Department of Molecular Microbiology, Tokyo University of Agriculture, Tokyo, Japan; (3) Graduate School of Agriculture, Kyoto University, Kyoto, Japan; (4) Iwate Biotechnology Research Center, Kitakami, Japan; (5) The Sainsbury Laboratory, University of East Anglia, Norwich Research Park, Norwich, U.K.

NLR immune receptors that function in pairs typically work through negative regulation, where one NLR represses the activity of the second, and this repression is relieved on detection of a pathogen effector leading to signaling process. However, whether this is a global mechanism by which all NLR pairs function is not known. Here, we show that the rice NLR pair Pikp-1/Pikp-2, which confers resistance to the rice blast pathogen Magnaporthe oryzae expressing the AVR-PikD effector, functions via cooperation with cell death requiring both NLRs and the effector. To explore the mechanism of NLR cooperation we overexpressed truncated variants of Pikp-1 and Pikp-2 and made mutations in key motifs previously identified as important for NLR function. We did not find a truncation of either NLR that could support cell death in planta in the presence of AVR-PikD, revealing that all domains are required for signaling. Mutations in the highly conserved P-loop and MHD motifs in the NB-ARC domains of either Pikp-1 or Pikp-2 leads to loss of activity, showing these core motifs are required for receptor function. Moreover, we demonstrate that Pikp-1 and Pikp-2 associate to form homo- and hetero-complexes in planta in the absence of AVR-PikD. On co-expression, the effector binds to Pikp-1, generating a tri-partite complex. Taken together, we provide evidence that Pikp-1 and Pikp-2 form a fine-tuned system, based on cooperation, which is distinct from other NLR pairs described to date.

What determines the composition of receptor complexes in nanodomains of the plasma membrane? A. EHINGER (1), S. Z. Oven-Krockhaus (1,2), N. Glöckner (1), F. Ladwig (1), K. Harter (1), B. Kemmerling (1), (1) ZMBP, University of Tuebingen, Tübingen, Germany; (2) Institute for Physical and Theoretical Chemistry, University of Tuebingen, Tübingen, Germany

The small leucine-rich repeat receptor-like kinase (LRR-RK) BRASSINOSTEROID INSENSITIVE 1 (BRI1)-ASSOCIATED KINASE (BAK1) is known as a general regulator of many other LRR-RKs by acting as a co-receptor and positive regulator of different ligand-binding receptors. The best-studied BAK1 interaction partners are FLAGELLIN SENSING 2 (FLS2), which senses bacterial flagellin and BRI1, the major Arabidopsis brassinosteroid receptor. FLS2 and BRI1 reside in different nanodomains of the plasma membrane, indicating that receptor-specific nanoclusters are formed with BAK1. In our lab, two novel LRR-RKs have been identified that interact with BAK1-BAK1 INTERACTING RECEPTOR-LIKE KINASE 2 (BIR2) and BIR3. In mass spectrometry interactome screens with BIR2 and BIR3 a SMALL MEMBRANE ASSOCIATED PROTEIN (SMAP), a common membrane localized interactor, was identified. SMAP interacts with diverse immune receptor classes, affects immune receptor signaling and might function as the determinant of immune receptor nanocluster composition. To investigate the involvement of SMAP in nanocluster formation of BAK1, BIR2, BIR3, and ligand binding receptors such as FLS2 or BRI1, we will analyze the mobility, the specific localization and the interaction of the individual receptor kinases in smap mutant background compared to wild type by single particle tracking photoactivated localization microscopy (sptPALM) and single molecule Förster resonance energy transfer (smFRET).

Peptides produced by diferent plants induce resistance against fungi in phylogenetically distant species J. PASTOR FERNÁNDEZ (1), P. Sánchez Bel (1), V. Pastor Fuentes (1), J. Gamir Felip (1), N. Sanmartín Martínez (1), V. Flors (2), (1) Universitat Jaume I, Castellón, Spain; (2) Universidad Jaume I, Castellón, Spain

Peptides are important plant regulators that participate in most plant events, from plant growth and development to plant defense. Recently an increasing number of plant peptides were described as defence elicitors that act as damage associated molecular patterns (DAMPs). They are released upon pest or pathogen attack and bind to pattern recognition receptors (PRRs) triggering a cascade of plant defences. In this work our goal is to find peptides with plant resistance inducing activity against fungal pathogens. We found that exogenous application of peptides from Solanaceous species (Systemins and HypSys) and antimicrobial peptides from radish (AFPs) are perceived and induce resistance to the necrotrophic fungus Plectospharella cucumerina in the taxonomically distant species Arabidopsis thaliana. Interestingly we found that Systemins at the low concentration of $0,1 \mathrm{nM}$ reduced the infection symptoms of the necrotrophic fungus. However, peptides from Soybean failed to protect Arabidopsis plants. Bioassays showed that peptides of study do not have antifungal activity, suggesting that they are protecting the plant through the promotion of the plant immune system. On the other hand, an analytical method to identify and quantify peptides is being implemented for subsequent quantitative studies by using High-performance liquid chromatography (HPLC) coupled to mass spectrometry (MS).

Role of a LysM-domain containing protein in the virulence of a necrotrophic plant-pathogen fungus M. CRUMIĖRE, A. De Vallée, C. Bruel, N. Poussereau, M. Choquer, University Lyon 1, CNRS, INSA, BAYER, Lyon, France

Due to its immunogenicity, the polysaccharide chitin that is part of the fungal cell wall plays an important role in the molecular interaction between plants and their fungal parasites. In order to circumvent perception of chitin fragments by their host plants, fungi have developed camouflage strategies. One of these strategies involves secretion of proteins arboring a LysM domain capable of binding fragments of chitin. In biotrophic fungi, LysM-domain containing proteins interact with chitin to prevent its degradation by plant chitinases or interfere in the recognition of chitin fragments by the plant. Such camouflage has never been considered for necrotrophic fungi but recent results obtained in the model Botrytis cinerea suggest its putative occurrence. Transcriptomic data collected during the early phase of a plant infection by this fungus showed a strong expression of the BcLysM1 gene encoding a putative LysM-domain containing protein. Transcriptional induction was measured 
after the differentiation of infection cushions by $B$. cinerea, structures dedicated to the penetration of the host. Based on this result, a $\triangle B c L y s M 1$ deletion mutant was constructed that showed delayed virulence in pathogenicity tests. This study aims at addressing the role of the BcLysM1 protein in the virulence of $B$. cinerea.

Evolutionarily distinct $R$ proteins detect Phytophthora infestans effector PiAVR2 through its action on different target proteins H. WANG (1), D. Turnbull (2), C. Aguilera Galvez (3), S. Breen (4), S. Naqvi (5), Z. Tian (6), F. Brunner (7), J. D. Jones (8), V. G. A. A. Vleeshouwers (3), E. M. Gilroy (1), P. R. J. Birch (9), (1) The James Hutton Institute, Dundee, U.K.; (2) University of Dundee, Dundee, U.K.; (3) Plant Breeding, Wageningen University and Research, Wageningen, Netherlands; (4) The University of Warwick, Coventry, U.K.; (5) Division of Plant Sciences, University of Dundee, Dundee, U.K.; (6) Huazhong Agricultural University, Wuhan, China; (7) Univ Tuebingen, Tuebingen, Germany; (8) The Sainsbury Laboratory, University of East Anglia, Norwich Research Park, Norwich, U.K.; (9) Cell and Molecular Sciences, James Hutton Institute, Dundee, U.K.

Understanding the roles of effectors in causing disease and the mechanism(s) by which R proteins detect them allows us to better combat plantpathogen interactions. We recently showed that Phytophthora infestans effector PiAVR2 targets all BSU1-like (BSL) family members in potato and transient expression of BSL1, 2, and 3 enhances $P$. infestans infection. BSL1 and BSL3 suppress INF1-triggered cell death (ICD), showing that they negatively regulate immunity. ICD suppression is dependent on the brassinosteroid-responsive host transcription factor CHL1. Thus, these phosphatases act as susceptibility factors in late blight infection. PiAVR2 is recognised by two evolutionarily distinct R proteins, R2 and MCQ1. Our data show that silencing BSL1 significantly decreases R2 CD, whereas MCQ1 CD is compromised only in BSL2/3 silenced plants. Interestingly, okadaic acid, a protein phosphatase (PP1 and PP2A) inhibitor suppresses both recognition events, indicating that phosphatase activities are required in each case. Transient overexpression of BSL1 increased R2 CD, whereas a BSL1 phosphatase-dead mutant suppressed it. In contrast, expression of BSL3 accelerated MCQ1 CD, whereas a BSL3 phosphatase-dead mutant did not. Our data suggest that R2 monitors the action of PiAVR2 on BSL1, whereas MCQ1 monitors its action on BSL3. We conclude that R2 and MCQ1 have evolved distinct mechanisms to detect PiAVR2 activity.

Identification of factors involved in foliar resistance to bacteria in potato D. A. HALTERMAN, USDA ARS, Madison, WI, U.S.A.

Solanaceous crops including tomato, pepper, and eggplant are susceptible to many foliar bacterial pathogens. However, cultivated potato is immune to most pathogenic Pseudomonas and Xanthomonas species. The purpose of this project is to understand the mechanisms involved in limiting infection of bacteria in the foliage of potato. We have previously found that many popular potato cultivars are immune $P$. syringae pv. tomato DC3000, while many wild species accessions of potato are susceptible or tolerant, suggesting that resistance to foliar pathogens may have been selected during cultivation of potato as a food crop. We have used various DC300 strains defective in pathogenesis $(\Delta \mathrm{hrcC}, \Delta \mathrm{fliC},-$ coronatine). Populations between wild and cultivated potato have also been developed to map the resistance locus using SNP genotyping. The identification of novel genes involved in bacterial resistance will facilitate the development of new varieties of Solanaceous crops.

Balanced nuclear and cytoplasmic distribution is required for activation of Gpa2-mediated resistance against potato cyst nematode Globodera pallida.

Q. ZHENG, E. Slootweg, O. Sukarta, C. C. Van Schaik, G. Smant, A. Goverse, Laboratory of Nematology, Wageningen University and Research, Wageningen, Netherlands

The resistance gene Gpa2 encodes a CC-NB-LRR protein in potato and confers resistance to the potato cyst nematode Globodera pallida. Gpa2 is able to recognize specific variants of the secretory effector protein RBP-1 from G. pallida. The activation of Gpa2-mediated cell death requires RanGAP2, which is known as an interactor of the Gpa2 CC domain. RanGAP2 serves as a cytoplasmic retention factor of the close homologue $\mathrm{Rx} 1$, which confers resistance to Potato Virus X. However, the effect of RanGAP2 on the nucleocytoplasmic distribution of Gpa2 in plant cells and how this contributes to Gpa2-mediated resistance remains unclear. Here, we show that Gpa2 has a nucleocytoplasmic distribution pattern despite the absence of canonical nuclear localization signals similar to Rx1. Cytoplasmic or nuclear targeted Gpa2 shows a reduced cell death response, suggesting that both the cytoplasmic and nuclear compartment are required for Gpa2-mediated cell death response. Besides, nuclear targeted RBP-1 results in loss of Gpa2-mediated cell death, which indicates that RBP-1 recognition takes place in the cytoplasm. Currently, we are exploring the role of RanGAP2 in the balanced nucleocytoplasmic partitioning of Gpa2 pre- and post-activation by RBP-1. Co-localization studies complemented with structure-informed approaches on complex formation among Gpa2, RBP-1 and RanGAP2 will give us more insights about how RanGAP2 is involved in Gpa2-mediated resistance against potato cyst nematode G. pallida.

The role of RPW8 and RPW8-NLRs in plant immunity

B. CASTEL, J. M. Feehan, P. M. Ngou, P. Ding, J. D. Jones, The Sainsbury Laboratory, University of East Anglia, Norwich Research Park, Norwich, U.K.

Plant pathogens cause crop losses, often necessitating chemical protection, which is costly for farmers and creates environmental impacts. Genetic protection of crops is preferable but requires knowledge. The plant immune system relies in part on an arsenal of Resistance-genes $(R$ genes) that encode Nucleotide-binding (NB), Leucine-rich Repeat (NLR) proteins. This superfamily is divided in three classes: the TIR-NLRs, the CC-NLRs and the RPW8-NLRs. The RPW8-NLRs are present in low copy number but are highly conserved in all plant genomes. They function as helper NLRs, required for signaling of many sensor TIR- and CC-NLRs. In addition, RPW8 carries the N-terminal domain, without NB or LRR domains. RPW gene family members are present in all Arabidopsis accessions and many plant genomes. They are involved in at least powdery mildew resistance and are targeted to the extra-haustorial membrane upon powdery mildew infection. We used CRISPR to knockout RPW8 copies in Arabidopsis and NRG1 copies in Arabidopsis and Nicotiana benthamiana. We found that NRG1 is indeed required for the activity of many TIR-NLRs (e.g. WRR4, RPP1, RRS1-RPS4), either totally or partially. We are now investigating possible mechanisms of action of NRG1 and RPW8. 
A natural stack of $\mathbf{R}$ genes maintains immunity in barley to non-adapted stripe rusts

S. HOLDEN (1), J. Bettgenhaeuser (2), P. Green (1), I. Hernández-Pinzón (1), M. J. Moscou (1), (1) The Sainsbury Laboratory, University of East Anglia, Norwich Research Park, Norwich, U.K.; (2) King Abdullah University of Science and Technology, Thuwal, Jeddah, Saudi Arabia

Formae speciales are lineages of the same species, specialised to infect a particular host and incompatible with other plant species. We aim to dissect the incompatibility between wheat stripe rust (Puccinia striiformis f. sp. tritici: Pst) and cultivated barley, and deploy these genes in other crops. We previously screened diverse barley germplasm and identified three critical accessions susceptible to Pst. Using 31 mapping populations we identified three major effect resistance loci, designated $R p s 6, R p s 7$, and $R p s 8$, which are widespread throughout barley. Using a doubledhaploid population segregating for all three genes we established that Rps6 and Rps 7 limit early hyphal colonisation, while Rps 8 permits colonisation but prevents the completion of the fungus' lifecycle (pustule formation). Using map-based cloning and GWAS we resolved the Rps 8 locus to $0.1 \mathrm{cM}(900 \mathrm{~kb})$ and identified two candidate genes in the interval. Ongoing work aims to validate all candidates through transgenic complementation in susceptible barley, Cas9-mediated knockouts of all three resistance loci, and a TILLING population segregating for loss of Rps 8. Marker-assisted fixation of $\mathrm{R}$ genes in breeding programs is routine, but the risks of incorporating susceptibility genes or removing genes providing incompatibility to non-adapted pathogens is often unknown and has led to host jumps including those of Magnaporthe oryzae onto wheat and Bipolaris victoriae onto oat.

Evidence for massive evasion of MAMP-triggered immunity in commensal bacteria

N. R. COLAIANNI (1), K. Parys (2), Y. Belkhadir (3), J. L. Dangl (4), (1) UNC Chapel Hill Biology, Chapel Hill, NC, U.S.A.; (2) Gregor Mendel Institute, Vienna, Austria; (3) Gregor Mendel Inst of Molecular Plant Biology GmbH, Vienna, Austria; (4) Department of Biology, University of North Carolina at Chapel Hill, Chapel Hill, NC, U.S.A.

Plants have an immune system capable of detecting bacterial derived molecules through pattern recognition receptors (PRRs). The first line of defense against colonizing bacteria is the recognition of Microbial Associated Molecular Patterns (MAMPs) through their binding to external PRRs. Recognition of the flg22 MAMP halts bacterial growth through the induction of MAMP-Triggered Immunity (MTI). Flg22 is a 22 amino acid portion of the bacterial fliC gene, an important component of the flagella. Flagella are enriched in plant-associated bacterial genomes, which led us to question how bacteria are able to colonize the plant in the face of a plant immune system capable of mounting a rapid response against flagella. To study this, we use an Arabidopsis derived commensal database containing 628 bacterial genomes. We initially found that $94.5 \%$ of our genomes lacked a T3SS, meaning that most bacteria contain another method to colonize the plant in the face of MTI. We hypothesized that most bacteria are evading MTI in order to colonize the plant. We identified flg22 variants and performed ROS burst assays on a subset of variants to determine their activity. We found that not all commensal bacteria have flg22 sequences capable of evading MTI, however the evasion of MTI by non-activating flg22 variants is widespread. Thus, evasion is a common strategy employed by bacteria to colonize the plant, however it is not the only strategy used by invading bacteria to evade MTI.

Could rice be a source of cereal rust resistance genes?

Z. MA (1), R. K. Shrestha (1), T. Song (1), T. Kroj (2), E. Thynne (1), A. Hinchliffe (3), H. J. Schoonbeek (4), C. J. Ridout (3), S. Fairhead (1), P. F. Sarris (5), M. J. Moscou (1), K. V. Krasileva (6), D. MacLean (1), W. Harwood (3), J. D. Jones (1), (1) The Sainsbury Laboratory, University of East Anglia, Norwich Research Park, Norwich, U.K.; (2) INRA Montpellier, Montpellier, France; (3) John Innes Centre (JIC), Norwich Research Park, Norwich, U.K.; (4) John Innes Centre, Norwich, U.K.; (5) Microbiology \& Plant Biotechnology Group, IMBB-FORTH, Greece; (6) University of California Berkeley, Berkeley, CA, U.S.A.

Most plants are resistant to most plant pathogens. The plant's ability to detect and induce defense against a potential pathogen involves cell surface immune receptors and intracellular immune receptors, the nucleotide-binding, leucine-rich repeat (NLR) proteins. NLRs activate effectortriggered immunity (ETI). Heterologous expression of surface immune receptors such as Arabidopsis EFR can elevate pathogen resistance. However, NLR genes from one plant family are often non-functional in another plant family ("restricted taxonomic functionality (RTF)"). An emerging concept of NLR function is that many "sensor" NLR proteins are paired with "helper" NLRs to initiate immune signaling. Thus, RTF for one NLR may arise from a requirement for the appropriate helper or partner NLR. Puccinia spp. cause major losses in cereal crops, but they do not infect rice. Rice presumably evolved from a rust-susceptible progenitor grass. Genetic and functional studies investigating resistance to rust pathogens showed the involvement of post-haustorial resistance, which suggest intracellular immune receptors may play an important role in non-host resistance (NHR) of rice to cereal rust. We investigate the basis of rice NHR to cereal rusts with the goal of transferring this rust resistance into other cereals. I will present our approach to defining rice NLR genes that might be suitable candidates for transferring to cereals to confer rust resistance.

Regulation of PAMP-induced transcriptional reprogramming by the active DNA demethylase ROS1

T. HALTER (1), J. Wang (1), D. Amesefe (1), E. Lastrucci (1), M. Charvin (2), M. Singla Rastogi (2), A. L. Perez Quintero (3), R. Birkenbihl (4), I. E. Somssich (5), L. Navarro (1), (1) IBENS, Paris, France; (2) IBENS-CNRS UMR8197, Paris, France; (3) Colorado State University, Fort Collins, CO, U.S.A.; (4) Max Planck Institute, Koln, Germany; (5) Max Planck Institute for Plant Breeding, Koeln, Germany

DNA methylation is known to silence transposable elements (TEs) as well as some genes carrying TE-derived repeats in their vicinity. The demethylase ROS1 actively erases DNA methylation preferentially at the border of TEs or TE-derived repeats to avoid methylation spreading. By using whole genome mRNA-, small RNA- and bisulfite-sequencing analyses, we have identified the whole set of flg22-responsive genes that are directly controlled by ROS1. Among those, we retrieved genes encoding the disease resistance proteins RMG1 and RBA1, as well as two RLPs, which might be relevant for PAMP detection. By analyzing regions hypermethylated in ros 1 at a subset of candidates, we found an overrepresentation of WRKY binding sites. Furthermore, we showed that several WRKYs can bind to the RLPs and RMG1 promoter DNA regions that are subjected to active demethylation in WT plants, while this binding was impaired in the ros 1 mutant. We have additionally shown that the artificial targeting of hypermethylation at these promoter regions in WT plants was sufficient to limit flg22-triggered induction of these genes. 
These data indicate that ROS1 plays a central role in facilitating the recruitment of WRKYs at these promoters, thereby ensuring a rapid and pervasive gene induction upon pathogen detection. I will present this ROS1-dependent regulatory process in the context of PTI. I will also show how this epigenetic-based transcriptional control can fine-tune the plant immune response in nature.

$\mathrm{ABC}$ transporter RaxB is required for processing and secretion of sulfated RaxX to yield a biologically active form that resembles a plant hormone peptide

A. JOE (1), D. D. Luu (1), Y. Chen (2), K. Parys (3), O. Bahar (4), R. Pruitt (5), C. J. Petzold (2), V. Stewart (1), Y. Belkhadir (6), P. C. Ronald (7), (1) University of California Davis, Davis, CA, U.S.A.; (2) Joint Bioenergy Institute, Emeryville, CA, U.S.A.; (3) Gregor Mendel Institute, Vienna, Austria; (4) ARO - Volcani Center, Rishon LeZion, Israel; (5) Univ of California, Davis, CA, U.S.A.; (6) Gregor Mendel Inst of Molecular Plant Biology GmbH, Vienna, Austria; (7) University of California, Davis, CA, U.S.A.

The rice XA21 immune receptor is activated upon recognition of the tyrosine sulfated peptide, RaxX (required for $a$ ctivation of $X$ A21-mediated immunity X) produced by Xanthomonas oryzae pv. oryzae (Xoo). RaxX shares remarkable similarity to the plant hormone PSY ( $p$ lant peptide containing sulfated tyrosine) family and mimics PSY's growth promotion activity. We have hypothesized that sulfated RaxX is processed and secreted by RaxABC type I secretion system to manipulate host cellular processes. Here, our mutational studies and targeted proteomics revealed that the RaxX precursor peptide (proRaxX) is processed and secreted by the protease/transporter RaxB, whose function can be partially fulfilled by a noncognate peptidase-containing transporter B (PctB). proRaxX is cleaved at a Gly-Gly motif, yielding a mature peptide that retains the necessary elements for RaxX function as an immunogen and host peptide hormone mimic. These results indicate that RaxX is the first prokaryotic member of a previously unclassified and understudied group of eukaryotic tyrosine sulfated RiPPs (ribosomally synthesized, posttranslationally modified peptides). We further demonstrate that sulfated RaxX directly binds XA21 with high affinity. We will present a complete, previously uncharacterized biological process: bacterial RiPP biosynthesis, secretion, binding to a eukaryotic receptor and triggering of a robust host immune response.

New insights into the role of a pseudokinase in stomatal immunity

A. GUZMAN, M. B. Mudgett, Department of Biology / Stanford University, Stanford, CA, U.S.A.

Pseudokinases are prevalent in plant genomes and their role in host-pathogen interactions is not well understood. The tomato atypical receptor kinase 1 (TARK1) is a pseudokinase required for immunity and is a target of the effector XopN from the bacterial pathogen Xanthomonas euvesicatoria $(\mathrm{Xe})$. To elucidate TARK1's functional role in immune signaling, a proteomic approach was used to isolate TARK1-associated protein complexes that accumulate at the plasma membrane during $X e$ leafinfection. Immunoprecipitation of TARK1-GFP from transgenic tomato leaves led to the identification of candidate proteins linked to receptor biogenesis, membrane trafficking and plant immunity. Notably, several of these proteins have homologs in Arabidopsis which are implicated in stomatal immunity, a pre-invasion defense response. Physiological studies of tomato leaves with altered levels of TARK1 indicate that this pseudokinase plays a role in stomatal movements triggered by bacteria and elicitors associated with biotic stress. In addition, TARK1-dependent changes in stomatal aperture in tomato leaves treated with bacteria impacts the severity of disease symptoms. Collectively, our results suggest that TARK1 functions in a protein complex that regulates guard cell movement in response to pathogen invasion.

Overexpression of the NBS-LRR receptor from N. benthemina confers resistance to HLB bacteria in transgenic citrus Y. DUAN, M. Pitino, N. Li, E. Stover, USDA-ARS, Fort Pierce, FL, U.S.A.

"Candidatus Liberibacter asiaticus" (Las) is the causal agent of citrus huanglongbing (HLB), a devastating disease of citrus worldwide. As phloem-limited plant pathogen with a small genome, Las attracts all citrus varieties, and causes a systemic disease. To reveal the molecular interactions between Las and plants, we identified putative Las effectors via bioinformatics, and transiently expressed them in Nicotiana benthamiana. Intriguingly, one of them, Las $5315 \mathrm{mp}$ (mature protein) was localized in the chloroplast, and induced cell death at 3 days postinoculation, while Las $\Delta 5315$ induced a massive accumulation of starch in the infiltration zone in $N$. benthamiana, mimicking a key physiological disorder of HLB. Furthermore, we identified a few host proteins that interact with Las $5315 \mathrm{mp}$ using a yeast two-hybrid system. One of them belongs to the NBS-LRR family, and is a putative receptor. By silencing the NBS-LRR receptor gene and SGT1 of $N$. benthamiana using TRV-based VIGS, cell death no longer displayed along with expression of Las $5315 \mathrm{mp}$. We then cloned the $N b N B S-L R R$ gene from $N$. benthamiana, and transformed Carrizo citrange via agrobacterium-mediated transformation. Transgenic plants expressing the $N b N B S-L R R$ were evaluated for resistance to Las via graft- and psyllid inoculation. The results indicated highly enhanced HLB resistance in the transgenic Carrizo rootstock plants.

Whole genome sequencing reveals an NLR-rich expansion at the Xo1 bacterial leaf streak resistance locus in Carolina Gold Select rice and identifies an Xo1 candidate

A. READ (1), M. Hutin (2), G. Pertea (3), L. Triplett (4), A. Zimin (3), M. J. Moscou (5), S. Salzberg (3), A. J. Bogdanove (1), (1) Cornell University, Ithaca, NY, U.S.A.; (2) Institut de Recherche Pour le Développement, Montpellier, France; (3) Johns Hopkins University, Baltimore, MD, U.S.A.; (4) The Connecticut Agricultural Experiment Station, New Haven, CT, U.S.A.; (5) The Sainsbury Laboratory, University of East Anglia, Norwich Research Park, Norwich, U.K.

Xanthomonas oryzae pv. oryzae (Xoo) and pv. oryzicola (Xoc) respectively cause bacterial blight and leaf streak of rice, leading to yield loss in Asia and Africa. The first leaf streak resistance locus, Xo1, was identified in American rice cultivar Carolina Gold Select (CGS). Xo1 maps to a $1.1 \mathrm{Mb}$ region of the rice reference (cv. Nipponbare) genome that encodes several nucleotide-binding leucine-rich repeat (NLR) resistance genes and overlaps the only cloned NLR conferring blight resistance, $\mathrm{Xal}$. Transcription activator-like effectors (TALEs) non-specifically are the trigger for both Xo1 and Xa1, and both Xo1 and Xa1 are suppressed by truncated TALEs encoded in Asian Xoo and Xoc. Because NLRs rapidly evolve, the reference genome is of limited utility in identifying the gene or genes responsible for Xo1 resistance. We used Nanopore long reads and Illumina data to generate a genome assembly of CGS. The CGS assembly shows a 200kb expansion at the Xol locus encoding twice as many 
predicted NLRs as the Nipponbare genome. Phylogenetic analysis shows that one of these, Xo1-11, is orthologous to Xa1. Both encode an Nterminal zfBED domain and conserved tandem repeats in the LRR region. Based on the similarities in activity and structure, we have tentatively identified Xol-11 as the Xol gene. Analysis is ongoing to confirm the role of Xol-11 as well as to determine the mechanistic and structural basis for activation and suppression of Xo1-mediated immunity.

Signaling in resistance mediated by TIR-type NLR receptors in Nicotiana benthamiana

J. Gantner (1), J. Ordon (1), R. Guerois (2), J. STUTTMANN (1), (1) Martin Luther University, Halle (Saale), Germany; (2) CEA, France

EDS1, PAD4 and SAG101 form a small family of proteins characterized by presence of a unique C-terminal 'EP' domain and an N-terminal lipase-like domain. EDS1 forms similar, but mutually exclusive, heterodimers with either PAD4 or SAG101. In the Arabidopsis model system, the EDS1-PAD4 complex is required for basal resistance and signaling downstream of TIR domain-containing immune receptors, while SAG101 appears to have little relevance in plant immunity. However, two decades after identification of EDS1 and PAD4, molecular functions yet remain obscure. We used genome editing, biochemical assays and reconstitution experiments to establish Nicotiana benthamiana $(\mathrm{Nb})$ as a novel system for analysis of EDS1 immune activities. As a key advantage, EDS1 immune functions can be uncoupled from complex biotic interactions in $\mathrm{Nb}$ by Agrobacterium-mediated transient protein expression. This was exploited for comprehensive structure-function analyses in the $\mathrm{Nb}$ model system. Important differences between functions of EDS1 complexes in Arabidopsis and $N b$ will be reported, which provide novel leads towards a molecular understanding of EDS1 immune activities.

The plasma membrane-associated Ca2+-binding protein PCAP1 plays a role in immunity and response to elicitors B. MATTEI (1), M. Giovannoni (2), S. Ferrari (3), T. Ott (4), G. De Lorenzo (2), (1) University of L'Aquila, L'Aquila, Italy; (2) Sapienza University of Rome, Rome, Italy; (3) Sapienza University Of Rome, Rome, Italy; (4) University of Freiburg, Germany

Early signaling events that occur in response to elicitation include reversible protein phosphorylation and relocalization of plasma membrane (PM) proteins. Oligogalacturonides (OGs), oligoanionic DAMPs released from the plant cell wall by pathogen-secreted polygalacturonases (PGs) during infection or by plant PGs upon wounding, are key signals in both immunity and development. In a previous study of early phosphoproteome changes in Arabidopsis after OG perception, we identified PCaP1, a PM-anchored Ca2+ binding protein with actin filamentsevering activity. We have uncovered that PCaP1 plays a role in Arabidopsis thaliana immunity and response to elicitors. Although basal resistance is not affected, protection against $B$. cinerea induced by OGs is defective in two pcap1 mutants indicating that PCaP1 is required for OG-induced priming of resistance against pathogens. Consecutive OG treatments lead to a desensitization, acting as a suppressor of elicitor response, that is much more pronounced in KO mutants compared to WT seedlings. Our data show that PCaP1 is localized in PM nanodomains and is rapidly internalized in endocytic vesicles in response to OGs, and a constitutive endocytic turnover is likely required for maintaining $\mathrm{PCaP} 1$ at the PM. OG-induced phosphorylation in combination with $\mathrm{Ca} 2+$ increase may affect PCAP1 localization from PM to other compartments, and the OG-induced endocytosis may be necessary to regulate the turnover of ligand-bound receptor(s) from the PM.

Perception of microbial features by the citrus innate immune system T. TORUNO (1), J. Franco (1), C. Ramadugu (2), G. L. Coaker (1), (1) University of California, Davis, CA, U.S.A.; (2) University of California, Riverside, CA, U.S.A.

Citrus is susceptible to multiple pathogens including Xanthomonas axonopodis pv. citri and Candidatus Liberibacter asiaticus. These pathogens cause bacterial canker and citrus greening respectively, two of the most devastating diseases that have severely impacted the citrus industry worldwide. Currently, the molecular basis of citrus immune responses to bacterial pathogens is poorly understood. We investigated the ability of the citrus and other genera within the Rutaceae family to mount an immune response after perception of the conserved bacterial MAMPs flagellin and cold shock protein. Ninety accessions, including cultivated and wild citrus relatives, were tested for their ability to induce an oxidative burst after treatment with flg22 and csp22 peptides. Differences in MAMPs perception among accessions, as well as the magnitude of the response, were observed. In order to identity putative immune receptors, we mined available citrus genomes for homologs to characterized receptors from other plant species. We will report efforts towards validation of putative citrus immune receptors for their ability to perceive bacterial MAMPs in Nicotiana benthamiana and Arabidopsis thaliana. A better understanding of MAMPs perception and immune receptor repertoire can be used to introduce novel receptors from model plants or wild citrus relatives to susceptible citrus genotypes to confer resistance against bacterial pathogens.

A putative zinc-binding motif in the rice blast effector AVR-Pii contributes to a stable and functional conformation of the protein. K. FUJISAKI (1), K. Yoshida (2), H. Saitoh (3), Y. Abe (4), J. C. De la Concepcion (5), M. J. Banfield (5), S. Kamoun (6), R. Terauchi (4,7), (1) Iwate Biotechnology Research Center, Japan; (2) Graduate School of Agricultural Science, Kobe University, Kobe, Japan; (3) Department of Molecular Microbiology, Tokyo University of Agriculture, Tokyo, Japan; (4) Iwate Biotechnology Research Center, Kitakami, Japan; (5) John Innes Centre, Norwich Research Park, Norwich, U.K.; (6) The Sainsbury Laboratory, University of East Anglia, Norwich Research Park, Norwich, U.K.; (7) Graduate School of Agriculture, Kyoto University, Kyoto, Japan

AVR-Pii is an effector from the blast fungus that is recognised in rice cultivars carrying the Pii NLR immune receptor pair, leading to disease resistance. Amino acid sequence alignments between AVR-Pii, and other small secreted proteins which show weak similarity to this effector, revealed the presence of two conserved motifs, an LxAR (Leucine, any amino acid, Alanine, Arginine) motif, and a CCH (Cysteine, Cysteine, Histidine) motif. The latter represents a putative zinc-binding motif. Using rice infection assays with transgenic blast fungus carrying mutated $A V R-P i i$, we determined that the CCH motif, but not the LxAR motif, is important for recognition of the effector by Pii in rice. Live cell imaging and cell death assays in rice protoplasts showed that the AVR-Pii CCH motif mutant was translocated into rice cells but failed to induce Piidependent cell death. We also found lower protein accumulation of the AVR-Pii CCH motif mutant compared to wild-type effector in rice, and on overexpression in Nicotiana benthamiana. Finally, the AVR-Pii CCH motif mutant failed to interact with rice OsExo70F3, a known interactor 
of AVR-Pii. Taken together, these results suggest an important role for a putative zinc-binding motif in AVR-Pii. Most likely, this motif is necessary for the protein to adopt a stable and functional conformation in planta.

Phosphorylation-dependent sub-functionalization of the calcium-dependent protein kinase CPK28

M. BREDOW (1), K. Bender (2,3), D. Holmes (1), A. Thomson (1), A. Johnson Dingee (1), S. Huber (2), J. Monaghan (1), (1) Queen's University, Kingston, ON, Canada; (2) Univeristy of Illinois-Urbana-Champaign, Champaign, IL, U.S.A.; (3) Current address: University of Zurich, Department of Plant and Microbial Biology, Zurich, Switzerland

Plant innate immunity relies on the detection of microbes by cell surface receptors that initiate signaling to prevent disease. In order to achieve optimal immune competence, regulatory mechanisms are needed to control the activation and attenuation of immune signals. The Arabidopsis calcium $\left(\mathrm{Ca}^{2+}\right)$-dependent protein kinase CPK28 helps maintain immune homeostasis by promoting the degradation of the immune kinase BOTRYTIS INDUCED KINASE 1 (BIK1). CPK28 is also required for generative growth with $c p k 28$ plants displaying stunted stem elongation associated with an accumulation of jasmonic acid. Our epistasis analysis suggests that different signaling components are required for CPK28mediated immunity and development. Site-specific phosphorylation was explored as a regulatory mechanism for directing CPK28 activity in these two pathways. Phosphoablative mutant plants were generated for previously identified in vivo autophosphorylation sites and in vitro BIK1 trans-phosphorylated sites identified on the rice CPK28 ortholog. Mutation of one site had no effect on CPK28 function in development but was uniquely non-functional in immune homeostasis. Biochemical analysis revealed that ablating this site increased $\mathrm{Ca}^{2+}$-dependence for in vitro autophosphorylation activity. This preliminary data presents the interesting possibility that phosphorylation at this site could be important for the activation of CPK28 by $\mathrm{Ca}^{2+}$-influxes following the perception of pathogens.

Plant recognition of Phytophthora PAMP XEG1

Y. WANG, Y. Xu, Y. Sun, F. Liu, W. Ye, S. Dong, Y. Wang, Nanjing Agricultural University, China

Phytophthora species are notorious plant pathogens that cause great damage on crops. Soybean root rot caused by Phytophthora sojae is destructive to soybean and often leads to an annual loss up to tens of billions of dollars. In our previous work, we identified XEG1 from $P$. sojae that functions as a novel pathogen-associated molecular pattern (PAMP). XEG1 belongs to the glycoside hydrolase family 12 and is widely distributed across microbial taxa. In this study, we employed Nicotiana benthamiana as a model plant and characterized the function of membrane-localized receptors in XEG1 recognition. In this way, we identified the recognition receptor RXEG1 that is responsive to XEG1 and multiple GH12 proteins. In addition, we profiled the receptor-like kinases downstream of RXEG1 that participate in XEG1 defense signal. Together, this study provides novel insights on plant innate immunity against Phytophthora pathogens and will contribute to the development of durable disease resistance.

Self-association and an intact NADase active site are required for plant TIR domain immune signaling J. CHEN (1), X. Zhang (2), H. Burdett (3), M. X. Rank (3), T. Ve (3), J. Rathjen (4), B. Kobe (3), P. N. Dodds (5), (1) Commonwealth Scientific and Industrial Research Organisation, Canberra, ACT, Australia; (2) The Australian National University, Canberra, ACT, Australia; (3) University of Queensland, Brisbane, QLD, Australia; (4) Research School of Biology, The Australian National University, Canberra, ACT, Australia; (5) CSIRO Agriculture and Food, Canberra, ACT, Australia

Plant NLR (nucleotide-binding leucine rich repeat) immune receptors recognise pathogen effectors to trigger plant immunity. The TIR (Toll/interleukin-1 receptor, resistance) domains of many plant NLRs are responsible for signaling. Isolated TIRs can self-associate through two interfaces, which are both required for signaling function. Although TIR domains of some NLRs do not signal autoactively as isolated fragments, we found that mutations in the self-interaction interfaces nevertheless disrupt effector dependent immunity of these full-length proteins, suggesting that this is a general model for TIR function. The TIR domain of the animal protein SARM1 is a functional NADase, and the catalytic glutamic acid (E) residue is highly conserved in plant TIRs. We found that introducing E to A mutants to this position of plant TIRs, abolished cell death signaling of both TIR-alone and full length NLR constructs. In addition, a double mutation of two R residues in the BB loop of the grapevine Run1 TIR resulted in both enhanced enzymatic activity and enhanced cell death signaling activity. Forced oligomerisation of plant TIRs by fusion to the SAM oligomerisation domain of SARM1 resulted in enhanced cell death signaling, while fusion to a non-oligomerising mutant version of SAM did not. Together these results suggest that formation of TIR oligomers is necessary and sufficient to induce TIR domain signaling and that NADase activity is required for this signaling function.

High-throughput functional analysis of Phytophthora infestans effectors in non-host plant immunity

S. LEE (1), J. H. Lee (1), E. Park (2), D. Choi (3), (1) Department of Plant Science / Seoul National University, Seoul, Korea, Republic of (South); (2) Department of plant science / Seoul National University, Seoul, CA, Korea, Republic of (South); (3) Department of Plant Science, Seoul National University, Seoul, Korea, Republic of (South)

Non-host resistance (NHR) is the most common form of resistance exhibited by plants against the majority of pathogens. Several elements of NHR have been identified including effector triggered immunity (ETI), and often leads to cell death. Pepper is a non-host plant of Phytophthora infestans and multiple effectors of $P$. infestans induce cell death in multiple pepper accessions, suggesting that pepper has several target proteins of $P$. infestans effectors. In this study, we compared functional pattern of targets of $P$. infestans effectors between host and nonhost plant during infection. Thirteen effectors of $P$. infestans showing cell death phenotype in pepper were selected. Co-immunoprecipitation and LC-MS/MS were accomplished to investigate host factor candidates of effectors both in $N$. benthamiana and C. annumm. Using clusters of orthologous groups (COGs), we identified group of numerous host factor candidates of each effector. Host factor candidates derived from each effector have shown the variety of functional patterns. For example, AVRblb2 homologues, one of the conserved effectors in Phytophthora spp. were turned out to be involved in post translational modification, protein turn over and metabolic process. It indicates that effector proteins have diverse strategies for their successful infection. This study is expected to advance our knowledge of effector dynamics and plant immune system. 
Identification and characterization of putative effectors from Ascochyta lentis

B. M. HENARES (1), R. A. Syme (1), J. W. Debler (1), L. Farfan-Caceres (1), C. G. Grime (1), S. N. Blake (2), J. A. Davidson (2), R. P. Oliver (3), K. B. Singh (1,4), R. C. Lee (1), (1) Centre for Crop and Disease Management, Curtin University, Perth, WA, Australia; (2) South Australian Research and Development Institute (SARDI), Urrbrae, SA, Australia; (3) Curtin University, Perth, WA, Australia; (4) UWA/CSIRO, Wembley, WA, Australia

Ascochyta lentis is a necrotrophic fungal pathogen that causes ascochyta blight in lentil. Our research has focussed on the discovery of necrotrophic effectors in A. lentis, and host specificity in two Australian lentil cultivars, Nipper and PBA Hurricane XT. Recent studies have found many isolates in the $A$. lentis population in Australia separate in response to reactions on these two cultivars. Group 1 isolates are virulent on Nipper and avirulent on PBA Hurricane XT and group 2 is the converse. The isolate AlKewell and reference isolate Al4 belong to group 2 and through genome comparisons between these two isolates we identified a putative effector (NIK) that was present in Al4 but absent from AlKewell. This gene encoded a small secreted protein that when agroinfiltrated in lentil, caused necrosis, making it a likely effector. However, we did not find cultivar differences in sensitivity. We have further investigated the response of $A$. lentis using a biparental A. lentis population generated with group 1 and 2 strains. Here we present the segregation of the disease response for 96 biparental A. lentis strains on PBA Hurricane XT and Nipper, and QTL mapping using ddRADseq genotyping. Preliminary characterisation of a second putative effector gene will be presented.

Multiple knock-outs of NLRs using CRISPR-Cas9 mediated mutagenesis in Arabidopsis and a functional test with abiotic stresses S. T. KIM, M. Choi, S. J. Bae, J. S. Kim, Institute for Basic Science, Daejeon, Korea, Republic of (South)

Nucleotide-binding domain leucine-rich repeat (NLR) proteins play an important role not only in specific recognition pathogenic molecules but also in response to abiotic stresses in plants. Plant NLRs are encoded by one of the most diversified gene families which often are located in the genome in tandem repeats. Knocking-out such multi NLR gene clusters, either individually or in combination, poses challenge as these clusters often carry highly repetitive sequence elements within, hindering functional annotation of such NLRs. Here we exploited the CRISPR-Cas9 genome editing tool to establish a reliable multiple knockout system as tandemly repeated NLRs in Arabidopsis thaliana as targets. Specific guide-RNAs were designed to maximize the number of simultaneous mutations within a NLR cluster yet to distinguish one cluster from another. We found that one guide-RNA was sufficient to induce mutations of an NLR cluster of which members share high sequence similarity, For example, a stable transgenic line was built carrying a whole $R P P 4 / R P P 5 / S N C 1$ cluster null (ca. $65 \mathrm{~kb}$ ) along with numerous partial knockouts of members in combination. Additionally, the ACQOS/VICTR cluster which functions to sense osmotic stress was engineered and the loss of cluster function was confirmed. Furthermore, additive and epistatic interaction between members of a cluster and moreover between clusters will be addressed upon producing the successive lines.

MAPK involves in BSK1-mediated plant immunity in Arabidopsis

H. SHI, D. Tang, Fujian Agriculture and Forestry University, Fuzhou, China

Perception of microbes by plant cell surface PRRs (pattern recognition receptors) triggers PTI (PAMP-triggered immunity). The Arabidopsis receptor-like cytoplasmic kinase BSK1 interacts with PRR FLS2 and is important for the activation of PTI. To understand how BSK1 transduces the signals from the FLS2 complex to downstream components, we performed whole-genome phosphorylation assay to identify the potential substrates of BSK1. We found Ser-511 residue of a family member of MAPK is not phosphorylated in the bsk1-1 mutant as it was in wild type, which indicates functional BSK1 is critical for the phosphorylation of MAPK and that MAPK may functions as a substrate of BSK1 in plant immunity. Through co-immunoprecipitation and split-luciferase complementation assays, we found BSK1 interacted with MAPK. In vitro kinase assay indicated S511 is the major autophosphorylation site of MAPK. Try to elucidate the function of MAPK in PTI signaling and the biological significance of the phosphorylation modified by BSK1 will make a further connection of defense signaling pathway from receptor complex recognition to MAPK activation.

Proteomic analysis of leaf epidermis inoculated with Fusarium graminearum in Arabidopsis ein 3 mutant D. TAMAOKI (1), D. Ikeda (2), Y. Sidiq (3), I. Karahara (1), T. Nishiuchi (4), (1) Graduate School of Science and Engineering, University of Toyama, Toyama, Japan; (2) Department of Biology, Faculty of science, University of Toyama, Gofuku Suehirotyo 2626-9, Toyama, Japan; (3) Graduate School of Natural Science and Technology, Kanazawa University, Kanazawa, Japan; (4) ASRC, Kanazawa University, Kanazawa, Japan

A detailed mechanism of host plant resistances against penetration of a phytopathogenic fungus Fusarium graminearum has unresolved questions. The representative Japanese strain (H3) of $F$. graminearum does not form appressorium and enters mainly into plant leaves via wounds and opened stomata. It was known that the phytohormone ethylene negatively regulates disease resistance of host plant against $F$. graminearum. Since hyphal entry rate into Arabidopsis leaves decreased in the ethylene-insensitive ein3-1 mutant, ein3-1 was selected as a candidate having penetration resistance against $F$. graminearum in this study. To reveal the mechanism of penetration resistance against $F$. graminearum, we performed shotgun proteomic analysis using epidermis of WT and ein3-1 mutant leaves inoculated with F. graminearum. More than two thousand proteins were identified in each sample. Proteomic analysis showed that proteins related to abscisic acid response or stomatal movement were up-regulated at $24 \mathrm{~h}$ postinoculation (hpi) in ein3-1 mutant compared to WT. Actually, stomata aperture of infected leaves decreased in ein $3-1$ mutant compared to WT at 24 hpi. These results suggested that stomatal closure prevents penetration of $F$. graminearum thereby enhancing disease resistance of ein3-1 mutant against $F$. graminearum.

Characterization of HopA1-triggered RPS6-mediated Immune Response

S. H. KIM (1), H. Kang (1), Q. M. Nguyen (1), G. H. Son (1), W. Gassmann (2), (1) Gyeongsang National University, Jinju, Korea, Republic of (South); (2) University of Missouri, Columbia, MO, U.S.A. 
Plants defend themselves for survival against pathogens. Generally, pathogen recognition and defense responses occur either in response to widely distributed pathogen-associated molecular patterns (PAMPs), or to very specific virulence effectors. Previously, we identified hopAl from Pseudomonas syringae pv. syringae strain 61 as an avirulence (effector) gene and cloned a hopAl-specific TIR-NBS-LRR class disease resistance gene, RPS6. As a large number of resistance proteins indirectly recognize effectors of pathogens, RPS6 is thought to indirectly recognize HopA1 and be activated by modification of effector target. However, the presence of HopA1 target protein is not yet known even though HopA1 interacts with EDS1. Here we showed both RPS6 and HopA1 are localized to the nucleus and cytoplasm, and nuclear localization of HopA1 is necessary to induce the cell death response in Nicotiana benthamiana. HopA1 induced a dwarf morphology, a cell death response, inhibition of bacterial growth and increased accumulation of defense marker proteins in transgenic Arabidopsis line expressing hopA1. In order to find target proteins of the HopA1 effector, we performed a yeast two-hybrid screen and isolated class I members of the TEOSINTE

BRANCHED1/CYCLOIDEA/PCF (TCP) transcription factor family. Specific interactions were verified in planta. Functional characterization of HopA1 interactors will provide an important piece of the plant immunity puzzle.

Molecular mechanism of CPK5 regulates CaMBP involving in plant immunity

N. LIU (1), W. Wang (1), D. Tang (2), (1) Fujian Agriculture and Forestry University, China; (2) Fujian Agriculture and Forestry University, Fuzhou, China

Calcium-dependent protein kinases (CPKs) function as calcium sensors and play important roles in plant immunity. EXO70B1 is a subunit of the exocyst complex and loss-of-function of EXO70B1 leads to autoimmunity. CPK5 is necessary for exo70B1-mediated immune responses. Overexpressing CPK5 lines display cell death and resistance, similar autoimmune responses in exo70B1. These defence phenotypes in both signaling require a truncated nucleotide binding domain and leucine-rich repeat (NLR) protein TIR-NBS2 (TN2). CPK5 can interact with TN2NBS domain. CPK5 directly links with an atypical immune receptor, indicating that CPK5 play important roles in plant immunity. To elucidate the molecular mechanism in $C P K 5$-mediated immune pathway, we compare the phosphorylation proteins between Col- 0 and $c p k 5$. We found that $\mathrm{CaM}$-binding protein (CaMBP) phosphorylations have been changed. The gain-of-function mutant cambp-D can suppress CPK5-OE-mediated cell death, suggesting that CaMBP is an important component in the CPK5-mediated downstream immune signaling. Further, we will use the combination of molecular and biochemistry approaches to study the relationship between CaMBP and CPK5 and CPK5/TN2/EXO70B1 signaling pathway and their function in immunity to understand the plant immune system.

Ethylene signaling negatively regulates the stomata movement by infection of Fusarium graminearum D. IKEDA (1), T. Nishiuchi (2), I. Karahara (3), D. Tamaoki (3), (1) Department of Biology, Faculty of science, University of Toyama, Toyama, Japan; (2) ASRC, Kanazawa University, Kanazawa, Japan; (3) Graduate School of Science and Engineering, University of Toyama, Toyama, Japan

Phytopathogen fungi Fusarium graminearum enters plant tissues though wounds or opened stomata of leaves. An Arabidopsis ethyleneinsensitive ein3-1 mutant showed enhanced disease resistance against $F$. graminearum. Additionally, our proteomic analysis using leaf epidermis inoculated with $F$. graminearum showed that stomata movement-related proteins were up-regulated in ein $3-1$ mutant when compared with WT, suggesting that regulation of stomatal movement is involved in enhanced resistance of ein3- 1 mutant to $F$. graminearum. To elucidate this possibility, we measured stomatal aperture and hyphal entry rate into leaves at $24 \mathrm{~h}$ postinoculation in WT and ein $3-1$ mutant. The results showed that stomatal aperture and the entry rate were decreased in ein $3-1$ mutant when compared to WT. These results indicated that the ein $3-1$ mutant tended to close stomata, thereby preventing penetration of $F$. graminearum. Next, we prepared secreted proteins from $F$. graminearum conidia cultured medium and measured stomatal aperture after co-application of these proteins and chitin oligosaccharide. Chitin oligosaccharide-induced stomatal closure of WT leaves was suppressed by application of secreted proteins, whereas this suppression wasn't observed in ein 3-1 leaves. These results suggest that $F$. graminearum secreted proteins such as effectors to keep stomata of host plants open via ethylene signaling and to enter into plant tissues.

Overexpression of Nicotiana benthamiana BAX inhibitor-1a suppress hypersensitive cell death in tobacco H. Y. YOON, Y. Kim, H. A. Jang, S. K. Oh, Department of Applied Biology, Chungnam National University, Daejeon, Korea, Republic of (South)

Programmed cell death (PCD) is a typical biological process that occurs under various biotic and abiotic stresses in both plants and animals. One candidate regulator for PCD is $B A X$ inhibitor-1(BI-l) which is evolutionary conserved endoplasmic reticulum resident protein. In this study, a Nicotiana benthamiana BAX inhibitor-1a ( $N b B I-1$ a) was isolated, and its role in PCD was studied. According to our founding NbBI-1a localized to the endoplasmic reticulum of $N$. benthamiana when expressed as an NbBI-1a-mGFP. We also performed gain of function studies using $N$. benthamiana, transformed with $N b B I-1$ a. The results revealed, suppression of both type of cell death; $B A X$-induced cell death as well as hypersensitive cell death (PCD) when transformed plants challenge against the Avrblb2/Rpiblb2 and AvrPto/Pto genes carrying agrobacteria. On contrary, loss-of-function studies of $\mathrm{NbBI}-1$ a using virus-induced gene silencing in $N$. benthamiana resulted in increased BAX-induced cell death. Moreover, the $\mathrm{C}$ - and $\mathrm{N}$ - terminal of NbBI-1a resulted in accelerated BAX-induced cell death. Hence, our results provide molecular evidence for a role of $\mathrm{NbBI}-1 \mathrm{a}$ as a negative regulator for HCD responses triggered by various biotic and abiotic stresses in $N$. benthamiana plants

Tomato I-2 homologues (I-2h) gene ; a new partial resistance gene to Fusarium wilt disease M. R. KIM, J. J. Lee, S. K. Oh, Department of Applied Biology, Chungnam National University, Daejeon, Korea, Republic of (South)

Fusarium wilt disease, caused by Fusarium oxysporum f. sp. lycopersici (Fol), is one of the most devastating disease in tomato. Fol is known for secreting Avr2 effector protein during infection. Avr2 effector is recognized by the tomato $I-2$ resistance $(R)$ genes and triggers hypersensitive cell death (HCD) in tomato plants carrying I-2 gene. In the present study, we successfully isolated Avr2 effector, secreted by Fol (KACC 40044), which is responsible for wilt disease in tomato plants. To analyze the resistance responses of tomato plants against Fol infection, we challenged 
128 tomato cultivars with $\mathrm{Fol}$ and selected R113 tomato cultivar. Since Avr2 is recognized by the tomato $\mathrm{I}$-2, we also performed transient expression of Avr2 in R113 tomato leaves. However, HCD was not induced when R113 tomato leaves were infiltrated with Avr2. Accordingly, we isolated $I-2$ homologues gene $(I-2 h)$ by PCR-based cloning from R113 tomato genomic DNA to compare with $I-2$. I- $2 H$ shows $99 \%$ structural similarity to the group of recently isolated $I-2$ that contain a coiled-coil nucleotide binding site leucine-rich repeat protein. Our results have revealed that co-expression of $I-2 h$ with $A V R 2$ constructs in Nicotiana benthamiana leaves is not required for HCD. These results indicated that $I-$ $2 h$ gene is not essential for the HCD but is required for $I-2 h$-mediated resistance to Fol. Further work will be focused on the characterization of $I$ $2 h$ gene and its regulation by another pathway in plants.

The xopJ6 gene encodes a PopP2-like acetyl transferase from Xanthomonas campestris pv. campestris recognized by RRS1/RPS4 R genes in Arabidopsis

E. Lauber (1), M. Gonzalez-Fuente (1), M. Escouboué (1), C. Vicedo (1), J. S. Luneau (1), A. Jauneau (2), L. Deslandes (1), L. D. NOEL (1), (1) Université de Toulouse, LIPM, CNRS-INRA, Toulouse, France; (2) Université de Toulouse, FRAIB 3450, UPS-CNRS, Toulouse, France

Xanthomonas campestris pv. campestris (Xcc) is the causal agent of black rot disease of Brassicaceae and an Arabidopsis pathogen. Xcc relies on a cocktail of type III effectors for its pathogenicity. We identified the xopJ6 type III effector gene encoding a close homologue of the Ralstonia solanacearum acetyltransferase PopP2. xopJ6 is located on a transposable element found in 1 to 4 copies on chromosome or plasmids of Xcc. XopJ6 recognition causes hypersensitive response in specific Brassica olearacea cultivars and avirulence on given Arabidopsis ecotypes. Similar to PopP2, XopJ6 acetyltransferase activity triggers activation of the RPS4/RRS1-R immune receptor complex, through manipulation of RRS1-R WRKY DNA-binding domain. Importantly, a natural single amino-acid polymorphism in XopJ6 allows Xcc to evade RRS1/RPS4 recognition in Arabidopsis. This study identifies a cognate RRS1/RPS4 avirulence determinant in a bioagressor of Brassicaceae. Latest results will be presented.

Development of SNP marker set related to ripe rot disease resistance in Grapevine by GWAS.

D. G. KIM (1), H. A. Jang (1), K. S. Lee (1), Y. Y. Hur (2), S. K. Oh (1), (1) Department of Applied Biology, Chungnam National University, Daejeon, Korea, Republic of (South); (2) Rural Development Administration, Wanju, Korea, Republic of (South)

The grape (Vitis spp. L.) is the fourth most produced fruit in the world. Grape ripe rot disease caused by Colletotrichum spp., a notorious disease in grape, causes fruit rot and is one of the economically important disease in world. Generally, the management of ripe rot disease largely depends on the breeding of resistant varieties; accordingly, the search for resistant varieties and molecular marker analysis of ripe rot disease resistance should be done in parallel. In this study, 350 grape varieties were analyzed by Genotyping-By-Sequencing (GBS) and Genome-Wide Association Study (GWAS) to achieve two goals; First to search for resistance genes against grape ripe rot disease and second to develop SNP maker. As a result of GBS based genotyping analysis, about 70,000 SNP data was obtained. In addition, phenotypic assay revealed that $64.7 \%$ of the susceptible and $35.2 \%$ varieties were resistant. Moreover, after confirmation of resistance analogous genes (RGA) with the result of GWAS analysis utilizing the GBS result and phenotypic data, luna probe melting analysis was utilized to identify the melting peak of representative varieties. Our results suggest that RGA genes as a molecular marker will be useful for screening of grape ripe rot disease resistant varieties and for the breeding of grapevine.

Molecular and functional characterization of the plant immune receptor RLP32 and its ligand K. FROEHLICH, E. Melzer, L. Fan, T. Nuernberger, Centre for Plant Molecular Biology, Eberhard Karls University of Tübingen, Tübingen, Germany

Plants are able to detect pathogens via pattern recognition receptors (PRRs), binding pathogen-associated molecular patterns (PAMPs), thereby inducing PAMP triggered immunity (PTI). We identified the translation initiation factor (IF1) as a new PAMP which is conserved in all proteobacteria. IF1 is able to trigger early immune responses in A. thaliana, e.g. ethylene production. A next generation sequencing (NGS)-based genomics approach identified the receptor-like protein RLP32 as the pattern recognition receptor of IF1 in A. thaliana. RLP32 is a leucine-rich repeat receptor protein lacking an intracellular kinase domain. Co-immunoprecipitation experiments showed an interaction of RLP32 with SOBIR1 as well as members of the SERK family. Solanaceous crops lack an RLP32-homolog and are susceptible towards many pathogenic proteobacteria. After stable transformation of $N$. benthamiana with RLP32 immune responses are induced when elicited with IF1, indicating that $N$. benthamiana gained responsiveness to pathogenic proteobacteria. $N$. benthamiana expressing RLP32 showed enhanced resistance after infection with the hrcC- mutant strain of Pseudomonas syringae. IF1 successfully primed immunity in $A$. thaliana leading to an enhanced resistance to $P$. syringae. In contrast pretreatment with IF1 did not protect rlp32 knock-out mutant genotypes. Deletion construct analysis revealed that virtually the entire IF1 protein (or its tertiary structure fold) are required for its immunogenic activity.

Multiple Pathogen Recognition at the Mla locus in Barley

H. J. BRABHAM (1), I. Hernández-Pinzón (1), J. M. Lorang (2), T. J. Wolpert (2), P. M. Hayes (3), K. Sato (4), H. Saitoh (5), M. Shimizu (6), R. Terauchi (6,7), M. J. Moscou (1), (1) The Sainsbury Laboratory, University of East Anglia, Norwich Research Park, Norwich, U.K.; (2) Department of Botany and Plant Pathology, Oregon State University, Corvallis, OR, U.S.A.; (3) Department Crop and Soil Science, Oregon State University, Corvallis, OR, U.S.A.; (4) Institute of Plant Science and Resources, Okayama University, Kurashiki, Japan; (5) Department of Molecular Microbiology, Tokyo University of Agriculture, Tokyo, Japan; (6) Iwate Biotechnology Research Center, Kitakami, Japan; (7) Graduate School of Agriculture, Kyoto University, Kyoto, Japan

The majority of NLR-encoding resistance genes recognise single pathogen species; few NLRs have the capacity to recognise multiple pathogens. The Mla locus has over 30 described alleles conferring isolate-specific resistance to Blumeria graminis f. sp. hordei (powdery mildew), contains three NLR encoding gene families ( $R G H 1, R G H 2$, and $R G H 3$ ), and is associated with resistance to multiple pathogens including Puccinia striiformis f. sp. tritici (wheat stripe rust; Rps7) and Magnaporthe oryzae (rice blast; Rmol). In addition, sensitivity to the Cochliobolus victoriae toxin victorin (HvLov1) is in coupling with Mla3. In Arabidopsis, sensitivity to victorin is mediated by an NLR-dependent (Lov1) plant immune response. We performed a high-resolution recombination screen and confirmed the genetic coupling of Mla3, Rmol, and HvLov1. Using 
sequence capture and RNAseq, we discovered copy number variation and high expression levels for Mla3 (RGH1; three copies), with one expressed copy (Mla3D6) containing a 6 bp deletion in the LRR region. Evaluation of stable transgenic barley found that Mla 3 conditions powdery mildew and rice blast resistance, whereas Mla3D6, RGH2, and $R G H 3$ do not confer resistance to either pathogen. This work, coupled with the recent discovery of direct interaction of MLA and $A_{V R}$ effectors (Saur et al. 2019), suggests that MLA has the capacity to recognise molecular structures conserved among plant pathogen effectors.

Searching for resistance genes against Leptosphaeria maculans late effectors, involved in systemic colonization of Brassica napus. A. JIQUEL (1,2), J. Gervais (1), I. Fudal (1), A. Pitarch (1), S. Faure (3), M. H. Balesdent (1), T. Rouxel (1), (1) INRA BIOGER, ThivervalGrignon, France; (2) Euralis Semences, Mondonville, France; (3) Innolea, Mondonville, France

The fungal pathogen Leptosphaeria maculans, causal agent of the blackleg disease, is responsible for important yield losses in oilseed rape crops After primary infection of cotyledons and leaves, L. maculans develops an asymptomatic endophytic stage in the stem. This infection stage lasts several months before necrosis develops at the stem base. While numerous effector genes are known to be expressed during the primary infection, little was known on the stem colonization stage. With an RNAseq approach, we identified different waves of effector expression, with one specific of early colonization stage and a second one specific of late colonization stage. These two categories of effector genes also differed by their genomic location (Gervais et al. 2017). Among late effector candidates, we selected 6 genes specifically overexpressed in petiole or stem in controlled conditions, and which are also differentially expressed in field conditions. The functional characterization of these genes is in progress, including CRISPR-Cas9 inactivation. To find resistance genes against late effectors, we are creating transgenic isolates expressing late effector candidates at an early stage of infection. With this strategy, we already identified a specific resistance to a late effector candidate. This will now be expanded to screen 250 diversified B.napus genotypes with the transgenic isolates expressing late effectors at the cotyledon stage.

The function of the ToBRFV movement protein in the breakdown of Tm-2(2) resistance in tomato H. Hak (1), M. Vaisman (1,2), Z. SPIEGELMAN (1), (1) Agricultural Research Organization, The Volcani Center, Israel; (2) The Hebrew University of Jerusalem, Israel

A recent outbreak of a new resistance-breaking tobamovirus named tomato brown rugose fruit virus (ToBRFV) has substantially damaged the tomato industry in Israel and Jordan, with symptoms that include severe plant yellowing, stunting, and reductions in yield and fruit quality. The lack of genetic resistance against ToBRFV makes it a major risk for Israeli tomato production, and a potential threat to the global tomato industry. Indeed, reports of recent ToBRFV outbreaks from around the world indicate an emerging global epidemic. ToBRFV overcomes the current tobamovirus resistance in tomato, facilitated by the $R$ gene $T m-2^{2}$, which has not been broken for over 50 years. $T m-2^{2}$ encodes a coiledcoil NB-LRR protein that binds directly to the $A v r$ factor - the tobamovirus movement protein (MP). Here, we show that the resistance-breaking factor in ToBRFV is the viral MP. Sequence analysis shows that the ToBRFV MP is altered in elements required for triggering $T m-2^{2}$ resistance response. Transient co-expression assays establish that the ToBRFV MP is not recognized by $\mathrm{Tm}-2^{2}$ resistance and is therefore the resistancebreaking factor. Using virus hybrids, MP fusions and truncations, we further characterize the motifs responsible for breaking $T m-2^{2}$. In addition, we provide evidence that altering the same motifs in tobacco mosaic virus (TMV) results in the restriction of viral infection. This finding may explain why the $T m-2^{2}$ resistance has not been broken by tobamoviruses other than ToBRFV.

Proteomic Study of Plant Leaf Epidermis Challenged with Fusarium graminearum

Y. SIDIQ (1), D. Tamaoki (2), T. Nishiuchi (3), (1) Graduate School of Natural Science and Technology, Kanazawa University, Kanazawa, Japan; (2) Graduate School of Science and Engineering, University of Toyama, Toyama, Japan; (3) ASRC, Kanazawa University, Kanazawa, Japan

The plant epidermis is the first line of plant defense against pathogen infection and likely contains important proteins related to the plantpathogen interaction. In this study, we performed comparative proteomic study using the leaf epidermis to identify differentially expressed proteins at the plant-pathogen interface. A phytopathogenic fungus, Fusarium graminearum is the causal agent of Fusarium Head Blight (FHB) in cereal plants. F. graminearum also can infect both leaves and inflorescence tissues in Arabidopsis thaliana. The representative Japanese strain of $F$. graminearum does not form appressorium and enters mainly into plant leaves via wounds and opened stomata. The conidia solution was dropped onto the Arabidopsis leaf surface and then collect the epidermis tissues from inoculated leaves. Extracted proteins were digested by trypsin, and digested peptides were analyzed using Nano-LC and Thermo Orbitrap QE plus. Among about 5000 identified Arabidopsis proteins, label free quantification methods showed that many epidermal proteins were differentially expressed by inoculation with $F$. graminearum. Most of them were not observed as differentially expressed proteins in whole leaves. Fungal proteins expressed at the Arabidopsis epidermis were also identified. These results suggested that our method is useful for understanding the early response of protein expression at plant and pathogen interface.

What ' $R$ ' you doing here? Investigating the role of $S$-acylation in regulating plant disease resistance proteins. D. TURNBULL (1), P. R. J. Birch (2,3), P. A. Hemsley $(1,4)$, (1) University of Dundee, Dundee, U.K.; (2) Division of Plant Science, James Hutton Institute, University of Dundee, Dundee, U.K.; (3) Cell and Molecular Sciences, James Hutton Institute, Dundee, U.K.; (4) The James Hutton Institute, Dundee, U.K.

Plants are constantly surrounded by potential pests and pathogens, threatening the health of the plant and the security of our food production. Unable to move to escape challenge, plants must defend themselves in situ, and possess a complex, multi-layered innate immune system. A crucial aspect of the plant immune response is the recognition of specific pest and pathogen 'effector proteins'-secreted molecules that manipulate plant processes to promote infection. Effector recognition is facilitated by plant resistance (R) proteins, largely belonging to the nucleotide-binding leucine-rich repeat (NB-LRR) family. Recent work on a group of R-proteins from potato, required for resistance to the late blight pathogen Phytophthora infestans, has revealed that they undergo S-acylation - a reversible fatty acid-based post-translational modification. S-acylation is particularly known for its role in membrane anchoring and localisation but, due to its reversibility, is also linked to regulating 
aspects of protein function such as activation, trafficking, and protein-protein interaction. Ongoing investigation has shown that S-acylation occurs at multiple conserved sites, in multiple domains, within R proteins, suggesting a complex role for this dynamic post-translational modification. Our latest data on the functional consequences of R-protein S-acylation during plant defence responses will be presented, with this modification shown to be essential for full immune signaling.

A novel family of mixed lineage kinase domain-like proteins mediates resistance to obligate biotrophic pathogens in plants L. K. Mahdi (1,2), M. Huang (3), X. Zhang (3), R. T. Nakano (1), I. M. L. Saur (1), F. Jacob (1), P. Schulze-Lefert (1), J. Chai (2,3,4), T. MAEKAWA (1), (1) Max Planck Institute for Plant Breeding Research, Cologne, Germany; (2) University of Cologne, Germany; (3) Tsinghua University, Beijing, China; (4) Max Planck Institute for Plant Breeding Research, Germany

A characteristic four-helical bundle structure, called the HeLo domain after the fungal HET and LOPB proteins, is found in proteins involved in cell death associated-immunity in plants, animals and fungi. One salient example of a HeLo domain-containing protein is MLKL (mixed lineage kinase domain-like protein), which mediates necroptosis in vertebrates. We have found in flowering plants a new family of HeLo domaincontaining proteins that are fused to a pseudokinase. Strikingly, this modular structure is identical to the vertebrate MLKL. Demonstrating the pathogen selectivity of the plant MLKL, an Arabidopsis null mutant was highly susceptible to Golovinomyces orontii and partially to Hyaloperonospora arabidopsidis but neither to Botrytis cinerea nor to virulent and avirulent Pseudomonas syringae strains. These data imply that plant MLKL plays a pivotal role specifically in immunity to obligate biotrophic pathogens. Arabidopsis MLKLs were cable of eliciting cell death in protoplasts and a phosphomimetic mutation in the activation loop of the kinase domain potentiated its cell killing activity as well as immunity to $G$. orontii in stable transgenic lines. The Cryo-EM structures of a tetrameric Arabidopsis MLKL complex do not only corroborate the structural similarity of plant MLKLs to the animal counterpart but also implicate autoinhibiton of HeLo domain-mediated signaling as important for the function of both plant and vertebrate MLKLs.

Effector-dependent changes in protein-protein interactions in the RPS4/RRS1 immune receptor complex H. K. AHN (1), P. Ding (1), H. Guo (1), H. Brown (1), S. U. Huh (2), J. D. Jones (1), (1) The Sainsbury Laboratory, University of East Anglia, Norwich Research Park, Norwich, U.K.; (2) Kunsan National University, Department of Biology, Gunsan, Korea, Republic of (South)

Plant intracellular immune receptor (NLR) proteins confer resistance to pathogens by detecting their effectors. We study the Arabidopsis NLR protein pair RPS4 and RRS1; RRS1 carries an integrated WRKY domain. The RRS1-R allele paired with RPS4 responds to bacterial effectors AvrRps4 and PopP2, whereas the RRS1-S allele with RPS4 responds to effector AvrRps4 only. Here, we have characterized RRS1 and RPS4 proteins expressed in Arabidopsis by using various extraction methods and determined the changes of the RPS4-RRS1 complex in the presence or absence of its cognate effector AvrRps4. Extraction of RPS4 requires more stringent conditions than that of RRS1, consistent with previous reports that RPS4 may associate with membrane fractions. The type of detergents may also alter the abundance of extracted RPS4 proteins. The presence of DTT also alters the migration of RPS4 and RRS1 proteins in SDS-PAGE gels, suggesting redox state or disulphide bond formation of RPS4 and RRS1 may be occurring in planta. We have also observed changes of the RPS4-RRS1 complex upon effector-triggered immunity, mimicked by induced effector expression in Arabidopsis. Blue-native PAGE results indicated changes in oligomeric state of RPS4 and RRS1 upon effector expression induction. The changes of RPS4 and RRS1 in oligomerization by effector suggests conformational changes and subsequent changes in stoichiometry of the RPS4-RRS1 complex may be essential for downstream immune signaling.

Recognition of immunoactive compounds from rhizosphere bacteria in Solanum pennellii M. BOEHME (1), P. Schulze-Lefert (2), T. Nuernberger (3), (1) Center for Plant Molecular Biology, Eberhard Karls University of Tübingen, Tübingen, Germany; (2) Max Planck Institute for Plant Breeding Research, Cologne, Germany; (3) Centre for Plant Molecular Biology, Eberhard Karls University of Tübingen, Tübingen, Germany

The recognition of biotic threats and subsequent activation of basal immunity is of critical importance for plants. For this reason, plants evolved pattern recognition receptors localized in the plasma membrane to sense their environment. These receptors are able to detect highly conserved microbial structures, so called microbe associated molecular patterns (MAMPs), such as flagellin or peptidoglycan. Pattern recognition leads to physiological and morphological changes, such as stomata closure, release of secondary metabolites and ethylene production. Although many putative receptors have been identified by genome sequencing, few have been assigned a function. In my work, I am looking for novel MAMPs which can be recognized by the wild type tomato Solanum pennellii, but not the modern variety S. lycopersicum cv. M82. Therefore, I screened a collection of rhizosphere bacteria for their ability to trigger immunity using the ethylene production as readout. Screening revealed two compounds, both proteins of more than $6 \mathrm{kDa}$. Here I present my ongoing efforts to purify and identify the immunogenic compounds. With the pure active substance, we will proceed to identify the receptor location using introgression lines, cross-breedings between the responding $S$. pennellii and the non-responding S. lycopersicum cv. M82. A novel receptor-ligand pair could enhance the recognition capacities for microbial patterns and therefore facilitate the improvement of crop resistance.

Molecular basis for ligand recognition specificity and activation of a plant NLR

M. HU (1), J. Qi (1), J. Wang (2), J. Wang (2), G. Wang (1), H. W. Wang (2), J. Chai (2,3), J. M. Zhou (1), (1) Institute of Genetics and Developmental Biology, Chinese Academy of Sciences, Beijing, China; (2) Tsinghua University, Beijing, China; (3) Max Planck Institute for Plant Breeding Research, Germany

Intracellular nucleotide binding, leucine-rich repeat receptors (NLRs) activate plant immunity upon recognition of specific pathogen effector proteins. We previously showed that the Xanthomonas campestris campestris effector AvrAC uridylylates the Arabidopsis PBL2 kinase, and the latter is specifically recruited to the coiled-coil (CC) NLR ZAR1 through the pseudokinase RKS1 to activate immunity. Here, we show that ZAR1 and RKS1 form an oligomeric complex in plant cells when induced by AvrAC. By collaborating with structure biologists, we show that AvrAC induces ZAR1 oligomerization to form a wheel-like pentamer termed ZAR1 resistosome, which mediates the immunity activation. The activated ZAR1 undergoes striking structural reorganization and releases the very N-terminal $\alpha$-helix of the CC domain to form a funnel-shaped 
structure. This structure associates with plasma membrane (PM) and is required for cell death and disease resistance. Moreover, we found a mutation at two negatively charged residues on the inner surface of the funnel structure did not affect the PM association, but impaired disease resistance, and addition of $\mathrm{LaCl}_{3}$, a calcium channel blocker, severely impedes AvrAC-triggered cell death, suggesting that the ZAR1 resistosome acts as a cation channel at PM to activate immunity. In addition, we have identified specific motif in PBL2 that is required and sufficient for AvrAC recognition, a finding useful for designing new recognition specificities for a given NLR.

Tomato Sw-5b NLR immune receptor mediated recognition of tospovirus invasion M. Zhu, J. Li, L. Jiang, H. Chen, X. Chen, X. TAO, Nanjing Agricultural University, Nanjing, Jiangsu 210095, China

Plant uses both cell surface-resident pattern-recognition receptors (PRRs) and intracellular nucleotide-binding leucine-rich repeat (NLR) receptors to detect various pathogens. Plant PRRs typically recognize conserved pathogen-associated molecular patterns (PAMPs) to provide a broad-spectrum resistance. In contrast, plant NLRs generally detect pathogen strain-specific effectors and confer race-specific resistance. We showed previously that tomato Sw-5b NLR immune receptor confers broad-spectrum resistance to multiple tospoviruses through recognition with a conserved 21-amino-acid PAMP-like region in the viral effector $\mathrm{NSm}\left(\mathrm{NSm}^{21}\right)$. We identified that four polymorphic sites and an adjacent R927 residue in the LRR domain are critical for translating recognition of $\mathrm{NSm}^{21}$ into activation of Sw-5b. Recently, we demonstrated that Sw-5b has evolved a two-step recognition mechanism to detect NSm or $\mathrm{NSm}^{21}$. Both the N-terminal non-canonical Solanaceae domain (SD) and the NBARC-LRR region of $\mathrm{Sw}-5 \mathrm{~b}$ interact with $\mathrm{NSm} / \mathrm{NSm}^{21}$. The $\mathrm{Sw}-5 \mathrm{~b} \mathrm{SD}$ and the NB-ARC-LRR functions as an integrated sensor a switch activator, respectively. This "integrated sensor and switch activation" mechanism adopted by Sw-5b NLR significantly enhances its sensitivity to detect viral effector. Furthermore, we found that the Sw-5b SD also functions as an intracellular translocation modulator, allowing Sw-5b to translocate from the cytoplasm to the nucleus to dictate the plant immunity against tospovirus invasion.

Players and mechanisms in dsRNA-induced pattern-triggered immunity in plants

A. NIEHL (1,2), I. Wyrsch (2), T. Boller (2), M. Heinlein (3), (1) Julius Kühn-Institute, Institute for Epidemiology and Pathogen Diagnostics, Braunschweig, Germany; (2) Department of Environmental Sciences, Plant Physiology, University of Basel, Basel, Switzerland; (3) Institut de Biologie Moléculaire des Plantes, UPR2357 CNRS, France

Plant viruses are recognized by different layers of plant immunity. While it is well established that plants recognize viruses and defend themselves against virus infection via RNA silencing and effector-triggered immunity, it was only recently discovered that also pattern-triggered immunity (PTI) is active against virus infection in plants. PTI relies on the perception of conserved pathogen associated molecular patterns (PAMPs) by pattern-recognition receptors (PRR). Recently, we identified double-stranded (ds) RNAs as elicitor to induce PTI responses in plants dependent on the co-receptor kinase SERK1. The signaling cascade involves activation of typical PTI responses including activation of mitogen activated protein kinases, ethylene production, and defense gene expression. However, in contrast to microbial PAMPs, which occur extracellularly, dsRNAs produced during virus replication occur intracellularly. Thus, whether dsRNAs are perceived by plasma membrane bound PRRs as described for microbial PAMPs, by PRRs located on endomembranes, or rather in the cytosol, is still an open question, which we hope to answer by further elucidating the molecular mechanisms underlying dsRNA-mediated antiviral immune signaling. Our new data implicate phosphorylation of the translation initiation factor eIF2 alpha in dsRNA signaling, thus opening the possibility that virus infection may be controlled by mechanisms inhibiting protein translation.

Arabidopsis Transcription Factor RTP3 Negatively Regulates Resistance Against Phytophthora parasitica W. LU (1), F. Deng (1), W. X. Shan (2), (1) College of Plant Protection, Northwest A\&F University, Yangling, China; (2) College of Agronomy, Northwest A\&F University, Yangling, China

Phytophthora species include dozens of destructive plant pathogens that cause significant crop losses worldwide. To understand plant susceptibility to oomycete pathogens and to explore novel disease resistance strategies, we employed Arabidopsis thaliana - $P$. parasitica model pathosystem and screened T-DNA insertion $A$. thaliana mutant lines for resistant mutants to $P$. parasitica. This led to identification of mutant 267-31 that showed enhanced resistance to $P$. parasitica. Southern blot and TAIL-PCR results showed that 267-31 carries a T-DNA insertion site in the promoter region of RTP3 (Resistance to Phytophthora parasitica 3) gene. which encodes an ethylene response factor. Additional rtp3 mutants were generated by CRISPR/Cas9 technology and were confirmed to be increased with resistance to P. parasitica. Further RNA-seq, ChIP-seq and IP-MS experiments will be useful to identify the direct target genes of RTP3 and the interacting proteins of RTP3.

Molecular mechanisms regulating race specific wheat-yellow rust interactions in winter wheat cultivars

S. AKHTER, K. Lundén, S. Bourras, L. Sjöholm, A. Berlin, Department of Forest Mycology and Plant Pathology/ SLU, Uppsala, Sweden

Wheat (Triticum aestivum) is one of the most important crops for global food security and is one of the most important crops in Swedish agriculture. Yellow rust is a devastating disease of wheat, caused by the obligate biotrophic fungus Puccinia striiformis. The disease can cause significant yield losses and occurs in all wheat producing areas in the world. Gene expression studies during the host-pathogen interactions are the key to a better understanding of molecular mechanisms of pathogenicity by the fungus and defence response by the host plant. We utilise RNA sequencing approach to unravel the transcript profiles underlying the molecular mechanisms that regulate wheat-yellow rust interactions. We infected winter wheat cultivar, Brons, using two Puccinia stiiforminsf. sp. triticiraces Kranich and Warrior. In order to follow the transcriptional changes that occur in the host-pathogen interaction, we collected infected and non-infected samples as well as germinated spores of the fungus. By comparing transcriptomes between these infected and non-infected samples, we aim to characterise the regulation of races with different virulence pattern on wheat and regulation of the race-specific responses of wheat to yellow rust. Hence, we hypothesise that such response can be based on differential expression of candidate effector genes in the pathogen, and genes specifically controlling race-specific resistance to yellow rust in the wheat host. 
Potential of Pseudomonas cyclic lipopeptides to control downy mildew in grapevine by induced resistance and direct antagonism L. HEYMAN (1), E. Ferrarini (1), O. O. Omoboye (1), L. Sanchez (2), E. A. Barka (2), M. Hofte (1), (1) Ghent University, Ghent, Belgium; (2) Université de Reims Faculté des Sciences, BP 1039 Reims Cedex 2, France

Grapevine downy mildew, caused by Plasmopara viticola, leads to serious losses in vineyards. Plasmopara viticola has been shown to build up fungicide resistance and to overcome grapevines' genetic resistance, rendering the current control strategies, the heavy use of fungicides and the cultivation of resistant varieties, unsustainable. Therefore, additional control methods are being sought. Cyclic lipopeptides have proven to be powerful assets in the sustainable biocontrol of many plant pathogens. We assessed the potential of several Pseudomonas cyclic lipopeptides to control downy mildew in grapevine. The crude extracts of some cyclic lipopeptides were found to be directly antagonistic to Plasmopara viticola, thus reducing disease incidence and severity. Also, some crudes were able to induce resistance through leaf treatment. To elucidate the mode of action, we are exploring what rapid defense responses are being triggered by the cyclic lipopeptides. Oxidative burst, enzyme activities and transcriptional alterations are being tested in plant cell suspension cultures, before scaling up to plant roots and leaves. Our research indicates that Pseudomonas cyclic lipopeptides could be valuable tools in the sustainable integrated control of grapevine downy mildew.

ChIP-seq analysis of SA-responsive transcription cofactor NPR1 in Arabidopsis thaliana

M. NOMOTO (1), Y. Kitagawa (2), T. Itaya (2), T. Suzuki (3), Y. Tada (1), (1) Center for Gene Research, Nagoya University, Nagoya, Japan; (2) Division of Biological Science, Graduate School of Science, Nagoya University, Nagoya, Japan; (3) Chubu Univ., Japan

Plants fine-tune immune systems in response to diverse environmental stimuli to best fit themselves to the environment. Upon pathogen infection, plants produce an immune hormone salicylic acid (SA) to activate the transcription cofactor NPR1, which plays a major role in SA-induced immunity. The active form of NPR1 is translocated into the nuclear and interacts with TGA transcription factors to up- and down-regulate its targets including pathogenesis-related genes. Although the majority of SA-responsive genes are coordinated by NPR1, the molecular basis of its transcriptional regulation has not been fully elucidated. Here we performed genome-wide detection of NPR1-binding sites by ChIP-seq analysis. SA significantly increased the binding of NPR1 to the promoter regions of 476 genes, among which 124 genes were expressed in a SA/NPR1dependent manner while the expression levels of 350 genes were not affected by SA compared to mock treatment. The MEME-ChIP analysis identified TGA-box as the most overrepresented motif in the former gene set. However, the latter genes significantly possess TGA-box and Gbox in the promoters, suggesting that NPR1 mostly targets TGA transcription factors independent of transcriptional output. Interestingly, GO analysis revealed that a gene set constitutively targeted by NPR1 could be involved in programmed cell death. We discuss novel target transcription factors of NPR1 responsible for not only plant immunity but also for environmental responses.

Recognition of the type III effector HopF2a by the Arabidopsis resistance proteins ZAR1 and ZRK3 D. SETO (1), D. Desveaux (1,2), (1) University of Toronto, Toronto, ON, Canada; (2) Centre for the Analysis of Genome Evolution and Function, Canada

Pseudomonas syringae is a Gram-negative bacterial pathogen capable of infecting a wide range of plant species, including important crop species such as wheat, bean, and tomato. P. syringae possesses a needle-like type III secretion system, which is required for injecting effector proteins into plant cells. These effectors can disrupt the signaling components of the plant immune system, thereby promoting pathogen virulence. However, plants have evolved nucleotide-binding leucine rich repeat (NLR) proteins to recognize effectors and activate effector-triggered immunity (ETI) to counter pathogenesis. For example, the NLR ZAR1 indirectly recognizes the effector HopZ1 a by monitoring the host kinase ZED1 for HopZ1a-induced modifications. Similarly, ZAR1 recognizes AvrAC by detecting the formation of an effector-induced complex between the kinase PBL2 and the ZED1-related kinase, ZRK1 (aka RKS1). The ADP-ribosyltransferase effector HopF2a is also recognized by ZAR1 and requires the host kinase ZRK3 for this recognition. However, HopF2a has not been found to interact with or modify ZRK3, and therefore may require another component for its recognition. Here we show that like AvrAC, HopF2a can promote interactions between ZRK3 and several PBL kinases as well as modify them. This provides evidence that ZAR1/ZRK complexes have evolved to monitor multiple host kinases for effector-induced perturbations, thereby broadening recognition specificity, while also guarding the plant kinome.

Further Expansion of the ZAR1 Type III Effector Recognition Profile Through ZED1-Related Kinases A. MARTEL (1), B. Laflamme (1), M. M. Dillon (1), R. N. Almeida (1), D. S. Guttman (1,2), D. Desveaux (1,2), (1) University of Toronto, Toronto, ON, Canada; (2) Centre for the Analysis of Genome Evolution and Function, Canada

Phytopathogenic bacteria such as Pseudomonas syringae carry distinct repertoires of type III secreted effectors (T3SEs), which are important virulence factors used to subvert host basal immunity in order to cause successful infections. In response, plants have developed mechanisms to detect a subset of these effectors through the intermediary of nucleotide-binding leucine-rich repeat receptors (NLRs) in order to mount a robust immune response termed effector-triggered immunity (ETI). A recent attempt to represent the diversity of T3SEs originating from 494 sequenced P. syringae strains has been used in order to probe the ETI landscape of the P. syringae - Arabidopsis pathosystem. This screen has highlighted the importance of the ZAR1 NLR, which was found to be a novel component in the recognition of three distinct effectors, in addition to the previously published role of ZAR1 in the HopZ1a and HopF2a ETI responses. ZAR1 uses ZED1-related kinases (ZRKs) to sense type III effectors and our novel ZAR1-dependent ETIs also require ZRKs. These include previously elucidated ZAR1/ZRK complexes as well as a novel ZAR1/ZRK combination. In order to further characterize our novel ETI responses we have developed a high-throughput pathology assay coupled with a GWAS approach, which is uncovering novel facets of ZAR1-dependent immunity.

Unravelling the molecular basis of the cell death response of new allelic wheat NLR PM2 variants to powdery mildew effectors B. MANSER (1), T. Koller (1), C. R. Praz (1), B. B. Wulff (2), B. Keller (1), J. Sanchez-Martin (1), (1) University of Zurich, Zurich, Switzerland; (2) John Innes Centre, Norwich, U.K. 
The wheat NLR protein PM2A confers resistance against Blumeria graminis $f$. sp. tritici upon the perception of the RNase-like effector AVRPM2. In this work, we present five new Pm2 alleles isolated from the wild wheat ancestor Ae. tauschii, several of which encode immune receptors that recognize AVRPM2 in the heterologous Nicotiana benthamiana system. Furthermore, four of the PM2 allelic variants recognize a second RNase-like effector of the AVRPM2-protein family, showing a different specificity and recognition spectra compared to PM2A. Based on the amino acid changes among the PM2 variants, combined with data from site-directed mutagenesis, we could determine the amino acids of PM2 variants that play a key role in the recognition of the AVRPM2 family members and characterize the allele-specific responses to wheat powdery mildew. Besides, a structure-based mutagenesis screen on AVRPM2 was performed to gain further insights into the molecular AVRPM2-PM2A interaction. The results indicate a region in AVRPM2 which is important for recognition by PM2A. This region was swapped on a closely related AVRPM2 family member and the novel, engineered effector was recognized by PM2A. By unravelling the interaction between PM2 variants and corresponding AVRPM2 variants, we contribute to a better understanding of the wheat-powdery mildew pathosystem and the evolution and specific recognition of avirulence effectors by NLRs, which could be employed in long-term resistance breeding strategies.

Dissecting $\beta$-glucan-triggered immunity in different plants

A. WANKE (1,2), H. Rövenich (2), S. Wawra (2), F. Schwanke (2), A. Zuccaro (2), (1) Max Planck Institute for Plant Breeding Research, Germany; (2) University of Cologne, Germany

The primary detection of both symbiotic and pathogenic fungi by plants takes place via the recognition of conserved molecular structures by invasion pattern receptors (IPR). Although the perception of chitin as invasion pattern and the corresponding plant IPRs are well characterized, chitin often depicts a minor component of fungal cell walls. The main building blocks are composed of $\beta$-glucans, mainly connected via $\beta-1,3$ and $\beta-1,6$ O-glycosidic bonds. A variety of $\beta$-glucan fragments differing in length and linkage patterns was shown to lead to invasion patterntriggered immunity responses in different angiosperms covering monocotyledonous and dicotyledonous plants. Although this ability of $\beta$-glucans to elicit plant defense responses was already described in the 70ies, less is known on the corresponding plant $\beta$-glucan receptors and their downstream molecular network. Here, we present our recent findings on the $\beta$-glucan-triggered immune response network in plants, including approaches for the identification of components involved in the perception of $\beta$-glucan.

Homeostasis of cell wall-derived DAMPs is regulated through oxidation by Berberine-Bridge like proteins F. LOCCI (1), D. Pontiggia (2), M. Benedetti (3), M. Citterico (2), S. Costantini (2), B. Mattei (4), F. Cervone (2), G. De Lorenzo (2), (1) Sapienza, University of Rome, Rome, Italy; (2) Sapienza University of Rome, Rome, Italy; (3) University of L'Aquila, L'aquila, Italy; (4) University of L'Aquila, L'Aquila, Italy

In order to cause a disease, pathogens need to break the plant cell wall and to this purpose they secrete degrading enzymes towards various cell wall components. Several oligosaccharides released during pathogenesis, such as the Oligogalacturonides (OGs) and Cellodextrins (CDs) upon the breakdown of the homogalacturonan and cellulose respectively, act as Damage-Associated Molecular Patterns (DAMPs) and activate immunity. However, an over-accumulation of DAMPs may lead to a hyper-immunity characterized by a reduction of growth and, sometimes, cell death; for example, an over-accumulation of OGs leads to a hyper-immunity phenotype. Therefore, it is conceivable that the response to DAMPs must be controlled through homeostatic mechanisms to prevent hyper-immunity. We have discovered that four Arabidopsis Berberine Bridge Enzyme-like (BBE-like) proteins (OGOX1-4) oxidize OGs and impair their elicitor activity. We have also discovered another member of the BBE-like enzymes (CELLOX) which is expressed coordinately with OGOX1 during immunity and that specifically oxidizes CDs, also impairing their elicitor activity. Moreover, plants overexpressing OGOX1 or CELLOX display an enhanced resistance to Botrytis cinerea, likely because oxidized OGs and CDs are a less valuable carbon source for Botrytis. Thus, the capacity of impairing the activity of the cell wall-derived DAMPs is one important feature of BBE-like proteins that it may serve for the homeostatic control of the level of DAMPs.

NLR-IDs: Windows into the hidden world of plant-pathogen interactions

E. THYNNE (1), E. L. Baggs (2), A. Deatker (3), M. J. Moscou (1), K. V. Krasileva (3,4,5), (1) The Sainsbury Laboratory, University of East Anglia, Norwich Research Park, Norwich, U.K.; (2) UC Berkeley, Berkeley, CA, U.S.A.; (3) University of California Berkeley, Berkeley, CA, U.S.A.; (4) Earlham Institute, U.K.; (5) The Sainsbury Laboratory, Norwich, U.K.

Nucleotide Binding Leucine Rich Repeat domain proteins (NLRs) represent a major class of intracellular plant immune receptors. It was recently discovered that NLRs can incorporate integrated domains, some of which are involved in pathogen effector recognition. These integrated domains (IDs) can act as decoys of the true effector target. We propose that NLR-IDs spot-light plant proteins likely to be targeted by effectors (directly or indirectly), and so can be used as tools to design hypotheses in the study of plant-pathogen interactions. Our two questions of interest are: 1) If the target of a pathogen's effector is known, can we identify NLR-ID homologues that can be used as sources of resistance to the pathogen; 2) Do homologues of IDs function in plant-microbe interactions, and are plant pathways represented by IDs heavily targeted by pathogens? To address these questions we developed a new pipeline to rapidly identify NLRs with IDs of interest. From the list of NLR-IDs identified, we have cloned and screened multiple IDs against a novel wheat yellow rust library enriched for effectors that we have developed as well as several known effectors. Simultaneously, we have screened various NLR-IDs for ability to induce cell death, transiently, in $N$. benthamiana. In addition to functional testing, we have successfully mapped ID homologs to different plant pathways, identifying ones highly represented by NLR-IDs, and highlighting them as likely hubs of plant-pathogen interactions.

Towards the mechanistic understanding of immunity mediated by distinct resistance proteins in Arabidopsis against Albugo candida T. Parkes, R. Ofoe, V. CEVIK, Department of Biology and Biochemistry, University of Bath, Bath, U.K.

The biotrophic oomycete Albugo candida causes white rust disease in many Brassicaceae. Some A. candida races can also infect Arabidopsis accessions. We identified distinct Arabidopsis White Rust Resistance genes that arrest the development of $A$. candida at different infection stages. The paired TIR-NB-LRR genes WRR5A and WRR5B confer chlorotic resistance to $A$. candida. WRR5A encodes a typical TNL protein while $W R R 5 B$ encodes a TNL protein with C-terminal zinc-binding LIM and peptidase (PEP) domains. We also cloned an additional gene named 
WRR7 that provides necrotic resistance. WRR7 codes for $\mathrm{CC}_{\mathrm{R}}-\mathrm{NB}$ type of resistance protein with C-terminal LIM-PEP domains. To gain mechanistic insights into immunity mediated by WRR5A\&B paired NLRs, we obtained transgenic Arabidopsis plants expressing tagged versions of both proteins and carried out immunoprecipitation followed by mass spectrometry. We identified WRR5A\&B associated proteins with novel functions in immunity against $A$. candida. We used ethylmethanesulfonate mutagenesis approach to identify signaling components required for the WRR7 immune response. The mutant plants characterised so far revealed that a specific calmodulin-binding transcription activator (CAMTA) as well as a member of the Mediator kinase module are required for WRR7 mediated resistance. Thus, decoding of calcium signatures by a specific CAMTA and the kinase module of Mediator play important roles in effector triggered immunity in Arabidopsis.

Identification and cloning of disease resistance genes from wild barley using sequence capture and association genetics G. BETHKE (1), S. Arora (2), B. Steuernagel (2), K. Gaurav (2), A. Sallam (1), M. J. Moscou (3), B. B. Wulff (2), G. J. Muehlbauer (1), B Steffenson (1), (1) University of Minnesota, Saint Paul, MN, U.S.A.; (2) John Innes Centre, Norwich, U.K.; (3) The Sainsbury Laboratory, University of East Anglia, Norwich Research Park, Norwich, U.K.

To engineer broad-spectrum resistance in domesticated barley (Hordeum vulgare), we have launched a comprehensive project to identify and clone novel disease resistance (R) genes from wild barley (Hordeum vulgare spp. spontaneum). The Wild Barley Diversity Collection (WBDC), consisting of 318 accessions, was evaluated for resistance against eight different diseases including powdery mildew (Blumeria graminis f. sp. hordei), stem rust (Puccinia graminis), leaf rust (Puccinia hordei), spot blotch (Cochliobolus sativus) and stripe rust (Puccinia striiformis f. sp. hordei), septoria speckled leaf blotch (Septoria passerinii), scald (Rhynchosporium commune), and net blotch (Pyrenophora teres). Over 200 of the WBDC accessions exhibiting isolate-specific reactions to the pathogens were subjected to association genetics-resistance gene enrichment sequencing (AgRenSeq) to identify the associated resistance genes. We will present an update on the resistance genes identified and comment on the progress made to clone them.

Cloning new soybean cyst nematode resistance QTLs in soybean: Convergent evolution toward unusual SNAP and NSF processes J. ZAYAS-RIVERA (1), K. Butler (1), C. Fliege (2), M. Riga (1), M. Hudson (2), B. Diers (2), A. F. Bent (1), (1) University of WisconsinMadison, Madison, WI, U.S.A.; (2) University of Illinois at Urbana-Champaign, Urbana, IL, U.S.A.

Soybean cyst nematode (SCN) is the most economically damaging pathogen of soybean. The key resistance locus Rhgl utilizes a novel alphaSNAP ( $\alpha$-SNAP). $\alpha$-SNAP and NSF are core eukaryotic housekeeping proteins that sustain cellular vesicle trafficking. We previously found that the Rhgl $\alpha$-SNAP is a toxic variant that accumulates specifically in nematode feeding sites. More recently, we also discovered depletion of wild-

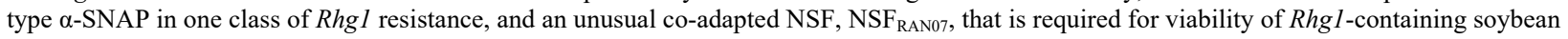
lines (Bayless et al. 2018 PNAS). We now report that another resistance source also modifies SNAP expression. Pyramiding Rhgl with other QTLs for SCN resistance is a breeding priority. Map-based cloning of $c q S C N-006$, which was recently introgressed into elite soybeans from Glycine soja, has led us to a gamma-SNAP protein. CRISPR/Cas9-mediated disruption of the resistant allele reduces SCN resistance. There is no amino acid polymorphism between the products of resistant and susceptible $c q S C N-006$ alleles, but we identified promoter and gene body DNA variants, variation in alternative splicing, and differing induction of $\gamma$-SNAP protein between resistant and susceptible infection sites. This presents an independent example of disease resistance against cyst nematodes that once again involves alteration of cellular SNAP and/or NSF processes. We will also report our work to clone another resistance QTL, cqSCN-007.

Recognition of herbivore-associated molecular patterns by a novel Leucine-Rich Repeat Receptor-Like Protein (LRR-RLP) A. D. STEINBRENNER (1,2), M. Muñoz-Amatriaín $(3,4)$, T. J. Close (3), E. A. Schmelz (2), (1) University of Washington, Seattle, WA, U.S.A.; (2) University of California San Diego, La Jolla, CA, U.S.A.; (3) University of California Riverside, Riverside, CA, U.S.A.; (4) Colorado State University, Fort Collins, CO, U.S.A.

Plants detect molecular patterns from a diverse array of pests and mount inducible defense responses. Specific receptors recognize molecular patterns from bacteria, fungi, oomycetes, parasitic plants and nematodes; however, receptors for chewing herbivores have remained elusive. To bridge this void, we utilized a set of elicitors found in caterpillar oral secretions, termed inceptin-related peptides, to characterize a receptor for chewing herbivory. Inceptin-induced response variation across cowpea (Vigna unguiculata) germplasm was leveraged for both QTL mapping and a Genome Wide Association Study (GWAS) to identify genetic loci containing candidate leucine-rich repeat receptor-like kinases (RLKs) and proteins (RLPs). A single RLP gene, termed Inceptin Receptor (INR), was sufficient to impart inceptin responses to Nicotiana benthamiana. INR binds acridinium labeled inceptin peptide and associates with SOBIR1 and SERK family coreceptors from both Arabidopsis and cowpea. Wildtype tobacco ( $N$. tabacum) plants do not respond to inceptin; however, stable transgenic lines expressing INR gain inceptin-induced defenses and reduce the growth of beet armyworm (Spodoptera exigua) caterpillars. Our results demonstrate that a novel receptor for herbivory, currently restricted to legume genomes, can enhance immunity to caterpillars in inceptin-insensitive plant species. Elucidating INR function will help to establish signaling mechanisms upstream of herbivore-induced defenses.

Engineering a Decoy Substrate in Soybean to Enable Recognition of the Soybean Mosaic Virus NIa Protease M. HELM (1), M. Qi (2), S. Sarkar (2), H. Yu (2), S. A. Whitham (2), R. W. Innes (1), (1) Indiana University, Bloomington, IN, U.S.A.; (2) Iowa State University, Ames, IA, U.S.A.

In Arabidopsis, recognition of the AvrPphB effector protease from Pseudomonas syringae is mediated by the disease resistance protein RPS5, which is activated by AvrPphB-induced cleavage of the Arabidopsis protein kinase PBS1. The recognition specificity of RPS5 can be altered by substituting the AvrPphB cleavage site within PBS1 with cleavage sequences for other proteases, including proteases from viruses. AvrPphB also activates defense responses in soybean, suggesting that soybean may contain an R protein analogous to RPS5. It was unknown, however, whether this response is mediated by cleavage of a soybean PBS1-like protein. Here we show that soybean contains three PBS1 orthologs and that their products are cleaved by AvrPphB. Further, transient expression of soybean PBS1 derivatives containing a five-alanine insertion at their AvrPphB cleavage sites activated cell death in soybean protoplasts, demonstrating that soybean likely contains an AvrPphB-specific resistance protein that 
is activated by a conformational change in soybean PBS1 proteins. Significantly, we show that a soybean PBS1 decoy protein modified to contain a cleavage site for the Soybean mosaic virus (SMV) NIa protease triggers cell death in soybean protoplasts when cleaved by this protease, indicating that the PBS1 decoy approach will work in soybean using endogenous PBS1 genes. Lastly, we show that activation of the AvrPphBdependent cell death response effectively inhibits systemic spread of SMV in soybean.

$R$ gene regulation mediated by miRNA/phasiRNA during plant defense response against $P$. syringae A. DEL-ESPINO (1), D. Lopez Marquez (2), N. Lopez-Pagan (3), E. A. Rodríguez-Negrete (4), I. Rubio Somoza (5), J. Ruiz-Albert (6), E. Rodríguez Bejarano (7), C. R. Beuzon Lopez (6), (1) Univ of Malaga, Spain; (2) Univ de Malaga, Malaga, Spain; (3) University of Malaga, Spain; (4) Instituto Politécnico Nacional, Sinaloa, Mexico; (5) CENTRE FOR RESEARCH IN AGRICULTURAL GENOMICS, Spain; (6) Univ of Malaga, Malaga, Spain; (7) IHSM-UMA-CSIC, Málaga, Spain

In plants, two main types of noncoding small RNA molecules have been found: microRNAs (miRNAs) and small interfering RNAs (siRNAs), differing these in their biogenesis and mode of action, but sharing similar sizes (20-24 nt). In plants, their mature forms are products of the activity of DCL proteins and can act as negative regulators of gene expression. In recent years, the role of miRNAs in regulation of gene expression in plant responses against bacterial pathogens is becoming clearer. Comparisons carried out in our lab between expression profiles of different Arabidopsis thaliana mutants affected in gene silencing, and plants challenged with Pseudomonas syringae pathovar tomato DC3000, led us to identify a set of uncharacterized R genes, belonging to the TIR-NBS-LRR gene family, as differentially expressed in these conditions. By bioinformatics tools, we found a miRNA* of $22 \mathrm{nt}$ putatively responsible for down-regulating expression of these R genes. We have also found that the corresponding pri-miRNA is down-regulated after PAMP-perception. We demonstrate that plants with altered levels of this miRNA* (knockdown lines or overexpression lines) exhibit altered PTI-associated phenotypes, suggesting a role for this miRNA* in this defence response against bacteria. We have characterized the expression pattern of both primiRNA and its best target R genes. Finally, we identify phasiRNAs that arise from the transcript of this R gen in a miRNA*-RDR6-DCL4-dependent manner.

Mapping of NLP Recognition and Immune Response in Brassica napus by Bulk-Segregant Analysis H. A. YALCIN (1), R. H. Ramirez-Gonzalez (1), H. J. Schoonbeek (1), C. J. Ridout (2), (1) John Innes Centre, Norwich, U.K.; (2) John Innes Centre (JIC), Norwich Research Park, Norwich, U.K.

Brassicas are important crops susceptible to significant losses caused by disease. Breeding resistant lines can mitigate the effects of pathogens. The first level of the plant immune response is PAMP-triggered immunity (PTI). PAMPs (Pathogen associated molecular pattern) are conserved molecules of pathogens that elicit host defence response. We studied the response to NLPs in Brassica napus. NLPs are found in a wide range of phytopathogens, including major disease-causing fungal species that infect Brassicas. Therefore, improved understanding of the NLP recognition mechanisms could enable disease resistant crops to be developed. The aim of the study is to map the responsible regions and genes in B.napus genome linked to NLP recognition and understand their contribution to the immune system. To identify genes controlling NLP response, Bulk Segregant Analysis (BSA), has been used. A segregating population was developed from a cross between two NLP responsive and nonresponsive oilseed rape accessions. To create the diverse DNA pools, the production of Reactive Oxygen Species (ROS) in response to NLPs was measured in $925 \mathrm{~F} 2$ lines. The segregation ratio indicates there is both a qualitative and quantitative aspect to the NLP-response. Illumina sequencing was performed on pooled DNA. The outcome will be analysed by comparing to parental genotypes. Results will enable identification of genetic markers that could be used to develop more durable disease resistance in Brassicas.

Rice RPM1-like gene OsRPML1 mediates in rice blast resistance

G. Ma, Q. Xing, Z. Guo, X. CHEN, China Agricultural University, Beijing, China

Rice blast caused by Magnaporthe oryzae, one of the top 10 fungal pathogens, is a widely spread rice disease. In this study, we report a nucleotide-binding site (NBS)-leucine rich repeat (LRR) gene OsRPML1, which confers resistance to M. oryzae in rice. OsRPML1 is rapidly induced by flg22 and chitin treatments, as well as by both virulent and avirulent pathovars of M. oryzae. Quantitative real time PCR assays showed that the expression levels of OSRPML1 is significantly different between japonica and indica rice varieties, consistant with rice blast disease resistance. Knockout of OsRPML1 by means of CRISPR/Cas9 exhibited enhanced susceptibility against M. oryzae compared to the wild type control. Further, cis elements were verified through $O S R P M L 1$ promoter deletion and mutant analysis in tobacco leaves through transient expression system. We found that different $O s R P M L 1$ expression levels due to cis-element base difference between japonica and indica rice varieties and the $\mathrm{C} 2 \mathrm{H} 2$-type transcription factor could interact with the above cis-element by yeast one-hybrid assay. Taken together, these results suggest that $O S R P M L 1$ play a role in rice resistance against $M$. oryzae.

Involvement of Miraculin-Like Protein genes in the coffee immune response to nematode L. A. Vidal (1,2), P. Grynberg (1), A. S. Petitot (3), A. Z. Mota (1,4), R. Togawa (1), D. FERNANDEZ (1,3), E. V. S. Albuquerque (1), (1) Embrapa Genetic Resources and Biotecnology, Brasilia, Brazil; (2) Universidade de Brasilia, Brasilia, Brazil; (3) IRD- French Research Institute for Development, UMR IPME, IRD, Cirad, UM, Montpellier, France; (4) UFRGS, Porto Alegre, Brazil

Meloidogyne incognita root-knot nematode (RKN) is a very harmful pathogen in coffee (Coffea arabica) plantations. Resistant coffee roots exhibiting hypersensitive-like (HR) response to $M$. incognita showed deregulation of miraculin-like genes in our RNA-seq transcriptome analysis. Miraculin-like proteins (MLPs) are members of the plant Kunitz serine trypsin inhibitor (STI) family of proteinase inhibitors (PIs). Some evidences in the literature point out to MPL involved in coffee reaction to biotic stresses. We aimed to study the expression of Kunitz type PI genes in coffee by i) homologous searches in coffee genomic and transcriptomic data and ii) real-time qPCR validation of candidate genes. In silico differential expression and GO enrichment results identified 14 genes of the Kunitz type PIs, similar to MLPs that were activated in the coffee inoculated plants. We found 32 gene members for the STI family in coffee and designed specific qPCR primers. Effectively, we observed differential expression in our qPCR analysis of resistant coffee roots upon M. incognita infection. MLPs are probably important players in the coffee-RKN incompatible interaction. 
It pays to be different: A non-transcriptional TAL dependent resistance mechanism in rice

A. I. HUERTA (1), T. Borland (1), L. Triplett (2), J. E. Leach (3), (1) Colorado State University, Fort Collins, CO, U.S.A.; (2) The Connecticut Agricultural Experiment Station, New Haven, CT, U.S.A.; (3) Colorado State University, CO, U.S.A.

Plant pathogenic Xanthomonasspp. secrete transcription activator-like effectors (TALEs). TALEs induce expression of host susceptibility genes via a highly specific TALE-DNA binding mechanism, which is conferred by the repeat variable diresidue (RVD) in the central repeat region (CRR) of the protein. To circumvent pathogen infection, plants have evolved TALE-associated resistance, including loss of susceptibility, dominant TALE-dependent and dominant transcription-independent resistance. Bs4 from tomato and Xo1 from rice fall under the latter category, yet their mechanistic details remain unknown. Both proteins are independent of TALE-DNA binding and hypothesized to be dependent on cytoplasmic recognition of a pattern in the CRR. To better understand how TALEs trigger or escape Xo1 detection, we aligned the CRR protein sequences of Xo1-triggering TALEs with non-triggering effectors. We found consistent variation in four non-RVD amino acids (AA) in each repeat. These same AA were predicted to be solvent-exposed. To test their role in triggering Xoland Bs4, we swapped AA residues at these key positions in resistance (PthXo1)- and susceptibility (AvrHah1)-inducing TALEs. PthXo1 variant TALEs lost their resistance-triggering ability in both rice and tomato, indicating that Xo1 and Bs4 recognition is sensitive to solvent-exposed residues, expanding our knowledge of resistance mechanisms in Xanthomonas-plant interactions.

Sugar transporters contribute to defense activation in Arabidopsis K. YAMADA (1), Y. Takano (2), (1) Max Planck Institute for Plant Breeding Research, Germany; (2) Graduate School of Agriculture, Kyoto University, Kyoto, Japan

Sugar acts as not only a metabolic energy source but also a signaling molecule in most creatures. Plants, autotrophic organisms, fix atmospheric carbon dioxide to sugar via photosynthesis. On the other hand, heterotrophic organisms, such as microbial pathogens, need to acquire carbon from other organisms. We previously showed that host plants prevent sugar loss to bacterial pathogens by enhancing sugar uptake activity through phosphorylation-dependent sugar transporter regulation. It results in a restriction of bacterial energy source and sugar-inducible bacterial effector delivery. Here we presented that sugar uptake through sugar transporters also contributes to host defense activation. We found that loss of three sugar transporter genes caused enhanced susceptibility to bacterial pathogens and less activation of pattern-triggered immunity (PTI) in Arabidopsis. These results implied that sugar signaling and PTI converge to achieve full activation of defense responses. Taken together, our findings illuminated that sugar transporter plays a central role in host defense through limiting pathogen virulence and activating own defense responses.

Leaf rust induced, hypersensitive-like response in EMS mutated wheat J. P. FELLERS, USDA ARS, Manhattan, KS, U.S.A.

Puccinia triticina is a wheat obligate biotrophic pathogen causes severe yield losses worldwide. Generally, single, major gene resistance has been used to provide protection against infection, but because of high rates mutation in the pathogen, resistance genes have a limited window of effectiveness. Broad spectrum, minor resistance wheat genes appear to be involved in the biotrophic interaction of rust and wheat. Thus, an EMS mutagenesis approach was taken to identify other genes involved in host-pathogen interactions. $\mathrm{M}_{1}$ lines of leaf rust susceptible Thatcher were infected with $P$. triticina race BBBD to identify individuals with reduced infection. One line, 531, was found to have an induced hypersensitivelike response when infected with spores. The response was pronounced at $4 \mathrm{dpi}$ and further developed into necrotic lesions by $14 \mathrm{dpi}$. Newly emerged, non infected leaves did not have lesions. Bulk segregate analysis of cDNA from F2:3 families are being sequenced and the results will be presented.

Mechanistics of surfactin perception in plant immunity elicitation by Bacillus M. Deleu, L. Lins., M. ONGENA, Liege University, Belgium

The Bacillus velezensis species is of particular interest regarding its biocontrol activity which is mainly due to its potential to produce a variety of bioactive secondary metabolites acting either as signals or as antimicrobials. This includes cyclic lipopeptides involved in rhizosphere fitness, direct antagonism of pathogens or host resistance stimulation. The surfactin (SF) lipopeptide has been identified as main Bacillus product triggering immune-related defense responses and Induced Systemic Resistance (ISR). However, the mechanistics underlying perception of elicitors such as SF typically secreted by beneficial bacteria still remain quite obscure. In this work, novel insights were obtained by combining functional and biochemical assays together with in silico and experimental biophysics. We show that lipopeptide recognition by plant root cells does not involve high-affinity proteic receptors but primarily rely on an unsuspected process at the lipid phase of cell plasma membrane. We hypothesize that SF insertion into specific lipid domains induces some re-organization of this lipid bilayer which could in turn affect the localization and thereby activate key proteins involved in early immune signaling. Such a peculiar mechanism of recognition may explain why SF acts as a priming agent with no impact on fitness upon elicitation but with systemic stimulation of defenses upon infection.

\section{STRUCTURE-BASED STUDY OF THE SR33 COILED-COIL DOMAIN CHANGES PARADIGMS FOR COILED-COIL SELF- ASSOCIATION \\ X. HU, The University of Queensland, BRISBANE, QLD, Australia}

Plants utilize intracellular immunity receptors, known as NLRs (nucleotide binding [NB], leucine-rich repeat [LRR]/nucleotide-binding oligomerization domain [NOD]-like receptors) to recognize specific pathogen effector proteins and induce immune responses. These proteins provide resistance to many of the world's most destructive plant pathogens, yet many molecular details involving NLRs remain undefined. Some plant NLRs utilize an N-terminal coiled-coil (CC) domain to facilitate downstream signaling, which has been shown to be necessary and 
sufficient for cell death response in a number of plant immune systems. Hear we examined the active constructs of CC domain in wheat NLR protein $\mathrm{Sr} 33$ and demonstrate its oligomerization determining immune activation. Truncations and mutants are created at the end of the Cterminus of the final helix of the $\mathrm{Sr} 33 \mathrm{CC}$ domain, which demonstrate that this final helix is important for self-association in active $\mathrm{Sr} 33 \mathrm{CC}$ domain. Combining in situ proteolysis and mass spectrometry, we successfully crystallized a final helix containing form of Sr33 CC domain and collected a dataset to $\sim 3 \AA$ A resolution. Molecular replacement using the published Sr33 crystal structure has not been successful, suggesting that the structure of our crystallized form differs from the published one (without final helix).

Sweet orange expressing the immune receptor AtEFR is able to recognize and trigger immune defense responses against bacterial citrus diseases

L. Kuster Mitre (1,2), R. Rodrigues de Souza Neto (1,2), R. Caserta (1), N. DE SOUSA TEIXEIRA E SILVA (1), C. Zipfel (3,4), A. A. De

Souza (1), (1) Sylvio Moreira Citrus Research Center, Agronomic Institute (IAC), Cordeirópolis, Brazil; (2) University of Campinas, UNICAMP, Campinas, Brazil; (3) The Sainsbury Laboratory, University of East Anglia, Norwich Research Park, Norwich, U.K.; (4) Inst. of Plant and Microbial Biology, University of Zürich, Zurich, Switzerland

Previous work showed that heterologous expression of the EFR pattern recognition receptor from Arabidopsis thaliana, a leucine-rich repeat receptor kinase recognizing bacterial EF-Tu (or the derived peptides elf18/elf26), provides broad-spectrum resistance against bacterial pathogens in different species, such as tobacco, tomato, rice, wheat, and potato. Transgenic sweet orange constitutively expressing $A t$ EFR were generated to access whether this receptor would also confer increased anti-bacterial resistance in citrus. After genetic confirmation of transgenic events, we confirmed $A t$ EFR functionality by measuring reactive oxygen species (ROS) production, expression of immune marker genes and bacterial infection in ten transgenic events. Notably, detached leaves from transgenic plants infiltrated with Xanthomonas citri (the causal agent of citrus canker) showed less symptoms and reduced bacterial population. Some transgenic lines showed higher resistance to canker disease since no necrosis or petiole abscission were observed. Similar assays are under analysis for Xylella fastidiosa (the causal agent of CVC - Citrus Variegated Chlorosis). Altogether, our results in sweet orange indicate that EFR has great potential to improve broad-spectrum anti-bacterial disease resistance in Citrus.

The plant immune system constrains bacterial attempts to remain motile while staying undetected K. PARYS (1), N. R. Colaianni (2), H. S. Lee (3), N. Edelbacher (1), A. Trgovcevic (1), Z. Blahovska (4), M. Madalinski (5), N. Geldner (6), C. Jones (7), J. L. Dangl (8), Y. Belkhadir (9), (1) Gregor Mendel Institute, Vienna, Austria; (2) UNC Chapel Hill Biology, Chapel Hill, NC, U.S.A.; (3) Gregor mendel insititute, Vienna, Austria; (4) Gregor Mendel Institute, Austria; (5) Research Institute of Molecular Pathology, Austria; (6) University of Lausanne, Switzerland; (7) Carolina Center for Genome Sciences, Chapel Hill, U.S.A.; (8) Department of Biology, University of North Carolina at Chapel Hill, Chapel Hill, NC, U.S.A.; (9) Gregor Mendel Inst of Molecular Plant Biology GmbH, Vienna, Austria

Plants sense and respond to microbes with an arm of the plant immune system that relies on the detection of microbial-associated molecular patterns (MAMPs) by cell surface pattern-recognition-receptors (PRRs). Stereotypical MAMPs include several epitopes derived from flagellin molecules, the building-block of bacterial flagella. In Arabidopsis, the PRR FLAGELLIN-SENSING 2 (FLS2) detects a highly conserved 22amino acid epitope (flg22) from the $\mathrm{N}$-terminus of flagellin. flg22 is evolutionary conserved and mutations of conserved amino acids that subvert immune receptor detection have deleterious effect on flagellar function and bacterial motility. Here, we set out to define how tradeoffs between motility and host recognition have been molecularly balanced throughout evolution. To circumvent the shortcoming of working in a limited flg22 natural evolutionary space, we artificially evolved $>1000$ flg22 variants and tested their interaction with FLS2. We will present work defining the molecular constrains and associated mechanisms that have impinged on bacterial attempts to evade immune system activities while remaining motile.

Rpp1 encodes an NBS-LRR protein with an integrated Ubiquitin-Like Protease 1 (ULP1) domain that controls the immune response to Phakopsora pachyrhizi

K. F. PEDLEY (1), A. K. Pandey (2), A. Ruck (1), L. M. Lincoln (3), S. A. Whitham (2), M. A. Graham (3), (1) USDA ARS FDWSRU, Frederick, MD, U.S.A.; (2) Iowa State University, Ames, IA, U.S.A.; (3) USDA ARS CICGRU, Ames, IA, U.S.A.

Phakopsora pachyrhizi is the causal agent of Asian soybean rust. Although most soybean accessions are susceptible, seven loci (Rpp1 - Rpp 7) that provide varying degrees of resistance to $P$. pachyrhizi (Rpp) have been identified. Rppl maps to a region on soybean chromosome 18 between markers Sct_187 and Sat_064 in an isoline developed from Williams 82 and PI 200492 (Rpp1). Rpp1 is unique among the Rpp genes in that it confers an immune response (IR) to avirulent $P$. pachyrhizi isolates. The IR is characterized by a lack of visible symptoms, whereas resistance provided by all other known Rpp genes results in red-brown (RB) foliar lesions. To identify Rpp1, we constructed a bacterial artificial chromosome (BAC) library from soybean accession PI 200492. Sequencing a $324 \mathrm{~kb}$ region of contiguous BACs spanning the Rpp1 locus identified three homologous nucleotide binding site-leucine rich repeat (NBS-LRR) candidate resistance genes between Sct_187 and Sat_064 Each candidate gene is also predicted to encode an N-terminal ubiquitin-like protease 1 (ULP1) domain. Co-silencing of the Rppl candidates resulted in the formation of RB lesions in response to an avirulent isolate of P. pachyrhizi. This suggests that silencing Rppl compromised the IR, but not resistance. Further investigation into the role of the Rpp1 ULP1 domain is an important future goal that should provide additional insight into soybean defense mechanisms and $P$. pachyrhizi virulence determinants.

Evolution of a guarded decoy protease and its receptor in Solanaceae

J. KOURELIS (1,2), S. Malik (3), O. Mattinson (2), S. Krauter (2), P. Kahlon Singh (4), J. K. Paulus (3), R. A. L. Van Der Hoorn (5), (1) The Sainsbury Laboratory, Norwich, U.K.; (2) University of Oxford, U.K.; (3) University of Oxford, Oxford, U.K.; (4) Technische Universität München, Freising, Germany; (5) Department of Plant Sciences, University of Oxford, Oxford, U.K.

Cf-2-dependent recognition of the Cladosporium fulvum effector Avr2 requires the tomato papain-like cysteine protease Rcr3. It has been proposed that Rcr3 acts as a decoy for the recognition of Avr2, and that the Rcr3-related Pip1 is the operative target of Avr2. Here we show that 
Rcr3 and Pip1 are evolutionary conserved in Solanaceae, whereas Cf-2 belongs to a Solanum-specific gene family. Surprisingly, Nicotiana benthamiana is a natural knock-out for Rcr3 and Pipl, and both genes can be resurrected with minimal changes to encode for active proteases. Transient co-expression of Cf-2, Rcr3, and Avr2 in N. benthamiana triggers a hypersensitive response (HR). This HR correlates with the sensitivity of Rcr3 for inhibition by Avr2, which is determined by four residues in Rcr3. However, the inhibition of Rcr3 homologs from different Solanaceae species by Avr2 does not necessarily result in a HR. Instead, the ability of these Rcr3 homologs to induce a HR upon co-expression with Cf-2 and Avr2 correlates with the phylogenetic distance to tomato. Combined this shows that $C f-2$ evolved recently and co-opted $R c r 3$ for the recognition of Avr2. This highlights how genetically unlinked indirect recognition mechanism initially evolve and can aid in the application and rational engineering of such mechanisms.

Medicago truncatula genes involved in resistance against Asian Soybean Rust confers partial resistance in soybean Y. Ishiga (1,2), U. S. Gill (2), S. R. Uppalapati (2), B. Pant (2), K. S. MYSORE (2), (1) University of Tsukuba, Tsukuba, Japan; (2) Noble Research Institute, Ardmore, OK, U.S.A.

Tobacco retrotransposon, Tnt1, has been used to mutagenize and tag the whole genome of a model legume, Medicago truncatula. A forward genetics approach using Tnt 1 tagged M. truncatula lines has been established to identify M. truncatula genes that confer resistance to Asian Soybean Rust pathogen, Phakopsora pachyrhizi. Several M. truncatula Tnt1 mutants with altered response to P. pachyrhizi have been identified and being characterized. Identified mutants belong to four different classes namely inhibitor of rust germ-tube differentiation (irg), enhanced cell death upon rust infection (ecd), enhanced penetration of rust (epr), and sporulation of rust ( $(p p r)$. Genes mutated in some of these mutants have been identified. Characterization of these mutants will be presented. For example, an irgl mutant, which has a mutation in a Cys(2)His(2) zinc finger transcription factor, inhibited pre-infection structure differentiation of $P$. pachyrhizi and several other biotrophic pathogens. Overexpression of some of the identified M. truncatula genes in soybean confers partial resistance against Asian Soybean Rust.

Uncovering host adaptation of Phytophthora cactorum to strawberry and apple C. F. NELLIST, A. D. Armitage, M. K. Sobczyk, R. J. Vickerstaff, H. J. Bates, R. J. Harrison, NIAB EMR, East Malling, U.K.

Phytophthora cactorum $(P c)$, a water-borne oomycete causes disease in a variety of plant species, including two diseases in cultivated strawberry (Fragaria $\mathrm{x}$ ananassa), crown rot (CR; causing whole plant collapse) and leather rot (LR), affecting the fruit. In cultivated apple (Malus $\times$ domestica) $P c$ causes bark rots on the scion (collar rot) and rootstock (crown rot), as well as necrosis of the fine root system (root rot) and fruit rots. We investigated the basis of $P c$ pathogenicity on strawberry, through genome sequencing and comparative genomics at the species ( $P c$ vs Phytophthora idaei), pathotype (strawberry, CR and LR vs apple) and population (within strawberry CR) levels. Whole genome phylogenetic analysis showed resolution between CR and apple infecting isolates, providing genomic support for discrete lineages within $P c$. We further identified the gain and loss of gene compliments across the $P c$ phylogeny, including apoplastic and cytoplasmic effectors. This identified gains and losses of previously characterised and novel effector candidates, representing putative determinants of host boundaries. Transcriptome analysis has given insight into key effectors during strawberry infection. Functional validation of these candidates, alongside integration of this information with identified QTL for $P c$ resistance will lead to improved disease resistance in strawberry.

Antagonistic functions of selective SUMO paralogs regulate innate immunity in Arabidopsis thaliana K. D. INGOLE (1), M. Kasera (2), S. Bhattacharjee (3), (1) Regional Centre for Biotechnology, NCR Biotech Science Cluster, Faridabad., Faridabad, India; (2) SRF, Regional Centre for Biotechnology, NCR Biotech Science Cluster, Faridabad., Faridabad, India; (3) Assistant Professor, Regional Centre for Biotechnology, Faridabad, Faridabad, India

Signaling networks in plant innate immunity are orchestrated by extensive modulation of post-translational modifications (PTMs) of transducers and receivers. Covalent attachment by the Small Ubiquitin-Like Modifiers (SUMOs) on target proteins alter properties such as localization, stability, and activation/inactivation. In Arabidopsis thalianafour SUMO paralogs are expressed. Although partial redundancy is demonstrated between selective paralogs, clear demarcation of specificity remains unknown. Using well characterized defense signaling cascades induced upon infection with the hemi-biotrophic pathogen Pseudomonas syringae, we identify antagonistic functions between selective SUMO paralogs. While SUMI and SUM2additively function as negative regulators, SUM3positively regulates defenses. A lack of SUM1or SUM2primes expression of defense-related markers thereby resulting in enhanced defenses. Interestingly, most defensive genes induced comprise the salicylic acid signaling routes. The loss of SUM3in a SUMImutant tones down enhanced defenses suggesting functional contrasts between the paralogs. We further identify that SUMO3, but not SUMO1, not only forms non-covalent homo- but also hetero-dimers with SUMO1. Covalent interactions both as a donor or an acceptor are allowed only for SUMO1 whereas SUMO3 functions only as donor. Taken together we highlight extensive crosstalks between SUMO paralogs for regulation of plant innate immune responses.

\section{A pathogen turns 'immunity on' to 'immunity off' with a flick of the switch}

Q. HE (1), S. Naqvi (2), H. McLellan (3), L. Yang (4), M. R. Armstrong (5), E. M. Gilroy (6), P. R. J. Birch (7), (1) Division of Plant Science, James Hutton Institute, University of Dundee, Dundee, U.K.; (2) Division of Plant Sciences, University of Dundee, Dundee, U.K.; (3) Cell and Molecular Sciences, the James Hutton Institute, Dundee, U.K.; (4) University of Dundee, Dundee, U.K.; (5) The James Hutton Institute, Invergowrie, Dundee, U.K.; (6) The James Hutton Institute, Dundee, U.K.; (7) Cell and Molecular Sciences, James Hutton Institute, Dundee, U.K.

Plant pathogens, such as the potato blight agent Phytophthora infestans, deliver effectors into plant cells to promote disease. Recent evidence suggests that some effectors exploit endogenous negative regulators of immunity, or so-called susceptibility (S) factors, to inactivate the immune system. Previously, we found that $P$. infestans RXLR effector Pi02860 uses the potato protein StNRL1 as an S factor to target a positive regulator of immunity, the guanine exchange factor StSWAP70, for proteasome-mediated degradation (He and Naqvi et al 2018 PNAS 115:E7834-E7843). StNRL1 is a member of the NPH3 and RPT2-like family of CULLIN 3 ubiquitin E3 ligases that act downstream of the blue light regulators, phototropins. Remarkably, we found that StNRL1 and the effector Pi02860 interact in yeast and in planta with 14-3-3 proteins. We found that 14- 
3-3 acts to positively regulate plant immunity. 14-3-3 functions as a phosphoprotein molecular switch, interacting with StNRL1 in order to reduce or prevent its interaction with SWAP70. The effector Pi02860 potentially acts as a decoy to misdirect 14-3-3 away from its intended interaction sites in StNRL1, freeing StNRL1 to interact with and degrade SWAP70. This illustrates an example of the complex molecular battle between plant and pathogen in which an immunity 'on' switch is turned 'off' by an effector. Critically, as many plant pathogen effectors target 14-3-3 proteins, our results provide a model by which such effectors may operate.

Induction of autophagy requires MoPRMT1 methylation of MoSNP1 in Magnaporthe oryzae W. LIU (1), G. L. Wang (2), (1) Inst of Plant Protection, CAAS, Beijing, China; (2) Department of Plant Pathology, The Ohio State University, Columbus, OH, U.S.A.

Autophagy is essential for conidiation and appressorium-mediated plant infection by Magnaporthe oryzae, the causal agent of rice blast disease. Thus far, the regulatory mechanism of vegetative growth and pathogenicity-associated autophagy remains largely unknown. Here, we characterized the function of MoPRMT1, a homolog of yeast Hmtl that encodes a protein arginine methyltransferase (PRMT) in M. oryzae. Knock out of Moprmt1 results in inhibited growth and a decreased ability to cause disease lesions on rice seedlings. MoPRMT1 is localizes to autophagosomes, and the Moprmt1 mutants display defects on autophagy induction under nitrogen starvation. MoPRMT1 catalyzes the methylation of MoSNP1 in vitro, in a manner dependent on direct interaction. RNA-seq analysis revealed that deletion of Moprmt 1 leads to splicing defects in more than 500 genes involved in multiple biological processes. The splicing defects are identified in transcripts of several autophagy-related (ATG) genes involved in control of the formation of autophagosomes. Our findings suggest that MoPRMT1 regulates the formation of autophagosomes through methylation of MoSNP1 protein to control the pre-mRNA splicing process of ATG genes in M. oryzae. Our study depicts that arginine methylation plays an essential role in accurate pre-mRNA splicing necessary for diverse developmental processes, including autophagosome formation.

An acetyltransferase required for modulation of innate immunity in rice N. A. ESPINAS (1), N. T. Le (2), Y. Shimajiri (3), K. Shirasu (1), H. Saze (2), (1) RIKEN Center for Sustainable Resource Science, Yokohama, Japan; (2) Okinawa Institute of Science and Technology Graduate University, Onna-son, Japan; (3) University of Kyushu, Fukuoka, Japan

$C B P / p 300$ family of genes are well-known for its functions in bridging DNA-binding proteins, recruiting transcription factors, and exhibiting acetyltransferase activities on target proteins in differentiation and developmental processes. How $C B P / p 300$ functions in transcriptional reprogramming and modulation of the chromatin state of plant defense genes are unclear. Here, we report that $r C B P$ [rice Cyclic adenosine monophosphate response element-binding protein (CREB) Binding Protein], a member of the rice $C B P$ family, was earlier identified to be responsive to flg22 induction. Isolation of $r C B P$ CRISPR-Cas9 mutant lines revealed variable disease phenotype responses to a number of rice pathogens, while having wild type number of effective grains. Analysis of histone acetyltransferase activity in RNAi transgenic and CRISPRCas9 mutant lines showed that $\mathrm{H} 3$ lysine sites are possibly targeted by rCBP with very high acetylation specificity on H3K9. Thus, our study reveals a possible role of $\mathrm{rCBP}$ in regulating innate immunity in rice.

Quantitative phosphoproteomic analysis reveals common regulatory mechanisms between NLR- and PRR-triggered immunity in plants. Y. KADOTA (1,2), T. W. H. Liebrand (3), Y. Goto (1), J. Sklenar (2), P. Derbyshire (2), F. L. H. Menke (2), M. A. Torres (4), A. Molina (5), C. Zipfel (2,6), G. L. Coaker (3), K. Shirasu (7), (1) RIKEN Center for Sustainable Resource Science, Plant Immunity Research Group, Yokohama, Japan; (2) The Sainsbury Laboratory, University of East Anglia, Norwich Research Park, Norwich, U.K.; (3) University of California, Davis, CA, U.S.A.; (4) Universidad Politécnica de Madrid, Pozuelo de Alarcon, Spain; (5) Centro de Biotecnología y Genómica de Plantas, Universidad Politécnica de Madrid, Pozuelo de Alarcón, Spain; (6) The Sainsbury Laboratory, University of East Anglia, Norwich Research Park,, Norwich, U.K.; (7) RIKEN Center for Sustainable Resource Science, Yokohama, Japan

Plant immunity consists of two arms: surface-localized pattern recognition receptor (PRR-triggered immunity (PTI) and intracellular nucleotidebinding domain leucine-rich repeat receptor (NLR-triggered immunity. Despite structural similarity, both receptor types activate similar responses with different dynamics. To better understand phosphorylation events during NLR-triggered immunity, we employed a screen using an inducible expression system of the bacterial effector avrRpt2 in Arabidopsis thaliana, and identified 109 residues of membrane-associated proteins on activation of the intracellular RPS2 receptor. Interestingly, several RPS2-regulated overlap with those that are regulated during PTI, suggesting that these may be convergent points of both signaling arms. Moreover, some of these sites are residues of important defense components, including the NADPH oxidase RBOHD, ABC-transporter PEN3, calcium-ATPase ACA8, G $\alpha$ protein XLG2 and $\mathrm{H}^{+}$-ATPases. In particular, we found common phosphorylation sites of RBOHD during both immunities. These sites are required for the production of reactive oxygen species and immunity against bacteria and a virulent necrotrophic fungus. We provide, for the first time, large-scale phosphoproteomic data of NLR-triggered immunity, thereby suggesting crucial roles of common in plant immunity.

SUMOylation is important for fungal development and pathogenicity in the rice blast fungus, Magnaporthe oryzae Y. J. LIM (1), K. T. Kim (1), Y. H. Lee (1,2,3,4), (1) Department of Agricultural Biotechnology, Seoul National University, Seoul, Korea, Republic of (South); (2) Plant Immunity Research Center, Seoul National University, Seoul, Korea, Republic of (South); (3) Center for Fungal Genetic Resources, Seoul National University, Seoul, Korea, Republic of (South); (4) Research Institute of Agriculture and Life Sciences, Seoul National University, Seoul, Korea, Republic of (South)

Among the post-translational modifications, SUMOylation is a conserved and essential process to regulates many biological mechanisms including transcriptional regulation, protein stabilization, cell cycle, DNA repair, and pathogenesis in eukaryotes. However, the functional role of SUMOylation is not well understood in plant fungal pathogens including Magnaporthe oryzae. In this study, we elucidated the roles of four SUMOylation-associated genes that encode one SUMO protein (MoSMT3), two E1s (MoAOS1 and MoUBA2), and one E2 (MoUBC9) in fungal development and pathogenicity. Western blot assay showed that SUMO modification was abolished in all deletion mutants. MoAOS1 and MoUBA2 were mainly localized in the nucleus whereas MoSMT3 and MoUBC9 were localized in both the nucleus and cytoplasm. However, 
when exposed to oxidative stress, the four SUMOylation-associated proteins were localized in the nucleus. All deletion mutants were viable, but showed significant defects in mycelial growth, conidiation, septum formation, conidial germination, appressorium formation, and pathogenicity. In addition to infection-related development, SUMOylation is also required for nutrient starvation, DNA damage, and oxidative stresses tolerance. Taking together, SUMOylation is important for fungal development, stress responses, and pathogenicity in M. oryzae. This study provides new insight into the role of SUMOylation in the molecular mechanisms of pathogenesis of the rice blast fungus and beyond.

Shining a light on the black box of effector-triggered immunity signaling

S. L. JOHNSON, P. Ding, H. Guo, P. Derbyshire, J. D. Jones, F. L. H. Menke, The Sainsbury Laboratory, University of East Anglia, Norwich Research Park, Norwich, U.K.

Conserved pathogen-associated molecular patterns (PAMPs), are detected by cell-membrane receptors, which activate PAMP-triggered immunity (PTI). Pathogen effectors, which usually carry virulence roles in manipulating host immunity, are monitored by intracellular NLR protein receptors, which can activate effector-triggered immunity (ETI) and lead to the hypersensitive cell death response (HR). The first NLR gene was cloned twenty-five years ago, but while activation of NLR proteins can initiate a rapid accumulation of defence-related genes (within two to four hours postinfection), the downstream signaling from NLR proteins is still largely unknown. Post-translational modifications (PTMs) are crucial for PTI signaling, and protein phosphorylation plays a prominent role. Whether similar mechanisms are required for ETI is not known. Recently, changes in protein phosphorylation have been observed upon triggering ETI and showed some overlap with PTI-induced phosphorylation. We aim to identify the regulatory mechanisms at the earliest time point upon activation of the RRS1/RPS4 receptor complex through profiling changes in protein phosphorylation. In a pathogen-free inducible ETI system, we can robustly trigger ETI signaling and immediate early defense gene expression. We are building a comprehensive phosphoproteome library and are using targeted proteomics to map the earliest phosphorylation events that occur upon effector recognition.

\section{CDC48A protein level is regulated by SCF-SNIPER7}

K. AO, M. Tong, X. Li, University of British Columbia, Vancouver, BC, Canada

The homeostasis of many plant proteins is tightly regulated for proper development and stress responses. Many important signaling components in plant immunity are regulated by complex E3 ligase complexes that specifically target and ubiquitinate their substrates. The $26 \mathrm{~S}$ proteasome complex then degrades the poly-ubiquitinated substrates. CDC48A, a protein evolutionarily conserved in eukaryotes, can aid in this process by specifically extracting ubiquitinated target proteins from complexes. Mutations in these protein homeostasis regulation components can result in autoimmunity due to overaccumulation of their substrates. For example, a partial loss-of-function mutant of CDC48A, $c d c 48 a-4$ is dwarfed and exhibits enhanced resistance to pathogens. The exact role CDC48A plays in plant immunity is unclear. Using a reverse-genetics approach, we identified an F-box protein, SNIPER7, that exhibits autoimmune phenotypes similar to $c d c 48 a-4$ when overexpressed. Through IP-MS using SNIPER7 as bait, we identified CDC48A as a strong interactor of SNIPER7. Interestingly, biochemical assays revealed that CDC48A levels are reduced and stabilized in plants expressing SNIPER7 and dominant negative SNIPER7, respectively, suggesting that CDC48A levels are regulated by SCF-SNIPER7. Overall, our data supports a model where SNIPER7 is induced after infection for degradation of CDC48A in order to accumulate sufficient immune proteins necessary for a robust defense response.

MAPK regulation of chromatin-associated factors during biotic stress in Arabidopsis: A phosphoproteomic approach Arsheed Sheikh, Naganand Rayapuram, Heribert Hirt

King Abdullah University of Science and Technology (KAUST), Saudi Arabia

Pathogen recognition triggers Mitogen Activated Protein Kinase (MAPKs) activation, which coordinates external cues to changes in plant gene expression. Given the robust subcellular localization of MAPKs, we focused on the chromatin phosphoproteomics of WT and $m p k 3, m p k 4$, and mpk6 mutant plants. A number of novel nuclear targets were identified for MPK3, MPK4 and/or MPK6. These targets include key regulatory proteins of cell division, development and immunity. Out of the targets, 165 proteins were identified to be differentially phosphorylated after Microbe Associated Molecular Pattern (MAMP) treatment. Using different criteria, a number of MAPK substrates were validated as true substrates of MAPKs. Signaling network analysis revealed their potential role in general cellular processes like RNA metabolism, transcription and chromatin organization, and also specific roles in immunity. Overall, this study unravels a set of chromatin-associated targets of MAPKs and a potential link between MAPK signaling to a number of chromatin-related events.

A ubiquitin E3 ligase regulatory cascade controls defence by modulating the abundance of an immune-suppressive RNA binding protein H. MCLELLAN (1), Q. He (1), P. Boevink (2), A. Sadanandom (3), Z. Tian (4), P. R. J. Birch (2), (1) Division of Plant Science, James Hutton Institute, University of Dundee, Dundee, U.K.; (2) Cell and Molecular Sciences, James Hutton Institute, Dundee, U.K.; (3) Durham university, Durham, U.K.; (4) Huazhong Agricultural University, Wuhan, China

Ubiquitination is a post-translational modification which regulates many processes in plants. Several ubiquitin E3 ligases have been shown to act as either positive or negative regulators of immunity via the degradation of different substrates. StPUB17 is an E3 ligase which has previously been shown to be a positive regulator of immunity to the oomycete pathogen Phytophthora infestans. Silencing of PUB17 promotes pathogen colonisation and attenuates Cf4/avr4 cell death and flg22 signaling. Using yeast-2-hybrid and co-immunoprecipitation we identified the putative K-homology $(\mathrm{KH})$ RNA binding protein, StKH17, as a target for degradation by PUB17. KH17 acts as a negative regulator of immunity which promotes $P$. infestans infection and suppresses Cf4/avr4 Cell death. As StPUB17 is a known target of the ubiquitin E3 ligase, StPOB1, we reveal the final step in an E3 ligase regulatory cascade that controls plant defence. 
Multiple mechanisms regulate the degradation of steady-state and activated CNL immune receptor RPM1

F. EL KASMI (1), E. H. Chung (2), Z. Namazi (3), J. L. Dangl (4), (1) Center for Plant Molecular Biology (ZMBP), University of Tuebingen, Tübingen, Germany; (2) University of North Carolina, Chapel Hill, NC, U.S.A.; (3) ZMBP/University of Tübingen, Tübingen, Germany; (4)

Department of Biology, University of North Carolina at Chapel Hill, Chapel Hill, NC, U.S.A.

Nucleotide-binding site leucine-rich repeat receptors (NLR) perceive the presence of pathogen-derived effector proteins and activate effectortriggered immunity (ETI) leading to a programmed cell death, termed the hypersensitive response (HR). Deregulation of or mutations in NLRs or in regulators of NLRs often leads to autoimmunity, resulting in severe growth reduction and in some cases lethality. Thus, NLR function needs to be tightly regulated in order to maintain an adequate trade-off between growth and immunity and to ensure a specific and efficient immune response. Regulating the abundance and turnover of NLRs is one way to control NLR activity. Steady state protein levels of Arabidopsis NLRs, SNC1 and RPS2, are regulated through proteasomal degradation. Loss of function mutations in the F-box protein CPR1 result in elevated levels of SNC1 and RPS2, and a severe autoimmune phenotype. Intriguingly, we still lack fundamental knowledge about the regulation and turnover of activated NLR proteins and the influence of the protein degradation machinery on HR and ETI. Arabidopsis CNL RPM1 is rapidly degraded upon activation and the initiation of HR. Interestingly, disappearance and function of activated RPM1 cannot be blocked by proteasome inhibitors. We analyzed whether CPR1 is involved in regulation of steady-state and activated RPM1 protein abundance or if other protein degradation mechanisms, such as autophagy, are operating to regulate the disappearance of activated RPM1.

Characterization of the Pseudomonas syringae (Psy) Effector Network

K. BROWNSTEIN, J. Jelenska, J. T. Greenberg, University of Chicago, Chicago, IL, U.S.A.

Bacterial infection of crops is a major concern for farmers. The model bacterial pathogen Pseudomonas syringae pv syringae B728a (Psy) uses an infection strategy that involves a type III secretion system (T3SS). The T3SS injects effector proteins into plant cells. These effectors may not only modify the host, but also modify themselves to evade the immune response of the host. Individual Psy effectors have been extensively studied in plants; however, research is limited on their biochemical interactions and their consequences within Psy and plant hosts. To date, we have shown that HopZ3 can modify several other effectors to impact immune activation. We have evidence that HopZ3 can interact with at least three other effectors and post-translationally modify them. Currently, we are investigating the biochemical interactions of the effector network to discern whether they make multi-protein effector complexes in vitro, in bacteria, and in plants. The complex sizes are being investigated with size exclusion chromatography using fast protein liquid chromatography (FPLC) and other methods. Our findings revealed the nature of the effector network enabling us to further understand infection outcomes between bacteria and their hosts.

The UBIQUITIN PROTEIN LIGASE (UPL) family regulates salicylic acid-dependent plant immunity Z. WANG, B. Orosa, S. H. Spoel, School of Biological Sciences, University of Edinburgh, Edinburgh, U.K.

The HECT family of E3 ubiquitin ligases are critically important for a variety of cellular processes in eukaryotes. These ligases contain a conserved HECT domain often located in the C-terminus that is responsible for substrate ubiquitination. In Arabidopsis, members of the UBIQUITIN PROTEIN LIGASE (UPL) family have been identified as HECT-domain containing ubiquitin ligases. Among them, UPL1, UPL3 and UPL5 were shown to be involved in plant immunity mediated by the defence hormone salicylic acid (SA). However, little is known about how these UPLs regulate plant immunity. Here we show that UPL1, UPL3 and UPL5 associate with and recruit E3 ligase activity to proteasomes. Moreover, global cellular polyubiquitination levels decreased in $u p l 1, u p l 3$ and $u p l 5$ mutants, suggesting these UPLs control ubiquitination of wide range of substrates. Furthermore, RNA-Seq showed that $u p l$ mutants failed to reprogram the transcriptome in response to SA. SA-induced transcription reprogramming is dependent on the coactivator NPR1. Interestingly, we found that accumulation of NPR1 upon SA treatment or pathogen infection was significantly reduced in $u p l$ mutants compared to WT. Overall, our results indicate UPL1, UPL3 and UPL5 regulate SAinduced cellular ubiquitination levels, control cellular homeostasis of NPR1 protein, and are indispensable for SA- and NPR1-mediated plant immunity.

Chitosan oligosaccharides induce resistance to Pseudomonas syringae pv. tomato DC3000 in Arabidopsis thaliana via distinct Nglycosylation regulation pattern

H. YIN, Dalian Institute of Chemical Physics, Chinese Academy of Sciences, Dalian, China

As an essential posttranslational modification of proteins, glycosylation is involved in a number of biological processes. And evidence is accumulating that protein glycosylation play important roles in plant defense response. However, the function mechanism of $\mathrm{N}$-glycosylation in plant defense response and chitosan oligosaccharide (COS) induced resistance still remains unclear. In the present study, glycomics analysis of Arabidopsis proteins during Pst DC3000 infection and COS induced resistance were carried out by LC-PGC-ESI-MS/MS. Sixty N-glycans were successfully quantified from three biological replicates of four treatment groups Clustering analysis revealing that $\mathrm{N}$-glycans were changed significantly during Pst DC3000 infection and COS pretreatment, moreover, COS pretreatment could reverse the effect of Pst DC3000 on some $\mathrm{N}$-glycans, suggesting $\mathrm{N}$-glycosylation is differently regulated during plant innate immunity and COS induced resistance. In order to further confirm the involvement of N-glycosylation in plant innate immunity and COS induced resistance, and reveal the distinct N-glycosylation regulation pattern during this two processes, N-glycosylation impaired mutants ManI and stt3a were used. stt3a and ManI were shown more severe symptoms than WT when Pst DC3000 infection, and stt3a shown more impaired plant immunity than ManI, suggested that both highmannose N-glycans and complex N-glycans are essential for Arabidopsis defense to Pst DC3000.

Diverse Ubiquitin Chain Linkage Types Orchestrate the Immune Response

B. OROSA (1), J. Strachan (2), C. Spanos (3), J. Rappsilber (3), E. Bayne (4), S. H. Spoel (1), (1) School of Biological Sciences, University of Edinburgh, Edinburgh, U.K.; (2) Institute of Cell Biology, Edinburgh, U.K.; (3) Wellcome Trust Centre for Cell Biology, U.K.; (4) Institute of Cell Biology, U.K. 
The covalent attachment of ubiquitin molecules to proteins is a crucial mechanism for rapid regulation of cell signaling in eukaryotic cells. Ubiquitin can form various homo- and heterotypic polymers on substrate proteins, termed ubiquitin chain linkages. To date, eight different ubiquitin linkage types have been described and the identity and length of the chain plays critical roles in determining the fate of modified proteins. Albeit it is well known that ubiquitin signaling is important for cellular responses against infection, little is known about the role of ubiquitin chain diversity in immunity. Here we employ a linkage-specific affinity purification approach, coupled to mass-spectrometry, to purify and identify proteins modified with different ubiquitin chain linkages in response to infection by the bacterial leaf pathogen Pseudomonas syringae. Our results show that activation of immunity increased the levels of some but not all ubiquitin linkage types, suggesting particular linkage types are specifically associated with immune responses. Furthermore, MS analyses indicate that each linkage is associated with different cellular defence roles. Finally, mutation of immune-associated ubiquitin ligases dramatically altered linkage diversity. Taken together, our findings suggest that multiple ubiquitin linkage types contribute to plant immunity and that linkage diversity is associated with activities of specific immune-associated ubiquitin ligases.

Pathogen-inducible Nucleoredoxin protects plant cells against oxidative stress

J. R. BLEAU, S. Kneeshaw, S. H. Spoel, School of Biological Sciences, University of Edinburgh, Edinburgh, U.K.

Accumulation of reactive oxygen species (ROS) in the cell is associated with several biological processes, including pathogen infection. Although plants have evolved to exploit ROS as signaling molecules, they can cause severe cellular damage if unregulated. The pathogeninducible oxidoreductase Nucleoredoxin1 (NRX1) protects the cell in ROS-rich environments by guarding the activity of antioxidant enzymes involved in the hydrogen peroxide $\left(\mathrm{H}_{2} \mathrm{O}_{2}\right)$ scavenging pathway, including catalase. However, little is known about the role NRX1 plays in curbing oxidative stress independent of antioxidant enzymes. Here we show that NRX1 selectively rescues oxidative stress in antioxidantdeficient plants. While expression of NRX1 was able to rescue oxidative cell death in catalase mutants, it failed to rescue cell death in glutathione-deficient plants, indicating NRX1 functions in specific ROS detoxification pathways. In catalase deficient plants, expression of NRX1 prevented cell death induced by high light and the oxidative stressor methyl viologen. Rescue was associated with suppression of ROSinduced gene expression despite unaltered levels of the key cellular antioxidant glutathione. These results suggest that in addition to protecting the activity of antioxidant enzymes, pathogen-induced NRX1 may control specific ROS signaling pathways during infection.

Ribosome heterogeneity in the control of plant immunity

Y. IZQUIERDO, B. López, C. Castresana, National Centre of Biotechnology, Madrid, Spain

Regulation of protein synthesis is crucial to redistribute cell resources in response to stress. The idea that specialized ribosomes in yeast and metazoans regulate protein synthesis is an old hypothesis that has now gained much attention, however, the higher multiplicity of ribosomal protein (RP) paralogs in plants makes them the ideal model to challenge it. Additionally, we show that higher inter-paralog RP diversity in Arabidopsis increases the potential to generate heterogeneous ribosomes; we thus explore RP diversity as a regulatory mechanism in plant immunity. By combining structural, sequence and expression data we identified low-expression, sequence-divergent RP isoforms as candidates to drive functional ribosome heterogeneity. We found that knock out mutants on these genes show differences in pathogen growth and PR1 accumulation, even when the contribution of these proteins to total ribosomes is presumed negligible. Hence, these altered defense responses indicate specific functions. Interaction of a sub-population of ribosomes with regulatory proteins can also lead to specificity. We show that predicted ribosome interactors GCN1 and GCN20 promote defense and influence protein synthesis changes in response to Pseudomonas. We therefore propose that specific ribosomes can influence the outcome of plant-pathogen interactions. Defining the composition and translational properties of such "defensive" ribosomes should open novel strategies to combat plant diseases.

Identification of a RING E3 ligase that regulates NADPH oxidase stability

B. A. CASTRO ESPARZA (1), D. Lee (1), M. A. Gomez (2), N. Lal (1), S. P. Dinesh-Kumar (1), B. J. Staskawicz (3), G. L. Coaker (1), (1) University of California, Davis, CA, U.S.A.; (2) Innovative Genomics Institute, University of California, Berkeley, CA, U.S.A.; (3) Department of Plant and Microbial Biology, University of California, Berkeley, Berkeley, CA, U.S.A.

Plants produce limited resources which must be tightly regulated between growth and immune responses. Surface localized receptors recognize conserved microbial patterns, leading to pattern triggered immunity (PTI). A pivotal event post PTI activation is the production of reactive oxygen species (ROS) by NADPH oxidases. ROS function as antimicrobials, can strengthen the plant cell wall and act as signals that lead to stomatal closure. NADPH activity must be tightly regulated to balance resistance and developmental defects due to ROS overproduction. PIRE is an Arabidopsis RING E3 ubiquitin ligase. PIRE knockouts display enhanced resistance to Pseudomonas syringae, enhanced ROS production, and higher basal accumulation of the NADPH oxidase RBOHD. PIRE ubiquitinates RBOHD's C-terminus, suggesting an important role of RBOHD ubiquitination for stability and disease resistance. PIRE is conserved across land pants. The results of genome editing of PIRE homologs in tomato and their effect on disease resistance will be reported. We hypothesize that PIRE homologs can be effectively targeted using genome editing for enhanced pathogen resistance.

Involvement of phosphorylation in cucumovirus-Nicotiana benthamiana interaction B. Wrzesinska (1), A. Zmienko (2), A. OBREPALSKA-STEPLOWSKA (1), (1) Institute of Plant Protection - National Research Institute, Poznań, Poland; (2) Institute of Bioorganic Chemistry, Polish Academy of Sciences,, Poznań, Poland

Virus infection causes plant response which influences the fate of infected host and the virus maintenance and spread in infected cells. Signalling is an integral part of plant-virus interaction. Posttranslational protein modifications, especially protein phosphorylation play very important role in cellular signaling. Peanut stunt virus (PSV) is (+)ssRNA virus which belongs to the Cucumovirus genus (Bromoviridae family) and infects many plants, including legumes. Virus causes wide range of symptoms on infected plants, including chloroses, mosaics and stunting. Some PSV strains are associated with non-coding satellite RNAs (satRNAs) influencing plant-virus interaction. In this study we analyzed transcriptome of the Nicotiana benthamiana infected with PSV using species-specific microarrays in order to analyze the expression of genes involved in signaling 
during plant-PSV interaction. The analyses showed that infection caused significant number of changes in the expression of genes associated with plant defence and primary metabolism. We revealed also that virus infection caused differential expression of many genes associated with phosphorylation (biological process) and ATP binding. Among them were several kinases (e.g. MPK20, MAP kinase kinase kinase, SnRK2) and phosphatases. This study was funded by the Polish National Science Centre Grant 2014/13/B/NZ9/02002

Exploring HIGS technology for reducing Fusarium graminearum infection in model and crop plants A. K. MACHADO (1), E. Yamazaki-Lau (2), M. Urban (1), J. M. C. Fernandes (2), K. Kanyuka (3), K. Hammond-Kosack (1), (1) Rothamsted Research, Harpenden, U.K.; (2) Embrapa Wheat, Passo Fundo, Brazil; (3) Department of Biointeractions and Crop Protection, Rothamsted Research, Harpenden, U.K.

Fusarium graminearum is a major fungal pathogen of cereals worldwide, causing seedling, stem base and floral diseases, including Fusarium Head Blight (FHB). Currently FHB control using fungicides is only partially effective whilst crop cultivars with acceptable level of resistance are scarce. Host-Induced Gene Silencing (HIGS) is a transgenic RNAi technology whereby small interfering RNAs produced by the plant induce targeted silencing of the fungal genes during attempted infection. Our aim is to identify Fusarium pathogenicity and essential for life genes whose silencing through HIGS is robust and leads to reduced disease levels. For this, we have carried out $F$. graminearum inoculation of detached Arabidopsis leaves expressing various HIGS constructs. HIGS of six selected $F$. graminearum genes rendered Arabidopsis plants less susceptible to fungal infection. We noticed that the effect of silencing was not the same for every HIGS construct tested. This variation could be the consequence of differing role(s) of each selected sequence and/or differing construct efficacies. We will discuss how these data obtained using model plant species could be used to develop RNAi-based Fusarium control strategies in cereal crops.

An insight into the apoplast of rice during Rhizoctonia solani AG1-IA infection

R. DATTA (1), A. Kumari (1), S. N (2), U. Acharya (1), S. Saha (2), A. Ghosh (1), (1) Division of Plant Biology, Bose Institute, Kolkata, India; (2) Division of Bioinformatics, Bose Institute, Kolkata, India

Rice is consumed as a staple food in many countries in the world. One of the major threats to rice production is the infection with necrotrophic, basidiomycetes fungi called Rhizoctonia solani AG1-IA that causes sheath blight of rice. Worldwide, a substantial proportion of the total yield of rice is lost every year due to Rhizoctonia solani AG1-IA infection. Rhizoctonia solani AG1-IA invades rice by degrading the plant cell wall and thereby establishing an intimate contact with individual host cells. During this invasion, the plant apoplastic region is the primary site of molecular crosstalk between the plant and the fungal defense signals. An insight to such crosstalk can be obtained by looking at the protein functions within the apoplast. In this study we present an overview of the proteome composition of the rice apoplast during sheath blight infection. A comparison of the apoplastic proteome of uninfected rice with that of the infected one revealed increased abundance of proteins belonging to different functional classes in the later. Among the enzymatic activities that were found to be differentially expressed between infected and uninfected apoplasts were peroxidase, protease and glycosidase. This study also demonstrates the identification of Rhizoctonia solani AG1-IA proteins that are secreted only in response to host invasion. Data related to evaluation of these proteins as potential pathogen virulence factors will also be presented.

Functional analysis of a Cercospora zeina effector using Agrobacterium transient assays C. A. SEGAL (1), D. K. Berger (2), (1) Dept. Plant \& Soil Sciences, FABI, University of Pretoria, South Africa; (2) Dept. Plant \& Soil Sciences, FABI, University of Pretoria, Pretoria, South Africa

Cercospora zeina, a foliar pathogen of maize, is a poorly understood fungus. Not much is known on a molecular level about how it causes grey leaf spot (GLS), a yield limiting disease of maize that threatens food security. Studies showed that effector proteins contribute to fungal virulence and pathogenicity. To try understanding effector protein influence on C. zeina virulence and pathogenicity, a candidate effector, extracellular protein 2 (Ecp2) has been identified in the $C$. zeina genome sequence and found to be expressed in planta. C. zeina Ecp2 (CzEcp2) was cloned by RT-PCR from cultures grown in vitro under conditions that simulated in planta conditions. Sequence analysis confirmed that the correct gene had been amplified and that the intron predicted from the genome sequence had been spliced out as expected. $C z E c p 2$ was cloned into a binary vector for Agrobacterium transient transformation in tobacco. This effector is predicted to be exported to the apoplast after cleavage of its signal peptide. Since it is not known if its native signal peptide will be correctly cleaved in tobacco it was cloned in two configurations, with its own signal peptide sequence, and only the mature coding sequence which will be fused to a plant signal peptide that will direct it to the apoplast. We hypothesize that after Agroinfiltration, the CzEcp2 will induce necrosis, indicating interaction with the tobacco defense machinery.

Discovery and characterisation of two novel necrosis-inducing effectors in Fusarium graminearum C. H. WALKER, Rothamsted Research, Harpenden, U.K.

Fusarium graminearum is the causal agent of Fusarium Head Blight (FHB) - a highly hazardous wheat disease resulting in crop loss and mycotoxin contamination. A recent transcriptomic investigation of the $F$. graminearum-wheat interaction revealed an up-regulation in hundreds of genes encoding small secreted proteins (SSPs), or putative effectors, during infection which we hypothesise could contribute towards pathogenicity. The aim of this study was therefore to identify and characterise bioinformatically-selected $F$. graminearum SSPs. Promising candidates were transiently expressed in Nicotiana benthamiana via the pEAQ-HT vector system enabling the identification of two necrosisinducing SSPs, FgSSP32 and FgSSP33, both of which were found to preferentially induce necrosis around the vasculature. Expression of proteins lacking a signal peptide did not result in necrosis suggesting that these proteins function apoplastically. To explore the contribution of these proteins during FHB development, FgSSP32 and FgSSP33 were overexpressed in wheat using the Barley Stripe Mosaic Virus-mediated overexpression system leading to a significant reduction in Fusarium pathogenicity. Single gene deletions of FgSSP32 and FgSSP33 were found not to result in any change in fungal pathogenicity which we hypothesise is a result of genetic redundancy. Further research is required to uncover the precise function of these Fusarium-specific SSPs and to identify the corresponding plant receptors. 
Differential plant defense responses of lettuce genotypes against human pathogenic bacteria

C. JACOB (1), M. Melotto (2), (1) University of California, Davis, Davis, CA, U.S.A.; (2) University of California, Davis, CA, U.S.A.

Every year, disease outbreaks related to the consumption of fresh produce cause a significant number of illness, hospitalization, and death episodes affecting both public health and agribusiness. In this study, we sought to assess and understand the effect of genetic variation of lettuce on the interaction between the plant and strains of the two most common bacterial species identified in these outbreaks: Salmonella enterica serovar Typhimurium and Escherichia coli O157:H7. Using plants grown under controlled environment, we observed significant variation in the bacterial persistence in the leaf apoplast among eleven lettuce genotypes (Lactuca sativa L. and L. serriola L.) after surface- and syringeinoculation methods. Three lettuce genotypes with contrasting bacterial persistence phenotypes also exhibited significant differences in the level of plant defense responses (i.e., reactive oxygen species burst and callose deposition) against S. enterica 14028s and E. coli O157:H7. Simultaneous and temporal RNA-sequencing analysis of the plant and bacterium transcriptomes revealed an active interaction between these organisms and a significant modulation of diverse metabolic pathways, including plant defense response. Overall, this study demonstrates the relevant impact of plant genotypic diversity on the leaf - human pathogenic bacteria interaction and exhibits determinant biological processes underlying this phenotypic variation.

Verticillium dahliae effector SCP8 suppresses plant immunity activated by LRR-domain containing cell surface receptors C. HUA (1), L. Zhang (1), H. S. Guo (2), T. Nuernberger (1), (1) Centre for Plant Molecular Biology, Eberhard Karls University of Tübingen, Tübingen, Germany; (2) Institute of Microbiology, Chinese Academy of Science, Beijing, China

Verticillium dahliae is a soil-borne fungal pathogen that infects over 200 plant species. As many other pathogens $V$. dahliae has to modulate the host immune system mostly by secreting effector proteins during infection. Here we describe $V$. dahliae SCP8, a secreted small cysteinecontaining protein that accumulates at the fungus-host interface during infection. SCP8 is required for full virulence of $V$. dahliae on different host plants. Transgenic Arabidopsis plants expressing SCP8 exhibited enhanced susceptibility to both fungal and bacterial infections.

Interestingly, SCP8 suppresses plant immune responses activated by Leucine-rich repeat (LRR)-domain containing cell surface receptors, such as FLS2, RLP23 and RLP42 that recognize PAMP molecules flg22, nlp20 and fungal endopolygalacturonase (PG), respectively. Immune activation through LysM-domain containing receptors, such as CERK1 (recognizes fungal chitin), is not blocked by SCP8. In planta analysis of SCP8 suggests that the effector functions in the plant apoplastic space. Truncation analysis of SCP8 revealed a 49-aa-domain in the conserved region to be sufficient for PTI-suppression. We propose that SCP8 targets a hub component mediating PTI activation through LRR-RK/RP-type immune receptors.

Pathogen induced pectin remodelling contributes to plant immunity A. GUZHA (1), R. McGee (2), D. Hartken (1), M. Wiermer (1), G. Haughn (2), T. Ischebeck (1), (1) Georg-August University of Goettingen, Germany; (2) University of British Columbia, Canada

The temporal and spacial remodelling of the cell wall is a critical component of plant growth and development as well as for a response to biotic and abiotic pressures. In Arabidopsis thaliana, pectin is the most complex and abundant polysaccharide component of the primary cell wall and its synthesis and modification involves numerous proteins. Various pectin-modifying proteins are upregulated upon infection of Arabidopsis by different pathogens. Here we report the activity of a cell wall localised Pectin Modifying Protein (PMP) that is upregulated upon infection of Arabidopsis by various pathogens. Mutant complementation assays coupled with monosaccharide analysis of pectin using GC-MS show that PMP is a bifunctional (alpha)-L-arabinosidase and (beta)-D-xylosidase. Expression of PMP upon wounding is partly Jasmonic acid (JA) dependent. We also show that the knockout mutant pmp has a compromised immunity against the necrotrophic pathogen Botrytis cinerea compared to wildtype Col-0. PMP mutants show a reduction in the biosynthesis of JA upon infection with $B$. cinerea and wounding. The mutants also show a reduction in the expression of PAD3 after $B$. cinerea infection. Arabidopsis plants with ectopic PMP expression show a heightened basal and induced expression level of JA marker gene Plant defensin 1.2 (PDF1.2). The remodelling of pectin is thus an important component of plant immunity partly due to its contribution to JA signaling and activation of immune response.

Mining and systematic analysis of the effector repertoire of Ustilago hordei during host colonization

B. OKMEN (1), D. Mathow (2), A. Hof (2), U. Lahrmann (2), D. Aßmann (3), G. Doehlemann (1), (1) University of Cologne, Cologne, Germany; (2) Max Planck Institute for Terrestrial Microbiology, Germany; (3) Max Planck Institute for Terrestrial Microbiology, Marburg, Germany

The success of phytopathogens relies on their arsenal of virulence factors that are delivered into host tissues during infection. The biotrophic fungal pathogen Ustilago hordei causes covered smut disease on barley. In this study, we combined cytological, genomics and molecular biological methods to achieve a better understanding of the molecular interactions in the $U$. hordei-barley pathosystem. Microscopic analysis showed that $U$. hordei densely colonizes barley leaves upon penetration, in particular the vascular system, and forms haustorium-like structures. Transcriptome analysis of $U$. hordei at different infection stages revealed differential expression for 273 effector gene candidates. Furthermore, we showed that $U$. hordei transcriptionally activates core-effector genes that may suppress even non-host early defense responses. Knock-out studies of 14 effector candidates in $U$. hordei resulted in identification of four virulence factors (named as Uvi1 to 4) required for host colonization. Yeast-two-hybrid screening performed for the Uvi1 and Uvi2 identified potential barley targets for these effectors. Moreover, we have also characterized a secreted $U$. hordei ribonuclease protein that induces plant cell death in tobacco leaf by means of hypersensitive response. Together, this study provides a first systematic analysis of the effector repertoire of $U$. hordei and identified four virulence factors.

Exploring Apoplastic Sulfate Restriction as a Component of Pattern Triggered Immunity in Arabidopsis thaliana A. H. LOVELACE, B. H. Kvitko, University of Georgia, Athens, GA, U.S.A. 
Transcriptome analysis of the pathogen Pseudomonas syringae pv. tomato DC3000 (DC3K) during early infection and exposure to Pattern Triggered Immunity (PTI) in Arabidopsis thaliana provided insights into the earliest transcriptional events corresponding with immune exposure. We observed that the induction of sulfur starvation response genes such as alternative sulfur importers in DC3K is a rapid and specific response to PTI exposure. We confirmed observations that $A$. thaliana SULTR1;2, a high affinity sulfate transporter gene, is induced in leaves during PTI. This led us to hypothesize that the apoplast may become sulfate depleted during PTI thus restricting pathogen access to sulfur. We optimized a method for extracting high purity apoplastic wash fluid from $A$. thaliana free of cytoplasmic contamination. We used ion chromatography analysis to determine that apoplastic fluid from naïve $A$. thaliana contains approximately $2 \mathrm{mM}$ sulfate. DC $3 \mathrm{~K}$ growth in vitro is inhibited at sulfate concentrations below $10 \mu \mathrm{M}$. In addition, we are conducting mutagenesis of five sulfate transporters in DC3K, to determine whether sulfate uptake is important for growth in planta. Taken together, our research suggests that apoplastic sulfate limitation could be a mechanism for PTI.

Discovering effector proteins in necrotrophic fungal onion pathogens

M. B. F. STEENTJES, Wageningen University, Wageningen, Netherlands

Botrytis aclada (neck rot), Botrytis squamosa (leaf blight) and Sclerotium cepivorum (white rot) are the most important necrotrophic fungal pathogens of onion (Allium серa) in the Netherlands. Recent research on other necrotrophic fungi has highlighted the importance of secreted host cell death-inducing effector proteins required for virulence. This project aims to characterize effector proteins of B. aclada, B. squamosa and $S$. cepivorum that induce host cell death in $A$. cepa (onion). For $B$. squamosa, a crude extract was produced that upon infiltration in onion leaves caused a necrotic response. Purification of the crude extract was performed using ion exchange chromatography and yielded protein fractions that caused, upon infiltration, a cell death response in onion but not in tested dicots. Furthermore, only one out of the two tested onion cultivars showed this response, suggesting an absence of the receptor for the active protein in the cultivar not showing symptoms. Active fractions were analysed by mass spectrometry resulting in the identification of potential effector genes. To validate the candidate effectors, proteins will be individually produced by heterologous expression in the yeast Pichia pastoris, and their cell-death inducing capacity will be tested by infiltrating the proteins in onion leaves. After the identification of effector proteins, knock-out mutants will be made by homologous recombination to test the role of the effectors in virulence.

\section{A Sclerotinia sclerotiorum effector protein specifically interacts with and negates the inhibitory effect of plant polygalacturonase- inhibiting protein (PGIP) \\ W. Wei (1), L. Xu (2), W. CHEN (3), (1) Washington State University, Pullman, WA, U.S.A.; (2) Northwestern A\&F University, Yangling,} China; (3) USDA ARS, Washington State University, Pullman, WA, U.S.A.

Fungal pathogens use polygalacturonases (PGs) to degrade plant cell wall in infection. Plants have developed polygalacturonase-inhibiting proteins (PGIPs) to limit fungal PG activity. The functions of PGs in fungal virulence and PGIPs in plant defense have been well documented. However, there have been no reports of pathogens' ability to counteract plant PGIPs. The necrotrophic pathogen Sclerotinia sclerotiorum causes the white mold disease with tissue maceration as the prominent symptom. Here we show a small effector peptide (SsE1) secreted by $S$. sclerotiorum that specifically interacts with Arabidopsis thaliana PGIP1, as demonstrated using techniques of yeast two-hybrid, coimmunoprecipitation and bimolecular fluorescence complementation assays. Expression of SsE1 is significantly induced during early stages of infection. Deletion of SsE1, as well as of SsPG1, resulted insignificant reductions in virulence. In enzymatic assays using purified SsE1, SsPG1and AtPGIP1 from heterologous expression in yeast cells, SsE1 exhibited no PG enzyme activity, but reduced effectiveness of AtPGIP1 in inhibiting SsPG1 activity in degradation of polygalacturonic acid. Expression of SsE1 in Arabidopsis thaliana plant increased susceptibility to infection. Our results show that $S$. sclerotiorum secretes a special effector that interferes with plant PGIP, mitigating PGIP inhibitory effect and enhancing fungal PG activity in supporting the maceration effect of Sclerotinia infection.

Solanaceae-specific secretory peptide SAR8.2m is essential for non-host resistance of Nicotiana benthamiana to a variety of taxonomically distant Phytophthora species.

S. IMANO (1), Y. Kondou (1), Y. Shibata (1), T. Kondo (1), A. Tanaka (1), I. Sato (1), S. Chiba (1), K. Kageyama (2), K. Kawakita (1), D. Takemoto (1), (1) Nagoya Univ., Japan; (2) Gifu Univ., Japan

Phytophthora infestans, the causal agent of potato late blight, is one of the most devastating and historically significant pathogens in agricultural production. Using virus-induced gene silencing-based screening, we identified a gene encoding small secretory peptide SAR8.2m as an essential factor for the resistance of Nicotiana benthamiana against P. infestans. SAR8.2m have been isolated from tobacco as an elicitor-inducible gene over 20 years ago, but their function in disease resistance is still unknown. SAR8.2m-silenced plants became highly susceptible to $P$. infestans causing systemic disease symptom, while gene silencing of SAR8.2m showed no effect on the resistance to bacterial and fungal pathogens so far tested. In $S A R 8.2 m$-silenced plants, resistance reactions induced by inoculation of $P$. infestans, but not elicitor treatment, was significantly suppressed, suggesting that SAR $8.2 \mathrm{~m}$ might be involved in the suppression of effector function(s) of $P$. infestans. SAR8.2m-silenced plants also became susceptible to various Phytophthora species such as $P$. palmivora (isolated from fig fruit), $P$. hedraindra (from tulip) and $P$.

niederhauserii (from European ivy), however, $R$ gene-dependent resistance of $N$. benthamiana expressing Solanum demissum $R 1$ gene against $P$. infestans was not affected by gene silencing of SAR8.2m. These results suggested that $N$. benthamiana SAR $8.2 \mathrm{~m}$ is involved in non-host resistance against taxonomically distant Phytophthora species.

The Role of Rhynchosporium commune Effectors in Colonisation of Barley Apoplast

L. Gamble, L. L. Griffe, K. Ford, A. Okpo, A. O. AVROVA, James Hutton Institute, Dundee, U.K.

Rhynchosporium commune has remained one of the most destructive and economically important pathogens of barley for over a century. Following conidia germination on the leaf surface and cuticle penetration, this hemibiotrophic fungus spreads between the host epidermal cells. Sequencing of the R. commune genome, and RNA from germinated conidia and an early stage of barley infection, led to identification of putative effectors with roles in the interaction with the host plant. The $R$. commune genome contains 7 genes encoding proteins with one to four LysM 
domains. Another two genes code for enzymes containing LysM domain pairs, a subgroup C chitinase and a putative peptidoglycan lytic transglycosidase. Transcriptional upregulation of $R$. commune secreted chorismate mutase and several genes encoding LysM domain proteins at the onset of barley colonisation suggests their importance during the early stages of interaction with the host. RcLysM3 protein was detected in apoplastic fluid from barley leaves inoculated with R. commune. The ability of RcLysM2 and RcLysM3 produced using Pichia pastoris and Escherichia coli, respectively, to bind chitin and chitosan suggest their role in prevention of the host immune response to chitin. Chorismate mutase on the other hand might be involved in manipulation of SA mediated defences resulting in a compatible interaction. Targeted silencing of the secreted chorismate mutase gene suggests its importance for pathogenicity.

Proteome characterization of the cell wall, apoplast and extracellular space of sugarcane infected with Sporisorium scitamineum, the causal agent of smut disease

M. J. CALDERAN-RODRIGUES (1), A. Peres Bini (2), C. B. M. Vitorello (3), (1) "Luiz de Queiroz" College of Agriculture. University of São Paulo., Piracicaba, Brazil; (2) Centro de Cana, IAC, Ribeirão Preto, Brazil; (3) Univ of São Paulo, ESALQ, Piracicaba, Brazil

The smut disease is one of the most damaging to sugarcane, caused by the fungus $S$. scitamineum. Upon infection, plants and pathogens release proteins that determine the disease progression. Pathogens secrete molecules associated with infection and pathogenicity, whilst the plant related to resistance. Although secreted proteins play a fundamental role and advances were made to elucidate the interaction of sugarcane and $S$. scitamineum, the proteomics of the cell wall, apoplast and extracellular spaces is lacking. This work identified secreted proteins released upon $S$. scitamineum infection in a sugarcane variety with an intermediate level of resistance to smut, RB925345. Sugarcane buds were infected, grown, and sampled thirty days after infection. Two salt-based proteins extraction methods were used to target the cell wall, a destructive and a nondestructive. Protein identification used UPLC-MS/MS. In both methods, proteins related to carbohydrates, lipids, oxidorreductases and proteases were the most represented classes. In the two methods, the inoculated samples presented specific exclusive peroxidases and chitinases, or in more and less abundance, respectively. Results help to elucidate the responses after infection, such as the release of chitinases to degrade $S$.

scitamineum cell walls. Besides providing information on the extracelular role in the interaction, this work might identify new resistance markers. Proc. FAPESP 2017/13268-2,2018/13054-5 and CNPq 443827/2014-1.

\section{Cysteine proteases and their inhibitors in microbe-maize root interactions}

J. SCHULZE HÜYNCK (1), F. Kaschani (2), K. van der Linde (3), S. Ziemann (4), A. Müller (5), T. Colby (6), M. Kaiser (2), S. Glaeser (7), J. Kumlehn (8), J. Misas Villamil (4), G. Doehlemann (1), (1) University of Cologne, Cologne, Germany; (2) University of Duisburg-Essen, Germany; (3) University of Regensburg, Germany; (4) University of Cologne, Germany; (5) Max Planck Institute for Terrestrial Microbiology, Germany; (6) Max Planck Institute for Plant Breeding Research, Cologne, Germany; (7) University of Giessen, Germany; (8) Leibniz Inst of Plant Genetics \& Crop Plant Research, Gatersleben, Germany

Apoplastic papain-like cysteine proteases (PLCPs) are hubs in plant-microbe interactions. We propose that disruption of PLCP activity is a crucial component to establish a stable plant-microbe interaction. On the plant site, we have identified and biochemically characterized CP1C, a novel maize root specific apoplastic PLCP, which shares structural and sequence similarity with known apoplastic CP1-like PLCPs but shows distinct substrate specificity. Using quantitative mass spectrometry of root apoplastic fluids, we found a cystatin being induced on protein level and three previously uncharacterized PLCPs being post-translationally activated by salicylic acid (SA). Comparison of these results with previous findings in leaves is pointing to distinctly different interactions of SA, PLCPs and their regulating cystatins in root versus shoot of maize plants. In a screening approach we identified four root-endophytic bacteria that secrete inhibitors of maize PLCPs. To identify these inhibitors, bacterial genomes were sequenced to search for putative PLCP inhibitors, while in parallel fractionation of the bacterial culture supernatants using mass spectrometry is performed. Further experiments aim to reveal the role of PLCPs and their microbial inhibitors for microbe-maize root interactions in a community context.

The lush life: Escherichia coli 0157 colonising the apoplast A. HOLMES, K. Wright, J. Marshall, N. Holden, The James Hutton Institute, Dundee, U.K.

The consumption of fresh produce, such as spinach, lettuce and tomato, is increasingly being linked to foodborne outbreaks of verotoxigenic $E$. coli (VTEC) and Salmonella enterica. We want to understand the mechanisms these human pathogens use for plant colonization, from initial attachment to plant tissues to bacterial survival in the apoplastic space. Transcriptomic analysis by microarray was used to assess the changes of VTEC strain Sakai in apoplast washings from spinach and Nicotiana benthamiana (naïve and defense response primed by purified H7 flagella). We are comparing these two plant species as VTEC can proliferate to high numbers in the apoplastic spaces of $N$. benthamiana but have limited replication in the spinach apoplastic space. VTEC Sakai differentially regulates approximately $14 \%$ of its genome in response to apoplast washings in vitro. From this data, we can identify and further characterize metabolic pathways the bacteria use for survival in the plant apoplastic space and secreted proteins potentially used to counteract the plant defense response.

Functional analysis of a novel, in planta highly expressed, LysM effector of Zymoseptoria tritici

C. I. MACKENZIE (1,2), H. Tian (3), L. Rodríguez-Moreno (4), J. Rudd (5), B. P. H. J. Thomma (6), (1) Wageningen University and Research, Wageningen, Netherlands; (2) Corpus Christi College, University of Cambridge, Cambridge, U.K.; (3) Wageningen University \& Research, Wageningen, Netherlands; (4) CEBAS-CSIC, Murcia, Spain; (5) Rothamsted Research, Harpenden, U.K.; (6) Wageningen University, Wageningen, Netherlands

Plant pathogens secrete effector proteins to establish their infection. Many fungi secrete effectors containing lysin motifs (LysMs) that bind the fungal cell wall constituent chitin that acts as a MAMP in plants. The hemi-biotrophic pathogen Zymoseptoria tritici, causal agent of the yieldreducing foliar disease septoria tritici blotch of wheat, was previously shown to express in planta two LysM effectors, $M g 1 L y s M$ and $M g 3 L y s M$, that contain one and three LysMs, respectively. We now demonstrate that a third Z. tritici LysM effector that contains a single LysM is induced in wheat leaves during transition from the biotrophic to necrotrophic infection stage, named Mg1LysM-b. We demonstrate that Mg1LysM-b binds 
chitin, protects fungal hyphae from hydrolysis by plant chitinases and suppresses chitin-triggered immune responses in vitro. We are presently generating deletion strains for single LysM effector genes or combinations thereof to assess their contribution to fungal virulence. Our findings support a function of $Z$. tritici LysM effectors in the transition from biotrophic to necrotrophic infection stages.

Photoaffinity profiling to unveil the role of metalloproteases in plant immunity

K. MORIMOTO (1), D. Krahn (2), D. Hopkinson-Woolley (1), S. Mohammed (3), R. A. L. Van Der Hoorn (1), (1) Department of Plant Sciences, University of Oxford, Oxford, U.K.; (2) Department of Chemistry, University of Oxford, Oxford, U.K.; (3) Department of Biochemistry, University of Oxford, Oxford, U.K.

Plant genomes encode approximately 100 metalloproteases that belong to 19 families. These families are diverse and are divided over 11 evolutionarily unrelated clans. All metalloproteases contain catalytic metal ions (i.e. $\mathrm{Zn}^{2+}, \mathrm{Co}^{2+}$ and $\mathrm{Mg}^{2+}$ ) that activate water for nucleophilic attack of the peptide bond while stabilizing the oxyanion hole. Metalloproteases are involved in development and regulation of root and shoot meristem size, senescence and plant innate immunity. However, the regulatory mechanisms of metalloproteases are poorly understood. Here, we synthesized photoaffinity chemical probes that label metalloproteases to uncover the role of metalloproteases in plants (i.e. Arabidopsis thaliana, Nicotiana benthamiana) by activity-based protein profiling (ABPP). We have characterized the labeling conditions and identified the labeled proteins by mass spectrometry analysis. Metalloprotease labeling of apoplastic fluids of Nicotiana benthamiana treated with different Pseudomonas syringae strains displayed interesting differential activity profiles. We are currently further characterizing the roles of these metalloproteases in plant immunity.

Global gene expression profiling of maize genes in defense responses to Puccinia sorghi S. B. KIM, P. Balint-Kurti, North Carolina State University, Raleigh, NC, U.S.A.

Common rust, caused by the obligate biotroph Puccinia sorghi, is one of the most destructive diseases of maize. Different alleles of the Rp1 resistance gene confer resistance to different common rust isolates. Among them, the Rp1D allele confers resistance to the $P$. sorghi IN2 isolate, mediating a hypersensitive response (HR- a localized rapid cell death at the point of pathogen penetration). Resistance genes of the class to which $R p 1$ belongs, recognize the products of specific genes in the pathogen termed avirulence (Avr) genes. Although Rp1D was cloned from maize in 1999, the corresponding AvrRp1D recognized by Rp1D has not been identified. Additionally, the host defense mechanism induced by infection by $P$. sorghi in maize is thus far uncharacterized. Here we present our progress on a strategy to characterize the defense response induced by $P$. sorghi, identify AvrRp1D and to understand the genetics controlling the HR induced by Rp1D.

The SCAR/WAVE complex protein API controls the timely establishment of infection-conducive cell wall properties.

A. GAVRIN (1), R. Thomas (2), T. Torode (3), J. Toulotte (1), A. Chatterjee (1), J. L. Kaplan (1), H. Takagi (4), V. Charoensawan (5), D. Rengel (6), F. Debellé (7), E. P. Journet (8), F. De Carvalho-Niebel (9), R. Terauchi (10), S. Braybrook (1), S. Schornack (11), (1) Cambridge University, Cambridge, U.K.; (2) University of Cambridge, Cambridge, U.K.; (3) Cambridge University, Sainsbury Laboratory (SLCU), Cambridge, U.K.; (4) Iwate Biotech Res Ctr, Kitakami Iwate, Japan; (5) Mahidol University, Bangkok, Thailand; (6) Université de Toulouse, Toulouse, France; (7) Laboratoire des Interactions Plantes Microorganismes, INRA-CNRS, Castanet-Tolosan, France; (8) CNRS INRA, Castanet Tolosan, France; (9) Toulouse University, Toulouse, France; (10) Graduate School of Agriculture, Kyoto University, Kyoto, Japan; (11) Sainsbury Laboratory, University of Cambridge, Cambridge, U.K.

The cell wall is a primary interface between plant cells and their immediate environment and thus must balance multiple functionalities including development, structural support, entry of beneficial microbes, and protection against pathogens. To identify genes commonly required for the accommodation of pathogenic and symbiotic microbes, we carried out root oomycete infection assays on Medicago truncatula mutants affected in rhizobia-root nodule/arbuscular mycorrhiza symbiosis development. We identified and characterised the Medicago gene API, as a susceptibility factor contributing to the microbial infection. API encodes a SCAR2 protein, a subunit of the SCAR/WAVE actin regulatory complex. We demonstrate that API controls establishment of cell wall properties required for efficient root infection by the pathogen Phytophthora palmivora. Resistance was caused by modification of the cell wall architecture. We demonstrated that altered endomembrane trafficking dynamics in api mutants results in the distortion of secreted cell wall remodelling factors. This leads to changes in biochemical and rheological properties of root cell walls that impair pathogen root entry without affecting plant development. Our work demonstrates that alteration in cell wall architecture at a specific stage of root development contributes to disease resistance during compatible interactions without compromising root growth, offering a potential route to quantitative root resistance against Phytophthora.

Soybean stem canker caused by Diaporthe phaseolorum var. caulivora; colonization process and plant defense activation E. MENA (1), S. Stewart (2), M. Montesano (3), I. Ponce De Leon (1), (1) Clemente Estable Research Institute, Montevideo, Uruguay; (2) Instituto Nacional de Investigaciones Agropecuarias, Colonia, Uruguay; (3) Centro de Investigaciones Nucleares, Facultad de Ciencias, Montevideo, Uruguay

Soybean is an important crop in South America and its production is limited by fungal diseases caused by species from the genus Diaporthe, including seed decay, pod and stem blight, and stem canker. In this study we focused on Diaporthe phaseolorum var. caulivora $(D p c)$ isolated from soybean plants with stem canker lesions in Uruguay. Three different isolates were selected for pathogenicity assays, showing different virulence on susceptible soybean plants. Further inspection of the infection and colonization process showed that $D p c$ hyphae were associated with trichomes in leaves and stems, acting probably as physical adhesion sites of the hyphae and entry point for fungal infection. $D p c$ hyphae colonized the stem rapidly reaching the phloem and the xylem at 72 hours after inoculation (hpi) and after 96 hpi the stem was heavily colonized. After fungal infection soybean plants induce reinforcement of the cell walls, although they were not sufficient to stop fungal colonization. In addition, several defense genes were induced in inoculated stems, including a Pathogenesis-Related protein- 1 (PR-1), $\beta$-1,3-glucanase, chitinases, PR-10, lipoxygenases, a basic peroxidase, a phenylalanine-ammonia lyase and a chalcone synthase. This work allowed us to generate knowledge on the infection process of $D p c$ in soybean plants and the activation of plant defense mechanisms in response to this pathogen. 
L-amino Acid Oxidase Secreted by Trichoderma Elicits Systemic Resistance by Transporting to Chloroplasts of Host Cells and Targeting Chlorophyll a/b Binding Proteins

K. C. Peng (1), C. C. Lin (1), C. F. Liao (1), H. C. Yu (1), C. T. Lo (2), H. H. Yang (3), K. C. LIN (1), (1) Department of Life Science, National Dong Hwa University, Hualien, Taiwan; (2) Department of Biotechnology, National Formosa University, Yunlin, Taiwan; (3) Department of Medical Research, Buddhist Tzu Chi General Hospital, Hualien, Taiwan

Trichoderma spp. may promote plant growth and act as biocontrol agents to antagonize phytopathogens. L-Amino acid oxidase (ThLAAO) secreted by $T$. harzianum have been proven to exhibit antimicrobial effects. In this study we reported that ThLAAO can induce systemic resistance by promptly entering cabbage roots and translocating into leaves via vascular bundles. Chlorophyll a/b binding protein (CAB) was isolated by ConA-ThLAAO affinity chromatography. Pull down assay confirmed the binding between CAB and ThLAAO. Application of ThLAAO-FITC on tobacco protoplast or expression of ThLAAO-GFP fusion protein in transgenic tobacco revealed the localization of ThLAAO in chloroplasts. Transgenic tobacco expressing ThLAAO antagonized Botrytis cinerea and Sclerotinia sclerotiorum, and activated expression of defense-related genes and the genes involved in biosynthesis of salicylate, jasmonate, and ethylene. $\mathrm{H}_{2} \mathrm{O}_{2}$ were highly accumulated in chloroplasts of transgenic tobacco. Scavenge of $\mathrm{H}_{2} \mathrm{O}_{2}$ with ascorbate abolished disease resistance and expression of defense-related genes. Overall, ThLAAO, a microbe-associated molecule particle (MAMP) secreted by $T$. harzianum, is an exogenous mobile signaling molecule which may cause $\mathrm{H}_{2} \mathrm{O}_{2}$ generation in chloroplasts, induce systemic resistance, and confer pattern-triggered immunity (PTI) against various pathogens through $\mathrm{H}_{2} \mathrm{O}_{2}$ signaling. Furthermore, $\mathrm{CAB}$ in chloroplast is the potential pattern recognition receptor (PRR) of ThLAAO.

Role of chitosan and chitin deacetylases during development of Ustilago maydis

Y. S. RIZZI (1), S. Lenz (1), P. Happel (2), S. Reissman (2), S. Cord-Landwehr (3), B. M. Moerschbacher (3), R. Kahmann (4), (1) Dept of Organismic Interactions, Max Planck Inst for Terrestrial Microbiology, Germany; (2) Max Planck Institute for Terrestrial Microbiology, Germany; (3) Institut für Biologie und Biotechnologie der Pflanzen, Germany; (4) Max Planck Institute for Terrestrial Microbiology, Marburg, Germany

The basidiomycete Ustilago maydis causes smut disease in maize. This non-obligate pathogen penetrates the plant cell wall using appressoria and then establishes an extensive biotrophic interaction, where the hyphae are tightly encased by the plant plasma membrane. The fungal cell wall is the first structure to interact with the host and some of its components like chitin are well-known elicitors and trigger plant immune responses. To avoid this from happening in a compatible interaction, pathogens have developed strategies to modify or hide chitin. One of these strategies discussed in other pathosystems could be the conversion of chitin to chitosan by chitin deacetylases (CDAs). Chitosan is a poor substrate for plant chitinases and a weak inducer of plant immunity. The aim of this project is to determine whether chitosan is produced by $U$. maydis and which role it plays during colonization. U. maydis possesses six putative $c d a$ genes which are differentially expressed during development. Using a specific probe for chitosan, a strong signal was detected in appressoria and in biotrophic hyphae. To evaluate the function of chitosan during colonization, we are inactivating single and multiple $c d a$ genes. To see whether CDAs in U. maydis have distinct roles, we are assessing effects on morphology and virulence of the different mutants and elucidate, whether biotrophic hyphae still expose chitosan. Results from these studies will be presented.

Remorin: A membrane raft plasmodesmal protein limiting or promoting virus cell-to-cell movement of two distinct virus genera A. Perraki (1), J. Gronnier (2), A. F. Deroubaix (3), V. Simon (4), P. Gouguet (3), M. Boudsocq (5), E. Bayer (3), S. GERMAN-RETANA (4), S. Mongrand (3), V. Germain (3), (1) Department of Plant Sciences, University of Cambridge, Cambridge, U.K.; (2) Institute of Plant and Microbial Biology, University of Zurich, Zurich, Switzerland; (3) Laboratoire de Biogenèse Membranaire (LBM), CNRS/Universite de Bordeaux, Bordeaux, France; (4) INRA, UMR BFP 1332, Villenave d'Ornon, France; (5) IPS2, CNRS, INRA, Universite Paris-Sud, Plateau du Moulon, France

Remorin proteins belong to a plant specific multigene family, that localize in plasma membrane specialized sub-compartments called "membrane rafts" and for some of them in plasmodesmata. The first remorin (Solanum tuberosum Remorin StREM1.3) was discovered as a plasma membrane protein differentially phosphorylated in the presence of oligogalacturonates. We previously showed that in tomato and $N$. benthamiana, StREM1.3 limits the cell-to-cell spread of the potexvirus Potato virus X (PVX) without affecting viral replication, that the Cterminal anchor of REM is necessary for its membrane localization and restriction of PVX movement. We also showed that $A$. thaliana Calciumdependent protein kinase 3 is able to interact with group 1 REM in vivo, phosphorylates the N-terminal domain of StREM1.3 and restricts PVX cell-to-cell movement in $N$. benthamiana. However, for a distinct virus genus (potyvirus) and contrarily to the results obtained with PVX, we showed that StREM1.3 has an agonistic effect on the propagation of Turnip mosaic virus (TuMV). Membrane anchoring of StREM1.3 seems to be important for promoting TuMV propagation. However, StREM1.3's phospho-status shown to be important for restriction of PVX cell-to-cell movement does not seem to be important for the positive effect observed on TuMV cell-to-cell movement. These results suggest that StREM1.3 phospho-code probably leads to distinct plant responses against potexvirus and potyvirus.

Immune response induction in rice due to co-expression of $X o p Q$ and $X o p X$

S. DEB (1), H. K. Patel (2), R. V. Sonti (3), (1) CSIR- Centre for Cellular and Molecular Biology, Hyderabad, India; (2) CSIR-Centre for Cellular and Molecular Biology (CSIR-CCMB), Hyderabad, India; (3) National Institute of Plant Genome Research (NIPGR), New Delhi, India

Xanthomonas oryzae pv. oryzae (Xoo) causes bacterial blight, a serious disease of rice. Xoo uses the type III secretion system (T3SS) to suppress rice immune responses. The T3SS secreted effectors XopQ and XopX suppress rice immune responses by interaction with different rice 14-3-3 proteins. Sub-cellular localisation of XopQ and XopX mutants that are defective in 14-3-3 binding and suppression of immune responses indicates that, for suppression, XopQ requires a cytoplasmic localisation whereas XopX requires nuclear localisation. Hence, both XopQ as well as XopX individually act as suppressors of rice immune responses, probably by targeting unique pathways in different subcellular compartments. However, we find that when XopQ is delivered through Agrobacterium along with XopX, it becomes an inducer of immune responses and that it 
now localizes in the nucleus. We also find that XopQ and XopX can interact with each other. This raises the possibility that besides being a suppressor of immune responses, XopQ can under certain circumstances also function as an inducer of immune responses.

Host-induced gene silencing of Aspergillus flavus p2c gene reduced aflatoxin contamination in field grown maize Y. Raruang (1), O. Omolehin (1), Q. Wei (2), K. Rajasekaran (2), Z. Y. CHEN (1), (1) Louisiana State University AgCenter, Baton Rouge, LA, U.S.A.; (2) USDA, ARS, SRRC, New Orleans, LA, U.S.A.

Maize is frequently infected by Aspergillus flavus before harvest and subsequently contaminated with aflatoxins due to lack of resistance in commercial maize cultivars and limited success in biocontrols. In the present study, host-induced gene silencing (HIGS) was employed to mitigate aflatoxin contamination in maize, an A. flavus gene encoding polygalacturonase $(p 2 c)$, a key enzyme involved in the colonization of maize kernels. An RNAi vector containing a portion of the $p 2 c$ gene was constructed and introduced into B104 immature embryos through Agrobacterium transformation. Twenty-eight transgenic plants were produced from fifteen independent transformation events. Thirteen of fifteen transformation events were confirmed by PCR to have the $p 2 c$ gene. The T2 generation kernels containing the transgene from six out of eleven examined events had less aflatoxin than those without the transgene. Field-inoculated, four events of homozygous T3 transgenic kernels also revealed lower aflatoxins $(\mathrm{p} \leq 0.02)$ than kernels from the null or B104 controls. Two events were crossed with six elite breeding lines and the resulting crosses supported less aflatoxin $(\mathrm{p} \leq 0.02)$ than the crosses made with non-transgenic lines. In addition, significantly higher levels of $\mathrm{p} 2 \mathrm{c}$ gene-specific small RNAs were detected in the transgenic leaf and kernel tissues, indicating that the enhanced aflatoxin resistance in the homozygous transgenic kernels is likely due to suppression of $p 2 c$ expression through HIGS.

Towards Molecular Mechanism for the Functional Activation of Effector RipAY from Ralstonia solanacearum S. RHEE, Seoul National University, Department of Agricultural Biotechnology, Seoul, Korea, Republic of (South)

Phytopathogen Ralstonia solanacearum represents the causal agent of bacterial wilt disease of plants. Recent in vivo and in vitro studies suggested that type III effector RipAY from $R$. solanacearum suppresses plant immunity by degrading glutathione in the host plants that plays an important role in host immune system. Interestingly, RipAY's proposed activity becomes activated only after plant cytosolic thioredoxin acts on RipAY, although molecular mechanism of how thioredoxin activates RipAY has remained to be resolved. Furthermore, functional activation of RipAY is achieved by plant or yeast thioredoxin, but not by bacterial thioredoxin. Protein structure-based functional assignment could serve as one of the robust experimental approaches in characterizing biochemical function of effectors. By employing x-ray crystallographic approaches we have proposed a possible functional role of effector XopQ from Xanthomonas oryzae and HopA1 from Pseudomonas syringae. Here, in order to understand molecular insights on the functional activation of RipAY, we aim to determine a three-dimensional structure of RipAY protein in the absence and/or presence of plant cytosolic thioredoxin. To this end, we are currently carrying out purification and crystallization of RipAY and those progresses will be reported in the presentation. This work was supported by Next Generation BioGreen 21 program of Rural Development Administration (Plant Molecular Breeding Center) of Republic of KOREA.

\section{TFV1 is preferentially expressed in plant infection phase and is required for full virulence of Colletotrichum orbiculare on cucurbit} plants

R. ZHANG, Y. Inoue, M. Kaido, K. Mise, Y. Takano, Graduate School of Agriculture, Kyoto University, Kyoto, Japan

In general, plant pathogenic fungi preferentially express a set of effector genes during host infection. To investigate regulatory mechanisms for effector expression during host invasion by plant pathogenic fungi, we here focused on the transcription factors of cucumber anthracnose fungus Colletotrichum orbiculare that are preferentially expressed at the pre-invasion stage on the host plant. Based on RNA sequencing data of $C$. orbiculare under various conditions, we selected 9 candidate genes and generated the knock-out mutants of $C$. orbiculare for each gene. The deletion of one candidate gene caused the reduction in virulence on both cucumber and melon but not on Nicotiana benthamiana. We named this gene TFV1 (transcription factor for virulence 1). TFVI encodes a putative transcription factor containing the fungal $\mathrm{Zn}$ (II)2-Cys6 binuclear cluster domain. The phenotypes of the tfvl $1 \Delta$ mutants in vegetative growth, conidiation, and appressorium formation were similar to those of the wild-type strain. In contrast, the $t f v 1 \Delta$ mutants delayed appressorium-mediated host invasion on cucumber and melon. Subsequently, we performed comparative RNA sequencing analyses between the wild-type strain and the $t f v 1 \Delta$ mutant, the results showed that more than half of down-regulated genes in the $t f v l \Delta$ mutant were genes encoding secreted proteins. Currently, we are focusing on target genes of $T F V 1$, especially putative effector genes required for full virulence.

Nitric oxide and life in the xylem: How Ralstonia solanacearum uses a toxic metabolite to thrive in its host environment C. G. HENDRICH (1), A. N. Truchon (2), B. L. Dalsing (3), C. Allen (2), (1) University of Wisconsin - Madison, Madison, WI, U.S.A.; (2) University of Wisconsin-Madison, Madison, WI, U.S.A.; (3) Syngenta, Durham, NC, U.S.A.

The xylem-dwelling bacterial plant pathogen Ralstonia solanacearum $(R s)$ is the cause of bacterial wilt disease on a wide variety of plant hosts, including crops like potato, tomato, and banana. $R s$ infects through the roots and travels to the xylem, where it spreads through the plant, eventually blocking water transport and causing the host to wilt. A consortium of virulence factors allow $R s$ to thrive in its host. In vitro, Rs is slow growing and sensitive to stress, but in the plant $R s$ is a powerhouse, able to quickly grow to extremely high cell densities despite low nutrients, low oxygen, and an onslaught of plant defenses. If we understand of how $R s$ achieves this resilience, and what signals and traits allow it to transition from the soil to the plant environment, we may be able to better disrupt the disease. Data suggest that nitric oxide (NO) is one signal used by Rs to adapt to a host environment. NO is a ubiquitous molecule that is produced by both plants and Rs during infection. Although commonly used by eukaryotes as a signaling molecule, $\mathrm{NO}$ is toxic, and $R s$ upregulates many NO-degrading genes in planta to deal with its negative effects. We analyzed the transcriptomes of $R s$ in vitro and in planta in the presence of varying amounts of nitric oxide to identify the $R s$ traits regulated by NO. These included one of the most important bacterial wilt virulence factors, the Rs Type III Secretion System and several effectors. 
Barley RIC157 is involved in RACB-mediated susceptibility to powdery mildew S. ENGELHARDT (1), C. McCollum (2), R. Hueckelhoven (3), (1) TU Munich, Phytopathology, Germany; (2) Phytopathology, Technical University Munich, Germany; (3) Technical University of Munich, Freising, Germany

Biotrophic fungal pathogens like Blumeria graminis f. sp. hordei (Bgh) take advantage of certain cellular host processes in order to successfully establish an infection. Barley RACB, a small monomeric G-protein (ROP, RHO of plants), is required for full susceptibility to penetration by $B g h$, potentially due to its role in cell polarization and reorganization of the cytoskeleton. However, since RACB acts basically as a molecular switch transferring signals to downstream interactors, the exact mode-of-action of RACB-mediated susceptibility remains unknown. RIC proteins, a specific class of adapters, have been previously described to interact only with activated ROPs via a conserved CRIB domain and are considered to link ROPs to diverse downstream targets. In the present work we describe a yet uncharacterized RIC protein, RIC157, that interacts in yeast and in planta directly with RACB. Interestingly, RIC157 undergoes a recruitment to the cell periphery and plasma membrane in the presence of the activated form of RACB and transient overexpression of RIC157 rendered barley epidermal cells more susceptible to penetration by $B g h$ in a RACB-dependent manner. Preliminary data suggest a role for RIC157 in microtubule organization, as transient overexpression of RIC157 leads to a higher disorder of cortical microtubules. Together with published data, this might indicate that RIC157 is involved in effectortriggered and RACB-mediated support of fungal entry into barley epidermal cells.

The role of Avr4 effector in Cercospora cf. flagellaris virulence, cercosporin biosynthesis, and infection of soybeans Z. Y. CHEN, J. Santos Rezende, M. Zivanovic, M. I. Costa de Novaes, Louisiana State University AgCenter, Baton Rouge, LA, U.S.A.

The Avr4 effector, secreted by Cladosporium fulvum, was shown to be involved in pathogen virulence. Recent studies demonstrated that Avr4 is highly conserved among several Cercospora species, indicating a potentially important role of this gene in their virulence. Therefore, it is of great interest to investigate the presence and role of this effector in Cercospora cf. flagellaris, the causal agent of Cercospora leaf blight (CLB) of soybean. In this study, the Avr4 gene from C. cf. flagellaris was disrupted through homologous recombination. The $\Delta$ avr4 mutants grew faster, produced little to no cercosporin in vitro and had significantly reduced expression of cercosporin toxin biosynthesis genes. In culture with Avr4specific dsRNA, cercosporin production by the wild type was reduced by $88 \%$ and CTB1 and CTB 8 expression was reduced to $25 \%$ and $42 \%$, respectively, which confirmed the role of Avr4 in cercosporin biosynthesis. Furthermore, $\Delta$ avr4 mutants were more sensitive to chitinase than the wild type and cercosporin was found to directly suppress chitinolytic activity in vitro. When inoculated onto detached soybean leaves, the $\Delta$ avr 4 cf. flagellaris on soybean by regulating cercosporin biosynthesis in addition to protecting fungal hyphae from plant chitinases through chitinbinding activity as previously reported.

Fusarium graminearum virus 1 ORF2 protein is required for suppression of RNAi by transcriptional repression of FgDICER-2 and FgAGO-1

J. YU (1), J. I. Heo (2), J. Y. Park (2), K. H. Kim (3), (1) Plant Genomics and Breeding Institute, Seoul National University, Seoul, Korea, Republic of (South); (2) Department of Agricultural Biotechnology, Seoul National University, Seoul, Korea, Republic of (South); (3) Research Institute of Agriculture and Life Sciences, Seoul National University, Seoul, Korea, Republic of (South)

Fusarium graminearum virus 1 (FgV1), which infects Fusarium graminearum, confers hypovirulence-associated traits such as reduced mycelial growth, increase pigmentation, and reduced pathogenicity. F. graminearum has RNA interference (RNAi) that acts as a defense response against virus infections and exogenous dsRNA. In this study, we demonstrated that $\mathrm{FgV} 1$ has RNA silencing suppression ability by interfering inductions of $F g D I C E R-2$ and $F g A G O-1$ that are involved in RNAi antiviral defense and hairpin RNA/RNAi pathway in $F$. graminearum. To clarify how transcriptional inductions of $F g D I C E R-2$ and $F g A G O-1$ were suppressed following FgV1 infection, we conducted $F g A G O-1$ - or FgDICER-2promoter/GFP-reporter expression assay. As a result, we showed that transcript level of GFP driven by $F g A G O-1$ or $F g D I C E R-2$ promoter was down-regulated in FgV1-infected mutant strain. To determine which FgV1 viral gene product(s) repress transcriptional of those RNAi-related host genes, we generated the fungal mutants expressing each ORFs of FgV1 with or without a hairpin RNA construct. FgDICER-2 and FgAGO-1 transcript levels induced by a hairpin RNA construct were significantly decreased in FgV1 ORF2 expressing mutant compare to those of other ORF expressing mutants. Taken together, results of this study indicate that the ORF2 of FgV1 is able to counteract RNAi defense response in $F$. graminearum through interfering induction of $\mathrm{FgDICER}-2$ and $\mathrm{FgAGO}-1$ with promoter-dependent manner.

The roles of phytohormones in LsGRP1-mediated resistance against Botrytis pathogens

T. W. CHEN (1), C. Y. Chen (2), (1) Department of Plant Pathology and Microbiology, National Taiwan University, Taipei City, Taiwan; (2) National Taiwan University, Taipei, Taiwan

Perception of pathogen by plants triggers metabolic reprogramming via phytohormone signaling transduction. Previous reports indicated that salicylic acid treatment increased resistance against Botrytis elliptica and expression of LsGRP1 in Lilium cv. Star Gazer. To understand the roles of phytohormones in the interaction between Lilium cv. Star Gazer and B. elliptica, sequences with length about $300 \mathrm{bp}$ to $700 \mathrm{bp}$ from putative EIN3, COI1, MPK6 and SID2 of Lilium cv. Star Gazer were constructed into TRV vector for virus-induced gene silencing (VIGS) assay. Silencing of MPK6 and SID2 homologs of Lilium cv. Star Gazer showed an increased susceptibility against B. elliptica. In parallel, VIGS assay with different Arabidopsis phytohormone-related genes were performed in LsGRPl-transformed Arabidopsis against B. cinerea. The results would facilitate the understanding of the roles of phytohormones in LsGRP1-mediated resistance against Botrytis pathogens.

Exploration of interaction between Botrytis elliptica subtilase and plant defense protein LsGRP1

T. C. LIN, C. Y. Chen, National Taiwan University, Taipei, Taiwan 
In the experiment of co-immunoprecipitation, fungal protein subtilase was considered a candidate interacting with LsGRP1 of Lilium cv. Stargazer. The partial amino acid sequence of subtilase was also found in the transcriptome database of B. elliptica-infected Lilium 'Stargazer'. In this study, we aimed to provide evidence of the interaction between $B$. elliptica subtilase and LsGRP1. Firstly, the full-length cDNA of $B$. elliptica subtilase was cloned and named BeSerp. The gene expression of BeSerp in lily leaves was analyzed and shown a decrease $48 \mathrm{~h}$ post fungal inoculation. In addition, differences of $L S G R P 1$ expression and lesion development post fungal inoculation on HIGS-BeSerp lily were demonstrated as compared with that of the control. When histidine-tagged BeSerp expressed in E. coli system was co-incubated with LsGRP1, an upshifted protein band was detected on the western blot. A transient expression assay showed that BeSerp could reduce lesion development caused by B. elliptica. In brief, the up-to-date evidences support a high possibility of interaction between BeSerp and LsGRP1, which may affect the fungal infection process and symptom development of gray mold in Lilium.

It's All In The Delivery: Identifying and Functionally Characterising Yellow Rust Effectors in Wheat C. JENSEN (1), D. G. O. Saunders (2), (1) John Innes Centre, U.K.; (2) John Innes Centre, Norwich, U.K.

Yellow rust (YR) of wheat, caused by the obligate biotrophic fungus Puccinia striiformis $f$. sp. tritici (PST), remains one of the largest biotic limitations to wheat production worldwide. Despite this, no effectors that are recognized by a particular resistance protein in wheat have been characterized for PST. Here, we describe an in-silico pipeline to mine effector candidates of PST that are specifically recognized by YR2. To this aim, we identified SNPs in the genomes of near isogenic isolates in which one PST isolate had spontaneously gained virulence on YR2 wheat. The near isogenic nature of the isolates eliminates genetic variation due to evolutionary divergence, instead highlighting SNPs associated with changes in virulence. This pipeline produced seven candidates which are now of high priority to confirm experimentally in the lab. Unfortunately, reliable methods for delivering and functionally characterizing candidate effectors of PST in the native wheat host are limited. To address this issue, we are developing a system using heterologous expression in wheat blast (Magnaporthe oryzae). We have produced a single Magnaporthe transformant expressing AvrSr50 from stem rust (as a positive control) that elicits a Sr50 dependent hypersensitive response in wheat. These results provide a valuable starting point for developing a consistent system to deliver rust effectors in wheat, eventually leading to the functional confirmation of candidate effectors identified in-silico.

Deregulated expression of E2Fa and E2Fb transcription factors hampered the cell cycle leading to reduced nematode-induced gall development

D. C. DO NASCIMENTO (1), L. de Veylder (2), J. De Almeida-Engler (1), (1) INRA, Université Cote D’Azur, CNRS, UMR 1355-7254, Sophia Antipolis, France; (2) Department of Plant Systems Biology, VIB, Ghent University, Ghent, Belgium

Root-knot nematodes (RKN) infect a large number of plant species triggering the formation of galls by inducing vascular cells to reenter the cell cycle. These nematodes infect the host root and usurp the plant cell cycle machinery by inducing multinucleate giant-feeding cells surrounded by asymmetrically dividing neighboring cells. Cell cycle activation is a basis for gall induction and maintenance. Giant cell development is characterized by an acytokinetic mitotic phase, followed by an endoreduplication stage leading to polyploidization of nuclei during cell expansion. The endocycle involves genome duplication without cell division, resulting in polyploidy. E2Fa and E2Fb transcription factors are positive regulators of the mitotic as well the endocycle being essential to drive $\mathrm{S}$ phase. Via functional and molecular analysis, we unraveled the role of $A t E 2 F a$ and $A t E 2 F b$ for gall growth and development. In situ transcript localization has shown that $A t E 2 F a$ and $A t E 2 F b$ are highly expressed in these nematode feeding sites. A detailed analysis of $E 2 F$ knockout and overexpressing lines suggests that despite both genes seem to have slightly different timing and spatial localization during gall expansion, both are essential for gall development.

ROP INTERACTIVE PARTNER b (RIPb) supports penetration of barley by Blumeria graminis $\mathrm{f}$. sp. hordei C. MCCOLLUM (1), S. Engelhardt (2), R. Hueckelhoven (2), (1) Technische Universität München, Freising, Germany; (2) TU Munich, Phytopathology, Germany

Blumeria gramninis f. sp. hordei (Bgh) is a biotrophic ascomycete causing the powdery mildew disease on barley (Hordeum vulgare). Bgh grows mainly on the plants surface, but in the beginning of its life cycle $B g h$ has to penetrate an epidermal host cell to establish the haustorium, a special cell for nutrient uptake. RACB is a ROP (Rho of Plants) G-protein in barley and a susceptibility factor in the interaction of barley and Bgh. Overexpression of constitutively activated RACB renders plants more susceptible to fungal attack, while silencing of RACB decreases susceptibility. RACB is involved in cell polarization processes during plant development. It was shown that RACB-silenced plants show defects in root hair development, as well as stomatal subsidiary cell development. Since RACB further interacts with a putative effector of $B g h$, we hypothesize that $B g h$ takes control over a developmental polarization process of barley by manipulating RACB signaling pathways. In order to study possible signaling pathways of RACB we addressed ROP INTERACTIVE PARTNER $b$ (RIPb). RIPb possibly acts as a scaffold protein. We show that $\mathrm{RIPb}$ localizes to microtubules and the plasma membrane. We are further able to show that over-expression of RIPb also increases the susceptibility of barley to $\mathrm{Bgh}$. Co-localization experiments show that RACB and RIPb co-localize at the site of fungal attack. Data suggest that $\mathrm{RIPb}$ connects RACB to further downstream proteins that support accommodation of haustoria in barley cells.

Pathogen resistance mediated by modular dirigent-jacalin proteins are solely invented by monocots

C. Kirsch, L. Esch, L. Vogel, J. Czichowlas, U. SCHAFFRATH, RWTH Aachen University, Aachen, Germany

Plants of the Poaceae family express modular proteins with a dirigent and a jaclain-related lectin (JRL) domain which are unique for monocots We have shown that overexpression of a member of these proteins (OsJAC1) confers broad-spectrum disease resistance to bacterial and fungal plant pathogens in rice, barley and wheat. We further established that both domains are required for pathogen resistance. Consistently, FRET-, split-luciferase- and BiFC-analyses indicated that both domains physically interact when expressed as separate proteins. Dicot plants do not contain modular proteins with these two domains rather single domain proteins are present. Following the Rosetta-stone-hypothesis, we predicted that pairs of dirigent/JRL-proteins act in Arabidopsis resistance in an OsJAC1-similar manner. We challenged this hypothesis by selecting a set of Arabidopsis proteins with dirigent or JRL-domain and tested them for their capability to promote resistance in barley against powdery mildew. 
While this was successfully demonstrated for at least three pairs of proteins, we unexpectedly could not determine a contribution of these dirigent/JRL-proteins in pathogen resistance. This finding was substantiated in an experiment revealing that overexpression of OsJAC1 in Arabidopsis also did not promote resistance against pathogens. In sum our results account for an evolutionary unique event in ancestral monocots which led to the establishment of the dirgent/JRL-related resistance pathway.

Highly branched ROP signaling acts in effector-triggered susceptibility to powdery mildew

R. HUECKELHOVEN (1), C. McCollum (2), A. Trutzenberg (3), L. Weiß (3), J. Mergner (4), B. Küster (4), G. Hensel (5), J. Kumlehn (5), M. Kopischke (3), S. Engelhardt (3), (1) Technical University of Munich, Freising, Germany; (2) Phytopathology, Technical University Munich, Germany; (3) TU Munich, Phytopathology, Germany; (4) TU Munich, Proteomics and Bioanalytics, Germany; (5) Leibniz Inst of Plant Genetics \& Crop Plant Research, Gatersleben, Germany

The barley ROP GTPase RACB is a susceptibility factor in interaction with the barley powdery mildew fungus Blumeria graminis f. sp. hordei $(B g h)$. In healthy plants, RACB is involved in cell polarity and cytoskeleton organization and we hypothesize that $B g h$ profits from these functions in effector triggered susceptibility. Indeed, a non-conventional effector of $B g h$ interacts with RACB and destabilizes microtubules. We now identified further possible upstream and downstream interaction partners of RACB by yeast two hybrid assays, co-immunoprecipitation and FRET FLIM assays. This identified another possible effector of $B g h$, several scaffold proteins that might link RACB to downstream executer pathways and proteins of membrane signaling and organization. Several of those RACB-interaction partners show the potential to support susceptibility to $B g h$ and display RACB-dependent subcellular localization at sites of fungal invasion or at microtubules. Together, data strongly suggest that RACB is a central hub in a network of susceptibility factors that is directly addressed by fungal effector proteins.

Uncovering cell specific immune responses in plants using the GFP-strand system S. LOLLE (1), E. Henry (2), B. H. Kvitko (3), G. L. Coaker (2), (1) University of California, Davis, Davis, CA, U.S.A.; (2) University of California, Davis, CA, U.S.A.; (3) University of Georgia, Athens, GA, U.S.A.

As a primary step in infection, gram-negative bacterial pathogens, such as $P$. syringae, use the Type 3 Secretion System (T3SS) to deliver effectors into host cells. Delivery of effectors disturbs normal physiological and biochemical plant processes. During natural infection, effectors are not uniformly delivered into plants and, as a result, cells within a single leaf do not uniformly respond to pathogen infection. To study single cell responses in plants we have developed a GFP-strand reporter system. Due to size limitations of the T3SS, it is not possible to tag effectors with conventional fluorophores. Tagging effectors with only the $11^{\text {th }}$ strand of GFP allows for secretion though the T3SS and infection of plants expressing $\mathrm{GFP}_{1-10}$ with bacteria delivering effector-GFP ${ }_{11}$ results in reconstitution of GFP and enables direct effector detection in planta. We have developed a reporter that is expressed and delivered together with native effectors from $P$. syringae. The reconstitution of GFP enables differentiation between GFP+ cells (effector delivery) and the GFP- cells (no effector delivery). This reporter system thus enables us to identify single cells subjected to general effector delivery. After sorting GFP+/- cells transcriptional profiling allow us to gain insight in single cell responses in plant immunity. I will show results on the use of the GFP-strand system for mapping effector delivery and strategies for the use of our system to analyze cell specific host responses.

The gibberellic acid insensitive protein from Nicotiana benthamiana positively regulates Bamboo mosaic virus replication C. H. TSAI (1), I. H. Chen (2), Y. P. Huang (2), Y. H. Hsu (1), (1) Graduate Institute of Biotechnology, National Chung Hsing University, Taichung, Taiwan; (2) National Chung Hsing University, Taichung, Taiwan

Bamboo mosaic virus (BaMV) has one single-stranded positive-sense RNA genome of about $6366 \mathrm{nt}$. One of the upregulated genes after BaMV inoculation in Nicotiana benthamiana, ACCT7-1, identified by cDNA-AFLP was involved in BaMV infection. The accumulation levels of $\mathrm{BaMV}$ in the knockdown plants and protoplasts were increased to that of the controls. These results suggested that the gene could have an assisting role in viral infection. The full-length cDNA was obtained and revealed as a gibberellic acid insensitive (GAI). Therefore, this gene was designated $N b G A I$. To localize NbGAI in the cell, the NbGAI was subcloned into the pEpyon vector from which the fusion protein NbGAI-OFP was produced in plant cells. Since NbGAI is one of the DELLA proteins that regulate plant hormones, NbGAI was localized in the nucleus as expected. To reveal how a nuclear protein could assist virus RNA replication, mutants were created with the nuclear-localization signal (NLS) removed, $\mathrm{NbGAI} / \Delta \mathrm{NLS}$, or chloroplast transit peptide inserted, TP-NbGAI/ $\Delta \mathrm{NLS}$. The expression of NbGAI/ $\Delta \mathrm{NLS}$ was shown to have a similar level of BaMV accumulation as that of wild-type NbGAI. However, the other mutant TP- NbGAI/ $\Delta \mathrm{NLS}$ was revealed to have a significantly increased level of BaMV accumulation to those of NbGAI and NbGAI/ANLS. Overall of these results implied that NbGAI localized in the chloroplasts, where BaMV replicates, has a higher impact on assisting BaMV accumulation possibly for the replication.

Plant class II glycine-rich protein LsGRP1 playing roles in defense-growth tradeoffs and disease resistance C. H. LIN, N. H. Yeh, C. Y. Chen, National Taiwan University, Taipei, Taiwan

Plant optimizes its fitness costs via regulating growth-defense tradeoffs. Some mechanisms governing growth-defense tradeoffs presumably contribute to plant induced disease resistance from the primed state of defense against pathogen attack. Pathogen-responsive LsGRP1 is a plant class II glycine-rich protein (GRP) highly accumulating in the leaves of lily which exhibits salicylic acid-induced systemic resistance to gray mold disease. By using lily gene silencing system and Arabidopsis transformants, LsGRPI expression was found to confer plant disease resistance via enhancing types of defense responses, and to our surprise, also slightly promote plant growth in the absence of pathogens. Interestingly, the continuous exposure to pathogen-associated molecular patterns (PAMPs) and defense-related phytohormones reduced the growth vigor of $L s G R P 1$ transformants as that in wild type Arabidopsis. However, the treatment of PAMPs or host R protein-recognized effectors elicited earlier and stronger defense responses of callose deposition and reactive oxygen species accumulation or hypersensitive response, respectively, in LsGRP1 transformants rather than wild type Arabidopsis. Thus, disease resistance-related LsGRP1 involves defense-growth tradeoffs probably via a mechanism of immediately activating defense upon PAMP- and effector-triggered signaling, which is a novel function firstly expounded in plant class II GRPs. 
Live cell imaging of chitin induced callose deposition in Arabidopsis

M. KOHARI, D. Chen, S. Ohashi, N. Shibuya, H. Kaku, Dept. Life Sciences, Sch. Agriculture, Meiji University, Tama-ku, Kawasaki, Kanagawa, Japan

Plants are known to generate a structural barrier known as "papillae", which consists of a beta-1,3-linked glucan, callose, and other components at the tip of invading fungal hyphae as a part of immune response. It has been indicated that the host components required for callose synthesis/transportation are localized at the site of fungal invasion. However, the details of this process have been still unclear. Knowing that similar callose deposition could also be induced by chitin, a component of fungal cell wall, we proposed that the chitin induced response mediated by Arabidopsis chitin receptor (AtCERK1) was a useful system to investigate plant immune response against fungi. The purpose of this study is to establish the experimental conditions for the cytological analysis of chitin-induced callose deposition in live cells. After the evaluation of the factors affecting chitin-induced callose deposition in Arabidopsis, we could establish the conditions for live cell callose imaging in pavement cells of Arabidopsis leaf epidermis. For future prospects, we hope to clarify the mechanism of the callose deposition from the aspect of cell biology using this system.

A protein complex formed by Ustilago maydis effectors is essential for virulence

N. LUDWIG (1), L. Liang (1), K. Schipper (2), S. Reissman (3), D. Aßmann (1), C. Gonzalez (1), M. Moretti (1), T. Glatter (1), K. Snetselaar (4), R. Kahmann (1), (1) Max Planck Institute for Terrestrial Microbiology, Marburg, Germany; (2) Heinrich-Heine-Universität, Düsseldorf, Germany; (3) Max Planck Institute for Terrestrial Microbiology, Germany; (4) Saint Joseph's University, Philadelphia, PA, U.S.A.

Ustilago maydis is a biotrophic fungal pathogen, which causes smut disease in its host plant maize. During colonization U. maydis secretes effector proteins to suppress plant defense responses and manipulate the host physiology for its own benefit. A majority of these proteins lack functional annotations and their role in virulence remains to be determined. We systematically deleted effectors, whose expression is linked to the stage where biotrophy is established and thereby identified mutants which failed to cause disease. Mutants of these effectors, named $s t p 1, s t p 2$, stp 3 , stp4 and $\operatorname{stp} 5$ (stop after penetration) were still able to form appressoria and penetrate the plant, but arrested in the epidermal cell layer and elicitated plant defense responses and cell death. A similar phenotype was observed for the previously described effectors pep 1, pst 1 and pst 2 . Co-IP/MS experiments of plant tissue infected by U. maydis expressing tagged essential effectors revealed that all eight effectors form a complex. We provide evidence that not only the presence of the individual complex members, but the formation of the complex itself is necessary for successful colonization. We speculate that the complex is part of the structural machinery for the contact between fungus and host and will discuss what supports this proposition.

Differential nuclear dynamics underpin hyphal network organisation in a plant-pathogenic oomycete E. EVANGELISTI, L. Shenhav, T. Yunusov, M. Le Naour-Vernet, P. Rink, S. Schornack, The Sainsbury Laboratory, Cambridge University (SLCU), Cambridge, U.K.

Oomycete infections of plants involve coordinated pathogen development steps such as cyst germination, appressorium formation as well as the switch from biotrophic to necrotrophic growth. How nuclear-controlled processes are coordinated in multinucleate oomycetes remains to be fully understood. We investigated oomycete nuclear dynamics of the cocoa killer Phytophthora palmivora during plant infection as well as in axenic culture using time-lapse imaging. We observed coordinated bi-directional nuclear movements during plant infection and changes in nuclear dynamics upon appressorium development. During mycelial growth, active and passive movements achieve a near-equal distribution of nuclei along hyphae. This notably involves nuclei moving against the main cytoplasmic flow direction or into hyphal side branches. Such nuclei often change their shape, from near-globular up to extensively stretched. Interestingly, centrosome-labeling Centrin2 protein localizes at the stretched end of actively moving nuclei. This is supporting the hypothesis that astral microtubule-guided movements contribute to nuclear distribution within hyphae. Taken together, these results shed light on oomycete nuclear dynamics and provide a basis for computational modelling of their movement within branched hyphal networks.

Qualitative and quantitative analyses of the colonization characteristics of Bacillus subtilis NCD-2 on cotton root Q. GUO, L. Dong, S. Li, P. Ma, Plant Protection Institute, Hebei Academy of Agricultural \& Forestry Sciences, Baoding, China

Plant soil-borne diseases cause vast economic lost for agriculture, and microbial fungicides are confirmed as efficient and environment friendly method for suppressing plant soil-borne diseases. Bacillus subtilis is an important resource for developing microbial fungicides, and root colonization is prerequire for successful suppressing plant soil-borne disease. Bacillus subtilis NCD-2 is an excellent biocontrol agent against plant soil-borne diseases. With the purpose of determining the colonization characteristics of strain NCD-2 on different parts of cotton root, a constitutive expression promoter was cloned from strain NCD-2 and used to construct green fluorescent protein-tagged strain NCD-2. The colonization characteristics of strain NCD-2 on different root parts were qualitatively observed by confocal laser scanning microscopy and quantitatively analyzed using a traditional plating-PCR count method and a real-time PCR analysis. Results indicted the green fluorescent protein-tagged NCD-2 strain was successfully constructed, and confocal laser scanning microscopy observations revealed that it mainly colonized the differentiation and elongation zones of cotton root. A quantitative analysis confirmed that root tip is the primary area for colonization in autoclaved and non-autoclaved soil. This study provides a clearer understanding of the interactions between biocontrol agent and plant, as well as with the indigenous microorganisms in the soil.

Root-Knot Nematodes Induce Cell Cycle Check Point Activation During Parasitism on Plant Hosts

J. DE ALMEIDA-ENGLER (1), D. C. Do Nascimento (1), L. de Veylder (2), (1) INRA, Université Cote D’Azur, CNRS, UMR 1355-7254, Sophia Antipolis, France; (2) Department of Plant Systems Biology, VIB, Ghent University, Ghent, Belgium 
Root-knot nematodes (RKN; Meloidogyne spp.) are amongst the utmost damaging plant pathogens infecting plant roots and triggering the formation of tumor like structures named galls. Both, the plant mitotic cycle and the endocycle, are indispensable targets for a successful susceptible interaction and key cell cycle genes, as well as inhibitor genes are important components to allow the induction and maintenance of gall development. Among them, the expression of the WEE1 kinase in galls suggest cell cycle check point activation induced by nematode infection. WEE1 is involved in the phosphorylation and inactivation of cyclin-dependent kinase 1-cyclin B complex resulting in cell cycle arrest in response to DNA damage in Arabidopsis. The WEE1 protein is present early during nematode infection most likely due to stress. Functional analysis shows that the lack of WEE1 results in ectopic mitotic activity in galls leading to cumulative mitotic defects possibly due to the inaccurate timing for proper DNA replication and repair in giant cells. Our data suggest a conserved plant WEE1 function in galls triggering cell cycle arrest in response to DNA damage.

A TnSeq approach to study the genetic bases of Xanthomonas campestris pv. campestris adaptation to in vitro and in planta conditions J. S. LUNEAU (1), M. Baudin (2), S. Carrere (3), O. Bouchez (4), M. F. Jardinaud (5), J. Ray (6), A. M. Deutschbauer (6), J. D. Lewis (2), L. D. Noel (1), E. Lauber (1), A. Boulanger (1), (1) Université de Toulouse, LIPM, CNRS-INRA, Toulouse, France; (2) University of California, Berkeley, Albany, CA, U.S.A.; (3) Université de Toulouse, INRA, CNRS, Toulouse, France; (4) Genotoul Genome \& Transcriptome, INRA, Toulouse, France; (5) Laboratory of Plant-Microbe Interactions, Toulouse, France; (6) Lawrence Berkeley National Laboratory, Berkeley, CA, U.S.A.

Xanthomonas campestris pv. campestris $(X c c)$ is the causal agent of the black rot disease on wild and cultivated Brassicaceae worldwide. For decades, the major determinants of $X c c$ pathogenicity have been investigated and characterized, but yet, to this day, little is known about genes contributing to microbial fitness in the plant, especially at early steps of infection. Fortunately, thanks to the quick progression of Next Generation Sequencing techniques, new tools such as Transposon insertion site sequencing (TnSeq) have been developed and now allow the exploration of these aspects. By using TnSeq to perform a fitness analysis on a bacterial mutant population, we initiated a study of Xcc adaptation to both in vitro and in planta conditions. This fairly recent approach has been successfully used over the past few years to identify genes required for the virulence of numerous human bacterial pathogens and very recently produced interesting results in the field of plant pathology. We will explain how, by counting the relative abundance of a barcoded library of Tn-mutants, we will be able to identify genes important or specific to key environments of the in planta life of the bacteria. Thanks to this high-throughput genomic screening method, we address all components of bacterial fitness (metabolic capacities, defense evasion, stress tolerance...) and focus on three biologically relevant plant environments: hydathodes, xylem and mesophyll. Our progress will be presented.

Investigating the role of the early wheat immune system to Zymoseptoria tritici fungi using bio-imaging approaches F. VALENTE (1), C. Rodrigues (1), E. Wallington (2), G. Romana (3), D. Herring (4), G. Thomas (5), M. J. Deeks (1), (1) University of Exeter, Exeter, U.K.; (2) NIAB, Cambridge, U.K.; (3) University of Palermo, Palermo, Italy; (4) University of Birmingham, Birmingham, U.K.; (5) University of Exeter, U.K.

Despite great progress in the agrochemical industry and the high fungicide input in the past 50 years, phyto-pathogenic fungi still represent the main biotic threat for crop security. Zymoseptoria tritici is the main causal agent of the wheat foliar disease Septoria tritici blotch, characterised by a symptomless biotrophic phase lasting 7-11 days, followed by a necrotrophic period with development of fungal asexual sporulation structures called pycnidia throughout the leaf surface. However, several aspects of the first latent phase, including the active role of the early wheat immune system, and the spatiotemporal perception of the hyphae at the sub-cellular scale at the stomatal complex, remain still uncertain. Herein, we are testing the hypothesis that the early wheat immune system responds to pathogenic detection via early changes in cell wall architecture and organelle dynamics at the stomatal complex. In order to address our biological question, we propose a model of host-microbe interaction using bio-imaging approaches based on live-cell imaging and fluorescent proteins labelling plant organelles to investigate early subcellular responses occurring in wheat during the early contact with Z.tritici strains. Our findings could elucidate the role that the early wheat immune system might deploy at the stomatal apparatus in hyphal perception through cell wall reinforcement and organelle clustering at the site of fungal penetration during the initial host-microbe interaction.

Who are you rooting for? Investigating Phytophthora rubi infection of raspberries A. BEZANGER (1), L. Welsh (1), I. Hein (2), S. C. Whisson (1), R. J. Harrison (3), E. M. Gilroy (1), (1) The James Hutton Institute, Dundee, U.K.; (2) The James Hutton Institute/University of Dundee, Invergowrie, Dundee, U.K.; (3) NIAB EMR, East Malling, U.K.

Phytophthora rubi, causing raspberry root rot, is a widespread pathogen in the U.K., with more than $70 \%$ of the U.K.'s soil-based raspberry production affected by Phytophthora Root Rot (PRR). Although PRR first appeared in the U.K. in the 1930s, serious outbreaks across the world only occurred in the 1980s. Little is known about $P$. rubi and thus this project aims to better understand this under-studied pathogen by combining phenotypic and genotypic analyses.

We have successfully developed a hydroponic growth system for raspberry. This allows direct observation of infection in real-time with minimum damage to the roots for a more accurate assessment of disease development. $P$. rubi has been transformed to express fluorescent proteins (eGFP and tdTomato). Infection of hydroponically grown raspberries with fluorescently labelled $P$. rubi showed infection at root tips, hyphal progression along the root and production of reproduction structures (sporangia and zoospores). Phytophthora species are known to deploy a diverse array of effector proteins to facilitate infection. The diversity of effectors in $P$. rubi is being studied using Pathogen Enrichment Sequencing (PenSeq) on a variety of isolates dated from 1945 to 2017, and from a range of locations worldwide. This will yield valuable information on the arsenal of $P$. rubi effectors at an inter and intra-species level. Findings from this work will help to reveal how this pathogen causes disease, and how resistance functions in raspberry.

A poplar rust effector protein associates with the Arabidopsis protein disulfide isomerase-11 to enhance plant susceptibility M. H. MADINA (1), H. Zheng (2), H. Germain (3), (1) University of Quebec at Trois-Rivieres, Trois-Rivieres, QC, Canada; (2) McGill University, Montreal, QC, Canada; (3) University of Quebec at Trois-Rivières, Trois-Rivières, QC, Canada 
Melampsora larici-populina (Mlp), the causal agents of rust disease in Populus, secretes an array of effectors into the host through haustorium. The specific mechanisms by which these effectors promote virulence remains unclear. To address this question, we developed a stable transgenic Arabidopsis line expressed the candidate effector Mlp124357 in fusion with GFP. Confocal microscopy revealed that Mlp124357 targets the tonoplast, transvacuolar strands, and bulbs; constitutive expression of the effector increases plant susceptibility to pathogens. A predicted GXXXG motif present in the protein sequence of Mlp124357 is required for proper subcellular localization since replacement of the glycine residues with alanine (Mlp124357 $7^{\mathrm{GA}}$ ) led to the delocalization of Mlp124357 into the nucleus and cytoplasm. We used immunoprecipitation and mass spectrometry (MS) to identify Mlp124357 interaction partners. We tested five proteins obtained from the MS list and assessed if their absence in planta would lead to a delocalization of Mlp124357. A single knock-out line caused delocalization of the effector confirming that protein disulfide isomerase-11 (PDI-11) is required for the effector tonoplastic localization, which was further confirmed by a complementation test and yeast-two hybrid assay. Moreover, infection assay demonstrated that $A t$ PDI-11 act as a helper for Mlp124357. Our findings established that an effector of Mlp resides at the vacuole surface and modulates plant susceptibility.

Rsv3 modulates abscisic acid defensive roles in the extreme resistance against soybean mosaic virus

M. Alazem (1), R. Jeong (1), K. H. KIM (2), (1) College of Agriculture and Life Sciences, Seoul National University, Seoul, Korea, Republic of (South); (2) Research Institute of Agriculture and Life Sciences, Seoul National University, Seoul, Korea, Republic of (South)

Extreme resistance (ER) is a type of $R$-gene-mediated resistance that rapidly induces a symptomless resistance phenotype, which is different from the phenotypical $R$-resistance manifested by the programmed cell death, accumulation of reactive oxygen species, and hypersensitive response. In soybean cultivar L29, Rsv3 gene is responsible for ER against the avirulent strain G5H of soybean mosaic virus (SMV) but is ineffective against the virulent strain G7H. Our RNA-seq analysis revealed that this ER is achieved through fast trigger of few defence mechanisms; i) induction of abscisic acid (ABA) which results in the accumulation of callose and trapping SMV-G5H at the point of infection, ii) triggering the antiviral RNA silencing pathway, iii) partial activation autophagy pathway, and iv) Suppression of jasmonic acid pathway and large number of WRKY factors. In close examination of ABA's role in ER, ABA treatment increased the expression of several antiviral RNA silencing genes, as well as $P P 2 C 3 a$ which is responsible for the accumulation of callose. These conditions rendered L29 more resistant to G7H. However, in an rsv-null cultivar (Sumyumkung), ABA application did not affect plant resistance to both SMV strains, with PP2C3a and most the RNA silencing genes remained unaffected. Collectively, our data suggest that Rsv3 modulates ABA's effect on these two resistance pathways; callose and the antiviral RNA silencing pathway, and attribute large role for ABA in the Rsv3-mediated ER.

JAZ4 functions as positive or negative regulator of jasmonate signaling in different Arabidopsis organs L. DEMOTT (1), P. R. Oblessuc (2), M. Melotto (2), (1) University of California, Davis, Davis, CA, U.S.A.; (2) University of California, Davis, CA, U.S.A.

JAZ proteins are transcriptional repressors, governing control of plant growth, development, and defense responses. As we have previously reported, jaz4 mutants display hyper-susceptibility to Pseudomonas syringae pv. tomato strain DC3000, whereas overexpression of the dominantnegative JAZ4 $\triangle$ Jas leads to enhanced host resistance. Here, we characterized JAZ4 function pertaining to phenotypes related to secondary metabolites, growth, and development. Jasmonate signaling has been shown to suppress plant growth rate by reducing cell size and number. Surprisingly, jaz4 plants show enhanced leaf surface area and number of leaves compared to WT. However, JAZ4 $\Delta$ Jas plants display enhanced petiole growth, as expected. Next, we analyzed JA-induced root growth inhibition, in which JA-insensitive JAZ4 $\Delta$ Jas plants have elongated roots under normal conditions but indeed respond to JA treatment, inhibiting root growth to WT levels. JAZ proteins are known to reduce accumulation of secondary metabolites, in which we analyzed using spectrophotometric overlay and found JAZ4AJas plants have lower levels of flavonoids and chlorophyll, whereas jaz4 plants accumulate more anthocyanin. Histochemical analysis shows tissue-specific promoter activity corresponding with observed mutant and dominant-negative phenotypes. Our results suggest that JAZ4 functions as a positive and negative regulator within JA signaling, providing insight into specificity of JA signaling at the JAZ4 node.

Chitin-deacetylase activity has important roles in inducing appressorium formation S. SHOTA, Y. Shiga, K. Misa, A. Takayuki, K. Takashi, Tokyo University of Science, Japan

Magnaporthe oryzae, the plant pathogenic fungus that causes rice blast, forms an appressorium to infect plant cells. Chitin binding protein 1 (Cbp1), with its two chitin-binding domains, chitin deacetylase (CDA) domain, signal peptide, and ST cluster, is a putative GPI-anchored CDA protein. Appressorium formation is dramatically delayed in the $C B P 1$ deletion mutant $(\triangle \mathrm{cbp} 1)$ relative to the wild type strain (Kamakura et al., 2002). In addition, the CDA activity of $\mathrm{Cbp} 1$ is required for appressorium formation (Kuroki et al., 2017). Here, we analyzed the Cbp1 homologs and the role of the CDA-catalyzed byproduct in appressorium formation. The expression of six CDA homologs (non-GPI-anchored protein), was not up-regulated in the $\Delta c b p l$ mutant, while that of several homologs was induced by adding cutin monomer. Since chitosan accumulation was observed at the tip of the germ tube in the wild type strain, the $\mathrm{Cbp} 1$ activity and location might be important as an activator of these homologs for cell wall degradation. The CDAs convert chitin into chitosan and release acetic acid as a byproduct. We verified the effect of acetic acid for appresoriumformation. By adding extremely low concentration of acetic acid (1 fM), appressorium formation was restored in the $\Delta c b p 1$ mutant, although high concentration $(1 \mathrm{mM})$ showed cell toxicity. These results suggest that $\mathrm{Cbp} 1$ plays a role in not only cell wall degradation but also in the induction of appressorium formation via release of acetic acid.

NbRLP1 plays a role in plant immunity toward the oomycete plant pathogen Phytophthora parasitica Y. H. LI (1), S. Wei-che (1), C. W. Wang (2), R. F. Liou (1), (1) National Taiwan University, Taipei, Taiwan; (2) Academia Sinica, Taiwan

In response to pathogen infection, plants employ pattern recognition receptors to recognize PAMPs, thereby eliciting pattern triggered immunity. Recently, we found that leucine-rich repeat (LRR) protein kinase SISOBIR1 plays a key role in plant immunity toward Phytophthora parasitica infection as well as plant response to its elicitin ParA1. Here, we provide further evidence to explore how SISOBIR1 is involved in these 
processes. Using co-immunoprecipitation (Co-IP) coupled with mass spectrometry, we identified a LRR-containing receptor-like protein termed NbRLP1, which may associate with SISOBIR1. Indeed, Co-IP analysis revealed that NbRLP1-GFP pulled down NbSOBIR1-mCherry in the presence or absence of ParA1 and the BiFC assay further supported the interaction. Imaging results indicated that NbRLP1-GFP expressed by agroinfiltration is located in the membrane of endoplasmic reticulum (ER). Interestingly, NbRLP1-GFP is translocated to ER-plasma membrane contact sites (EPCS), accompanied by the movement of SISOBIR1-mcherry from plasma membrane to endosomes upon ParA1 treatment. Moreover, NbRLP1-GFP overexpression appeared to increase the number of NbSOBIR1-containing endosomes. Finally, we found that NbRLP1 overexpression enhanced the ParA1-induced necrosis on Nicotiana benthamiana, which suggests a role of NbRLP1 in plant immunity toward $P$. parasitica infection. Together, we propose that NbRLP1 mediates plant immunity through its interaction with NbSOBIR1.

A small protein secreted by Sclerotinia sclerotiorum is a new necrosis-inducing effector that requires BAK1 and SOBIR1 for its activity S. SEIFBARGHI (1,2), M. H. Borhan (1), Y. Wei (2), C. Coutu (1), D. Bekkaoui (1), D. D. Hegedus (1,2), (1) Agriculture and Agri-Food Canada, Saskatoon, SK, Canada; (2) University of Saskatchewan, Saskatoon, SK, Canada

Sclerotinia sclerotiorum, the causative agent of canola stem rot, is dependent on necrosis-inducing effectors to induce cell death, a hallmark of the necrotrophic stage of infection. In investigation of S. sclerotiorum necrosis-inducing proteins, a small, secreted protein was characterized. The 152 amino acids long predicted protein has four cysteine residues. The conversion of the cysteine residues to alanine reduced incidence and severity of necrosis symptoms, indicating that cysteine residues might play crucial roles in the function of this protein. Subcellular localization studies using fusion to the green fluorescent protein revealed that this protein targeted to the endomembrane system. This protein induced strong cell death in Nicotiana benthamiana via Agrobacterium tumefaciens-mediated infiltration that required a signal peptide and secretion to the periplasmic space for its activity. Investigating the biological function of orthologous proteins in four other fungal pathogens showed the similar activities to induce cell death. Furthermore, virus-induced gene silencing revealed that necrosis-inducing ability of this protein was dependent on the presence of plant receptor-like kinases BAK1 and SOBIR1, indicating the involvement of cell surface receptors in recognition of the protein. This study enabled the discovery of a novel necrosis-inducing effector that might facilitate the effector-guided breeding to identify germplasm with improved resistance to S. sclerotiorum.

\section{A virulence-related protein produced by Botrytis elliptica}

Y. J. WANG, C. Y. Chen, National Taiwan University, Taipei, Taiwan

Botrytis elliptica, a lily murderer causes necrosis symptoms on leaves and flowers called Botrytis blight. B. elliptica is considered as a necrotrophic pathogen and assumed secreting a plenty of plant-cell-death-inducing factors like the relative pathogenic fungus, $B$. cinerea. In this study, the B. elliptica conidia culture medium with lily leaf extract was collected as the source of secreted fungal proteins and demonstrated its lethal ability by electrolyte leakage assay on lily leaf discs. Owing to the binding capacities and preferences of PVDF membrane and MCE membrane, the lethal strength examinations of both filtrates were conducted. The PVDF-filtrated culture fluid maintained lethal ability on lily leaves, which was then examined by SDS-PAGE for differential bands. LC-Mass/Mass analysis indicated one hit to pectin methyl esterase of $B$. cinerea, BcPME1. Referring to the crucial contribution of BcPME1 to the virulence of $B$. cinerea Bd90, we tried to clone the pectin methyl esterase gene from $B$. elliptica and prove its contribution to fungal virulence.

Immune signals at the endoplasmic reticulum (ER) control broad-spectrum acquired resistance.

R. E. SCHWARZENBACHER, D. Pardo, G. E. Wardell, J. Stassen, J. Ton, The University of Sheffield, Sheffield, U.K.

Beta-aminobutyric acid (BABA) is a plant-endogenous stress metabolite that induces broad-spectrum resistance. Binding of BABA to the ERassociated receptor protein IBI1 primes its translocation to the cytosol upon pathogen attack, where it mediates augmented defence expression. Integrated transcriptome-metabolome analysis of wild-type and ibil-1 plants revealed that IBI1 suppresses abscisic acid (ABA) signaling during expression of BABA-induced resistance (BABA-IR) against Hyaloperonospora arabidopsidis (Hpa). Yeast-two hybrid screens identified several ER- and cytosol-localised IBI1 interactors. Bimolecular fluorescence complementation (BiFC) analysis confirmed that IBI1 interacts with the ABA-inducible transcription factors (TFs) VOZ1/2. These cytosol-localised TFs control Hpa resistance by suppressing ABA-dependent signaling and promoting callose-mediated immunity, indicating that $\mathrm{VOZ1} / 2$ act in a negative feedback loop to channel ABA signaling towards cell wall defence. BiFC analysis also confirmed the interaction between IBI1 and the ER-localised fatty acid hydroxylase FAH2, which controls the biosynthesis of ER stress-related lipids. Furthermore, BABA induces ER stress response genes in an IBI1-dependent manner and mutations in both FAH2 and the IRE Ia/b-dependent ER stress pathway reduce BABA-IR, indicating a role for ER stress signaling in BABA-IR. Collectively, our work reveals an important role for the ER in cellular defence signaling during acquired immunity.

OsWRKY10 is involved in defense signaling of rice to Xanthomonas oryzae pv. oryzae

N. Choi (1), J. H. Im (1), S. Park (2), D. J. HWANG (1), (1) National Institute of agricultural science, Korea, Republic of (South); (2) National Institute of agricultural Sciences, Korea, Republic of (South)

WRKY proteins are well known to play essential roles in regulation of pathogen defense as negative or positive regulators. Based on our genomewide expression profiling of OsWRKYs upon the infection of Xanthomonas oryzae pv. oryzae (Xoo), many OsWRKY TFs were induced in both compatible and incompatible pathogen interaction. Among them we characterized the function of OsWRKY10 in this study. Ectopic expression of OsWRKY10 resulted in reduced susceptibility to a bacterial pathogen, Xanthomonas oryzae pv. oryzae; this reduction was compromised in transgenic lines under-expressing OsWRKY10 generated by RNAi strategy. Defense-associated genes were highly up-regulated in OsWRKY10overexpressing (ox) lines whereas reduced in OsWRKY10 knock-down (kd) lines, suggesting that OsWRKY10 activates defense responses. It was revealed that OsWRKY10 was a transcriptional activator in yeasts and was localized to the nucleus in plants. OsWRKY10 bound directly to the promoter of OsPR1a, a gene associated with defense, and activated its transcription in vivo. Take together OsWRKY10 acts as a positive regulator that activates the defense response to Xoo by directly binding to the promoter region of OsPR1a. 
Analysis of type II secretion systems from Xanthomonas campestris pv vesicatoria S. DREHKOPF, D. Buettner, Martin-Luther Universität, Halle, Germany

Xanthomonas campestris pv. vesicatoria $(\mathrm{Xcv})$ is the causal agent of bacterial spot disease on pepper and tomato plants. During the host-pathogen interaction, $X c v$ injects effector proteins via a type III secretion (T3S) system into the plant cells to manipulate cellular pathways to its own benefit. The successful infection also depends on a type II secretion (T2S) system, which secretes degradative enzymes into the extracellular milieu. Virulence functions have been attributed to type II-secreted proteases, xylanases and a lipase of $X c v$. Notably, comparative analyses in the related pathogen $X$. campestris pv. campestris revealed that T2S systems are highly substrate specific. Thus, T2S substrates from $X c v$ and homologous proteins from $X c c$ are secreted independently of the T2S systems in $X c c$. Furthermore, the absence of functional T2S systems in $X c v$ did not completely abolish the secretion of T2S substrates, suggesting that these proteins can also be targeted to alternative transport routes such as outer membrane vesicles. Domain swapping experiments and complementation studies with T2S substrates and T2S mutants from $X c v$ and $X c c$ will help to identify protein regions in type II-secreted proteins or components of the T2S system which are involved in substrate recognition.

Natural Variation in AGO2 determines susceptibility to Potexvirus and Potyvirus but not to Cucumovirus. A. ADURogBAngBA (1), N. Montes-Casado (2), C. Brosseau (1), F. Garcia-Arenal (2), P. Moffett (3), (1) Univ de Sherbrooke, Sherbrooke, QC, Canada; (2) UPM, Madrid, Spain; (3) Université de Sherbrooke, Sherbrooke, QC, Canada

RNA silencing is a major mechanism of constitutive antiviral defense in plants. Arabidopsis encodes four Dicer-like (DCL) proteins and ten different Argonaute (AGO) proteins that are specialized to function in different RNA silencing-related mechanisms. We have previously shown that AGO2 plays an important role in protecting plants against viruses, including Potato virus X (PVX) in Arabidopsis. However, despite having a functional AGO2 protein, Nicotiana benthiana is highly susceptible to PVX. Using a transient expression system in $N$. benthamiana leaves, we found that AtAGO2, but not $\mathrm{NbAGO}$, possesses antiviral activity against PVX. Consistent with this, we find that the activity of NbAGO2, but not AtAGO2, is suppressed by the PVX suppressor of RNA silencing, P25. Therefore, we generated transgenic tomato and $N$. benthamiana plants containing AtAGO2 and NbAGO2 constructs. These transgenic lines were inoculated with PVX and accumulation levels were assessed, and our results indicate that transfer of $\mathrm{AGO} 2$ between species can confer a degree of resistance to viruses. In addition, we have observed natural variation in AtAGO2 in naturally occurring Iberian Arabidopsis ecotypes and found that certain polymorphisms correlate with the degree of susceptibility to PVX and to Turnip mosaic virus (TuMV) but not with susceptibility to Cucumber mosaic virus (CMV). Our results indicate that natural variation in $A G O 2$ can have important effects on infection for some, but not all viruses.

Stealthy infections - Pseudomonas syringae AlgU downregulates flagellin expression, helping to minimize plant immune activation B. SWINGLE, USDA-ARS and Cornell Universty, Ithaca, NY, U.S.A.

Flagella power bacterial movement to access or avoid certain environments, ultimately increasing a cell's probability of survival and reproduction. In some cases, flagella and chemotaxis are key virulence factors, enabling pathogens to access suitable host tissues. However, plant and animal immune systems have evolved receptors to sense flagellar filament proteins as signatures of bacterial infection. Microbes poorly adapted to avoid or counteract these immune functions are unlikely to proliferate in host environments, and this selective pressure has driven the evolution of diverse and often redundant pathogen compensatory mechanisms. We tested the role of AlgU, the Pseudomonas stress response sigma factor, in regulating flagellar expression in the context of Pseudomonas syringae-plant interactions. We found that AlgU is necessary for downregulating flagellin gene expression in planta and reduced plant immune elicitation. This AlgU-dependent regulation of flagellin is beneficial to bacterial growth in the course of plant infection and eliminating the plant's ability to detect flagellin suppresses the requirement for AlgU. Together, these results add support to an emerging model in which P. syringae AlgU functions at a key control point that serves to optimize expression of bacterial functions during host interactions, including minimizing expression of immune elicitors and concomitantly upregulating beneficial virulence functions.

An efficient strategy for obtaining mutants by targeted gene deletion in Ophiostoma novo-ulmi J. L. SARMIENTO VILLAMIL, T. Campos de Oliveira, E. S. Naruzawa, L. Bernier, Faculté de foresterie, de géographie et de géomatiqueUniversité Laval, Québec, QC, Canada

Dutch elm disease (DED) caused by Ophiostoma novo-ulmi is the most devastating disease of elm trees. The DED pathogen is transmitted from diseased to healthy elms by elm bark beetles. According to the current model of infection, $O$. novo-ulmi adopts different cell shapes and produces an array of specific metabolites to induce DED. In fungi, control of cell differentiation and secondary metabolism is often coordinated by the same molecular mechanisms. Understanding the infection mechanisms used by phytopathogen may help the development of novel control strategies. One of the most powerful tools for studying gene function is the generation of null mutants. However, gene deletion methods in $O$. novo-ulmi are laborious and deficient in null mutant production, with rates less than $1 \%$. We developed a gene knockout strategy that allowed us to obtain auxotrophic mutants of this vascular wilt fungi at rates higher than $88 \%$. This strategy is based on 1 ) using a $\Delta$ mus 52 O. novo-ulmi strain, which is deficient in NHEJ pathway; 2) transforming this strain with the latest version of OSCAR plasmids that generate deletion constructs containing the toxin-coding HSVtk gene which prevents ectopic integration of the T-DNA; and 3) crossing derived mutants with a sexually compatible wild type $O$. novo-ulmi strain in order to recover targeted deletion mutants that are wild-type for NHEJ. Our improved strategy is being used for deleting candidate genes for parasitic fitness in O. novo-ulmi.

A plant RabGAP negatively regulates autophagy and defence against the Irish potato famine pathogen Phytophthora infestans. A. Y. LEARY (1), Y. F. Dagdas (2), S. Kamoun (3), T. O. Bozkurt (1), (1) Imperial College London, U.K.; (2) Gregor Mendel Institute of Molecular Plant Biology, Vienna, Austria; (3) The Sainsbury Laboratory, University of East Anglia, Norwich Research Park, Norwich, U.K. 
A variety of autophagy adaptors and cargo receptors mediate selective autophagy pathways in plants. These components are tightly regulated to bind core autophagy protein ATG8 for coordinating diverse selective autophagy pathways. However, the molecular intricacies of these regulatory networks and how they contribute to immunity remain largely unknown. In this study, we identified Rab3GAP, a Rab GTPase activating protein that directly binds ATG8 through a canonical ATG8-interacting motif (AIM). Overexpression of Rab3GAP reduced the number of autophagosomes in an AIM and GAP domain dependent manner. Furthermore, overexpression of Rab3GAP counteracted the plant cargo receptor Joka2 and the Phytophthora infestans effector protein PexRD54 triggered autophagy. Interestingly, Rab3GAP overexpression also enhanced susceptibility to $P$. infestans in a GAP dependent but AIM independent manner. These results suggested that Rab3GAP negatively regulates autophagy and immunity through small GTPases implicated in these processes. We identified a RabGTPase, Rab8a that interacts with Rab3GAP in vivo, positively contributes to autophagosome formation, and localises to the autophagosome biogenesis sites. Rab8a also positively contributes to immunity against $P$. infestans by directing vesicle trafficking to the pathogen interface. Altogether, our results implicate Rab3GAP as a negative regulator of plant autophagy and immunity against $P$. infestans and provide insights into autophagosome biogenesis.

BAK1-based immunity controls hydathode colonization and vascular spread of Xanthomonas campestris pv. campestris in Arabidopsis. M. Van Hulten, S. Chatterjee, M. Kwaaitaal, E. Enderlee, H. A. VAN DEN BURG, University of Amsterdam, Amsterdam, Netherlands

The bacterial plant pathogen Xanthomonas campestris pv. campestris, the causal agent of Black Rot in Brassicaceae, has a unique entry strategy via hydathodes, which are specialized water pores on the leaf blade. From here the bacterium colonizes the vasculature and spreads systemically. Whereas bacterial host entry via wounds or stomata is well understood, surprisingly little is known of the resistance and virulence mechanisms that operate in these natural openings. We will present data on the interaction between Xanthomonas-Arabidopsis thaliana interaction focusing on a "guttation-based natural entry" assay that permits specific colonization of the hydathodes and in a compatible interaction outbreak to the xylem. Our data imply that $B A K 1$-dependent immunity against Xanthomonas operates in both the hydathodes and the xylem. Furthermore, this hydathodal immunity requires EDS1/PAD4 and accumulation of Salicylic acid. During early infection stages, this immunity controls bacterial levels in hydathodes, while later it prevents bacterial spread into the vasculature. As this immunity was independent of type III effectors, it can be classified as a Pattern-triggered immunity (PTI). However, effectors were essential for hydathode ingress, even in a hypersusceptible or immunocompromised background, eluding that they are required for full pathogenicity. To our knowledge, this is the first evidence that PTI operates in hydathodes to contain and restrict bacterial proliferation.

Pseudomonas mediates the crosstalk between autophagy and intracellular trafficking processes during plant immunity P. B. GOUGUET (1), S. Richter (2), J. Sklenar (3), D. Hofius (4), F. L. H. Menke (3), S. Kamoun (3), G. Jürgens (2), S. Üstün (5), (1) Universität Tübingen, Tübingen, Germany; (2) Universität Tübingen, Germany; (3) The Sainsbury Laboratory, University of East Anglia, Norwich Research Park, Norwich, U.K.; (4) Swedish University of Agricultural Sciences (SLU), Uppsala, Sweden; (5) Center for Plant Molecular Biology (ZMBP), Tübingen, Germany

Autophagy is a conserved intracellular recycling pathway that has been shown to be involved in plant-pathogen interactions, notably at the level of bacterial Type-III-secreted effectors (T3Es). T3Es have been previously found to target the host's proteasome in order to increase virulence. Yet the precise mechanism of how Pseudomonas effectors alter proteasome function persists to be equivocal. The Pseudomonas syringae T3E HopM1, in addition to inhibiting the proteasome, was recently shown to activate autophagic flux resulting in proteasome degradation. A known interactor of HopM1 is the ARF-GEF MIN7/BIG5 which localizes to the trans-Golgi Network (TGN). Under Pseudomonas syringae infection, we have identified that MIN7/BIG5, along with proteasome-subunits, associate with the autophagy pathway. Given the fact that HopM1 mediates degradation of MIN7/BIG5 during infection, we hypothesized that the loss of MIN7/BIG5 alters autophagy. Indeed, we have discovered that the absence of MIN7/BIG5 or inhibitor-mediated perturbations of vesicle trafficking activated the autophagy pathway and hence resulted in enhanced degradation. This preliminary data suggests that the HopM1-MIN7/BIG5 interaction at the TGN mediates a possible crosstalk between autophagy and intracellular trafficking processes.

The fungal effector Mlp37347 unlock the gateway to intercellular trafficking

M. S. RAHMAN (1), H. Germain (2), J. F. Laliberte (3), (1) University of Quebec at Trois-Rivieres, Trois-Rivières, QC, Canada; (2) University of Quebec at Trois-Rivières, Trois-Rivières, QC, Canada; (3) Institut National de la Recherche Scientifique-Institut Armand-Frappier, Laval, QC, Canada

Melampsora larici-populina is the most devastating pathogen of poplar trees; it causes poplar leaf rust. Genomic studies revealed that it possesses a repertoire of 1184 small secreted proteins. Some of them are characterized as candidate effectors. How these effectors promote virulence is still a blurred picture. This study was designed to investigate the candidate effector Mlp37347's role during infection. We developed a stable transgenic Arabidopsis line expressing the effector tagged with the GFP. In confocal microscopy image, we found that the effector accumulated exclusively in plasmodesmata (PD). Transcriptome profiling and gene ontology (GO) analysis of transgenic lines and wild-type (WT) revealed that glucan catabolic process genes are specifically up-regulated in effector expressed lines, suggesting that the deposition of the polysaccharide callose at PD will control the movement of endogenous macromolecules. This effector has previously been shown to interact with glutamate decarboxylase 1 (GAD1) and in silico docking of Mlp37347-GAD1 also showed strong binding affinity. The virulence activities of the effector in WT, and transgenic lines using bacterial and oomycete pathogens disclosed that the effector promotes oomycete (fungus-like) growth but bacterial. Further diffusion assay established that effector makes the dilation of PD and facilitates PD flux level. To conclude, the effector Mlp37347 targets PD in host cells, promote infection by regulating PD flux.

Functional characterization of Arabidopsis thaliana macrophage migration inhibitory factor/D-dopachrome tautomerase-like (AtMDL) proteins and their potential to exert conserved cross-kingdom functions

K. GRUNER (1), D. Sinitski (2), M. Brandhofer (2), P. Bourilhon (2), C. Coustau (3), M. Jaouannet (3), A. Kapurniotu (4), H. Keller (3), K. H. Kogel (5), C. Kontos (4), A. Reinstädler (1), B. T. Werner (6), P. Winkler (1), J. Bernhagen (2), R. Panstruga (1), (1) RWTH Aachen University, Aachen, Germany; (2) LMU Munich, Munich, Germany; (3) INRA Sophia Antipolis, Sophia Antipolis, France; (4) Technical University of 
Munich, Munich, Germany; (5) Institute of Phytopathology, Justus-Liebig-Universität Gießen, Germany; (6) Justus Liebig University Giessen, Giessen, Germany

Human macrophage migration inhibitory factor (MIF) is a versatile regulator of innate and adapted immune responses. As MIF is involved in diverse pathophysiological processes, it serves as a prominent human disease marker. Despite its small protein size and lack of any recognizable modular domains, MIF surprises with functional complexity, including catalytic and signaling activities. MIF is an ancient molecule, evolutionarily conserved across kingdoms and hence also present in plants. To date, the roles of MIF proteins in plants are unknown. Based on the identification of three MIF-like orthologs in Arabidopsis (termed AtMDLs), our analyses shed first light on their functions in plants. Utilizing transient and stable expression of fluorophore-tagged AtMDLs, we studied their subcellular localizations. We further selected a set of single, double and triple Atmdl mutants, challenged them with various biotic stresses and found an altered Pseudomonas syringae infection phenotype. Alongside, we noticed a delay in the onset of flowering in these mutants. Finally, cross-kingdom complementation studies revealed conserved and divergent activities of recombinant human MIF and Arabidopsis MDL proteins in characterized human MIF functions. Together, our analyses indicate an involvement of $A t$ MDLs in developmental processes in Arabidopsis as well as conserved immunological functions across kingdoms.

Tracking a cereal killer: How does Fusarium graminearum utilise the plasmodesmata to further infection? L. BAGGALEY (1), A. Huttly (1), C. Sparks (1), S. Kurup (1), C. Faulkner (2), M. J. Deeks (3), K. Hammond-Kosack (1), (1) Rothamsted Research, Harpenden, U.K.; (2) John Innes Centre, U.K.; (3) University of Exeter, U.K.

Fusarium graminearum, a fungal ascomycete, is the primary causal agent of Fusarium Ear Blight (FEB), a devastating disease of small grain cereals worldwide. Economic losses are accounted for by reductions in grain quality - through the production of mycotoxins - grain yield and ecosystem health. How $F$. graminearum infection transitions from an asymptomatic phase to a symptomatic one, in wheat, remains unclear. This transition in infection corresponds with one from intercellular to intracellular hyphal growth, resulting in rapid tissue colonisation and cell death. We therefore hypothesise that successful intracellular hyphal growth relies upon thin streams of cytoplasm between adjacent cells, vital for cellto-cell communication, namely plasmodesmata. Like the rice pathogen, Magnaporthe oryzae, detailed microscopic studies have demonstrated $F$. graminearum undergoes hyphal constriction to pass through plasmodesmata. Utilising a range of imaging techniques, we aim to discover what happens at the $F$. graminearum-plasmodesmata interface. Transgenic wheat plasma membrane reporter lines have been produced, to reveal at both the cellular and molecular level, the dynamics of this interaction. Simultaneously, the establishment of a wheat coleoptile infection assay has created a high-throughput screening system. Genes associated with plasmodesmatal function and callose deposition have also been identified and will be silenced to test their effect on $F$. graminearum virulence.

How NorA and HmpX collaborate to protect Ralstonia solanacearum cellular machinery from plant and bacterial oxidative stress A. N. TRUCHON (1), C. G. Hendrich (2), C. Allen (1), (1) University of Wisconsin-Madison, Madison, WI, U.S.A.; (2) University of Wisconsin - Madison, Madison, WI, U.S.A.

Ralstonia solanacearum $(R s)$ is a soilborne vascular pathogen of many economic crops, including tomato. Rs survives low- $\mathrm{O}_{2}$ environments like flooded soils and plant xylem because it can transition between aerobic and anaerobic respiration. $R s$ prefers to respire with $\mathrm{O}_{2}$ and quickly depletes it, so $R s$-colonized xylem vessels become hypoxic. $R s$ then respires on the $30 \mathrm{mM}$ nitrate $\left(\mathrm{NO}_{3}{ }^{-}\right)$present in tomato xylem sap, reducing it to an inert gas. Bacterial genes encoding denitrifying respiration are up-regulated in planta and this metabolic pathway is required for full virulence on tomato. But denitrifying respiration comes at a cost, producing destructive oxidative radicals like nitric oxide (NO), which also act as plant defense signals. To mitigate oxidative stress and protect its delicate cellular machinery, Rs must metabolize or immobilize toxic radicals. We found that a NO-binding protein, NorA, and a di-oxygenase, HmpX, are also highly upregulated in planta and work in concert to mitigate NO stress. Mutating either nor $A$ or $h m p X$ had little effect on oxidative radical tolerance or virulence, but a $\Delta$ nor $A / \Delta h m p X$ double mutant had severe defects in both traits. To identify the cellular processes affected by NorA and HmpX, we profiled transcriptomes of $R s$ wild-type and $\Delta n o r A$ or $h m p X$ mutants growing under denitrifying conditions or oxidative stress in culture and while infecting tomato plants.

Key region(s) of LsGRP1 for plant defense against grey mold Y. R. LAI, C. H. Lin, C. Y. Chen, National Taiwan University, Taipei, Taiwan

Lily grey mold caused by Botrytis elliptica is a severe disease in Taiwan. Drench with salicylic acid could systemically enhance resistance to grey mold in Lilium oriental hybrid cv. Stargazer accompanied with the accumulation of a leaf-specific plant class II glycine-rich protein LsGRP1. LsGRP1 consists of a signal sequence $\left(\right.$ LsGRR1 $\left.{ }^{\mathrm{SS}}\right), \mathrm{N}$-terminal $\left(\mathrm{LsGRP} 1^{\mathrm{N}}\right)$, glycine-rich (LsGRR1 ${ }^{\mathrm{G}}$ ) and cysteine-rich C-terminal $\left(\mathrm{LsGRR} 1^{\mathrm{C}}\right.$ ) regions and was proven to confer plant disease resistance via enhancing defense responses as assayed in transgenic Arabidopsis and genesilencing system of lily. To identify the key region(s) of LsGRP1 involved in plant defense, host necrosis and defense-related callose deposition upon grey mold challenge were examined post agroinfiltration with enhanced green florescence protein (EGFP) fusions of LsGRP1 or its partial deletions. Among all treatments, LsGRP1 exhibited the best protection and defense-enhancing efficiencies. LsGRR1 ${ }^{\text {SS }}$ deletion was severely reduced LsGRP1-conferred defense function. Similarly, the differences in grey mold resistance were also indicated in Arabidopsis transformants of $L s G R P 1$ and its deletions. Presumably, LsGRP1-mediated defense enhancement is highly correlated to its localization in plant cells. Subcellular distribution of LsGRP1 is examined by EGFP imaging on LsGRP1-EGFP transgenic Arabidopsis. This research will facilitate the elaboration of the working mechanism of LsGRP1 in plant resistance.

Lignified Apoplastic Structure Spatially Confines Pathogens and Enhances Disease Resistance in Plants S. H. Kim, M. H. LEE, H. S. Jeon, O. K. Park, Department of Life Sciences, Korea University, Seoul, Korea, Republic of (South)

Plants are exposed to diverse microorganisms having capability to invade and proliferate in the apoplastic space. Despite great advances in the study of plant immunity, the molecular mechanism how plants prevent the spread of pathogens remains unclear. Several reports have shown that 
lignin accumulates during the plant immune response against bacterial pathogens. In this study, we demonstrate that the lignin-deposited structure formed during plant-pathogen interactions inhibits the spread and growth of pathogens. Our results suggest that pathogen-induced lignin deposition plays a critical role in disease resistance.

Understanding the wheat Lr67 multipathogen resistance gene: A novel gain of function underpins resistance R. MILNE (1), K. Dibley (1), J. Bose (2), A. Ashton (1), P. Ryan (1), S. Tyerman (2), E. Lagudah (1), (1) CSIRO Agriculture and Food, Canberra, ACT, Australia; (2) School of Agriculture, Food and Wine, The University of Adelaide, Adelaide, SA, Australia

Fungal pathogens are a major constraint to global crop production, hence plant genes encoding pathogen resistance are important tools for combating disease. Many of the pathogen-resistance genes already identified contain nucleotide binding-leucine rich repeat (NLR) domains and, because they are pathogen- or race-specific, they are prone to being overcome by pathogens. A small number of resistance genes provide a partial, durable resistance to multiple pathogens. One example is the $L r 67$ gene in wheat which confers partial resistance to multiple biotrophic pathogens including stem rust, stripe rust, leaf rust and powdery mildew. Lr67 belongs to the STP13 subfamily of monosaccharide transporters and two variants exist (susceptible and resistant) which differ by two amino acids located in transmembrane regions (G144R, V387L). The susceptible variant (Lr67sus) functions as a high affinity hexose/proton symporter whereas the resistant variant (Lr67res) is incapable of transporting hexoses. Our recent findings indicate that Lr67res has a novel gain-of-function over Lr67sus which is linked with the resistance phenotype. Moreover, only a single mutation (G144R) is required to confer this phenotype. This gain of function was characterised using yeast and Xenopus laevis oocyte expression systems. We hypothesise that this Lr67res gain-of-function may cause physiological changes in planta that hinder pathogen development.

A nuclear protein NbTFIISL from Nicotiana benthamiana is involved in the movement of Bamboo mosaic virus

L. Y. CHIU, Graduate Institute of Biotechnology, National Chung Hsing University, Taiwan

Bamboo mosaic virus (BaMV) is a single-stranded positive-sense RNA virus belonging to the Potexvirus genus of Alphaflexiviridae. To identify the host factors involved in BaMV infection, we used cDNA-AFLP to screen the differentially expressed genes. A downregulated gene ACAC5-2 was revealed to affect the accumulation of BaMV in the inoculated and systemic leaves when the expression of ACAC5-2 was knocked down. However, the accumulation of BaMV in the ACAC5-2-knockdown protoplasts was shown no significant effect. Furthermore, the size of the fluorescent foci derived from the inoculated virion carrying GFP on the ACAC5-2-knockdown leaves was smaller than that of the control. These results suggested that ACAC5-2 could assist in BaMV cell-to-cell and systemic movement. In addition, the full-length cDNA of ACAC5-2 was obtained by rapid amplification of cDNA ends and was compared to the Nicotiana benthamiana database. This gene could encode a 992 -amino acid polypeptide possessing a TFIIS N-terminal domain, a conserved protein binding domain often being found at the transcription elongation factors. Therefore, this gene was designated as $N$. benthamiana transcription elongation factor TFIIS-like protein, NbTFIISL. The fusion protein NbTFIISL-OFP was revealed to mainly localize at the nucleus by confocal microscopy. How a nuclear protein is involved in assisting the movement of BaMV will be discussed.

Type II phosphatidate phosphatase gene CoPAP2 of Colletotrichum orbiculare is involved in fungal pathogenesis and host defense responses

S. KODAMA (1), M. Sakakiya (1), S. Takeyama (1), Y. Toyoda (1), T. Nishiuchi (2), Y. Kubo (1), (1) Kyoto Prefectural University, Kyoto, Japan; (2) ASRC, Kanazawa University, Kanazawa, Japan

The hemibiotrophic fungal pathogen Colletotrichum orbiculare forms appressoria as infection structures and causes anthracnose disease in cucumber. Screening of T-DNA insertional mutants deficient in pathogenicity led to the identification of a gene encoding a type II phosphatidate phosphatase domain protein CoPap2. Target gene deletion of CoPAP2 caused decrease in the frequency of host penetration by appressoria and subsequent lesion formation, although morphogenesis of appressoria of $\triangle$ copap 2 was normal and indistinguishable from those of the wild type. CoPap2 conserves lipid phosphate binding motifs conserved among yeast lipid phosphate phosphatases (LPPs). Accordingly, point mutations of the motifs resulted in reduced pathogenicity. Interestingly, the Acopap 2 formed lesions on heat-shocked cucumber cotyledons compared to the non-heat-shocked plants, suggesting that host defense responses may play a role in the defect of $\triangle$ copap 2 pathogenesis. The deposition of callose and ROS by host cells inoculated $\triangle$ copap 2 were similar to those induced by the wild type. In addition, $\mathrm{H}_{2} \mathrm{O}_{2}$ sensitivity of $\triangle$ copap 2 was similar to those of the wild type. Transcriptome analyses of $\triangle$ copap 2 during infection revealed that CoPAP2 is responsible for the expression of WSC domain proteins, which is involved in cell wall integrity and environmental stress responses in yeast. These results suggest the possible link between $C O P A P 2$ and fungal cell wall remodeling in response to stress relevant to infection.

In planta transcriptome analyses reveal three novel core effectors of the cucumber anthracnose fungus, Colletotrichum orbiculare, essential for full virulence on Cucurbitaceae host plants

Y. INOUE (1), T. T. P. Vy (1), K. Yamada (2), S. S. Ogawa (1), P. Gan (3), K. Shirasu (3), Y. Takano (1), (1) Graduate School of Agriculture, Kyoto University, Kyoto, Japan; (2) Graduate School of Technology, Industrial and Social Sciences, Tokushima Univ, Tokushima, Japan; (3)

RIKEN Center for Sustainable Resource Science, Yokohama, Japan

To establish basic compatibility with its host, successful pathogens use virulence factors, including secreted effector proteins that modulate plant immunity. However, our understanding on eukaryotic pathogen effectors that are essential for infection are limited. Here we report the identification of three novel effectors required for full virulence of the cucumber anthracnose fungus, Colletotrichum orbiculare (Co), on its Cucurbitaceae host plants. By using RNA-seq data from six Co isolates at 24 hpi of cucumber, 28 Co effectors highly induced at host penetration stage in all isolates were selected as effector candidates. Through targeted gene knockout (KO) of the 28 candidates and subsequent evaluation of the virulence of KO mutants, we identified three novel effectors, EPC1, EPC2, and EPC3 that are essential for full virulence of Co on cucumber. To further address the contribution of each effector to fungal virulence, double KO mutants of EPC1 and EPC2 were generated. Resulting double KO mutants significantly reduced virulence on cucumber, revealing the additive effect of the two effectors to fungal virulence. The KO mutants, 
$\triangle \mathrm{EPC} 1, \triangle \mathrm{EPC} 2$, and $\triangle \mathrm{EPC} 3$, were also less virulent on another Cucurbitaceae host, melon, while none of them showed reduced virulence on Nicotiana benthamiana, a Solanaceae plant that $C o$ can infect. These results suggest that three novel effectors identified here are $C o$ virulence factors specifically important for infection of cucurbits.

\author{
Identification and characterization of EFR-mediated immune signaling genes of Arabidopsis seedlings in response to Agrobacterium \\ infection \\ P. Y. Shih, C. J. LIN, E. M. Lai, Academia Sinica, Taipei, Taiwan
}

Agrobacterium tumefaciens is a useful tool to generate transgenic plants, but the transformation efficiency is often compromised by plant innate immunity. EFR is an immune receptor recognizing the conserved microbial signature, elongation factor Tu (EF-Tu), to trigger the first layer of immune response. Previous reports showed that the Arabidopsis mutant efr-1 was more susceptible to Agrobacterium-mediated transient transformation, but little is known about the regulatory factors and mechanisms underlying EFR-mediated immunity against Agrobacterium infection. In this study, we compared the gene expression profiles between shoots and roots of Arabidopsis thaliana Col-0 and efr-1 seedlings respectively, aiming to identify the genes involved in EFR-mediated immune signaling network during Agrobacterium infection. A total of 113 Agrobacterium responsive genes that are differentially regulated between shoots of Col-0 and efr-1 seedlings were identified. The Arabidopsis mesophyll protoplast transient expression assay was used to dissect their roles in regulating the immune marker genes such as FRK1. To this end, several genes were identified to affect the $F R K 1$ promoter-driven luciferase activity upon elf18 treatment, suggesting their regulatory roles in EFR-mediated immune signaling pathway. We hope that our findings may identify new factors involved in both Agrobacterium-mediated immune responses and transformation efficiency.

Mapping the tomato protein network exploited by type III effectors involved in bacterial wilt J. J. R. DE RYCK, VIB/UGent, Zwijnaarde, Belgium ILVO, Belgium

Worldwide, bacterial wilt is responsible for wilting and death of various ornamental plants and economically important solanaceous crops such as tomato and potato. The disease is caused by the phytopathogen Ralstonia solanacearum, which secretes type III effectors (T3E) into the host cell to suppress plant immunity and cause disease. However, the target plant proteins of these effectors are largely unknown. We aim at unraveling the protein-protein interaction network between these T3E and the proteins of tomato. Both direct interactions and indirect interactions will be investigated through GFP immunoprecipitation and proximity-dependent biotin identification (BioID). BioID relies on the fusion of a mutated form of the biotin ligase enzyme BirA (BirA*) with a protein of interest, in this case the T3E. BirA*is capable of biotinylating nearby interacting proteins, also those transiently interacting with the bait, when biotin is present. Ultimately, the results will be combined to construct a large interaction network of the investigated T3E and the tomato proteins. Validation methods such as RNAi or overexpression experiments will further optimize this network. These results could then be used to identify key tomato proteins that, when changed in expression or protein structure, influence susceptibility or resistance to bacterial wilt.

Xanthomonas type III effectors manipulate stromules

J. L. ERICKSON (1), N. Adlung (1,2), C. Lampe (1), M. H. Schattat (3), U. Bonas (1), (1) Department of Genetics, Martin Luther University, Halle, Germany; (2) Department of Bioproducts and Biosystems, Aalto University, Helsinki, Finland; (3) Department of Plant Physiology, Martin Luther University, Halle, Germany

Stroma-filled tubules (stromules) are characteristic of plastids in plant cells under stress and have been observed in species throughout the plant kingdom. These tubules are highly dynamic, and may elongate, branch, and recede within seconds. Stromules have been suggested to participate in the transfer of pro-defense signals from plastids to nuclei during effector-triggered immune (ETI) responses which result in programmed cell death. Currently, there is no mutant available which lacks stromules, making it challenging to evaluate the importance stromules to plant responses to pathogens. We used type III-secreted effector proteins (T3Es) from Xanthomonas campestris pv. vesicatoria (Xcv) as probes for stromule-pertinent cellular processes and plant signaling pathways. $X c v$ translocates T3Es directly into the plant cell where they target specific plant cell components to benefit the pathogen. Transient expression of 21 T3Es in N. benthamiana leaves revealed suppression of stromules by XopL, an E3 ubiquitin ligase. In addition, two other effectors induced stromules, XopQ and the transcription activator-like (TAL) effector AvrBs3.

Identification of Cellular Pathways involved in the RACB-mediated Susceptibility of Barley towards the Barley Powdery Mildew Fungus Blumeria graminis f. sp. hordei

L. WEIß (1), J. Mergner (2), B. Küster (2), G. Hensel (3), J. Kumlehn (3), T. Reiner (1), S. Engelhardt (1), R. Hueckelhoven (1), (1) TU Munich, Phytopathology, Germany; (2) TU Munich, Proteomics and Bioanalytics, Germany; (3) Leibniz Inst of Plant Genetics \& Crop Plant Research, Gatersleben, Germany

Pathogens employ effectors to suppress or bypass plant defenses, but plant proteins can also facilitate infection. Such plant proteins, so-called susceptibility factors, can have diverse physiological functions and are essential for full establishment of the pathogen. Barley RACB, a small monomeric ROP GTPase, has been shown to be such a susceptibility factor. RACB has been extensively studied in the interaction with the powdery mildew fungus Blumeria graminis f. sp. hordei (Bgh), which might target RACB directly to suscessfully infect plant cells. However, the molecular mechanisms behind RACB-mediated susceptibility remain incompletely understood. We used transgenic RACB overexpression plants to find new interaction partners and elucidate the cellular pathways involved in establishment of disease susceptibility. Co-immunoprecipitation and MS-analysis of putative RACB interactors revealed a strong overrepresentation of membrane-trafficking and phospholipid-binding proteins and potential fungal effectors. First functional results support a role of these proteins in the barley- $B g h$ interaction and might provide insight into how phospholipids and membrane-trafficking can support fungal accommodation in barley epidermal cells. 
Small Secreted Proteins of the root oomycete pathogen Aphanomyces euteiches, a new effector family targeting host nucleus L. CAMBORDE, B. Dumas, E. Gaulin, LRSV UMR5546 CNRS Université Toulouse III, Castanet-Tolosan, France

Among the oomycete lineage, the Aphanomyces genus includes plant and animal pathogenic species. By comparative genetic analyses, we found that the legumes pathogen Aphanomyces euteiches genome is characterized by a large repertoire of small secreted protein coding genes (SSP), highly induced during plant infection, and not detected in other oomycetes. Functional analyses of several AeSSPs revealed that AeSSP1256, which contains a nuclear localisation sequence, is able to bind plant RNA, to affect legumes development and increase plant susceptibility to microbial infection. Yeast two hybrid screening identify few nuclear Medicago truncatula proteins as putative targets for AeSSP1256 like a DEAD-box RNA helicase. FRET-FLIM analyses showed that AeSSP1256 binds the RNA helicase and prevents its association to plant RNA, probably to affect cellular processes such as pre-mRNA processing. Molecular experiments are in progress to decipher the impact of this interaction and on the outcome of the host infection.

Detect, deploy, defend: Chloroplasts at the forefront of the host pathogen interface A. Toufexi (1), C. Duggan (1), P. Pandey (1), Z. SAVAGE (1), M. E. Segretin (2), L. H. Yuen (1), D. C. A. Gaboriau (1), A. Y. Leary (1), V. Khandare (1), A. D. Ward (3), S. Botchway (3), I. Pan (1), I. Pan (1), M. H. Schattat (4), I. Sparkes (5), T. O. Bozkurt (1), (1) Imperial College London, U.K.; (2) INGEBI-CONICET, Buenos Aires, Argentina; (3) Central Laser Facility, Science and Technology Facilities Council Harwell, U.K.; (4) Department of Plant Physiology, Martin Luther University, Halle, Germany; (5) University of Bristol, U.K.

Chloroplasts are light harvesting organelles that arose from ancient endosymbiotic cyanobacteria. Upon immune activation, chloroplasts switch off photosynthesis, produce anti-microbial compounds, and develop tubular extensions called stromules, some of which are observed to extend toward the nucleus. The Irish potato famine pathogen Phytophthora infestans produces intracellular infection structures known as haustoria. These provide the key interface between host and pathogen where nutrients are taken from the host and exchanged for effectors which sabotage plant immune responses. We report that chloroplasts navigate to the pathogen interface to counter infection by the Irish potato famine pathogen Phytophthora infestans, physically associating with the specialised membrane that engulfs pathogen haustoria. Outer envelope protein, chloroplast unusual positioning1 (CHUP1), anchors chloroplasts to the host-pathogen interface. Stromules are induced during infection in a CHUP1-dependent manner, embracing haustoria and reaching between chloroplasts, to form dynamic organelle clusters. Infection-triggered reprogramming of chloroplasts relies on surface immune signaling, whereas pathogen effectors subvert these immune pulses. Chloroplast are deployed focally, and coordinate to restrict pathogen entry into plant cells, a process actively countered by parasite effectors.

Dynamic Chloroplast-Nucleus Communication in Plant Immunity

S. KIM (1,2), S. Jung (1,2), S. In (1,2), J. Woo (1,2), S. P. Dinesh-Kumar (3), D. Choi (1,2), E. Park (1,2,4), (1) Department of Plant Science, Seoul National University, Seoul, Korea, Republic of (South); (2) Plant Immunity Research Center, Seoul, Korea, Republic of (South); (3) University of California, Davis, CA, U.S.A.; (4) Department of Molecular Biology, University of Wyoming, Laramie, WY, U.S.A.

Plants have developed well-orchestrated strategies to defend against pathogen attack. Subcellular compartments play an essential role in the activation of a successful immune response against invading pathogens. Chloroplasts are a primary production site of pro-defense molecules. Also, they communicate and coordinate with other organelles during defense via stromules, dynamic tubular extensions from chloroplasts. Recently stromules have been proposed to transport pro-defense signals into the nucleus during immunity. Stromules extend along MTs and connect to $\mathrm{AF}$ anchor points surrounding nuclei, facilitating pulling of chloroplasts to nuclei during innate immunity. Although the stromule has been proposed as a communication path between organelles to transfer signaling molecules during plant stress responses, molecular mechanisms of stromule production remain elusive. To understand the stromule regulatory mechanism, we used effectors from Phytophthora infestans as a tool to control stromule production. Further study to identify the target of effectors in the plant will provide a clue for stromule signaling mechanisms.

A plausible role of mitochondria in epigenetic priming

A. LOPEZ SANCHEZ, C. Castresana, National Centre of Biotechnology, Madrid, Spain

In the response to pathogen attack plants induce immune responses which include production of oxylipins and activation of defence signaling. In the case of a recurrent infection, the responses activated are faster and stronger. This is referred as acquired resistance or priming and involves a certain memory of the stress. Different priming processes have been described in terms of durability; if transmitted to the next generations is known as transgenerational acquired resistance (TAR). Epigenetic mechanisms such as histone modifications and DNA methylation play a role in priming. However, the signaling cascade between the pathogen perception and the changes in the epigenetic marks acting as priming fingerprints still elusive. In this work, we present our results pointing to a role of the mitochondria in this process. We found that mutants altered in synthesis or signaling of 9-LOX-derived oxylipins are impeded in TAR and display differences in DNA methylation at specific nuclear loci. Given that a) oxylipin signaling involves mitochondrial changes, b) oxylipin defective mutants are altered in mitochondrial proteins and c) the production of methyl-groups donors for epigenetic marks partially take place at the mitochondria; we are currently assessing whether the signaling triggered by the pathogen recognition and mediated by oxylipins, induces mitochondrial changes affecting the deposition of epigenetic marks.

Resistance mechanisms to Botrytis in wild tomato

Y. YOU, J. Van Kan, Wageningen University, Wageningen, Netherlands

Tomato (Solanum lycopersicum) is susceptible to the grey mould Botrytis cinerea, while quantitative resistance in wild species Solanum habrochaites accession LYC4 has been reported. Artificial inoculation requires sugars in the inoculum to achieve synchronized infection. In most cases, the fungus causes dispersed necrotic lesions on LYC4, but these lesions do not expand ("incompatible interaction"). Remarkably, resistance of LYC4 is most pronounced when inoculated with high spore density (1000 spores/ $\mu \mathrm{L})$ in low sugar medium $(20 \mathrm{mM})$. Reducing spore density $(30$ spores $/ \mu \mathrm{L})$ or increasing sugar content $(60 \mathrm{mM})$ can lead to lesion expansion ("compatible interaction"). As a necrotrophic 
fungus, $B$. cinerea benefits from host cell death. It is unknown how the necrotic lesions confine the fungus. The balance between two programmed cell death pathways, autophagy and apoptosis, is important for successful infection. We hypothesize that $B$. cinerea suppresses autophagy in the initial stage and actively induces apoptosis later to enable lesion expansion. Besides PCD, resistance has been shown to relate to induction of $\mathrm{H}_{2} \mathrm{O}_{2}$ and cell wall modifications. The manipulation of outcomes (compatible and incompatible) with different inoculation conditions (medium and spore density) is a good setup to better understand B. cinerea infection. Differences between compatible and incompatible interactions, regarding PCD and defense responses will be further studied to shed light on resistance mechanisms.

Stylosanthes Phenylalanine ammonia lyase 1 (SgPAL1) positively regulates the resistance against Collectotrichum gloeosporioides P. Guo (1), H. Wang (1), Q. Liu (1), P. Liu (2), L. JIANG (1), L. Luo (1), (1) Hainan University, Haikou, China; (2) Chinese Academy of Tropical Agriculture Sciences, Danzhou, China

Stylosanthes is an important legume forage with largest cultivated area in tropical region worldwide. Anthracnose caused by Colletotrichum gloeosporioides (C. gloeosporioides) places major threats on Stylosanthes production. Phenylalanine ammonia lyase (PAL) catalyzes the first step of the phenylpropanoid pathway, which generates precursors for multiple important secondary metabolites contributing to plant defense. To isolate the regulators involved in the resistance, we performed a time-course transcriptome analysis of Stylosanthes following challenge with $C$. gloeosporioides at 24, 48, 60, $96 \mathrm{hrs}$. Using differential transcriptome and pathway analyses, we find that most transcripts are altered at $60 \mathrm{hrs}$ post-infection, and the majority of differential expressed genes were associated with phenylpropanoid metabolic pathways. The induction of Stylosanthes PAL ( $S g P A L 1, S g P A L 2, S g P A L 3)$ gene expression and enzyme activities are verified by RT-PCR and enzyme assay respectively. These results indicate that PALs and phenylpropanoid metabolic pathways play important roles in plant defense. To investigate the role of SgPAL1 in regulating the resistance against $C$. gloeosporioides, the transgenic Stylosanthes overexpressing SgPAL1 is generated. The transgenic lines show the increased resistance as evidenced by smaller lesion diameter postinfection, indicating that SgPAL1 is a positive regulator of plant's resistance against $C$. gloeosporioides.

Efficient CRISPR/Cas9 genome editing and tissue culture techniques for tetraploid local varieties and diploid potato M. LENMAN (1), E. S. Wang (2), N. Kieu Phuong (2), E. Andreasson (3), (1) Swedish University of Agricultural Science, Alnarp, Sweden; (2) Swedish Univ of Agric Sciences (SLU), Alnarp, Sweden; (3) Swedish University of Agricultural Sciences, Alnarp, Sweden

Potato is one of the best producers of edible biomass in terms of yield per hectare and production of potato is increasing globally. It is however attacked by several diseases including late blight caused by Phytophthora infestans and early blight caused by Alternaria solani. The stacking of resistance genes (R-genes) from wild Solanum relatives and the mutation of susceptibility genes (S-genes) are interesting prospects for the sustainable management of these diseases. We have developed a pipeline for the efficient generation and screening of R-gene containing transformants and S-gene CRISPR/Cas9 deletion mutants in tetraploid local varieties and diploid hybrid potato. A third of the putative CRISPR/Cas9 edited regenerated shoots were found to be mutated in at least one allele, and 13 percent were mutated in all four alleles. Several factors affecting regeneration following Agrobacterium transformation, such as the quality of starting plant material and the media composition were scrutinized. Exogenous Indole-3-butyric acid was not necessary for the maintenance of stock lines and long-term use led to aberrant morphological phenotypes. A protocol for refreshment of in vitro stock material proved highly beneficial and increased the transformation efficiency more than 4 -fold.

Extracellular low molecular weight phosphonates of Pectobacterium atrosepticum: Identification and role in pathogenesis O. PARFIROVA (1,2), V. Gorshkov (1,2), N. Tarasova (1), N. Gogoleva (1,2), A. Smolobochkin (3), O. Petrova (1), Y. V. Gogolev (1,2), (1) Kazan Institute of Biochemistry and Biophysics FRC "KSC of RAS”, Kazan, Russian Federation; (2) Kazan Federal University, Kazan, Russian Federation; (3) A.E.Arbuzov Institute of Organic and Physical Chemistry FRC "KSC of RAS", Kazan, Russian Federation

Phosphonates belong to an extensive group of compounds containing carbon-phosphorus bond in their structure. Phosphonates can be included in macromolecules or represent low-molecular-weight compounds for which antibacterial and herbicidal properties are described. Plant soft rotcausing phytopathogen Pectobacterium atrosepticum $(\mathrm{Pba})$ possess genes encoding the enzymes for phosphonate metabolism. By RNA-Seq analysis we have shown that $P b a$ phosphonate-related genes are up-regulated during soft-rot development compared to latent infection or cultures in vitro. However, in Pectobacterium, phosphonates are not described. The aim of the present study was to obtain information on low-molecularweight phosphonates in $\mathrm{Pba}$. To check if $\mathrm{Pba}$ indeed produces extacellular low-molecular-weight phosphonates, in vitro conditions that induce the expression of phosphonate-related genes were selected. After culturing under these conditions, the supernatants were separated, and the expected low-molecular-weight phosphonates were extracted. ${ }^{31} \mathrm{P}$ NMR-spectra for the obtained samples revealed the $21.91 \mathrm{ppm}$ signal typical of phosphonates as well as $59.69 \mathrm{ppm}$ signal characteristic of phosphonium salts. Mutant for phosphonate biosynthetic gene has been obtained and its virulence is characterized. Thus, we identified for the first time the low-molecular-weight phosphonates in plant pathogenic bacteria and obtained information on their role in pathogenesis. This study was supported by RSF (19-14-00194).

Not the end of the story: Identifying novel players controlling intracellular rhizobial infections M. BATZENSCHLAGER (1), T. Vernié (2), J. Keller (2), P. M. Delaux (2), T. Ott (1), (1) University of Freiburg, Faculty of Biology - Cell Biology, Freiburg, Germany; (2) LRSV UMR5546 CNRS/Université de Toulouse III, Castanet-Tolosan, France

Legumes engage in mutualistic interactions with rhizobia, allowing them to establish a nitrogen-fixing root nodule symbiosis (RNS). A fundamental but yet unresolved question is how bacterial symbionts successfully colonize host cells in an intracellular fashion. While forward genetic screens have identified most, if not all essential single gene candidates these approaches have been unable to sufficiently address the presence of functionally redundant paralogues. Largely available datasets from transcriptomic, comparative genomic and phylogenomic analyses enabled us to specifically select gene families with a particular evolutionary pattern within the nodulating clade. Since we aim to primarily target proteins that reside on infection thread membranes, we assumed that the corresponding genes are transcriptionally upregulated upon rhizobial infection. Phenotypic characterization following RNA interference-mediated multigene silencing of several candidates highlighted so far undescribed genes, whose simultaneous knock-down in Medicago transgenic roots strongly impaired epidermal infection. High-resolution 
subcellular localization studies in heterologous systems and legume host cells revealed that these candidates are indeed primarily targeted to the plasma membrane. This shows that systematically approaching genetic redundancy and functional dissection of individual paralogues will provide mechanistic insights to understand intracellular accommodation of symbiotic microbes.

Phytohormone crosstalk is important for Argonaute-mediated defense against virus infection G. S. MARTINS (1), A. Adurogbangba (1), C. Roussin-Léveillée (1), P. Moffett (2), (1) Univ de Sherbrooke, Sherbrooke, QC, Canada; (2) Université de Sherbrooke, Sherbrooke, QC, Canada

Previously, we have shown that the Argonaute 5 (AGO5) protein, in conjunction with Argonaute 2 (AGO2), plays an important role in restricting virus infection in Arabidopsis. Whereas an ago2 mutant shows susceptibility to Potato virus X (PVX) mostly in inoculated leaves, an ago2ago5 double mutant shows a high level of PVX accumulation in systemic tissue as well. Accordingly, AGO5, which is normally not expressed in vegetative tissues, is upregulated in the systemic leaves of plants infected with PVX or Plantago asiatica mosaic virus (PIAMV). Thus, although $A G O 5$ appears to play a role in defense against virus infection, it is unclear what induces its expression as a systemic defense. When different Arabidopsis phytohormone mutants were infected with PIAMV, a jasmonic acid (JA) mutant, dde2-2, showed strong expression of the $A G O 5$ gene. In agreement with this observation, JA-treated $d d e 2-2$ and wild-type plants showed lower expression of $A G O 5$. Interestingly, JA-mediated repression of $A G O 5$ expression resulted in enhanced infection by PIAMV and PVX. In contrast to the role of JA in $A G O 5$ induction, salicylic acid (SA), an important phytohormone in systemic defense, appears to positively regulate $A G O 5$. Therefore, our results suggest that the SA-JA crosstalk is important in Argonaute-related defense against systemic virus infection.

COLORFUL-ABA sensor reveals cell-specific activation of ABA signaling during plant-microbe interactions

M. EL SAYED (1), E. Schliekmann (1), J. N. Lübbers (1), K. Hanika (2), V. Lipka (1), H. Ghareeb (1), (1) Georg-August-University, Goettingen, Germany; (2) Wageningen University, Wageningen, Netherlands

A growing body of evidence suggests that abscisic acid (ABA) is an important modulator of plant innate immunity. However, the spatio-temporal cellular activities of $\mathrm{ABA}$ in plant defence have so far not been adequately addressed. Here, we developed a multi-modular reporter system termed "COLORFUL-ABA" that we used to map ABA signaling outputs in transgenic Arabidopsis wildtype and mutant plants at unprecedented single-cell resolution. Reporter readout quantification was supported by our recently established high-throughput imaging software "COLORFUL-Spotter". Using this platform, we demonstrated high sensitivity and rapid responsiveness of our reporter system to exogenously applied ABA and observed intrinsic COLORFUL-ABA activity patterns that are consistent with previously described roles of ABA in particular cellular domains of leave and root tissues. Next, we used our COLORFUL-ABA reporter lines to map the spatio-temporal dynamics of ABA signaling activities during biotrophic interactions of Arabidopsis with the oomycete $H$. arabidopsidis and the powdery mildew fungus G. orontii. Our studies revealed a cross kingdom-conserved induction of a cellularly confined ABA signaling signature at Arabidopsis interaction sites with these two pathogens. Interestingly, mutant analyses supported the idea that ABA functions as an important and common susceptibility factor. We are currently addressing the cell-specific contribution of selected ABA signaling components for this process.

Thecaphora thlaspeos infects Brassicaceae plants via novel penetration structures

L. PLÜCKER (1), K. J. Courville (2), V. Goehre (2), (1) Heinrich-Heine University, Universitaetsstraße 1, Building 26.12.01.31, Duesseldorf, Germany; (2) Heinrich-Heine University, Duesseldorf, Germany

T. thlaspeos is a dicot-infecting smut fungus of the Thecaphora-clade, related to the model smut $U$. maydis. While the grass smut fungi infect genetically complex crop plants, T. thlaspeos infects Brassicaceae plants, in which it establishes a symptomless, systemic and long-lasting biotrophic interaction, and overwinters with perennial hosts as an endophyte. Notably, T. thlaspeos can infect the roots, an infection route that has not been described for smut fungi. Molecular microscopic investigations of the early infection stages allowed the identification of specific penetration structures on the plant root as well as the leaf surface. Hypopodia-like structures are formed on the root surface resulting in rhizodermis cells filled with fungal hyphae, although this colonization does not seem to be essential for successful infection via the roots. On the leaf surface of cotyledons and true leaves, we observed that hyphae of germinated $T$. thlaspeos teliospores form either appressoria-like structures on the guard cells or growth directly through the stoma. In contrast to other plant-pathogenic fungi, the guard cells are subsequently colonized before T. thlaspeos continues to grow further towards the vasculature. These colonized guard cells seem to die during the process. This dataset broadens our understanding of the $T$. thlaspeos life cycle, thus providing the foundation for the analysis of tissue resolved molecular infection processes.

Generation of a versatile toolbox for multicolor labelling of Phytophthora subhyphal compartments L. SHENHAV, E. Evangelisti, T. Yunusov, M. Le Naour-Vernet, P. Rink, S. Schornack, The Sainsbury Laboratory, Cambridge University (SLCU), Cambridge, U.K.

Oomycetes are filamentous organisms that differ from fungi by structural, biochemical and genetic features. This group contains prominent plant pathogens, such as species from the genus Phytophthora. Despite several protocols being established for oomycete transformation, it still remains challenging and hinders research into their biology. Using the cocoa killer Phytophthora palmivora as a model system, we optimized existing electro-transformation protocols to achieve an efficient and reproducible transformation of Phytophthora. Next, we generated multisite Gateway vectors for dual labelling of hyphae and organelles as well as rapid testing of multiple promoter-reporter combinations. We showcase the usefulness of this new toolbox by identifying novel native $P$. palmivora promoters and by developing new reporter lines for organelle labelling. In particular, nucleus labelling allowed us to investigate nuclear movements within oomycete hyphae. In addition, we generated lifestyle transition marker lines for in vivo imaging of lifestyle transitions in Phytophthora. These technical advances unravel yet under-explored aspects of Phytophthora biology and will bring knowledge whose interest expands beyond the oomycete research community. 
Identification of three tomato proteins recruited into viral replication complexes of pepino mosaic virus

E. MÉNDEZ-LÓPEZ, L. Donaire, R. N. Sempere, L. Rodríguez-Moreno, M. A. Sánchez-Pina, M. A. Aranda, CEBAS-CSIC, Murcia, Spain

Pepino mosaic virus (PepMV; genus Potexvirus) causes large economic losses in tomato crops worldwide. To identify target genes to engineer loss-of-susceptibility mutants, tomato proteins that interact with the PepMV coat protein (CP) and the triple gene block protein 1 (TGB1) were identified using a yeast two-hybrid (Y2H) screening. Here we present results for three of them: a glutathione S-transferase (IP24) interacting with the CP, a protein involved in transcriptional regulation (IP9) interacting with the TGB1, and an RNase H like protein (IP10) interacting with both $\mathrm{CP}$ and TGB1. Interactions were validated by directed $\mathrm{Y} 2 \mathrm{H}$ and/or coimmunoprecipitation (CoIP). Confocal laser scanning microscopy (CLSM) of $N$. benthamianalive cells showed that the 3 IPs relocalized to cytoplasmic structures resembling virus replication complexes (VRC) of infected cells. For IP9 and IP10 this phenomenon was especially dramatic as they localize to the nucleus when expressed alone. We hypothesized that the TGB1, which is also localized to the nucleus when expressed alone, recruits IP10 and IP9 to the VRC suggesting a role of the IPs during the virus replication, and that IP24 is recruited to the VRC to create an optimal redox environment for the virus replication. PepMV susceptibility assays were carried out with IPs-transiently silenced tomato plants, and with IPs micro-tom knock-out mutants generated using the CRISPR/Cas9 technology, showing in some cases a reduction in virus accumulation.

Phosphatidylinositol 4-kinases contribute to defence responses triggered by actin cytoskeleton disruption in Arabidopsis T. KALACHOVA (1), O. Iakovenko (1,2,3), H. Leontovyčová (1), R. Pospichalova (1), M. Janda (4,5), J. Martinec (1), D. Kocourkova (1), L. Burketova (1), E. Ruelland (6,7), (1) Institute of Experimental Botany Czech Academy of Sciences, Prague, Czech Republic; (2) University of Chemistry and Technology, Czech Republic; (3) Institute of Bioorganic Chemistry and Petrochemistry NAS of Ukraine, Kyiv, Ukraine; (4) University of Chemistry and Technology Prague, Prague, Czech Republic; (5) Ludwig-Maximilians-Universität München, Genetics, Martinsried, Germany; (6) CNRS, Institut d'Ecologie et des Sciences de l'Environnement de Paris, UMR7618, France; (7) Université Paris-Est, UPEC, Creteil, France

Actin cytoskeleton is crucial for various cellular processes in plants. Early after infection, actin filaments get rapidly reordered to support transport of the stress-related compounds. Chemical drugs affecting actin polymerisation influence the infection process, and support pathogen growth while applied together with pathogen. On the contrary, being applied previously to infection, they can trigger defence-like responses and even induce resistance. Actin cytoskeleton remodelling cannot be separated from phospholipid turn-over and vesicular trafficking. In Arabidopsis thaliana, phosphatidylinositol-4-kinases beta1 and beta2 participate in vesicle formation, modulate callose deposition and regulate the biosynthesis of defence-related hormone salicylic acid (SA). Here, we studied the impact of actin disrupting compound latrunculin B (latB) on actin filaments organization, callose content, gene expression and resistance to Pseudomonas syringae in Arabidopsis seedlings. LatB treatment induced a transcription of several defence and SA-related genes. Besides, independently on SA, latB triggered callose accumulation through callose synthase PMR4, and this did not require transcriptional activation. The constitutive deficiency in PI4KB1B2 enhanced susceptibility to actin disruption drugs at different levels, in a connection with SA-signaling. Here, we add another puzzle to the picture of interactions between actin cytoskeleton and phospholipids in the context of plant immunity.

Plant and human bacterial pathogens trigger similar SA-mediated defense in Arabidopsis P. R. OBLESSUC, C. C. Matiolli, M. Melotto, University of California, Davis, CA, U.S.A.

Food contamination with Salmonella enterica and enterohemorrhagic Escherichia coli is among the leading cause of foodborne illnesses worldwide and crop plants are associated with $>50 \%$ of the disease outbreaks. We have observed that S. enterica serovar Typhimurium (STm) transcriptionally modulates plant innate immune responses in Arabidopsis leaves, providing opportunities to explore plant resistance as a mechanism to improve food safety. Among the STm-induced genes, we found NIMIN1, a repressor of the salicylic acid (SA) regulator NPR1, and $E X O 70 H 4$, involved in callose deposition. Arabidopsis nimin 1-1 and exo70h4-3 mutants showed impaired apoplastic and stomatal defenses against STm. NIMIN1 and EXO70H4 contributed to stomatal defense against E. coli O157:H7 and apoplastic immunity against the phytopathogen Pseudomonas syringae pv. tomato (Pst) DC3118. The SA signaling and biosynthesis proteins NPR1 and ICS1 were required for apoplastic and stomatal resistance to all three bacteria. Defense against STm and Pst DC3118 was associated with NIMIN1- and NPR1-mediated control of reactive oxygen species (ROS) burst. Callose deposition was induced after inoculation with all three bacteria, and required EXO70H4, NPR1, and ICS1 proteins. Our data indicate that inside the Arabidopsis leaf mesophyll, similar SA-mediated defense mechanisms are activated against STm and Pst DC3118, whereas on the leaf surface, stomatal defense mechanisms are shared against STm and E. coli O157:H7.

Activation of the susceptibility factor RACB in the interaction between barley with the powdery mildew fungus A. TRUTZENBERG, S. Engelhardt, R. Hueckelhoven, TU Munich, Phytopathology, Germany

RAC/ROP GTPases are molecular switches that are crucial in the regulation of plant development and immunity. We identified that in domesticated barley (Hordeum vulgare) ROPs play a major role in the interaction with the powdery mildew fungus Blumeria graminis $\mathrm{f}$. sp. hordei $(B g h)$. Over-expression of the constitutively activated barley GTPase RACB-G15V supports fungal penetration into host epidermis cells, whereas knock-down of its expression renders the plant more resistant to pathogen attack. Consequently, RACB functions as a susceptibility factor in this interaction. Endogenous activation of GTPases is typically catalysed by their interaction with guanine nucleotide exchange factors (GEFs), by facilitating the exchange of GDP with GTP and thereby turning ROPs into an active signaling state. Plants possess an independent class of GEFs with a plant-specific RAC/ROP nucleotide exchange domain (PRONE). In rice, GEFs are important activators of immunity-related ROPs. Hence, barley PRONE-GEFs might act as regulators of RACB in the interaction with Bgh. Additionally, PRONE-GEFs might link RACB with upstream cell surface components of the susceptibility pathway, some of which we found to be expressed in a RACB-dependent fashion. Here, we present data on barley PRONE-GEF expression and function in interaction with $B g h$.

Growth in host plants increases Ralstonia solanacearum resistance to eradication treatments and environmental stresses M. HAYES (1), C. Allen (2), (1) UW-Madison, Madison, WI, U.S.A.; (2) University of Wisconsin-Madison, Madison, WI, U.S.A. 
Ralstonia solanacearum (Rs) is a soil and water-borne phytopathogen that causes lethal bacterial wilt (BW) disease of over 200 species worldwide, including tomato, potato, and many ornamentals. While $R s$ is primarily a tropical pathogen, the R3bv2 subset of cool tolerant strains are tightly regulated quarantine pathogens subject to Select Agent rules in the United States. While developing and validating protocols to eradicate this bacterium from irrigation water, plant material, and laboratory surfaces, we discovered unexpected differences between $R s$ grown in planta and cells of the same strain grown in culture. Cells extracted from diseased plant tissue had dramatically higher tolerance of heat and ultraviolet (UV) radiation compared to cells grown in rich media. No cultured $R s$ cells survived 10 min exposure to $50^{\circ} \mathrm{C}$, but 240 min at $99^{\circ} \mathrm{C}$ was required to kill $R s$ cells extracted from plant tissue. $R s$ cells grown in planta were eliminated by 300 sec ( 5 min) exposure to UV, but only 30 sec UV exposure left no surviving cultured $R s$ cells. However, cultured and in planta cells had similar tolerance of reactive oxygen species (bleach, H2O2) and desiccation. Similar results were obtained for both R3bv2 and non-Select Agent Rs strains. These findings demonstrate that growing in plants increases $R s$ stress tolerance, meaning higher temperatures and longer exposures are needed to effectively eradicate this pathogen from plant material.

The role of Phytophthora infestans transglutaminases in appressoria formation, pathogenicity and PAMP-Triggered Immunity in potato. M. BRUS-SZKALEJ (1), M. Lenman (2), R. R. Vetukuri (3), L. I. Moushib (4), E. Alexandersson (5), E. Andreasson (1), L. J. Grenville-Briggs (1), (1) Swedish University of Agricultural Sciences, Alnarp, Sweden; (2) Swedish University of Agricultural Science, Alnarp, Sweden; (3) Swedish Univ of Agric Sciences (SLU), Alnarp, Sweden; (4) Lund University, Sweden; (5) Swedish University of Agricultural Sciences (SLU), Alnarp, Sweden

Late blight of potato caused by Phytophthora infestans is the most damaging oomycete disease worldwide. Effective disease control involves frequent sprayings with chemical pesticides which are harmful to the environment and can be rendered ineffective by development of pathogen resistance to the active substances. A promising new approach is to study the morphology and development of the pathogen in search of possible new targets for next generation chemical pesticides. During a proteomics screen of $P$. infestans development we identified several cell wall proteins as highly expressed during appressorium formation and during initial contact between $P$. infestans and the potato leaf (early infection). One such protein is a cell wall transglutaminase containing the Pep13 motif. This 13-amino acid peptide acts as a Pathogen Associated Molecular Pattern (PAMP) and activator of PAMP-triggered immunity in potato. Our aims are to investigate the role of Pep13-containing transglutaminases in the development and pathogenicity of $P$. infestans and to test if in planta expression of the peptide enhances potato resistance to late blight. We show that Pep13 containing transglutaminases are necessary for the formation of normal appressoria and pathogenicity. Pep13 expression in planta leads to reduced disease symptoms in both the laboratory and field conditions. Field microarray analysis gives us insight into plant defence and stress responses associated with the expression of Pep13.

Arabidopsis Lipid Biosynthesis Genes required for successful Colonization by the pathogenic Oomycete Hyaloperonospora arabidopsidis A. Keymer (1,2), C. CARDOSO (2), M. Messerer (3), M. Brands (4), A. Banhara (1), M. Parniske (1), K. F. X. Mayer (3), P. Dörmann (4), C. Gutjahr (1,2), (1) Genetics, Faculty of Biology, University of Munich (LMU), Biocenter Martinsried, Martinsried, Germany; (2) Plant Genetics, TUM School of Life Sciences Weihenstephan, Freising, Germany; (3) Plant Genome and Systems Biology, Helmholtz Center Munich, Neuherberg, Germany; (4) Institute of Molecular Physiology and Biotechnology of Plants, Bonn University, Bonn, Germany

Lipid biosynthesis and signaling has been shown to be implicated in plant immunity responses to biotrophic pathogens. To investigate the importance of lipid biosynthesis in plant-oomycete interactions we have characterized the infection of Arabidopsis mutants defective in fatty acid biosynthesis (kasI) and beta-monoacyl glycerol and cutin biosynthesis (gpat6 and gpat4x8 double mutant) with the oomycete Hyaloperonospora arabidopsidis (Hpa). Cotyledons of kasI, gpat6 and gpat4x8 are colonized to the same extent as the wild-type. However, on the mutant hosts the production of spores is reduced. Hpa morphology is also altered in the mutant host plants, with thickened hyphae and multilobed haustoria, which occur at much lower frequency in the wild-type plants. The three Arabidopsis mutants responded to Hpa infection with increased cell death events at the contact points with the pathogen. To further investigate how KASI, GPAT6 and GPAT4x8 control Hpa development and the plant immune response, RNAseq analyses of $\mathrm{Hpa}$ and mutant host plants were performed. This revealed distinct transcriptional profiles in the pathogen depending on the colonized Arabidopsis mutant, suggesting that the same morphological output may be based on different molecular responses to perturbed host lipid biosynthesis.

Establishing the roles and interactions of E3 Ubiquitin Ligase 1 (E3L1) and MAP Kinase Phosphatase 1 (MKP1) in pattern-triggered immunity

J. K. ELLINGSEN, L. Jiang, W. Costigan, S. Peck, Univ of Missouri, Columbia, MO, U.S.A.

When a pathogenic bacterium encounters a plant, it induces its Type III Secretion System (T3SS) in an attempt to infect the plant to obtain its nutrients. The T3SS serves as a method for the bacteria to inject effector proteins into the plant cell, helping it inactivate the plant's immune system. In Arabidopsis thaliana, we have identified a novel mutant, a knockout of map kinase phosphatase 1 (mkpl), as one that confers increased resistance to the plant against Pseudomonas syringae pv tomato DC3000 (DC3000). Upon further study, it was determined that DC3000 treated with $\mathrm{mkpl}$ plant exudate was less successful in inducing its T3SS than when treated with exudate from wild type plants. Metabolomic studies identified decreased abundance of specific metabolites in $\mathrm{mkpl}$ plants that are required by DC3000 to induce its T3SS, and the decrease in these bioactive chemicals was sufficient to explain the enhanced resistance in $m k p 1$ mutants. In this work, we report the identification of E3 Ubiquitin Ligase 1 (E3L1) as a novel component required for PTI. Knockout mutants (e3l1) do not properly manifest PTIrelated resistance, even though they correctly manifest a number of typical defense-related molecular responses. Infection of a double knockout mutant of E3L1 and MKP1, e3l1 mkp1, shows suppression of $m k p 1$-related resistance, genetically placing E3L1 in the MKP1-dependent pathway. Possible functions of E3L1 in MKP1-dependent resistance will be discussed.

Characterizing mechanisms of tolerance in the Clavibacter-Solanum pathosystem 
C. PERITORE-GALVE (1), C. Miller (2), J. L. Gonzalez Giron (1), C. D. Smart (1), (1) Cornell University, Geneva, NY, U.S.A.; (2) North Carolina State University, Raleigh, NC, U.S.A.

Clavibacter michiganensis subsp. michiganensis $(\mathrm{Cmm})$ is a gram-positive bacterium that causes bacterial canker of tomato, an economically devastating disease with a worldwide distribution. Cmm colonizes the xylem, leading to unilateral wilt, stem canker, and plant death. Splash dispersal of Cmm can also infect developing tomato fruit, forming bird's-eye lesions. There are no documented sources of qualitative resistance in Solanum spp., however, QTL conferring tolerance in Solanum arcanum and S. habrochaites have been identified. Mechanisms underlying tolerance of these species to Cmm have not been deciphered. We hypothesized that Cmm colonization in tolerant Solanum spp. would differ from S. lycopersicum, and that complex morphological, genetic, and metabolic factors may contribute to tolerance. Colonization patterns of WT Cmm and a hypervirulent expansin mutant were characterized in Solanum spp. during vascular and fruit infection stages. In planta colonization and spread was measured and compared to Cmm growth in extracted xylem sap. Cmm aggregates in xylem vessels were observed using scanning laser confocal microscopy and SEM. Sap was extracted from the most tolerant and most susceptible genotypes inoculated with water and WT $\mathrm{Cmm}$, and $\mathrm{Cmm}$ changes to the xylem environment are being analyzed using untargeted metabolomics. Foundational studies of Cmm-tolerant host interactions may contribute to development of strategies for prevention and management of bacterial canker.

\begin{abstract}
Abscisic acid and auxin are early activated in Arabidopsis Atseor1ko mutants during phytoplasma infection C. BERNARDINI (1), L. Pagliari (1), V. De Rosa (1), M. Almeida-Trapp (2), M. Martini (1), A. Mithofer (2), R. Musetti (1), (1) Dep. of Agricultural Food Environmental and Animal Sciences, University of Udine, Udine, Italy; (2) Dep. of Bioorganic Chemistry, Max Planck Institute for Chemical Ecology, Jena, Germany
\end{abstract}

Phytoplasmas are phloem-confined pathogens associated to several diseases of economically important plants. Plant response to the infection implies many physiological alterations, cytological modifications and modulation of global transcription and protein profile. We previously demonstrated that in Arabidopsis the absence of AtSEOR1 gene, involved in sieve-element filamentous protein formation in association with AtSEOR2, allowed the plant to face phytoplasma invasion more effectively. This led to speculate that AtSEOR2 protein, free from its bond with AtSEOR1, could act in defense mechanism. Nevertheless, no explication of this phenomenon was provided. In this study wild-type and Atseor $1 \mathrm{ko}$ lines were analyzed at early and late infection stages, performing microscopic, molecular and physiological analyses to identify possible key aspects of phytoplasma-AtSEOR2-plant defense interactions. According to transmission electron microscope and gene expression analyses, at the first stage of the infection, sieve-element filamentous proteins are not involved in plant response to the phytoplasmas. On the other hand, stress-related phytohormones, measured in both leaf midribs and laminae through HPLC-MS/MS highlighted that site-specific auxin and abscisic acid response takes place precociously in the Atseorlko plant infection site. Little is still known about the initial steps of the plant response against phytoplasma, but phytohormones seem to play a role in the first defense line.

Monitoring changes to the plant secretory pathway during cyst nematode feeding site formation A. HAYES (1), J. Denecke (2), P. E. Urwin (1), (1) University of Leeds, Leeds, U.K.; (2) University of Leeds, U.K.

Cyst nematodes cause billions of dollars' worth of crop loss each year. To obtain nutrients from their host, cyst nematodes re-program root cells to form a highly specialised feeding site, the syncytium. To form the syncytium, vast changes to the plant secretory pathway occur. This includes the proliferation of the endoplasmic reticulum (ER) and Golgi apparatus, loss of the large central vacuole and the formation of numerous smaller vacuoles. To monitor these changes, a portfolio of Arabidopsis double fluorescent reporter lines has been made. Each double expression vector contains a Golgi marker fused to red fluorescent protein, and an additional plant secretory pathway marker fused to yellow fluorescent protein. Therefore, these vectors label the Golgi, in addition to either the ER, trans-Golgi network, prevacuolar compartment or the vacuole. When infected with the beet cyst nematode Heterodera schachtii, the Arabidopsis reporter lines provide a model system to monitor the plant secretory pathway during syncytial formation in vivo. These Arabidopsis reporter lines could also be infected with other syncytial forming nematodes, such as Rotylenchulus reniformis, or root-knot forming nematodes such as Meloidogyne incognita. Furthermore, the double expression constructs could be used as a toolkit to characterise the plant secretory pathway within other plant-pathogen interactions.

Immune-responsive feedback between the cytoskeleton and plasma membrane S. Sassmann, C. Rodrigues, J. Hembrow, D. Richards, D. W. Horsell, M. J. DEEKS, University of Exeter, Exeter, U.K.

Filamentous actin plays a key role in the assembly of host Cell Wall Appositions (CWAs) that are produced reactively by the plant immune system. CWA construction is achieved with consistent precision at sites of assault to arrest microbial invasion and requires the controlled invagination of the plasma membrane (PM) in co-ordination with the export of barrier material to the volume between the plant PM and cell wall. Molecular, genetic or chemical interference of actin dynamics during this process lowers penetration defence, but it remains unclear how exocytosis and the cytoskeleton are linked in space and time to form functional CWAs. To gain mechanistic insight into this process we searched for Arabidopsis thaliana genes encoding known and predicted actin-binding proteins (ABPs) that responded at the transcriptional and translational level to microbial assault. We have found that membrane-integrated ABP FORMIN4 is delivered to immune response sites and contributes to local cytoskeletal dynamics at CWAs. Deactivation of FORMIN4 and its close homologues compromises subsequent defence and alters filamentous actin (F-actin) distribution at mature CWAs. Moreover, the tessellation of FORMIN4 at the PM with meso-domains of PEN3 reveals a fine spatial segregation of destinations for actin-dependent immunity cargo. This supports a model in which FORMIN4 is a spatial feedback element in a multi-layered, temporally defined sequence of cytoskeletal response.

Structural restriction to Ralstonia solanacearum colonization and invasion in resistant tomato varieties M. Planas-Marquès (1), J. Kressin (2), A. Kashyap (3), D. R. Panthee (4), F. J. Louws (5), N. SANCHEZ COLL (6), M. Valls (7), (1) Centre for Research in Agricultural Genomics, Spain; (2) North Carolina State University, Raleigh, U.S.A.; (3) Centre for Research in Agricultural Genomics (CRAG), Cerdanyola del Vallès, Spain; (4) North Carolina State University, Mills River, NC, U.S.A.; (5) Department of Entomology 
and Plant Pathology, North Carolina State University, Raleigh, NC, U.S.A.; (6) Centre for Research in Agricultural Genomics, Barcelona, Spain; (7) Univ of Barcelona, Barcelona, Spain

$R$. solanacearum, the causing agent of bacterial wilt, one of the most destructive bacterial diseases in plants due to its aggressiveness, its broad geographical distribution and its long persistence in soil and water. $R$. solanacearum infects plants through wounds in the roots and migrates intercellularly through the apoplast until it reaches the xylem vessels, where it multiplies and spreads systemically. Grafting approaches using resistant cultivars such as Hawaii 7996 as rootstocks have been the most effective method to control bacterial wilt in tomato due to limited bacterial growth in resistant rootstocks. Here, we describe the spatio-temporal bacterial dynamics on two resistant (Hawaii 7996 and Shield) and one susceptible (Marmande) tomato varieties using novel assessment techniques. Our results show that that resistant varieties reduce bacterial invasion and multiplication in the roots and that shoot colonisation is also restricted at various levels. We show that vascular bundle invasion, radial apoplastic pith/cortex invasion and vertical bacterial spread in the shoot are all restricted in Hawaii 7996 and the last two in Shield, highlighting the importance of structural constraints to bacterial spread as a resistance mechanism. Our results demonstrate that, besides the known limitation in root colonisation in resistant tomato varieties, resistance is also expressed in the shoot tissues.

Studies on the potential role of prolyl cis/trans isomerization of signaling proteins in the regulation of Bacillus species social development and biocontrol activity

M. DIMOU, A. Venieraki, P. Katinakis, Lab of General and Agricultural Microbiology, Agricultural University of Athens, Greece

Bacillus species respond to various signals and as a result they activate and regulate different developmental pathways that lead to distinct coexisting cell types in multicellular communities. Different sensor kinases phosphorylate at least three master regulators and specific phosphatases dephosphorylate them or block DNA binding. In some cases, not only the specific threshold levels of the phosphorylated regulator but also the dynamics through which it achieves these levels seem to be important for the entry into a differentiation program. Prolyl cis/trans isomerization is a rate limiting step in protein folding that is catalyzed by prolyl isomerases. As a post translational protein modification, it has been involved among others, in the control of the duration and the amplitude of certain cellular processes. We have inspected the available threedimensional structural data of key B. subtilis regulatory proteins and identified certain proteins with heterogeneous prolines in their structures that represent potential sites for prolyl isomerase binding. We have isolated endophytic Bacillus spp. strains from medicinal plant seeds and currently characterize them regarding various social behaviors and biocontrol activity. The functional characterization of prolyl isomerases from these strains as well as from various type strains will help to understand the possible involvement of protein dynamics in the kinetics of developmental processes.

Control of virulence by sirtuins in Ustilago maydis

B. NAVARRETE, J. I. Ibeas, R. R. Barrales, CABD Universidad Pablo de Olvide/ CSIC/ Junta de Andalucía, Sevilla, Spain

Ustilago maydis is a pathogenic fungus that infects maize causing tumors in several plant tissues. As most of plant fungal pathogens, $U$. maydis develops many morphological changes in order to ensure proper infection. These sequential morphological changes imply a rigorous regulation of different transcription programs controlling fungal development. The control of genetic programs involved in developmental changes has been typically shown to be regulated by chromatin modifying factors. In terms of chromatin biology, U. maydis has a peculiarity that is the lack of some of the main proteins involved in heterochromatin formation. A similar scenario is shown by Saccharomyces cerevisiae, where heterochromatin-like structures are performed by the SIR complex. The catalytic subunit of this complex is the sirtuin (NAD-dependent histone deacetylase) Sir2. We have identified a Sir2 homolog in U. maydis and observed that it is involved in the regulation of the infection process, as the overexpression of Sir2 during the pathogenic program drastically reduces infection symptoms in the plant. In addition, we observe that Sir 2 is controlling cell to hypha transition and is repressing some filamentation and pathogenic related genes during non-pathogenic condition. We are currently studying in depth the role of Sir2 in the transcriptional control of pathogenesis and its possible role in chromatin modification in this fungus.

Organelle Trafficking during Appressorium Development in Magnaporthe oryzae

A. B. ESEOLA (1,2), X. Yan (2), L. S. Ryder (2), M. Oses-Ruiz (2), N. J. Talbot (2), (1) School of Biosciences, University of Exeter, Geoffrey Pope Building, Exeter, U.K.; (2) The Sainsbury Laboratory, University of East Anglia, Norwich Research Park, Norwich, U.K.

Magnaporthe oryzae (syn. Pyricularia oryzae), the causal agent of the devasting rice blast disease, poses a significant threat to global food security, mostly in developing countries. The fungus develops a specialised infection structure called an appressorium in which turgor pressure is built and translated to mechanical force to breach the tough cuticle of the rice plant. We are studying the fate of organelles during conidial germination and appressorium development. Programmed conidial cell death, which requires autophagy, is a pre-requisite for development of a functional appressorium. Autophagy is a cell survival response to starvation stress and is used to selectively recycle contents of the conidium for appressorium maturation. In this study, we have designed molecular markers tagged with green fluorescence protein to localise specific organelles in M. oryzae to elucidate their fate during appressorium development. We have found that a proportion of organelles are trafficked to incipient appressoria and others degraded by autophagy. We are investigating the sorting mechanisms that operate in $M$. oryzae to determine the fate of organelles during plant infection. Our results provide insight into the group of organelles that are translocated and the group that are degraded during appressorium development

Pro-viral functions of the host proteins ARGONAUTE1 and VARICOSE in potato virus A infection K. MÄKINEN, M. Pollari, S. De, Department of Microbiology, University of Helsinki, Finland

The potyviral helper component-proteinase (HCPro) acts as a viral RNA silencing suppressor and is involved in aphid transmission, polyprotein maturation, systemic spread and the assembly of virus particles. Furthermore, HCPro is crucial for the formation of potyvirus-induced granules (PGs), which serve potentially as shelters for vRNA against the host's silencing machinery. One of the factors underlying the multifunctionality 
of HCPro is its ability to interact with a wide range of host factors. Previously, we have identified $N$. benthamiana ARGONAUTE 1 (AGO1) and VARICOSE (VCS) as components of PGs and showed that HCPro and AGO1 are potential interaction partners on polysomes. In this study, we identified AGO1 and VCS binding sites in potato virus A (PVA; genus Potyvirus) HCPro. Mutations at these sites significantly reduced the sequestration of AGO1 and VCS into PGs. The AGO1-binding defect in HCPro did not alter either PVA gene expression or viral cell-to-cell movement in local leaves, whereas both of them reduced to some extent because of the VCS-binding defect. Intriguingly, both mutations had an effect on PVA particle accumulation, which suggests a pro-viral role for AGO1 and VCS in virus particle production. Furthermore, we report the relevance of these interactions for the systemic spread of PVA.

Studying effector secretion by the rice blast fungus Magnaporthe oryzae C. RODRIGUEZ HERRERO (1), M. Martin-Urdiroz (2), X. Yan (3), M. J. Kershaw (2), D. Soanes (4), N. J. Talbot (3), (1) The Sainsbury Laboratory, University of East Anglia, Norwich Research Park, U.K.; (2) University of Exeter, U.K.; (3) The Sainsbury Laboratory, University of East Anglia, Norwich Research Park, Norwich, U.K.; (4) University of Exeter, Exeter, U.K.

Magnaporthe oryzae is the causal agent of rice blast disease, the most destructive disease of cultivated rice. During host colonization the rice blast fungus secretes a repertoire of effector proteins to suppress plant immunity. Effectors in M. oryzae are classified as cytoplasmic effectors or apoplastic effectors, depending on their site of delivery. Apoplastic effectors are found in the apoplastic space between the fungal cell wall and plant plasma membrane, while cytoplasmic effectors accumulate in a membrane-rich plant structure called the biotrophic interfacial complex (BIC). Cytoplasmic effectors are secreted via an unconventional route that appears to be Golgi-independent, while apoplastic effectors follow the conventional ER-Golgi secretion pathway. The BIC is involved in translocation of cytoplasmic effectors into the rice cytoplasm, but little is known about sorting mechanism of effectors into the correct secretory systems. To investigate this process, we generated a library of chimeric effector gene fusions that are being tested for effector translocation after expression in M. oryzae. We found that the promoter and signal peptide regions of effector-encoding genes are important to determine the cellular destination of effectors. We can therefore misdirect cytoplasmic effectors to the apoplast and vice versa. This suggests that signals in the 5'end of genes are necessary for effector delivery by the rice blast fungus.

Powdery mildew effectors target endoreduplication modulators in Arabidopsis A. G. MCRAE, E. Applegate, M. Kan, M. C. Wildermuth, University of California, Berkeley, Berkeley, CA, U.S.A.

Endoreduplication has been observed in the Golovinomyces orontii-Arabidopsis interaction site and induction is directly correlated with the extent of fungal reproduction. Microbe-induced endoreduplication of host tissue is common in plant biotrophs and thought to enhance the metabolic activity at these interaction sites to support nutrient acquisition. As regulators of plant growth, many TCP transcription factors are involved in cell cycle regulation including endoreduplication. In Weßling, et al. 2014 a yeast-2-hybrid (Y2H) screen revealed TCP transcription factors are among the most targeted Arabidopsis proteins by pathogen effectors from diverse pathogens including G. orontii. Using Agrobacterium-mediated transient expression in Nicotiana benthamiana we show that 14 out of 16 TCP-G. orontii effector pairings identified via Y2H colocalize to the nucleus in planta. G. orontii infection assays confirm Arabidopsis tcp13 and tcp14 and reveal tcp20 T-DNA mutants support enhanced fungal reproduction. Current investigations explore cellular phenotypes associated with constitutive expression or silenced expression of TCP-targeting effectors. In addition, we developed spray-induced gene silencing (SIGS) to limit powdery mildew gene expression. To our knowledge, this is the first report of SIGS against an obligate plant biotroph. This approach is now being used to identify G. orontii effectors that contribute to asexual reproduction.

9-LOX derived oxylipins activate mitochondrial signaling to trigger plant defense

L. Muñiz, Y. Izquierdo, C. CASTRESANA, National Centre of Biotechnology, Madrid, Spain

Studies in Arabidopsis using pure oxylipins and a series of 9-HOT- and 9-KOT-insensitive noxy mutants (non-responding to oxylipins) revealed the importance of the mitochondria as a component of the 9-LOX signaling and that its defect is accompanied by the alteration in the response of plants to infection of Pseudomonas syringae. Map-based cloning of noxyl and noxy5 mutants revealed that the corresponding NOXY genes encode uncharacterized Pentatricopeptide repeat (PPR) mitochondrial proteins that are likely functioning in the editing or the splicing of mitochondrial transcripts. The noxy 1 and noxy 5 mutations displayed altered mitochondrial function and formation of mitochondrial aggregates. Moreover, noxyl and noxy 5 mutants displayed AA resistance, being able to maintain meristem viability and function at wild type-lethal AA doses. We found that noxy1 and noxy5 mutants are not impaired in perception or signaling mitochondrial damage as their respond to AA by inducing similar levels of Alternative oxidase $(A O X)$ gene expression and AOX protein accumulation than wild type plants. By contrast, our results indicated that the insensitivity of noxyl and noxy 5 mutants to AA was due to constitutive activation of a resistance response. The identification of RNA or protein changes in mitochondrial enriched fractions will help to understand the actions of oxylipins on the mitochondria and the role of these organelles in plant immunity.

Subcellular dynamics of red clover necrotic mosaic virus (RCNMV) double-stranded RNAs in infected plant cells S. Takata, K. Mise, Y. Takano, M. KAIDO, Graduate School of Agriculture, Kyoto University, Kyoto, Japan

RNA viruses form virus replication complex (VRC) on endomembrane system and replicate their genomic RNAs.VRCs include virus-encoded replication proteins, host factor proteins, and genomic replication intermediate product that is double-stranded RNA (dsRNA).Red clover necrotic mosaic virus (RCNMV) is a positive-strand RNA virus with a bipartite genome. We previously reported that a large number of VRCs were formed at the cortical endoplasmic reticulum (ER) in the RCNMV-infected protoplasts of Nicotiana benthamianaand the viral movement protein (MP) colocalized with VRCs. In this study, using the transgenic Nicotiana benthamiana expressing a fusion protein of the dsRNA-binding domain of B2 protein of Flock House virus and GFP, we investigated the dynamics of VRCs in RCNMV-infected living plant cells by confocal laser scanning microscopy.VRC of RCNMV was observed as approximately 200 fine microspot-like structures of approximately 3 square micrometers by 5 hours postinoculation (hpi), and later as a huge structure of approximately 40 square micrometers by 14 hpi. The number of 
VRCs decreased from 5 to 14 hpi. These results suggest that small VRCs collide and merge to form larger ones. Using an $M P$ gene-frameshift mutant virus, we also showed that the RCNMV MP was not involved in the formation of VRC, although the expression of some mutant MPs affected the morphology of VRC.

Effects of symbiotic interactions with Gigaspora margarita on disease resistance in tomato plants M. FUJITA (1), M. Kusajima (1), K. Akiyama (2), K. Yoneyama (3), T. Asami (4), H. Kato (1), H. Nakashita (1), (1) Fukui Prefectural University, EIHEIJI, Japan; (2) Osaka Prefectural University, Sakai, Japan; (3) Utsunomiya University, Utsunomiya, Japan; (4) University of Tokyo, Tokyo, Japan

Plants have various types of interactions with microbes. Among them, some of symbiotic interactions are speculated to have some benefits to the host plants, however, which have been poorly understood. In this study, we analyzed the effects of interaction with an arbuscular mycorrhizal (AM) fungus, Gigaspora margarita, on disease resistance in tomato plants. Tomato plants (Solanum lycopersicum cv. Momotaro) inoculated with G. margarita (25 spores/plant) exhibited enhanced resistance against diseases caused by Pseudomonas syringaepv. tomato and Botrytis cinerea. The expression of pathogenesis-related $(P R)$ genes was not induced by interaction with this AM fungus. The levels of SA in leaf tissues were not influenced by symbiosis with $G$. margarita. These results indicated the possibility that $G$. margaritais able to induce disease resistance in tomato by activating "priming", a novel type of resistance mechanism independent of SA-mediated defense signaling. Recent progress on the analyses of priming effects will be also presented.

Heat-shock treatment induces SA-dependent NPR1-independent disease resistance in Arabidopsis H. NAKASHITA (1), M. Kusajima (1), M. Inoue (1), T. Ichinose (2), M. Fujita (1), C. Yamaguchi (2), T. Nishiuchi (3), A. Maruyama (2), (1) Fukui Prefectural University, EIHEIJI, Japan; (2) Kyushu University, Fukuoka, Japan; (3) ASRC, Kanazawa University, Kanazawa, Japan

The induction of disease resistance by high temperature treatment has been reported in cucumber, in which SA is likely to take part. To determine whether heat shock (HS) treatment can induce SAR, we analyzed the effects of HS on Arabidopsis. Heat shock treatment $\left(45^{\circ} \mathrm{C}, 2 \mathrm{~min}\right)(\mathrm{HS})$ induced disease resistance against a bacterial pathogen Pseudomonas syringae pv. tomato in Arabidopsis, which lasted about 3-5 days after the treatment. Enhanced expression of PR genes and increase in SA levels was observed in wild-type plants 2 days after HS treatment. However, disease resistance and $P R$ gene expression were not induced by HS treatment in sid 2 mutant, indicating that HS treatment induces systemic acquired resistance (SAR). However, this resistance was also induced in $n p r 1$ mutant, which suggesting an SA-dependent NPR1-independent disease resistance. To characterize the HS-induced resistance, gene expression and metabolite production were compared between wild-type and npr1 mutant, which indicated so far no significant differences in expression of many SA signal-related genes and amounts of glucosinolates and glutathiones. In both plants, different from SAR, HS induced the expression of some JA signal-related gens, suggesting some hormonal crosstalk may take part in HS-induced resistance. Recent progress of understanding the mechanism of NPR1-independency will be presented.

How do Ralstonia solanacearum type III chaperones modulate the secretion of type III substrates and play essential roles in virulence? F. Lonjon (1), M. Turner (1), D. Lohou (1), C. Henry (2), D. Rengel (1), N. S. Razavi Ebrahimi (1), C. R. R. Sabbagh (3), A. Le Ru (4), N. Peeters (1), F. Roux (1), S. Genin (1), F. VAILLEAU (1), (1) LIPM, Université de Toulouse, INRA, CNRS, INPT, Castanet-Tolosan, France; (2) UMR 119 MICALIS, PAPPSO, Domaine de Vilvert, 78352 Jouy en Josas, France; (3) LIPM, Université de Toulouse, UMR INRA-CNRS 4412594, 31326 Castanet Tolosan, France; (4) Research Federation Agrobiosciences Interactions et Biodiversité, Castanet-Tolosan, France

Ralstonia solanacearum is a worldwide major crop pathogen whose virulence strongly relies on a type III secretion system (T3SS) that allows the translocation of type III effectors (T3Es) directly into the host cells. To identify groups of effectors collectively promoting disease on susceptible hosts, we investigated the role of putative post-translational regulators in the control of type III secretion. We analyzed four Hpa (hypersensitive response and pathogenicity associated) proteins. Combining a shotgun secretome analysis with label-free quantification using tandem mass spectrometry, the search for Hpa-T3Es direct interactions, and the analysis of the pathogenicity of hpa mutants on several host plants, we have demonstrated that Hpa are main players acting in $R$. solanacearum to control type III substrate secretion, sometimes via direct interactions with T3Es. We showed that these four Hpa proteins are differentially implicated in R. solanacearum pathogenicity, according to the host plant. With recent data, we demonstrated an unexpected dichotomy of secretion pattern between HpaB and HpaP, both important for $R$. solanacearum virulence, data reinforcing the hypothesis of a hierarchy of delivery of the type III substrates. The results obtained during these last five years will be outlined, providing a better view of type III secretion control in $R$. solanacearum.

Helper NLRs - How is their cell death and resistance function regulated?

S. SAILE (1), V. Bonardi (2), L. Wünsch (3), P. M. Jacob (4), P. L. Teixeira (4), E. Sauberzweig (1), J. L. Dangl (4), F. El Kasmi (3), (1) Center for Plant Molecular Biology (ZMBP), University of Tuebingen, Tuebingen, Germany; (2) Novozymes North America, Durham, NC, U.S.A.; (3) Center for Plant Molecular Biology (ZMBP), University of Tuebingen, Tübingen, Germany; (4) Department of Biology, University of North Carolina at Chapel Hill, Chapel Hill, NC, U.S.A.

Plants have evolved a multi-faceted immune system to defend themselves against a wide variety of pathogens. The activation of ETI depends on the presence of intracellular receptors, so called NLRs. NLRs are molecular switches that bind nucleotides in order to activate disease resistance responses. Recent work suggests that NLRs function in an integrated network: Some NLRs act as the effector-specific sensor, whereas others function as so called helpers downstream of these sensor NLRs in signal transduction. The Arabidopsis genome encodes 5 full-length helper NLR (hNLR) genes that can be divided into two families, both belonging to the $\mathrm{CNL}_{\mathrm{R}}$ subclass of CNLs: ADRIs and NRG1s. Previously, it has been shown that the ADR1-L2 helper function during ETI responses and basal defense is independent of nucleotide binding, and hence independent of what is considered the canonical nucleotide switch activation. Interestingly, ADR1-L2 works additionally as a typical, activated disease resistance protein in cell death regulation in a canonical way. We have performed a suppressor screen, in which we identified suppressors of the constitutive pathogen response ( $\mathrm{cpr}$ ) like phenotype caused by expression of the auto-activated ADR1-L2. Characterizing the identified intragenic and 
extragenic suppressor mutations will help us to dissect the molecular mechanism of hNLR activation and cell death induction, and will contribute to the construction of a genetic network required for NLR (auto-)activity.

\begin{abstract}
4 Plants and A Pathogen: Host-specific Gene Expression in Phytophthora capsici may underpin dynamic adaptation on diverse host crops.

M. CUMMins (1), A. Howden (2), R. Stam (3), J. Pham (1), E. Huitema (1), (1) Division of Plant Sciences, The University of Dundee, Dundee, U.K.; (2) Cell Signalling and Immunology, University of Dundee, Dundee, U.K.; (3) Technische Universität München, Freising, Germany

Phytophthora capsici is a broad host range oomycete pathogen with global reach, threatening a worldwide vegetable crop valued at well over 1 billion USD each year. With populations growing and global temperatures set to rise, the role of plant pathogens as a threat to food security is going to become an ever more pressing issue. Controlling broad host range pathogens such as $P$. capsici, requires management of disease on diverse host crops. Therefore, it is critical that we understand the mechanisms which underpin the ability of $P$. capsici to adapt to different host plants whilst maintaining virulence. Here, we provide evidence that $P$. capsici modulates the expression of its gene complement in a host-specific manner. Comparative gene expression analyses revealed that at least $10 \%$ of $P$. capsici genes feature Host-Specific Gene Expression (HSGE). Critically, a large proportion of genes that feature HSGE encode secreted effectors, suggesting dynamic adaptation to a given host. Moreover, phenotyping of selected genes reveals those which contribute to host specific virulence. Here we will present our progress in characterizing HSGE in $P$. capsici and the role of those genes in host specific virulence to help achieve sustainable control of $P$. capsici disease.
\end{abstract}

Effects of strigolactone signaling on disease resistance in Arabidopsis

M. KUSAJIMA (1), H. Nakashita (2), K. Yoneyama (3), K. Akiyama (4), T. Nomura (3), T. Asami (1), (1) University of Tokyo, Tokyo, Japan; (2) Fukui Prefectural University, EIHEIJI, Japan; (3) Utsunomiya University, Utsunomiya, Japan; (4) Osaka Prefectural University, Sakai, Japan

Strigolactones (SLs) are phytohormones that inhibit shoot branching and function in the rhizospheric communication with symbiotic fungi and parasitic weeds. The details of the effects of SL on disease resistance have not been clarified. We have shown that treatment of SL analogs (4BD, GR24) in rice, tomato and Arabidopsis thaliana will not affect the induction of systemic acquired resistance (SAR). However, SL-related mutants of Arabidopsis exhibit weaker disease resistance compared to wild-type plant. We analyzed the influence of SL signal on disease resistance in Arabidopsis. Wild-type plant treated with GR24 exhibited enhanced resistance to the bacterial pathogen Pst DC3000, however, its effect was weaker compared to the treatment with SAR inducer. In addition, GR24 treatment induced disease resistance also in npr1-2 mutant. On the other hand, expression SAR marker gene was not observed in wild-type plant after GR24 treatment. These results suggested that GR24 induces disease resistance by a mechanism different from salicylic acid-mediated SAR. Recent progress of understanding the roles of SL in disease resistance will be presented.

ADR1-L2 helper NLR signals through a TIR-domain containing NLR.

P. M. JACOB (1), V. Bonardi (2), F. El Kasmi (3), L. Wünsch (3), J. J. Hige (1), B. J. Mckinney (1), P. L. Teixeira (1), J. L. Dangl (1), (1) Department of Biology, University of North Carolina at Chapel Hill, Chapel Hill, NC, U.S.A.; (2) Novozymes North America, Durham, NC, U.S.A.; (3) Center for Plant Molecular Biology (ZMBP), University of Tuebingen, Tübingen, Germany

Pathogens have evolved effectors capable of inhibiting the plant's immune response. In turn, plants developed the ability to recognize those pathogen effectors, thereby producing effector-triggered immunity (ETI). ETI is mediated by specific NLRs (nucleotide-binding, leucine-rich repeats receptors). In Arabidopsis, three NLRs, ADR1, ADR1-L1 and ADR1-L2, were found to be required for inducing cell death and defense after the activation of several other NLRs, mostly TIR-domain containing NLR (TNL). Moreover, the ADR1s were shown necessary for defense and salicylic acid (SA) accumulation in the context of PAMP-triggered immunity and ETI. To learn more about the genetic requirements for ADR1-L2 signaling, we performed a suppressor screen on the auto-active MHD mutant ADR1-L2 ${ }^{\mathrm{D} 484 \mathrm{~V}}$. We identified 5 different loss of function mutations able to suppress ADR1-L2 ${ }^{\mathrm{D} 484 \mathrm{~V}}$ auto-immunity in a previously uncharacterized TNL, consequently termed SAD1 (Suppressor of ADR1-L2 DV 1). Allelism tests confirmed that SAD1 alleles were responsible for suppressing ADR1-L2 ${ }^{\mathrm{D} 484 \mathrm{~V}}$ auto-immunity. Preliminary data showed that SAD1 is required downstream of the activation of ADR1s, including basal resistance to the virulent pathogen Pst DC3000 and Pst DC3000-avrRpt2. Inversely, SAD1 cannot suppress the autoimmune phenotype triggered by the TNL allele snc1. Interestingly, PR1 expression analyses suggest that SAD1 is involved in SA signaling in the area bordering the infection site but not in the infection site.

Signalling of membrane-localized immune receptors in Arabidopsis

S. SUNIL (1), F. Wilhelm (1), S. Beeh (1), M. J. Cann (2), F. El Kasmi (1), (1) Center for Plant Molecular Biology (ZMBP), University of Tuebingen, Tübingen, Germany; (2) School of Biological and Biomedical Sciences, Biophysical Sciences Institute, Durham, U.K.

Pathogen attack is an inevitable environment, a plant has to with-stand throughout its life cycle. To defend against these attacks, plants evolved specific extracellular and intracellular immune receptors. Plant intracellular NOD-like immune receptors (NLRs) perceive pathogen-derived effector proteins either directly or indirectly and activate a strong effector-triggered immune response (ETI). NLRs either localizes in the cytoplasm and nucleus or at cellular membranes. Studies have shown that cytoplasmic localized NLRs translocate to the nucleus to initiate transcriptional reprogramming that leads to ETI. However, membrane localized NLRs might not do so. As such we are interested in how are these membrane localized NLRs signal, in order to activate ETI. We also aim to understand the regulation of membrane NLRs. We identified an auto-active endomembrane localized, N-terminal truncated CNL, named PM5, in Arabidopsis. Interestingly this NLR is genetically linked to another CNL, named PM12.We performed an $\mathrm{Y} 2 \mathrm{H}$ screen with the $\mathrm{CC}$ domain of PM12 as a bait and identified putative interactors/regulators. We are also analyzing if these NLRs physically interact and if this is necessary for their function, since they are genetically linked. Through our study, we intent to draw a clear model of membrane CNL activation and signaling. Further, how are the various interaction partners involved in this regulation and function. 
ERF transcription factors provide opportunities for resistance breeding towards the vascular fungal pathogen Verticillium longisporum by activating Indole-glucosinolate biosynthesis

C. FRÖSCHEL, C. Weiste, W. Dröge-Laser, Julius-von-Sachs-Institute, Würzburg, Germany

This study defines valuable resources for smart breeding or genetic engineering. As transcription factors (TFs) often control sets of functionally related genes, they are suitable candidates for such approaches. We propose a novel gain-of-function screen to identify TFs enhancing resistance against plant pathogens. Frequently, severe crop loss is caused by pathogens, like the fungus Verticillium longisporum that damages oilseed rape cultures. By using this pathogen as a model, we established a simple and robust strategy to screen for reduced fungal colonisation in plants of a TF overexpressing collection (Arabidopsis TF Open-Reading-Frame-Expression collection). Seven closely related members of group IX of the ETHYLENE-RESPONSE-FACTOR (ERF) family were found to enhance resistance, namely ERF96 and ERF102-ERF107. Verticillium infected root cells produce indole-glucosinolates (IGs). Interestingly, ERF overexpressors displayed an enrichment of transcripts from genes responsible for the biosynthesis of IGs, such as CYP81F2 (CYTOCHROME-P450-MONOOXYGENASE81 F2). Moreover, they showed increased levels of 4 methoxy-indole 3-ylmethylglucosinolate (4MI3G), the product of CYP81F2. The promoter of this gene is a direct target of the studied ERFs, which activate transcription via two GCC-like boxes. Taken together, the IGs biosynthesis can be driven by ERFs and as the cyp81f2 mutant displayed significantly enhanced susceptibility, IGs are involved in restricting Verticillium propagation.

Zymoseptoria tritici pangenome-based functional screens of candidate effector proteins in wheat

H. CHEN (1), C. Bayon (1), R. King (2), C. Bouton (1), D. Smith (2), S. Torriani (3), S. Bieri (3), K. Kanyuka (1), K. Hammond-Kosack (4), J. Rudd (4), (1) Department of Biointeractions and Crop Protection, Rothamsted Research, Harpenden, U.K.; (2) Department of Computational and Analytical Sciences, Rothamsted Research, Harpenden, U.K.; (3) Syngenta Crop Protection AG, Schaffhauserstrasse, Stein, Switzerland; (4) Rothamsted Research, Harpenden, U.K.

Septoria tritici blotch (STB), caused by the ascomycete Zymoseptoria tritici, is one of the most important foliar diseases of wheat worldwide. Due to poor genetic resistance of wheat to this pathogen and emerging fungicide resistance in field population, new sources of plant genetic resistance are needed to improve varieties performance against STB. In this study, we performed a virulence screen of $43 \mathrm{Z}$. tritici isolates on 21 wheat cultivars. 25 isolates were subjected to genome sequencing and 12 of them to transcriptomic analysis. A pangenome was constructed and 100 effector candidates were selected and tested for activities in wheat leaves using a Foxtail mosaic virus overexpression system (FoMV VOX). Our results showed a diverse interaction profile between isolates and cultivars. Effector overexpression by FoMV was notably improved by using fertilised compost. Four effector candidates induced necrosis in Nicotiana benthamiana, but none in wheat. However, we found some examples of effector interactions with wheat genotypes which resulted in either a block on systemic viral spread (and symptoms) or a more rapid and pronounced systemic spread of viral symptoms. Our study reports the first use of the recently established FoMV VOX for candidate effector discovery. This system offers the potential for more rapid screening in wheat and other important crop plants.

Potyvirus And Potexviruses Small Membrane Binding Proteins Regulate bZIP60, bZIP28 and bZIP17 and Programmed Cell Death. M. GAYRAL (1), O. Arias Gaguancela (2), E. Vasquez (3), F. Flores (3), M. Pang (1), J. Verchot (1), (1) Texas A\&M AgriLife Research, Dallas, TX, U.S.A.; (2) Texas A\&M AgriLife Research, U.S.A.; (3) Universidad de las Fuerzas Armadas-ESPE, Ecuador

Plant potyviruses and potexviruses change ER morphology during infection which can create conditions that are adverse for the cellular protein synthesis and protein folding machinery, resulting in ER stress. Three sensors of ER stress include the inositol requiring enzyme (IRE1), bZIP17, and bZIP28 which respond to adverse conditions in the ER and activate a transcription cascades leading to increased expression of genes that are essential for restoring cellular homeostasis. Under conditions of chronic ER stress, this machinery may switch toward programmed cell death activation. While we have reported that IRE1 proteins plan critical roles in recognition of the potyvirus $6 \mathrm{~K} 2$ and potexvirus TGB3 proteins, our recent investigations determined that bZIP17 and bZIP28 also play differential roles in responding to these viral proteins and in regulating the levels of virus accumulation in whole plants. In this study we show that NAC089 and NAC103 i.e. two of the secondary ER stress signaling transcription factors, are differentially activated by potyviruses and potexviruses which also regulate pro-death and pro-survival responses to virus infection.

Pattern-triggered immunity affects Rx1 mediated defence responses

M. KNIP, M. M. Richard, M. Beijaert, F. L. Takken, University of Amsterdam, Amsterdam, Netherlands

Intracellular NLR (nucleotide-binding leucine-rich repeat)-type immune receptors are a fundamental part of plant immune systems. As pathogens infect plants at foci, activation of NLR-mediated immune responses is typically non-uniform and non-synchronized. Moreover, the NLR response is likely affected by the presence of a pathogen that induces PAMP Triggered Immunity (PTI). To untangle both responses and their potential crosstalk, we devised the CESSNA (Controlled Expression of effectors for Synchronized and Systemic NLR Activation) system. CESSNA mediated Potato Virus X coat protein (CP) expression allows monitoring Potato NLR Rx1-mediated immune responses in a quantitative, and reproducible manner. Because CESSNA relies solely on genetically encoded transgenes it is "PAMP-free" enabling the exclusive study of Effector-Triggered Immunity (ETI). Both early and late ETI responses following Rx1 activation were monitored, among which the Reactive Oxygen Species (ROS)-burst and cell-death. These outputs were studied with and without a PAMP pre-treatment. We found that PAMP pretreatment has a marked effect on ETI-responses; suppressing some and enhancing others. Our data reveals crosstalk between PTI and ETI and the implications of this for the study of immune signaling will be presented.

A Jasmonate signaling network activates root stem cells and promotes regeneration

W. Zhou (1,2), J. L. LOZANO TORRES (3), I. Blilou (1,4), X. Zhang (2), Q. Zhai (2), G. Smant (3), C. Li (2), B. Scheres (1,5), (1) Laboratory of Plant Developmental Biology, Wageningen University and Research, Wageningen, Netherlands; (2) Institute of Genetics and Developmental Biology, Chinese Academy of Sciences, Beijing, China; (3) Laboratory of Nematology, Wageningen University and Research, Wageningen, Netherlands; (4) King Abdullah University of Science and Technology, Saudi Arabia; (5) Rijk Zwaan Breeding, Fijnaart, Netherlands 
Plants have evolved an elaborate repertoire of responses when challenged with environmental damage. Mounting an immune response often results in a growth-defense trade-off. The stress hormone jasmonate (JA) has well-known roles in the biochemical response to tissue damage. JA also affects plant growth and development. However, the precise role of JA in tissue regeneration and stem cell activation upon wounding is not well understood. We have recently discovered a molecular network triggered by wound-induced JA that promotes stem cell activation and regeneration. JA regulates stem cell activation through the RBR stem cell regulatory network and the stress response protein ERF115. Furthermore, JA induces the transcription of ERF109 which stimulates the expression of CYCD6;1, functions upstream of ERF115 and promotes regeneration. Soil penetration and infections with the root-knot nematode Meloidogyne incognita induce and are dependent on this JA mediated regeneration response. Altogether, we conclude that the JA tissue damage response pathway plays a key positive role in stem cell regeneration.

Improved resistance to pathogens by overexpressing a transcription factor with an AP2/ERF domain in the moss Physcomitrella patens G. Reboledo (1), A. Agorio (1), J. M. Franco (2), I. PONCE DE LEÓN (1), (1) Instituto de Investigaciones Biológicas Clemente Estable, Montevideo, Uruguay; (2) Centro Nacional de Biotecnología-CSIC, Madrid, Spain

Plants activate different defense mechanisms once they detect the presence of pathogenic microorganisms. One of these mechanisms involves the participation of transcription factors, such as AP2/ERF (Apetala2/Ethylene Responsive Factor), which activate the expression of defense genes. Overexpressing one of these transcription factors in the moss Physcomitrella patens, PpAP2, allowed us to increase resistance to important crops pathogens, including Botrytis cinerea and Colletotrichum gloeosporioides. In the present work we performed a functional characterization of PpAP2 in order to understand the involvement of this gene in plant defense. We analyzed the transcriptomes of wild-type and PpAP2 overexpressing plants both infected or not with the pathogen B. cinerea. We've found that 45 genes from the AP2 superfamily are induced when moss plants are infected with this fungus, and the overexpression of only one, PpAP2, is enough to increase the resistance of this plant to several pathogens. In some cases, the cellular damage observed in pathogen-infected transgenic lines is similar to that of control plants without infection. The relation of PpAP2 and the activation of defense genes leading to resistance are discussed. The main objective of this work is to study novel defense mechanisms present in $P$. patens that could be used in biotechnological application in other plants.

Chloroplasts, a major hub of immune signaling.

S. BREEN (1), H. Brown (2), M. de Torres Zabala (3), D. Arnaud (4), R. F. Hussain (1), S. Kulasekaran (1), N. Smirnoff (4), M. Schwarzländer (5), G. Littlejohn (6), M. R. Grant (7), (1) The University of Warwick, Coventry, U.K.; (2) University of Exeter, Exeter, U.K.; (3) University of Exeter, Exceter, U.K.; (4) The University of Exeter, Exeter, U.K.; (5) Institute for Plant Biology and Biotechnology, Münster, Germany; (6) University of Plymouth, Plymouth, U.K.; (7) University of Warwick, Warwick, U.K.

Chloroplasts perform many other functions besides photosynthesis, including synthesis of phytohormone precursors, amino acids, nucleotides and generation of signaling molecules in the form of reactive oxygen species. Chloroplasts play pivotal roles in the perception, decoding and finetuning of responses to constantly varying environmental signals and metabolic demands. Recently chloroplasts have emerged as unexpected, yet key players in plant immune responses - Microbe Associated Molecular Pattern (MAMP) triggered immunity (MTI), effector triggered immunity (ETI) (Caplan et al. 2015) and systemic immunity, with some effectors specifically targeting the chloroplast or chloroplast components. To unravel the role of chloroplasts in plant immune signaling we are taking a two-pronged approach. Firstly, we are utilising genetically encoded reporters targeted to different sub-cellular locations to understand the role of $\mathrm{H}_{2} \mathrm{O}_{2}$ and ATP in defence and disease progression during Pseudomonas syringae infection. Secondly, we are identifying host interacting proteins of effectors with known localisation to the chloroplast. It is known that effectors modify chloroplast physiology so by elucidating their targets we can unpick their mode of action to reduce the photosynthetic capability of the chloroplast. These approaches will provide new insight into the importance of the chloroplasts in regulating immunity.

Arabidopsis IRE1 Regulates Pathogen-Mediated ER Stress Signaling

K. M. MUKHTAR, University of Alabama at Birmingham, Birmingham, AL, U.S.A.

In eukaryotic cells, the endoplasmic reticulum (ER) is essential for protein synthesis, folding, and processing. Diverse physiological and pathological stimuli can disrupt this ER homeostasis resulting in the activation of complex signaling network known as the unfolded protein response (UPR). The most conserved eukaryotic UPR sensor, Inositol-requiring enzyme 1 (IRE1), responds to ER stress by activating pathways that typically up-regulate cellular pro-survival signals, but under extreme conditions can lead to pro-death signals. My lab showed that pathogen infection exerts stress on ER protein folding capacity resulting in the activation of IRE1. We also demonstrated that IRE1 catalyzes unconventional cytoplasmic splicing of bZIP60 mRNA, giving rise to a transcriptionally active form that promotes the pro-survival response. Here we present novel findings on the structure-function analyses of IRE1. We determined that IRE1 undergoes N-glycosylation following the onset of ER stress. We also discovered dynamic changes in the interaction patterns of IRE1 with key cellular regulators of apoptosis highlighting its roles in cell fate decisions. We identified a novel microRNA that is up-regulated upon acute ER stress and can attenuate pro-survival signaling. These newly discovered signaling components uncovered the nature of novel molecular switches in ER stress signal transduction and will expand our understanding of the roles of IRE1-mediated ER stress upon biotic stresses.

The Relationship Between Flower Color and Disease Resistance Against Septoria gentianae in Japanese Gentian, Possible Involvement of Flavonoids and Related compounds in Blue Pigmentation

C. TATEDA, M. Nishihara, M. Iwai, K. Obara, K. Fujisaki, Iwate Biotechnology Research Center, Japan

So far, we have shown that stomatal density of adaxial side of leaves of Japanese gentians determines resistance against Septoria gentianae which uses stomata for their invasion. On the other hand, we found the presence of stomatal density-independent disease resistance mechanism. Gentiana scabra cv. Momokorin with pink flowers is susceptible to S. gentianae, while G. scabra cv. Aokorin with blue flowers shows resistance to $S$. gentianae. Both are sibling cultivars originated from the same breeding parents and have similar levels of stomatal density. To analyze the 
stomatal density-independent resistance in gentian, we first focused on the difference of flower color between Momokorin and Aokorin, and evaluated the involvement of metabolic pathways related to flower pigmentation in the resistance to $S$. gentianae. Expression analysis showed that $F 3^{\prime} 5$ ' $H$ (flavonoid 3', 5'-hydroxylase), which encodes a key enzyme in biosynthesis of delphinidin (blue color pigment), was up-regulated after $S$. gentianae infection in cv. Aokorin. Cv. Momokorin was previously identified as a $F 3^{\prime} 5^{\prime} H$-deficient mutant. Notably, the mutations in $F 3^{\prime} 5^{\prime} \mathrm{H}$ gene led to flower color change (from blue to pink) and enhanced susceptibility to $S$. gentiane. Thus, $F 3^{\prime} 5$ ' $H$ and probably it's related metabolites are important for the resistance to $S$. gentiane.

Functional study of rice calcium-dependent protein kinase family

Baohui Mu, Jiyang Wang, Shanzhi Wang, Wenxian Sun. Department of Plant Pathology and the Ministry of Agriculture Key Laboratory for Plant Pathology, China Agricultural University, Beijing, China

Calcium-dependent protein kinases (CPKs) are a typical calcium-binding protein involved in plant growth and development, biotic and abiotic stress responses, and intracellular hormone regulation.Some CPKs play a role in disease resistance signaling.Previous studies shown that OsCPK4 can participate in rice innate immunity by regulating the stability of OsRLCK176.On the other hand, according to the MPSS database analysis, OsCPK17 is involved in rice immune regulation and plays an important role.This study found that the lesions formed by oscpk17 mutants after inoculation with Xanthomonas oryzae pv. oryzae were significantly increased compared with wild-type;and rice protoplasts were treated with pathogen-associated molecular patterns (PAMPs) such as flg22 and chitin, OsCPK17 exhibits a more pronounced phosphorylation migration band. When the rice seedlings were treated with chitin and flg22, the activation of wild type DJ and oscpk17 mutant MAPK did not change significantly.Finally, in vitro phosphorylation demonstrated that both OsCPK17 and OsRLCK176 phosphorylate OsRbohB.Based on this research, we will further explore the interaction protein of OsCPK17 in rice and the function of this protein in innate immunity.

Exploring how plant nutrient transport affects resistance and susceptibility to oomycete pathogens U. Sonnawala (1), J. Herlihy (1), T. Long (2), G. Pilot (1), J. M. MCDOWELL (3), (1) Virginia Tech, U.S.A.; (2) North Carolina State University, U.S.A.; (3) Virginia Tech, Blacksburg, VA, U.S.A.

All plant pathogens obtain nutrients from the plant host to fuel their own growth. At the same time, plants must reprogram their own metabolic pathways to fuel their immune responses. These two critically important functions are accomplished in environments where key nutrients are either limiting, sufficient, or in excess. Physiological experiments have demonstrated that changes in host nutrient status either promotes or retards pathogen growth or plant resistance. However, these data are fragmentary; particularly for plant interactions with oomycetes (e.g., Phytophthora species, downy mildews) that account for tens of billions of dollars in annual crop losses. We are investigating this knowledge gap with pathosystems comprised of Arabidopsis and two oomycete pathogens: the foliar pathogen Hyaloperonospora arabidopsidis (obligate biotroph) and the root pathogen Phytophthora capsici (hemi-biotroph). We have developed a hydroponic system in which we can manipulate specific nutrients to observe effects on resistance/susceptibility. We are using this system in combination with phenotyping and transcript profiling to understand how plants and pathogens respond to nutrient stress. We have also used a reverse genetic approach to identify mutant alleles of nutrient transporters that promote or retard pathogen growth, and we are currently investigating the physiological and molecular basis of these phenotypes.

Interaction mechanisms of rice (Oryza sativa L.) to defense against root-knot nematode Meloidogyne graminicola under treatment with Aspergillus welwitschiae

W. K. Huang, L. Kong, H. Peng, D. PENG, Institute of Plant Protection, Chinese Academy of Agricultural Sciences, Beijing, China

Root-knot nematode Meloidogyne graminicola has been a serious threat to rice production in Southeast Asia. In recently years, it has been a virtual way to develop organic agriculture and ensure the safety of grain production by using biological agents to control nematode diseases in crops. Aspergillus welwitschiae AW2017 is a newly biocontrol fungus against plant parasitic nematodes, which has been verified to be effectively in decreasing root-knot nematode number in rice. The bioagent AW2017 displayed good nematicidal potential via its ovicidal and larvicidal action. The best larvicidal activity and ovicidal activity was observed at a concentration of $2 \times 10^{6}$ conidia $^{-1}{ }^{-1}$, which caused an $86.2 \%$ mortality rate of juveniles at $48 \mathrm{~h}$ postinoculation and an approximately $47.3 \%$ reduction in egg hatching after $8 \mathrm{~d}$ compared to the control. Under greenhouse conditions, the application of $A$. welwitschiae significantly reduced the root galls and nematodes in rice roots compared to the control. Juveniles and root galls in rice roots at $14 \mathrm{dpi}$ were reduced by $24.5 \%$ and $40.5 \%$, respectively. In addition, the attraction of M. graminicola to rice roots was significantly decreased in the AW2017 treatment. The development of nematodes in the AW2017-treated plants was slightly delayed compared with that in the PDB-treated control plants. The results indicate that $A$. welwitschiae is a potential biological control agent against $M$. graminicola in rice.

Characterising powdery mildew resistance in oats

A. REILLY (1,2), S. Kildea (2), J. Finnan (2), A. Feechan (3), (1) UCD; School of Agriculture and Food Science, UCD Earth institute, Ireland; (2) Teagasc Crops Environment and Land Use Programme, Teagasc, Ireland; (3) UCD, School of Agriculture and Food Science, UCD Earth institute, Dublin, Ireland

Blumeria graminis f. sp. avenae, the causal agent of powdery mildew disease, is one of the most important diseases of oat production. Identifying resistant oat lines could be used to improve levels of resistance to powdery mildew. Eleven commercial oat cultivars were scored in the field for infection at different growth stages. The results suggest that most commercial oat cultivars studied are susceptible to powdery mildew, while only four cultivars have near-complete resistance to the fungus in the field. However, microscopic assessment of attempted powdery mildew infection showed a reduction in penetration for only two of these cultivars. Twenty-two Irish heritage lines have also been screened for powdery mildew susceptibility under glasshouse conditions, as well as microscopic assessments. From these screens, one line with seedling resistance was 
identified, in which auto-fluorescent compounds were found at sites of haustorial development on this line. Three lines with adult resistance were also identified, which are currently undergoing microscopic analysis.

Evaluating the potential of beneficial Gaeumannomyces species for the control of take-all disease in wheat T. J. CHANCELLOR, Rothamsted Research, Harpenden, U.K.

Take-all disease, caused by the soil-borne ascomycete fungus Gaeumannomyces tritici, is the most economically important root disease of wheat in the U.K. and worldwide. Yield losses of up to $60 \%$ have been reported in the U.K. but are typically in the range of 5-20\%. The fungus invades the roots and destroys the vascular tissue, hindering the plants ability to uptake water and nutrients from the soil. Closely related Gaeumannomyces species occur naturally in arable and grassland soils and have considerable potential to suppress take-all disease. Studying these taxonomically closely related species also helps us to understand the responses of wheat to nonmycorrhizal endophytic, weakly-pathogenic and strongly pathogenic root invading fungi from the same genus. The main aims of this project are threefold: 1 . To further characterise the ability of elite wheat genotypes to build-up and sustain beneficial Gaeumannomyces populations under controlled environment and field conditions. 2. To characterise in detail the infection biology and molecular aspects occurring during root colonisation using beneficial Gaeumannomyces species and the take-all fungus. 3. Following characterisation of this infection mechanism, to develop a virus induced gene silencing system (VIGS) for wheat root systems, providing a much-needed technique for studying gene function and potentially permitting highthroughput functional genomics in wheat root systems.

Exploring virulence factors of Pseudomonas cannabina pv. alisalensis

N. SAKATA (1), H. Saito (1), T. Ishiga (1), Y. Ichinose (2), Y. Ishiga (1), (1) University of Tsukuba, Tsukuba, Japan; (2) Okayama University, Okayama, Japan

Bacterial blight of Brassicaceae caused by Pseudomonas cannabina pv. alisalensis (Pcal) in one of the most economically important bacterial diseases in Japan, especially in Nagano prefecture. Pcal can infect not only in wide range of Brassicaceae but also in green manure crops, such as oats. To identify genes involved in pathogenicity of $P$ cal, we carried out a screen for $T n 5$ mutants of $P c a l$ KB 211 with reduced virulence on cabbage after dip-inoculation and found 60 mutants showing reduced virulence. Furthermore, dip-inoculation with these mutants on oat plants showed not all mutants exhibited reduced virulence, indicating that there are common and specific virulence factors to success for infecting on monocotyledonous and dicotyledonous plants. Successful identification of $\operatorname{Tn} 5$ insertion sites in all mutants revealed novel virulence-related genes in Pcal. We determined several genes involved in bacterial infection process, including type IV pili, type III secretion system, multidrug resistance efflux pump, extracellular polysaccharides. Moreover, we identified regulators including transcription factors and small signal molecules essential for expression of genes required for survival during environmental stress condition. In summary, our study indicates an unravel Pcal adaptation to both monocot and dicot hosts and a mechanism of Pcal pathogenicity.

Acibenzolar-S-methyl activates stomatal-based defense against Pseudomonas cannabina pv. alisalensis in cabbage T. ISHIGA (1), Y. Iida (1), N. Sakata (1), T. Ugajin (2), T. Hirata (2), S. Taniguchi (2), K. Hayashi (2), Y. Ishiga (1), (1) University of Tsukuba, Tsukuba, Japan; (2) Syngenta Japan, Ushiku, Japan

Pseudomonas cannabina pv. alisalensis (Pcal), which causes bacterial blight disease of Brassicaceae, is an economically important pathogen worldwide. Copper fungicide and antibioticsare major strategies to manage the disease caused by Pcal, however a Pcal strain resistant against these chemicals has already been found, and severe outbreaks of bacterial blight disease have been reported on cabbage in Japan. Therefore, there is an urgent need to develop new Pcal management strategies. Plant defense activators could be useful not only to protect plants against invading pathogens, but also to reduce copper fungicides and antibiotics utilized against $P c a l$. However, the mechanisms by which plant defense activators contribute to controlling diseases remains unclear. In this work, we focused on cabbage and acibenzolar-S-methyl (ASM), a well-known plant defense activator. Expression profiles revealed that ASM induced systemic acquired resistance (SAR) marker gene transcripts including PRI, $P R 2$, and $P R 5$ in cabbage plants. We also demonstrated that 2 hours pre-treatment with ASM directly to soil via the drench method clearly showed reduced bacterial blight disease symptoms as well as reduced Pcal bacterial populations in cabbage. Furthermore, ASM application was able to prime cabbage for $P$ cal resistance by activating stomatal-based defense. Our findings highlight that ASM protects plants from bacterial pathogens by activating stomatal-based defense.

Molecular suppressive mechanism of a biocontrol agent Rhizobium vitis VAR03-1 against grapevine crown gall disease Y. NOUTOSHI (1), T. Ishii (2), M. Watanabe (3), K. Saito (3), H. Matsui (2), M. Yamamoto (3), Y. Ichinose (3), K. Toyoda (3), A. Kawaguchi (4), (1) Okayama Univ, Okayama, Japan; (2) Okayama University, Okayama, Japan; (3) Okayama University, Japan; (4) Western Region Agricultural Research Center, NARO, Japan

Rhizobium vitis VAR03-1 is a non-pathogenic bacterium with biologically control activity against grape vine crown gall disease caused by a pathogenic Rhizobium vitis Ti. We reported that both of growth and induction of virulence genes of Ti were suppressed when it was cultivated in the culture fluid of VAR03-1, suggesting that substance responsible for the suppressive activity should be secreted from VAR03-1. Then we successfully identified four mutants of VAR03-1 which lost halo formation to Ti strain using transposon-mediated random mutagenesis. The transposons of three of them were inserted into a phage-related gene and the other one was in its neighboring gene. Comparative genome analysis with VAR03-1, Ti, VAR06-30, a non-pathogenic and non-antagonistic strain, and S4, a virulent strain in Europe, revealed that the causal substance for VAR03-1 antagonism would be a tailocin, a prophage without head part. This novel tailocin seems to be generated by a union of two different types of prophage genomes by genomic rearrangement. Gene knockout analysis revealed that both genomic regions of this tailocin derived from two different origins is required for the growth-suppressive activity to Ti. The transcriptions of this tailocin genes are upregulated by UV-induced SOS response. Accordingly, the growth-inhibitory activity in the culture fluid of VAR03-1 to Ti was also increased after UV irradiation, suggesting that expressions of this tailocin genes are regulated as an operon. 
Genomics of the rose-infecting soil-borne pathogen Rosellinia necatrix

E. A. CHAVARRO CARRERO (1), R. Garcia Velasco (2), M. F. Seidl (1), B. P. H. J. Thomma (1), (1) Wageningen University, Wageningen, Netherlands; (2) Centro Universitario UAEM Tenancingo, Universidad Autonoma del Estado de Mexico, Tenancingo, EM, Mexico

Little is known about pathogenesis of the soil-borne pathogen Rosellinia necatrix that causes disease in many woody plant species, including rose, avocado, apple, pear, grape, orange, olive and coffee. Typical symptoms vary from wilting to host plant death. The aim of the present study is to identify genes that mediate pathogenicity on rose. To this end, strains were obtained from rose in Mexico (6 strains) and avocado in Spain ( 3 strains). The pathogenicity of these strains was tested on rose, revealing one strain that was originally collected from avocado plants to be nonpathogenic while the other strains showed differential degrees of aggressiveness. All strains were sequenced using Nanopore technology, and genome assemblies of $\sim 49 \mathrm{Mb}$ were obtained. Next, comparative genomics is performed, in which the non-pathogenic strain is compared with the other strains. First, we will query for genes that are shared among the pathogenic strains and that are absent in the non-pathogenic one for functional analysis. Next, comparative genomics between more and less aggressive strains will be performed to identify additional virulence factors. With this approach, we hope to identify $R$. necatrix genes that are crucial for the infection of rose plants.

Comparative secretome analysis identifies conserved motifs and protein families in the secretory proteins of plant fungal pathogens R. JASWAL (1), H. Dubey (2), K. Kiran (2), H. Rawal (2), S. C. Bhardwaj (3), P. Prasad (3), N. Gupta (4), T. R. Sharma (1,2), (1) National Agri food Biotechnology Institute (NABI), Mohali, India; (2) ICAR-National Research Centre on Plant Biotechnology, Pusa Campus, Delhi, India; (3) ICAR-Indian Institute of Wheat and Barley Research, Regional Station, Flowerdale, Shimla, India; (4) Department of Microbiology, Panjab University,, Chandigarh, India

The public availability of genomic resources for various plant pathogens has led to identification of several secretory proteins and few conserved motifs in their secretome. The identification of two conserved motifs; RxLR-dEER in oomycetes and $\mathrm{Y} / \mathrm{F} / \mathrm{WxC}$ in powdery mildews are the prime examples that provides motivation for identifying conserved motifs in other fungal pathogens. In the present study, we have identified and compared the secretomes of 23 fungal pathogens with diverse lifestyle. In addition to standard secretomics pipeline and parameters used in previous studies, we used other parameters like small repeats, effectorP \& presence of transit peptide for effectors prediction. Our analysis showed in studied pathogens highest effector proteins were present in Puccinia striiformis. The de novo motif analysis using predicted effectors revealed conserved motif sequences in multiple effector proteins of P.strifromis and Claviceps pupurea that has not reported in any previous studies. The phylogeny of conserved region of these proteins showed that these proteins might belong to two different conserved families. Multiple candidates in P.striformis also showed high expression in transcriptomic analysis suggesting their role in fungal virulence and being characterized to find role in fugal pathogenesis. This study provides first report showing the presence of conserved motifs and putative secretory proteins families in P.strtiiformis and in C.pupurea plant fungal pathogens.

Regulation of TOR pathway in context of pathogenic and symbiotic bacterial interactions with nematodes

V. Phani, C. G. Bhat, A. Raj, A. Taiyab, V. S. Somvanshi, U. RAO, ICAR- Indian Agricultural Research Institute, New Delhi, India

The target-of-rapamycin (TOR) kinase engaged in cell growth, development and metabolism in multicellular organisms belongs to phosphatidylinositol-kinase family. The activation of TOR/raptor complex $(\mathrm{Trc})$ requires energy resources, nutrients and oxygen abundance for mRNA translation, leading to protein synthesis. The mechanistic regulation of Trc has been studied in mammals, yeast, plants and Caenorhabditis elegans. However, no knowledge exists on the involvement of Trc in host-bacterium interactions. Here, we investigated the role of Trc in parasitic and symbiotic bacterial interactions using nematode models. The C. elegans Trc homologues [DAF-15 (raptor), LET-363] were amplified and cloned from plant-parasitic nematode, Meloidogyne incognita (root-knot nematode) and entomopathogenic nematode, Heterorhabditis bacteriophora. Both the genes were expressed in all life-stages of $M$. incognita. Encumbrance of Pasteuria endospores (hyperparasite of $M$. incognita) caused downregulation of daf-15 and let-363 expression in M. incognita, indicating suppression of Trc-related pathway(s). RNAi-silencing of $M$. incognita mucin-like gene (Mi-muc-1) also repressed daf-15 expression, possibly representing the crosstalk between TOR and mucin-dependent pathways. Work is in progress to explore involvement of Trc expression in Heterorhabditis-Photorhabdus system. This study will elucidate role of TOR/raptor-complex in context of interaction of animals with pathogenic and symbiotic bacteria.

Comparative studies of diverse Actinobacteria identifies new biofungicide candidates

L. F. THATCHER (1), C. A. O'Sullivan (2), K. Belt (1), D. M. Gardiner (2), J. P. Anderson (1), M. M. Roper (1), (1) CSIRO Agriculture and Food, Floreat, WA, Australia; (2) CSIRO Agriculture and Food, St Lucia, QLD, Australia

Necrotrophic fungal pathogens cause many destructive diseases of global agricultural crops. Management options can be limited due to the lack or complexity of host resistance, or ineffective and/or harmful chemical controls. The loss or deregistering of chemical controls owing to 1) toxicity, 2) increasing regulation or 3) increased incidence of resistance in pathogen populations due to over usage necessitates the need for alternate or auxiliary controls. To address these concerns, we curated a collection of beneficial soil and plant endophyte microbes of the Actinobacteria phylum isolated from the biodiversity hotspot of south-west Australia. Actinobacteria are known for their ability to produce bioactive secondary metabolites such as antimicrobials however genome sequencing indicates many compounds remain 'undetected' or nonexpressed under standard laboratory conditions. This hidden chemical potential can be tapped for the discovery of new biofungicides. Combining omics approaches with traditional microbial fermentation processes, and in vitro and in planta screening for suppression of fungal pathogens, we screened a subset of the Actinobacteria collection for inhibitory activity against a fungal pathogen diversity panel and are interrogating genome and transcriptome data to identify potent antifungal isolates and candidate biosynthetic pathways underpinning their activity. Knowledge transfer to product development is underway. 
Proteomics of different immune reactions in Potato leaves

M. A. ZAHID (1), S. Resjo (1), D. D. Burra (1), M. Lenman (1), F. Levander (2), E. Andreasson (1), (1) Swedish University of Agricultural Science, Alnarp, Sweden; (2) Department of Immunotechnology, Lund University, Lund, Sweden

Plants have a variety of ways to defend against pathogens. A commonly used model of the plant immune system divides it into a general response triggered by pathogen associated molecular patterns (PAMPs), and a specific response triggered by effectors. The first type of response is known as PAMP triggered immunity (PTI) and the second as effector-triggered immunity (ETI). We have performed comparative proteomic analysis of a PTI and two different ETI models (relating to Phytophthora infestans) in potato. Several proteins showed higher abundance in all immune reactions, such as a protein annotated as sterol carrier protein 2 that could be interesting since Phytophthora species are sterol auxotrophs. We also identified some PTI-specific changes of protein abundance, such as for a DUF26 domain-containing protein and a glyoxysomal fatty acid beta-oxidation multifunctional protein. The proteins specifically upregulated in ETI included several catalases. Few proteins were regulated in only one of the ETI interactions. For example, histones were only downregulated in the ETI-Avr2 interaction, and a putative multiprotein bridging factor was only upregulated in the ETI-IpiO interaction. We also analyzed protein methylation. One example of a methylated protein that increased in the ETI interactions was a serine hydroxymethyltransferase. These methods and results might be used in new pre-breeding and prediction of sustainable combinations of resistance genes.

Genome-wide translatome studies reveal cell-layer specific responses of the Arabidopsis root towards pathogenic and mutualistic microorganisms

W. DRÖGE-LASER, C. Fröschel, Julius-von-Sachs-Institute, Würzburg, Germany

As the plant root is built of concentric cell-layers, it is anticipated that these layers respond towards microbial infection by employing specific, genetically defined programs. Applying the Arabidopsis model, we established a TRAPseq method (Translating Ribosome Affinity Purification followed by RNA sequencing) to study root responses towards different microorganisms (MOs) on a cell-layer resolution. Due to cell-type specific expression of FLAG-tagged ribosomal proteins, ribosome/m-RNA complexes can be co-immunoprecipitated to obtain cell-layer specific expression profiles (translatomes) by subsequent RNA sequencing. To study root responses, we compared pathogenic and mutualistic rootinfecting MOs, which differ considerably in their lifestyles: the oomycete Phytophthora parasitica switches from an early biotrophic to a necrotrophic phase, Verticillium longisporum performs as a vascular fungal pathogen and the mutualist Piriformospora indica colonizes the cortex as an endophyte. In support of our hypothesis, we identified root cell-layer specific expression patterns, which differ depending on the MO's lifestyle. Here, we present genome-wide analyses, as well as selected examples covering biosynthesis of antimicrobial compounds, barriers in the endodermis or cell-cycle responses in the central cylinder. Mining the complex datasets will enable to generate experimentally testable hypotheses which will broaden our understanding of the plant MO interactions in the rhizosphere.

Oryza sativa LysM receptor kinases regulate intracellular rice root colonisation by Magnaporthe oryzae A, N. IBE (1), N. J. Talbot (2), U. Paszkowski (3), (1) University of Cambridge, Cambridge, U.K.; (2) The Sainsbury Laboratory, University of East Anglia, Norwich Research Park, Norwich, U.K.; (3) University of Cambridge, U.K.

Very few examples exist for shared signaling components between mutualistic and parasitic root interactions. The Chitin Elicitor Receptor Kinase 1 (CERK1) is presently the only known gene required for early signaling in arbuscular mycorrhizal (AM) symbiosis and in plant innate immunity in rice, where it interacts with the Chitin Elicitor Binding Protein (CEBiP) to induce plant defense responses. The closest homolog of OsCERK1 in Lotus japonicus, Nod Factor Receptor 1 (NFR1), interacts with LjNFR5 to activate rhizobia symbiotic signaling pathways and mycorrhizal colonisation. However, unlike OsCERK1, which is required for symbiosis, OsCEBiP and OsNFR5 are not required. Here, we report a novel role of three LysM receptor-like kinases (OsCERK1, OsCEBiP and OsNFR5) in regulating intracellular accommodation of the pathogen Magnaporthe oryzae in rice roots. Molecular quantification of M. oryzae biomass showed an overall reduction in fungal colonisation in all three mutants. A particularly striking phenotype was seen in inoculated cerkl mutant rice root cells, where a large proportion of proliferating invasive M. oryzae hyphae appeared either dead or restricted. These results suggest that LysM receptor-like kinases may be involved in signaling pathways that promote intracellular accommodation of $M$. oryzae in rice roots, with OsCERK1 defining a signaling function in AM symbiosis that has been adopted by M. oryzae to trick, invade and gain control of the plant host machinery.

Intra-species variations in root fungus Colletotrichum tofieldiae cause transitions between beneficial and parasitic lifestyles K. HIRUMA (1,2), A. Uchiyama (2,3), R. Sugita (4), T. Sato (5), K. Tanoi (2,4), Y. Saijo (1), (1) Nara Institute of Science and Technology, Japan; (2) JST PRESTO, Japan; (3) NARA Institute of Science and Technology, Ikoma, Japan; (4) Isotope Facility for Agricultural Education and Research, The University of Tokyo, Tokyo, Japan; (5) National Agriculture and Food Research Organization, Tsukuba, Japan

Association with different host plants in various habitats is predicted to cause substantial genetic variations within the same fungal species. However, it is not clear whether and how intra-species variations affect beneficial associations between fungal endophytes and the host plants. The endophyte Colletotrichum tofieldiae $(\mathrm{Ct}$ ) colonizes roots of brassica plants including A. thaliana and promotes plant growth in low phosphate via transferring phosphorus to plants. Here we report intra-species variations in $C t$ for the interactions with $A$. thaliana. In contrast to the relative species, majority of the tested $C t$ strains promote plant growth in low-Pi, suggesting that beneficial traits are well conserved in $C t$ lineage. Notably, root colonization by one of the $C t$ strains from Japan causes severe plant growth inhibition with reduced Pi content in shoots. Thus, intra-species variations could even cause transitions to parasitic lifestyles that disturb plant growth. To uncover the genetic basis of the transitions between beneficial and parasitic lifestyles, we have conducted comparative genomics among $C t$ including 3 new $C t$ strains from Japan as well as time-resolved transcriptome analysis during root colonization by $C t$. The data has revealed candidate plant and fungal genes that modulate various $C t$ lifestyles. We will also talk about the mechanisms by which these plant and fungal genes regulate interactions in the context of interplay between plant nutrient and hormone responses. 
A satellite RNA outcompetes replicase-encoding RNAs of the helper virus for replication-associated host factors via a three waybranched RNA structure

L. He, Y. Gao, Z. Gu, Q. Liao, Z. DU, Zhejiang Sci-Tech University, Hangzhou, China

As a class of parasitic, non-coding RNAs, satellite RNAs (satRNAs) have to compete with their help virus for limited amounts of viral and/or host resources for efficient replication, by which they usually reduce viral accumulation and symptom expression. Here we report a cucumber mosaic virus-associated satRNA (sat-T1) that ameliorated CMV-induced symptoms, accompanied with a significant reduction in the accumulation of viral genomic RNAs 1 and 2 that encode viral replicase. In trans replication assays indicate that the reduced accumulation is the outcome of replication competition. Specific inhibition to the replication of RNAs 1 and 2, but not RNA3 suggests that sat-T1 competes for limited amounts of host factor(s) required for the replication of RNAs 1 and 2 . The structural basis of sat-T1 responsible for the competition was determined to be a three way-branched secondary structure that contains two biologically important hairpins. One is indispensable for the competition, and the other engages in formation of a tertiary pseudoknot structure that is essential for sat-T1 survival. This secondary structure containing the pseudoknot is the first RNA element with a biological phenotype experimentally identified in CMV RNAs. We propose a model that CMV satRNAs reduce viral accumulation and symptom expression by outcompeting replicase-encoding RNAs of the helper virus for replication-associated host factor(s) via the newly identified structure.

Identification and characterisation of SMT, a biosynthetic gene cluster from Epichloë spp. involved in stroma development D. Berry (1), K. Grage (1), A. Ram (1), P. Nagabhyru (2), C. Schardl (2), C. A. Young (3), P. Dijkwel (1), B. D. SCOTT (4), (1) Massey University, New Zealand; (2) University of Kentucky, U.S.A.; (3) Noble Research Institute, U.S.A.; (4) Massey Univ, Palmerstown North, Manawatu, New Zealand

Fungal species from the genus Epichloëare symbionts of grasses that systemically infect intercellular spaces of aerial tissues. Asexual Epichloë spp. exclusively transmit vertically through the host seed but sexual Epichloë spp. can also form stromata, which are pre-sexual structures that envelop emerging host inflorescences. We have identified afive-gene biosynthetic cluster surrounding a single module NRPS-like gene that is involved in stroma formation. This SMT cluster is universally conserved in sexual Epichloë spp., whereas asexual species often contain SMTpseudogenes or whole cluster deletions. RNAseq analysis of stromata and vegetative tissues demonstrated that $S M T$ genes are dramatically and specifically upregulated in stromata across three diverse grass/endophyte associations. We reconstituted this pathway inPenicillium paxilli through heterologous expression of SMT genes. This enabled characterisation of the reaction order, intermediates, and secondary metabolite product of the SMT pathway. The products identified by heterologous expression are identical to those found in extracts of stromata harvested from the field. We have also deleted each of the genes within this cluster and determined the host interaction phenotype. We propose a mechanism where this SMT pathway controls initiation of stroma formation

The positively selected effector MiL648 of Meloidogyne incognita contributes to nematode virulence through its interactions with the 12oxophytodienoate reductase OPR2 in tomato

A. VERHOEVEN (1), A. Finkers-Tomczak (1), P. Prins (1), D. Valkenburg-van Raaij (1), K. Varossieau (1), H. Overmars (1), E. Slootweg (1), A. Goverse (2), G. Smant (2), (1) Laboratory of Nematology, Wageningen University and Research, Netherlands; (2) Laboratory of Nematology, Wageningen University and Research, Wageningen, Netherlands

Insight in the molecular basis of virulence can help to identify new and more durable sources of resistance to the root-knot nematode Meloidogyne incognita in tomato. In nematode genomes, evidence of positive, diversifying selection can point to loci involved in a molecular arms race with other organisms. We used evidence of positive selection as a first criterion to identify novel virulence genes in the genome of $M$. incognita, one of which encodes the effector MiL648. Bioassays with M. incognita on tomato plants overexpressing an RNAi construct matching the MiL648 sequence showed that this gene is required for nematode virulence. Likewise, tomato plants overexpressing MiL648 were more susceptible to $M$. incognita, demonstrating that it functions as a bona fide effector. We identified six likely host targets of MiL648 in a yeast twohybrid screen of nematode-infected roots of tomato, including the 12-oxophytodienoate reductase SIOPR2. We confirmed the interaction between SIOPR2 and MiL648 in planta by transient expression and co-immunoprecipitation in leaves of Nicotiana benthamiana. We therefore concluded that the effector MiL648 enhances the virulence of M. incognita through its interactions with S1OPR2.

Interaction of nematode-resistant plant Solanum torvum and virulent/avirulent root-knot nematodes

K. SATO (1), Y. Kadota (1), P. Gan (2), T. Uehara (3), T. Bino (4), K. Yamaguchi (4), Y. Ichihashi (5,6), H. Iwahori (7), N. Maki (1), S. Shigenobu (4), T. Suzuki (8), K. Shirasu (2,9), (1) RIKEN Center for Sustainable Resource Science, Plant Immunity Research Group, Yokohama, Japan; (2) RIKEN Center for Sustainable Resource Science, Yokohama, Japan; (3) National Agriculture and Food Research Organization, Tsukuba, Japan; (4) National Institute for Basic Biology, Okazaki, Japan; (5) RIKEN BioResource Research Center, Tsukuba, Japan; (6) JST, PRESTO, Kawaguchi, Japan; (7) Ryukoku University, Otsu, Japan; (8) Chubu Univ., Japan; (9) The University of Tokyo, Tokyo, Japan

Plant parasitic nematodes, such as root-knot nematodes (RKNs), are among the most devastating pests in agriculture. Solanum torvum is horticulturally used as a rootstock for eggplants, which confers resistance against RKNs such as Meloidogyne incognita and Meloidogyne arenaria Okinawa isolate (M.a. Okinawa). However, M.a. Japanese Main island isolate (M.a. Main island) can infect S. torvum. To understand the molecular basis of plant immunity and virulence of RKNs, we performed dual RNA-sequencing of S. torvum root tissues invaded by M.a. Okinawa and M.a. Main island, as well as genomic sequencing of these two M.a. isolates using PacBio RS II. We revealed that $S$. torvum infected with M.a. Okinawa, but not with M.a. Main island, dramatically upregulated the expression of genes involved in defense responses such as lignin biosynthesis. We confirmed that M.a. Okinawa infection induced lignin accumulation at the infection site in S. torvum. Additionally, we have screened virulent effector candidate genes based on genomic and transcriptomic information. The commonalties and differences of effectors in these two M.a. isolates will be discussed. 
The evolutionary origins of effectors in plant-parasitic nematodes.

C. Lilley (1), L. M. Jones (1), A. Maqbool (2), M. J. Banfield (3), P. R. J. Birch (4), J. T. Jones (5), P. E. Urwin (6), S. EVES-VAN DEN AKKER (7), (1) University of Leeds, Leeds, U.K.; (2) John Innes Centre, Norwich, U.K.; (3) John Innes Centre, Norwich Research Park, Norwich, U.K.; (4) Cell and Molecular Sciences, James Hutton Institute, Dundee, U.K.; (5) The James Hutton Instittue/University of St. Andrews, Invergowie Dundee, U.K.; (6) University of Leeds, U.K.; (7) University of Cambridge, U.K.

Many effectors are present in gene families, yet, their evolutionary origins are unclear. In exception to this are the glutathione synthetase (GS)like effectors of plant-parasitic nematodes. A clear, two-step evolutionary process gave rise to the present three clade family where it appears: Clade one are common to nematodes; Clade two are common to endoparasites; while the effector genes of Clade three are specific to a lineage of plant-parasitic biotrophs. How endogenous housekeeping genes are co-opted as effectors, as demonstrated by effector gene birth in the GS family, involves the gain of the "effector promoter", which we identify, name and validate. Once recruited as an effector, the Clade 3 progenitor underwent massive replication, and the active site residues diverged from that of a canonical GS. GS-like effectors no longer synthesise the same products as endogenous GS in vitro. To explore the structural basis for effector diversification, we solved the high-resolution structures of two GS-like effectors, representing the first crystal structures of any kind for a plant-parasitic nematode. We hypothesise that this re-deployment and re-purposing of an endogenous housekeeping gene constitutes a "weaponization programme" that is common to the genesis of other plantparasitic nematode effectors.

A Nuclear Localized Aphid Effector Contributes to Aphid Virulence S. LIU (1), C. J. G. Lenoir (1), E. Huitema (2), J. I. B. Bos (1), (1) University of Dundee, Dundee, U.K.; (2) Division of Plant Sciences, The University of Dundee, Dundee, U.K.

Aphids are phloem-feeding insects that, similar to plant pathogens, deliver effectors inside their host to manipulate host cellular processes to enable infestation. Effector discovery and characterization has led to significant conceptual advances and provided evidence for the effector paradigm in plant-aphid interactions. To understand how, at the fundamental level, aphids interact with host plants to promote susceptibility, it is essential to explore the activities of their effectors. Transcriptomic and saliva proteomics approaches facilitated the prediction of effector repertoires from several aphid species, including Myzus persicae (green peach aphid), which is a major agricultural pest. Among the effectors we have characterized, two nuclear-localized effectors have been identified, one of which was named MpSec75. We found that ectopic expression of MpSec75 in Arabidopsis thaliana enhances susceptibility to M. persicae. In addition, silencing of MpSec75 in aphids reduces aphid performance, indicating this effector is essential for virulence. Protein-protein interaction studies identified an E3 SUMO ligase SIZ1 as a potential target of MpSec75. Aphid performance assays on siz1-2 Arabidopsis line support a role of SIZ1 in negatively regulating plant immunity to aphids. Our preliminary results suggest that M. persicae deploys a nuclear effector to target an important immune regulator in plants.

The role of epigenetics in the interaction between rice and parasitic nematodes T. KYNDT, M. R. Atighi, B. Verstraeten, A. Meijer, T. De Meyer, UGent, Belgium

Rice is the most important staple food grain worldwide. The root-knot nematode Meloidogyne graminicola is one of its most destructive belowground pathogens, inducing the formation of root galls. Transcriptome analyses on galls have revealed major reprogramming and pointed to a role for epigenetic regulation. We are investigating the major epigenetic pathways in infected rice roots. First, global DNA methylation assays, confirmed and extended by whole genome bisulfite sequencing, revealed major DNA hypomethylation of cytosine in the CHH context in galls. Mutants in the RNA-directed DNA methylation (RdDM) pathway, and azacitidine treatment revealed that loss of DNA methylation leads to reduced susceptibility. Long non-coding RNAs (lncRNAs) and small RNAs (smRNAs) are major players in RdDM. Using total RNAsequencing, covering smRNAs as well as poly-adenylated and non-adenylated lncRNAs, we revealed more than 1000 differentially expressed non-coding rice transcripts that respond to nematode infection. Next to that, ChIP-seq showed major hypermethylation of histones in nematodeinduced galls, consistent with strong transcriptional activation of histone lysine methyltransferases. Finally, transgenerational experiments revealed that offspring of nematode-infected rice plants are significantly less susceptible. These results demonstrate that epigenetic changes are a major regulator of rice defence against nematodes, and that they may even be inheritable.

Long-lasting jasmonic acid induced resistance against a generalist herbivore comes at the cost of enhanced susceptibility to pathogens S. W. WILKINSON (1), R. S. Wilson (1), M. A. Henderson (1), J. Stassen (1), A. Lopez Sanchez (2), M. H. Magerøy (3), J. Ton (1), (1) The University of Sheffield, Sheffield, U.K.; (2) National Centre of Biotechnology, Madrid, Spain; (3) Norwegian Institute of Bioeconomy Research, Norway

The phenomenon of induced resistance (IR), whereby exposure of plants to specific stimuli makes them more resistant to subsequent attack, could be more widely used in crop pest management if it was long-lasting. Previous studies have shown that long-lasting resistance can be induced by a variety of stimuli including jasmonic acid (JA). We aimed to better understand the mechanisms underpinning long-lasting IR by treating two-week old Arabidopsis thaliana seedlings with JA and then challenging these plants three weeks later. Interestingly, while we found that JA seedling treatment induced resistance against a generalist herbivore, it induced susceptibility against both necrotrophic and hemibiotrophic pathogens. Transcriptome analysis demonstrated that JA treatment primed herbivore defences for a faster and stronger upregulation post challenge. Whereas defences effective against pathogens were repressed long-term. JA induced susceptibility was compromised in DNA (de)methylation mutants. Thus, the repression of pathogen defences and subsequent induced susceptibility appears linked to JA induced epigenetic changes. To better understand the role of DNA methylation in long-lasting IR, we are analysing the long-lasting JA-induced changes to the Arabidopsis methylome. This study highlights the importance of assessing how resistance inducing stimuli effect plants long-lasting defence responses against a diversity of biotic stresses, before using these stimuli in crop pest management. 
Ascorbate oxidation primes the rice plants for enhanced defence against root-knot nematode Meloidogyne graminicola through jasmonate/ethylene activation

R. R. SINGH (1), B. Verstraeten (2), S. Siddique (3), A. M. Tegene (4), R. Tenhaken (5), M. Frei (6), A. Haeck (4), K. Demeestere (7), S. Pokhare (6), G. Gheysen (8), T. Kyndt (2), (1) Gent University, Gent, Belgium; (2) UGent, Belgium; (3) INRES Molecular Phytomedicine, Bonn, Germany; (4) Gent University, Belgium; (5) University of Salzburg, Salzburg, Austria; (6) University of Bonn, Germany; (7) Ghent University, Ghent, Belgium; (8) Ghent Univ, Ghent, Belgium

Ascorbic acid (AA) is known to play a vital role in plant growth and detoxification of reactive oxygen species, however little is known about the significance of AA oxidation in plant defence against pathogens. The role of ascorbate oxidation in rice defence against root-knot nematodes, Meloidogyne graminicola, was tested with application of AA, ascorbate oxidase (AO), dehydroascorbic acid (DHA), biosynthesis inhibitors and use of mutants. Transcriptome analysis was done on AO treated plants, and hormone measurements were executed to confirm the results. Biochemical analyses were used to study oxidative stress markers, including accumulation of hydrogen peroxide $\left(\mathrm{H}_{2} \mathrm{O}_{2}\right)$, malondialdehyde (MDA) and AA/DHA. AO and DHA treated plants are significantly less susceptible to M. graminicola, while AA mutants are significantly more susceptible. Transcriptome data, corroborated by hormone measurements, show that ethylene (ET) and jasmonic acid (JA), are activated in rice roots upon ascorbate oxidation, while Methyl-JA, ethephon or AO can complement the susceptibility phenotype of the vitamin C (vtc)mutant. Additionally, oxidative stress markers are accumulating in nematode-induced root galls. Our data further demonstrate a novel pathway showing that induced defence by ascorbate oxidation is dependent on a functional JA and ET pathway and leads to primed accumulation of $\mathrm{H}_{2} \mathrm{O}_{2}$.

The role of ncRNAs in the interaction between root-knot nematode and rice B. VERSTRAETEN, M. R. Atighi, T. De Meyer, T. Kyndt, UGent, Belgium

In this study, the involvement of non-coding RNAs (ncRNAs) in the infection of rice (Oryza sativa) by the root-knot nematode (Meloidogyne graminicola) is being investigated. Meloidogyne graminicola, is a major economic threat to worldwide rice production. By performing small and total RNA sequencing on healthy roots and galls at 3 days postinfection, we identified 15 differentially expressed miRNAs and thousands of lncRNAs likely to play a role in the infection process. Putative targets for the miRNAs were predicted and cross referenced with the total RNA dataset. The expression profile of differentially expressed targets was validated by RT-qPCR. To ensure the quality of the validation, several candidate reference miRNAs were tested for expression stability and the two stablest transcripts were chosen. Among the predicted targets is a gene encoding ascorbate oxidase, which could play a role in the plant defense response. Furthermore, two miRNAs are putatively involved in a miRNA-decoy-target system. Clustering analysis revealed that hundreds of differentially expressed lncRNAs are clustered together with differentially expressed protein coding genes, suggesting potential in cis expression regulation. Furthermore, overexpression lines of four differentially expressed miRNAs are currently tested in infection assays. This research will contribute to a deeper understanding of ncRNA involvement in rice immunity which, in turn, may contribute to new nematode control strategies.

DNA hypomethylation confers enhanced immunity against parasitic nematodes in rice M. R. ATIGHI, B. Verstraeten, T. De Meyer, T. Kyndt, UGent, Belgium

Epigenetics plays an important role in the plant response to adverse environmental conditions. A role for DNA hypomethylation has recently been suggested in the pathogenic interaction between bacteria and plants, yet it remains unclear whether this phenomenon reflects a conserved and general plant immunity response. We therefore investigated the role of DNA methylation in the interaction between rice and one of its most damaging pathogens, Meloidogyne graminicola $(\mathrm{Mg})$. Global DNA methylation analysis of nematode induced gall tissue at 3 days postinoculation demonstrated general hypomethylation in galls. Treatment of uninfected roots by a pathogen-associated molecular pattern (PAMP) revealed similar hypomethylation, suggesting causal impact on immunity. This was confirmed by a reduced plant susceptibility upon azacytidine treatment. Whole genome bisulfite sequencing of gall tissue 3 dpi revealed that hypomethylation was massively present in the $\mathrm{CHH}$ context, while absent for $\mathrm{CpG}$ or $\mathrm{CHG}$ nucleotide contexts. CHH hypomethylated regions were significantly enriched for gene promoter regions, leading to gene overexpression at $7 \mathrm{dpi}$, but not at $3 \mathrm{dpi}$. Finally, the relevance of CHH hypomethylation in plant defence was confirmed in mutants of the RNA-directed DNA methylation pathway (RdDM). We demonstrated that DNA hypomethylation confers enhanced defence in rice towards root-parasitic nematodes and is likely to be part of the basal PAMP-triggered immunity response in plants.

The potato cyst nematode effector RHA1B is a ubiquitin ligase and uses two distinct mechanisms to suppress plant immune signaling F. XIAO, University of Idaho, Moscow, ID, U.S.A.

Plant pathogens, such as bacteria, fungi, oomycetes and nematodes, rely on wide range of virulent effectors delivered into host cells to suppress plant immunity. Although phytobacterial effectors have been intensively investigated, little is known about the function of effectors of plantparasitic nematodes, such as Globodera pallida, a cyst nematode responsible for vast losses in the potato and tomato industries. Here, we demonstrate using in vivo and in vitro ubiquitination assays the potato cyst nematode (G. pallida) effector RHA1B is an E3 ubiquitin ligase that employs multiple host plant E2 ubiquitin conjugation enzymes to catalyze ubiquitination. RHA1B was able to suppress effector-triggered immunity (ETI), as manifested by suppression of hypersensitive response (HR) mediated by a broad range of nucleotide-binding leucine-rich repeat (NB-LRR) immune receptors, presumably via E3-dependent degradation of the NB-LRR receptors. RHA1B also blocked the flg22triggered expression of Acre 31 and WRKY22, marker genes of pathogen-associated molecular pattern (PAMP)-triggered immunity (PTI), but this did not require the E3 activity of RHA1B. Moreover, transgenic potato overexpressing the RHA1B transgene exhibited enhanced susceptibility to G. pallida. Thus, our data suggest RHA1B facilitates nematode parasitism not only by triggering degradation of NB-LRR immune receptors to block ETI signaling but also by suppressing PTI signaling via a yet unknown E3-independent mechanism.

Discovery of the first pectin methylesterase gene in a plant-parasitic nematode

C. S. L. VICENTE (1), L. G. Nemchinov (2), M. Mota (1), J. D. Eisenback (3), K. K. Kamo (2), P. Vieira (3), (1) ICAAM / University of Evora, Evora, Portugal; (2) USDA-ARS, Beltsville, MD, U.S.A.; (3) Virginia Tech, Blacksburg, VA, U.S.A. 
The root lesion nematode (RLN) Pratylenchus penetrans is considered one of the most damaging plant-parasitic nematode (PPN) species worldwide affecting important agronomic and horticultural crops (e.g. potato, corn). The successful invasion of roots by RLNs is related to their ability to overcome the barrier imposed by the plant cell wall. Like other PPNs, RLNs are equipped with a protrusible stylet that mechanically disrupts the cell wall and through which cell wall-degrading enzymes are secreted to facilitate penetration and migration of the nematode through host roots. Herein, we identified and characterized a pectin methylesterase gene for $P$. penetrans. Sequence analysis confirm the eukaryotic gene structure of Pp-pme. Expression of the Pp-pme gene was localized in the esophageal glands of $P$. penetrans as determined by in situ hybridization. The possible function and activity of the gene were assessed by transient expression of Pp-pme in plants of Nicotiana benthamiana plants via a Potato Virus X-based vector. PMEs have been so far described for plants, fungus, bacteria, and to a restrict number of insects. To our knowledge, this is the first report a PME within the phylum Nematoda.

Investigating early aphid-induced calcium signals and their role in determining aphid-host compatibility J. R. JOYCE, J. Canham, M. Giolai, A. J. Miller, D. Sanders, S. A. Hogenhout, John Innes Centre, Norwich, U.K.

As hemipteran pests and vectors of many significant plant viruses, aphids pose a substantial and increasing threat to plant health. Despite this, little remains known about the mechanisms by which plants can detect and defend themselves against aphid attack. Cytosolic calcium, a fundamental plant second messenger, has recently been implicated in the interactions between Arabidopsis thaliana and the generalist aphid, Myzus persicae. Elevations in cytosolic calcium concentrations occur as rapid and local signals within the epidermal and mesophyll tissues during probing by the aphid before it locates its stylet within the phloem for feeding. By using live in vivo imaging of early calcium signaling responses to aphid species of different host compatibilities, we are now gaining a unique insight into the key features of these signals and their functions within aphid-plant interactions. These findings will prove fundamental in understanding the colonization ability of different aphid species and the mechanisms underlying aphid-host compatibility.

Aphid elicitors induce plant immunity in a cysteine protease-dependent manner

J. CANHAM (1), J. Sklenar (2), M. Giolai (1), M. Gravino (1), R. Terauchi (3), H. Kato (4), S. T. Mugford (1), F. L. H. Menke (2), S. A. Hogenhout (1), (1) John Innes Centre, Norwich, U.K.; (2) The Sainsbury Laboratory, University of East Anglia, Norwich Research Park, Norwich, U.K.; (3) Graduate School of Agriculture, Kyoto University, Kyoto, Japan; (4) Nagoya Univ, Nagoya Aichi, Japan

PAMP-triggered immunity (PTI) is the first layer of plant defence response to pathogens and pests, and involves the recognition of a conserved molecule (PAMP) of the pathogen by a plant cell surface Pattern Recognition Receptor (PRR). In investigations of aphid-plant interactions, we previously demonstrated that the PRR co-receptor BAK1 is required for the plant defence response induction during aphid feeding and upon application of aphid extract. Here, we present an immunoactivity-led purification strategy to identify aphid PAMPs that trigger PTI in plants using an Arabidopsis luciferase reporter. Interestingly, we show that low molecular weight components in aphid-derived fractions can rapidly induce defence pathways in a cysteine protease-dependent manner. The immuno-activity of these fractions elutes as two major peaks from a reverse-phase $\mathrm{C}_{18}$ HPLC column. We have identified several candidate putative PAMPs within these peaks via LC-MS/MS which appear to be previously uncharacterised as elicitors of plant immunity. This study aims to underpin future research on the identification of sources of genetic resistance in crops to aphids and potentially other piercing-sucking insect pests.

Understanding the role of strigolactones in cyst nematode-plant interaction

N. GUARNERI (1), C. M. Escudero-Martinez (2), M. G. Sterken (1), G. Smant (1), A. Goverse (1), (1) Laboratory of Nematology, Wageningen University and Research, Wageningen, Netherlands; (2) Plant Sciences, School of Life Sciences, University of Dundee, Dundee, U.K.

Cyst nematodes are obligate, biotrophic parasites of many important agricultural crops that have the fascinating ability to reprogram plant cells and form a unique feeding structure called syncytium. The syncytium functions as a metabolic sink from which feeding nematodes acquire their nutrients for several weeks. Establishment of a successful cyst nematode-plant interaction strongly depends on plant hormone homeostasis. We have recently discovered that strigolactones play distinct roles in the interaction of beet cyst nematode (Heterodera schachtii) with Arabidopsis Strigolactone signaling mutant $\max 2$ negatively affects nematode attraction to the roots, whereas both $\max 2$ and the strigolactone biosynthesis mutant $\max 4$ enhance syncytium development. Moreover, the exogenous application of the synthetic strigolactone GR24 expands the zone above the root tip where $H$. schachtii invades the host. We want to dissect the role of strigolactone pathway in the overall susceptibility of Arabidopsis to $H$. schachtii. Furthermore, we aim at understanding the molecular mechanisms underlying the effect of strigolactone signaling and responses in nematode attraction, host invasion, and syncytium development.

Effectors of specific C13 peptidases induce host pathogenic programmed cell death - A molecular pathogenic mechanism of Bursaphelenchus xylophilus

B. XIE (1), X. Cheng (2), X. Zhang (2), (1) Institute of Vegetables and Flowers, Chinese Academy of Agricultural Sciences, Beijing, China; (2) College of Life Sciences, Beijing Normal University, Beijing, China

The molecular mechanism of the pinewood nematode (Bursaphelenchus xylophilus) killing pine trees is unclear. Early histopathological studies observed parenchyma cell vacuolization and vacuoles breakdown resulting in a rapid cell death in pine host at the initial stage of pathogenesis, which similar to VPE-mediated programmed cell death (PCD) in plants. By comparative genome analysis, we found cysteine peptidases $\mathrm{C} 13$ family (legumain/VPE /AEP) are significantly abundant in PWN genome. We introduced BxVPEs into the Arabidopsis yvpe-nul mutant and detected cell death triggered by fungal toxin FB1. The result showed that specific BxVPEs could best complement the function of the Arabidopsis $\gamma$ VPE. Effector properties of BxVPEs were determined by hybridization in situ and yeast secretion assay. Comparative transcriptome analysis of Arabidopsis yvpe-null mutant, wild-type and BxVPE transformed plants identified 59 differentially expressed genes (DEGs), with 26 upregulated and 33 downregulated in WT and BxVPE transformed plants, compared to $\gamma v p e$-null mutant. Functional annotation showed that these genes are mainly encoding transcription factors, E3 ubiquitin ligases and related proteins, F-box proteins, protein kinases, etc., which involved in 
transcription regulation, ubiquitin mediated proteolysis, signal transduction, carbohydrate metabolism, and so on. We suggest that PWN secretes specific BxVPEs into host to produce pathological PCD, resulting in host's cell rapid death.

A class of cell wall-derived damage-associated molecular patterns affects plant-aphid interactions

M. GRAVINO (1), S. T. Mugford (1), F. Cervone (2), G. De Lorenzo (2), S. A. Hogenhout (1), D. Pontiggia (2), (1) John Innes Centre, Norwich, U.K.; (2) Sapienza University of Rome, Rome, Italy

Aphids are among the most destructive pests in agriculture, causing direct damage to crops by feeding on phloem, as well as indirect losses by transmitting more than half of all described plant viruses. During feeding aphids secrete effector proteins into their hosts to manipulate cellular processes and promote infestation. Aphids are largely controlled by pesticides that are environmentally damaging and are being withdrawn from the market. The development of insect-resistant crops represents a viable alternative for aphid control and can be greatly enhanced by knowledge on plant resistance mechanisms against these pests. Cell wall and cell wall-derived oligogalacturonides (OGs), a well-known class of damageassociated molecular patterns (DAMPs), are important components of the plant immune system against fungal and bacterial pathogens, but their role against aphids is unknown. We show that OGs enhance Arabidopsis thaliana resistance to the green peach aphid Myzus persicae. This resistance response is mediated by calcium-dependent protein kinases. Intriguingly, the aphid effector Mp10 suppresses the OG-induced immunity. A. thaliana transgenic plants with increased methyl esterification level of cell wall pectin are more susceptible to aphid colonisation. Our work shows that cell walls and OGs are components of the plant immune response to aphids. This is important, because aphids are likely to release these DAMPs when they penetrate plant cell walls with their stylets.

An integrative outlook at protein and RNA level to understand the mechanism of resistance in a pigeonpea wild relative, Cajanus platycarpus to herbivory by Helicoverpa armigera

B. Roschitzki (1), J. Grossmann (1), P. Nanni (1), C. Panse (1), W. Wolski (1), M. Rathinam (2), P. Mishra (2), V. Rai (2), R. Schlapbach (1), N. K. Singh (2), U. Rao (3), R. SREEVATHSA (2), (1) University of Zurich \& ETH Zurich, Switzerland; (2) ICAR-National Institute for Plant Biotechnology, India; (3) ICAR- Indian Agricultural Research Institute, New Delhi, India

Pigeonpea (Cajanus cajan L.), one of the most important legume crops is attacked by almost 200 species of insects, pod borer Helicoverpa armigera being the most devastating. Wild relatives, the ancestors of crop plants have been challenged by nature and hence hardy towards various vagaries vis a vis cultivated counterparts. Pigeonpea wild relatives too portray relatively higher levels of resilience to various biotic and abiotic stresses including resistance to $H$. armigera. Understanding their mechanism(s) of resistance can provide leads for better management of the pest. We performed a comparative transcriptome and proteome analyses (18h, 38h, 96h) of Helicoverpa challenged-wild relative (Cajanus platycarpus) and $C$. cajan and comprehensively analysed the global changes in mRNA and protein profiles in the two contrasting pigeon pea species. All genes and proteins of C. cajan and C. platycarpus that showed differential expression between $96 \mathrm{~h}$ and $18 \mathrm{~h}$ of infection were correlated which depicted 2604 and 2054 correlation pairs. The correlation coefficient (r) between RNAseq and protein expression was around 0.2 for both, C. cajan and C. platycarpus. Further bioinformatics analysis of the clustered pairs showed fascinating integration and over representation of differentially expressed RNA and proteins involved in insect resistance. Further functional analysis of shortlisted proteins is in progress to assess the potential relevance with respect to pest resistance.

Evolution of effector function in an aphid chemosensory protein that supresses plant immunity. S. T. MUGFORD, C. L. Drurey, Y. Chen, R. Corkill, T. C. Mathers, S. A. Hogenhout, John Innes Centre, Norwich, U.K.

Along with other hemipteran herbivores, aphids feed from plants via specialised mouthparts (stylets) that pierce the plant tissue and suck the sap from vascular cells. Plants respond to the presence of insects through activation of the pathogen-associated molecular pattern (PAMP)- triggered immunity (PTI) signaling pathway, leading to enhanced resistance to the aphids. A growing body of evidence shows that aphids are able to supress PTI through the action of effector proteins in the saliva that modulate the defence responses of the plant. We show that one of these proteins Mp10, is delivered into the host plant mesophyll cells during the early stages of feeding. We show that Mp10 supresses the PTI signaling pathway, enabling the aphids to successfully colonise the plant. Mp10 belongs to the chemosensory protein family (CSP). Interestingly we have found that the origin of effector function in the Mp10 lineage of CSPs occurred early in the evolution of plant-feeding hemiptera, and the effector function is shared by orthologues from whiteflies and leafhoppers. By combining phylogenetic and functional studies we now have a better understanding of how the effector function evolved.

A novel aphid-specific gene family plays an important role in host colonization Y. CHEN (1), A. Singh (2), G. G. Kaithakottill (2), S. T. Mugford (1), C. Van Oosterhout (3), D. Swarbreck (2), S. A. Hogenhout (1), (1) John Innes Centre, Norwich, U.K.; (2) Earlham Institute, Norwich, U.K.; (3) School of Environmental Sciences, University of East Anglia, Norwich, U.K.

The green peach aphid Myzus persicae is a major agricultural pest. Unlike the majority of plant feeding insects that tend to specialize on a single plant species or family, M. persicae colonizes over 100 plant species from divergent families. We previously found that $M$. persicae is able to colonize distantly related host plants, including Arabidopsis thaliana and Nicotiana benthamiana, by rapidly modulating expression of genes belonging to aphid-expanded gene families. Here, we investigated transcriptional plasticity of a single asexually reproducing $M$. persicae clone on nine divergent plant species from five families, including dicots and the monocot maize. The aphids were able to colonize the nine plant species confirming that the M. persicae clone is polyphagous. We confirmed previous findings that specific aphid gene families are differentially regulated in $M$. persicae on different plant species. In addition, we identified effector candidates belonging to a novel aphid-specific gene family that are co-regulated. RNAi-mediated knock down of these effector genes in M. persicae decreased fecundity of this aphid, whereas the fecundity was increased on plants that stable express one member of this family. Our work demonstrates that $M$. persicae produces effectors that belong to aphid-specific families and that are co-regulated in the M. persicae clone in response to host plant species. 
The plant response to aphid feeding is similar to when a pathogen infects. One such economically important aphid is Diuraphis noxia that is a highly specialised aphid pest on a limited number of grasses. When it feeds on wheat, phenotypic responses similar to pathogen infection are observed in the wheat plants. Although this interaction have been studied, we are only starting to understand the molecular interaction and have yet to identify any of the wheat resistance genes. From wheat harbouring the Dn1-resistance gene we have identified a WRKY integrated domain nucleotide-binding leucine-rich repeat (NLR-ID) protein (TaADNR1) that plays an extensive role in D. noxia detection. Knockdown studies of resistant cultivars show that the loss of this protein result in increased aphid fitness and a highly susceptible wheat phenotype. This was observed for four different biotypes of $D$. noxia indicating that biotypification is not based on the loss of recognition by TaADNR1. The integration of the WRKY domain onto the NLR core protein is a very recent Triticum specific event as this WRKY domain only occurs in T. aestivum and none of the hexaploid precursors. This indicates that WRKY domain integration happened in the last ten thousand years. The integration of a WRKY domain onto TaADNR1 and one of its two homoeologs highlights the possible significance of these NLR-IDs. This significance is reinforced as the TaAdnrl transcript is targeted by a miRNA during this interaction.

Towards the characterisation of a novel resistance gene to control the rice root-knot nematode S. MANTELIN (1), H. Nguyen Thi (2), H. Ho-Bich (3), M. R. Armstrong (4), L. Xiong (5), J. T. Jones (6), I. Hein (4), S. Bellafiore (7), (1) The James Hutton Institute, Invergowrie, Dundee, U.K.; (2) University of Science and Technology of Hanoi, IRD-LMI RICE at AGI, Hanoi, Viet Nam; (3) IOIT/University of Science and Technology of Hanoi, VAST and UMMISCO, IRD, Hanoi, Viet Nam; (4) The James Hutton Institute/University of Dundee, Invergowrie, Dundee, U.K.; (5) National Key Laboratory of Crop Genetic Improvement, Huazhong Agicultural Uni., Wuhan, Hubei, China; (6) The James Hutton Instittue/University of St. Andrews, Invergowie Dundee, U.K.; (7) IRD-CIRAD-University of Montpellier, UMR IPME, Montpellier, France

The rice root-knot nematode, Meloidogyne graminicola, is considered a major threat to rice agriculture, particularly in Asia where changes in agricultural practices in response to environmental and socioeconomic conditions have led to a dramatic increase in the nematode disease pressure. Development of resistant rice varieties is essential to mitigate the spread of the disease and to promote sustainable control. Resistance to M. graminicola was identified in the Chinese tropical Oryza sativa japonica cultivar Zhonghua 11 (Zh11). Histological characterisation of the infection indicated that the resistance kicks in shortly after penetration and impairs the development of the nematode feeding site. Notably, phenolic compounds accumulate in the nematodes' neighbouring root cells during migration and a hypersensitive reaction occurs, leading to necrosis. Genetic characterisation of crosses between Zh11 and the susceptible indica cultivar IR64 suggested the presence of a major dominant resistance gene. As Zh11 demonstrated the hallmarks of a typical NB-LRR-type of resistance, a targeted resistance gene-enrichment sequencing (RenSeq) approach was developed in rice, which combined with bulk-segregant analysis of the F2 plants, identified one major resistance locus at the bottom of the rice chromosome 11. KASP markers are in development to screen the recombinants and identify resistance candidate genes.

Root-knot nematode effectors and their role in the tomato root interactome during infection B. STOJILKOVIC (1), G. Gheysen (2), (1) Faculty of Bioscience Engineering, Ghent University, Gent, Belgium; (2) Ghent Univ, Ghent, Belgium

Tomato (Solanum lycopersicum) is one of the widely consumed vegetable crops all over the world, as well as an important source of nutrition in the human diet. Tomato roots interact with beneficial or pathogenic organisms such as Root-Knot Nematodes (RKN). These interacting organisms manipulate the host cell metabolism by secreting different proteins, called effectors, in order to suppress the plant's immune response. The average tomato yield loss due to Meloidogyne spp. is $10 \%$, hence it is important to develop new strategies to investigate this plant-pathogen relationship. Despite this remarkable co-evolution, a molecular understanding of the nature of both, effectors, as well as the mechanism of their action is still missing. Based on literature and unpublished results, $20 \mathrm{RKN}$ effectors, shown to be important for successful parasitism, were selected for further investigation. Although many RKN effectors have been partially characterized, to date only five host targets of RKN effectors have been identified. For the selected effectors, we have started to identify tomato proteins as interaction partners and aim to elucidate the network of protein-protein interactions, and unravel the connection between the effectors, the plant immune system and defense related JA and/or SA pathways. Studying the interactome in tomato roots will also provide an insight into important biological questions that remained unexplored so far.

Resistance-gene independent variation in susceptibility to the root-knot nematode Meloidogyne incognita in Solanum lycopersicum M. G. STERKEN, S. Warmerdam, N. Guarneri, C. C. Van Schaik, M. Oortwijn, J. J. M. van Steenbrugge, J. L. Lozano Torres, A. Goverse, G. Smant, Laboratory of Nematology, Wageningen University and Research, Wageningen, Netherlands

Root-knot nematodes (Meloidogyne spp.) are among the most devastating plant parasites in global food production. For example, M. incognita control is important in tomato production, yet depends on a few resistance (R-) genes. Hence, the rise of resistance breaking (virulent) $M$. incognita populations is an ever-increasing concern, incentivizing the search for novel natural variation for virulence control. Here, we investigated susceptibility to $M$. incognita in tomato accessions without R-genes. Initially, 179 tomato accessions were screened for $M$. incognita susceptibility by challenging at least 10 plants per accession with 100 infective juveniles. We found large, accession-dependent, variation in susceptibility. To uncover underlying mechanisms, a high-resolution time-series RNAseq experiment on 10 accessions differing in susceptibility was performed. By isolating galls and corresponding tissue in mock-infected plants at 1,2, 3, 4, 7, and 10 days post-inoculation, feeding-site establishment was followed. We found $>700$ genes differential expressed related to feeding-site establishment and $>100$ genes correlating with accession susceptibility (Bonferroni-corrected $\mathrm{p}<0.05$ ). In conclusion, we found R-gene independent variation in susceptibility to $M$. incognita in tomato. This can help to identify genes associated with feeding site formation and loss-of-susceptibility. Furthermore, it opens the possibility to study the role of natural variation on feeding-site establishment. 
Host-induced RNAi-based gene silencing (HIGS) in rice for identifying Meloidogyne javanica essential genes for nematode infection M. Grossi de Sa (1), A. S. Petitot (1), I. Mezzalira (1), M. A. Beneventi (1,2), M. E. L. Sa (1,2,3), D. X. Amora (1,2), S. Lacombe (1), E. V. S. Albuquerque (4), M. F. Grossi de Sa (2), D. FERNANDEZ (1,4), (1) IRD- French Research Institute for Development, UMR IPME, IRD, Cirad, UM, Montpellier, France; (2) EMBRAPA CENARGEN, Brasilia, Brazil; (3) EPAMIG, Brazil; (4) Embrapa Genetic Resources and Biotecnology, Brasilia, Brazil

Root-knot nematodes (Meloidogyne spp.) are endo-parasitic worms with a wide host range. They induce the differentiation of some roots cells into specialized nurse cells to feed and to complete their life-cycle inside host roots. Successful infection is likely achieved by effector proteins produced in the nematode esophageal gland cells and released in the host plant cells. The aim of this study was to assess the potential of hostinduced RNAi-based gene silencing (HIGS) to control Meloidogyne javanica development in rice (Oryza sativa). HIGS was tested on essential genes for the nematode, including two putative effector genes (MSP2 and MSP19) and the Isocitrate lyase (IsoLy) metabolism gene as comparison. We produced $O$. sativa Nipponbare homozygous transgenic plants expressing artificial 21-bp micro-RNAs (amiRNAs) able to silence the nematode genes. All rice RNAi lineages displayed less nematodes infecting roots than the untransformed control rice at 21 days after inoculation. These data showed that the MSP2 and MSP19 genes are important for M. javanica development and infection in rice. A higher reduction in nematode number was observed for the RNAi lines against the metabolism IsoLy gene than for the RNAi lines against the MSP2 and MSP19 genes. HIGS in rice against nematode genes may then represent a robust and efficient method for identifying essential genes and could be used in a highly targeted specific manner to combat Meloidogyne spp. infecting crop plants.

Ethylene participate in plant defense response to whitefly Bemisia tabaci C. ZOU (1), C. Liu (1), P. Li (1), S. S. Liu (2), X. W. Wang (1), (1) Zhejiang University, China; (2) Institute of Insect Sciences, Zhejiang University, Hangzhou, China

To cope with biotic stresses, plants often initiate defense responses, one of which is achieved via phytohormone pathways. However, crosstalk among different phytohormone signaling pathways in plant can be synergistic or antagonistic. Ethylene has been recognized as a crucial phytohormone that directly or indirectly regulates the biosynthesis of secondary compounds through the crosstalk with other plant hormone signaling pathways. However, few case studies have been carried out on ethylene-related plant defense against small sap-sucking insects. Here, we report that the expression levels of genes of ethylene-signaling pathway were increased in the whitefly-infested tobacco compared to the control. To the contrary, genes of the jasmonic acid signaling pathway were decreased. Moreover, exogenous application of ethylene significantly increased the fecundity of whitefly and decreased the expression levels of genes in the jasmonic acid signaling pathway. Taken together, these results indicate that ethylene likely participates in the plant defense against whitefly through the crosstalk with the jasmonic acid signaling pathway. Our findings provide novel information for the exploration of mechanisms underlying plant defense response against herbivorous insects.

Manipulation of plant defence response by a whitefly salivary protein

F. WANG (1), Y. Hong (1), H. X. Xu (1), S. S. Liu (2), X. W. Wang (1), (1) Zhejiang University, China; (2) Institute of Insect Sciences, Zhejiang University, Hangzhou, China

Many phloem-feeding insects are important agricultural pests that directly feed on plant phloem using their stylets. While ingesting phloem sap, phloem-feeding insects may secrete saliva into plant to regulate plant defense. Previous studies have shown that, to facilitate their feeding, many phloem-feeding insects can elicit plant salicylic acid (SA) signaling pathway and suppress effective jasmonic acid (JA) defenses. However, the molecular basis of the regulation of plant JA-SA defense by phloem-feeding insects remains largely unknown. Here we show that BtQ0F1, a whitefly-secreted salivary protein which we previously identified from a whitefly, increases the susceptibility of host plant by deceiving the latter into suppressing SA signaling pathway as well JA signaling pathway. Overexpression of BtQ0F1 gene in planta promotes the susceptibility of tobacco to whitefly and suppresses SA signaling pathway as well as JA signaling pathway. In contrast, silencing whitefly BtQ0F1 gene significantly decreases whitefly performance on host plants. These data indicate that the whitefly salivary effector BtQ0F1 is able to enhance insect virulence by regulating plant SA-JA defense.

A Novel Ha18764 Effector from Heterodera avenae Suppresses Plant Defenses and Promotes Parasitism S. Yang, H. Jian, Q. LIU, China Agricultural University, Beijing, China

We identified a G16B09-like effector protein from the transcriptome of Heterodera avenae, and then further characterized its function. Our experimental evidence suggested that Ha18764 was specifically expressed in the dorsal gland and was dramatically upregulated in the J4 stage of nematode development. A Magnaporthe grisea secretion system in barley showed that the signal peptide of Ha18764 had secretion activity to deliver mCherry into plant cells. Arabidopsis thaliana overexpressing Ha18764 or Hs 18764 was more susceptible to Heterodera schachtii. In contrast, BSMV-based host-induced gene silencing (HIGS) targeting Ha18764 attenuated H. avenae parasitism and its reproduction in wheat plants. Transient expression of Ha18764 suppressed PsojNIP, Avr3a/R3a, RBP-1/Gpa2 and MAPK kinases (MKK1 and NPK1 ${ }^{\mathrm{Nt}}$ )-related cell death in Nicotiana benthamiana. Co-expression assays indicated that Ha18764 also suppressed cell death triggered by four $H$. avenae putative cell-death-inducing effectors. Moreover, Ha18764 was also shown strong PTI suppression such as reducing the expression of plant defenserelated genes, the burst of reactive oxygen species, and the deposition of cell wall callose. Together, our results indicate that Ha18764 promotes parasitism, probably by suppressing plant PTI and ETI signaling in the parasitic stages of $H$. avenae.

Heterodera avenae GLAND5 effector interacts with pyruvate dehydrogenase subunits of plants and promotes nematode parasitism S. Yang, L. Pan, Y. Chen, Q. Liu, H. JIAN, China Agricultural University, Beijing, China 
The cereal cyst nematode Heterodera avenae is a major pathogen of wheat that causes huge yield losses. As a sedentary endoparasitic nematode, $H$. avenae secretes many effectors into plant tissues to suppress plant defense and facilitate parasitism. In this study, we showed that $H$. avenae has evolved a novel effector, HaGland5, which was exclusively expressed within the nematode dorsal gland cells and upregulated in the early parasitic stage. Transgenic Arabidopsis thaliana lines expressing HaGland5 became significantly more susceptible to $H$. schachtii infection than wild-type control plants. Conversely, the silencing of HaGland5 in wheat through barley stripe mosaic virus-medicated host-induced gene silencing techniques substantially reduced $H$. avenae infection in wheat. Moreover, HaGland5 could suppress the plant defense responses including the repression of plant defense-related genes, reducing deposition of cell wall callose and the burst of reactive oxygen species. Mass spectrometry, co-immunoprecipitation, and firefly luciferase complementation imaging assays confirmed that HaGland5 interacted specifically with Arabidopsis pyruvate dehydrogenase subunit (AtEMB3003). We infer that $H$. avenae may utilize a novel mechanism based on a fatty-acidderived signal to modulate the defense signaling pathways in plants to suppress plant defenses and promote parasitism.

An endosymbiont increases the retention of a plant virus in its whitefly host

T. LEI (1), H. L. Wang (1), S. S. Liu (2), (1) Zhejiang University, Hangzhou, China; (2) Institute of Insect Sciences, Zhejiang University, Hangzhou, China

Many crops are threatened by viruses in the genus Begomovirus (family: Geminiviridae) that are transmitted by whiteflies of the Bemisia tabaci species complex. The ability of $B$. tabaci in virus transmission may be affected by its endosymbionts. In a survey of endosymbiont diversity among 21 cryptic species of the B. tabaci complex, we identified a new Rickettsia strain in the whitefly species Asia II 1, one of the major virus vectors of begomoviruses in the cotton field in many regions of Asia. The purpose of this study is to find out whether this new Rickettsia strain could affect the capacity of Asia II 1 in virus transmission. We first established two cultures of Rickettsia-infected and Rickettsia-free Asia II 1 lines and then compared the ability between the whitefly lines in transmitting cotton leaf curl Multan virus, one of the major causing agents of cotton virus disease in the field in many regions of Asia. Quantitative real-time PCR analysis of the quantity of the virus in the test whiteflies showed that the Rickettsia-infected Asia II 1 line retained significantly more virus than the uninfected line. This result implies that this Rickettsia endosymbiont of Asia II 1 may enhance the host's ability to transmit cotton leaf curl Multan virus. Further experiments of comparing the ability of the two Asia II 1 lines in transmitting begomoviruses are underway.

\section{The transcription factor TCP9 regulates responses in root architecture to the beet cyst nematode Heterodera schachtii in Arabidopsis} thaliana

J. J. WILLIG, W. D. Jong, M. G. Sterken, J. L. Lozano Torres, A. Goverse, J. Bakker, G. Smant, Laboratory of Nematology, Wageningen University and Research, Wageningen, Netherlands

Low levels of infection by cyst nematodes on some resistant crop varieties result in significant loss in yield, while other heavily infected susceptible varieties show hardly any symptoms at all. This suggests that some plants tolerate biotic stress by plant parasitic nematodes better than others. We hypothesize that tolerant plants accommodate nematode infections by altering the architecture of their root system. Our current objective is to investigate whether the transcription factors TCP9 and TCP20, which are known regulators of plant root architecture, are involved in tolerance to cyst nematodes in Arabidopsis. To this end, we first tested whether TCP9 and TCP20 regulate susceptibility of Arabidopsis to the beet cyst nematode Heterodera schachtii. Next, we analysed the root architecture of nematode-infected roots of Arabidopsis tcp 9 and tcp 20 knock-out mutants to assess whether these genes are involved in alterations in primary and secondary roots associated with nematode infections. Finally, we tested whether $t c p 9$ and tcp 20 mutants are less sensitive for methyl jasmonate, which is required for the recovery of damaged plant tissue after host invasion by nematodes. Altogether, our data demonstrates that TCP9 regulates responses in root architecture in Arabidopsis to $H$. schachtii.

Functional analysis of core effectors of syncytium forming nematodes

K. LESLIE (1), S. Mantelin (2), J. T. Jones (3), S. Eves-van den Akker (4), (1) The James Hutton Institute/University of St Andrews, Dundee, U.K.; (2) The James Hutton Institute, Invergowrie, Dundee, U.K.; (3) The James Hutton Instittue/University of St. Andrews, Invergowie Dundee, U.K.; (4) University of Cambridge, U.K.

Plant parasitic nematodes infect many major food crops worldwide, causing damage globally valued at approximately 80 billion U.S. dollars per year. Some nematodes form a feeding site called a syncytium in the roots of their host. Relatively little is known about how nematodes initiate and maintain these feeding structures. However, specialised proteins and small molecules, known as effectors, secreted into the plant by the pathogen, are thought to play critical roles in these processes. It is important to establish a greater understanding of the role of effectors in syncytium formation and maintenance alongside host invasion by the nematode. Exploiting the genomic and transcriptomic data available from four syncytium-forming species; G. rostochiensis, G. pallida, R. reniformis \& N. abberans we have identified a series of candidate core effectors conserved in syncytia-forming nematodes. A subset of three of these core effectors (Gr20E03, GrGLAND11 and GrGLAND15) of unknown function are being studied in more detail. Two of these, GrGLAND11 and GrGLAND15 localised to the actin cytoskeleton when expressed as fusions with fluorescent proteins. Yeast two-hybrid has identified a predicted arginine N-methyltransferase as a potential interacting host protein of GrGLAND11. We are currently investigating the role of this interaction in more detail.

Comparing genomes of (a)virulent inbred lines of the cyst nematode Globodera rostochiensis regarding the potato resistance gene $\mathrm{H} 1$ J. J. M. VAN STEENBRUGGE, M. Holterman, S. van den Elsen, G. Smant, J. Helder, Laboratory of Nematology, Wageningen University and Research, Wageningen, Netherlands

Two common potato cyst nematodes, Globodera rostochiensis and G. pallida, largely reduce potato production worldwide. Juveniles of potato cyst nematodes have a protrusible stylet that delivers a mixture of effector proteins into selected root cells. Effectors enable the nematode to penetrate the plant, to suppress host-immunity, and to form a feeding site. Due to their limited mobility and their long generation time, host plant resistances are an effective and durable means to manage potato cyst nematodes. The potato resistance gene $H 1$ was introgressed in the late 
1960ies into commercial potato cultivars and has been highly effective in managing two pathotypes of G. rostochiensis for decades. In the past, we generated two nematode inbred lines that are either fully virulent or avirulent with regard to $H 1$. For both lines, high-quality genome assemblies were created using PacBio sequencing. By comparing the effector gene families in both inbred lines, we aim to discover effector variants that are responsible for gain of virulence. Ultimately, insights on effector variants are essential in our understanding why host plant resistance proteins loose resistance against certain potato cyst nematode populations. This understanding may provide ways to develop new breeding strategies and hence reduce the damages caused by these pathogens in a specific and environmentally sound manner.

Salicylic acid biosynthesis in rice and nematode interference

H. LEFEVERE (1), G. Gheysen (2), (1) UGent, Ghent, Belgium; (2) Ghent Univ, Ghent, Belgium

Salicylic acid (SA) is an important plant hormone that mediates host responses upon pathogen infection. While it is widely accepted that this hormone is synthesized by either the phenylalanine ammonia-lyase (PAL) or the isochorismate synthase (ICS) pathway, some of the enzymes involved have not yet been identified. Furthermore, the importance of each of these pathways in pathogen-induced SA accumulation differs between plants. In Arabidopsis thaliana, it is the ICS pathway that is mainly induced upon pathogen infection, while in potato (Solanum tuberosum) there seems to only be PAL pathway upregulation under similar circumstances. In rice (Oryza sativa) there is no clear consensus on whether the ICS or PAL pathway is the most important or whether they work in tandem, as is the case in soybean (Glycine max). Using CRISPR/Cas9 methods, we are creating stable knock-out rice plants for our genes of interest and will analyze those mutants for transcription levels of relevant genes and SA accumulation upon pathogen infection. We have also identified a chorismate mutase and isochorismatase in nematodes that could interfere with the biosynthesis of SA. Therefore, we will also perform infection experiments with nematodes on these plants, to elucidate how they can alter the biosynthetic pathway. Using enzyme activity assays, we are elucidating the role and function of known and unknown genes to map the mechanisms of the two biosynthesis pathways in detail.

Silencing of an integrated domain nucleotide-binding leucine-rich repeat (NLR-ID) gene, Adnr1, indicates that it underlies Diuraphis noxia resistance in Dn7-mediated resistance in wheat

N. S. MKHIZE (1), V. Nicolis (2), E. Venter (2), (1) University of Johannesburg, Johannesburg, South Africa; (2) University of Johannesburg, Auckland Park, South Africa

The Russian wheat aphid (Diuraphis noxia Kurdjumov, RWA) is a major pest of bread wheat (Triticum aestivum L.) that has contributed to the major crop losses of susceptible cultivars over the past three decades. However, plants have a continuously evolving innate immune systems to counter various evolving pathogens and pest virulence strategies. Wheat cultivars carrying inbred resistance genes provided resistance against $D$. noxia until the recent development of at least three resistance breaking biotypes (RWASA1-4). TaAdnrl, a nucleotide-binding leucine-rich repeat gene (NLR) carrying an integrated WRKY-domain, was identified to play a crucial role in the Dn1-resistance gene-based wheat response against the RWA. The aim of this research was to ascertain the role of TaAdnrl in imparting resistance to the three resistance-breaking RWASA biotypes. Virus induced gene silencing (VIGS) of TaAdnrl resulted in the Dn7-mediated resistance response becoming a susceptible phenotype. This was quantified through determining the silencing efficiency as reduced transcript abundance, increased aphid reproduction rates, decrease in dry weights of the plants and the loss of the oxidative response. These results indicated that the NLR-ID underlies aphid recognition in wheat cultivars harbouring different resistance genes. Furthermore, ADNR1 is suggested to recognise effectors from all four biotypes indicating that these effectors are not changed during biotypification of $D$. noxia.

RanGAP1 and RanGAP2 are common virulence targets of two independently evolved effectors from the potato cyst nematode Globodera pallida and Potato Virus $X$

O. Sukarta (1), A. Diaz Granados (2), E. Slootweg (3), G. Smant (1), A. GOVERSE (1), (1) Laboratory of Nematology, Wageningen University and Research, Wageningen, Netherlands; (2) Wageningen University, Wageningen, Netherlands; (3) Laboratory of Nematology, Wageningen University and Research, Netherlands

The closely related potato resistance genes Gpa2 and $R x 1$ encode canonical intracellular CC-NB-LRR immune receptors. They belong to the same $R$ gene cluster but evolved to defend against two unrelated pathogens. Gpa2 detects specific GpRbp-1 effector variants secreted by the potato cyst nematode Globodera pallida, whereas Rx1 recognizes the viral coat protein (CP) of Potato Virus X. How effector recognition by these receptors occurs has yet to be demonstrated. However, artificial tethering studies suggest that recognition may occur indirectly through the shared co-factor, Ran GTPase Activating Protein 2 (RanGAP2). Using a combination of Co-IP and cellular imaging studies, we could show that both the eliciting and non-eliciting variants of GpRbp-1 and PVX-CP can interact with RanGAP2 in planta through its WPP domain. Moreover, we could show that the RanGAP1 homolog can also associate with these effectors in the cell. From these data, we conclude that RanGAP1 and RanGAP2 are a common host target for two distinct pathogen effectors with a possible role in virulence. Interestingly, infection assays on mutants and TRV-VIGS silenced plants could show that RanGAP2 and RanGAP1 contribute to the pathogenicity of both cyst nematodes in Arabidopsis and PVX in N. benthamiana. From these data, a picture emerges that RanGAP1 and RanGAP2 are shared virulence targets of two evolutionary distinct pathogens, which are guarded by two closely related CC-NB-LRR immune receptors in potato.

Identification, cloning and RNAi silencing of selected genes for management of pigeon pea pod borer, Maruca vitrata M. Chatterjee, P. K. Papolu, J. Yadav, R. Sreevathsa, V. S. Somvanshi, U. RAO, ICAR-Indian Agricultural Research Institute, New Delhi, India

Pigeon pea (Cajanus cajan) is a widely cultivated perennial legume crop in tropical and sub-tropical regions of the world. Pod borer, Maruca vitrata, is one of the major biotic stress in pigeon pea. Due to the emergence of resistance to chemical pesticides, new management methods are required. Based on transcriptome analysis of $M$. vitrata, 12 genes responsible for growth and development, and six genes expressed in midgut were selected, cloned and used for both in vitro and host delivered RNAi. In vitro RNAi via feeding of dsRNA of selected nine genes resulted in phenotypic and behavioural changes such as sluggishness, nonfeeding and discoloration of $M$. vitrata larvae. Quantitative RT-PCR confirmed the silencing of two of the growth and development related genes (chymotrypsin-like serine protease and serine protease S3 subunit) and three 
midgut expressed genes ( $H^{+}$transporting ATP synthase delta subunit, triose phosphate isomerase, acyl CoA delta desaturase). Further, hairpingene constructs were prepared and used for pigeon pea transformation. The putative transformants identified by antibiotic selection were subjected to PCR and Southern analysis for confirmation. The transgenic pigeon pea plants are also being evaluated against $M$. vitrata for their efficacy. This is the first report of using RNAi for silencing $M$. vitrata genes for its management.

The root-knot nematode effector MiEFF18 targets the host plant splicing machinery to favor parasitism.

J. MEJIAS (1), N. M. Truong (2), P. Abad (3), B. Favery (4), M. Quentin (5), (1) INRA/ Sophia Agrobiotech institute, Sophia-antipolis, France; (2) Kumamoto University, Kumamoto, Japan; (3) INRA, Antibes CEDEX, France; (4) INRA UNS CNRS, Sophia-Antipolis, France; (5) INRA/ UMR / CNRS, France

Meloidogyne incognita is a root-knot nematode (RKN) that triggers an intimate relationship with their host plant. After penetrating the plant root, Meloidogyne species secrete effectors protein synthesized from esophageal glands, via their stylet, into the host cells. RKN effectors reprogram vascular root cells into a feeding structure consisting of five to seven giant cells. These effectors target diverse subcellular compartments and manipulate essential developmental processes, e.g. cell cycle and transcriptional regulations. MiEFF18 was identified as a putative effector using genomic and transcriptomic data, combined with in situ hybridization. The MiEFF18 protein is predicted to carry a signal peptide for secretion, and a nuclear and a nucleolar localization signal. MiEFF18 localized into the nucleus and the nucleolus, when transiently expressed in tobacco leave cells. To identify MiEFF18 function during parasitism, we search for its direct targets in tomato roots using a yeast two hybrid approach. We found a core spliceosomal Sm protein as the best scored target of MiEFF18. We showed this Sm protein is an essential plant protein for the nematode parasitic success. This Sm protein is known to be involved in splicing of mRNA and to facilitate silencing in plants. We are investigating the outcomes of MiEFF18 interaction with its target, and the cellular functions, including alternative splicing, RNA quality control or PTGS modulation that may be hijacked by MiEFF18.

Caught in the act: The butterfly egg-associated molecular pattern that activates plant defense

L. CAARLS (1), R. Mumm (2), R. de Vos (2), N. Bassetti (1), E. Schranz (1), N. Fatouros (1), (1) Biosystematics Group, Wageningen University \& Research, Netherlands; (2) Business Unit Bioscience, Wageningen University \& Research, Netherlands

Plants can defend against insect eggs deposited on their leaves with the formation of necrotic tissue underneath the eggs, a so-called hypersensitive-like response (HR-like). Oviposition by cabbage white butterflies (Pieridae) induces cell death and ROS underneath eggs in several species of the Brassicaceae family. Of these, the wild mustard Brassica nigra shows a strong egg-killing HR-like to Pieris eggs in nature. Knowledge on how plants detect insect eggs and activate defense is minimal. We aim to isolate and identify the egg-associated molecular pattern (EAMP) that triggers necrosis in B. nigra. First, we show that a wash of Pieris eggs elicits the same response as eggs, suggesting that the elicitor is in secretions covering the eggs or in the egg glue released with the eggs. In addition, this induction was shared between washes of four tested Pierid species. Dissection of eggs from the ovaries of $P$. brassicae showed that the active compound is only present on fertilized eggs that have passed the oviduct and its associated gland containing egg glue. We then analysed the chemistry of egg washes by LC/MS and compared inducing and non-inducing washes. Furthermore, active washes were fractionated. One compound was shared between all inducing washes and the active fraction and is thus a candidate for necrosis inducing EAMP. Validation of this compound is ongoing and when accomplished will aid us in identifying the corresponding plant receptor of this egg-killing response.

Pathogens and pests on plants: Plant attackers impact each other's performance when co-occurring on a host L. J. A. VAN DIJK, Stockholm University, Solna, Sweden

Both pathogens and herbivores exploit plants for nourishment, and they commonly co-occur on the same host plant. As a consequence, attackers may impact not only performance of their host plant but also of one another, for example via altered host plant chemistry. The outcome of multispecies interactions is highly context-dependent and a general consensus on the topic is currently lacking. In this study, we investigated the outcome of the interaction between oak trees (Quercus robur), two types of herbivorous insects (aphids, Tuberculatus annulatus, and caterpillars, Phalera bucephala) and a biotrophic pathogen (powdery mildew, Erysiphe alphitoides) in a greenhouse experiment. We found that the impacts of aphids and powdery mildew on each other's performance were in opposite directions: Aphids performed less well when co-occurring with mildew, whereas mildew performed better. Caterpillars were disadvantaged when eating mildew-infected leaves. Interestingly, early arriving attackers modified the interaction outcome between two later arriving attackers. Host plant performance was only minimally impaired when exposed to attackers. Our findings highlight the complexity of plant-attacker interactions: Attacker species identities as well as their time of arrival matters for performance outcomes, which may, in natural settings, have far-reaching consequences for the ecology and evolution of plantattacker interactions.

Transient Expression of Whitefly Effectors in Nicotiana benthamiana Leaves Activates Systemic Immunity Against the Leaf Pathogen Pseudomonas syringae and Soil-Borne Pathogen Ralstonia solanacearum

H. R. Lee (1), S. Lee (1), P. J. M. V. Kleeff (2), R. C. Schuurink (2), C. M. RYU (1,3), (1) KRIBB, Daejeon, Korea, Republic of (South); (2)

University of Amsterdam, Amsterdam, Netherlands; (3) University of Science and Technology, Daejeon, Korea, Republic of (South)

Infestation of plants with the phloem-feeding whitefly Bemisia tabaci modulates root microbiota and both local and systemic immunity against microbial pathogens. Specifically, aboveground whitefly infestation suppresses pathogen propagation and symptom development caused by the soil-borne pathogens Agrobacterium tumefaciens and Ralstonia solanacearum in the root system through systemic signal transduction. Therefore, we hypothesized that secreted protein(s)/non-protein factors from whitefly saliva (referred to as candidate effectors) might function as insect determinants that activate systemic acquired resistance (SAR) in the host plant. Here, we intensively screened a cDNA library constructed from mRNA from whitefly feeding on Nicotiana benthamiana leaves and selected three candidate effectors 2G4, 2G5, and 6A10, that appear to reduce disease development caused by the aboveground pathogen Pseudomonas syringae pv. tabaci and the soil-borne pathogen $R$. solanacearum. Transient expression of the three candidate effector cDNAs in leaves primed the expression of SAR marker genes $N b P R I a$ and $N b P R 2$ in local 
and systemic leaves against $P$. syringae $p v$. tabaci, while leaf infiltration with $2 \mathrm{G} 4$ or $6 \mathrm{~A} 10 \mathrm{cDNA}$ elicited strong defense priming of SAR markers following drench application of $R$. solanacearum on plant roots. This is the first report of whitefly effectors that prime SAR against aboveground and belowground bacterial pathogens.

AgriVectors: A systems biology portal for plant pathosystems and arthropod vectors of plant diseases S. SAHA (1), W. Hunter (2), L. A. Mueller (1), A. Consortium (1), (1) Boyce Thompson Institute, Ithaca, NY, U.S.A.; (2) USDA-ARS, U. S. Horticultural Research Laboratory, Fort Pierce, FL, U.S.A.

Arthropod vectors of pathogens cause enormous economic losses and are a fundamental challenge for sustainable increases in food production. To more effectively fight plant diseases, data pertaining to a disease system needs to be consolidated, made searchable and amenable to data mining. The proposed AgriVectors platform is an open access and comprehensive resource for growers, researchers and industry working on plant pathogens and pathosystems spread by arthropod vectors. The portal connects established public repositories with pathosystem-specific data repositories. The AgriVectors system will provide tools to enable technologies such as RNAi, CRISPR, screening bioassays, etc. to leverage current and emerging knowledge across disciplines. The portal will be based on the Citrusgreening.org (https://citrusgreening.org/) community resource for the Huanglongbing pathosystem that was developed as a model for systems biology of tritrophic disease complexes. It includes a biochemical pathway database for each organism in this disease complex, and an expression atlas for the psyllid vector and citrus host. The AgriVectors portal will extend this model beyond gene-centric omics data to the broader Pathosystem-wide information, with integrated pest management, behavioral, plant health, soil health and climate data to incorporate rapid phenotyping information from research trials, building a foundation for more effectively identifying solutions to combat plant diseases.

Uncovering the molecular basis of virulence and avirulence in the Medicago - pea aphid system S. ALTMANN (1), R. M. Lopez-Cobollo (2), P. Thorpe (3), M. Gierliński (1), S. Kanvil (2), C. Turnbull (2), J. I. B. Bos (1), (1) University of Dundee, Dundee, U.K.; (2) Imperial College London, U.K.; (3) University of St Andrews, U.K.

Aphids are important agricultural pests, and control heavily relies on insecticides. Genetic crop resistance, mediated by major plant resistance (R) genes, to aphids is generally lacking, and where available, its molecular basis is not well understood. Medicago truncatula resistance to pea aphid (Acyrthosiphon pisum) biotypes conforms to the "gene-for-gene" model. Here, we used two divergent pea aphid genotypes that differ in their virulence phenotype on M. truncatula plants carrying the RAPI (resistance to Acyrthosiphon pisum 1) resistance gene to gain insight into the determinants of (a)virulence. We undertook a bulk segregant analysis approach of the N116 (virulent) and PS01 (avirulent) parents, and their F1/F2 progeny after crossing. High throughput DNA- and RNA-sequencing of parents and pools of virulent and avirulent progeny was performed to potentially associate sequence variation and expression differences with the (a)virulence phenotype. Initial data point to a high number of single nucleotide polymorphisms (SNPs) between the two strains and showed that the avirulent genotype expresses a higher number of genes compared to the virulent genotype. We are optimizing and applying RNAi-mediated silencing in aphids to further validate the role of candidate genes identified through our approach in (a)virulence.

Comprehensive analysis of a large group of CLE effectors encoded by Globodera cyst nematodes S. Chen (1), P. Lang (1), M. G. Mitchum (2), X. WANG (1,3), (1) Cornell University, Ithaca, NY, U.S.A.; (2) University of Missouri, Columbia, MO, U.S.A.; (3) USDA-ARS, Ithaca, NY, U.S.A.

Plant-parasitic cyst nematodes (Heterodera and Globodera spp.) are agriculturally important pests that cause significant annual yield losses worldwide. These sedentary endoparasites secrete various effector proteins into selected root cells to create a unique feeding cell structure that serves as the only nutrient source for the nematode to develop and reproduce. CLAVATA3/ESR (CLE)-like effector genes are expressed in several cyst nematode species and their encoded proteins function as mimics of plant CLE peptides that modulate host signaling pathways to promote feeding cell formation. We have cloned a large group of $C L E$ genes from four closely related Globodera species, including G. rostochiensis, G. pallida, G. ellingtonae, and G. tabacum. These Globodera CLE genes primarily encode multiple-domain CLE proteins that could result in $>30$ distinct CLE peptides when proteolytically processed in planta. Characterization of these Globodera CLE peptides using exogenous peptide assays identified a subgroup of CLEs that could affect host root growth and vascular development. Receptor mutant screening with these unique CLE peptides confirmed a role of the CLV2 receptor in root growth but not vascular development. Further studies are underway to discover host receptors that are responsible for perceiving this novel group of Globodera CLE peptides to function in vascular development in host roots.

Characterisation of transthyretin-like proteins of the plant-parasitic nematode Pratylenchus penetrans suggests involvement in oxidative stress response.

M. ESPADA (1), C. S. L. Vicente (1), J. Branco (1), M. Mota (1), P. Vieira (2), (1) ICAAM / University of Evora, Evora, Portugal; (2) Virginia Tech, Blacksburg, VA, U.S.A.

Transthyretin-like proteins (TTL) belong to a widely conserved family present only in the Phylum Nematoda. Although several TTLs (protein domain PF01060) have been identified for both animal and plant-parasitic nematodes most of their function(s) remains unclear. In Pratylenchus penetrans, a migratory plant-parasitic nematode, transcriptomic data revealed that some members of this family are abundantly expressed during plant infection. In silicoanalysis identified twenty-four predicted TTLs members for this species, all of them containing a predicted signal peptide.In situ hybridisation assays demonstrate that some of these members are specifically localised in the nematode esophageal gland cells (parasitism specialised cells), suggesting their potential involvement during parasitism. Oxidative stress assays demonstrate that some of these TTLs have a strong upregulation when nematodes are exposed to an external oxidative agent (hydrogen peroxide) stimulus compared to a secreted nematode catalase - an enzyme that directly degrades hydrogen peroxide. The results suggest that some of these TTLs might have a potential role during ROS scavenging activity. Other functional analyses are underway to provide more insights into the functional role of these TTLs during plant parasitism of $P$. penetrans. 
The role of root exudates in the interaction between plant parasitic nematodes and coffee plants

A. CASEY (1), P. E. Urwin (1), J. Mccarthy (2), (1) University of Leeds, Leeds, U.K.; (2) Nestlé, France

Coffee is major crop which supports the economy of over 60 countries. A detriment to coffee production is the damage caused by plant parasitic nematodes, reducing yields by up to $15 \%$. The most damaging species include root knot (Meloidogyne spp.) and root lesion nematodes (Pratylenchus spp.). The endoparasitic lifestyle of root knot and lesion nematodes requires the recognition of, and movement towards, host roots followed by invasion for the establishment of a food source. We are investigating how root exudates mediate these interactions between nematodes and host roots, and characterising the components of neuronal signaling that underlie the behavioural responses. Chemotaxis assays have been used to demonstrate attraction of both Meloidogyne and Pratylenchus species to compounds exuded by the roots of coffee. As coffee is often intercropped, we are also exploring how the host status of intercrop plants affects the behaviour of the nematodes in response to their root exudates. We have evidence that nematodes are differentially attracted to root exudates from a range of plant species and to those from different coffee cultivars. A comparative metabolomic approach will be used to analyse exudate components for the different cultivars. These findings will better inform both breeders and growers on the best attributes for resistant coffee cultivars and offer potential targets for the control of plant parasitic nematodes.

Architecture and dynamics of $\mathrm{JA} / \mathrm{ABA}$ - and thrips-induced gene regulatory networks

S. C. VAN WEES (1), M. Steenbergen (1), N. Aerts (1), S. Sarde (2), M. Dicke (2), R. Hickman (1), C. M. J. Pieterse (1), (1) Plant-Microbe Interactions, Department of Biology, Utrecht University, Utrecht, Netherlands; (2) Laboratory of Entomology, Wageningen University, Wageningen, Netherlands

To survive attack by insects, plants have evolved sophisticated immune signaling networks that enable them to mount an effective defence response upon recognition of the invader. Plant hormones, especially jasmonic acid (JA) and abscisic acid (ABA) emerged as core players in the orchestration of the signaling networks that underlie activation of anti-herbivore defences. To advance our understanding of the different modes of regulation of JA- and ABA-induced defence responses, we performed high-resolution RNA-Seq time series analyses of JA- and ABA-treated Arabidopsis to build dynamic gene regulatory network (GRN) models and infer novel regulators of the individual and combinatorial hormoneinduced responses. Using a similar approach, we analyzed the GRN induced upon infestation by the insect thrips. The significance of the novel regulatory components predicted from our analyses was validated by bioassays using Arabidopsis and thrips. We extended our analysis of the thrips interaction by monitoring the response of pepper and cabbage crop plants to feeding by thrips, allowing us to compare the GRNs induced by thrips feeding across different host plant species. This revealed overlaps in JA and ethylene signaling, in engagement of specific transcription factor families, and in activation of phenylpropanoid and flavonoid biosynthesis, but also highlighted differences in multiple sectors of the GRNS, like terpenoid and glucosinolate biosynthesis.

Watery Saliva Protein 1 (NIWSP1) of brown planthopper (Nilaparvata lugens) attenuates salicylic acid defense in rice Y. JIANG (1,2,3), X. Zhang (4), C. Zhang (4), S. Y. He (1,5,6,7), (1) MSU-DOE Plant Research Laboratory, U.S.A.; (2) Xishuangbanna Tropical Botanical Garden, Chinese Academy of Sciences, China; (3) Howard Hughes Medical Institute, U.S.A.; (4) Zhejiang University, China; (5) Plant Resilience Institute, U.S.A.; (6) Howard Hughes Medical Institute, East Lansing, MI, U.S.A.; (7) Department of Plant Biology, Michigan State University, U.S.A.

The brown planthopper (BPH) Nilaparvata lugens is the most destructive insect pest of rice; it poses a serious threat to global rice production. In the past decade, a number of BPH resistant genes have been cloned, which sheds light on the molecular basis of incompatible interactions between BPH and rice plants. However, how BPH infects genetically susceptible rice plants remains largely a mystery. Being a monophagous insect that feeds exclusively on the phloem of rice plants, BPH is hypothesized to deliver salivary proteins into the phloem tissue as part of its strategy to subvert rice plants. We have previously identified Watery Saliva Protein 1 (WSP1) as being critical for the survival of BPH on rice. Here, we characterized the biological function of WSP1 in rice plants. We hypothesized that WSP1 functions in the plant cell and produced transgenic rice plants expressing WSP1. WSP1-expressing plants exhibit no significant growth or reproductive phenotypes. Strikingly, however, they restored the survival of WSP1-silenced BPH insects, which grow poorly on wild-type rice plants. Salicylic acid (SA) level as well as SA pathway gene expression are significantly decreased in the WSP1-expressing plants. Moreover, WSP1 interacts with a member of the rice phenylalanine ammonia-lyase (OsPAL) family. Together, these data suggest that the essential virulence protein WSP1 manipulates the SA signaling pathway as a mechanism to ensure BPH growth and survival in rice.

A putative R gene RESISTANT TO MYZUS PERSICAE is required for Arabidopsis defense against the green peach aphid M. Twayana, J. SHAH, University of North Texas, Denton, TX, U.S.A.

Resistance $(R)$ genes have important functions in plant defense against pests. Several $R$ genes that confer resistance against pathogens have been described. However, very few $R$ genes that confer resistance against insects, in particular insects that feed from the phloem, are known. We have identified a non-canonical $R$ gene, RESISTANT TO MYZUS PERSICAE (RMP), in Arabidopsis thaliana that confers resistance to the green peach aphid (GPA; Myzus persicae), an important pest of a wide variety of plants. The rmp mutant accumulates lower levels of an antibiosis activity against the GPA, which correlated with the increased fecundity of GPA on the rmp mutant compared to the wild-type (WT) plants. When given a choice, GPA preferred settling on the rmp mutant than the WT plants. Similarly, dispersal assays indicate that in comparison to the WT plants, emigration of GPA from the rmp mutant was reduced, thus indicating that the insect prefers the rmp mutant compared to the WT plant. RMP is required for turning on premature senescence and cell-death in response to extracts derived from the GPA. We hypothesize that $R M P$ is required for Arabidopsis response to an aphid-derived elicitor. Future efforts are directed towards understanding the mechanism underlying $R M P$ 's function in defense against the GPA. 
A genome-wide association study uncovers novel SNPs that affect the parasitic ability of Meloidogyne graminicola in rice A. Hada (1), T. K. Dutta (1), N. Singh (2), V. Rai (2), N. K. Singh (2), U. RAO (1), (1) ICAR- Indian Agricultural Research Institute, New Delhi, India; (2) ICAR- National Research Centre on Plant Biotechnology, New Delhi, India

Rice root-knot nematode (RRKN), Meloidogyne graminicola is one of the major biotic constraints in rice-growing countries of South-East Asia. Host plant resistance is an environmentally friendly and cost-effective mean to mitigate RRKN damage to rice. Considering the limited availability of genetic resources in Asian rice (Oryza sativa) cultivars, exploration of novel sources of RRKN resistance and dissection of the genetic basis is necessary. Herein, we screened 272 diverse (wild rice, landraces and improved cultivars) accessions to identify genotypes resistant to RRKN. We dissected the genetic basis of RRKN resistance using a genome-wide association study with SNPs genotyped by using $50 \mathrm{~K}$ "OsSNPnks" genic Affymetrix chip. Population structure analysis revealed that these accessions were stratified into three major subpopulations. Overall, 40 resistant accessions (nematode multiplication factor/MF $<2.5$ ) were identified, with 14 novel SNPs being significantly associated with phenotypic traits such as number of galls, egg masses, eggs per egg mass and MF per plant. SNPs were localised to the quantitative trait loci (QTL) on chromosome 1, 2, 3, 6, 8, 11 and 12 with putative functions of bZIP and WRKY transcription factor, ADPribosylation, NB-ARC domain, vacuolar ATPase etc. The identified SNPs enrich the repository for future marker-assisted breeding program to alleviate the damage of $\mathrm{RRKN}$ in rice.

Softening the Breaks: Releasing a Key Negative Immune Regulator Results in Enhanced Resistance to Nematodes M. A. Teixeira (1), D. Zhou (2), J. He (1), D. Godinez-Vidal (1), J. Van Norman (1), I. KALOSHIAN (1), (1) University of California Riverside, CA, U.S.A.; (2) Jiangsu Academy of Agricultural Sciences, China

Recent research has shown that plant-parasitic nematodes are perceived by plants similar to the perception of microbial pathogens. This perception in both tomato (Solanum lycopersicum) and Arabidopsis (Arabidopsis thaliana) requires the well-known co-receptor BAK1/SERK3, involved in the perception of a number of pathogens and herbivores. Using RNA-seq analysis of Arabidopsis bak1-5 mutant and wild-type Col-0 roots, infected with the root-knot nematode Meloidogyne incognita, we identified a lectin receptor-like kinase (LecRK; AtG-LecRK-VIII.8) null mutants of which displayed enhanced resistance to M. incognita infection. AtG-LecRK-VIII.8 belongs to G-type LecRKs (G-LecRK) encoding the typical G-LecRK domains, including the S-domain, in addition to the atypical EGF domain. Compared to wild-type plants, the $A t G$-LecRK-VIII. 8 mutants did not exhibit altered plant or root growth phenotypes. The enhanced resistance in the AtG-LecRK-VIII.8 mutants was manifested by faster and higher levels of ROS burst and increased transcript levels of immune-related genes. Complementation of a mutant with AtG-LecRKVIII.8, either behind the native or $35 \mathrm{~S}$ promoters, reversed the enhanced resistance phenotype. Taken together, AtG-LecRK-VIII. 8 is a negative regulator of nematode-triggered immunity providing a simple target for engineering resistance in crops to root-knot nematodes.

Egg-killing plants: Unravelling the genetics mechanisms of a butterfly egg-induced necrosis in Brassica crops N. BASSETTI (1), L. Caarls (1), G. Bukovinszkine Kiss (2), G. Bonnema (3), B. Zwaan (2), E. Schranz (1), N. Fatouros (1), (1) Biosystematics Group, Wageningen University \& Research, Netherlands; (2) Laboratory of Genetics, Wageningen University \& Research, Netherlands; (3) Laboratory of Plant Breeding, Wageningen University \& Research, Netherlands

Biotic stresses determine about a third of crop yield losses worldwide. Identification of resistance (R) genes has been successful against pathogens and partly against phloem-feeding insects such as aphids, whiteflies and planthoppers. However, R gene-mediated resistance against caterpillars (butterflies and moths) is still lacking. An alternative is to understand the insect egg-plant interaction, before that the feeding damage has occurred. Eggs of cabbage white butterflies (Pieris spp.) induce a necrotic lesion in different plant species of the Brassicaceae family. The necrosis resembles a programmed-cell death usually triggered by pathogens and it promotes egg mortality through egg desiccation and/or dropping off. We studied the genetic mechanisms of this Pieris egg-induced necrosis as it potentially represents a novel defense trait against these major cabbage pests. A germplasm screening of 58 Brassica rapa genotypes revealed genetic variation for egg-induced necrosis. Consequently, a phenotyping protocol based on image analysis was developed to measure lesions size and color. Finally, a panel of 160 Brassica rapa RILs was infested with eggs and the egg-induced necrotic lesions were phenotyped by imaging for QTL mapping. This study provides the first genomic regions associated with the Pieris egg-induced necrosis, possibly a step closer towards unravelling the first plant receptor triggering an eggkilling plant response.

Identification of genes involved on Arabidopsis thaliana response to Meloidogyne paranaensis J. P. TOMAZ, C. D. F. P. de Brito, A. C. Z. Machado, IAPAR, LONDRINA, Brazil

Besides of the importance of Meloidogyne paranaensis for coffee yields, studies with this pathosystem are time- and space-cosuming. Arabidopsis thaliana could be used to access knowledge about plant $\mathrm{x}$ nematode interactions by DNA site-directed mutant banks analysis. The aim of this work was to investigate the involvement of genes analysing A. thaliana mutants challegend with M. paranaensis. We tested 3 single mutants: rps5-1 (SALK_127201, Atlg12220), atpepr2-1 (SALK_036564, At1g17750), and a pseudo-nbs (SALK_080562, At4g14610); 1 double mutant: atpepr1-1 (SALK_059281, At1g73080) x atpepr2-1; and Col-0 as control. Plants were grown under 16 hours of light, at $22^{\circ} \mathrm{C}$ for 12 days. After this, plants were challenged with 500 specimens of M. paranaensis and grown at $25^{\circ} \mathrm{C}$ for 35 days. Roots were collected and nematodes were quantified to estimate nematode reproduction factor, which was submited to Scott-Knott test at 5\% significance. atpepr 2-1 and pseudo-nbs were grouped with Col-0, indicating that these genes are not involved in $A$. thaliana response to the nematode. rps $5-1$ was classified in a second group, with RP means higher than that presented by Col-0, which indicates that this gene has some role in plant response against the nematode attack. Moreover, the double mutants were grouped together with $r p s 5-1$, and since atpepr $2-1$ were grouped with Col-0, it indicates that AtPEPR1 could also have a role in plant defense or even acts sinergisctly with AtPEPR 2 in nematode recognition.

Effectors from poplar rust modulate Arabidopsis transcriptome

K. C. GONÇALVES DOS SANTOS, I. Desgagné-Penix, H. Germain, University of Quebec, Trois-Rivieres, QC, Canada 
Melampsora larici-populina (Mlp) causes the poplar rust and encodes at least 1184 candidate effectors (CEs), but their functions are poorly known. Our objective is to analyze the transcriptome of Arabidopsis constitutively expressing CEs of Mlp to discover processes targeted by these fungal proteins. For this, we extracted total RNA of Arabidopsis plants expressing one of 14 selected CEs and of a control line and sequenced their transcriptome with Illumina HiSeq. We aligned the reads to the Arabidopsis genome and then aligned the unmapped reads to the transgenes (CE::GFP and resistance gene). We observed high variation on the expression of the CEs, from 36.57 Transcripts Per Million (TPM) to 4992.48 TPM. We proceeded with the differential expression analysis using the most stably expressed genes as controls. We found 1752 deregulated genes in at least one of the 14 transgenic lines. These genes are related to defense, photosynthesis and growth. Four lines expressing CEs from different families showed a similar pattern of gene deregulation. This group contained downregulated genes associated to response to insects. We also found that plants expressing the CE Mlp124518 show an exclusive set of upregulated genes related to photosynthesis and transcription. Taken together our results demonstrate that effectors that have completely different sequences can alter expression of similar genes, while effectors of the same family can target completely different gene sets.

A novel auto-immune jasmonic acid mutant affects root hormone-defense crosstalk and associations with the microbiome Y. SONG, The University of British Columbia, Vancouver, BC, Canada

Plant roots are constitutively exposed to a nutrient and microbe rich soil environment. However, the molecular mechanisms underlying root immune responses to commensals and pathogens are largely unknown. Here we describe a forward genetic approach to reveal novel component of plant root immunity that shapes associations with the rhizosphere microbiome. Pseudomonas syringae produces a phytotoxin coronatine (Cor) to suppress pattern triggered immunity (PTI) in roots and leaves. A previous screen yielded $17 \mathrm{hsm}$ (hormone-mediated suppression of MAMPtriggered immunity) mutants that are impaired in COR-mediated suppression of root PTI marker genes. Interestingly, hsm 13 is an auto-immune mutant which is dwarf in soil, and transcriptome profiling revealed the constitutively activation of JA-responsive genes in $h s m 13$. Remarkably, we found that $h s m 13$ shows growth stunting after colonization of beneficial microbe Pseudomonas sp. WCS365, which indicates hsm 13 is incompatible with rhizosphere commensals. Through microbiome sequencing, we confirmed that hsm 13 shifted the rhizosphere microbiome composition and Pseudomonas sp. were enriched in hsm 13's rhizosphere. Ongoing mapping of HSM13 will elucidate the more details about the roles of HSM13 in JA signaling and plant-microbiome interactions.

Parallel Phytophthora and Pseudomonas effector screens to identify targets of effectors in soybean S. B. EASTMAN (1), J. R. Alfano (2), (1) University of Nebraska-Lincoln, Lincoln, NE, U.S.A.; (2) University of Nebraska, Lincoln, NE, U.S.A.

Soybeans are subject to infection by diverse plant pathogens such as the oomycete Phytophthora sojae and the bacterium Pseudomonas syringae pv. glycinea. Despite their evolutionary distance and distinct mechanisms of infection both $P$. sojae and $P$. syringae pv. glycinea utilize effectors to suppress soybean immunity. The $P$. sojae effector inventory is dominated by RXLR-domain effectors while the $P$. syringae effector inventory consists primarily of type III secreted effectors. We have cloned the repertoire of type III effectors from the $P$. syringae pv. glycinea strain LN10 and are screening these effectors for suppression of the hypersensitive response, callose deposition, or production of reactive oxygen species. By generating effector deletions mutants in $P$. syringae we are screening each effector for contributions to pathogenicity. Simultaneously, we are evaluating $P$. sojae RXLR effectors for their potential to contribute to immune suppression and are generating gene knockouts in $P$. sojae using CRISPR-Cas9. We are screening to research effectors of both $P$. sojae and $P$. syringae pv. glycinea using an integrated approach to identify both shared and unique effector targets. A shared target identified using this approach may represent a soybean protein with conserved function in immunity, while a target unique to effectors of a pathogen may represent a soybean protein important in a pathogen-specific immune response. We will present our current results in our poster.

Powdery mildew infection alters host metabolism to fuel fungal sporulation J. JAENISCH (1), J. Sasse (2), T. R. Northen (2), M. C. Wildermuth (1), (1) University of California, Berkeley, Berkeley, CA, U.S.A.; (2) Lawrence Berkeley National Laboratory, Berkeley, CA, U.S.A.

As obligate biotrophic pathogens, powdery mildew fungi are entirely reliant on their host for nutrients to fuel reproduction. The asexual spores that are produced have energy reserves in the form of large lipid droplets and glycogen reserves that are mobilized upon infection of a new host. I present how mutant host plants that restrict lipid metabolism affect fungal reproductive output, and how defects in carbon metabolism and FA trafficking affect label incorporation from plant tissue into storage lipids in fungal spores, as measured by LC/MS-MS. In addition, the analysis of mutants lacking transcription factors promoting seed development and associated lipid storage suggests their activation in leaves during infection contributes to fungal reproductive fitness. This work provides insight into the phase-specific requirements for culturing an obligate biotroph and highlights the induced change in host metabolism required to meet the demands of fungal asexual reproductive development.

Reduction of Phakopsora pachyrhizi infection of soybean through host and spray induced gene silencing D. Hu (1), Z. Y. CHEN (2), C. Zhang (3), M. Ganiger (4), (1) Louisiana State University, Baton Rouge, LA, U.S.A.; (2) Louisiana State University AgCenter, Baton Rouge, LA, U.S.A.; (3) Alcorn State University, Lorman, MS, U.S.A.; (4) University of Minnesota, St Paul, MN, U.S.A.

Host induced gene silencing (HIGS) is a mechanism in which small interfering RNAs (siRNAs) of pathogen are produced in the host plant that can silence their targets in the invading pathogen, which has become a novel way to manage plant diseases. In order to determine whether HIGS can be used to control soybean rust disease caused by the obligate fungal pathogen Phakopsora pachyrhizi, eight $P$. pachyrhizi genes were selected and cloned into a bean pod mottle virus (BPMV) based vector, which was introduced into soybean through bombardment. Soybean plants expressing three of these modified BPMV constructs suppressed the expression of their corresponding target gene, disease symptom development and fungal biomass. To further simplify the procedure, double-stranded RNAs (dsRNAs) for two of the genes, were synthesized in vitro and co-incubated with P. pachyrhizi urediniospores for $10 \mathrm{hrs}$ before inoculating onto detached soybean leaves, which also significantly 
reduced rust accumulation on the detached leaves compared to controls. In addition, direct spraying of the above two synthesized dsRNAs and another one targeting P. pachyrhizi GCS_H protein on soybean leaves prior to rust inoculation also resulted in an average of over $75 \%$ reduction of pustule numbers compared to control. To the best of our knowledge, this is the first report of suppressing $P$. pachyrhizi infection in soybean through both HIGS and spray induced gene silencing (SIGS).

Azospirillum brasilense is a member of the seed microbiome that forms an endophytic relationship with plant roots where it alters root development through production of auxin and cGMP

C. E. BAUER, K. E. Thum, E. Malinich, Indiana University, Bloomington, IN, U.S.A.

Azospirillum brasilense is a plant growth promoting bacterium that is used as a bio-fertilizer world-wide. Plants inoculated with $A$. brasilense display increased root length, root branching, and root hair formation via A. brasilense's ability to produce several plant hormones such as auxin and cytokines. Stimulation of root development is thought to account for reported increases in plant fitness and crop productivity in plants grown from seeds that are surface inoculated with $A$. brasilense. Until recently, A. brasilense was considered to be a rhizosphere mutualist only, however, using quantitative PCR, deep sequencing and confocal microscopy with a GFP expressing strain of $A$. brasilense, we have recently shown that $A$. brasilense associates with the entire plant. In particular, we have shown that $A$. brasilense is a significant component of the plant seed microbiome among several crop plants. We have further shown that that $A$. brasilense migrates from roots to flowers early in seed development to populate the seed coat. Furthermore, we have also shown that $A$. brasilense that is naturally present between seed coat cells is more effective at root colonization then is $A$. brasilense that is artificially inoculated on the surface of seeds. Finally, we have also explored the role of $A$. brasilense excreation of auxin and cGMP on Arabidopsis seedling root development.

Expression analysis-based selection of candidates to screen for reduced susceptibility to Verticillium dahliae in tomato K. HANIKA, H. Schouten, B. P. H. J. Thomma, Y. Bai, Wageningen University, Wageningen, Netherlands

Worldwide, vascular wilts caused by the soil-borne pathogen Verticillium dahliae are devastating fungal diseases that affect a wide range of crops. They are particularly difficult to control due to the long viability of fungal resting structures in the soil, the broad host range, and the ineffectiveness of fungicide treatment. Hence, disease management is highly dependent on host plant resistance. However, sources of dominant resistance have not been found for most host species. The use of recessive resistance resulting from impaired susceptibility genes ( $S$-genes) offers an alternative strategy for resistance breeding. $S$-genes are host genes that are used by the pathogen to facilitate a compatible host-pathogen interaction. As hardly any knowledge on $S$-genes is available for $V$. dahliae, this study aims at identifying $S$-genes of tomato (Solanum lycopersicum). Gene expression data of compatible and incompatible interactions were analysed to identify genes that were preferentially induced during the compatible interaction in the roots or in the leaves. Subsequently, the top 120 of these candidate genes were silenced by virus-induced gene silencing and plants were challenged with $V$. dahliae to screen for reduced susceptibility. A set of four promising candidate genes was selected and stable RNA interference and CRISPR/Cas transformants are generated to further study these candidates.

Unravelling a potential pathway linking plasma membrane to chloroplasts and its role in the regulation of plant defence responses. L. MEDINA-PUCHE, Shanghai Center for Plant Stress Biology (PSC), Shanghai, China

Plant pathogens need to suppress plant defence responses in order to establish a successful infection. Plants have diverse strategies against viral invasion, which include production of defensive hormones such as salicylic acid (SA). C4, a small protein encoded by Tomato yellow leaf curl virus and essential for infectivity, contains two overlapping localization signals, an N-myristoylation motif and a chloroplast transit peptide, and consistently localizes in two distinct subcellular compartments, namely plasma membrane (PM) and chloroplasts (1). PM localization overrides chloroplast localization, since a non-myristoylable mutant version of $\mathrm{C} 4\left(\mathrm{C}_{\mathrm{G} 2 \mathrm{~A}}\right)$ localizes to chloroplasts exclusively; moreover, activation of defence responses leads to a re-localization of $\mathrm{C} 4$ from $\mathrm{PM}$ to chloroplasts. We have found that $\mathrm{C}_{\mathrm{G} 2 \mathrm{~A}}$ interferes with retrograde signaling from the chloroplast through the interaction with the chloroplast calcium sensing receptor protein, CAS, which is required for the pathogen-associated molecular patterns (PAMP)-induced expression of defence genes, SA biosynthesis, and regulation of cytoplasmic calcium oscillations (2), all of them repressed by $\mathrm{C}_{\mathrm{G} 2 \mathrm{~A}}$. Interestingly, this dual localization is also present in a number of pathogen effectors and in a subset of defence-related plant proteins. These findings suggest that an intrinsic route in plants delivering proteins from the PM to chloroplasts might be hijacked by plant pathogens to suppress defence responses.

The Arabidopsis receptor-like kinase IOS1 links filamentous pathogen attack to endoplasmic reticulum stress L. GIORDANO, INRA, UMR ISA, sophia antipolis, France

To sense the environment, plant cells possess more than 200 plasma membrane-spanning receptors, which are composed of extracellular leucinerich repeats (LRRs) and an intracellular kinase domain. We previously identified a member of this LRR-RLK family in Arabidopsis, which contributes to the infection success of filamentous, biotrophic pathogens such as oomycetes. The extracellular region of the LRR-RLK "Impaired Oomycete Susceptibility 1" (IOS1), is composed of LRRs and a domain, which shares similarities with malectin from animals. Animal malectins interact with ribophorins of the Oligosaccharyltransferase (OST) complex in the endoplasmic reticulum (ER), where they bind carbohydrates and monitor proper folding of glycoproteins. We observed retention of IOS1 in the ER, which is mediated through the "malectin-like" (ML) domain. We show that the extracellular ML domain of IOS1 interacts in yeast with a ER membrane-localized plant ribophorin. We assessed the role of IOS1, and of it's exodomain for plant responses to both downy mildew infection and ER stress using mutant analyses, and by overexpressing individual IOS1 domains in Arabidopsis. We show that the "Unfolded Protein Response" (UPR) is affected in both the iosl mutant and in transgenic Arabidopsis over-expressing the extracellular ML domain. Our data suggest that the individual domains of IOS1 convey dual functions to the receptor in different subcellular compartments. 
Microbial small molecules - weapons of plant subversion

R. DE JONGE, E. Li, H. Zhang, C. M. J. Pieterse, P. A. H. M. Bakker, Plant-Microbe Interactions, Department of Biology, Utrecht University, Utrecht, Netherlands

Plant roots are associated with a diverse microbial community containing detrimental as well as mutualistic organisms. While mutualistic plantmicrobe interactions are essential for plant performance, the evolutionary origin of rhizosphere mutualism remains mysterious. Here we examined the evolutionary dynamics of an originally detrimental rhizosphere plant-bacterium interaction. Bacteria rapidly diversified on plant roots into de novo phenotypes ranging from detrimental to mutualistic, with the latter becoming dominant after a few plant cycles. Genetically, transition from antagonism to mutualism was associated with mutations in global regulators controlling phytotoxic bioactive compound synthesis. Moreover, we find that mutations affecting lipopolysaccharide modifications, via disruption of multiple different genes on a small 16-gene genetic island, are selected rapidly and repeatedly. We conclude that plants on the one hand control their microbiota over evolutionary time scales by domesticating wild microorganisms, while microorganisms on the other hand can adapt rapidly by altering their external appearance.

The bacterial root microbiota of Arabidopsis thaliana influences the trade-off between plant growth and defense R. T. NAKANO, R. Garrido-Oter, P. Schulze-Lefert, Max Planck Institute for Plant Breeding Research, Cologne, Germany

Healthy plants in nature are densely populated by taxonomically diverse microbes called the plant microbiota. The genomes of these commensal microbes encode conserved microbial epitopes, designated MAMPs, which are predicted to be perceived by the plant immune system. Chronic activation of MAMP-triggered plant responses results in root growth inhibition (RGI), which is thought to be consequence of a plant growthdefense trade-off. However, it remains unclear how plants coordinate growth and immunity in natural environments in the presence of associated commensal microbes. We here describe commensal bacteria from the root microbiota of Arabidopsis thaliana that interfere with host responses to the well-characterized MAMP flg22 peptide and suppress flg22-triggered RGI. Physical attachment of the commensal to roots is dispensable for MAMP response suppression, suggesting a type-III and type-IV secretion system-independent interference mechanism and the involvement of diffusible bacterial molecules. Transcriptomic analyses of $A$. thaliana roots revealed that expression of immune marker genes is retained despite a clear suppression of RGI. Overall our findings suggest that commensal bacteria can either directly or indirectly uncouple the trade-off between plant growth and defense, demonstrating an important role of the microbiota for plant growth and fitness in nature.

The Xanthomonas type-three effector XopS interferes with proteasomal turnover of a WRKY transcription factor to dampen the induction of plant defence responses

M. RAFFEINER (1), T. Guerra (1), S. Üstün (2), F. Börnke (1), (1) Leibniz Institute of Vegetable and Ornamental Crops, Großbeeren, Germany; (2) Center for Plant Molecular Biology (ZMBP), Tübingen, Germany

In plants, WRKY transcription factors play important roles as positive and negative regulators of defence gene expression. Many Gram-negative plant pathogenic bacteria rely on so called type-III effector (T3E) proteins as virulence factors to interfere with the induction of defence responses at different levels. We show here, that the T3E XopS of Xanthomonas campestris pv. vesicatoria, causal agent of bacterial spot in pepper, interacts with the transcription factor WRKY40 in vitro and in planta. Transient expression in leaves of Nicotiana benthamiana suggests that WRKY40 undergoes rapid proteasomal protein turnover. However, WRKY40 protein strongly accumulates upon co-expression with XopS, indicating that XopS interferes with proteasomal turnover of WRKY40 and thus could interfere with defence gene induction that requires degradation of this negative regulator of plant defence. Furthermore, it is shown that XopS interferes with the expression of several defence related genes during infection. Different strategies to unravel the mode of action of XopS and to assess the role of the XopS-WRKY40 interaction in suppression of plant immunity are discussed here.

The Verticillium dahliae effector Ave1 manipulates the host microbiome

N. C. SNELDERS (1), M. Rocafort Ferrer (2), J. R. Mesters (3), M. F. Seidl (1), R. Nijland (1), B. P. H. J. Thomma (1), (1) Wageningen University, Wageningen, Netherlands; (2) Massey University, New Zealand; (3) University of Lübeck, Germany

Pathogen-secreted effectors are typically studied in a particular plant-pathogen interaction, while largely ignoring the (a)biotic context. However, plants associate with an overwhelming number of microbes that collectively form the microbiome, which represents a key determinant for plant health. Moreover, plants actively shape the composition of their root microbiome by recruiting beneficial microbes to suppress pathogen invasion. Consequently, it can be anticipated that plant pathogens have evolved mechanisms to affect other microbes within that plant microbiome to successfully establish infection. Previously, we identified the effector protein VdAve1 as virulence factor of the broad host-range soil-borne vascular wilt pathogen Verticillium dahliae. Using antimicrobial activity assays combined with electron microscopy we reveal that VdAve1 is an antimicrobial protein with bactericidal activity that most effectively affects Gram positive bacteria. Metagenomic characterization of the tomato root microbiome revealed that secretion of VdAve1 by $V$. dahliae changes the host microbiome. Collectively, our data suggests that $V$. dahliae exploits VdAve1 to affect the composition of the host microbiome, presumably to promote the establishment of infection.

Functional analysis of the RsRIpA effector in the soil borne pathogen Rhizoctonia solani AG2-2IIIB revealed a potential biotrophic stage upon infection

F. Dölfors (1), L. Holmquist (2), P. Moschou (3), C. Dixelius (4), G. TZELEPIS (1), (1) Department of Plant Biology, Swedish University of Agricultural Sciences, Uppsala, Sweden; (2) MariboHilleshög Research AB, Sweden; (3) Department of Biology, University of Crete, Greece; (4) Swedish University of Agricultural Sciences, Department of Plant Biology, Uppsala, Sweden

Rhizoctonia solani (Rs) is a soil borne pathogen with a broad host range causes mainly damping-off in seedlings, although older plants can also be infected. It belongs to Basidiomycota class and its isolates are categorized to anastomosis groups (AGs), further subdivided to subgroups. Isolates from the AG2-2IIIB subgroup mainly attack sugar beets. Genome analysis of an isolate in this subgroup revealed 11 unique effector genes as compared to already published genomes from other AG strains. A gene candidate encoding a rare lipoprotein-A-like protein (RsRplA), 
contain a double-psi beta-barrel domain (DPBB, IPR009009) and its predicted 3D structure showed high similarities to papain-like inhibitors. The RsRplA gene was highly induced at early stage of sugar beets infection and heterologous expression in Cercospora beticola led to increased fungal colonization as compared to WT strain. RsRlpA was localized to $C$. beticola hyphal periphery and to nucleoplasm and plasma membrane in plant cells. RsRlpA can suppress hypersensitive response (HR), triggered by the Avr4 effector in Cf- 4 transgenic plants. The amino acid serine at position 120 in the DPBB domain seems to be crucial for HR suppression, since substitution with threonine abolished this function. Overall, this study showed that $R$. solani, which has been considered as a necrotroph, relies on effectors others than those causing necrosis in order to establish a successful infection, suggesting a hemibiotrophic lifestyle.

PhoP of Pectobacterium carotovorum subsp. brasiliense (Pcb1692) represses T6SS and carbapenem production in planta N. P. NKOMO, university of Pretoria, Pretoria, South Africa

Pathogenicity of Pectobacterium carotovorum subspecies brasiliense 1692 (Pcb1692), the causal agent of soft rot disease on many plants, is a complicated process encompassing several virulence factors regulated by a complex regulatory network. In this study we characterized the role of the Pcb1692 PhoP transcriptional regulator in terms of virulence and fitness. RNA-seq analysis was used to determine the full regulon of PhoP in planta at 12 and $24 \mathrm{hrs}$ postinfection. Transcriptome analysis showed that a total of 500 genes, representing about $20 \%$ of the $P c b 1692$ genome, were differentially regulated at both 12 and $24 \mathrm{hrs}$. The results showed that $P c b 1692 \Delta p h o P$ was less virulent compared to the wildtype strain when inoculated into potato tubers. In addition, genes associated with the type VI secretion system and carbapenem were upregulated in $P c b 1692 \triangle p h o P$ relative to the $P c b 1692$ wild type strain. In planta bacterial competition assays demonstrated that the $P c b 1692 \Delta p h o P$ mutant outcompetes Dickeya dadantii five-fold more compared to the wild-type strain. Apart from a role in antibacterial competition, PhoP was shown to play a role in motility and production of plant cell wall degrading enzymes.

Comparative analysis of rice sensing of plant-beneficial Burkholderia

E. KING (1), L. Moulin (2), P. Czernic (1), (1) Univ Montpellier, IRD, Cirad, IPME, Montpellier, France, Montpellier, France; (2) IRD, Cirad, Univ Montpellier, IPME, Montpellier, France, Montpellier, France

In the context of plant-pathogen and plant-mutualists interactions, the underlying molecular bases associated with host colonization are well described. However, it is not the case for non-mutualistic beneficial interactions or associative symbiosis. Particularly little is known about the transcriptional regulations associated with the "immune tolerance" of crop plants towards beneficial microbes. In this context, the study of the Burkholderia-rice model is very promising to describe the molecular mechanisms implicated in associative symbiosis. Indeed, several species of the Burkholderia genus can colonize rice tissues and have beneficial effects. We produced the transcriptome at 7 dpi of rice plants inoculated with two rice root-associated Burkholderia s.l. (B. vietnamiensis, P. kururiensis) through RNA-Seq and proceeded to their comparative analysis. This study revealed that extensive transcriptional regulations occur in leaves throughout the establishment of the interaction at the root level. However important differences were observed, although the studied Burkholderia species are phylogenetically closely related, especially the defense response of rice roots during bacterial colonization were very different. We were also able to identify hormonal signaling pathways showing opposite regulations in leaves depending on the inoculated species of which the expression of related genes was analyzed throughout the establishment of the interaction.

Xylem sap proteomics reveals distinct differences between $\boldsymbol{R}$ gene- and endophyte-mediated resistance against Fusarium wilt disease in tomato

F. J. DE LAMO (1), M. E. Constantin (1), D. H. Fresno (1), S. Boeren (2), M. Rep (1), F. L. Takken (1), (1) University of Amsterdam, Amsterdam, Netherlands; (2) Wageningen University \& Research, Wageningen, Netherlands

Both Resistance $(\mathrm{R})$ genes and endophytes can confer disease resistance. We set out to phenotypically and molecularly characterize the underlying resistance mechanisms in the tomato (Solanum lycopersicum) - Fusarium pathosystem. As resistant control tomato cultivars were used that contain the $I-2 R$ gene that confers resistance to the vascular wilt fungus $F$. oxysporum f. sp. lycopersici (Fol). The endophytic Fusarium oxysporum (Fo) strain Fo47 that confers protection against Fol was used for comparison. Fol colonizes the xylem vessels of susceptible and I-2 tomato plants, but only causes disease in the former. Fol was also found to colonize the vasculature of endophyte-colonized plants, and could be isolated from stems of non-diseased plants co-inoculated with Fo47 and Fol. Because the xylem vessels form the main interface between plant and pathogen, the xylem sap proteomes during $R$ gene- and Endophyte-Mediated Resistance (EMR) were compared by label-free quantitative nLC-MS/MS. Both proteomes were remarkably similar to the mock, revealing only one and two differentially accumulated proteins in the respective interactions. The role of these proteins in Fol resistance is currently being studied and the findings will be reported. Notably, over $54 \%$ of the identified proteins lack discernable secretion signatures implying that they might be present in exosomes.

Characterization of the effect of Ralstonia solanacearum NLS-containing type III effectors on plant immunity and development H. JEON (1), W. Kim (1), S. Lee (1), J. Jayaraman (2), K. H. Sohn (3), S. Cécile (1), (1) Seoul National University, Seoul, Korea, Republic of (South); (2) The New Zealand Institute for Plant and Food Research Ltd, Auckland, New Zealand; (3) Pohang University of Science and Technology, Pohang, Korea, Republic of (South)

Ralstonia solanacearum (Rso) causes lethal bacterial wilt in a wide range of food crops. Rso injects type III effectors (T3Es) directly into host cells through the type III secretion system that is essential for pathogenicity. T3Es can dampen plant immunity and affect various cell processes to provide a favorable environment for the bacterial proliferation. Owing to the diversity and complexity of Rso T3E repertoire, the functional characterization of Rso T3Es remains scarce. Here, we selected 10 Rso T3Es that are predicted to carry a nuclear localization signal motif and investigated their impact on plant immunity and development. Using Agrobacterium-mediated transient expression, we found that several effectors triggered programmed cell death, which suggests possible recognition by the host immune system. Other effectors impaired pathogenassociated molecular pattern-triggered reactive oxygen species burst and defense-marker gene expression, indicating that they interfere with pattern-triggered immunity. To confirm the immuno-suppressive activity and to examine the effect of the selected effectors on root development, 
we generated Arabidopsis thaliana effector-expressing lines (EELs). EELs will be further examined to identify molecular targets of Rso T3Es and assess their contribution to Rso virulence. Unraveling the molecular mechanisms through which Rso T3Es perturb certain host cellular processes will help to design and engineer bacterial wilt resistant crops.

Phytophthora RXLR-WY effectors cooperate to modulate host vesicle trafficking

M. P. CONTRERAS (1), B. Petre (1,2), T. O. Bozkurt (3), J. Win (1), S. Kamoun (1), (1) The Sainsbury Laboratory, University of East Anglia, Norwich Research Park, Norwich, U.K.; (2) Université de Lorraine, Nancy, France; (3) Imperial College London, U.K.

Pathogens secrete dozens to hundreds of effector proteins to reprogram multiple host processes and facilitate infection. However, the mechanisms through which effectors subvert these processes and the extent to which they cooperate to achieve this reprogramming are not fully understood. In this work, we studied how two RXLR-WY effectors from the oomycete pathogen Phytophthora infestans, PexRD31 and PexRD54, cooperate to alter host vesicle trafficking. While PexRD54 has an established role in modulating selective autophagy, we report that PexRD31 perturbed vesicle trafficking, leading to an increase in endosome numbers. Transient co-expression in Nicotiana benthamiana showed that PexRD31 and PexRD54 co-localize in mobile punctate bodies that accumulate at haustoria during infection. In-planta co-immunoprecipitation showed that both effectors associate and share host interactors. We hypothesize that PexRD31 and PexRD54 cooperate to alter vesicle trafficking during infection. Current work focuses on further characterizing the association between these two effectors and how they function in concert. By dissecting the molecular mechanisms of these virulence proteins, we hope to gain a better understanding of how host processes are reprogrammed during infection and exploit this knowledge to engineer resistance in crops.

New effectors from the multihost blast fungus Magnaporthe oryzae target HMA domain containing host proteins Y. PETIT (1,2), J. Win (1), T. Langner (1), A. Harant (1), R. Terauchi (3,4), M. J. Banfield (5), S. Kamoun (1), (1) The Sainsbury Laboratory, University of East Anglia, Norwich Research Park, Norwich, U.K.; (2) UMR BIOGER, INRA, AgroParisTech, Université Paris-Saclay, Thiverval-Grignon, France; (3) Graduate School of Agriculture, Kyoto University, Kyoto, Japan; (4) Iwate Biotechnology Research Center, Kitakami, Japan; (5) John Innes Centre, Norwich Research Park, Norwich, U.K.

Magnaporthe oryzae is a multihost pathogen that infects more than 50 species of grasses, including the cereal crops wheat, barley and rice. This pathogen deploys an arsenal of secreted proteins called effectors to infect its host plants and counteract plant defences. How $M$. oryzae effectors have adapted to various grass hosts is poorly understood. Three effectors, AVR-Pia, AVR1-CO39 and AVR-Pik are known to bind sHMA proteins but can also be recognized by NLR resistance proteins with integrated HMA domains. The aim of this project is to discover new $M$. oryzae effectors that interact with sHMA proteins and determine the diversification of these effectors in host-specific lineages of $M$. oryzae. First, we predicted candidate effectors from the secretomes of $M$. oryzae strains infecting different grass hosts. We used two major criteria: i) proteins with sequence similarity to known avirulence effectors; ii) Hidden Markov Model profile matches to the MAX structural fold. This resulted in 185 potential effector candidates, which we synthesized and cloned as level 0 Golden Gate vectors, and publicly released as a community bioresource. We have been testing these candidates for their ability to interact with sHMA proteins using yeast two-hybrid screens. We have now identified and validated several new effector-HMA interacting pairs. We will build on this work to improve our understanding of the evolution and diversification of HMA-targeting effectors in M. oryzae.

Phytoplasma effectors have converged onto degrading plant transcription factors with fundamental roles in plant development and defense to insects W. HUANG, S. A. Hogenhout, John Innes Centre, Norwich, U.K.

Phytoplasmas are intracellular bacterial parasites of plant phloem sieve cells and are transmitted by phloem-feeding insects. Being dependent on both hosts, they have evolved unique strategies to modulate these divergent organisms and their interactions. A recurring feature of phytoplasma disease is the generation of Zombie plants that show dramatic symptoms and are sterile and stay alive only for the spread of phytoplasmas. Previous research showed that phytoplasma effectors SAP11 and SAP54 destabilize plant TCP and MADS-box transcription factors (TFs) and SAP54 does this via the proteasome shuttle factor RAD23. Here, we found that phytoplasma effector SAP05 degrades plant SPL and GATA TFs thereby disturbing plant phase transitions, reproductive organ development and lateral branching, inducing symptoms that are common in Zombie plants. The SAP05-mediated SPL and GATA degradation relies on hijacking the plant ubiquitin receptor RPN10 and 26S proteasome. Intriguingly, SAP05 homologs are present in divergent phytoplasmas and have evolved to differentially degrade SPLs or GATAs, and only SAP05 homologs that degrade SPLs promote phytoplasma insect vector fecundity. Notably, SAP05 does not interact with insect vector RPN10, and only minor mutations of the plant RPN10 generated plants that are resistant to SAP05 actions. In summary, phytoplasma effectors have converged to degrade diverse plant TFs. Moreover, we discovered how to generate plants that have better Zombie resistance.

Identification of novel downy mildew proteins with diverse effects on the plant innate immune system K. J. WOOD (1), K. Fletcher (1), J. Gil (1), Y. Zhai (2), W. Ma (3), R. W. Michelmore (1), (1) University of California, Davis, CA, U.S.A.; (2) University of California, Riverside, CA, U.S.A.; (3) University of California, Riverside, Riverside, CA, U.S.A.

Identification of effectors in pathogen genomes is foundational for understanding the mechanisms of pathogenesis, monitoring field pathogen populations, and for breeding for resistance. Using comparative genomics and bioinformatics, we identified candidate effectors from downy mildew of lettuce, Bremia lactucae, by searching for WY domains, which are implicated in the function of Phytophthora effectors. These candidate effectors have characteristics of pathogen effectors, including a secretion signal, lineage specificity, and evidence of gene duplication and gene family expansion. Unexpectedly, only a minority of WY effectors contained the canonical N-terminal RXLR motif, which is reported to be a conserved feature in Phytophthora effectors. Functional characterization of 21 of the WY domain effectors revealed 11 effectors that elicited an immune response on resistant lettuce genotypes. Several of the recognized WY effectors were found to have RNA silencing suppression activity, while others were found to have immune suppression activity. Our results help characterize the effector repertoire in lettuce downy 
mildew and also suggest that there has been evolutionary divergence in sequence motifs between genera that has important implications for effector prediction in downy mildews and other oomycetes.

Ralstonia solanacearum subverts plant metabolism to support bacterial nutrition and cause bacterial wilt disease L. Xian, G. Yu, Y. Wei, Y. Li, H. Xue, J. Chen, R. Morcillo, Y. Sang, J. S. Rufian, A. P. MACHO, Shanghai Center for Plant Stress Biology, China

Plant diseases caused by bacterial pathogens pose an important threat to food security worldwide. Ralstonia solanacearum is one of the most destructive bacterial plant pathogens, and is able to infect important crops, including potato, tomato, tobacco, banana, pepper and eggplant, among others. To induce disease, bacterial pathogens have to suppress plant immunity and manipulate plant cells to shape a niche suitable for bacterial replication. Although it is assumed that bacterial pathogens need to manipulate plant metabolism to support bacterial nutrition, the specific components of this manipulation and their molecular mechanisms of action remain unknown. In this work, we uncover a bacterial effector protein that, when delivered inside plant cells, interacts with metabolic enzymes to enhance the biosynthesis of GABA. Besides being a signaling molecule in eukaryotic cells, GABA constitutes an excellent nutrient to support the growth of $R$. solanacearum during the infection process. We have performed a detailed molecular characterization of this phenomenon and revealed that this process is essential for the development of bacterial wilt disease.

Integrated strategy to unravel the biological role of evolutionary conserved type III effectors from xylem-colonizing bacteria M. GONZALEZ-FUENTE (1), C. Remblière (2), S. Carrere (2), N. Arroyo-Vélez (2), N. Peeters (3), L. D. Noel (1), (1) Université de Toulouse, LIPM, CNRS-INRA, Toulouse, France; (2) Université de Toulouse, INRA, CNRS, Toulouse, France; (3) LIPM, Université de Toulouse, INRA, CNRS, INPT, Castanet-Tolosan, France

Xylem-colonizing bacteria are among the most devastating plant pathogens worldwide. Xanthomonas campestris pv. campestris (Xcc), the causal agent of black rot disease on Brassicaceae, and Ralstonia solanacearum (Rs), the causal agent of bacterial wilt on more than 200 plant species, are both xylem-colonizing bacteria. Despite their differences in host range, infection strategy and effectome repertoire, reference strains Xcc 8004 and $R s$ GMI1000 share six orthologous type III effectors (T3Es). This provides a valuable opportunity for comparative studies as it is likely that these orthologous T3Es target orthologous processes in the host plants. Our strategy combines protein-protein interaction screenings,

physiological analyses of transgenic plants, genetic screens and reverse genetics approaches to identify 'biologically relevant' interactors of these orthologous T3Es focusing on Arabidopsis thaliana, common host of both pathogens. Altogether, this project will provide a better understanding of the biological role of these T3Es conserved through evolution.

Screening of small secreted proteins of $\mathrm{Z}$. tritici reveals a candidate protein that interacts with host ubiquitin system S. J. KARKI, UCD; School of Agriculture and Food Science, UCD Earth institute, Dublin, Ireland

Septoria Tritici Blotch, caused by the ascomycete fungus Zymoseptoria tritici, is a major threat to wheat production worldwide. The Z. tritici genome encodes for many small secreted proteins $(Z t S S P)$ that likely play a key role in the successful colonisation of host tissues. In this study, we screened thirty of these ZtSSP for the induction of cell death in the non-host model plant Nicotiana benthamiana. We identified five novel candidates that resulted in the rapid accumulation of $\mathrm{H}_{2} \mathrm{O}_{2}$ and cell death. Transient overexpression of these five candidates also resulted in the upregulation of defense marker genes in $N$. benthamiana. These five candidates were further tested for cell death induction in the host wheat. However, our assay showed that none of these induce cell death in wheat, which might suggest host specificity of ZtSSP function and lack of recognition in the host. Additionally, yeast two-hybrid assay using one of the cell death-inducing candidate ZtSSP2 showed that it interacts with wheat E3 ubiquitin ligase in yeast and in planta. Expression analysis of wheat ubiquitin ligase suggests that wheat infected $Z$. tritici plants showed significant downregulation of TaE3UBQ expression at specific time points (4 dpi and $21 \mathrm{dpi}$ ) suggesting its potential role in regulating defense signaling during the wheat-Z. tritici interaction.

The two faces of an effector. Does HopAG1 exert contrasting activities?

M. GÓRECKA (1), P. B. Zembek (2), K. Pawłowski (3), J. Hennig (2), M. Krzymowska (2), (1) Institute of Biochemistry and Biophysics PAS, Warsaw, Poland; (2) Institute of Biochemistry and Biophysics, PAS, Warsaw, Poland; (3) Faculty of Agriculture and Biology, Warsaw University of Life Sciences, Warsaw, Poland

Pseudomonas syringae uses type three secretion system to deliver proteins called effectors into host cells. The effectors affect a variety of cellular processes in order to take over the host defence systems and to avoid recognition. Over a hundred of various effector families have been so far identified in $P$. syringae, but their mechanisms of action remain poorly understood. HopAG1, belonging to one of those effector families, is present in an array of $P$. syringae pathovars and its homologues are found in other bacterial species.

Bioinformatics analyses of HopAG1-like sequences revealed three putative catalytic domains ART (ADP-ribosyltransferase), kinase, and Nudix (nucleoside diphosphate-linked to some moiety-X) hydrolase. The presence of putative ART and Nudix domains seems to be especially intriguing as they were reported to display oppose activities.

In order to characterise the effector properties we split the HopAG1 encoding sequence from P. syringae pv syringae B728a into three segments corresponding to the respective domains and expressed them in plant and bacterial systems The single domains exhibited defined subcellular localisation in Nicotiana benthamiana epidermal cells, while the intact HopAG1 was observed in the cytoplasm, chloroplasts and nucleus, which may suggest that different biochemical activities of HopAG1 are exhibited in particular compartments of the cell. Our studies focus on identification of host targets specific for these cellular compartments. 
Bacillus strains modulate immune response, biomass production and flowering time in Arabidopsis and Brachypodium distachyon. C. JIMENEZ-QUIROS (1), Ö. Baysal (2), P. Nicholson (3), M. Tor (4), (1) University of Worcester, Worcester, U.K.; (2) Muğla Sitkı Koçman University, Turkey; (3) John Innes Centre (JIC), Norwich Research Park, Norwich, U.K.; (4) School of Science and the Environment, Worcester, U.K.

Bacillus strains have been used on crop protection on many different crops for long time, due to their PGP activity as well as biological controls of plant diseases. Both characteristics are desirable as a strategy to protect environment since there is a potential contribution in the reduction of pesticide use. We hold three Bacillus strains capable to control Fusarium graminearum growth in vitro. Assays in vivo revealed that, these bacteria interact with Arabidopsis plants to promote a better response to pathogens attack, increase biomass production and modify the flowering time. Plants treated with these Bacillus strains showed significant difference in the level of growth characteristics in comparison to the control. Moreover, we found that these same bacteria, as PGPs, can modulate the flowering time, head number and biomass growth of the small grass Brachypodium distachyon. Lyons et al. (2015) highlighted how the infection by Fusarium oxysporum accelerate the flowering time on Arabidopsis plants. In our system, the pathogen Fusarium graminearum induced some responses on plants but the interaction with the Bacillus strains changed that response. We will present the latest results in which we investigated the expression of defence genes within this system.

Crispr-engineered Bs4 mutants facilitate identification of TALE target genes in tomato K. SCHENSTNYI, R. Morbitzer, A. Strauss, T. Lahaye, ZMBP, Tübingen, Germany

Transcription Activator-Like Effectors (TALEs) from the bacterial plant pathogen Xanthomonas bind to Effector Binding Elements (EBEs) present in host promoters and activate transcription of downstream host genes to promote disease or to trigger resistance. The DNA-binding specificity of TALEs is defined by tandem-arranged 34/35-amino acid repeats, each binding one DNA base in a given EBE. TALEs with desired sequence specificity (dTALEs) can be generated by assembly of individual TALE repeats to activate transcription of user-defined genes of interest (GOIs). However, in tomato plants the nucleotide-binding leucine-rich repeat (NB-LRR) resistance protein Bs4, recognizes TALEs and initiates a plant immune response. Thus, in tomato dTALEs cannot be used to activate desired GOIs. To overcome this limitation, we engineered a tomato Bs4 mutant using the CRISPR/Cas9 system that now facilitates the use of dTALEs. Moreover, delivery of native TALEs into the Bs4knockout tomato and subsequent transcriptome profiling can now be used to uncover otherwise hidden TALE host target genes.

Equilibrium» in the Pectobacterium-Plant Pathosystem: Physiological Bases and Molecular Players

V. GORSHKOV (1,2), O. Petrova (1), R. Gubaev (1), M. Ageeva (1), A. Daminova (1), N. Gogoleva (1,2), O. Parfirova (1,2), B. R. Islamov (1), I. Tsers $(1,2)$, V. Vorob'ev $(1,2)$, Y. V. Gogolev $(1,2),(1)$ Kazan Institute of Biochemistry and Biophysics FRC "KSC of RAS”, Kazan, Russian Federation; (2) Kazan Federal University, Kazan, Russian Federation

Plant soft rot caused by pectobacteria (PB) is a result of the collapse of host parenchymatous tissues. However, PB spend most part of their life not in parenchyma, but in the xylem vessels without causing the disease. In this case, the pathogen behaves stealthy and the pathosystem is in equilibrated state. But as PB colonize parenchyma applying their brute force, the equilibrium is disturbed. The aim of our study is to elucidate the criteria of stealth and brute force behavior of PB. By NGS transcriptomics, several molecular players (including the novel ones) have been suggested as the "switches" that turn the peaceful coexistence of host and pathogen to the war-like one. By using several approaches (involving mutagenesis, cloning, chromatography, NMR-spectroscopy, microscopy, phytohormone application, immunodetection, etc), some of those "switches" have been proved to be valid. Phytohormones (ethylene, and jasmonic and salicylic acids), ROS, PB exopolysaccharides, coronafacic acid, and low molecular weight extracellular phosphonates, plant and bacterial enzymes and proteins for plant cell wall modification are among those "switches". The obtained results allow us to reveal the induced susceptible plant responses that drive PB behavior in planta, to find new criteria of PB aggressiveness, and to draw a general picture of the mechanisms that support/disturb equilibrium in plant-microbe pathosystem. The study is supported by RSF № 19-14-00194.

Yellow rust infected field data reveals temporal differences in the expression of host primary metabolic pathways P. CORREDOR MORENO (1), P. E. Davey (2), D. G. O. Saunders (2), (1) John Innes Centre, NORWICH, U.K.; (2) John Innes Centre, Norwich, U.K.

Yellow rust (YR) is a major threat to wheat production worldwide and can lead to total crop loss when untreated. Identifying which host pathways are targeted by the pathogen is crucial to get a better understanding of the host mechanisms involved in disease response. We used infected field RNA-seq data coupled with controlled infections of susceptible varieties using different YR strains. Differential expression analysis revealed changes in the expression of primary metabolic pathways including photosynthesis and metabolism of amino acids. One of the genes encodes a branched-chain aminotransferase (BCAT) and time-course experiment data revealed a peak in its expression at $24 \mathrm{~h}$ post-inoculation. The most susceptible interactions showed higher BCAT expression levels. We obtained a double tetraploid TILLING knock-out line and, upon infection, the bcat knock-out line showed reduced susceptibility to yellow and stem rust. We hypothesise that knockout mutants have higher levels of basal salicylic acid (SA) due to the accumulation of isoleucic acid (activator of the SA pathway) caused by the impairment of BCAT. Accordingly, SA-associated pathogenesis-related (PR) gene expression was significantly higher in either double or single uninfected mutant lines in comparison to WT plants, suggesting a connection of BCAT in SA signaling. We are using Virus-Induced Gene Silencing (VIGS), CRISPR/Cas9 and Metabolomics to further understand the pathway and its link to in disease progression.

Analysis of Argonoute 1-associated small RNAs in Phytophthora infestans and potato

K. PERSSON HODÉN (1), X. Hu (1), C. Dixelius (2), (1) Department of Plant Biology, Swedish University of Agricultural Sciences, Uppsala, Sweden; (2) Swedish University of Agricultural Sciences, Department of Plant Biology, Uppsala, Sweden

We are studying small RNA (sRNA) biology events in the interaction between Phytophthora infestans and potato. The present work is a continuation of the characterization of Argonaute 1 (Ago1) in P. infestans (Pi) and the proof-of-concept on host-induced gene silencing in this 
pathosystem. We used the PiAgo1-GFP strain to analyze sRNA-associated events during potato infection. Potato (St) sRNAs could be detected in our PiAgo1 pull-down material indicating a potential incorporation of host sRNAs in the oomycete RNAi machinery. We found extensive targeting of endogenous and pathogen derived sRNAs to a large number of genes including resistance $(R)$ genes in the potato genome. A new unique host target of one $P i$-sRNA clearly promotes infection of the pathogen. In parallel, StAgol was tagged, overexpressed in transgenic potato and used in pull-down experiments. Among all data generated, a striking generation of U2 spliceosomal RNA fragments was seen. One site, spanning less than $7000 \mathrm{nt}$ of potato chromosome 2, was covered by approximately one third of the data, altering the sRNA fingerprint during infection. Further details on our different sources of sRNAs and their implications for the $P$. infestans - potato interaction will be presented.

Pseudomonas fluorescens LBUM677 enhances total lipid and stearidonic acid yields in corn gromwell (Buglossoides arvensis). A. NOVINSCAK, M. Filion, Université de Moncton, Moncton, NB, Canada

n-3 polyunsaturated fatty acid (PUFA) consumption is important for human growth and development. Currently, most available n-3 PUFA sources are of marine origin, however their supply is becoming limited. Plant sources of n-3 PUFA appear to be a viable alternative. Stearidonic acid (SDA) is a n-3 PUFA produced in large quantity by certain plants belonging to the Boraginaceae family, which can provide health benefits similar to those of marine oils. The aims of this study were to identify a bacterial inoculant able to enhance oil and SDA yield in Buglossoides arvensis under field conditions and characterize the strain's colonization ability. Forty bacterial strains were screened for the ability to increase oil and SDA yield under soil conditions. One strain (Pseudomonas fluorescens LBUM677) was able to significantly enhance oil yield by $24 \%$ and SDA yield by $33 \%$. A qPCR assay was developed to specifically characterize LBUM677's rhizosphere colonization ability on different $B$. arvensis cultivars and determine the minimum inoculation threshold to achieve biological activity. Overall, inoculation with at least $10^{9}$ bacteria per B. arvensis seed was found to be required for increased accumulation of oil and SDA. The colonization ability of LBUM677 was different between the $B$. arvensis cultivars, which also impacted oil and SDA accumulation. In conclusion, progress is being made to optimize the use of LBUM677 as a biofertilizer to increase seed oil and SDA yields in B. arvensis.

Auxin plays multiple roles during Pseudomonas syringae pathogenesis

B. N. KUNKEL (1), A. Thierry Djami-Tchatchou (1), G. A. Harrison (1), C. P. Harper (1), R. Wang (2), M. Prigge (2), M. Estelle (2), (1) Washington University in St. Louis, St. Louis, MO, U.S.A.; (2) University of California, San Diego, San Diego, CA, U.S.A.

The bacterial plant pathogen Pseudomonas syringae pv. tomato strain DC3000 (PtoDC3000) manipulates host auxin physiology to promote pathogenesis. For example, PtoDC3000 can synthesize the auxin indole-3-acetic acid (IAA) and it modulates host auxin signaling via secreted virulence factors. Auxin inhibits salicylic acid (SA)-dependent host defenses, and IAA synthesis by PtoDC3000 promotes pathogen growth by suppressing SA defenses. However, we have also shown that auxin promotes PtoDC3000 growth in planta via a mechanism independent of SA. Thus, auxin plays multiple roles during pathogenesis. To test if host auxin signaling is important during pathogenesis, we used transgenic plants carrying an inducible, dominant negative allele of $A X R 2$, GR-axr2-1, that impairs auxin signaling. We observed both repression of auxinresponsive genes and significant reduction of bacterial growth in GR-axr2-1 plants compared to wild type, indicating that host auxin perception is required for normal susceptibility. To explore additional roles for auxin during pathogenesis, we investigated whether IAA can directly affect PtoDC3000 and found that IAA down-regulates Type III secretion genes, but induces expression of other genes likely involved later during infection. We observed a similar pattern in PtoDC3000 growing in plant tissue. Our results suggest that, in addition to suppressing host defenses, IAA acts as a microbial signaling molecule to coordinate expression of PtoDC3000 virulence genes.

Identification of targets of the Pmk1 MAP kinase during plant colonisation by the rice blast fungus Magnaporthe oryzae N. CRUZ-MIRELES (1), M. Oses-Ruiz (1), W. Sakulkoo (2), P. Derbyshire (1), F. L. H. Menke (1), N. J. Talbot (1), (1) The Sainsbury Laboratory, University of East Anglia, Norwich Research Park, Norwich, U.K.; (2) Biosciences, University of Exeter, Exeter, U.K.

Rice blast disease is among the most devastating diseases affecting global agriculture. The disease is caused by the fungal pathogen Magnaporthe oryzae. This fungus gains entry to the plant by forming a dome-shaped infection structure called an appressorium. Once inside the plant cell, $M$. oryzae develops intracellular invasive hyphae that facilitate cell-to-cell movement through pit field sites where plasmodesmata are situated. The Pmk1 (MAP) kinase signaling pathway, plays a key role during appressorium formation as well as host colonisation. However, how Pmk1 regulates such downstream processes remains unclear. Here, we report a phosphoproteomic analysis that we are using to identify direct downstream targets of the Pmk1 MAPK during all stages of infection. To understand the role of Pmk1 during rice tissue invasion we used a chemical genetics approach to generate a $M$. oryzae $p m k l^{A S}$ mutant in which we are able to conditionally inactivate the kinase in the presence of napthyl-PP1. We have identified phosphorylated candidate proteins related to cellular processes such as cytoskeleton remodelling, vesicular trafficking and cell cycle control. We are currently validating their roles and defining the signaling network that operates downstream of the Pmk1 MAPK during plant infection by the rice blast fungus.

Elucidating the localization and function of Ustilago hordei avirulence effector UhAVR1, during compatible and incompatible host interactions

A. P. MONTENEGRO ALONSO (1), G. Bakkeren (2), (1) The University of British Columbia, Vancouver, BC, Canada; (2) Agriculture \& AgriFood Canada, Summerland, BC, Canada

Ustilago hordei $(U h)$ causes smut disease in barley and oats. Uh secretes effector UhAVR1 which is not essential for complete virulence towards susceptible barley cv. Odessa but triggers a fast HR in cv. Hannchen having corresponding resistance gene Ruhl. To study the localization and role of this effector in both types of interaction, a multisystems approach was taken, using Nicotiana benthamiana (Nb), Arabidopsis thaliana (At) and barley. Secretion from Uh seems affected by Brefeldin A and, when expressed without its signal peptide (SP) from the 35S promoter and linked to GFP, localizes to confined foci in the host cell cytoplasm in $N b$, transgenic $A t$, and upon transfection into barley protoplasts. Transgenic At lines expressing UhAVR1-SP show increased susceptibility to Pseudomonas syringae pv. tomato (Pst) DC3000 and Uh growth, suggesting a 
possible role in defense suppression. Transcriptome data comparing infection of cv. Hannchen by $U h$ versus a $\Delta U h A v r l$ strain, show a defense response at 48hpi including up-regulation of peroxidases, PR proteins, chitinases and cell wall modifying enzymes. Ruhl was previously mapped to the end of barley chromosome 7, a region where many predicted resistance genes are located. BAC clones carrying the candidate Ruhl gene are being tested for HR triggering by co-expression with UhAvrl in $\mathrm{Nb}$. This data, along with in planta UhAVR1:GFP co-immunoprecipitation experiments results we have obtained may provide a clue of this effector target.

Functional study of type III effector AvrE during plant infection

M. YUAN, B. Cai, Y. Hu, X. Xin, Institute of Plant Physiology \& Ecology, Chinese Academy of Sciences, Shanghai, China

The type III effectors are the key virulence weapons for Gram-negative pathogens to successfully infect plants. Our previous study showed that bacterial pathogens such as Pseudomonas syringae can establish an aqueous living space in the apoplast of the plant leaf by effectors like HopM1 and AvrE. Establishment of the aqueous apoplast is a key virulence mechanism. However, how AvrE promotes this process is not known. To address this question, we have performed protein pull-down assay coupled with mass spectrometry to identify AvrE-interacting plant proteins from Arabidopisis. Interestingly, multiple members of the calcium-dependent protein kinases (CPKs) family can interact with AvrE. BiFC and Co-IP assays were performed to confirm the interaction, and $\mathrm{N}$ terminus of AvrE protein and the kinase domain of CPK protein seem to mediate the interaction. Transient expression assay in tobacco plants showed that the N-terminal AvrE protein can stabilize CPK protein levels in vivo.We found that AvrE is phosphorylated in planta, as detected by a phospho-Ser/Thr antibody, and furthermore, in vitro kinase assays showed that CPKs can phosphorylate AvrE directly. Further research is needed to demonstrate how phosphorylation events of AvrE are linked to its virulence function and the formation of aqueous apoplast during diseases.

Dissecting the effect of AP2muA protein on Bamboo Mosaic Virus infection

C. P. CHENG, P. Y. Hou, H. J. Hsu, Department of Life Sciences, Tzu Chi University, Hualien, Taiwan

Clathrin-coated vesicle (CCV) system is one of the major pathways involved in endocytic and endosomal trafficking. The CCV system has been demonstrated to participate in the intracellular movement of several animal viruses. However, little is known about the role of CCV system in plant virus infection. The AP2muA protein is an adaptin which binds to the sorting receptor through interacting with its tyrosin-sorting signal, YXXF. In this study, we discovered that all of the movement proteins and coat protein of Bamboo mosaic virus (BaMV) contain the tyrosinesorting signal-like sequence which may interact with the AP2muA protein of $N$. benthamiana $(\mathrm{NbAP} 2 \mathrm{muA})$. Our data demonstrated that treatment with the inhibitor of $\mathrm{u}$ subunit as well as knocking down the expression of $N b A P 2 m u A$ gene reduced the accumulation of BaMV in $N$ benthamiana, suggesting a positive role of $\mathrm{NbAP} 2 \mathrm{muA}$ plays in BaMV infection. Based on the co-crystal structure of rat muA protein and the tyrosin-sorting signal, the interaction models of the NbAP2muA and tyrosine-sorting signal-like sequence of BaMV proteins are proposed. These tyrosine-sorting signal-like sequences of BaMV proteins were mutated accordingly to study the effect on BaMV infection. The full-length cDNA of $N b A P 2 m u A$ was cloned for further studies.

New insight to the role of the Xanthomonas core effector XopX in defense suppression and activation of cell death A. DUNCAN, M. B. Mudgett, Department of Biology / Stanford University, Stanford, CA, U.S.A.

A class of highly conserved, core type III effector (T3Es) proteins (e.g. XopX, AvrE1 and HopM1) from pathogenic bacteria are known to suppress some plant immune responses but also cause robust programmed cell death (PCD). One model to explain these conflicting phenotypes is that core T3Es may interfere with a critical plant cell process that impacts defense signaling and cellular homeostasis. To gain insight to the mechanisms underlying this "dual role", we have been investigating the biochemical function of XopX from Xanthomonas euvesicatoria (Xe) and its homologs found in almost every Xanthomonas species. XopX was previously shown to be a potent suppressor of flagellin-induced reactive oxygen species (ROS) production. In this work, we performed an unbiased proteomic screen to identify XopX protein complexes in Nicotiana benthamiana leaves. Strikingly, we identified several proteins that play roles in ROS production and regulation. We are currently studying these complexes using functional genomics and metabolite profiling. Progress in elucidating the mechanisms by which XopX alters host signaling impacting ROS and cell viability will be discussed.

Identification of Pseudomonas syringae Effectors Responsible for Sugar Efflux in Arabidopsis Reveals a Bacterial Feeding Strategy C. ROUSSIN-LÉVEILLÉE (1), G. M. Lajeunesse (1), P. Moffett (2), (1) Univ de Sherbrooke, Sherbrooke, QC, Canada; (2) Université de Sherbrooke, Sherbrooke, QC, Canada

Multiple types of microbes induce transcriptional reprogramming in the cells of their plant host. Successful pathogens must inhibit host defenses, while at the same time establishing an environment favourable to pathogen growth at the host-pathogen interface. For example, the rice pathogen Xanthomonas oryzae, uses TAL (transcription activator-like) effectors to induce the expression of SWEET sugar transporters, which in turn export sugars to the apoplast to the benefit of the pathogen. However, how other important bacterial pathogens obtain nutrients from their host plants remains unresolved. We find that infection by Pseudomonas syringae pv. tomato DC3000 (Pst) induces a massive upregulation of SWEET transporters in Arabidopsis thaliana. This effect depends on type III secretion and is accompanied by a depletion of host starch stores over time. By using a repertoire of $P$ st type III effector mutants, we identified individual candidate effector proteins responsible for this phenomenon. Furthermore, pattern-triggered immunity appears to reduce the expression of SWEET sugar transporters to restrict sugar availability in the apoplast. Altogether, our data support a general model by which pathogens hijack their host's sugar export machinery to promote their virulence.

Functional investigation of Ralstonia solanacearum PPR-containing Effector in Plant Development and Immunity W. KIM (1), H. Jeon (1), S. Lee (1), J. Jayaraman (2), K. H. Sohn (3), S. Cécile (1), (1) Seoul National University, Seoul, Korea, Republic of (South); (2) The New Zealand Institute for Plant and Food Research Ltd, Auckland, New Zealand; (3) Pohang University of Science and Technology, Pohang, Korea, Republic of (South) 
Ralstonia Solanacearum (Rso) is plant pathogenic bacterium that causes lethal bacterial wilt in a wide range of crops including potato, tomato, and pepper. Rso effectors (T3Es) are injected into host plant by type III secretion system to manipulate various host cell processes and dampen plant immunity, providing a favorable environment for infection. Here, we investigated the function of pentatricopeptide repeat (PPR)-containing Rso effector (PRE) in plant immunity and development. PRE-overexpressing transgenic plants showed delayed flowering phenotype in long day conditions. This delayed flowering is mediated by decrease of flowering time regulator genes such as $A P 1, F U L, L F Y$ and $S O C 1$. Interestingly, miR156 target genes SPLs are also downregulated in this plant, indicating that flowering time regulation by PRE function is linked with miRNA pathway. We also observed that PRE-YFP protein localized to lipid bodies. We are currently investigating the effect of PRE on the host immune system using Agrobacterium-mediated transient expression in $N$. benthamiana and $N$. tabacum. Taken together, PRE might regulate plant development via miRNA pathway and/or association with lipid body compartment. This study will contribute to elucidate the molecular mechanisms of Rso T3Es function in plant development and immunity.

\section{Nuclear effectors of Magnaporthe oryzae modulate host susceptibility via transcriptional reprogramming of immunity-associated target} genes

S. KIM (1), C. Y. Kim (1), S. Y. Park (2), K. T. Kim (1), J. Jeon (3), H. Chung (1), S. Kwon (4), J. Choi (3), J. Jeon (5), Y. H. Lee (1,3,6,7,8), (1) Department of Agricultural Biotechnology, Seoul National University, Seoul, Korea, Republic of (South); (2) Department of Plant Medicine, Sunchon National University, Suncheon, Korea, Republic of (South); (3) Interdisciplinary Program in Agricultural Genomics, Seoul National University, Seoul, Korea, Republic of (South); (4) Heinrich-Heine-Universität Düsseldorf, Düsseldorf, Germany; (5) Yeungnam University, Gyeongsan, Gyeongbuk, Korea, Republic of (South); (6) Research Institute of Agriculture and Life Sciences, Seoul National University, Seoul, Korea, Republic of (South); (7) Center for Fungal Genetic Resources, Seoul National University, Seoul, Korea, Republic of (South); (8) Plant Immunity Research Center, Seoul National University, Seoul, Korea, Republic of (South)

Plants evolve immune systems to overcome pathogen invasion, but the pathogens deploy effectors to evade host defense responses. Despite importance of the effectors in plant-pathogen interactions, revealing molecular mechanism is still elusive especially in fungal pathogens, the most destructive plant invaders. Here, we characterized two fungal nuclear effectors, named as Host Transcription Reprogramming (MoHTR), in the rice blast fungus. The MoHTR effectors are actively translocated to host nuclei during pathogenesis, and also move to adjacent host cells prior to hyphal invasion. In addition, the effectors are required for full virulence of the fungus without other phenotypic defects. The function of MoHTRs as transcriptional regulator is potentiated by domain structure, and effector binding elements of MoHTR were investigated by protein binding microarray (PBM) to find host target genes. Consequently, the effectors affect expression of immunity-associated target genes, which were downregulated in transgenic rices expressing MoHTRs. Furthermore, the action of MoHTR confers increment of host susceptibility to rice blast and bacterial leaf blight, the most serious two rice diseases. Our finding is first case of fungal nuclear effectors directly regulating host transcription, and we propose that fungal nuclear effectors affect broad-spectrum resistance of host plant.

Identification and function analysis of a conserved RxLR effector from Phytophthora parasitica Q. ZHANG (1), W. X. Shan (2), (1) College of Plant Protection, Northwest A\&F University, Yangling, China; (2) College of Agronomy, Northwest A\&F University, Yangling, China

During plant infection, Phytophthora pathogens employ an arsenal of effectors to manipulate plant immunity. The class of RxLR proteins is the most extensively studied effectors in Phytophthora, which contain a conserved RxLR motif (Arginine-Any amino acid-Leucine-Arginine) in the $\mathrm{N}$-terminus. RxLR proteins were presumed to target cytoplasmic host proteins to alter plant process and enable parasitic colonization by the pathogen. $P$. parasitica is a typical soil-born pathogen and causes numerous notorious diseases in more than 255 genera of plants. However, little is known about the pathogenesis mechanism of $P$. parasitica. Using the publicly available $P$. parasitica genomes, we identified a conserved RxLR effector- $P$ p 18, which was determined in this study to promote $P$. parasitica infection and suppress PAMP-triggered immunity (PTI). Using the anti-GFP coimmunoprecipitation-liquid chromatography-tandem mass spectrometry (coIP-LC/MS-MS) approach, we further identified a candidate host target protein MST35, a $\mathrm{H}_{2} \mathrm{O}_{2}$ scavenging enzyme. The interaction between Pp18 and the target protein MST35 was further confirmed. The MST35 was determined to be involved in resistance to Phytophthora, suggesting that hydrogen peroxide metabolic process is critical to plant immunity.

The Xanthomonas effector protein XopI suppresses the stomatal immunity

O. NAGEL (1), U. Bonas (2), (1) Martin-Luther-University Halle-Wittenberg, Germany; (2) Department of Genetics, Martin Luther University, Halle, Germany

Our laboratory studies the plant pathogen Xanthomonas campestris pv. vesicatoria $(X c v)$ and its host plants pepper and tomato. Essential for pathogenicity is the type III secretion system (T3SS) which translocates at least 35 effector proteins (T3Es) into the plant cell cytoplasm. T3Es suppress the plant innate immunity and alter the plant metabolism to the pathogen's advantage. Due to a eukaryotic F-box motif in the N-terminal region, the T3E XopI likely integrates into plant SCF (Skp1-Cullin-F-box protein) complexes which target proteins for ubiquitination. Interaction studies in yeast showed that XopI specifically interacts with one out of 21 Arabidopsis thaliana Skp1-like proteins (ASK) suggesting that XopI integrates into particular plant SCF complexes. A yeast-two-hybrid screen with XopI as bait identified five proteins that presumably are involved in the regulation of stomatal immunity. Silencing of two interactors confirmed that they mediate stomatal closure after PAMP treatment in Nicotiana benthamiana. In tomato plants, virulence of Xcv85-10 $\triangle x o p I$ strains is dramatically reduced. The stomatal aperture is also reduced suggesting that XopI is essential for $X c v$ entry into the plant apoplast. Stomata assays with stable xopI transgenic $N$. benthamiana lines showed that XopI suppresses stomatal closure. 
Identification and functional characterisation of genes regulated by the PnPf2 Zn2Cys6 transcription factor in Parastagonospora nodorum

K. C. TAN (1), D. Jones (1), K. Rybak (1), H. Phan (1), E. John (1), K. B. Singh (2), R. P. Oliver (1), (1) Curtin University, Perth, WA, Australia; (2) UWA/CSIRO, Wembley, WA, Australia

The fungus Parastagonospora nodorum causes septoria nodorum blotch (SNB) of wheat. SNB is largely dictated by interactions between necrotrophic effectors (NEs) and host dominant susceptibility genes. Three of the best characterised NE-sensitivity gene interactions are ToxATsn1, Tox1-Snn1 and Tox3-Snn3. ToxA and Tox3 are positively regulated by a Pleosporales-specific zinc finger transcription factor PnPf2. Mutants deleted in PnPf2 lost the ability to infect Tsn1 and Snn3 wheats but remained infectious on hosts carrying Snn1. A comprehensive RNAseq transcriptomic approach was used to compare the transcriptome of the $P$. nodorum wild type (SN15) and a PnPf2-deficient strain ( $p f 2$ $69)$ to identify other targets of PnPf2 regulation during infection. Gene ontology enrichment analysis of the differentially expressed (DE) genes revealed genes associated with plant cell wall degradation and proteolysis were enriched in the DE gene sets and displayed a down-regulated trend in $p f 2-69$. In contrast, genes associated with nutrient and ion transport tended to be up-regulated in $p f 2-69$. In addition, changes in the regulome was also observed in the absence of PnPf2. We concluded that in addition to modulating effector gene expression, PnPf 2 may play a broader role in establishment of a necrotrophic lifestyle by orchestrating the expression of genes associated with plant cell wall degradation and nutrient assimilation.

Modulation of Defense-Related Autophagy at the Pathogen Interface Y. TUMTAS (1), I. Morianou (1), C. Molinari (1), Y. F. Dagdas (2), S. Kamoun (3), T. O. Bozkurt (1), (1) Imperial College London, U.K.; (2) Gregor Mendel Institute of Molecular Plant Biology, Vienna, Austria; (3) The Sainsbury Laboratory, University of East Anglia, Norwich Research Park, Norwich, U.K.

The Irish famine pathogen Phytophthora infestans penetrates host cells and forms haustoria to enable translocation of effector proteins. Invaded plant cells respond by focalizing immune responses at pathogen contact sites. This includes defense related host autophagy machinery such as the autophagy receptor Joka2/ NBR1. P. infestans counteracts this by deploying PexRD54, an effector that antagonizes Joka2-mediated immunity. However, the molecular mechanisms underlying how Joka2 contributes to defense-related autophagy remain unknown. By using a coimmunoprecipitation approach, we identified two defense-related kinases implicated in immune signaling, which interact with Joka2. We show that these interactions do not require the ubiquitin binding domain of Joka2 and are mediated through different domains of Joka2. Consistent with this, autophagy suppression or down regulation of Joka2 gene expression did not affect the turnover of the interacting kinases, indicating that these are not autophagic substrates recruited by Joka2. Confocal microscopy uncovered that Joka2-interacting immune proteins colocalize with Joka2 puncta accumulating around the $P$. infestans haustoria. Our data support a model where Joka2 contributes to plant defense by forming immune complexes at the pathogen interface. These results implicate autophagy machinery in plant focal immunity and suggest more complex functions for autophagy than the known degradative roles.

Assessing early resistance responses of sugarcane to Xanthomonas albilineans

A. PERES BINI (1), M. C. D. Serra (1), H. R. V. Silva (2), G. T. Ragagnin (3), C. B. M. Vitorello (2), L. E. A. Camargo (2), M. A. Van Sluys (3), S. Creste (1), (1) Centro de Cana, IAC, Ribeirão Preto, Brazil; (2) Univ of São Paulo, ESALQ, Piracicaba, Brazil; (3) Departamento de Botânica, IB, USP, São Paulo, Brazil

Leaf scald disease caused by Xanthomonas albilineans affects sugarcane and is controlled by the use of resistant cultivars. Herein, we inoculated and quantified $X$. albilineans in two sugarcane genotypes with contrasting resistance levels. The pathogen was inoculated using the decapitation method and the bacteria was quantified in a time course TaqMan based experiment. X. albilineans was detected immediately after inoculation until 144 hours post-inoculation (hpi) in both genotypes. At 72 hpi pathogen quantification in not-yet-emerged apical leaf tissue was approximately 2.5 -fold higher in the susceptible genotype when compared to the resistant one. These results suggest the presence of an early defense response in the resistant genotype against $X$. albilineans. Increased production of reactive oxygen species (ROS) is an important early plant response to pathogen infection and contributes to resistance. We used a combination of genomics, transcriptomics and proteomics datasets related to sugarcane-pathogen interactions to select candidate genes associated to plant oxidative burst. The expression profile of these genes was evaluated by qPCR in both genotypes to elucidate sugarcane early responses to $X$. albilineans. This is an ongoing study by GEMACANA (Study Group on Sugarcane Associated Microorganisms) conducted to better understand the resistance responses associated with ROS production in sugarcane after infection with X. albilineans. FAPESP (2018/07615-4, 2016/17545-8).

Towards the identification of strategies to counteract RNA silencing suppression mediated by pathogen-encoded GW/WG effectors G. LEPÈRE (1), L. Scalisi (2), M. Charvin (3), O. Thiebeauld (4), M. Singla Rastogi (3), L. Navarro (5), (1) ENS CNRS-UMR 8197, Paris, France; (2) ENS-CNRS UMR 8197, France; (3) IBENS-CNRS UMR8197, Paris, France; (4) IBENS-ImmunRise, Paris, France; (5) IBENS, Paris, France

We have previously reported that a subset of type III effectors from Pst DC3000 can dampen the Arabidopsis miRNA pathway to cause disease. Among them, HopT1-1 was found to potently suppress both miRNA activity and PAMP-Triggered Immunity (PTI). This effector is additionally sensed by host cells, which results in a strong Effector-Triggered Immunity (ETI) response. Importantly, both HopT1-1-triggered RNA silencing/PTI suppression and ETI activation are fully dependent on the ability of this effector to interact with the silencing factor ARGONAUTE 1 (AGO1) through conserved glycine/tryptophan (GW) motifs. Here, we have conducted an unbiased forward genetic screen aiming at identifying Arabidopsis mutations that alter the ability of HopT1-1 to suppress miRNA activity and/or ETI activation. So far, 41 putative mutants have been selected and we are in a process of identifying the causal mutations using a mapping-by-sequencing approach. We are anticipating to retrieve mutations in (i) RNA silencing factors that alter their ability to interact with HopT1-1 but that do not interfere with their cellular function, (ii) immune receptors that impair their ability to detect HopT1-1, and (iii) negative regulators of RNA silencing that would counteract HopT1-1triggered suppression of miRNA activity. I will present the phenotypical and molecular characterization of some of these mutants. I will also discuss how these findings can be exploited to confer broad spectrum disease resistance. 
"The $\mathrm{S}$ factor" from rice in the interaction with Rhizoctonia solani

J. ACEVEDO-GARCIA (1,2), N. van Reekum (2), R. Gorkink (2), R. Hulzink (2), R. Peng Wang (2), K. Nijbroek (2), K. Huevenaars (2), R. van Daelen (2), H. van der Poel (2), H. Schneiders (2), S. White (2), A. Janssen (2), M. van Verk (2), M. de Vos (2), W. Ligterink (2), E. van der Vossen (2), W. Tameling (2), (1) European Union's Horizon 2020 programme, Marie Skłodowska-Curie grant No. 791867, Netherlands; (2) Keygene N.V., Wageningen, Netherlands

In resemblance to the famous reality TV competition (The X Factor), we want to find a new talent. S (susceptibility) factors encoded by plant $S$ genes facilitate the infection process or support compatibility with a pathogen. In rice (Oryza sativa), mutated $S$ genes may offer durable and broad-spectrum resistance to Rhizoctonia solani, a soilborne, necrotrophic fungus that causes the detrimental sheath blight disease (ShBD). The auditions to select the contestants follow two stages: 1) transcriptomics: an (adapted) reproducible nano-chamber method was used for an infection time course experiment (focused on the presumed biotrophic phase of the pathogen) for RNA-seq with a susceptible rice variety and a $R$. solani AG1 IA strain. From 1226 differentially expressed genes (contestant S factors), 135 and 1091 were down- or up-regulated, respectively. Selection of top contestants that are going to the live shows is in progress. 2) effectoromics: we aim to find pathogen-derived, conserved and early expressed effectors and their host targets. Currently, we are using Oxford Nanopore Technologies ${ }^{\mathbb{B}}$ to sequence five strains of $R$. solani AG1 IA (isolated from infected rice) from Japan, India and Arkansas. The effector host targets (contestant S factors) will be identified by using yeast two-hybrid- and pull-down assays. The winner of "The S Factor" will become the next target to generate rice varieties with improved resistance to ShBD using KeyGene's KeyPoint ${ }^{\mathbb{R}}$ technology.

Structure/function determination of JIP60 mode of action: The ribosome inactivating protein targeted by RNase-like powdery mildew effectors

M. Przydacz (1), M. Bruderer (1), G. Belmans (2), R. Greehill (1), E. Cota (1), P. D. SPANU (1), (1) Imperial College London, London, U.K.; (2) Imperial College London, U.K.

The fungi which cause powdery mildews encode a large superfamily of RNase-like effectors which aid infection and development of disease. Previously, we have shown that one of these effectors (BEC1054/CSEP0064) interferes with the host immunity associated with rRNA degradation and we hypothesised that the RNase-like effectors inhibit host ribosome inactivating action of jasmonate induced proteins (JIP). Here, we use structure prediction of the barley JIP60 to identify potential catalytic amino acids in the active site and test these by mutagenesis and in vivo assay of necrosis induction by expression in Nicotiana benthamiana. We have found that Tyrosine 96, Glutamate 202, Arginine 205, and Tryptophan 235, in the presumptive active site of JIP60 are conserved in 815 plant ribosome inactivating proteins. When these amino acid residues are individually mutated, the necrosis-inducing activity is impaired. We therefore propose that the action of JIP60 is based on hydrolytic depurination of adenine by the ribosome inactivating domain. We also confirm that the powdery mildew BEC1054/CSEP0064 is capable of inhibiting induction of necrosis by JIP60, when co-expressed in N. benthamiana.

Functional characterization of the "negatively selected" oafA gene in Pseudomonas protegens CHAO

H. ZHANG, E. Li, C. M. J. Pieterse, R. De Jonge, Plant-Microbe Interactions, Department of Biology, Utrecht University, Utrecht, Netherlands

Plants exude up to $40 \%$ of their photosynthetically fixed carbon sources into the rhizosphere, fostering a large microbial community. New members in such community rely on strong competitive ability as key ingredients to occupy this attractive niche. The bacterial genes involved in such adaptation, however, remain unknown. Here we report on an evolutionary experiment in which Pseudomonas protegens strain CHA0 evolved in the Arabidopsis thaliana Col-0 rhizosphere under axenic conditions. A transversion oafA mutant (oafA-1) and an indel oafA mutant $($ oaf $A-2)$ were found to be dominating in the rhizospheric bacterial population in two independent replicates after the first growth cycle. Both mutations result in a premature stop. The oafA protein is supposedly responsible for acetylation of the $\mathrm{O}$-antigen. No significant differences between wild-type CHA0 and the oafA- 1 mutant growth on Biolog Microplates were observed, emphasizing on carbon source utilization, and osmotic, $\mathrm{pH}$ or chemical sensitivity. Likewise, wild-type and mutants grew similar when they were inoculated on axenic Col-0 seedlings, separately. Intriguingly, mixed inoculation of wild-type and oafA-1 resulted in a rapid population decline to about fifty percent suggesting that bacterial cell death rather than decelerated growth affected the outcome. This population furthermore contains eighty percent oafA-1. These results suggest that a strong selection factor operates when bacteria are cultured with the plant.

Structure and functions of extracellular polysaccharides of the phytopathogenic bacterium Pectobacterium atrosepticum B. R. ISLAMOV (1), V. Gorshkov (1,2), O. Petrova (1), P. V. Mikshina (1), V. Vorob'ev (1,2), M. Ageeva (1), G. Burygin (3), Y. V. Gogolev $(1,2)$, (1) Kazan Institute of Biochemistry and Biophysics FRC "KSC of RAS”, Kazan, Russian Federation; (2) Kazan Federal University, Kazan, Russian Federation; (3) Inst of Biochemistry and Physiology of Plants and Microorganisms RAS, Saratov, Russian Federation

Bacterial extracellular polysaccharides (EPS) determine many properties microbial cells (adhesion, resistance, virulence, biofilm formation, etc). Plant soft rot-causing phytopathogen Pectobacterium atrosepticum $(\mathrm{Pba})$ is able to form various 3D biofilm-like structures (including bacterial emboli); however, an extracellular matrix and EPS have not been described for this bacterium. The aim of our study was to identify Pba EPS, determine their molecular structure and elucidate their role during host plant colonization. EPS were extracted and then purified and analyzed by size-exclusion and anion-exchange chromatography. To establish EPS structure, one-dimensional $\left({ }^{1} \mathrm{H},{ }^{13} \mathrm{C},{ }^{13} \mathrm{C}\right.$ JMOD), two-dimensional heteronuclear (HSQC, HMBC) and homonuclear (COSY, TOCSY, ROESY) NMR-specta were analyzed. The EPS backbone consisted of repeating trisaccharide $[\rightarrow 3)-\alpha$-D-Galp- $(1 \rightarrow 2)-\alpha$-D-Manp- $(1 \rightarrow 4)-\alpha$-L-Rhap- $(1 \rightarrow]$, the side chain of $\beta$-D-Erwiniose- $(1 \rightarrow 3)-\alpha$-D-Galp- $(1 \rightarrow$ was attached to mannopyranosyl residue. By immunocytochemistry, EPS were shown to be synthesized in the host plant during the formation of bacteria emboli. Using dynamic light scattering and viscosimetry, EPS were shown to increase the viscosity of liquids by forming supramolecular aggregates, thus facilitating the colonization of xylem vessels. In addition, Pba EPS had antioxidant properties. Herewith, EPS acted as "weak elicitors". The study is supported by RSF (No 19-14-00194). 
Toward identification of TAL effector targeted susceptibility genes in black rot of cabbage caused by Xanthomonas campestris pv. campestris

Z. DUBROW (1), H. Lange (1), I. Fuentes (2), C. Audran (2), B. Szurek (3), C. D. Smart (4), L. D. Noel (2), A. J. Bogdanove (1), (1) Cornell University, Ithaca, NY, U.S.A.; (2) Université de Toulouse, LIPM, CNRS-INRA, Toulouse, France; (3) IRD UMR IPME, Montpellier, France; (4) Cornell University, Geneva, NY, U.S.A.

Transcription activator-like effectors (TALEs) are a large family of type III secreted effectors present in some Xanthomonas species that specifically target and directly upregulate plant host genes. Xanthomonas campestris pv. campestris (Xcc) causes black rot of crucifers, one of the most important Brassica diseases worldwide. The first three complete Xcc genome sequences published had no TALE-encoding genes. However, more recent work and reclassification of Xanthomonas campestris pathovars have revealed that many Xcc strains do encode, and express, TALEs. By PCR and western and Southern blotting we surveyed 124 Xcc isolates from a 10-year New York State collection and found that 30\% encode and express TALEs. Across the New York Xcc isolates, TALE repertoires differ, even between isolates found by multi-locus sequence analysis to be closely related. By targeted mutagenesis, we have found that multiple TALEs from different New York Xcc strains contribute to virulence in cabbage. We are sequencing TALE containing strains and identifying the brassica susceptibility genes targeted by these TALEs by binding site prediction and by using RNA-Seq and qRT-PCR. Identification of susceptibility genes could inform breeding of cruciferous vegetable crops for resistance to black rot.

Early promoters of ATCV-1 show a broad cross-species activity K. Lindner, B. Wahl, A. J. P. PFITZNER, Universitaet Hohenheim, Germany

Acanthocystis turfacea Chlorella Virus-1 (ATCV-1) belongs to the Phycodnaviridae, a family of large dsDNA viruses known to infect marine and freshwater algae. Since algae are the most important contributors to $\mathrm{CO} 2$ fixation and $\mathrm{O} 2$ production on earth, it is of great importance to study viruses that have an impact on algal populations. Many phycodnaviruses have been sequenced so far. How algal viral genes are regulated at the molecular level is unknown. Here we provide evidence for transcriptional activity of several ATCV-1 promoters in tobacco protoplasts and Chlamydomonas reinhardtii cells, including promoters for viral DNA polymerase and promoters for other immediate early and early genes. We show that all tested ATCV-1 promoters active in tobacco protoplasts and Chlamydomonas contain at least one G-box-like element, a cisregulatory element found in many plant and algal genes. Our results suggest that early promoters of ATCV-1, and probably of other Phycodnaviridae, depend on ubiquitous host transcription factors for activation, thus indicating a broad virus host range at the cellular level.

Equilibrium between interchangeable redox intermediates shapes plant immunity

L. FRUNGILLO (1), S. U. Lee (2), M. Nomoto (3), Y. Tada (3), B. W. Yun (2), S. H. Spoel (1), (1) School of Biological Sciences, University of Edinburgh, Edinburgh, U.K.; (2) Kyungpook National University, Daegu, Korea, Republic of (South); (3) Center for Gene Research, Nagoya

University, Nagoya, Japan

Immunity relies on sophisticated hormonal signaling networks tightly associated with intracellular redox changes. Plant immune responses to the bacterial pathogen Pseudomonas syringae (Psm) is mediated by the phytohormone salicylic acid (SA) through a marked burst in the production of reactive oxygen and nitrogen intermediates. To counter plant immune responses, Psm secretes the phytotoxin coronatine, a phytohormone analogue that facilitates bacterial invasion through the stomatal aperture. Surprisingly, coronatine-deficient $P s m$ shows poor virulence even when stomata immunity is bypassed, indicating that coronatine have additional roles during disease establishment. The mechanisms by which coronatine disarms the plant immune system and promote virulence, however, remains largely obscure. Here we provide genetic and biochemical evidence that $P s m$-produced coronatine creates a short window of opportunity for virulence. Our data shows that coronatine targets specific branches of redox signaling in hosts to stagger pathogen infection and the onset of immune responses. We propose a model in which the outcome from plant-pathogen interaction is determined by a molecular battle between hosts and adapted pathogens to offset the equilibrium of interchangeable reactive oxygen and nitrogen intermediates.

The root invading pathogen Fusarium oxysporum targets PTI using cytoplasmic and apoplastic effectors N. TINTOR (1), M. Paauw (2), P. V. Dam (2), M. Rep (1), F. L. Takken (1), (1) University of Amsterdam, Amsterdam, Netherlands; (2) University of Amsterdam, Netherlands

Plant roots are exposed to an extraordinarily microbe-rich environment where the root interacts with both beneficial and pathogenic microorganisms. The virulence strategies of latter, as well as the complementary plant defense mechanisms, are little understood. Fusarium oxysporum $(F o$ ) is a ubiquitous root-colonizing fungus. Root colonization by $F o$ is often symptomless, but individual isolates acquired the ability to cause disease. These pathogenic isolates have specific sets of candidate effectors (small secreted proteins) that are presumably involved in host manipulation. We characterized eight predicted effectors of the Arabidopsis infecting Fo5176 strain. We found that four of them suppressed PAMP-triggered immune responses upon transient expression in Nicotiana benthamiana and one induced chlorosis. Two of these were shown to act intracellular and the other three in the apoplast. Next, we assessed which effectors are translocated into host cells upon Fo infection of Arabidopsis roots. Interestingly, all - but the apoplastic effectors - were observed inside host cells, indicating their specific uptake. Based on protein size, charge or sequence no differentiation could be made between translocated (cytoplasmic) and apoplastic effectors, implying that uptake relies on other, elusive features of these proteins.

A family of Xanthomonas harpin peptides effective against biotic and abiotic stresses

G. ZORNETZER, K. Staska, Z. Wei, Plant Health Care, Inc, Raleigh, NC, U.S.A.

T-Rex is a novel family of bioactive peptides derived from the HreX gene of Xanthomonas campestris. We have completed several greenhouse and field trials to evaluate the effect of these peptides on the plant response to stresses. Greenhouse and field tests were conducted evaluating T- 
Rex peptides as anti-nematode agents. Greenhouse tests of foliar application on tomatoes revealed reduction in the production of Root Knot Nematode eggs. Foliar tests in soy demonstrated reduction in cyst formation by Soybean Cyst Nematode. Further, we observed that secretions from the roots of peptide-treated plants reduced hatching of cyst nematode eggs. Additional testing with T-Rex peptides applied as a seed treatment for soy revealed excellent efficacy against both Soybean Cyst Nematode and Root Knot Nematode. Field tests in Brazil revealed robust control of Pratylenchus nematodes by T-Rex peptide variants. In summary, the T-Rex family of peptides elicits plant defense responses against nematodes to levels comparable to those of chemical methods, suggesting that elicitation of plant defenses is a viable mechanism for the management of nematode pests. In addition, greenhouse tests revealed that T-Rex also increased the tolerance of corn and soy to drought stress. Multiple years of field testing in corn have confirmed performance under drought stress, suggesting efficacy against both biotic and abiotic stresses.

Bacterial community maintenance of plant root development

O. M. FINKEL (1), I. Salas González (1), G. Castrillo (2), T. F. Law (1), J. L. Dangl (3), (1) University of North Carolina, Chapel Hill, NC, U.S.A.; (2) University of Nottingham, Nottingham, U.K.; (3) Department of Biology, University of North Carolina at Chapel Hill, Chapel Hill, NC, U.S.A.

Culture-independent studies are limited in their ability to infer causality of interactions within the rhizosphere. To model plant-microbe-microbeenvironment interactions in a controlled setting, we established a high-complexity plant-microbe microcosm. We inoculated plants with a defined 185-member bacterial synthetic community (SynCom), exposing each microcosm to one of 16 different abiotic contexts by manipulating four abiotic variables (salinity, temperature, phosphate concentration and $\mathrm{pH}$ ), and measured SynCom composition in roots, shoots and agar after 12 days by $16 \mathrm{~S}$ rRNA amplicon sequencing. These data provide a catalogue of the niche breadth of 185 isolates with respect to four abiotic parameters and the plant tissues that they occupy. By iterative deconstruction of the SynCom, we identified a single bacterial genus, Variovorax, which is able to revert strong phenotypic effects on root development induced by a wide diversity of bacteria, as well as by the whole community. Variovorax use mechanisms related to auxin degradation and ethylene manipulation to balance this realistic root community's effects on stereotypic root development. This work demonstrates metabolic signal interference from within a complex model community and stages Variovorax as key players in bacteria-plant communications networks, demonstrating an ability to block bacterial effects on root development, thus promoting typical root development, across a wide range of biotic and abiotic contexts.

Aspergillus flavus secondary metabolites and their roles in fungal development, survival and virulence J. W. CARY (1), M. D. Lebar (1), C. Carter-Wientjes (1), B. Mack (1), Q. Wei (1), R. Majumdar (1), S. De Saeger (2), J. Diana Di Mavungu (2), (1) USDA, ARS, SRRC, New Orleans, LA, U.S.A.; (2) Ghent University, Ghent, Belgium

Aspergillus flavus is primarily known for its ability to produce toxic and carcinogenic aflatoxins during colonization of susceptible crops such as maize and groundnut. A total of 56 secondary metabolite (SM) gene clusters are predicted to be present in the A. flavus genome. To date, metabolites have only been experimentally assigned to thirteen of these SM clusters. It is important to identify as many of these compounds as possible to determine their bioactivity with respect to fungal development, survival, and virulence. We have identified and functionally characterized metabolites associated with a number of previously uncharacterized SM clusters including those involved in fungal development and survival (asparasone A and leporins). We recently identified aspergillic acid (AA) as the product of a nonribosomal peptide synthetase (NRPS) present in SM cluster \#11. The hydroxamic acid functional group in both aspergillic acid and its hydroxy analog allows the molecules to bind to iron resulting in the production of a red pigment in A. flavus identified as ferriaspergillin. Knockout of the NRPS gene resulted in loss of AA production. An A. flavus NRPS mutant demonstrated significantly reduced levels of infection and growth in a laboratory assay with corn kernels resulting in reduction of aflatoxin B1 and cyclopiazonic acid compared to a control. This suggests a role for AA in iron homeostasis and virulence on crops.

Global novel effector-mining in the rice blast fungus Magnaporthe oryzae

X. YAN (1), V. M. Were (1), L. S. Ryder (1), B. Tang (1), M. Oses-Ruiz (1), D. Soanes (2), F. L. H. Menke (1), N. J. Talbot (1), (1) The Sainsbury Laboratory, University of East Anglia, Norwich Research Park, Norwich, U.K.; (2) University of Exeter, Exeter, U.K.

Blast disease is caused by the ascomycete fungus Magnaporthe oryzae and is one of the most devastating plant diseases threatening global food security. It is therefore important to investigate the interaction between the blast fungus and its host plant. Fungi secrete effector proteins into the apoplast and into plant cells, to facilitate invasive hyphal growth. Transcriptional profile analysis revealed differentially expressed genes of $M$. oryzae during rice infection. We classified 401 of these genes as candidate effector-encoding genes, including the well-characterized $A V R$-PITA, $A V R-P I K$ and SLP1 genes. Translational GFP fusions were expressed in M. oryzae to visualize expression and secretion of each effector candidate by live-cell imaging. Transient expression of effector candidates in Nicotiana benthamiana was used to visualize subcellular localization in plant cells and interference with plant defense. We functionally identified a group of novel MEP (Magnaporthe Effector Proteins) effector genes. Apoplastic Mep effectors outlined invasive hyphae, while cytoplasmic Mep effectors accumulated at the biotrophic interfacial complex (BIC) in infected rice cells. Early expressed Mep1 family effectors play an important role in the suppression of plant immunity, Mep3 family effectors are involved in the inhibition of ROS production, while the late expressed Mep2 family effectors are required for transition of the rice blast fungus from biotrophic growth to necrotrophic growth.

A single Verticillium dahliae effector induces defoliation of cotton plants G. L. FIORIN (1), J. Li (1), L. Faino (2), B. P. H. J. Thomma (1), (1) Wageningen University, Wageningen, Netherlands; (2) Sapienza Università di Roma, Italy

Verticillium dahliae is a soil-borne fungal pathogen that causes wilt disease on a wide range of dicotyledonous plants. Although symptoms such as chlorosis, stunting and withering are typically induced by pathogenic strains, differential aggressiveness towards a given host is often observed among isolates. On cotton and olive, $V$. dahliae strains either exhibit a highly aggressive phenotype, including defoliation, or milder symptoms 
that do not include defoliation. In order to gain insights into the molecular basis of this phenomenon, we sequenced and compared the genomes of $V$. dahliae strains belonging to either of the two pathotypes. Consequently, we identified a genomic region that is exclusively present in defoliating strains, comprising only two, identical, genes that are highly induced upon host colonization. Thus, we hypothesized that the effector that is encoded by these genes is responsible for the defoliation phenotype. Indeed, transgenic lines of a non-defoliating strain expressing the effector gene gained the ability to defoliate cotton plants, while deletion lines of a defoliating strain lacking both effector gene copies lost pathogenicity on cotton. Furthermore, cotton seedlings treated with heterologously-produced effector protein exhibited defoliation symptoms. Taken together, we identified a defoliation-inducing effector that is a pathogenicity factor of $V$. dahliae on cotton.

Identifying disease-critical effectors of a bacterial plant pathogen by knockout

J. JAYARAMAN, E. Applegate, M. D. Templeton, The New Zealand Institute for Plant and Food Research Ltd, Auckland, New Zealand

The kiwifruit bacterial canker pathogen Pseudomonas syringae pv. actinidiae Biovar 3 (Psa BV3) causes canker disease on kiwifruit (Actinidia spp.). Psa BV3 strain ICMP 18884 injects approximately 30 effector proteins into host cells via a canonical type III secretion system (T3SS). We tested effectors, both individually and in blocks, for reduced Psa BV3 virulence in the native kiwifruit host using gene knockouts. Mutants deleted for individual effector genes ( $\triangle h o p A Z 1$ and $\triangle h o p S 2)$, the entire exchangeable effector locus mutant ( $\triangle E E L ; 10$ effectors), and the conserved effector locus mutant ( $\triangle C E L ; 3$ effectors) were all independently significant contributors to virulence in bacterial growth and disease phenotype tests. All four contributors to virulence were additive in their effect on pathogenicity and a Psa BV3 multi-knockout of these four elements resembled a complete type III secretion system knockout mutant $(\triangle h r c C)$. We show that AvrE1 alone in the $C E L$ is required for full pathogenicity and that four EEL effectors (HopD1, AvrB4, HopAW1, and HopAO2) likely redundantly contribute to pathogenicity through suppression of PTI. These results support the hypothesis that, similar to other $P$. syringae pathovars, Psa carries robust effector redundancy for a large portion of its effectors with a few being truly critical to disease.

MiR398 plays a regulatory role in Bamboo mosaic virus infection

K. Y. Lin (1), Y. H. Hsu (2), N. S. LIN (3), (1) Institute of plant and microbial biology, Academia sinica, Taipei, Taiwan; (2) Graduate Institute of Biotechnology, National Chung Hsing University, Taichung, Taiwan; (3) Institute of Plant and Microbial Biology, Academia Sinica, Taipei, Taiwan

MicroRNAs (miRNAs) regulate gene expression involved in development and defense responses by transcript cleavage or translational repression in diverse eukaryotes. Numerous miRNAs were changed upon virus infection, however, the interaction between miRNAs and virus infection remains largely elusive. By comparing small RNA profiles of mock- and Bamboo mosaic virus (BaMV)- infected Nicotiana benthamiana, we confirmed that $\mathrm{Nb}$-miR398 was one of the upregulated miRNAs as BaMV infected over time. MiR398 is one of the wellstudied stress responsive miRNAs involved in oxidative stress, ultraviolet stress, copper and phosphate deficiency and bacterial infection. We have identified Nb-miR398 targeting copper/zinc superoxide dismutase (CSD) 1 and 2 by RNA ligase-mediated rapid amplification of cDNA ends. Overexpressing miR398 resulted in the decreased CSD1/2 and increased BaMV titer, indicating that miR398 can enhance BaMV accumulation. Surprisingly, higher CSDs in both RNA and protein levels were also detected upon BaMV infection. Besides BaMV infection, BaMV triple gene block protein 1 alone can also induce miR398 but not NbCSD2. Of note, when plants infected with asymptomatic BaMV $\triangle$ CPN35 mutant, a truncated but not full-length form CSD2 was detected in infected leaves. Taken together, our results suggest that the induction of miR398 by BaMV infection may facilitate titer accumulation while CSD2 expression level may contribute to BaMV symptom development in $N$. benthamiana.

A new putative effector Ha34609 of Heterodera avenae targeting to tonoplastc, is involved in the formation and maintenance of syncytia J. Jian, D. Wu, X. Li, H. Peng, J. Cui, W. Huang, L. Kong, D. PENG, Institute of Plant Protection, Chinese Academy of Agricultural Sciences, Beijing, China

Heterodera avenae is the most important namtode pest on wheat worldwide, it caused serious yield losses and threaten to wheat production and food security in China. A high-throughput transcriptome sequencing of the H. avenae were performed and 63 putative novel effectors were identified. Among then, A new putative effector Ha34609 was cloned. The cDNA full length is $837 \mathrm{bp}$, encoding one 194-amino-acids peptide. No transmembrane domain were found. It had a high similarity with G20E03 of H. glycines. In situ hybridization showed that the transcripts of Ha34609 were mainly expressed in subventral esophageal gland cells of H. avenae. Gene expression showed that Ha34609 transcriptions were expressed effectively at the preJ2 and parasitic J2 and J3. Subcellular localization assays revealed that Ha34609 was localized in the tonoplast of tobacco leaves cell. It is confirmed that Ha34609 is synthesized by subventral esophageal gland cells of $H$. avenae and then secretes into host tissues through the stylet, suggesting that Ha34609 invovled in the formation and maintenance of syncytia by acting on the tonoplast during infection and early parasitic process. After J2 soaking with dsRNA and inoculation plant, the female number and whole size were significantly reduced compared with controls. Yeast two-hybrid (YTH) has been using to identify the protein interaction with Ha36409, positive clones were obtained. This work was supported by the Natural Science Foundation of China (31772142)

The Phytophthora sojae effector Avh241 modulates host immunity by targeting soybean NDR1

B. YANG, Y. Wang, B. Guo, H. Wang, W. Ye, Y. Wang, S. Dong, Y. Wang, Nanjing Agricultural University, China

Phytophthora pathogens secrete an arsenal of effectors to manipulate host innate immunity and thus facilitating infection. Our previous work showed that Avh241 induces cell death in plants, but also promotes the infection of Phytophthora, indicating that Avh241 interacts with the plant immune system via at least two different mechanisms. By using coimmunoprecipitation (Co-IP) and liquid chromatography-tandem mass spectrometry (LC-MS/MS) analysis in Nicotiana benthamiana, we identified the NDR1 (non-race-specific disease resistance 1) isoforms as a host target of Avh241. We showed that Avh241 interacts with $N$. benthamiana and soybean NDR1 in vivo. Silencing of NDR1 in N. benthamiana increase plant susceptibility to $P$. capsici infection, and overexpression of $N D R 1$ induce resistance to $P$. capsici in $N$. benthamiana. However, 
silencing of $N b N D R 1$ does not affect the cell death triggered by Avh241, indicating that targeting of NDR1 involves the virulence function of Avh241. The molecular mechanisms underlying how Avh241 interferes with NDR1 function will be further studied.

A Xanthomonas effector suppresses host immunity possibly by inhibiting dimerization of host factor.

K. YAMAGUCHI (1), G. Yamamoto (1), S. Yoshimura (1), S. Tsuge (2), T. Kawasaki (1), (1) Dept. Adv. Biosci. Kindai Univ., Nara, Japan; (2) Kyoto Prefectural Univ, Kyoto, Japan

Xanthomonas oryzae pv. oryzae (Xоo) is a bacterium which causes a serious blight of rice. Previously we found that the transgenic rice plant expressing XopZ, one of the Xoo effectors showed severe susceptibility to the type III secretion system-deficient mutant of Xoo. It suggested that XopZ may inhibit host factors involved in PAMPs-triggered resistance (PTI). To examine the subcellular localization of XopZ, we transiently expressed XopZ-GFP in rice protoplasts. Despite its nuclear localization signal, XopZ was localized in nuclei. To elucidate the molecular mechanisms of XopZ-mediated PTI inhibition, we tried to identify host targets that interact with XopZ by yeast two-hybrid screens and found several XopZ Interacting Protein (ZIPs) for the host target, which include transcriptional factors. Among them, ZIP3 is hypothetical protein and localized in nucleus and cytosol in rice. ZIP3 contains Structural Maintenance of Chromosomes (SMC) domain at N terminus. XopZ also contains SMC domain at $\mathrm{N}$ terminus. The $\mathrm{Y} 2 \mathrm{H}$ assay showed that the $\mathrm{N}$ terminal of XopZ specifically interacted with $\mathrm{N}$ terminal of ZIP3. The $\mathrm{N}$ terminal of ZIP3 formed the dimer. Interestingly, the Y3H assay showed that XopZ inhibited the dimerization of ZIP3. In this study, the inhibitory mechanism of XopZ against rice immunity will be discussed.

The ethylene response factor StERF49 negatively regulates potato immune response during potato virus $\mathrm{Y}$ infection A. COLL (1), T. Lukan (1), H. McLellan (2), M. Petek (1), P. R. J. Birch (2,3), S. Prat (4), K. Gruden (1), (1) National Institute of Biology, Ljubljana, Slovenia; (2) Division of Plant Science, James Hutton Institute, University of Dundee, Dundee, U.K.; (3) Cell and Molecular Sciences, James Hutton Institute, Dundee, U.K.; (4) Centro Nacional de Biotecnología-CSIC, Madrid, Spain

Potato is the world's most widely grown tuber crop and Potato virus Y (PVY) is one of the major potato pathogens causing severe crop losses worldwide. It is thus important to improve our understanding of potato-PVY interaction for more efficient crop breeding strategies. The analysis of transcriptomics data previously obtained in our group suggested that the ethylene response factor StERF49 is an important player in $N y$ mediated hypersensitive response to PVY. Studies with stable transgenic potato lines in which StERF49 was knockdown revealed that the gene has a positive role in viral multiplication and symptom development and, consequently, can be considered as a susceptible factor in PVY infection. We performed RNAseq experiments on PVY infected and mock inoculated leaves of StERF49-knockdown potato plants. Several gens involved in the protein degradation pathway seem to be regulated by StERF49 after PVY infection. On the other hand, the StERF49 is strongly accumulated in cell nucleus only after PVY infection. Considering these results, we hypothesis that PVY might interfere in the host mechanisms for its own benefit by preventing degradation of StERF49. We are further studying the complex network of plant signaling pathways involved in this response using $\mathrm{Y} 2 \mathrm{H}$ and $\mathrm{Y} 1 \mathrm{H}$ assays. We identified two transcription factors, involved in the ethylene signaling pathway, that activate the expression of StERF49 gene.

Characterization of Pseudomonas putida L5 and P. fluorescence K1-3-1, two endophytic bacteria with ability for biohardening of micropropagated strawberry seedlings

N. C. LIN, M. C. Huang, National Taiwan University, Taipei, Taiwan

Strawberry (Fragaria $\times$ ananassa Duchesne) is an economically important herbaceous plant. Due to latent infection of Colletotrichum gloeosporioides and infection of daughter plants by Fusarium oxysporum f. sp. fragariae through runners, use of the micropropagated plantlets as the mother plants could be a good mean to propagate specific-pathogen-free (SPF) seedlings. Attempts to screen beneficial endophytic bacteria with potential to improve the hardening procedure (biohardening) for micropropagated strawberry seedlings were achieved by the plant growth promotion and pathogenicity assays using endophytic bacteria isolated from the roots of strawberry plants collected in the field. Among those endophytic bacteria, eighty-eight isolates could produce siderophores, 43 isolates were tested positive for nitrogen fixation and 55 isolates showed potential for solubilizing inorganic phosphate. The plant growth promotion assay revealed that inoculation of micropropagated strawberry plantlets with Pseudomonas putida L5 could increase biomass of the seedlings. Furthermore, $P$. fluorescens K1-3-1 could enhance strawberry resistance to anthracnose and inhibit mycelial growth of $C$. gloeosporioides and $F$. o. f. sp. fragariae in an antagonistic assay. Previous studies indicated that salicylic acid (SA) signaling pathway is important for strawberry resistance against anthracnose, and whether SA signaling pathway is involved in induced resistance by K1-3-1 is currently under investigation.

An effector protein from Ralstonia solanacearum targets plant $\mathbf{E} 3$ ligases to alter secondary metabolism in plant cells and promote disease development

J. S. RUFIAN (1), X. Liu (1), Y. Wang (1), H. Xue (1), Y. Sang (1), J. M. Zhou (2), A. P. Macho (1), (1) Shanghai Center for Plant Stress Biology, China; (2) Institute of Genetics and Developmental Biology, Chinese Academy of Sciences, Beijing, China

Bacterial wilt caused by Ralstonia solanacearum is a serious threat to several crops worldwide. In order to survive, proliferate and produce disease, $R$. solanacearum must suppress the plant defence responses and manipulate other physiological functions in plant cells, and this role is carried out by bacterial proteins called type III effectors. Deciphering the function of type III effectors is expected to uncover plant physiological functions important for bacterial pathogenesis and disease resistance. The $R$. solanacearum effector Rip 15 suppresses plant immunity in both $N$. benthamiana and Arabidopsis. A transcriptomic analysis of Arabidopsis transgenic lines expressing Rip15 showed a deregulation of genes related to specific secondary metabolism pathways, and further metabolomic analysis confirmed an altered concentration of their associated metabolites. We are currently validating the association of these metabolites with actual immune responses that lead to disease resistance. A Yeast-TwoHybrid screen identified several E3 ligases as targets for Rip15. Subsequently, we found that these E3 ligases regulate the accumulation of several proteins involved in early immune signaling, linking the biochemical activity of Rip15 with its impact in the suppression of defence 
responses. Altogether, our work may reveal a novel pathway linking early immune signaling to the alteration of metabolic pathways for the execution of efficient immune responses against bacterial pathogens.

The Ralstonia solanacearum PopP2 effector exploits immune-associated MAPKs to potentiate its virulence functions and evade host recognition

M. Escouboué (1), G. Huet (2), C. Vicedo (1), A. Jauneau (3), F. Roux (4), L. Xian (5), A. P. Macho (5), J. Lluch (6), Y. Couté (7), S. Carrere (8), D. Rengel (4), L. DESLANDES (1), (1) Université de Toulouse, LIPM, CNRS-INRA, Toulouse, France; (2) CNRS/INRA, Castanet-Tolosan, France; (3) Université de Toulouse, FRAIB 3450, UPS-CNRS, Toulouse, France; (4) LIPM, Université de Toulouse, INRA, CNRS, INPT, Castanet-Tolosan, France; (5) Shanghai Center for Plant Stress Biology, China; (6) INRA, GeT-Plage, Genotoul, Castanet-Tolosan, France; (7) CEA, Grenoble, France; (8) Université de Toulouse, INRA, CNRS, Toulouse, France

The Gram-negative Ralstonia solanacearum $(R s)$ is the causal agent of bacterial wilt disease. One of the best characterized effectors from $R s$ is the PopP2 acetyltransferase. PopP2 dampens basal immunity through acetylation of the DNA-binding domain of multiple WRKY defensive transcription factors, causing loss of their transactivating functions needed for defense gene expression. By contrast, PopP2 elicits disease resistance in Arabidopsis in presence of the RPS4/RRS1-R NLR receptor complex. Here, we found that PopP2 is phosphorylated in planta on three SP motifs located in its N-terminus. We identified in PopP2 a domain similar to previously described MAPK-docking domains and that is required for phosphorylation. Protein-protein interaction studies and bacterial phosphorylation assay demonstrated that PopP2 is a direct substrate of immune-associated MAPKs. Consistent with that, activation of MAPKs signaling cascades during PTI promotes PopP2 phosphorylation. Although being dispensable for its avirulence function in Arabidopsis expressing RPS4/RRS1-R, PopP2 phosphorylation is required for full virulence activity. Surprisingly, when delivered by $R s$, PopP2 phosphomutants, but not a PopP2 phosphomimic variant, trigger an RRS1independent resistance phenotype. Together these data highlight a virulence strategy where a bacterial effector benefits from early steps of PTI signaling activation to both promote its virulence functions and avoid recognition.

Sarocladium oryzae and Pseudomonas fuscovaginae, two very different pathogens causing rice sheath rot in a surprisingly similar way K. J. PEETERS, K. Demeestere, M. Hofte, Ghent University, Ghent, Belgium

Sheath rot is an emerging rice disease that can destroy the yield of a growing season. Diseased plants show necrotic lesions on the leaf sheath and produce empty seeds or no seeds at all. The main sheath rot pathogens are the fungus Sarocladium oryzae and the bacterium Pseudomonas fuscovaginae. They produce respectively the toxins helvolic acid and cerulenin and cyclic lipopeptides. As these pathogens cause similar symptoms, we compared their interaction with the host. Phytohormones were measured at different time points during infection using HPLC-ESIMS/HRMS. Both pathogens triggered abscisic acid (ABA), jasmonic acid (JA) and 12-oxo-phytodienoic acid (OPDA). For $S$. oryzae, these levels correlated with in planta helvolic acid production, severity of sheath rot symptoms and loss in grain production. Although ABA, JA and OPDA are known for their defense-regulatory role, they do not act as resistance factors during $S$. oryzae infection. Both the ABA and JA pathway, however, are known to interfere with seed setting and filling by interrupting source-sink relationships. We hypothesise that the toxins produced by S. oryzae and P. fuscovaginae increase ABA, JA and OPDA levels, resulting in lower grain yields. We are currently investigating the correlation of these phytohormones with symptoms caused by P. fuscovaginae. Moreover, we study the role of the microbial toxins in these host manipulations by using knock-out mutants in both pathogens.

Specificity of proteasome regulation by tail-anchored transcription factors during plant immunity G. LANGIN (1), R. S. Marshall (2), R. D. Vierstra (2), S. Üstün (1), (1) Center for Plant Molecular Biology (ZMBP), Tübingen, Germany; (2) Department of biology, Washington University in St. Louis, St. Louis, MO, U.S.A.

The ubiquitin proteasome system (UPS) is essential for many developmental and physiological process during plant life. Recent studies showed the importance of the UPS for plant immunity. However, the UPS is exploited by plant pathogenic bacteria Pseudomonas syringae pv. tomato (Pst), which suppresses proteasome function via autophagic degradation. This suggests the presence of a multi-layered fine-tuning mechanism of proteasome regulation during plant-microbe interactions. Here, we want to characterize the specificity of proteasome regulation during the interaction of Pseudomonas syringae and Arabidopsis thaliana. Recently a pair of tail-anchored transcriptions factors (TFs), NAC53 and NAC78, have been shown to be involved in the regulation of the proteasome complex. Our preliminary data indicate that these two NAC TFs participate in the proteasome stress response (PSR) during A. thaliana infection by $P$. syringae. The gene expression and protein abundance of proteasome subunits is partially affected in nac53 nac 78 mutants, suggesting that $P$ st induces proteasome stress regulon using both TFs. In addition, nac53 nac78 plants are more resistant against $P$ st infection, thus implying that the activation of the proteasome is required for virulence. Taken together, our analysis will provide new insights of how bacterial infection might influence the balance between up- and down regulation of proteasome function.

Pseudomonas syringae produces an inhibitor of a plant defence-related Beta-galactosidase

N. SANGUANKIATTICHAI, B. Chandrasekar, P. Buscaill, R. A. L. Van Der Hoorn, G. M. Preston, University of Oxford, U.K.

During infection, plants and bacteria participate in a dynamic interaction in the apoplast. Secreted hydrolases are among the prominent players in plant defence and are therefore targets of virulence factors. Using Nicotiana benthamiana and Pseudomonas syringae pv. tomato (Pst) DC3000 as a model system, we recently discovered a plant $\beta$-galactosidase (BGAL1) that functions in immunity against bacteria by facilitating the hydrolytic release of elicitor peptides from glycosylated flagellin, which activates plant defences. Interestingly, PstDC3000 produce an inhibitor of BGAL1. Here, we identify the biosynthetic gene cluster that is responsible for BGAL1 inhibitor production, which is under the control of type III secretion system regulators hrpR/S/L. Mutant bacteria lacking this gene cluster show reduced virulence in $N$. benthamiana. Comparative genomics suggests acquisition of this gene cluster via horizontal gene transfer and indicates a correlation between inhibitor production and the identity of flagellin glycans. Partial purification and characterization of the inhibitor has revealed that it is a hydrophilic, heat stable and basic small 
molecule, similar to a sugar derivative. Our work has uncovered a novel small molecule virulence factor that is secreted into the apoplast to inhibit a plant defence-related enzyme and highlights the role of glycans and apoplastic enzymes in plant-pathogen interactions.

Bioinformatics and transcriptomic analysis of the HrpL regulon in Pseudomonas savastanoi pathovars of woody host A. MORENO PEREZ (1), P. Rodriguez-Palenzuela (2), C. Ramos (1), (1) IHSM-UMA-CSIC, Malaga, Spain; (2) ETSIA UPM, Madrid, Spain

The species Pseudomonas savastanoi, a member of the Pseudomonas syringae complex, includes four pathovars causing knots or excrescences in woody hosts: P. savastanoi pv. savastanoi (Psv), pv. fraxini (Psf), pv. nerii (Psn) and pv. retacarpa (Psr), comprising isolates from olive, ash, oleander and broom plants, respectively. One of the better-characterized pathogenicity factor in this bacterial complex is the type III secretion system (T3SS) and its effector (T3E) repertoire. Transcription of the T3SS structural genes and that of most of its T3E genes is positively regulated by HrpL. We have constructed $\triangle h r p L$ mutants in a model strain belonging to each of these four pathovars and have analyzed the role of this gene in the pathogenicity of $P$. savastanoi. As previously reported for Psv, the hrpL gene is also necessary in Psn, Psf and Psr for both symptoms production during a compatible interaction and the induction of the hypersensitive response in a resistant host. To characterize the HrpL regulon, we are currently following two strategies, (i) the bioinformatics identification of HrpL-dependent promoters in the genomes of several P. savastanoi pathovars and, (ii) a comparative RNAseq analysis of a wild-type Psv strain and its $\Delta h r p L$ mutant. The results obtained will be compared with those published for other $P$. syringae strains isolated from herbaceous hosts, in order to identify specific HrpL-dependent genes involved in the interaction with woody hosts.

Natural variation of HopQ1 effector from Pseudomonas syringae

P. B. ZEMBEK (1), A. Danilecka (1), R. Hoser (1), L. Eschen Lippold (2), M. Benicka (1), M. Grech-Baran (1), M. Piechocki (1), J. Poznański (1), J. Lee (2), J. Hennig (1), M. Krzymowska (1), (1) Institute of Biochemistry and Biophysics, PAS, Warsaw, Poland; (2) Leibniz Institute of Plant Biochemistry, Halle (Saale), Germany

Sequence analysis of the HopQ1 effector family revealed a low amino acid diversity among Pseudomonas syringae strains. However, two HopQ1 variants from phaseolicola 1448A (Pph) and tomato DC3000 (Pto) pathovars, that differed only by six amino acids, showed considerable differences in their ability to suppress plant defences. The presence of serine 87 and leucine 91 rendered $P$ toHopQ 1 susceptible to N-terminal processing by plant proteases. Substitutions at these two positions did not strongly affect PtoHopQ1 virulence properties in a susceptible host, however, in a resistant host they reduced bacterial growth and accelerated onset of cell death. This suggests that presence of S87 and L91 rendered PtoHopQ1 susceptible to processing in planta and, thus, represents a mechanism of recognition avoidance. Furthermore, our data revealed that the co-expression of HopR1, another effector encoded within the same gene cluster, masks HopQ1-triggered hypersensitive response in a strain-dependent manner. Therefore, we have undertaken a large-scale analysis of $P$. syringae strains to reveal HopR 1 features that determine its ability to co-operate with the specific HopQ1 variant. Collectively, our findings suggest that $P$. syringae may have evolved at least two independent strategies of recognition avoidance based on differential effector processing or masking, and that the variability of HopQ1 sequences results from the high host-pathogen co-evolutionary selection pressure.

Cytokinin level manipulation in the ergot fungus Claviceps purpurea

S. KIND (1,2), J. Vrabka (3), P. W. Tudzynski (2), P. Galuszka (3), (1) RWTH Aachen University, Plant Physiology Department, Aachen, Germany; (2) WWU Muenster University, Germany; (3) Centre of the Region Haná for Biotechnological and Agricultural Research, Olomouc, Czech Republic

The ergot fungus Claviceps purpurea is a biotrophic phytopathogen with sophisticated host manipulation strategies. The plant hormones cytokinins $(\mathrm{CKs})$ are known to regulate diverse plant cell processes and several plant pathogens alter CK levels during infection. $C$. purpurea synthesizes CKs via two mechanisms and fungus-derived CKs influence the host-pathogen interaction. CK deficiency in fungi was only achieved to date by deletion of a tRNA-ipt which is also involved in translation. For a better understanding of the CK biosynthesis and the CK contribution to the plant-fungus interaction we applied different approaches. The first approach is based on the deletion of the two "Log" encoding genes which are supposed to be essential for the release of active CKs. Single and double deletion strains were able to produce all types of CKs. Apparently, $\log$ gene products are dispensable for the formation of CKs and alternative activation pathways must be present. The CK biosynthesis pathway remains unaffected in the second approach as it is based on heterologous expression of a CK degrading enzyme from maize (Ckx1). $z m c k x 1$ overexpressing C. purpurea strains have Ckx activity and drastically reduced CK levels. Evidently, expression of $z m c k x 1$ resulted in the formation of functional protein. The strains are impaired in virulence which reinforces the assumption that fungal-derived CKs are crucial for full virulence.

Investigating the barley proteome of powdery mildew haustorial complexes to identify host susceptibility factors promoting Blumeria infection

S. LAMBERTUCCI (1), K. M. Orman (1), S. Das Gupta (1), F. P. James (1), S. Gazal (1), R. J. Williamson (2), R. Cramer (2), L. V. Bindschedler (1), (1) Royal Holloway University of London, Egham, U.K.; (2) University of Reading, Reading, U.K.

The biotrophic fungal pathogen Blumeria graminis is responsible for powdery mildew infection in cereal crops. Blumeria forms specialized feeding structures exclusively within the plant epidermis called haustoria. Haustoria are surrounded by a host extrahaustorial membrane (EHM) which is in continuum to but distinct from the plant plasma membrane. Host proteins localized to the EHM are likely to modulate the infection process, but the EHM proteome remains unknown. A differential proteomics approach was undertaken to identify barley proteins more abundant in barley epidermis and enriched haustorial structures during infection. These proteins are putatively associated to haustorial structures and possibly to the EHM. Several pathogenesis-related proteins were more abundant in infected epidermis: cysteine-rich venom secreted proteins (PR1), peroxidases, chitinases, and two thaumatin-like proteins (TLP, PR5). TLP5 was shown to interact with the effector BEC1054. Transient induced gene silencing (TIGS) of TLP5 enhanced barley susceptibility to Blumeria. Proteins associated with enriched haustorial structures included: a $23 \mathrm{kDa}$ jasmonate induced protein, a macrophage migration inhibitory factor homolog, a xylosidase and xylanase inhibitors, TCA 
cycle enzymes, and proteins which generate proton gradients. This large-scale proteomics approach combined to TIGS was successful in identifying key mediators of barley susceptibility and resistance to Blumeria.

A structural perspective on the diversification and adaptation of oomycete and fungal plant pathogen effector proteins R. K. HUGHES, M. J. Banfield, John Innes Centre, Norwich Research Park, Norwich, U.K.

Studies of filamentous plant pathogen effector proteins have revealed unexpected structural similarities amid the sequence diversity. Conserved protein folds, such as the oomycete WY-domain and the fungal MAX and ToxA-like domains, act as building blocks to support structural integrity, while allowing plasticity in protein function. This plasticity is most likely encoded in the effectors' protein surfaces, allowing them to interact with different host partners. Effector evolution and diversification can develop via mutation, addition of short linear motifs, domain integration and duplications, and oligomerisation. Adaptations of the oomycete $\alpha$-helical WY-domain fold showing such changes include AVR3a11, PexRD2, PexRD54 and PiSFI3 from Phytophthora sp. Other work will be described addressing effectors from Magnaporthe oryzae, some of which share a six-stranded $\beta$-sandwich (MAX) fold but show variations that give rise to distinct surface properties. Intriguingly, there are also examples of filamentous plant pathogen effector folds that appear to be unique, and not part of larger families. The discovery and characterisation of effector protein folds, to explore diversity and adaptability in plant pathogen strategies to modulate host cell functions, remains an important objective in plant-microbe interactions. Understanding the interactions between effector proteins and their host protein targets may lead to new opportunities to restrict plant disease in key crops.

Functional characterization of a regulator for leaf tumor formation in Ustilago maydis

J. S. LIN (1), C. Pellegrin (2), P. Happel (1), S. Reissman (1), R. Kahmann (3), (1) Max Planck Institute for Terrestrial Microbiology, Germany; (2) University of Cambridge, U.K.; (3) Max Planck Institute for Terrestrial Microbiology, Marburg, Germany

The fungus Ustilago maydis causes corn smut disease with characteristic tumor formation. Previously, we analyzed the transcriptional changes during plant associated stages of $U$. maydis by RNAseq analysis and demonstrated a crucial role for leaf tumor formation and effector gene expression for one transcription factor Nlt1 (No leaf tumors 1). Microscopic analysis and fungal biomass determination now revealed that the $n l t 1$ mutant did colonize plants efficiently, could not complete karyogamy, and was attenuated in massive late proliferation. Although the nlt 1 mutant failed to induce tumors in leaves, small tumors were detected in cobs, male flowers as well as at the base of the stem in tissue that is actively dividing. To elucidate which genes and processes are regulated by Nlt1, RNAseq analysis was performed of mutant and wild type infected tissue and putative direct Nlt1 targets were identified by ChIP-seq analysis. A large of genes were regulated by Nlt1, and among these we identified 360 putative direct targets. These included 17 transcription factors as well as 35 effector genes. Interestingly, we also found that maize genes involved in auxin signaling and transport are positively regulated by Nlt1. We currently focus on the analysis of these effector genes with the aim of linking their function to leaf tumor induction and auxin signaling.

Regulation of Bacterial Pathogenesis of Plants by Two Component Systems A. PANDEY, CSIR-Central Institute of Medicinal and Aromatic Plants, Lucknow, India

Two component systems (TCSs) are one of the most prevalent ways by which bacteria sense, respond, and adapt to various changes in their environment. We are using Rice-Xanthomonas oryzae pv. oryzae (Xoo; the causal agent of rice disease called bacterial blight) pathosystem to understand the role of TCSs in plant-microbe interaction. Genome sequence analysis revealed that Xoo strains are equipped with large repertoire of TCSs. TCS constitute a signal transduction mechanism which is basically formed by two proteins: a membrane bound histidine kinase sensor and a cytoplasmic response regulator. Sensor histidine kinase responds to an environmental change or stimulus by phosphorylating a cognate response regulator which triggers changes in cellular physiology or gene expression in order to enhance adaptability. A system level mutagenesis in an Indian Xoo strain led to identification of several virulence genes encoding for response regulators. Here, work will be presented indicating importance for an orphan response regulator (encoded by BXO1_05645 gene) for Xoo virulence on rice. This response regulator contains the CheY-like receiver domain and is required for in planta growth as well as migration, suppression of host defense response, induction of non-host resistance, optimum expression of type 3 secretion system, EPS production, motility, biofilm formation and survival under specific stress conditions.

Functional characterization of the conserved effector AvrHar in Pyrenophora teres $f$. teres

N. A. WYATT (1), J. K. Richards (2), R. S. Brueggeman (3), T. L. Friesen (4), (1) North Dakota State University, Fargo, ND, U.S.A.; (2) Louisiana State University Agricultural Center, Baton Rouge, LA, U.S.A.; (3) Department of Plant Pathology, North Dakota State University, Fargo, ND, U.S.A.; (4) Cereal Crops Research Unit, USDA-ARS, Fargo, ND, U.S.A.

Pyrenophora teres f. teres is the causal agent of net form net blotch (NFNB) on barley. NFNB is an economically important disease in all barley growing regions globally, but little is known of the molecular mechanisms involved in the barley- $P$. teres f. teres interaction. Recently, a genomic locus was found to be associated with avirulence/virulence on Tifang barley using a genome wide association study (GWAS) approach with a global $P$. teres $\mathrm{f}$. teres population. This genomic locus was also identified in the P. teres $\mathrm{f}$. teres $15 \mathrm{~A} \times 0-1$ mapping population corresponding to the virulence/avirulence locus AvrHar. AvrHar is in the sub-telomeric region of chromosome 5 and RNAseq-supported gene models placed a small secreted protein (SSP) in this region. Comparative analysis of the effector gene between the avirulent isolate $15 \mathrm{~A}$ and the virulent isolate 0 1 revealed an insertion of an alanine and aspartic acid in the virulent 0-1 isolate. Gene disruption using CRISPR-Cas9-RNP mediated gene editing in isolate $0-1$ resulted in a loss of virulence on Tifang as well as 27 barley lines commonly used in differential sets. Site directed gene disruption in isolate 15A had no visible effect on virulence for the same barley lines. AvrHar is present in a global collection of P. teres f.teres and P. teres f. maculata isolates as well as the closely related wheat fungal pathogen Pyrenophora tritici-repentis. Further functional characterization is underway using CRISPR-Cas9 mediated gene editing. 
Association mapping to identify Sclerotinia sclerotiorum genes influencing aggressiveness on sunflower K. Belay (1), R. Sharma Poudel (1), R. S. Brueggeman (1), W. UNDERWOOD (2), (1) Department of Plant Pathology, North Dakota State University, Fargo, ND, U.S.A.; (2) Sunflower \& Plant Biology Research Unit, USDA-ARS, Fargo, ND, U.S.A.

Sclerotinia sclerotiorum is a necrotrophic fungal pathogen causing disease and economic loss on numerous crop plants. This fungus has a broad host range and can infect over 400 plant species, including important oilseed crops such as soybean, canola, and sunflower. S. sclerotiorum isolates vary in aggressiveness of lesion formation on plant tissues. However, the genetic basis for this variation remains to be determined. We sought to identify loci responsible for differences in aggressiveness among S. sclerotiorum isolates via an association mapping approach, with the assumption that the functions of underlying genes will reveal novel insights into S. sclerotiorum pathogenicity. We evaluated a diverse collection of 227 S. sclerotiorum isolates for aggressiveness of stem lesion formation on sunflower. In parallel, we performed genotyping-by-sequencing on the isolate collection to discover SNP markers for genome-wide association mapping to identify candidate genes associated with aggressiveness. These efforts identified a total of 9 loci associated with variation in aggressiveness. We are currently evaluating candidate genes at several loci, including a transmembrane transporter and several small secreted proteins, for roles in pathogenicity by marker-replacement mutagenesis. Functional characterization of these gene products will improve our understanding of the virulence strategies used by this pathogen to cause disease on a wide array of agriculturally important host plants.

Reference Genes for Transcript Profiling in Uromyces appendiculatus during Urediospore Infection of Common Bean T. I. LINK, University of Hohenheim, Stuttgart Baden-Wuerttemberg, Germany

Uromyces appendiculatus is a major pathogen in common bean. The most abundant spore form during the disease cycle that also mediates epidemic spread is the urediospore. From the urediospore the infection process proceeds through germination, appressorium formation, penetration and formation of infection hyphae, haustoria formation, and later sporulation combined with senescence of both the infected leave and fungal colonies on it. Analysis of gene expression during the infection process is a major part of dissecting the molecular mechanisms enabling this biotrophic interaction. For future transcript profiling using RT-qPCR suitable reference genes for normalization were determined. Samples representing seven stages of the infection process were used in RT-qPCR to measure the transcript levels of 14 candidate reference genes and to determine the best ones based on Cq range and on comparative calculations using the algorithms geNorm and NormFinder. Here $T u b$ and $U b c E 2$ were rated best by geNorm with $C y t B$ as a third. $U b c E 2$, Tub, and $E l f 3$ were the first choice of NormFinder. The Cq range was smallest for RPS14, followed by Elfla and Elf3. Combining these rankings with equal weight $U b c E 2, E l f 3$ and Tub can be considered the best reference genes for transcript profiling during the infection process of $U$. appendiculatus.

Host-interactor screens of RXLR effectors reveal plant processes manipulated by Phytophthora J. WIN (1), M. P. Contreras (1), B. Petre (2), T. O. Bozkurt (3), M. H. Schattat (4), J. Sklenar (1), A. Abd-el-Haliem (5), Y. F. Dagdas (6), R. Lozano-Duran (7), A. M. E. Jones (8), J. H. Vossen (9), S. Robatzek (10), S. Kamoun (1), (1) The Sainsbury Laboratory, University of East Anglia, Norwich Research Park, Norwich, U.K.; (2) Université de Lorraine, Nancy, France; (3) Imperial College London, U.K.; (4) Department of Plant Physiology, Martin Luther University, Halle, Germany; (5) University of Amsterdam, Amsterdam, Netherlands; (6) Gregor Mendel Institute of Molecular Plant Biology, Vienna, Austria; (7) Shanghai Center for Plant Stress Biology, China; (8) University of Warwick, Coventry, U.K.; (9) Plant Breeding, Wageningen University and Research, Wageningen, Netherlands; (10) Ludwig-Maximilians-Universität München, Genetics, Martinsried, Germany

Oomycete plant pathogens deliver RXLR effectors inside plant cells to alter host processes to their advantage. Effectors interact with plant proteins including their cognate targets and helpers to achieve their functions. This study aims to identify the plant proteins interacting with RXLR effectors and ultimately the function of the effectors in planta. We transiently expressed 75 FLAG-tagged RXLR effectors in Nicotiana benthamiana using the high-expression vector pTRBO. Effector fusions and associated host proteins were co-immunoprecipitated (co-IP) and identified by LC-MS/MS spectrum matching against $N$. benthamiana proteome database. Out of 75 effectors, 53 were expressed to sufficient levels for co-IP and identification by mass spectrometry. GO annotation of associated plant proteins revealed 48 plant processes that might be targeted by the effectors. We classified the candidate interacters with a scoring system. So far, we confirmed more than 20 effector-plant protein associations. Notably, we found that the PEXRD12/31 effectors associated and co-localized with components of vesicle trafficking machinery including VAMPs, VAC14, coatomers, vesicle fusing ATPase, syntaxin, exocyst, and Ras-related proteins. PEXRD31 increased the number of FYVE-labelled endosomal membrane compartments in plant cells, suggesting that the effector modulates a process related to endosomal trafficking. We will discuss these findings and their implications for plant-oomycete interactions.

Function and regulation of Verticillium longisporum-encoded putative effector genes

L. SCHÄFER, J. Schmitz, C. Thurow, C. Gatz, Georg-August-University Göttingen, Göttingen, Germany

The plant pathogenic fungus Verticillium spec. is a soil-borne pathogen that enters its host plants through the roots. Subsequently, it colonizes the xylem where it completes its life cycle. Verticillium longisporum, which infects mainly brassicaceae, is responsible for large economical losses in e.g. Brassica napus (reviewed by Depotter et al. (2016)). We are interested in the plant signals that induce fungal gene expression during its stay in the xylem. To identify plant induced fungal genes we have conducted transcriptome analysis of infected Arabidopsis thaliana roots at 10 days postinoculation. We have started to analyze four putative $V$. longisporum effector genes that are highly induced upon interaction with $A$. thaliana. Promoter:reporter gene fusions will now serve to spatially and temporally resolve the expression pattern of these genes. Additionally, one of these putative effector genes encoding a hypothetical protein was deleted to analyze its role during the infection process. Infection of $A$. thaliana with the corresponding knock-out lines led to reduced symptoms as compared to wild type. Further studies will have to unravel whether these reduced symptoms correspond with less pathogen growth and if so, at which stage pathogen growth is compromised. 
Crystal structure of rice blast effector AVR-Pii in complex with host Exo70 defines a new effector-target interface and a novel fold for fungal effectors

J. C. DE LA CONCEPCION (1), K. Fujisaki (2), N. Cruz-Mireles (1,3), V. Sanchez (1), J. Win (3), D. Lawson (1), S. Kamoun (3), R. Terauchi (4,5), M. J. Banfield (1), (1) John Innes Centre, Norwich Research Park, Norwich, U.K.; (2) Iwate Biotechnology Research Center, Japan; (3) The Sainsbury Laboratory, University of East Anglia, Norwich Research Park, Norwich, U.K.; (4) Graduate School of Agriculture, Kyoto University, Kyoto, Japan; (5) Iwate Biotechnology Research Center, Kitakami, Japan

Exocytosis is increasingly recognized as having an important role in plant-microbe interactions, both in pathogenesis and symbiosis. Exocyst subunit Exo70 is involved in pathogen virulence and is monitored by the plant immune system to detect invading pathogens. Exo70 proteins associate with RIN4/NOI containing proteins, which are well-known targets of pathogen effectors. Both Exo70 and NOI domains are frequently integrated into NLR receptors. To date, knowledge of how effectors perturb exocyst function is still limited. The Magnaporthe oryzae effector AVR-Pii binds rice Exo70F-2 and Exo70F-3. AVR-Pii association with Exo70F-3 is detected by the immune receptor pair Pii, via an integrated NOI domain. Here, we dissect the molecular basis of AVR-Pii interaction with rice Exo70s. We determined the crystal structure of AVR-Pii in complex with rice Exo70F-2, allowing us to define the interface between these proteins. In future work we will use structure-informed mutations to gain better understanding of how the AVR-Pii/Exo70 interface underpins protein function. Furthermore, the crystal structure of AVR-Pii revealed a new type of protein fold in fungal effectors, based on a Zinc-finger motif. We will exploit this information to explore the prevalence and function of Zinc-finger fold (ZIF) effectors across M. oryzae lineages.

Is the $\mathrm{pH}$ responsive transcription factor PacC required for the virulence of wheat pathogenic fungi and the regulation of effector expression in Blumeria graminis?

S. DAS GUPTA (1), M. Urban (2), S. Gazal (1), H. Blyth (2), J. Rudd (2), G. Scalliet (3), K. Hammond-Kosack (2), L. V. Bindschedler (1), (1) Royal Holloway University of London, Egham, U.K.; (2) Rothamsted Research, Harpenden, U.K.; (3) Syngenta Crop Protection AG, Stein, Switzerland

A battery of powdery mildew haustoria specific Candidate Secreted Effector Proteins (CSEPs) have been identified in Blumeria graminis f. sp. $h o r d e i$, the causal agent of barley powdery mildew. However, the regulation of CSEP gene expression is not known. Using an $a b$ initio prediction tool, we identified a conserved $\mathrm{pH}$ regulatory element (PRE) overrepresented in CSEP-specific promoter regions that might explain CSEPS' relative high expression in haustoria. PREs are targeted by the $\mathrm{pH}$ responsive transcription factor PacC/RIM101. PacC promotes growth and virulence of various fungal pathogens like Candida and Aspergillus sp. at alkaline or neutral pH, but negatively regulates virulence in Fusarium oxysporum. PacC is activated by high $\mathrm{pH}$, inducing genes needed for alkaline growth while repressing genes required for acid growth. The host induced gene silencing (HIGS) of Blumeria PacC homologue reduced powdery mildew infection, suggesting its role for Blumeria virulence. Moreover, PacC silencing reduced transcript levels of the known Blumeria effectors - BEC1019 and BEC1011. Thus, further suggesting the role of PacC in Blumeria effector regulation. Since BEC1019 has homologues in other fungi like pH regulated Candida PRA1, and in wheat pathogens Fusarium graminearum and Zymoseptoria tritici, the role of $\mathrm{PacC}$ in virulence of these wheat pathogens is also being investigated.

Next-generation interaction screening for discovery of host targets of fungal effectors J. M. Elmore (1), V. Velasquez-Zapata (2), S. Banerjee (2), P. Surana (2), G. Fuerst (1), R. WISE (1), (1) USDA-ARS/Iowa State University, Ames, IA, U.S.A.; (2) Iowa State University, Ames, IA, U.S.A.

Defining the temporal dynamics of fungal-host interactions is a critical step in understanding pathogen virulence and host defense in cereals. The genome of the causal agent of barley powdery mildew disease, Blumeria graminis f. sp. hordei (Bgh), encodes $>500$ candidate secreted effector proteins (CSEPs). Effectors target plant proteins to suppress plant immunity and/or manipulate host physiology to enable colonization. While many Bgh CSEPs contribute to infection, the molecular mechanisms of CSEP-mediated virulence are largely unknown. We have undertaken a large-scale yeast two-hybrid approach using next-generation sequencing to establish a protein-protein interaction framework of $B g h$ virulence and barley defense. First, we used deep RNA-Seq of barley lines containing the Mla6 resistance gene and isogenic immune system mutants inoculated with $B g h 5874\left(A V R_{a 6}\right)$ to profile pathogen gene expression during key early stages of fungal infection. Subsequently, 100 highly expressed CSEPs were selected based on divergent expression patterns on resistant and susceptible genotypes and used as baits to screen a comprehensive cDNA library derived from the infection time course. Our results have uncovered CSEP interactions with barley immune signaling proteins, cellular chaperones, transcriptional regulators, and proteins of unknown function. We will present current work towards validating and assaying these interactions for functional significance during $B g h$ infection.

Abscisic acid-mediated signaling is a nexus of host modulation by the A. tumefaciens Type VI Secretion System L. M. BANTA, R. Froom, R. Gorelov, W. Doyle, J. Troiano, J. E. Bravo, Williams College, Williamstown, MA, U.S.A.

In A. tumefaciens, as in several other bacterial species, the recently discovered type VI secretion system (T6SS) releases effectors that act as toxins in inter- and intra-bacterial competition. However, the contribution of the A. tumefaciens T6SS to virulence is not yet well understood. We discovered that inoculation of Arabidopsis thaliana stems with agrobacteria lacking the T6SS resulted in attenuated virulence. Ablation of the homologous secretion system in the closely related alpha-proteobacterium Rhizobium leguminosarum was previously shown to extend the bacterium's host range. Together, these and other data from our lab led us to postulate that the T6SS promotes bacterial virulence in mature plants by dampening $A$. thaliana defenses but may elicit host defenses that limit transient transformation of host seedlings. Gene expression studies identified the abscisic acid (ABA) hormone pathway as a likely direct or indirect target of the T6SS. While the T6SS did not alter transcription of the rate-limiting ABA biosynthetic gene, it did impact ABA-regulated gene expression. Seedlings with defects in ABA sensitivity exhibited altered susceptibility to transient transformation compared to control seedlings. Finally, our data implicate the receptor kinase Lyk3, a mediator of late ABA responses as well as rhizobial Nod factor-induced repression of host immunity, in T6SS recognition. 
Ca. Liberibacter asiaticus peroxiredoxin (LasBCP) is a secreted effector that suppresses ROS-mediated localized and systemic defense responses in citrus.

M. Jain, A. Munoz-Bodnar, D. W. GABRIEL, University of Florida, Gainesville, FL, U.S.A.

Two peroxiredoxin genes of uncultured $\mathrm{Ca}$. Liberibacter asiaticus (Las), causal agent of Huanglongbing (HLB or citrus greening) were functionally characterized in culturable proxy host L. crescens (Lcr). One of these, Lasbcp (CLIBASIA_RS00445), is expressed only in planta and was confirmed to be secreted from Lcr with substrate specificity for organic peroxides. Transient expression of Lasbcp in tobacco confirmed its role as a potential pathogenicity effector. LasBCP suppressed $\mathrm{H}_{2} \mathrm{O}_{2}$-mediated transcriptional activation of RbohB, the key gatekeeper of the systemic plant defense signaling cascade. LasBCP expression also interrupted Las flagellin-induced RBOHB activation and callose deposition. Quantitative RT-PCR analyses showed transcriptional downregulation of oxylipin-regulated jasmonic acid and salicylic acid signaling pathways in citrus leaves expressing LasBCP. Taken together, these data demonstrated a protective role of secreted LasBCP for Las survival and colonization by (1) alleviating ROS-induced lipid peroxidation in citrus host, (2) preventing localized accumulation of antimicrobial oxylipins, and (3) suppressing both localized and systemic immune responses in planta. A high throughput assay was used to identify 28 chemicals from two Prestwick small molecule libraries for inhibition of LasBCP enzymatic activity. Three lead chemicals are currently being examined for potential HLB control.

HopG1 targets the Arabidopsis thaliana nonhost resistance 2B (AtNHR2B) to interfere with plant immune responses upon bacterial infection.

C. M. RODRIGUEZ-PUERTO (1), R. Singh (2), C. M. Rojas (1), (1) University of Arkansas, Fayetteville, AR, U.S.A.; (2) University of Arkansas, FAYETTEVILLE, AR, U.S.A.

The pathogenicity of Pseudomonas syringae is associated with the type III secretion system (T3SS), a complex of proteins assembled in the inner and outer bacterial membranes that traverses the plant cell wall to deliver bacterial proteins into the cytoplasm of plant cells. The effector proteins translocated into the plant cells are called Hops (Hypersensitive response and pathogenicity outer proteins). Bacterial effectors target plant immune proteins to suppress defense responses and enhance bacterial parasitism. The Arabidopsis thaliana nonhost resistance 2B (AtNHR2B), a recently identified immune protein, is degraded after inoculation with the adapted pathogen of Arabidopsis $P$. syringae pv tomato DC 3000 (PstDC3000), but not by the non-adapted pathogen P. syringae pv. tabaci (Pstab). A yeast-two hybrid screening revealed that several PstDC3000 effectors, including HopG1, HopD1, HopAO1, HopR1, and HopC1, interact with AtNHR2B. Interestingly, transgenic plants expressing HopG1 in combination with AtNHR2B caused susceptibility to Pstab as shown by the development of disease symptoms and by a significant increase in bacterial growth. Co-localization experiments by live-cell imaging and cellular fractionation approaches using a fluorescent version of AtNHR2B (AtNHR2B-GFP) revealed that AtNHR2B-GFP localizes to the mitochondria, where HopG1 also localizes. Together, these results suggest that HopG1 targets AtNHR2B to interfere with plant immune response upon bacterial infection.

Uncovering soybean rust effectors and their interactions within the host

M. Qi (1), M. G. Elmore (1), H. Yu (1), T. I. Link (2), K. F. Pedley (3), S. A. WHITHAM (1), (1) Iowa State University, Ames, IA, U.S.A.; (2) University of Hohenheim, Stuttgart Baden-Wuerttemberg, Germany; (3) USDA ARS FDWSRU, Frederick, MD, U.S.A.

Phakopsora pachyrhizi, the causal agent of Asian soybean rust (ASR), secretes an arsenal of effector proteins to manipulate host immunity. Therefore, identifying $P$. pachyrhizi effectors and understanding their functions is crucial to curb soybean losses to ASR. Previously, $156 P$. pachyrhizi effector candidates (PpECs) were predicted from the haustorial transcriptome. These PpECs were cloned into various vectors that enabled expression in heterologous systems to perform screens for effector-like functions and in planta localization. Several of the PpECs suppressed and/or activated defense or defense-like responses in host and non-host plants. We further functionally characterized PpECs that belong to a small cysteine-rich family of proteins or have protease activity, because they strongly suppressed immune responses. PpECs were also screened for potential effector-effector interactions using the yeast two hybrid system to identify possible hierarchical relationships that might influence function. More recently, new PpECs were discovered from multiple stages/time points during infection using different transcriptome sequencing platforms. From this data set, a few hundred new PpECs and their subcellular localizations were predicted using EffectorP, LOCALIZER, and ApoplastP prediction tools. Together, these data are providing new insights into the network of protein-protein interactions that occur during infection to promote $P$. pachyrhizi pathogenicity.

A novel SPRYSEC effector RBP-2 from soybean cyst nematode Heterodera glyciens suppresses plant immunity to promote parasitism H. PENG, W. Huang, L. Kong, J. Cui, D. Peng, Institute of Plant Protection, Chinese Academy of Agricultural Sciences, Beijing, China

The soybean cyst nematode ( $\mathrm{SCN}$ ), Heterodera glycines, is one of the most economically important pest on soybean worldwide, and causing extensive annual yield losses. Effectors of phyto-nemaotdes are proved to play key roles during nematode-host interactions by modifying plant cell well and suppressing host deference as well as modulating development of host cells. A large effector family of Ryanodine receptor (SPRY) domain-containing proteins have been identified from potato cyst nematodes. In our study, a novel SPRYSEC effector candidate $H g$-r $r p$ - 2 was isolated and characterized. An in situ hybridization assay demonstrated that $H g-r b p-2$ is expressed specifically in the dorsal pharyngeal gland cell, $H g-r b p-2$ has the highest expression in parasitic J2 and J3 stages. Subcellular localization assays revealed that $H g$-rbp-2 is localized in the cytoplasm. Transgenic soybean hairy root expressing $H g-r b p-2$ become significantly more susceptible to $H$. glycines infection than control plants, and conversely, RNA interference-mediated gene silencing of the $H g-r b p-2$ significantly reduced infectivity of the pathogen. Evidence indicating that the effector $H g-r b p-2$ plays a role in nematode parasitism. Transient expression of $H g-r b p-2$ in Nicotiana benthamiana suppressed flg22induced ROS production in leaf explants. Yeast two-hybrid screening has been used to identify the host target protein. This work was supported by the Natural Science Foundation of China (31672012). 
Mapping of the key events in the development of the tomato hairy root disease E. CEULEMANS, VIB Center for Plant Systems Biology, Gent, Belgium

Cultivation of hydroponically grown tomatoes is currently facing problems due to the occurrence of the hairy root disease. This disease is characterized by an extensively expanded root system, leading to withering and yield losses. The microbes responsible for the hairy root disease are collectively referred to as rhizogenic Agrobacteria. In this group, Agrobacterium biovar 1 strains are the causative agent for hairy roots in tomato and other Solanaceae. To date, the molecular mechanisms behind the development of hairy root disease in an infected tomato plant remain virtually unknown. Therefore, we are performing transcriptomics, metabolomics and interactomics analysis to identify key genes and processes involved in rhizogenic Agrobacterium infection or the plant's defense response. Time-course RNA sequencing experiments together with elaborate metabolomics profiling will shed more light on how the infection evolves and which plant metabolic, signaling or other cellular processes are modulated. A yeast two-hybrid next generation sequencing (Y2H-seq) platform has been developed and is being used to screen for tomato proteins that interact with the Agrobacterium biovar 1 bait proteins encoded on the T-DNA of the Ri plasmid. The knowledge gained during this project will not only provide novel fundamental insights in plant disease and defense development but may ultimately also provide means to combat the hairy root disease or improve Agrobacterium-based transformation methods.

The Pathogen-Host Interactions phenotype database, PHI-base: Harnessing community expertise to fight plant diseases K. HAMMOND-KOSACK, Rothamsted Research, Harpenden, U.K.

PHI-base (www.phi-base.org) is a knowledge database accessed by researchers in over 125 countries. PHI-base contains expertly curated molecular and biological information on genes proven to affect the outcome of pathogen-host interactions reported in peer reviewed research articles. Genes not affecting the disease interaction phenotype are also curated. PHI-base data is linked to the genome browsers and advanced query tools in ENSEMBL and FungiDB. The data content provided comes from $>3000$ manually curated references and reports information on 6438 genes from 263 pathogens (fungal, protist and bacteria) tested on 194 hosts (plant, animal, others) in 11340 interactions. The direct targets of pathogen effector proteins are also included. The different use types (including simple queries, hypothesis testing, exploring newly sequenced pathogen genomes, comparative analyses as well as pathways and protein-protein network analyses) and the future directions of PHI-base, including the development of an online author curation tool called PHI-Canto, to capture new data sets at source, will be discussed.

Differentially expressed genes and metabolomic regulation induced by salt and Epichloë endophyte in Festuca rubra subsp. pruinosa E. PEREIRA (1), I. Zabalgogeazcoa (1), I. Nagy (2), A. Schilmiller (3), B. Hamberger (4), B. R. Vázquez de Aldana (1), T. Asp (2), (1) Institute of Natural Resources and Agrobiology of Salamanca (IRNASA-CSIC), Salamanca, Spain; (2) Dept. Molecular Biology and Genetics, Science and Technology, Aarhus University, Slagelse, Denmark; (3) Mass Spectrometry \& Metabolomics Core Facility, Michigan State University, East Lansing, MI, U.S.A.; (4) Department of Biochemistry and Molecular Biology, Michigan State University, East Lansing, MI, U.S.A.

Festuca rubra subsp. pruinosa (FRP) is a perennial grass that inhabits sea cliffs of the Atlantic coasts of Europe. In this unhospitable environment plants are exposed to abiotic stress factors such as salinity. These plants maintain a natural symbiotic association with the fungal endophyte Epichloë festucae, which colonizes the intercellular space of aerial tissues. The production of secondary metabolites is an important characteristic of this symbiosis. Epichloë species can provide benefits to their hosts but it is still unclear if $E$. festucae can be beneficial to FRP plants, and which mechanisms are involved. Clones of FRP infected by E. festucae (E+) and endophyte free (E-) were exposed to tap and saltwater treatments. Transcriptome response and metabolomic changes in response to salinity and Epichloë infection were investigated. E- plants subjected to salinity showed 18 and 32 times more transcripts accumulated to higher and lower level, respectively, than E+ plants exposed to the same conditions. Gene Ontology enrichment analysis showed changes in several biological pathways in response to salinity stress. A metabolic analysis identified two abundant compounds unique to plants infected by Epichloë with a mass consistent with terpenoids. Our results reveal that the salinity causes dramatic changes on the FRP gene expression but can be attenuated by the presence of Epichloë, suggesting that this endophyte infection has an important effect in the host metabolism.

Study of the functional domains of the PTGS suppressor V2 from geminivirus Beet curly top virus (BCTV) A. P. LUNA (1), E. A. Rodríguez-Negrete (2), G. Morilla (1), A. G. Castillo (1), E. Rodríguez Bejarano (1), (1) IHSM-UMA-CSIC, Málaga, Spain; (2) Instituto Politécnico Nacional, Sinaloa, Mexico

Geminiviruses constitute a group of plant viruses that infect vegetable crops all over the world. Among the Geminiviridae family, the genera Mastrevirus, Begomovirus and Curtovirus are the most abundant. Suppression of gene silencing is a key mechanism for viral infection in plants. In begomovirus, V2 is a strong posttranscriptional gene silencing suppressor. We recently showed that V2 from curtovirus Beet curly top virus (BCTV) is a PTGS suppressor by impairing the RDR6/SGS3 pathway, as V2 from begomovirus. In order to identify the domains involved in the suppression activity and viral pathogenicity, we performed an alignment of several begomovirus and curtovirus V2 proteins. A protein kinase $\mathrm{C}$ (PKC) phosphorylation motif essential for suppression activity in begomovirus (P1) was found in all analysed sequences. We also found similar hydrophobic profiles, with two hydrophobic domains $(\mathrm{H} 1$ and $\mathrm{H} 2)$ followed by a long hydrophilic domain. Then we generated BCTV V2 mutant proteins and performed transient assays in Nicotiana benthamiana plants to test their suppression activity. We also expressed them from a Potato virus $X$-derived vector to check the symptoms produced. Additionally, their subcellular localization was determined. Finally, we produced BCTV viruses mutated in the different domains and $N$. benthamiana plants were infected, analysing virus levels and symptoms produced. The results showed that $\mathrm{P} 1, \mathrm{H} 1$ and $\mathrm{H} 2$ are involved in the suppression activity and viral pathogenicity.

A genome-wide screen identifies Pseudomonas syringae B728a genes required for epiphytic and apoplastic leaf colonization T. C. HELMANN (1), A. M. Deutschbauer (2), S. E. Lindow (1), (1) University of California, Berkeley, CA, U.S.A.; (2) Lawrence Berkeley National Laboratory, Berkeley, CA, U.S.A. 
To compare the fitness contribution of individual genes in Pseudomonas syringae B728a across multiple phyllosphere habitats and hosts, we constructed a large, mapped, randomly barcoded transposon mutant library (median 21 insertions per gene). Changes in relative proportion of each mutant within the mixture enabled quantification of fitness of a given gene in the apoplast of the susceptible host Phaseolus vulgaris (green bean), in which the pooled population grew 1000-fold. Mutations in 108 genes moderately reduced growth (2- to 4-fold), and disruption of 65 additional genes severely reduced growth (>4-fold). On the leaf surface, 84 mutants grew 2 - to 4 -fold less than wild type, while 31 grew over $4-$ fold less. 55 genes contributed to growth both in and on the leaf. Genes within the functional categories of amino acid and polysaccharide (including alginate) biosynthesis contributed most to fitness in or on leaves, while genes in the type III secretion system and syringomycin synthesis were specifically important in the apoplast. Additionally, we revealed numerous hypothetical proteins and uncategorized glycosyltransferases with significant contributions to competitive fitness in leaf colonization. For most genes, no relationship was seen between fitness in planta and measured expression in planta in other studies. A lack of association of gene expression and fitness has important implications for our understanding of plant-microbe interactions.

Mapping and pyramiding adult plant resistance loci to manage oat crown rust caused by Puccinia coronata f. sp. avenae E. S. NAZARENO (1), M. E. Miller (2), M. Carson (3), S. Chao (4), M. Figueroa (1), S. F. Kianian (3), (1) University of Minnesota, St. Paul, MN, U.S.A.; (2) Pairwise, Durham, NC, U.S.A.; (3) USDA-ARS Cereal Disease Laboratory, St. Paul, MN, U.S.A.; (4) USDA-ARS Cereal Crops Research Unit, Fargo, ND, U.S.A.

The fungus Puccinia coronata f. sp. avenae ( $P c a$ ) causes crown rust, a disease that poses a threat to global oat production. Given the rapid evolution of $P c a$ to overcome race-specific resistance, adult plant resistance (APR) is regarded as a sustainable management strategy for this disease. Here, we are mapping APR loci in recombinant inbred line (RIL) populations generated from nine biparental crosses. Of these, three populations have been mapped to date. These RILs were phenotyped for three years under high disease pressure conditions at a buckthorn (the alternate host) nursery, where a natural sexually recombining crown rust population exists. Selected lines from each population were genotyped using the 6K Oat SNP Chip. For CI1712 $\times$ Otana, results showed that two loci are associated with the resistance phenotype in CI1712: one in Mrg (linkage group) 6 with five linked markers and one in Mrg 11 with 13 linked markers. For PI260616-1 × Otana and CI8000-4 × Otana, our analysis showed that both populations have a major QTL in Mrg 21 with 10 linked markers. Kompetitive Allele-Specific PCR (KASP) markers were used to validate the presence of these loci in the whole mapping population. For the latter two populations, two KASP markers explained $30-51 \%$ of the phenotypic variation. Crosses have been initiated to pyramid these loci into a single line for future cultivar development. This work will also help in cloning resistance genes from oat and elucidate the molecular basis of APR.

Effector distribution in Xanthomonas species that cause bacterial leaf spot of tomato, capsicum and chilli in Australia R. Roach (1), R. Mann (2), C. F. GAMBEY (3), R. Shivas (4), T. Chapman (5), B. Rodoni (2), (1) Horticulture and Forestry Science, Department of Agriculture \& Fisheries, QLD, Brisbane, QLD, Australia; (2) Microbial Sciences, Pests \& Diseases, Department of Jobs, Precincts and Regions, Bundoora, VIC, Australia; (3) Horticulture and Forestry Science, Department of Agriculture \& Fisheries, QLD, Applethorpe, QLD, Australia; (4) Department of Agriculture and Fisheries, Brisbane, QLD, Australia; (5) Plant Biosecurity Research NSW Department of Primary Industries, Menangle, NSW, Australia

Bacterial leaf spot is a disease that affects Australian tomato, capsicum and chilli production every year by reducing yield and spreading rapidly in wet weather conditions. Despite their prevalence in production areas, control options are limited to resistance breeding in capsicum and chilli lines and general chemical sprays. The species that cause this disease in Australia were recently determined to be Xanthomonas vesicatoria, $X$. euvesicatoria and X. perforans. Recent genomic studies of Australian Xanthomonas species have provided sequence data that allow for the investigation of genetic elements associated with host range. The distribution of these genetic elements, termed 'effectors', across the studied Xanthomonas species can indicate how they may interact with their host plant.Strains from the major in-field production areas of Australian tomato, capsicum and chilli were collected in addition to historical herbarium strains. Whole genome sequences of these strains were compared to publicly available Xanthomonas genomes from across the world. The taxonomy and genome structure of pathogenic and non-pathogenic Xanthomonas species from this collection was determined using multiple methods. Protein sequence with homology to established effectors were identified within this dataset, resulting in a hypothetical effector profile for $X$. vesicatoria, X. euvesicatoria and X. perforans. These profiles were then interrogated to reveal conserved effectors within these species.

Evolution of pathogen resistance in wild tomato populations

R. STAM, G. A. Silva-Arias, P. Kahlon Singh, A. Tellier, Technische Universität München, Freising, Germany

We study which genes and molecular processes are involved in the evolutionary arms race between plants and pathogens in a natural context. We investigate the diversity and selection of defence-associated genes within a wild tomato species, Solanum chilense. The species shows diversity in resistance properties against several main tomato pathogens throughout its range and we find that $\mathrm{R}$ protein-mediated recognition of Cladosporium fulvum effectors correlates geographically with high recognition frequencies in the centre of the species range and loss on the edges. Furthermore, we used R-gene enrichment sequencing (RENSeq) to obtain polymorphism data at Nucleotide binding site Leucine-rich repeat Receptors (NLRs), which are canonical resistance (R) genes in plants, fungi and animals. We studied NLRs of 14 populations covering the whole species range and identified small subsets of outlier NLRs exhibiting signatures of selection across the different populations. NLRs are known to function as central (helper) and peripheral (sensor) proteins in a signaling network. NLRs under selection between habitats are more often helper NLRs, while NLRs showing signatures of adaptation in single populations are more often sensor NLRs. Thus, centrality in the NLR network does not constrain NLR evolvability, and new mutations in central genes in the network are key for R gene adaptation during colonisation of different habitats. These findings help understand durability of resistance in nature.

Genomic characterization and effector identification in 'Candidatus Phytoplasma cynodontis', the causative agent of Bermudagrass white leaf (BGWL) disease 
S. T. Cho (1,2), H. J. Kung (1), C. P. Lin (2), C. H. KUO (1), (1) Academia Sinica, Taipei, Taiwan; (2) National Taiwan University, Taipei, Taiwan

'Candidatus Phytoplasma cynodontis' is the causative agent of Bermudagrass white leaf (BGWL) disease, which was first reported in Taiwan in 1972 and has been found worldwide. The symptoms include chlorosis and dwarfism, thus decreasing the quality of sports fields and pastures, as well as lowering the protection against soil erosion. This $\mathrm{Ca}$. species belongs to the $16 \mathrm{SrXIV}$ group and is highly divergent from those wellstudied phytoplasmas (e.g., ' $\mathrm{Ca}$. P. asteris' in the 16SrI group). To characterize its gene content, as well as the genomic diversity among phytoplasmas, we performed metagenome sequencing of a diseased plant for comparative analysis. The draft assembly contains five phytoplasma contigs totaling 502,218 bp (i.e., $>90 \%$ of the estimated chromosome size) and 425 protein-coding genes were annotated. Comparisons with six other representative phytoplasma genomes revealed extensive diversification in gene content. Notably, ' $\mathrm{Ca}$. P. cynodontis' harbors a new type of potential mobile unit (PMU) but none of the three characterized phytoplasma effectors (i.e., SAP11, SAP54/PHYL1, and TENGU). Bioinformatic prediction, metatranscriptomics, and empirical validation identified two novel effectors that induced abnormal development in Nicotiana benthamiana. These findings improved our understanding of these uncultivated plant pathogens. Our on-going work on the molecular interactions between these novel effectors and the plant host could further elucidate phytoplasma pathology.

A novel gene cluster involved in high tolerance to copper compounds has been detected in Pseudomonas syringae pv. syringae J. A. GUTIÉRREZ-BARRANQUERO (1), F. Aprile (2), F. Cazorla (1), A. De Vicente (1), (1) Department of Microbiology, Fac. of Science. University of Malaga, Málaga, Spain; (2) Department of Microbiology, Fac. of Science, University of Málaga., Málaga, Spain

Pseudomonas syringae pv. syringae (Pss), is the most polyphagous bacterium of the $P$. syringae complex based on its wide host range, that severely affects woody and herbaceous plants. Pss has been described as the causal agent of bacterial apical necrosis (BAN) on mango crops, an important threat that drastically reduces the mango fruit yield in the Mediterranean region. Copper compounds have traditionally been used as standard bactericides to manage woody plant diseases, being the spraying of copper compound known as Bordeaux mixture (BM), the conventional treatment for controlling BAN disease. The extensive use of copper compounds can lead to many problems, and among them, the emergence of copper-resistant strains is one of the most serious. Pss strains isolated from mango trees harbour at least, two different genetic structures (copABCD and copABCD modified) encoded by plasmids that are involved in copper detoxification. Determination of the minimum inhibitory concentration of copper, together with a phylogenetic distribution analysis performed in a collection of Pss strains isolated from mango trees revealed a phylogenetic group of Pss strongly associated with high levels of copper resistance. Genome mining and functional characterization of one Pss strain present in this group have uncovered a novel gene cluster of chromosomal location that could be playing a major role in high levels of copper resistance during the epiphytic survival of Pss on mango tree surfaces.

Mapping of a major quantitative trait locus for bakanae disease resistance in rice H. JI (1), K. S. Cheon (1), J. Oh (1), D. Y. Kang (1), H. Oh (1), S. L. Kim (1), Y. J. Won (2), J. Baek (1), I. Choi (1), I. S. Yoon (1), K. H. Kim (1), (1) National Institute of Agricultural Sciences, Jeonju, Korea, Republic of (South); (2) National Institute of Crop Science, Suwon, Korea, Republic of (South)

Bakanae disease (BD) is caused by a seed-borne fungal pathogen, Fusarium fujikuroi, and has become a serious threat in almost all rice cultivation regions worldwide. Nampyeong is a Korean japonica rice variety known to be resistant to BD. In this study, quantitative trait locus (QTL) mapping was performed with F2 and F3 plants derived from a cross between the Nampyeong variety and a susceptible Korean japonica variety, Junam. Using 205 kompetitive allele-specific PCR (KASP) markers, a genetic map was constructed from the genotypes of 188 plants from the Junam/Nampyeong F2 plants. The total length of the constructed genetic map was $1477.5 \mathrm{cM}$ with an average interval between markers of $7.66 \mathrm{cM}$. The $\mathrm{BD}$ mortality rate of each F2 plant was measured by testing $60 \mathrm{~F} 3$ progenies by in vitro seedling screening. QTL analysis based on the genetic map and mortality rate data revealed a major QTL, $q F f 1$, on rice chromosome $1 . q F f 1$ was located at $98.9 \mathrm{cM}$ with a logarithm of the odds (LOD) score of 21.4 and $\mathrm{R}^{2}$ value of 0.50 . The closest marker to this QTL was KJ01_075. The $q F f 1$ and its closely linked marker will be useful in breeding rice varieties resistant to BD.

A new role of retrotransposons in fungal virulence

A. WEIBERG, Ludwig-Maximilians University (LMU), Munich, Germany

Retrotransposons (RTs) are fascinating "alien DNA" populating genomes of all living species. RTs are mobile mutagens therefore dangerous for a hosting genome, while transient RT activation in pathogens seems to drive evolution of genomic regions comprising virulence genes, which contributes to host adaptation. RNA interference (RNAi) is a genome surveillance mechanism that controls RT activity, and RTs are hotspots of small RNA (sRNA) production. Botrytis cinerea is a broad host-range fungal pathogen thatdelivers sRNAs into plants to suppress host innate immunity, a mechanism called cross-kingdom RNAi. It stays illusive how this pathogen acquired sRNAs to suppress host genes in many plant species. Interestingly, most Botrytis virulence sRNAs derive from RTs. And Botrytis population studies reported on transposon-carrying strains outnumber transposon-less strains on host plants. These observations led us suggest RTs as important source of virulence, and we now provide first evidence. We found the two B. cinerea isolates D08 and D14 lack RTs and virulence sRNAs, and both strains showed low virulence. Genome and sRNA sequencing revealed RTs being heavily C-> T/G->A mutated by RIP (Repeat Induced Point mutation) that led to loss of RTsRNA accumulation. Remarkably, transgenic insertion of an unRIPed RT into the D08 genome resulted in RT-sRNA production, enhanced virulence, and plant target gene silencing. This new role of RTs in fungal virulence raises new intriguing questions.

Gene erosion and genome expansion in a group of highly host-specialized fungal phytopathogens

L. Frantzeskakis (1), M. Németh (2), M. Barsoum (3), S. Kusch (3), S. Takamatsu (4), L. Kiss (5), R. PANSTRUGA (3), (1) DOE Joint Genome Institute, U.S.A.; (2) Hungarian Academy of Sciences, Hungary; (3) RWTH Aachen University, Aachen, Germany; (4) Mie University, Japan;

(5) University of Southern Queensland, Australia 
Powdery mildew fungi have a strict host dependency (termed obligate biotrophy) and unusually large genomes ( $>100 \mathrm{Mb}$ ). This size expansion is largely due to the pervasiveness of transposable elements on $70 \%$ of the genomes and is associated with the loss of multiple conserved ascomycete genes required for a free-living lifestyle. To date, little is known about the mechanisms that drove this expansion, and information on ancestral powdery mildew genomes is lacking. We report the genome analysis of the early-diverged and exclusively sexually reproducing powdery mildew, Parauncinula polyspora. In contrast to other sequenced species of the group, the $P$. polyspora genome is surprisingly small $(<30 \mathrm{Mb})$, has a higher content of conserved ascomycete genes and is sparsely equipped with transposons $(<10 \%)$ despite the conserved absence of a common defense mechanism involved in constraining repetitive elements. We speculate that transposable element spread might have been limited by this pathogen's unique reproduction strategy and host features and further hypothesize that the loss of conserved ascomycete genes may promote the evolutionary isolation and host niche specialization of powdery mildew fungi. Limitations associated with this evolutionary trajectory might have been in part counteracted by the evolution of plastic, transposon-rich genomes and/or the expansion of gene families encoding secreted virulence proteins.

Genotypic and functional characterization of Streptomyces spp. causing potato common scab in Eastern Canada C. HUDEC, A. Novinscak, S. Lamarre, M. Filion, Université de Moncton, Moncton, NB, Canada

Streptomyces scabies is the main causal agent of common scab, a potato disease whose symptoms significantly decreases the market value of potato tubers. Closely related species, including $S$. acidiscabies and $S$. turgidiscabies have also been shown to cause the disease, and significant intraspecific variations among these species have also been reported. In an effort to better characterize the diversity of scab causing Streptomyces spp., as well as better understand how this diversity impacts virulence, isolation and characterization of two hundred and ninety-six pathogenic Streptomyces spp. isolates retrieved from diseased tubers were undertaken. The tubers were collected from commercial fields distributed along a longitudinal gradient in Prince Edward Island, the main potato-producing province in Canada. DNA was extracted from each isolate and genome fingerprinting analyses using repetitive elements polymerase chain reactions (ERIC- and BOX-PCR) were performed. They revealed 14 distinct genetic groups, which were further characterized using multi-locus sequence analysis. The geographical distribution of these groups was analyzed, and their pathogenicity and virulence were characterized using in vitro and in vivo bioassays. Finally, PacBio RSII genome sequencing was performed on the 14 groups and comparative genomic analyses are presently ongoing. This study will contribute to better understanding the interaction existing between taxonomy, functional diversity and virulence.

Whole-genome analyses reveals novel pathogenic features of the necrotrophic pathogen Alternaria brassicae S. RAJARAMMOHAN (1), D. Pental (2), J. Kaur (3), (1) National Agri-Food Biotechnology Institute, Mohali, India; (2) Centre for Genetic Manipulation of Crop Plants, University of Delhi South Campus, India; (3) Department of Genetics, University of Delhi South Campus, New Delhi, India

Alternaria brassicae, a necrotrophic fungal pathogen, causes Alternaria Leaf Blight, one of the most economically important diseases of Brassica crops. A reference-quality genome assembly of $A$. brassicae has been generated using minION sequencing with a N50 of $3.05 \mathrm{Mb}$. Wholegenome phylogenetic analysis reconfirmed the monotypic lineage of $A$. brassicae within the Alternaria genus. Genome alignments with other Alternaria sp. revealed high levels of synteny between most chromosomes with some intrachromosomal rearrangements. We show for the first time that $A$. brassicae, a large-spored Alternaria species, also contains conditionally dispensable chromosomes (CDCs). Gene prediction and annotation revealed a diverse inventory of secreted proteins, carbohydrate-active enzymes, and secondary metabolite (SM) gene clusters. Few of the carbohydrate-active enzymes are present exclusively in A. brassicae, which may explain the highly invasive nature of the pathogen. Furthermore, we have identified the SM gene clusters responsible for production of Destruxin B, a known pathogenicity factor of $A$. brassicae, and biosynthesis of the black pigment - melanin. Broadly, this study adds to the current genomic resources available for Alternaria and provides insights into the pathogenic lifestyle of an important necrotrophic pathogen.

The Compound 2-Hexyl, 5-Propyl Resorcinol has a Key Role in Biofilm Formation by the Biocontrol Rhizobacterium Pseudomonas chlororaphis PCL1606.

S. TIENDA SERRANO (1), Z. Heredia-Ponce (1), E. Arrebola (1), C. E. Calderon (1), G. Cárcamo-Oyarce (2), L. Eberl (3), A. De Vicente (1), F. Cazorla (1), (1) Department of Microbiology, Fac. of Science. University of Malaga, Málaga, Spain; (2) University of Zurich. Institut für Pflanzenbiologie, Switzerland; (3) Department of Plant and Microbial Biology/ UZH Zurich, Switzerland

The production of the compound 2-hexyl-5-propyl resorcinol (HPR) by the rhizobacterium Pseudomonas chlororaphis PCL1606 (PcPCL1606) is crucial for fungal antagonism and biocontrol activity against the phytopathogenic fungis. The production of HPR is also involved in plant root colonization. This pleiotrophic response prompted us to study the potential role of HPR production in biofilm formation. The swimming motility of PcPCL1606 is enhanced by the disruption of HPR production. Mutants deficient in HPR production, revealed that colony morphology, adhesion and typical air-liquid interphase pellicles were all dependent on HPR production. The role of HPR production in biofilm architecture was analyzed at the initial stage of biofilm maturation using flow-through flow cells chambers. These experiments revealed that the HPR mutant cells had less tight unions than those producing HPR, suggesting an involvement of HPR in the production of the biofilm matrix by PcPCL1606.

Adaptation to plant communities across the genome of Arabidopsis thaliana

B. MAYJONADE (1), L. Frachon (2), C. Bartoli (3), N. C. Hautekeete (4), F. Roux (5), (1) INRA-LIPM, Toulouse, France; (2) DSEB, University of Zurich, Switzerland; (3) IGEPP, Université de Rennes, France; (4) Evo-Eco-Paléo, Université de Lille, France; (5) LIPM, Université de Toulouse, INRA, CNRS, INPT, Castanet-Tolosan, France

Despite the importance of plant-plant interactions on plant community dynamics and crop yield, our understanding of the adaptive genetics underlying these interactions is still limited and deserves to be investigated in the context of complex and diffuse interactions occurring in plant assemblages. Studying the genetic architecture of adaptation while conserving an ecological realism is feasible through powerful ecological genomics approaches. Here, based on 145 natural populations of Arabidopsis thaliana characterized for plant communities, we conducted a 
Genome Environment Association analysis using more than 1.5 million SNPs to finely map adaptive genomic regions of $A$. thaliana associated with plant community descriptors. The candidate adaptive loci associated with species abundance were highly dependent on the identity of the neighboring species, suggesting a high degree of biotic specialization of $A$. thaliana to members of its plant interaction network. Moreover, the identification of adaptive loci associated with $\alpha$-diversity and composition of plant communities supports the ability of $A$. thaliana to interact simultaneously with multiple plant neighbors, which in turn can help to understand the role of community-wide selection. This study provides keys information that will allow a better understanding of the co-evolutionary processes related to plant-plant interactions and highlights the importance to study the genomics of these interactions in natural habitats.

Investigating the molecular interactions of Plasmodiophora brassicae with Arabidopsis thaliana through a genome-wide association study and gene expression analysis

J. C. OCHOA, S. Mukhopadhyay, W. M. D. Truman, R. Malinowski, Institute of Plant Genetics, Polish Academy of Science, Poznań, Poland

Plasmodiophora brassicae is the causal agent of clubroot disease in oilseed rape and other brassicas, and is responsible for significant crop losses. P. brassicae induces the formation of large galls on infected plants establishing a favourable environment for sequestration of host resources and spore multiplication. To better understand the genetic basis underpinning resistance and susceptibility to clubroot disease we carried out a screen of Arabidopsis accessions, quantifying their pathogen levels when infected with an aggressive pathotype (P1b) prevalent in Poland. We inoculated 142 accessions and collected the hypocotyl and upper $1 \mathrm{~cm}$ of root 19 dpi. Quantitative PCR was performed on DNA extracted from these galls using primers for the pathogen gene Pb18S and the host gene AtSK11 to determine relative infection levels. 12 accessions were resistant based on pathogen quantification and absence of gall formation. Genome wide association (GWA) analysis highlighted a SNP in $A T 1 G 32030$ present in 8 of the 12 resistant accessions, this gene is adjacent to the RPB1 resistance loci previously identified in Tsu- 0 . Confirmation of $R P B 1$ as the gene responsible for resistance in each incompatible interaction is ongoing. GWA analysis of the 130 susceptible accessions has implicated numerous genes and T-DNA knock-out lines for selected candidates are being evaluated. Gene expression responses in the compatible and incompatible interactions are being profiled using RNA-Seq and q-RT-PCR.

A Psl-like polysaccharide has a key role in the biofilm architecture of two plant-associated Pseudomonas

Z. HEREDIA-PONCE (1), G. Purtschert (2), J. A. Gutiérrez-Barranquero (1), L. Eberl (3), A. De Vicente (1), F. Cazorla (1), (1) Department of Microbiology, Fac. of Science. University of Malaga, Málaga, Spain; (2) University of Zurich. Institut für Pflanzenbiologie, Switzerland; (3) Department of Plant and Microbial Biology/ UZH Zurich, Switzerland

The Psl polysaccharide has only been studied in Pseudomonas aeruginosa, and its role in bacteria that interact with plants is still unknown. On the one hand, P. syringae is a model of study of plant-pathogen interactions. On the other hand, P. chlororaphis is a biocontrol agent of plantfungal diseases, as the white root rot caused by Rosellinia necatrix. In silico analysis have let us identify in both species a genomic region which is ortholog to the Psl-encoding region of $P$. aeruginosa PAO1. We constructed mutants of this region in both strains and analysed its phenotype in biofilm formation, both in static microwell plates and dynamic flow-cell chamber experiments. The results revealed a different and an important role of this polysaccharide in the biofilm architecture of both strains. Furthermore, in P. syringae this Psl-like polysaccharide has a very important role in swarming motility. All these striking phenotypes led us further study the implication of this polysaccharide in the lifestyles of both models of plant-bacteria interaction.

New insights into pathogenicity of the emerging tropical pathogen: Phytophthora colocasiae on taro D. SEN (1), S. Kushwaha (1), K. H. Lamour (2), S. Tripathy (3), L. J. Grenville-Briggs (1), R. R. Vetukuri (1), (1) Swedish University of Agricultural Sciences, Alnarp, Sweden; (2) Univ of Tennessee, Knoxville, TN, U.S.A.; (3) CSIR Indian Institute of Chemical Biology, Kolkata, India

Phytophthora colocasiae is an oomycete that causes leaf blight and corm rot of taro (Colocasia esculenta). Taro is a staple crop in many tropical countries and the emergence of $P$. colocasiae as a devastating pathogen is a matter of serious concern for food security. $P$. colocasiae strain 7290 was isolated from a diseased taro plant in Vietnam in 2010. A draft genome assembly was generated from Illumina paired-end and mate-pair libraries. The assembly is $57.6 \mathrm{Mb}$, close to the estimated genome size of $65 \mathrm{MB}$ and is syntenic with other sequenced Phytophthora genomes. It comprises 738 scaffolds. A total of 19,984 genes were predicted including RxLR and CRN effectors. Differential gene expression analysis of the $P$. colocasiae-taro interaction reveals many genes of importance for infection. RT-qPCR validated the upregulation of a 4-coumarate COA ligase gene. This gene is a homolog of oxalyl COA synthetase which breaks down oxalate in plants. The presence of this gene in six isolates of $P$. colocasiae confirms its importance to the species. This is the first report of an oxalic acid breakdown enzyme in the oomycetes. In order to confirm the role of 4-coumarate COA ligase in infection we are performing transient silencing with RNA interference (RNAi) followed by phenotyping assays. Our data represent the first step towards determining pathogenicity factors in $P$. colocaisae so that effective management strategies can be adopted to control taro blight.

Chromosome scale comparative analysis of two wheat cultivars unravels large structural variations and underpinning molecular mechanisms

A. K. THIND (1,2), T. Wicker (3), T. Muller (2), P. M. Ackermann (2), B. Steuernagel (4), B. B. Wulff (4), M. Spannagl (5), M. Felder (5), T. Lux (5), K. F. X. Mayer (6), I. W. G. S. Consortium (7), B. Keller (3), S. G. Krattinger (8), (1) The Sainsbury Laboratory, U.K.; (2) University of Zurich, Switzerland; (3) University of Zurich, Zurich, Switzerland; (4) John Innes Centre, Norwich, U.K.; (5) Helmholtz Zentrum Munich, Germany; (6) Plant Genome and Systems Biology, Helmholtz Center Munich, Neuherberg, Germany; (7) International Wheat Genome Sequencing Consortium (IWGSC), U.S.A.; (8) King Abdullah University of Science and Technology, Saudi Arabia

The ploidy, repeat content, and size represented a major challenge in generation of high-quality reference assembly of the wheat genome. Recent improvements in DNA sequencing and genome scaffolding have paved the way to generate high-quality de novo assemblies of pseudomolecules representing complete chromosomes of wheat and its wild relatives. We generated a high-quality sequence of chromosome 2D of elite Swiss 
spring wheat line ' $\mathrm{CH}$ Campala $\mathrm{Lr} 22 a$ ' using the short-read sequencing and Chicago long-range scaffolding and performed comparative sequence analysis with the chromosome 2D of old landrace Chinese Spring (IWGSC RefSeq v1.0). Both the chromosomes were assembled into megabasesized scaffolds for comparative analysis. We identified four InDels of 285, 494 and $765 \mathrm{~kb}$ in size. Based on the molecular signatures at the breakpoints, unequal crossing over and double-strand break repair were identified as the molecular mechanisms that caused these indels. Three of the large indels affect copy number of NLRs, a gene family involved in plant immunity. Comparison of the gene content showed that $99 \%$ of the genic sequences were conserved between the two genotypes with $0.4-0.7 \%$ representing the unique genes. This comparative analysis of two highquality chromosome assemblies enabled a comprehensive assessment of large structural variations and gene content, specifically highlighting the substantial variation present at NLR loci in wheat.

Bremia lactucae exhibits genomic signatures of somatic hybrid vigor due to heterokaryosis with phenotypic consequences. K. FLETCHER, J. Gil, L. D. Bertier, A. Kenefick, K. J. Wood, L. Zhang, S. Reyes-Chin-Wo, K. Cavanaugh, C. Tsuchida, J. Wong, R. W. Michelmore, University of California, Davis, CA, U.S.A.

Bremia lactucae causes lettuce downy mildew, the most important disease of lettuce globally. This highly variable oomycete has previously been shown to rapidly overcome resistance genes and fungicides. Genome assembly of $B$. lactucae was challenging, due to the biotrophic lifestyle exhibited by the pathogen, coupled with high levels of heterozygosity and a recently expanded repeat content. The combined use of multiple read types, including synthetic long reads, single molecule sequences, and $\mathrm{Hi}-\mathrm{C}$, resulted in a high-quality, near-chromosome-scale, consensus assembly of this diploid pathogen. A high incidence of heterokaryosis from B. lactcuae was demonstrated by flow cytometry plus resequencing of 30 field isolates, 37 sexual offspring, and 19 asexual derivatives from single, multinucleate sporangia. Heterokaryons have phenotypic differences and increased fitness compared to homokaryotic somatic derivatives. Consequently, B. lactucae exhibits somatic hybrid vigor and selection should be considered as acting on a population of nuclei within coenocytic mycelia. This provides evolutionary flexibility to the pathogen possibly enabling rapid adaptation to different repertoires of host resistance genes and other challenges.

Biosynthesis of IAA in Pseudomonas savastanoi: A comparative genomic approach A. PINTADO (1), J. Murillo (2), P. Rodriguez-Palenzuela (3), C. Ramos (1), (1) IHSM-UMA-CSIC, Malaga, Spain; (2) Univ de Navarra, Pamplona, Spain; (3) ETSIA UPM, Madrid, Spain

Bacteria belonging to the Pseudomonas syringae complex cause diseases in a variety of woody and herbaceous plants. Pseudomonas savastanoi is a member of this complex and produces tumors or excrescences in the aerial parts of woody plants. The species is classified into four pathovars, i.e. savastanoi (Psv, olive), nerii (Psn, oleander), retacarpa (Psr, Spanish broom) and fraxini (Psf, ash). Our research group have carried out the sequencing, annotation and comparative analysis of the genomes of several strains belonging to these pathovars. These analyses have identified the set of genes belonging to the $P$. savastanoi pan-genome and the collection of pathovar- and strain-specific genes. Through the web app PIFAR (Plant-bacterium Interaction Factors Resource) we have determined the presence of potential virulence factors involved in the interaction of these pathovars with their hosts. Special attention was given to genes putatively involved in the production of indole-3-acetic acid (IAA), a pathogenicity factor in these bacteria. With the exception of Psf, $P$. savastanoi strains produce IAA from tryptophan through the indole3-acetamide pathway (iaaM and $i a a H$ genes). We have shown that a Psv $\triangle i a a M H$ mutant drastically reduces IAA levels. However, this mutant produces amounts of this phytohormone similar to those synthesized by Psf strains. We are currently analyzing the role of several Psv genes in the biosynthesis of IAA through alternative pathways.

Genomic analysis of the Solanaceae NLRome

E. SEO (1), K. Seong (1), M. Li (1), B. J. Staskawicz (2), (1) University of California, Berkeley, CA, U.S.A.; (2) Department of Plant and Microbial Biology, University of California, Berkeley, Berkeley, CA, U.S.A.

As plants are sessile, they have evolved elaborate innate immune systems against invading pathogens. A gene family of Nucleotide-binding site and leucine rich repeat (NB-LRR, or NLR) is one of the largest families in plants. Most of known plant disease resistance genes belong to this family. They interact with effectors from pathogens directly or indirectly to induce defense response. In this study, we captured and identified NLRs using Resistance gene enrichment sequencing (RenSeq) from Solanaceae species (Solanum lycopersicum Heinz, 15 wild tomato species, Nicotiana benthamiana and Capsicum annuum ECW). The sequencing was conducted by PacBio platform to get longer reads. As results, we found 264 to 332 NLRs in each wild tomato, 307 NLRs in N. benthamiana, and 899 NLRs in pepper. In addition, we confirmed the annotation of NLRs has been improved compared to that from whole genome sequencing. These results might give us new resource for disease resistance and can be used for further evolutionary studies.

RIP mutations an important driver of genome variation in Fusarium circinatum

S. VAN WYK (1), B. D. Wingfield (2), N. A. van der Merwe (3), L. De Vos (4), E. T. Steenkamp (3), (1) University of Pretoria, Forestry \& Agricultural Biotechnology Institute, Pretoria, South Africa; (2) University of Pretoria, FABI, South Africa; (3) University of Pretoria, FABI, Pretoria, South Africa; (4) University of Pretoria, Pretoria, South Africa

The Repeat-Induced Point (RIP) mutation pathway is a fungal-specific genome defense mechanism that impedes the deleterious consequences of repeated motifs such as transposable elements (TE). RIP functions by permanently mutating the targeted sequences by introducing cytosine to thymine transitions. The aim of this study was to determine manner RIP affects the genome of the pitch canker fungus, Fusarium circinatum. For this purpose, multiple sequenced representatives were subjected to genome-wide and alignment-based RIP analyses to determine (i) the extent of RIP mutations, (ii) the most extensively affected genomic regions (iii), and the genetic changes brought about by RIP. Our results showed that RIP mutations were acquired in an isolate-specific manner and the hallmark traits of RIP were identified in both TEs, and endogenous genomic regions. RIP mutations further constituted large proportions of genome assemblies and were particularly prominent in the dispensable genomic compartments of these isolates. Extensively RIP affected genomic regions were characterized with a reduced GC content, diminished coding 
capacity, and RIP affected genomic regions were enriched for proteins predicted to be involved with host-pathogen interactions. These results show that RIP is an important and ongoing source of genetic variation and contributes to the evolution of this important fungal pathogen.

Updated analysis of Plasmodiophora brassicae genomes - MPMI 2019

S. STJELJA, J. Fogelqvist, C. Dixelius, Swedish University of Agricultural Sciences, Department of Plant Biology, Uppsala, Sweden

Unicellular eukaryotes or protists can be found in any habitat worldwide. Among these ancient organisms is the large and diverse group of Rhizaria in the kingdom Chromista. The plasmodiophorids within the Rhizaria include several plant pathogens. The most important ones are Spongospora subterranea causing powdery scab on potato and being the vector for Potato Mop Top Virus, Polymyxa betae which transmits the virus responsible for Rhizomania in sugar beets and Plasmodiophora brassicae, the soil-borne clubroot disease pathogen of crucifers. Our work has an emphasis on the genomes of the obligate biotroph P. brassicae. After considerable efforts, DNA was generated enabling PacBio sequencing to clarify number of chromosomes including telomere sequences. The former Illumina sequencing did not manage to completely cover the large mitochondrial genome. PacBio sequencing generated one single mitochondrial contig and identified sequences that were not found by applying Illumina technology. Combined automatic and manual annotation revealed a complex structure of the mitochondrial genome. The new data is presently used for comparative genomics where the challenge is to distinguish biological from technical sequence differences.

Rapid diagnostic method to identify bacteria associated with Acute Oak Decline

V. BUENO-GONZALEZ (1), C. Brady (1), J. Allainguillaume (1), S. Denman (2), D. L. Arnold (3), (1) University of the West of England, Bristol, U.K.; (2) Forest Research, Farnham, U.K.; (3) Univ of the West of England, Bristol, U.K.

British oaks, Quercus robur and Q. petraea, are under threat from Acute Oak Decline (AOD). Severe cases are lethal within five years. Symptoms include necrotic, longitudinal, bleeding cracks in the bark, from which dark fluid emanates. AOD is caused by the interaction of several bacterial species. The lesion microbiome shows a significantly similar composition pattern, where Brenneria goodwinii, Gibbsiella quercinecans and Rahnella victoriana are the predominant pathogens. Species belonging to the Enterobacterales and Pseudomonadaceae are also routinely isolated. Many field samples from affected oak need to be processed on a daily basis, therefore a rapid and cost-effective detection and identification method is needed. A multiplex high-resolution melt (HRM) analysis has been developed for the identification of $B$. goodwinii, $G$. quercinecans, R. victoriana and Lonsdalea britannica. HRM analysis is a real-time PCR-based technique in which single nucleotide polymorphisms can be identified in amplicons, without DNA sequencing. Our multiplex HRM can identify up to four bacterial pathogens in a single tube in only 40 minutes per HRM run. This diagnostic method will potentially be a useful tool for research institutions working with AOD. To further investigate the bacteria associated with AOD we have identified several novel species of Pseudomonas and described these following a polyphasic approach. Further investigation will establish if the novel Pseudomonas play a role in AOD.

Multiple quantitative trait loci contribute tolerance to bacterial canker incited by Pseudomonas syringae pv. actinidiae in diploid kiwifruit (Actinidia chinensis)

J. TAHIR, The New Zealand Institute of Plant and Food Research, Palmerston NORTH, New Zealand

Kiwifruit (Actinidia spp.) breeding programs select strongly for tolerance or complete resistance to Pseudomonas syringae pv. actinidiae (Psa), as the virulent, canker-inducing strain, Psa Biovar 3 is an economic threat to the kiwifruit industry world-wide. However, there is no information available on the genetic and molecular basis of Psa tolerance in Actinidia species. In this study we identified quantitative trait loci (QTLs) for Psa tolerance in a diploid kiwifruit (A. chinensis) population, derived from a cross between the ex-elite Psa susceptible 'Hort16A' cultivar and a moderately tolerant male breeding parent P1. A major QTL for Psa tolerance was identified in 'Hort16A' revealing candidate genes for Psa susceptibility and tolerance in kiwifruit. In combination with multiple minor QTLs identified in P1, the nature of Psa tolerance was found to be oligogneic and complex. Global transcriptomic analysis of field grown F1 Psa tolerant and susceptible genotypes further revealed that field resistance is associated with the expression of genes involved in basal defense. A few of these genes were also found to be induced in tolerant plants only during controlled-infection experiments. We propose that tolerance to Psa in diploid A. chinensis originates from divergent genetic pathways regulating innate immunity.

Distinct life histories impact dikaryotic genome evolution in the wheat stripe rust fungus Puccinia striiformis $\mathbf{f}$. sp. tritici B. SCHWESSINGER (1), Y. J. Chen (2), J. K. Vogt (3), C. K. Sørensen (4), T. Sicheritz-Ponten (5), M. S. Hovmøller (6), A. F. Justesen (6), J. Rathjen (1), (1) Research School of Biology, The Australian National University, Canberra, ACT, Australia; (2) University of Copenhagen, Frederiksberg C, Denmark; (3) Novo Nordisk Foundation Center for Basic Metabolic Research, Denmark; (4) 2Department of Agroecology Faculty of Sciences and Technology, Aarhus University, Aarhus, Denmark; (5) Natural History Museum of Denmark, Denmark; (6) Department of Agroecology, Faculty of Sciences and Technology, Aarhus University, Aarhus, Denmark

Wheat stripe rust, caused by the obligate biotrophic fungus Puccinia striiformis f. sp. tritici (Pst), is a major threat to wheat production worldwide with an yearly loss of $\sim$ US\$ 1 billion. Pst undergoes a complex multi-spore lifecycle and infects wheat during its asexual stage mostly via reinfection by dikaryotic urediniospores. The recent advances in long-read sequencing technologies and tailored-assembly algorithms enable us to disentangle the two haploid genomes of Pst. This provides us with haplotype-specific information at a whole-genome level. Exploiting this novel information, we perform whole genome comparative genomics of two Pst isolates with contrasting life histories. We compare one isolate of the old European linage (PstS0), which has been asexual for over 50 years, and a Warrior isolate (PstS7 linage) from a new incursion into Europe in 2011, which is derived from sexual populations in the Himalayan region. This comparison provides evidence that long-term asexual evolution leads to genome expansion, accumulation of transposable elements, and increased heterozygosity at the single nucleotide, structural and allele levels. At the whole genome level, the candidate effectors are not compartmentalized and the whole candidate effector repertoire is conserved at a similarly as all other genes. This detailed comparative analysis of two haplotype-aware assemblies of $P s t$ are the first steps to understand the evolution of dikaryotic rust fungi at a whole genome level. 
Characterization and Pathogenicity of New Morphospecies from Apple Bitter Rot Disease (Colletotrichum spp.) in Korea M. M. OO, S. K. Oh, Department of Applied Biology, Chungnam National University, Daejeon, Korea, Republic of (South)

Bitter rot caused by Colletotrichum species is one of the important apple fruit diseases in worldwide and causes significant yield losses to the apple producers. Presently, enormous evolutionary genetic divergence in the population biology of Colletotrichum spp. indicates that species are very complicated and should be intensely segregated into distinct species. In this study, typical bitter rot symptoms were observed in numerous apple orchards in Korea (2015-2016 yr) and 18 isolates were examined based on the morphological features, pathogenicity test, and nucleotide sequence data of glyceraldehyde-3-phosphate-dehydrogenase and internal transcribed spacer regions. The obtained 18 isolates were classified into four different species; C. fructicola, C. siamense, C. nymphaeae, and C. fioriniae, respectively. In addition, pathogenic comparison between these 4 species demonstrated that the current isolates belonging to the $C$. acutatum species complex (C. nymphaeae, C. fioriniae) produced smaller lesions than isolates belonging to the $C$. gloeosporioides species complex (C. fructicola, C. siamense). Additionally, $C$. siamense affiliated with the $C$. gloeosporioides complex species produced the biggest, widest lesions and was found to be the most aggressive among these 4 species. Taken together, our finding pointed out the correct classification of pathogens within a species complex of genus Colletotrichum and it will be helpful for the control of bitter rot disease on apple fruit.

Comparative analyses of compatible and incompatible host-pathogen interactions provide insight into host specialization of closely related pathogens

J. HAUEISEN (1,2), M. Möller (1,2), C. S. Small (1,3), A. Maliskat (1), M. Wilkens (1), A. Nommensen (1,2), E. H. Stukenbrock (1,2), (1)

Environmental Genomics, Botanical Institute, University of Kiel, Kiel, Germany; (2) Max Planck Institute for Evolutionary Biology, Plön, Germany; (3) Department of Phytopathology, Technical University Munich, Munich, Germany

Emerging pathogens need to adapt swiftly to exploit new hosts for growth and reproduction. Host shifts can be facilitated by adaptive changes in gene expression landscapes. In order to identify transcriptional patterns related to divergent host specialization in specialized fungal plant pathogens, we compared the development and expression profiles of the wheat pathogen Zymoseptoria tritici and two closely related species $Z$. pseudotritici and Z. ardabiliae during infection of wheat. Infections by Z. pseudotritici and Z. ardabiliae are incompatible: hyphae penetrate wheat stomata but are blocked in substomatal cavities involving plant defense responses. In contrast, $Z$. tritici can overcome host defenses to complete its lifecycle in wheat. Nevertheless, the three species show a highly similar developmental program during early host interaction. Gene expression programs are likewise conserved as only $6.5 \%$ of orthologous genes are differentially expressed between $Z$. tritici and the sister species. This indicates that wheat specialization is facilitated by a small number of key traits. We focused on ten differentially expressed candidate effectors and studied their role in wheat infections using Z. tritici mutants. The observed in planta phenotypes cover reduced and increased virulence and changes in temporal disease development indicating that the contribution of these effectors to wheat adaptation is complex and dynamic since speciation of $Z$. tritici 11,000 years ago.

Pangenome analysis of the wheat pathogen Zymoseptoria tritici from the U.K.

E. CURRAN (1), A. M. M. Tiley (2), A. Feechan (3), J. I. Burke (1), (1) University College Dublin; School of Agriculture and Food Science, Ireland; (2) UCD; School of Agriculture and Food Science, UCD Earth institute, Ireland; (3) UCD, School of Agriculture and Food Science, UCD Earth institute, Dublin, Ireland

Zymoseptoria tritici is the causal agent of Septoria tritici Blotch (STB), an agriculturally important disease of wheat. The objective of this study was to isolates $Z$. tritici races from infected wheat leaves in order to construct a pangenome of $Z$. tritici in the U.K. The isolates used in this study are derived from STB-infected wheat leaves from four sites across the U.K. Infected leaves from 30-40 cultivars from each site were collected in 2018 and will be collected again in 2019 and 2020. Fifteen isolates of $Z$. tritici were collected from each site. Isolate mixtures from the 4 sites were sprayed on blocks of five winter wheat cultivars (Andante, Exsept, Flame, Longbow, and Stigg) at growth stage 39. At 28 days postinoculation the percentage leaf area diseased was scored. The 2018 scores revealed differences between the site inoculums. An overall trend showed that the Swindon inoculum had high disease scores on all cultivars save Exsept. Longbow in general returned the highest disease scores of between $10.8 \pm 1.18 \%$ and $34.6 \pm 45.4 \%$. This was followed by Andante, Flame, Stigg, and Exsept. Exsept showed highest resistance to inocula from all sites with scores of $5 \pm 2.4 \%$ to $7 \pm 0.6 \%$. The $Z$. tritici isolates will be genotyped by next gen sequencing. The genomic information from this will be used to build a pangenome across the sites and years, allowing an examination of changes across site and time. We would like to acknowledge Origin Enterprises-SFI for funding this research.

Dissecting the key effectors involved in Pseudomonas cherry canker

M. T. HULIN (1), J. W. Mansfield (2), S. Lynn (1), K. Housley (1), F. Fernandez (1), M. Lipska (1), H. C. Neale (3), D. L. Arnold (3), R. W. Jackson (4), R. J. Harrison (1), (1) NIAB EMR, East Malling, U.K.; (2) Imperial College At Wye, Ashford, Kent, U.K.; (3) Univ of the West of England, Bristol, U.K.; (4) University of Reading, Reading, U.K.

Pseudomonas syringae pathovars are important pathogens globally. Individual strains are believed to be host specialised to a greater or lesser extent, which may be linked to their repertoire of type III effector proteins, involved in both virulence and avirulence in planta. It is hypothesised that effectors may form functionally redundant groups that allow $P$. syringae to suppress the immune response of different hosts whilst utilising contrasting effector sets. Multiple clades of $P$. syringae have independently converged to cause canker disease of cherry (Prunus avium). They include P.s pv. morsprunorum races 1 and 2 and P.s pv. syringae. Genomic analysis of the Prunus strains revealed highly divergent effector and toxin repertoires between the different clades, indicating they use distinct mechanisms to cause disease. Despite these differences, the presence/absence of several effectors was associated with the disease and phylogenetics revealed effectors have been frequently swapped between cherry pathogens via horizontal gene transfer, on both plasmid and phage sequences. By contrast, the HopAB effector family has been lost or truncated in cherry-pathogenic clades likely due to an avirulence reaction in cherry. Utilising a Bayesian co-occurrence analysis of effector gene evolution, we now aim to dissect which effectors act redundantly in cherry pathogens, determine the set required for virulence and complement this with a polymutant approach in the laboratory. 
Using population level diversity of the rice blast fungus Magnaporthe oryzae to identify new resistant varieties of rice for Sub-Saharan Africa.

V. M. WERE (1), D. T. Mwongera (2), A. Foster (3), X. Yan (1), D. Soanes (4), S. K. Mutiga (5), F. Rotich (6), M. J. Kershaw (7), B. Zhou (8), I. Ouédraogo (9), T. K. Mitchell (10), G. L. Wang (11), J. Correll (12), N. J. Talbot (13), (1) The Sainsbury Laboratory, University of East Anglia, Norwich Research Park, Norwich, U.K.; (2) Kenya Agricultural and Livestock Research Organization, NAIROBI, Kenya; (3) The Sainsbury Laboratory, Norwich, U.K.; (4) University of Exeter, Exeter, U.K.; (5) BecA-ILRI, Nairobi, Kenya, Nairobi, Kenya; (6) Dept. of Plant Pathology, University of Arkansas, AR, U.S.A.; (7) University of Exeter, U.K.; (8) 7Regional Office for Asia and Pacific, Bangkok, Thailand, Thailand; (9) 6Institute of Environment and Agricultural Research, Ouagadougou, Burkina Faso, Burkina Faso; (10) Ohio State University, Columbus, OH, U.S.A.; (11) Department of Plant Pathology, The Ohio State University, Columbus, OH, U.S.A.; (12) 4Dept. of Plant Pathology, University of Arkansas, U.S.A., AR, U.S.A.; (13) The Sainsbury Laboratory, Norwich, Norfolk, U.K.

The rice blast fungus, Magnaporthe oryzae, causes the most serious disease of cultivated rice. To understand population level diversity of the rice blast fungus in Sub-Saharan Africa, and guide resistance breeding, we analysed 500 rice blast isolates from 9 countries in the region and carried out extensive pathotype analysis on monogenic rice lines carrying 24 known rice blast resistance genes. We then used Pacbio sequencing and RNA-seq, to generate improved contiguous genome assemblies of two M. oryzae strains, Guy11 and the Kenyan isolate KE002. We used informatics tools to analyse the relationship between effector repertoire in the rice blast population and virulence spectrum and found that $P i-9$, Pita-2, Pi-12, Piz and Pi-z5 offer the greatest possibility to exclude the prevailing rice blast population. We also analysed a novel effector gene $P W L 2$, a host specificity determinant for infection of weeping lovegrass (Eragrostis curvula), that may identify a novel source of resistance for introduction into rice. We used CRISPR/Cas9 genome editing to generate Dpwl2 mutants in M. oryzae strain Guy11, which has three copies of the gene, resulting in gain of virulence towards weeping lovegrass. We are exploring the possibility of identifying a novel R-gene from Eragrostis curvula for deployment in rice along with a stack of native R-genes, to obtain durable rice blast resistance in sub-Saharan Africa.

Targeted nanopore sequencing for real-time plant disease diagnostics and strain-level identification of fungal pathogens G. V. RADHAKRISHNAN (1), N. M. Cook (1), V. Bueno-Sancho (1), A. Debebe-Mitiku (2), A. Persoons (1), C. M. Lewis (1), P. E. Davey (1), M. Heaton (1), D. P. Hodson (3), D. G. O. Saunders (1), (1) John Innes Centre, Norwich, U.K.; (2) Ethiopian Institute for Agricultural Research, Addis Ababa, Ethiopia; (3) International Maize and Wheat Improvement Center (CIMMYT), Addis Ababa, Ethiopia

Plant diseases come with enormous cost to food production, and it is vital that we protect our crops from diseases to ensure global food security. A robust disease prevention strategy requires that we can respond to rapidly evolving pathogen populations. For this, we need surveillance systems that provide real-time information about global pathogen populations. In the past, real-time surveillance was not technically feasible but advances in mobile sequencing technologies have now made this possible. For instance, the MinION sequencer was recently used to monitor the spread of Ebola and Zika epidemics in real-time. Despite the promise that these new technologies hold, it has not yet been possible to adapt such methods for plant pathogenic fungi due to their complex genomes. To address this, we used the yellow rust pathogen, Puccinia striiformis, as a model system to develop a computational approach that reduces the complexity of genomic data required for surveillance. Using this approach coupled with a sample preparation workflow requiring minimal equipment, we employed the MinION sequencer for real-time surveillance of yellow rust in Ethiopia. Using this system, we could accurately classify pathogen isolates to known phylogenetic groups within 48 hours of sample collection. Our MARPLE (Mobile And Real-time PLant disEase) diagnostics system allows real-time monitoring of important traits in pathogen populations and can be easily adapted to other filamentous plant pathogens.

Investigating the genetic diversity of the fungal wheat pathogens Zymoseptoria tritici and Fusarium graminearum from field site isolates P. PILO (1), J. I. Burke (2), A. M. M. Tiley (3), C. Lawless (1), A. Feechan (4), (1) UCD; School of Agriculture and Food Science, UCD Earth institute, Ireland; (2) University College Dublin; School of Agriculture and Food Science, Ireland; (3) UCD; School of Agricultural and Food Science, UCD Earth institute, Ireland; (4) UCD, School of Agriculture and Food Science, UCD Earth institute, Dublin, Ireland

The global demand for wheat is expected to increase by $33 \%$ by 2050 (FAO, 2006). In Ireland, wheat is the second largest cereal produced and constitutes $30 \%$ of the 2.4 million tonnes of cereal produced (CSO, 2017). Two major pathogens which threaten wheat production are the fungi Zymoseptoria tritici, causal agent of Septoria Tritici Blotch (STB) and Fusarium graminearum ${ }^{2}$ causal agent of Fusarium Head Blight (FHB). This project aims to use data collected from $Z$. tritici and $F$. graminearum field populations to investigate genetic diversity and identify effector gene candidates which may be required for disease. Fungal spore traps have been installed at four sites across the U.K. (Angus, Yorkshire, Wiltshire and Kent) and samples have been collected bi-weekly from May-August in 2018 and this will continue until 2022. The spore trap samples from 2018 are currently being microscopically examined for the presence of Z. tritici, F. graminearum, and other disease-causing fungi. Between the sites there is a difference in the abundance of these fungal species. DNA has been extracted from the samples and sent for sequencing to assess $Z$. tritici and F. graminearum strain diversity. The data collected from this project will be used alongside fungal field isolate studies in order to identify key virulence genes in $Z$. tritici and $F$. graminearum required for disease. The results from this research will be used to inform future control strategies against these major pathogens of wheat.

\section{Exploring NLR diversity in Oryza sativa}

S. FAIRHEAD (1), T. Kroj (2), P. Gladieux (3), A. Jouet (4), K. Witek (1), C. Van Oosterhout (5), J. Morel (6), J. D. Jones (1), (1) The Sainsbury Laboratory, University of East Anglia, Norwich Research Park, Norwich, U.K.; (2) INRA Montpellier, Montpellier, France; (3) INRA, Montpellier SupAgro, Montpellier, France, France; (4) The Sainsbury Laboratory, University of East Anglia, Norwich Research Park, Norwich, U.K.; (5) School of Environmental Sciences, University of East Anglia, Norwich, U.K.; (6) UMR BGPI, U.K.

We aim to deepen our understanding of diversity in the NLR-encoding disease-resistance $(R)$ gene complement in Oryza sativa (rice). We investigate NLR gene repertoire diversity rice landraces grown in Southwest China that show high levels of resistance to Magnaporthe oryzae (cause of rice blast). The two major cultivated groups of rice are indica and japonica, and the landraces we investigated are predominantly in the 
indica group. We have sequenced the NLR gene repertoire of 6-8 indivuals from 5 of these landraces, and also a diverse set of cultivated accessions using Resistance Gene Enrichment Sequencing (RenSeq). We demonstrate that NLR genetic diversity can be higher within landraces than between domesticated varieties, likely rendering them less susceptible to diseases, including rice blast. Analysis to date has further revealed that NLR diversity is elevated compared to a subset of non-NLR genes in both landraces and cultivated varieties. Funded by Gordon and Betty Moore Foundation

Genetic analysis of immune responses in Brassica species H. J. SCHOONBEEK (1), H. A. Yalcin (1), A. Gomez Gutierrez (1), R. Wells (1), C. J. Ridout (2), (1) John Innes Centre, Norwich, U.K.; (2) John Innes Centre (JIC), Norwich Research Park, Norwich, U.K.

Utilising multiple genomic resources, we have developed a novel approach to improve disease resistance in Brassicas to address the challenges facing agriculture. The multi-partner study on Mechanistic Analysis of Quantitative disease resistance in Brassicas by Associative Transcriptomics (MAQBAT) aims to achieve more durable control of the most important pathogens of Brassicas. The plant immune system influences resistance to multiple microorganisms and is modulated by environmental conditions. The recognition of pathogen-, or microbe-, associated molecular patterns (PAMPs/MAMPs) by receptors and co-receptors in plant cells results in the activation of defence and PAMPtriggered immunity (PTI). Increasing evidence indicates that PTI contributes to quantitative disease resistance (QDR) (Poland et al. 2009). Using methods we developed in B. oleracea and B. napus (Lloyd et al. 2014), we investigated whether PTI is active in Brassica crops and how variation in PTI-responses can be exploited for resistance breeding. Brassicas show multiple responses, including the production of reactive oxygen species (ROS) and gene-induction, to PAMPs such as flg22, NLPs and chitin, ultimately leading to induced resistance. The intensity and speed of responses varies between species and varieties. We found 100-fold differences in the ROS-responses within a diverse set of 192 B. napus lines, which we are using for Associative Transcriptomics (Harper et al. 2012) to identify causative gene loci.

The dark matter of genomics: Mini chromosomes as drivers of host adaptation in the blast fungus

T. LANGNER (1), L. B. Gomez-Luciano (2), A. Harant (1), J. Win (1), S. Kamoun (1), (1) The Sainsbury Laboratory, University of East Anglia, Norwich Research Park, Norwich, U.K.; (2) Biodiversity Research Center, Academia Sinica, Taipei, Taiwan

Eukaryotic genomes are often organized into compartments to facilitate transcriptional regulation, recombination, and adaptation. In many plant pathogens, growing evidence suggest that compartmentalization of effectors into mini- or accessory chromosomes facilitates adaptive evolution. The blast fungus Magnaporthe oryzae causes the most devastating rice disease worldwide and infects more than 50 grass species, including the staple crops rice, wheat and millet. Host adaptation is linked to presence/absence polymorphisms of effectors, possibly as the result of genetic material exchange and recombination associated with repeats and transposable elements. This gene flow can lead to new structural variants potentially involved in host jumps and disease outbreaks. We set out to study genomic structural variation in host specific lineages of $M$. oryzae combining nanopore MinION sequencing and pulse-field gel electrophoresis. Almost every isolate contained a specific set of mini-chromosomes ranging in size from $500 \mathrm{~kb}-3 \mathrm{MB}$ suggesting massive structural variation even in clonal lineages. We established a workflow to sequence isolated mini-chromosomes and a bioinformatics pipeline to identify them in nanopore assemblies. We found that mini chromosomes contain effector genes and seem to be involved in large scale genomic rearrangements. This raises the possibility that mini chromosomes are part of a mechanism that facilitates structural diversification of the blast fungus genome.

The genetics of plant-plant cooperation in Arabidopsis thaliana

H. J. SUBRAHMANIAM (1), C. Libourel (2), A. Chevalier-Mairet (2), D. Roby (3), F. Roux (4), (1) INRA, TOULOUSE, France; (2) INRA, France; (3) LIPM, INRA, CNRS, Université de Toulouse, Castanet-Tolosan, France; (4) LIPM, Université de Toulouse, INRA, CNRS, INPT, Castanet-Tolosan, France

The role of positive plant-plant interactions (i.e. mutualism at the interspecific level and cooperation at the intraspecific level) on overyielding in crops mixtures and in regulating the composition of natural plant communities has recently gained a lot of attention. However, there is a paucity of studies aimed at identifying the genetic and molecular bases underlying natural variation of positive interactions. In this study, we conducted a greenhouse experiment to study the genetics of intraspecific positive interactions in Arabidopsis thaliana. For this objective, we used a highly polymorphic local mapping population of 192 whole-genome sequenced accessions. All these accessions were grown alone or in presence of each of three other tester accessions from the same population. Maximal rosette diameter was estimated for both partners. For a significant portion of pairwise interactions, an increase in rosette diameter was detected for both interacting plants. In addition, only two of the cooperative accessions were shared among the three testers. A GWA mapping approach was adopted to detect QTLs associated with cooperation. We detected neat but different association peaks between the three testers, suggesting a highly flexible genetic architecture for cooperation. Interestingly, cooperative accessions are genetically distant from the tester for the detected QTLs, suggesting that cooperation is based on compatibility alleles in our study.

Genomic diversity and insights into virulence of the Huanglongbing pathogen Candidatus Liberibacter asiaticus S. P. THAPA (1), N. Wang (2), V. Ancona (3), W. Ma (4), G. L. Coaker (1), (1) University of California, Davis, CA, U.S.A.; (2) University of Florida, Lake Alfred, FL, U.S.A.; (3) Texas A\&M Kingsville Citrus Center, Weslaco, TX, U.S.A.; (4) University of California, Riverside, Riverside, CA, U.S.A.

Huanglongbing (HLB) is currently the most devastating disease of citrus. HLB is caused by the Gram-negative bacterial pathogen Candidatus Liberibacter asiaticus (CLas). CLas is psyllid transmitted and resides in the phloem. In order to gain greater insight into CLas biology and genetic diversity, we have initiated genome sequencing and comparative analyses of $C$ Las from different geographical regions, including Asia, Mexico, California, Texas and Florida. Seven hundred sixty-five orthologous genes were used to analyze $C$ Las phylogenetic relationships. Our results reveal diversity among $C$ Las isolates with different $C$ Las phylogroups present in the United States. Our phylogenetic analyses also indicate two different introduction events in California. Texas isolates were closely to Florida strain Psy62, while Asian isolates exhibited the most genetic variation. We have also identified conserved CLas proteins likely involved in bacterial survival and virulence and analyzed their expression in 
their plant and psyllid hosts. Individual SEC-dependent effectors exhibited differential expression patterns in their plant and psyllid hosts, indicating that $C$ Las utilizes its effector repertoire to differentially modulate diverse hosts. Taken together, these data will facilitate a greater understanding of CLas genetic diversity and biology that can be used to guide HLB control strategies.

Characterizing the growth and gene expression profile of Phytophthora agathidicida in (the soils of) alternate land managements P. PANDA (1), S. Sturrock (1), R. L. McDougal (1), P. M. Scott (1), K. Lewis (2), A. Black (2), L. M. Condron (2), M. O'Callaghan (2), N. M. Williams (3), (1) Scion, NZ Forest Research Institute Limited, Rotorua, New Zealand; (2) Bio-Protection Research Center, Lincoln University, Lincoln, New Zealand; (3) Scion (New Zealand Forest Research Institute, Ltd), Rotorua, New Zealand

New Zealand kauri (Agathis australis) is an iconic tree species of cultural significance, and an important forest ecosystem engineer and timber resource. Phytophthora agathidicida, an aggressive soilborne pathogen causes dieback in kauri trees of all ages. This pathogen infects roots and damages tissues that distribute nutrients and water within the tree. Surveillance studies between 2008 and 2017 have shown the widespread distribution of the disease in many of the regions where kauri is established illustrating the threat to the long-term survival of the species. This study attempts to interrogate the growth response and gene expression profile of $P$. agathidicida exposed to different soils taken from alternate land management regimes within and adjacent to kauri forests (indigenous kauri forest, pastureland and commercial pine forest). Our aim was to assess whether soils from these contrasting land-uses are conducive, suppressive or neutral to the pathogen growth, with implications for pathogen spread and establishment beyond kauri forests. Preliminary results suggest that there is a difference in sporangia production with time and between soils in association with observed variation in gene expression. Details of the most abundant gene classes, their associated molecular functions and biological processes, expressed with respect to the life stage development and at different soil conditions will be presented.

New Insights into mycoparasitism and microbial defence in oomycete-oomycete interactions revealed through comparative genomics and microbiome sequencing

D. Liang (1), R. R. Vetukuri (1), C. Faure (2), D. Sen (1), C. B. Andersen (1), M. Brus-Szkalej (1), C. Million (3), D. Dou (4), E. Gaulin (2), B. Dumas (2), T. Rey (5), L. J. GRENVILLE-BRIGGS (1), (1) Swedish University of Agricultural Sciences, Alnarp, Sweden; (2) LRSV UMR5546 CNRS Université Toulouse III, Castanet-Tolosan, France; (3) Swedish Univeristy of Agricultural Sciences (SLU), Alnarp, Sweden; (4) Nanjing Agricultural University, Nanjing, China; (5) De Sangosse, Pont-du-Casse, France

Pythium oligandrum and Pythium periplocum are mycoparasitic oomycetes with potential as biocontrol agents. Traits important for successful mycoparasitism are being revealed by comparative genomic and transcriptomic analyses of the hyper-aggressive mycoparasite $P$. oligandrum versus the weaker mycoparasite $P$. periplocum on oomycete and fungal hosts. These genomes lack the typical 2-speed architecture of their phytopathogenic counterparts. The CAZyzome of these species are expanded compared to phytopathogens and several subfamilies of cell wall degrading enzymes are rapidly evolving indicating their importance for mycoparasitism. The presence of a novel family of GH46 enzymes may be an indication of horizontal gene transfer from bacteria. $\mathrm{ABCb}$ and $\mathrm{ABCc}$ transporter subfamilies are undergoing expansion in the stronger mycoparasite $P$. oligandrum in a strikingly similar manner to mycoparasitic fungi. Thus, there are many features of the mycoparasitic oomycetes that are distinct from the phytopathogens. Dual interaction transcriptomics reveals that the plant pathogen Phytophthora infestans expresses RxLRs, elicitins and protease inhibitors to defend against mycoparasitic attack by $P$. oligandrum. Microbiome sequencing reveals the effects of $P$. oligandrum on soil health. Field application of $P$. oligandrum can affect changes within bacterial and fungal communities in the potato rhizosphere, an important consideration for the ecology and application of this species as a biocontrol agent.

TAL effector composition and characterization of Xanthomonas citri pv. mangiferaeindicae M. R. DOMMEL (1), J. C. Huguet-Tapia (2), F. F. White (1), (1) University of Florida, Gainesville, FL, U.S.A.; (2) Department of Plan Pathology, University of Florida, Gainesville, FL, U.S.A.

Xanthomonas citri pv. mangiferaeindicae $(\mathrm{Xcm})$ is the etiologic agent of bacterial canker in mango. The disease has been reported in Africa, southeast Asia, Hawai' $i$, and the continental United States and has become an important problem in mango cultivation. Characteristic symptoms of the disease are black, star-shaped erumpent lesions in leaves and fruits. Xanthomonas species produces disease by hijacking transcriptional mechanism of the host using transcription activator-like effectors (TALEs). Here, we report the complete genome sequence of Xcm strain L242, isolated from southern Florida U.S.A. The strain is virulent in at least two varieties of mango, Keitt and Lemon Zest. Strain Xcm L242 produces characteristic necrotic lesions in leaves in greenhouse conditions. Genome sequencing was performed using Nanopore and PacBio platforms, and the technologies were evaluated and compared. The genome of Xcm strain L242 is composed of a large contig of 5.3 Mb, corresponding to the chromosome, and three small contigs of 42,75 and $96 \mathrm{~Kb}$ with plasmid-like gene profiles. We found 13 TALes encoded in the chromosome. TAL effectors characterization and sequencing technology comparisons will be presented.

Effector profile analysis of the tomato and pepper pathogens Xanthomonas perforans and $X$. euvesicatoria F. IRUEGAS-BOCARDO (1), S. Timilsina (1), M. O. Jibrin (1), G. V. Minsavage (1), P. Abrahamian (2), D. S. Egel (3), T. Creswell (3), S. Miller (4), F. Rotondo (4), F. J. Louws (5), T. Adhikari (6), C. Trueman (7), R. Roach (8), T. Coutinho (9), O. Pruvost (10), N. T. T. Nga (11), L. Luo (12), M. Kebede (13), N. Potnis (14), G. E. Vallad (2), J. B. Jones (1), E. M. Goss (1), (1) Department of Plant Pathology, University of Florida, Gainesville, FL, U.S.A.; (2) Gulf Coast Research and Education Center, University of Florida, Wimauma, FL, U.S.A.; (3) Department of Botany and Plant Pathology, Purdue University, West Lafayette, IN, U.S.A.; (4) Department of Plant Pathology, The Ohio State University, Wooster, OH, U.S.A.; (5) Department of Entomology and Plant Pathology, North Carolina State University, Raleigh, NC, U.S.A.; (6) North Carolina State University, Raleigh, NC, U.S.A.; (7) Ridgetown Campus, University of Guelph, Guelph, Canada; (8) Horticulture and Forestry Science, Department of Agriculture \& Fisheries, QLD, Brisbane, QLD, Australia; (9) Department of Microbiology and Plant Pathology, University of Pretoria, Pretoria, South Africa; (10) CIRAD, UMR, PVBMT, St. Pierre, Réunion, France; (11) Department of Plant Protection, Can Tho University, Can Tho, Viet Nam; (12) Department of Plant Pathology, China Agricultural University, Beining, China; (13) School of Plant Science, Haramaya University, Dire Dawa, Ethiopia; (14) Department of Entomology and Plant Pathology, Auburn University, Auburn, AL, U.S.A. 
Bacterial plant pathogens secrete type III effectors (T3Es) to interact with and disrupt host cell defenses. Susceptibility or resistance of the host depends on their ability to recognize and restrict the establishment of the pathogen. Xanthomonas euvesicatoria (Xe) and X.perforans (Xp), causal agents of bacterial spot disease (BLS) on tomato and pepper, are informative models to study the roles of effectors in host-pathogen interactions. $X e$ infects tomato and pepper, $X p$ infects only tomato, however, $X p$ strains have been isolated from pepper. To understand factors that influence the presence and distribution of effectors between and within species, we sequenced and analyzed the effector profiles of 179 and 131 strains, collected in 13 countries over time ( 25 to $56 \mathrm{yrs}$ ) for $X p$ and $X e$, respectively. Both pathogens are distributed worldwide and follow a classical gene-for-gene interaction defining pathogenicity and virulence with respect to their host. Consequently, host, year and geographic origin were expected to influence the effector profiles of strains, including effector presence and allelic state. The core and non-core effectors within and between $X p$ and $X e$ strains are described, expanding previous knowledge of their effector content. However, host, year and country of collection do not explain the variation in effector profiles suggesting migration and distribution of strains impacts the population structure more than local demographic processes.

The evolution of Arabidopsis thaliana-associated Pseudomonas

H. ASHKENAZY, T. Karasov, O. Shalev Skriptchak, M. Neumann, D. Weigel, Max Planck Institute for Developmental Biology, Tuebingen, Germany

Members of the Pseudomonas genus of gram-negative bacteria are often highly abundant in metagenomic samples collected from plants. To better understand the process of Pseudomonas colonization, we study the evolution of a local collection of plant-associated Pseudomonas strains via comparative genomics. Specifically, we analyzed 1,524 genomes of Arabidopsis thaliana-associated Pseudomonas isolates, collected across several years and sites near Tübingen, Germany. We inferred the pan-genome for all isolates based on their orthologous groups. Out of the 72,397 orthologous groups specified, only $1.3 \%$ belonged to the bacterial 'core genome', while $36.3 \%$ were unique to individual isolates. Analyzing the dynamics of gain-and-loss events within and between orthologous groups with an evolutionary framework allowed us to infer a co-evolutionary network composed of orthology groups gained-and-loss together. Overall, $9.81 \%$ of the orthologous groups co-evolve with at least one partner. Further dividing this co-evolutionary network into 1,014 highly connected modules, we identified modules that are associated with specific cellular functions. By comparing closely related isolates we also discovered genomic islands and further characterized their evolutionary dynamics. Finally, by focusing on a subgroup of phylogenetically close isolates differing in their pathogenicity levels, we could associate specific orthologous groups and co-evolving modules with strain pathogenicity.

Genome-wide study identifies epistatic interactions between immune genes in Arabidopsis

W. ZHU, Max Planck Institute for Developmental Biology, Tuebingen, Germany

In Arabidopsis thaliana, the quantitative resistance gene ACCELERATED CELL DEATH 6 (ACD6) has been implicated in the trade-off between growth and defense in natural strains. $A C D 6$-Est-1-type alleles can lead to spontaneous activation of immune responses, including leaf necrosis. The extent of visible hyperimmunity varies, however, substantially between $A C D 6$-Est- 1 carriers. To identify genes that modulate $A C D 6$ activity, we performed a genome-wide association study (GWAS) of 84 strains that carry the $A C D 6$-Est-1 allele. GWA together with linkage analysis identified both MODULATORS OF HYPERACTIVE ACD6 1 (MHA1, an unknown gene) and MHA2 (an uncharacterized subtilase) as ACD6 modifiers for cell death regulation. MHA1, coding for a small protein of $\sim 7 \mathrm{kDa}$, and its paralog MHA1-LIKE (MHAL) differentially interact with different ACD6 protein variants, and MHA1/MHAL interactions with ACD6 are important for expression of autoimmunity as well as disease resistance. Haplotype analysis suggested that the epistatic interactions between the ACD6-Est-1 allele and its modifiers have been favoured by natural selection in the field. We propose that allelic diversity at MHA1 and MHA2 contributes to the maintenance of $A C D 6$-Est-1 in A. thaliana populations, and that epistatic interactions are an important component of quantitative disease resistance.

Molecular evolutionary characterizations of antagonistic Fusarium species identified from the soybean rhizosphere soil L. KONG, W. K. Huang, H. Peng, D. Peng, Institute of Plant Protection, Chinese Academy of Agricultural Sciences, Beijing, China

The soybean root rot disease was mainly caused by Fusarium oxysporum. Using non-pathogenic Fusarium spp. to control this disease is economic and has proved to be efficient in many crops. In this study, 529 fungi isolates were isolated from 34 soybean rhizosphere soil samples in five different regions and from which 208 isolates were identified as Fusarium spp. The dominant Fusarium spp. were different between different regions. During these 208 Fusarium isolates, 158 strains exhibited antagonistic activity against $F$. oxysporum $\mathrm{f}$. sp. glycines and the pathogenicity of these isolates were evaluated subsequently. The results showed that three antagonistic isolates were non-pathogenic on soybean seedlings. According to the morphology and molecular technologies such as PCR and sequencing, these three non-pathogenic isolates were identified as Fusarium redolens, Fusarium acuminatum, and Fusarium equseti, respectively, which indicating that these three isolates could be potentially used as biological antagonists. Their molecular antagonism mechanism is ongoing. Moreover, Phylogenetic tree analysis showed that the antagonistic $F$. oxysporum had an overall distant relationship with the tested $F$. oxysporum $\mathrm{f}$. sp. glycines. The genomes of these antagonistic pairs of $F$. oxysporum are going to be uncovered. Our results not only identified some potential biological Fusarium species, but also investigate their molecular evolutionary relationships.

Re-programming of Pseudomonas syringae pv. actinidiae gene expression during early stages of infection of kiwifruit M. D. TEMPLETON (1,2,3), P. A. McAtee (1), N. J. Nieuwenhuizen (1), X. Chen (1), B. Jose (3,4), J. Jayaraman (1,3), E. H. A. Rikkerink (1), P. P. Gardner (3,4), A. C. Allan (1,2), (1) The New Zealand Institute for Plant and Food Research Ltd, Auckland, New Zealand; (2) University of Auckland, Auckland, New Zealand; (3) Bioprotection Centre for Research Excellence, New Zealand; (4) Department of Biochemistry, University of Otago, Dunedin, New Zealand

Pseudomonas syringae pv. actinidiae (Psa) initiated a worldwide pandemic in 2008 on cultivars of Actinidia chinensis var. chinensis. RNA-seq was used to investigate gene expression in Psa in vitro and during the first five days after infection of kiwifruit plantlets. Principal component and 
heatmap analyses showed distinct phases of gene expression during the infection time course. The first phase was an immediate transient peak of induction around three hours postinoculation (HPI) that included genes that code for a Type VI Secretion System and nutrient acquisition. This was followed by a significant commitment, between 3 and 24 HPI, to the induction of genes encoding the Type III Secretion System (T3SS) and Type III Secreted Effectors (T3SE). Expression of these genes collectively accounted for $6.3 \%$ of the bacterial transcriptome at this stage. There was considerable variation in the expression levels of individual T3SEs but the majority followed the same temporal expression pattern. As infection progressed over the time course of five days, there was an increase in the expression of genes with roles in sugar, amino acid and sulfur transport and the production of alginate and colanic acid. Both polymers are involved in biofilm production. The results from this study indicate that there is a complex remodeling of the transcriptome during the early stages of infection, with at least three distinct phases of coordinated gene expression.

Metabarcoding diagnostics of Phytophthora species in environmental samples

L. PRITCHARD (1), P. J. Cock (1), P. Thorpe (2), E. Randall (1), S. Green (3), D. Cooke (1), (1) The James Hutton Institute, Dundee, U.K.; (2) University of St Andrews, U.K.; (3) Forest Research, Roslin, U.K.

Metabarcoding analysis of marker sequences is a very sensitive and widely used high-throughput technique for estimating the species composition of microbial populations and for determining the presence of potential pathogens in environmental samples on the basis of characteristic DNA regions. The ITS1 marker region is typically used to identify and taxonomically classify Phytophthora species and has been used previously in metabarcoding studies. Unlike rDNA markers in bacteria, which occur singly or at low copy numbers, a single Phytophthora isolate may contain over 40 distinct ITS1 sequences. This increases the difficulty of performing accurate and precise identification and quantification of Phytophthora spp. from environmental samples. We describe: design and use of novel synthetic control metabarcoding sequences to estimate and control for natural and artefactual sequence variation; development of multilabel classification metrics for evaluating classifier performance against complex microbial community mixtures; the influence of alternative methods of reference database construction on classification accuracy; and the relative performance of several alternative classification algorithms. We demonstrate that careful protocol design, including choice of algorithm and database construction, can improve accuracy and specificity when developing metabarcoding diagnostic methods, and we present a novel software tool to aid in metabarcoding identification of Phytophthora spp.

Co-infection shapes pathogen fitness in a genetically diverse population of Pseudomonas viridiflava isolates from Arabidopsis A. D. GLOSS (1), P. Laurin (1), X. Zhai (1), T. Karasov (2), J. Bergelson (1), (1) University of Chicago, Chicago, IL, U.S.A.; (2) Max Planck Institute for Developmental Biology, Tuebingen, Germany

Plants are often co-infected by multiple strains of a pathogen. Interactions between strains range from competitive to synergistic and can affect pathogen fitness. However, little is known about how the costs and benefits of co-infection vary across naturally co-occurring strains, the extent to which these costs and benefits are general or strain-specific, and the identity and evolutionary history of loci shaping these interactions. Quantifying these patterns is essential for understanding the importance of co-infection in pathogen ecology and evolution. We are measuring effects of co-infection in a population of a bacterial pathogen, Pseudomonas viridiflava, isolated from Arabidopsis thaliana, using reporter- and sequence-based methods for high-throughput quantification of pathogen abundance. Across co-infections with hundreds of pairwise strain combinations in gnotobiotic plants, we find that between-strain interactions can explain as much variation in pathogen fitness as focal strain genotype alone. Competition is pervasive and predicted by growth rate in single infections. However, we find combinations of strains that deviate from expectations based on growth rate, including both inhibitory and putatively beneficial interactions. We are now using GWAS to identify loci underlying these outcomes. The prevalence of strain-dependent effects of co-infection underscores the importance of interactions between pathogen genomes as a key force in pathogen ecology and evolution.

Gibberellin promotes fungal entry and colonization during Paris-type arbuscular mycorrhizal symbiosis in Eustoma grandiflorum T. TOMINAGA (1), C. Miura (1), N. Takeda (2), Y. Kanno (3), Y. Takemura (1), M. Seo (3), M. Yamato (4), H. Kaminaka (1), (1) Tottori University, Japan; (2) Kwansei gakuin university, Japan; (3) RIKEN CSRS, Japan; (4) Chiba University, Japan

Arbuscular mycorrhiza (AM) are morphologically divided into two types: Arum- and Paris-type AM depending on host species. Arum-type AM shows intercellular hyphae and highly branched structures (arbuscules) in the cortical cell for the exchange of nutrients. On the other hand, intracellular hyphal coils with or without arbuscules are found in Paris-type AM. Gibberellin (GA) is known to inhibit fungal entry to the epidermal cell and arbuscule formation by using model plants forming Arum-type AM, whereas the effects of GA on Paris-type AM symbiosis are unclear. To clarify the effects of GA on Paris-type AM symbiosis, Eustoma grandiflorum was used as the host plant for Paris-type AM establishment with the model AM fungus, namely, Rhizophagus irregularis. Here, we show that exogenous GA treatment significantly induced hyphopodium formation at the epidermis, thus leading to the promotion of fungal colonization and arbuscule formation in the root cortex. By contrast, the suppression of GA biosynthesis and signaling impaired fungal entry to E. grandiflorum roots. Moreover, the exudates from GAtreated roots strongly induced the hyphal branching of R. irregularis. Our results show that GA has an opposite effect on Paris-type AM symbiosis in E. grandiflorum compared with Arum-type AM symbiosis in model plants. This finding could be explained by the differential regulation of the early colonization stage, where fungal hyphae make contact with and penetrate the epidermis.

The metabolic root of beneficial rhizobacteria-induced systemic resistance

I. A. STRINGLIS (1), K. Yu (1), K. Feussner (2), R. De Jonge (1), S. Van Bentum (1), P. A. H. M. Bakker (1), I. Feussner (2), R. L. Berendsen (1), C. M. J. Pieterse (1), (1) Plant-Microbe Interactions, Department of Biology, Utrecht University, Utrecht, Netherlands; (2) Department of Plant Biochemistry, University of Goettingen, Goettingen, Germany

Probiotic members of the root microbiome promote plant growth and protect the host plant against pathogens and pests. In the Arabidopsis thaliana-Pseudomonas simiae WCS417 (hereafter WCS417) model system, the root-specific transcription factor MYB72 is an important regulator of rhizobacteria-induced systemic resistance (ISR) and iron uptake responses. Following iron deficiency, MYB72 regulates the 
biosynthesis and excretion of coumarins. We recently found that coumarins, such as scopoletin, apart from their iron-mobilizing capacity, can shape root microbiome assembly. The ISR-eliciting rhizobacterium WCS417 induces MYB72 in Arabidopsis roots, but is insensitive to the antimicrobial activity of scopoletin. We hypothesize that WCS417 induces coumarin production by plant roots to improve its own niche establishment and in return benefits the health and growth of the host plant. To dissect the metabolic signatures of this interaction, we compared the metabolomes of root-colonized wild-type Col-0 and mutant myb72 Arabidopsis plants. Our data show that WCS417 stimulates the biosynthesis of coumarins in Arabidopsis roots, and significantly affects the biosynthesis of glucosinolates and fatty acid metabolites with predicted functions in plant immunity. These results provide novel insight into the metabolites that play a role in the mutualistic interaction between plant roots and ISR-inducing rhizobacteria.

Novel insights to arbuscular mycorrhizal pre-symbiotic signaling - A transcriptomic approach to characterize fungal-triggered responses in rice

W. SUMMERS (1), J. Choi (1), S. Mueller (1), G. Oldroyd (2), U. Paszkowski (3), (1) University of Cambridge, Cambridge, U.K.; (2) Sainsbury Laboratory, Cambridge, U.K.; (3) University of Cambridge, U.K.

Prior to the establishment of arbuscular mycorrhizal (AM) symbiosis, early pre-symbiotic communication between fungus and plant takes place across the rhizosphere. Our understanding of this process has advanced in recent years. In rice, the receptor D14-LIKE has been found to be absolutely required for AM fungal perception, while another receptor CERK1 has been shown to mediate chitooligosaccharide-induced perinuclear calcium spiking, which is the central node of the common SYM pathway. However, while these components are all known to contribute to pre-symbiotic fungal perception, their interrelationship is unknown. To provide an insight into the architecture of pre-symbiotic signaling pathways, a large-scale transcriptomic project was conducted that has yielded fascinating data. By examining the responses of presymbiotic rice mutants to a range of fungal exudates, including germinated spore exudates and diverse chitinaceous fungal signals, the contributions and relationships of the D14-LIKE, CERK1 and common SYM pathways in host reprogramming have been elucidated. Furthermore, light has been shed on the requirement of these pathways for the perception of distinct fungal triggers. This has not only refined our understanding of early pre-symbiotic processes, but also raised exciting questions for the future.

The origin of new gene families and the convergence of molecular interactions during a niche transition in ectomycorrhizal Amanita Y. W. WANG (1), J. Slot (2), J. Hess (3), A. Pringle (1), (1) University of Wisconsin-Madison, Madison, WI, U.S.A.; (2) The Ohio State University, Columbus, OH, U.S.A.; (3) University of Vienna, Austria

While ectomycorrhizal (EcM) symbioses are among the most prominent mutualisms in nature and have independently evolved repeatedly across the fungal tree of life, the genetics enabling EcM symbiosis remain a mystery. Specifically, little is known about the functions and origins of gene families unique to EcM fungi. Using the genomes of three EcM Amanita fungi and three non-EcM species, we identified 109 gene families unique to EcM Amanita species. Expression profiles of Amanita muscaria demonstrate that genes in these families are more highly expressed during symbiosis, suggesting unique gene families play a functional role in symbiosis. We then explored the origins of these unique gene families by building pipelines to identify mechanisms of acquisition. Two, six and eight gene families were categorized as resulting from de novo gene creation, horizontal gene transfer, and fast evolution, respectively. Other unique families might appear unique because of multiple deletions in asymbiotic lineages or may remain enigmatic because of incongruent analytical results or a lack of statistical confidence. A unique gene encoding an ACC deaminase was expressed in the greatest relative abundance in symbiosis, compared to other genes. ACC deaminase can reduce the synthesis of ethylene, known to inhibit symbiosis, and may point towards a common signaling pathway among plant-microbe symbioses.

Raman Spectroscopy as a Tool to Study Genotype-Species Dependence of Tomato-Trichoderma Interaction Effects on Seed Germination I. Vukelic (1), D. Radic (1), I. Pecinar (2), S. Levic (2), G. Racic (1), D. PANKOVIC (1), (1) Educons University, Sremska Kamenica, Serbia; (2) University of Belgrade, Faculty of Agriculture, Belgrade, Serbia

In this paper, the effect of five Trichoderma species on seed germination of two tomato cultivars (Narvik and Gružanski zlatni) with contrasting resistance to diseases, was examined in vitro. Tomato seeds were co-cultured with T. harzianum, T. brevicompactum, T. virens, $T$. longibrachiatum and T. citrinoviride on Murashige and Skoog agar for 72 hours. The stimulation of seed germination of cultivar Gružanski zlatni ranged from $19 \%$ with $T$. virens, to $78 \%$ with $T$. longibrahiatum, and for the cultivar Narvik, from $140 \%$ with $T$. harzianum to $726 \%$ with $T$. brevicompactum. Emerging radicles of seed in co-culture with T. harzianum and T. brevicompactum, were cut longitudinally and recorded at room temperature, with Raman microspectroscope within wavenumber range from 250 to $1800 \mathrm{~cm}^{-1}$, using laser with a $785 \mathrm{~nm}$ wavelength. The most significant difference between analysed Raman spectra was observed in co-culture of cultivar Gružanski zlatni and T. harzianum. The band at around $400 \mathrm{~cm}^{-1}$ and spectral region from 1040 to $1120 \mathrm{~cm}^{-1}$ can be assigned to cellulose, high intensity bands at 1203 and $1219 \mathrm{~cm}^{-1}$ and weak band around $522 \mathrm{~cm}^{-1}$ indicate on hemicellulose. Some differences in the spectra at $\sim 1640 \mathrm{~cm}^{-1}$ and $\sim 1590 \mathrm{~cm}^{-1}$ could point out on presence of phenolic compounds. The observed correlation between increased cellulose signal as well as other root macromolecules and positive plantsymbiont interaction is under investigation.

Adaptations and evolution of a heritable leaf nodule symbiosis between Dioscorea sansibarensis and Orrella dioscoreae A. CARLIER, Ghent University, Ghent, Belgium

Various plant species establish intimate symbioses with bacteria within their aerial organs. The bacteria are contained within nodules or glands often present in distinctive patterns on the leaves in what is commonly referred to as leaf nodule symbiosis. We recently described a highly specific symbiosis between a wild yam species from Madagascar, Dioscorea sansibarensis and bacteria of the species Orrella dioscoreae. Using population genetic and laboratory experiments, we show that symbionts are transmitted vertically via vegetative propagules. Unique among leaf nodule symbioses, the bacteria can be cultured and are amenable to comparative transcriptomics and phenotypic characterization, revealing a potential role in complementing the host's arsenal of secondary metabolites. We leverage these unique features to reveal pathways and functions likely to play a role in the establishment of a permanent association in the phyllosphere. 
A legume actin depolymerization factor is required for rhizobia infection and nodule organogenesis

C. QUINTO (1), Y. Ortega-Ortega (2), J. Carrasco-Castilla (3), M. Juárez-Verdayes (4), R. Toscano-Morales (5), N. Nava (1), L. Cardenas (1), (1) Depto. de Biología Molecular de Plantas, Instituto de Biotecnología, UNAM, Cuernavaca, MR, Mexico; (2) Centro de Investigación en Química Aplicada-CONACYT, Saltillo, CU, Mexico; (3) Centro de Estudios Científicos y Tecnológicos No.17, IPN., León, GJ, Mexico; (4) Universidad Autónoma Agraria Antonio Narro, Saltillo, CU, Mexico; (5) Dept. of Plant Biology, College of Biological Sciences, Univ. of California, Davis, CA, U.S.A.

Actin plays a major role in the rhizobium-legume symbiosis. It participates in cytoskeletal rearrangements, actin polymerization and dynamics in response to Nod factors. These rearrangements might be mediated by diverse actin-binding proteins, such as actin depolymerization factors (ADFs). The functions of ADFs during bacterial infection and nodulation remain unknown. Herein, the function of an ADF in the Phaseolus vulgaris-rhizobia interaction was analyzed. Nine $A D F$ genes were identified in the $P$. vulgaris genome; $P v A D F E$ was preferentially expressed in rhizobia-inoculated roots and nodules. $P v A D F E$ promoter activity was observed in root hairs harboring growing infection threads, cortical cell divisions, and vascular bundles in mature nodules. Gene silencing of $P v A D F E$ using RNA interference (RNAi) in transgenic roots increased the number of infection threads, resulting in an increase of nodule numbers and nitrogen fixation. Conversely, over-expression of $P v A D F E$ decreased root-nodule number and nitrogen fixation and resulted in an altered accumulation of NIN and ENOD2 transcripts, revealing that actin changes modulated by PvADFE participate in nodulin signaling. Sub-cellular localization studies indicated that PvADFE was distributed in the rhizobial infected root hairs and vascular bundles of mature nodules. These data suggest that an excess of PvADFE might negatively control actin severing and dynamics during rhizobial infection and nodule organogenesis.

Transcriptome analysis of the antagonistic effect of NADPH oxidase gene RbohB in the common bean after rhizobial and arbuscular mycorrhizal inoculation

C. FONSECA (1), E. A. Zayas Del Moral (1), J. Montiel (2), N. Nava (1), F. Sánchez (1), C. Quinto (1), (1) Depto. de Biología Molecular de Plantas, Instituto de Biotecnología, UNAM, Cuernavaca, MR, Mexico; (2) Centre for Carbohydrate Recognition and Signaling, Aarhus University, Aarhus, Denmark, Mexico

The reactive oxygen species (ROS) mainly generated by NADPH oxidases called Respiratory Burst Oxidase Homologs (RBOH) in plants have critical roles in the symbiosis between legume and nitrogen-fixing bacteria and arbuscular mycorrhizal fungi (AMF). Studies in our group indicate that, downregulation of $P v R b o h B$ in Phaseolus vulgaris impairs rhizobia infection thread progression and nodule development, but also enhances the size of hypopodia and AMF hyphal colonization. These data suggest that $P v R b o h B$ exhibits an opposite mechanism that differentially regulates contrasting features between both symbioses. To explore this hypothesis, an RNA-Seq transcriptomic analysis was performed to identify the differentially expressed genes (DEG) in the PvRbohB-RNAi roots inoculated with Rhizobium tropici or Rhizophagus irregularis. Our results indicate that root nodule symbioses induce considerable transcriptomic reprogramming, with the AMF inducing less of a response than rhizobia during the early stages of their respective $P$. vulgaris symbioses. Additionally, a compendium of DEG related to ROSscavenging, cell wall-remodeling, and phytohormone homeostasis during nodulation and mycorrhization in $P$. vulgaris was found to be affected by $P v R b o h B$ silencing. These results provide important information on the symbiotic gene signaling networks involved in the early stages of rhizobial and mycorrhizal colonization, and the differential effects of a NADPH oxidase gene, $R b o h B$, on these processes.

Fusarium oxysporum endophytes and pathogens featuring as Dr. Jekyll and Mr. Hyde M. E. CONSTANTIN, F. J. de Lamo, F. L. Takken, M. Rep, University of Amsterdam, Amsterdam, Netherlands

The Fusarium oxysporum ( $F o$ ) species complex contains pathogenic strains that cause vascular wilt disease and endophytic strains that colonize plants without causing disease. Interestingly, some of the non-pathogenic strains confer endophyte-mediated resistance (EMR) in tomato against vascular wilt disease caused by $F o$ f. sp. lycopersici $(F o l)$. Tomato bioassays, using a set of 45 non-pathogenic $F o$ strains, revealed that EMR is a common feature of $F o$. How non-pathogenic $F o$ triggers EMR is currently unknown. Whole genome sequencing showed that non-pathogenic $F o$ strains have fewer putative effector genes as compared to pathogenic strains. It is hypothesized that Fusarium endophytes trigger EMR by inducing a plant immune response. Fol inoculation of tomato lines compromised in jasmonic acid (JA), ethylene (ET) or salicylic acid (SA) signaling revealed that EMR is independent from these phytohormones. These findings imply that the induced resistance response against Fusarium wilt disease differs from the classical Induced Systemic Response (ISR) or Systemic Acquired Resistance responses, suggesting the presence of a novel root-specific resistance mechanism against wilt disease.

Genetic tools in Frankia: Yes we can, from complementation to site-specific mutagenesis

C. PESCE, R. Oshone, V. A. Kleiner, S. G. Hurst IV, L. S. Tisa, University of New Hampshire, Durham, NH, U.S.A.

The filamentous actinobacteria, Frankia, forms a nitrogen-fixing symbiotic association with eight families of angiosperms. These actinorhizal plants have positive impacts on the environment by for example reclaiming salt or heavy metal contaminated soils. One of the major obstacles in this field is the absence of genetic tools for Frankia. We have developed an efficient and stable plasmid transfer protocol that uses filter mating. Four derivatives of the broad-host-range vector pBBR1MCS were introduced into 4 Frankia strains at frequency of $10^{-2}$ to $10^{-4}$ and confirmed by molecular approaches. These plasmids were stably maintained for over 2 years or through passage in a plant host. The salt-tolerance candidate gene from the salt-tolerance Frankia sp. strain CcI6 was cloned into pBBR1MCS-3. The resulting construct was introduced into the salt-sensitive F. casuarinae strain CcI3, providing an increased level of salt tolerance A targeted mutagenesis system based on a modified CRISPR/Cas9 system was develop and introduced into Frankia, which was stably maintained in the cells. End point RT-PCR results showed that the Cas 9 gene was expressed in Frankia. As a proof of concept, genes for the hopanoid biosynthesis cluster in F. casuarinae strain CcI3 were targeted. The first Frankia mutants generated are being confirmed by molecular approaches. These genetic tools, including the stable transformation and targeted mutagenesis, will open up new research avenues for this symbiosis. 
Symbiosis-induced rice phosphate transporter PT13 mediates cellular phosphate efflux

S. Y. YANG (1), Y. Wu (2), P. de Waard (3), H. van As (4), Y. Poirier (5), Z. Shang (2), U. Paszkowski (6), (1) National Taiwan University, Institute of Plant Biology, Taiwan; (2) Hebei Normal University, College of Life Science, China; (3) University of Wageningen, Magnetic resonance research facility, Netherlands; (4) University of Wageningen, Department of Agrotechnology and Food Sciences, Netherlands; (5) University of Lausanne, Department of Plant Molecular Biology, Switzerland; (6) University of Cambridge, U.K.

Most plants acquire phosphorus in the form of inorganic phosphate (Pi) via association with mutualistic arbuscular mycorrhizal (AM) fungi. Fungal hyphae take up Pi from the soil and deliver it to the plant inside inner cortex cells at highly branched ephemeral structures, the arbuscules. In rice, two PHosphate Transporter 1 (PHT1) type of proteins, PHT1;11 and PHT1;13 are specifically induced in arbusculated cells. Both transporters are required for fungal colonization of the root but only PT11 imported fungus-provided Pi, while the role of PT13 remained elusive. Here, we show that PT13 is a plasma membrane-localized Pi exporter that is induced in tissue with strongly elevated Pi concentration. Our data indicate that the formation of arbuscules is associated with a sharp increase in plant cellular Pi which is accompanied by the induction of PT13 promoter activity and suggests a role for PT13 in Pi homeostasis of arbuscule-containing cells.

Characterization of North American strains of the fungal endophyte PRB110

S. DÍAZ GONZÁLEZ (1), P. Marín (2), P. Olivares (2), K. Donohue (3), M. Borja (4), F. Brunner (2), S. Sacristan (5), (1) Plant Response Biotech, S.L./Universidad Politécnica de Madrid, Spain; (2) Plant Response Biotech, S.L., Spain; (3) Department of Biology. Duke University, Durham, NC, U.S.A.; (4) Universidad Complutense de Madrid, Spain; (5) Universidad Politécnica de Madrid, Madrid, Spain

The fungal endophyte PRB110 stablishes a mutualistic interaction with Arabidopsis thaliana, promoting plant growth and silique production under phosphate $(\mathrm{Pi})$ starvation. Until now, this interaction had only been observed in nature in Pi poor soils of Central Spain. In this work, we have detected PRB1 10 in 38\% of the sampled Arabidopsis plants from a wild population at North Carolina (U.S.A.), isolating new strains from different surface sterilised plant tissues. Retrotransposon-Microsatellite Amplified Polymorphism analyses including several Spanish and European strains showed different haplotypes, three of which were only found in American isolates. Four PRB110 American strains and two Spanish strains were co-cultivated with Arabidopsis at sufficient and starving Pi concentrations. Results showed that PRB110 strains displayed a similar behaviour, increasing the fresh weight of the plant both under sufficient and starving Pi conditions. Moreover, the expression of the gene Pht1;3, involved in plant Pi uptake, was upregulated in Pi starving conditions in plants treated with all the PRB110 strains. In a different assay, both the American and the Spanish strains increased the number of siliques produced by Arabidopsis plants grown in a poor-nutrient soil. These results indicate that the beneficial effect of PRB110 in Arabidopsis is independent of the geographical origin of the isolates.

Modulation of quorum sensing as an adaptation to nodule cell infection during experimental evolution of legume symbionts M. TANG (1), O. Bouchez (2), S. Cruveiller (3), C. Masson-Boivin (4), D. Capela (5), (1) LIPM, Université de Toulouse, INRA, CNRS, CASTANET TOLOSAN, France; (2) Genotoul Genome \& Transcriptome, INRA, Toulouse, France; (3) LABGeM, Génomique Métabolique, Genoscope, Institut François Jacob, CEA, CNR, France; (4) INRA-LIPM, Castanet-Tolosan, France; (5) CNRS INRA, Castanet Tolosan, France

Rhizobia, the nitrogen-fixing legume symbionts, have evolved via the horizontal transfer of essential symbiotic genes in diverse soil bacteria, followed by the optimization of the acquired symbiotic potential by genome remodeling under plant selection pressure. Transition towards legume symbiosis has recently been tested at the laboratory scale. The symbiotic plasmid of the rhizobium Cupriavidus taiwanensis was introduced into the strictly extracellular plant pathogen Ralstonia solanacearum GMI1000, and the resulting chimera evolved in intracellular symbionts of Mimosa pudica (the C. taiwanensis host) by experimental evolution. Here we showed that the Phe quorum sensing system required for the virulence of the ancestral bacterium was reconfigured to allow evolved Ralstonia to massively infect plant (nodule) cells. Mutations in the components of this system delayed the induction kinetics of the central regulator PhcA it controls in function of cell density. Change in the expression of a PhcA-target gene was only observed in infection threads progressing in root hairs, suggesting an early programming of late infection. Delayed switch to the quorum-sensing mode decreased the pathogenicity of the ancestral strain. This work illustrates the functional plasticity of regulatory systems and shows how a small modulation in signal response can operate drastic changes in bacterial lifestyle.

\section{Reexamining the phenomenon of chemotaxis to host exuded flavonoids in S. meliloti}

K. K. COMPTON III (1), B. E. Scharf (2), (1) Virginia Tech, Blacksburg, VA, U.S.A.; (2) Virginia Tech, Black, VA, U.S.A.

Past research past showed that several rhizobial species will exhibit chemotaxis, the directed movement of bacteria using flagella, to flavonoids, the inducers of nodulation genes. Flavonoids were therefore concluded to serve a dual role by both recruiting the symbiont and inducing nod gene expression. Recently, no research has followed up on this topic and the mechanism of flavonoid chemoattraction remains a mystery. In investigating chemotaxis of the cognate alfalfa symbiont, Sinorhizobium meliloti, we performed the traditional capillary assay to test chemotaxis of S. meliloti to several flavonoids. The traditional capillary assay is the "gold standard" for chemotaxis assays because it produces quantitative data and is independent of bacterial metabolism. Quercetin, hyperoside, pratensein, and luteolin were tested in the capillary assay because they are found in the extracts and exudates of alfalfa seeds. Additionally, luteolin acts as a nodulation inducer for S. meliloti. Due to a lack of solubility, certain flavonoids require cosolvents such as dimethylsulfoxide or methanol. We also examined the possibility that these solvents are themselves attractants. Tentatively, we found $S$. meliloti to be weakly attracted to methanol and that taxis to flavonoids is not distinguishable from that to the methanol cosolvent. In addition to these results, we will discuss the situational issues surrounding chemotaxis to flavonoids because this is a topic that merits revisiting.

The genomic bases for cheating among wild populations of nodule-associated Bradyrhizobium A. J. WEISBERG (1), J. H. Chang (1), J. Sachs (2), (1) Oregon State University, Corvallis, OR, U.S.A.; (2) University of California Riverside, Riverside, CA, U.S.A. 
Nitrogen-fixing rhizobia can form mutualistic relationships with legumes. In this interaction, rhizobia occupy plant nodules and fix nitrogen for the plant in exchange for carbon. The fitness cost associated with fixing nitrogen is high and given the large population sizes and short life cycle of the symbiont, can lead to the evolution of "cheaters" and a break down in mutualism. In this scenario, the bacteria can still induce nodules and gain carbon without reciprocating fixed nitrogen. To understand the bases for the cheater phenotype, we cultured and characterized 88 strains of Bradyrhizobium isolated from root nodules of Acmispon strigosus sampled across southern California. Most isolates could induce nodules and fix nitrogen. However, a number of isolates failed to elicit nodules. A third set were cheaters that could induce nodules on A. strigosus but did not fix nitrogen. Analyses of whole genome sequences revealed that cheaters are closely related to beneficial isolates and are predicted to be capable of fixing nitrogen on the basis of having all conserved nodulation (nod) and nitrogen fixation (nifffix) genes. However, multiple cheater lineages vary in their repertoire of secretion system loci, which have been previously shown to influence symbioses. Long-read DNA sequencing was also employed, and analyses revealed other large-scale changes, including variation in plasmids that are also implicated in influencing symbiosis.

Comparative genomic analysis reveals the convergent evolution of lichen-forming fungi

H. SONG (1), K. T. Kim (2), G. W. Lee (3), J. Choi (1), J. Jeon (1), K. Cheong (1), G. Choi (1), S. Y. Park (4), J. S. Hur (5), Y. H. Lee $(1,2,6,7,8),(1)$ Interdisciplinary Program in Agricultural Genomics, Seoul National University, Seoul, Korea, Republic of (South); (2) Department of Agricultural Biotechnology, Seoul National University, Seoul, Korea, Republic of (South); (3) Macrogen, Inc., Seoul, Korea, Republic of (South); (4) Department of Plant Medicine, Sunchon National University, Suncheon, Korea, Republic of (South); (5) Korean Lichen Research Institute, Sunchon National University, Suncheon, Korea, Republic of (South); (6) Research Institute of Agriculture and Life Sciences, Seoul National University, Seoul, Korea, Republic of (South); (7) Center for Fungal Genetic Resources, Seoul National University, Seoul, Korea, Republic of (South); (8) Plant Immunity Research Center, Seoul National University, Seoul, Korea, Republic of (South)

Lichen-forming fungi associated with green algae or cyanobacteria are known as mutualists. We sequenced five genomes of lichen-forming fungi in two different classes, Lecaronomycetes and Eurotiomycetes, and performed comparative analysis. The phylogenomic analysis revealed the convergent evolution of lichen-forming fungi. The evolutionary factors that contributed to be lichen seem to be not their core genes, but their lineage-specific and orphan genes. The expression analysis revealed that the lineage-specific and orphan genes were highly induced in the early 'resynthesize' stages, whereas the conserved genes were induced during the later 'growth together' stages of the lichen formation. In contrast, some gene families such as small secreted proteins, carbohydrate-active enzymes, and secondary metabolite synthesize genes underwent common changes, and they may also contribute to the convergent evolution. The distribution of these gene families was different with other symbionts. This comparative analysis revealed the genomic factors that led the convergent evolution of lichen-forming fungi, and this would provide a platform to understand a novel symbiotic lifestyle of lichen-forming fungi.

Physiological and molecular mechanisms of enhanced rice root development inoculated with Burkholderia vietnamiensis R. SHINJO (1), A. Tanaka (2), T. Suzuki (3), D. Takemoto (2), M. Kondo (1), (1) Nagoya University, Japan; (2) Nagoya Univ., Japan; (3) Chubu Univ., Japan

Introducing beneficial plant-growth-promoting-rhizobacteria (PGPR) to the agricultural field would improve crop yield, maintain soil sustainability, and ameliorate microbial communities in the long term. Burkholderia vietnamiensisis a nitrogen-fixing bacterium isolated from rice, sugarcane and paddy field soil.We recently isolated endophytic $B$. vietnamiensis strain RS1 from the surface-sterilized tuberous roots of sweet potato (Ipomoea batatascv.Beniazuma). Inoculation of the strain RS1 to rice seedlings (Oryza sativaL. cv. Nipponbare, 21-day-old) significantly increased the dry weight of shoot and root by $21 \%$ and $29 \%$, respectively. Total root length of inoculated seedlings increased by $35 \%$ associated with an increase in nitrogen content. The quantitativePCR analysis detected RS1 cells mostly in the root tissues. To better understand the mechanisms of plant growth promotion by the strain RS1, we conducted a next-generation sequencing of mRNA in rice roots inoculated with RS1. This analysis revealed that several genes related to plant hormone biosynthesis were significantly upregulated in the roots inoculated with RS1. The result indicatesthe possibility that inoculation of RS1 promotes root development by affecting phytohormone signaling in roots, which lead to enhanced nutrient uptake from soil and eventual improvement of shoot growth in rice.

\section{Fungal Endophytes Mediate Plant Drought Tolerance Through Alteration of Host Metabolism, Enzymatic Activities and Gene} Expression

K. M. G. DASTOGEER (1,2), H. Li (2), S. Wylie (2), M. G. K. Jones (2), (1) Bangladesh Agricultural University, Mymensingh, Bangladesh; (2) Murdoch University, Perth, Australia

Beneficial plant-microbe associations play significant roles in both natural and agricultural systems. For example, fungal endophytes have profound impacts on growth and physiology of plants, which may be particularly important under conditions of stress. To study the impact of endophyte infection, we inoculated seedlings of Nicotiana benthamiana with two ascomycetous fungal endophytes, an isolate of Cladosporium cladosporioides and an unidentified ascomycete fungus. Endophyte colonisation caused significantly differential accumulation of 17-21 metabolites including sugars, sugar alcohols, amino acids and other metabolites at various stages of plant growth and stress application. The water stress tolerance of fungus-inoculated plants was correlated with increases in plant biomass, relative water content, soluble sugar, soluble protein, proline content, increased activities of the antioxidant enzymes catalase, peroxidase and polyphenol oxidase, decreased production of reactive oxygen species, and decreased electrical conductivity. In addition, there was significant upregulation of several genes previously reported as drought-induced. The findings suggest a symbiotic relationship between these fungi and $N$. benthamiana plants, a species adapted to hot and unpredictable soil moisture conditions of northern Australia. Importantly, this work sheds light on the mechanisms underlying enhanced tolerance to water stresss provided by fungal endophyte infection.

A comparative transcriptome analysis reveals rhizobial infection-related pathways dependent on ERN AP2/ERF transcription factors J. FOURNIER (1), M. F. Guerrero-Molina (1), L. Frances (1), M. Renard (1), A. Dufour (1), D. Rengel (2), M. F. Jardinaud (1), F. De CarvalhoNiebel (1), (1) Laboratory of Plant Microbe Interactions (LIPM), INRA, CNRS, Univ Toulouse, Castanet-Tolosan, France; (2) LIPM, Université de Toulouse, INRA, CNRS, INPT, Castanet-Tolosan, France 
Plants establish symbiotic associations with microbes to overcome nutrient limitations. The legume-rhizobium symbiosis forms a specialized organ, the root nodule, where the bacteria fix nitrogen for the benefit of the host plant. Nodule development requires the coordinated reprogramming of different root tissues, to allow concomitant root infection by rhizobia and initiation of nodule organogenesis. In Medicago truncatula, rhizobial infection initiates in root hairs via the construction of a new apoplastic compartment called the infection thread (IT). ERF Required for Nodulation 1 (ERN1) and 2 (ERN2) encode AP2/ERF transcription factors that regulate in concert the formation of ITs in M. truncatula. To elucidate the infection-related pathways controlled by these regulators, we compared the root transcriptome of the WT A17 to the IT-defective (ern1) and IT-minus (ernlern2) mutant lines, before and after inoculation with rhizobia. The rhizobia-induced MtENOD11, $M t E N O D 12$ and MtENOD9 genes previously identified as ERN1/ERN2 putative targets ${ }^{1}$, are significantly down-regulated in the mutant contexts, validating our experimental approach. These and other candidate genes are currently being validated by quantitative RT-PCR and characterized by complementary tissue-specific expression and functional approaches, as will be presented.

How does the addition of isoflavones into the culture medium during bradyrhizobia growth impacts soybean-bradyrhizobia symbiosis? B. Riviezzi (1), C. Cagide (1), A. Pereira (1), C. Herrmann (2), R. Lombide (2), I. Sicardi (2), M. Lage (2), S. Castro-Sowinski (1,3), M. MOREL REVETRIA (1), (1) IIBCE, Montevideo, Uruguay; (2) LAGE y Cia SA, Uruguay; (3) Facultad de Ciencias, Udelar, Uruguay

New advances in the use of inoculants for legumes are based on the incubation of rhizobia with secondary metabolites. This strategy could raise yield over the traditional inoculation. Flavonoids produced by plants roots are secondary metabolites that mediate nodule organogenesis in rhizobia-leguminous symbiosis. The specific rhizobia recognition determines their synthesis, and they are the main inducer of Nod factors production by rhizobia. This work described the results related to the effects of the pre-incubation of bradyrhizobia with isoflavones on soybean (Glycine $\max$ (L.) Merr.) - bradyrhizobia symbiosis. B. elkanii strains were grown in culture medium treated or not with isoflavones, and the inoculum was applied to seeds. The project comprised in vitro, greenhouse, and field research trials. The results included the evaluation of agronomic parameters of production and the characterisation of plants response in nodulation and plant growth as well as the evaluation of bacterial survival on both seeds and liquid formulation. Improved plant response was observed in nodulation when the bacteria were grown in the presence of isoflavones in all tested plant growth conditions, and increased grain yield was observed on field experiments. We conclude that the combination of active bradyrhizobia with isoflavones provides synergistic or complementary benefits with significant advantages to soybean growers in comparison with its counterpart without isoflavones

Impact of Arabidopsis micronutrient status and root-associated bacterial communities on accommodation of host-beneficial fungal endophytes

A. S. PIRO (1), C. Uhlmann (1), K. Raj (1), A. Zuccaro (2), J. E. Parker (1), (1) Max Planck Institute for Plant Breeding Research, Cologne, Germany; (2) University of Cologne, Germany

Plants form mutualistic interactions with soil microbes to enhance their tolerance to biotic and abiotic stress. The most important of these relationships are formed with endophytic fungi which invade host tissues, requiring plants to balance mutualist-accommodation with defense to avoid exploitation by pathogens. Plants accommodate endophytes when the relationships prove beneficial, such as during nutrient scarcity. However, signaling events allowing nutrient deprivation to affect accommodation remain unknown. Furthermore, how microbiome inputs (PAMPs, biochemical capacity, virulence factors) impact accommodation is unclear. We are examining how micronutrient status interfaces with accommodation/defense decisions toward two closely related endophytic fungal mutualists, Serendipita vermifera and S. indica in Arabidopsis. We are also examining how accommodation decisions are modified by the presence of Synthetic Microcosms (SynComs) composed of rootassociated bacteria with defined PAMP/biochemical capacities to decipher the role of the microbiome in shaping accommodation.

A novel DNA-binding transcriptional regulator participates in the activation of RAM1 during arbuscule development M. PARIES (1,2), P. Pimprikar (1,2), K. V. Hobecker (1), A. Heutinck (2), E. Evertz (2), C. Gutjahr (1), (1) Technical University of Munich (TUM) - Plant Genetics, Freising, Germany; (2) Ludwig-Maximilians-University Munich (LMU), Planegg, Germany

During arbuscular mycorrhiza (AM) symbiosis the fungus forms highly branched hyphal structures, the arbuscules, inside root cortex cells. The colonized plant cells undergo extensive transcriptional reprogramming. This enables expression of factors needed for subcellular rearrangement to accommodate the fungus and for nutrient exchange. A key transcriptional regulator enabling arbuscule branching is the GRAS protein REDUCED ARBUSCULAR MYCORRHIZA1 (RAM1). We previously showed, that a complex of the calcium-calmodulin dependent protein kinase CCaMK, the central symbiosis transcription factor CYCLOPS and the repressor of gibberellin signaling DELLA, regulates the activity of the RAMI promoter during AM development. Intriguingly, RAMI can also be induced in mutants of CCaMK and CYCLOPS by ectopic expression of a stabilized DELLA protein, although DELLA on its own is thought not to bind to DNA. Thus, DELLA must interact with another DNA binding transcription factor (complex), which seems to become sufficient when DELLA is stabilized and highly abundant. We have identified a new DNA-binding transcriptional regulator, which specifically transactivates the RAM1 promoter in Nicotiana benthamiana leaves when fused to the VP16 activator domain. In addition, we mapped the $R A M 1$ promoter region, required for this transactivation. This new transcriptional regulator thus represents a promising candidate for the elusive factor that participates in activation of the RAM1 promoter.

noeM, a novel nodulation gene involved in symbiosis with Mimosa pudica B. DAUBECH (1), V. Poinsot (2), A. Klonowska (3), C. Chaintreuil (4), P. Tisseyre (3), L. Moulin (5), M. Marchetti (1), C. Masson-Boivin (1), (1) INRA-LIPM, Castanet-Tolosan, France; (2) IMRCP, Toulouse, France; (3) IRD, Montpellier Cedex 5, France; (4) IRD, Senegal; (5) IRD, Cirad, Univ Montpellier, IPME, Montpellier, France, Montpellier, France

The beta-rhizobium Cupriavidus taiwanensis is a nitrogen-fixing symbiont of Mimosa pudica. Its symbiotic plasmid (pRalta) carries 10 nod genes, the nodBCIJHASUQ genes preceded by a nod-box and one regulatory gene nodD divergently transcribed. A second nod-box was predicted upstream of a transmembrane protein bearing a fatty acid hydroxylase domain, pRalta_0471. We identified pRalta_0471 as a novel nod gene 
involved in Nod factor biosynthesis and renamed it noeM. noeM is expressed during symbiosis with $M$. pudica and requires NodD and luteolin for optimal expression. noeM is involved in the synthesis of an atypical Nod factor, where the reducing sugar is open and oxidized. Closest noeM homologs formed a separate phylogenetic clade containing rhizobial genes only, which are located on symbiosis plasmids downstream a nod-box. noe $M$ was mostly found in Mimoseae isolates, and specifically detected in all tested strains able to nodulate $M$. pudica. noeM deletion mutants of C. taiwanensis, B. phymatum STM815, and R. mesoamericanum STM3625 were affected for the nodulation of M. pudica, confirming the role of noe $M$ in the symbiosis with this legume.

Small regulatory RNAs and epigenetic control of Arbuscular Mycorrhizal Symbiosis A. DALlAIRE (1), N. Ramakrishna (1), B. Manley (2), U. Paszkowski (3), E. Miska (1), (1) The Gurdon Institute, University of Cambridge, Cambridge, U.K.; (2) University of Cambridge, Cambridge, U.K.; (3) University of Cambridge, U.K.

Research has revealed fascinating insights into mechanisms underlying the establishment of arbuscular mycorrhizal (AM) symbioses and the molecular dialogue between AM fungi and plants. However, until now, most studies focused on the plant perspective of symbiosis control, and major insights into the mechanisms of this regulation by the fungus are lacking. The recent sequencing of $R$. irregularis' genome revealed that it has dedicated a large number of genes to small non-coding RNA (sRNA) pathways. For example, it has 26 Argonaute (AGO) genes, while most other fungi have up to two. AGO proteins bind sRNAs and use them as guides to direct gene silencing in a sequence-specific manner at both transcriptional and post-transcriptional levels. This process called RNA interference (RNAi) is essential for genome defense and gene regulation. In AMF, the existence of epigenetic mechanisms involving sRNAs has yet to be demonstrated. This project aims to unveil the sRNA repertoire of $R$. irregularis and to explore its functional relevance in the establishment of AM symbiosis, with a focus on sRNA-associated functional partners, in vivo targets of fungal sRNAs and their molecular function in the regulation of symbiosis-related gene expression. We hypothesize that sRNAs play key roles in the cell biology of AMF and may be involved in the control of this most ancient form of mutualism with plants. To test this, we combine high-throughput sequencing, bioinformatic and biochemical approaches.

Relationships Between Arbuscular Mycorrhizal Fungi Association, Low Phosphate Tolerance and Root Architecture in Strawberry. H. M. COCKERTON (1), E. Stavridou (2), B. Li (2), A. Johnson (2), A. Karlstrom (1), A. D. Armitage (1), A. Martinez-Crucis (2), L. GalianoArjona (2), R. J. Harrison (1), (1) NIAB EMR, East Malling, U.K.; (2) NIAB EMR, U.K.

Root architecture plays an important role in phosphate $(\mathrm{P})$ acquisition where finer roots have an increased capacity to absorb nutrients. These roots are able to uptake $\mathrm{P}$ from the immediate root area, thus creating a nutrient depletion zone. Symbiotic association with Arbuscular Mycorrhizal Fungi (AMF) allows plants to acquire P from beyond the root nutrient depletion zone. We investigate the contrasting root architecture of 'Redgauntlet' and 'Hapil' strawberry cultivars through a mapping population and determine the relationship between root architecture and AMF association. The root architecture for each genotype was visualized using a rhizotron system and quantified using automated image analysis. The variation in root architecture of the population can be described by differences in the area the network explores. No phenotypic correlation or genotypic association was found between low P tolerance and root architecture. Experiments quantifying AMF colonisation within each genotype suggested a weak genotypic association with root diameter and AMF structures, however further work must determine whether this association is an artifact of the AMF analysis method. Roots are known to show phenotypic plasticity through increasing lateral root growth in response to low $\mathrm{P}$ environments. Therefore, future work should determine whether root plasticity under low $\mathrm{P}$ conditions is associated with P tolerance and the propensity to form AMF associations.

Thinking outside the plant: TAL effector-like proteins in a bacterial-fungal symbiosis M. E. CARTER, S. C. D. Carpenter, Z. Dubrow, T. E. Pawlowska, A. J. Bogdanove, Cornell University, Ithaca, NY, U.S.A.

To cause rice seedling blight, Rhizopus microsporus requires a toxin synthesized by symbiotic Burkholderia spp. that live within the fungus. The type III secretion system of Burkholderia rhizoxinica is critical for establishing symbiosis with R. microsporus, but its effector repertoire remains uncharacterized, with little known about endohyphal bacterial effectors. Previous whole-genome sequencing and biochemical analysis revealed that B. rhizoxinica encodes proteins similar to DNA-binding transcription activator-like effectors (TALEs), used by plant pathogenic Xanthomonas spp. to directly alter host gene expression. However, Burkholderia TALE-Like proteins (BTLs) have only a weakly predicted T3S signal, a cryptic nuclear localization signal, and no canonical eukaryotic activation domain. We demonstrate nonetheless that BTLs transit the type III secretion system of Pseudomonas syringae and have a nuclear localization signal functional in yeast. Further, we show that endohyphal Burkholderia from diverse $R$. microsporus isolates contain variable BTL repertoires each with at least one BTL gene. We hypothesize that, while Xanthomonas TAL effectors act on a plant genome during pathogenesis, BTLs act on the fungal genome to maintain the mutualism. Ongoing studies aim to determine if the type III regulator hrpB controls btl expression and whether BTLs alter the R. microsporus transcriptome.

Rice plants expressing the Rhizophagus irregularis RiPEIP1 gene show enhanced growth and incresed level of mycorrhizal colonization C. VOTTA (1), V. Fiorilli (1), J. Gómez-Ariza (2), F. Fornara (3), L. Lanfranco (1), (1) University of Torino, Torino, Italy; (2) crag, Spain; (3) University of Milan, Italy

The Rhizophagus irregularis RiPEIP1 (Preferentially Expressed In Planta) is a fungal gene strongly expressed in the intraradical phase, including arbuscules, in different host plants. When expressed as a GFP fusion in yeast cells, RiPEIP1 localizes in the endomembrane system, in particular to the endoplasmic reticulum, which is consistent with the in silico prediction of four transmembrane domains. In the absence of genetic transformation protocols for arbuscular mycorrhizal (AM) fungi, we exploited two different heterologous expression systems. Previous results showed that RiPEIPI expression in Oidiodendron maius, an ericoid endomycorrhizal fungus, led to enhanced colonization capacity compared to the O. maius wild type strain. To gain further insights on the biological role of RiPEIPl we also generated rice RiPEIP1-expressing lines under the constitutive $35 \mathrm{~S}$ promoter. A preliminary analysis showed that these transgenic lines have a higher number of crown roots, an increased shoot length, a higher root and shoot biomass, an early flowering phenotype, an increased number of panicles and seeds and a higher 
AM colonization level compared to wild type plants. Although the mechanism of action of RiPEIPl is still unknown, these findings suggest that RiPEIPI expression affects different host metabolic pathways which promote plant growth and mycorrhizal colonization.

Enhancement of ginsenoside production in ginseng by the treatment of formulated endophyte isolated from mountain-cultivated ginseng (Panax ginseng Meyer)

S. Salsabila, S. O. Rim, H. BAE, Yeungnam University, Gyeongsan, Korea, Republic of (South)

Plants harbor diverse communities of fungi and bacteria in their internal compartments as endophytes. We isolated endophytes from three different tissues (root, stem and leaf) of mountain-cultivated ginseng (MCG, Panax ginseng Meyer) grown in 24 different geographic locations in Korea to exploit their potentially beneficial properties toward plants. Ginseng is an invaluable medicinal plant containing various bioactive metabolites (e.g. ginsenosides). We evaluated the effect of endophytes (129 fungal and 252 bacterial isolates) on ginsenoside production in ginseng. The level of total ginsenosides was enhanced by approximately twofold under the formulated endophyte treatment in the controlled environment and mountain area. Our results revealed that endophyte has great potential as an elicitor of ginsenoside production.

Characterization of a cis-regulatory region in NIN promoter required for infection thread formation in Lotus japonicus A. AKAMATSU (1), M. Nagae (2), Y. Nishimura (1), N. Takeda (1), (1) Kwansei gakuin university, Japan; (2) NIAS, Japan

The establishment of the legume-rhizobial association is initiated by a molecular communication between the plant root and the rhizobial bacteria in the soil. Plants release flavonoids that are recognized by the rhizobial bacteria, which in turn produce Nod factors (NFs). Recognition of NFs by the plasma membrane-localized receptors turns on the switch for nodule symbiosis signaling in the host plants, resulting in expression of several transcription factors involving in a nodule-specific transcription factor NODULE INCEPTION $(N I N)$. NIN has essential roles for the formation of infection thread (IT), which is a tube-like structure in the root cells to promote colonization of rhizobia. Although much work has been done on the NIN, the precise regulation of NIN expression has not been clarified. Here, we attempted to characterize a cis-regulatory region in NIN promoter required for the IT formation in Lotus japonicus. The nin-9 mutant was transformed with NIN driven by multiple lengths of NIN promoter region, and we found that the region required for induction of IT formation locates around $1 \mathrm{~kb}$ upstream of NIN. To confirm this finding, we generated a deletion mutant of the promoter region by CRISPR/Cas9 system. The number of IT significantly decreased in the mutant. In addition, a promotor GUS analysis indicated that the region has an essential role to induce $N I N$ at the root hair cells infected by rhizobia. We will conduct further studies on the mechanisms of NIN expression.

Deciphering the cellular and molecular bases of rhizobial pre-infection reprograming in Medicago truncatula A. Kelner, L. Frances, M. Beck, J. Fournier, F. DE CARVALHO-NIEBEL, Laboratory of Plant Microbe Interactions (LIPM), INRA, CNRS, Univ Toulouse, Castanet-Tolosan, France

The symbiotic association of legumes with nitrogen-fixing rhizobia forms a specialized organ, the root nodule, where the bacteria have the optimal environment for fixing nitrogen for the plant. Nodule formation depends on interconnected paths and cell communication for coordinating nodule organogenesis and the construction of infection thread (IT) tubular structures, which guide the entry of rhizobia. ITs grow through a route pre-defined by the plant, where plant cells remodel and polarize their cytoplasm for creating a pre-infection (or PIT) bridge for the future passage of the IT. Despite its importance, PIT reprogramming is still poorly understood partially due to the difficulty of accessing the rare sites where it occurs. Here we developed new live-tissue imaging tools for visualizing PIT formation with cellular resolution in rhizobiainoculated $M$. truncatula roots. We determined the spatio-temporal dynamics of PIT cytoplasmic remodelling and polarization in vivo, which is associated with the dynamic subcellular re-localization of a calcium binding protein, whose importance for nodulation is currently being investigated by functional approaches. Applying this in vivo approach to infection-defective mutants enabled the identification of plant genetic components regulating the distinct phases of remodelling and polarization as will be presented.

A mutation in the E3-ubiquitin ligase PUB1 alters Nod factor structure recognition by Medicago truncatula M. D. MBENGUE (1), C. Gibelin (1), S. Exbrayat (1), V. Schilling (1), F. Robson (2), C. Herve (1), F. Debellé (1), (1) Laboratoire des Interactions Plantes Microorganismes, INRA-CNRS, Castanet-Tolosan, France; (2) Department of Cell and Developmental Biology, John Innes Centre, Norwich, U.K.

Rhizobial Nod factors (NFs), lipochitooligosaccharidic signals carrying specific substitutions such as sulfate in Sinorhizobium meliloti NFs, are crucial determinants of legume nodulation and host range but the molecular mechanisms controlling the specific recognition of NF substitutions are poorly understood. We thus looked for EMS mutants of the model legume Medicago truncatula able to nodulate in the presence of a nodH mutant of S. meliloti which produces non-sulfated NFs and is unable to nodulate wild-type M. truncatula. Genetic analysis of one such mutant identified a candidate causal mutation in $P U B 1$, a gene encoding an E3-ubiquitin ligase previously characterized as an interactor of receptor-like kinases (RLKs) involved in NF signaling. We validated the candidate mutation by transferring the mutated form into wild-type or PUB1 mutants of M. truncatula. Since the mutation in PUB1 is in the C-terminal part containing Armadillo repeats known to play a role in protein-protein interactions, we tested whether this mutation could affect interactions of PUB1 with its known partners. In the yeast two-hybrid system the mutation did not affect the interaction with DMI2 and LYK3, two RLKs with symbiotic roles, but abolished the interaction with NFP, a LysMRLK required for NF perception. We are currently using co-immunopurification and FLIM-FRET approaches to validate in planta the effect of the mutation on the interactions of PUB1 with its partners.

Optimising growth promotion and induced resistance in cucumber exposed to gaseous bacterial volatiles in a miniature greenhouse system

G. C. Song (1), M. RIU (1,2), C. M. Ryu (1,3), (1) KRIBB, Daejeon, Korea, Republic of (South); (2) Chungnam National University, Daejeon, Korea, Republic of (South); (3) University of Science and Technology, Daejeon, Korea, Republic of (South) 
Bacterial volatiles promote plant growth and elicit immunity responses in plants grown in two-compartment Petri dishes. Due to the limitations of bacterial volatile compound (BVC) treatments such as their high evaporation rates, it is convenient to apply BVCs in closed systems such as greenhouses. We therefore attempted to optimise BVC emissions from bacteria grown on solid medium and synthetic BVC treatment in order to maximise plant growth and induced resistance in a miniature greenhouse system. We cultivated the model BVC emitter Bacillus subtilis GB03 on complex medium for continuous treatment, which we placed near 1-week-old cucumber seedlings in a miniature greenhouse. Aboveground and belowground plant growth parameters were significantly increased at 1 and 2 weeks after treatment with BVCs released by B. subtilis GB03. Moreover, this treatment protected cucumber seedlings against the angular leaf spot pathogen Pseudomonas syringae pv. lachrymans. The mechanism of induced resistance appears to involve the activation of the jasmonic acid signaling pathway. To overcome the difficulties associated with treatment using a single application of BVC in the greenhouse, we optimised conditions for BVC application via consistent exposure in a slow-release system.

Evolution of a recently emerged heritable plant symbiont

B. DANNEELS, University of Ghent, Ghent, Belgium

The leaf nodule symbiosis between Orrella dioscoreae and its host Dioscorea sansibarensis is a very intimate one. The symbiont resides in special glands in the leaf tip and is also found to reside in the apical meristem and has never been sampled free-living in the environment. Interestingly, the symbiont can be cultured outside of the host, making this symbiosis an interesting potential model system. We investigated the presence, diversity, and evolution of the symbiont by sampling wild specimens of Dioscorea sansibarensis from Madagascar. We showed the presence of the symbiont in all wild-collected leaf nodules and proved that the symbiont is passed on to the plant's offspring by colonizing the aerial bulbils responsible for the vegetative reproduction of the host. Phylogeny of host and symbiont were mostly congruent, and showed separation based on geographical location of the samples. This indicates vertical transmission is likely the main mode of transmission. Nevertheless, the symbiont genome did not show signs of genome reduction, which could be attributed to a recent emergence of the symbiosis ( 0.02 to $3.1 \mathrm{Mya}$ ), or a high effective population size. We did however find signs of positive selection in genes likely involved in host-symbiont interaction and secondary metabolism. This could be indicative of ongoing diversification or adaptation of the symbiont to different (a)biotic factors affecting its host, very unusual for vertically transmitted symbionts.

Influence of the mutual symbiotic fungi Epichloë spp. on the grass host phyllosphere microbial community Y. BECKER, A. Popovska, Julius Kühn-Institut, Institute for Epidemiology and Pathogen Diagnostics, Braunschweig, Germany

Pathogens, commensals and mutualists occupy the rhizosphere and phyllosphere of plants. Several seed-transmitted Epichloë spp. are mutualistic symbionts of grasses (Pooideae), that protect the host plant from microbial pathogens by producing antibiotic and fungistatic alkaloids and toxins and by stimulating the host SAR. The fungus establishes a restricted hyphal network in the phyllosphere apoplast and on the surface of leaves. Endophytic hyphae exit the plant by means of an inverted appressorium, the expressorium. The purpose of the epiphyllous hyphae is still speculative. Next to excluding niches from pathogenic microbes, niches may be established for phylloplane microbes of the indigenous mirobiota. Epiphyllous hyphae produce secondary metabolites and antifungal proteins, provide bacteria with nutrients, and protect them from desiccation and UV radiation, thus offering a setting for coevolved bacteria. In addition, epiphyllous hyphae and expressoria might offer a pathway through cuticle and intercellular spaces between plant tissue and penetrating hypha for a coevolved bacterial pioneer endophyte community analogous to rhizosphere fungal highways. Here, we present our results on the influence of Epichloë on the phyllosphere microbial community using cultivation dependent approaches of Epichloë-colonized and Epichloë-free plants. The interaction of Epichloë with the isolated microbes from the native community is analysed in in vitro and in in planta co-culture experiments.

\section{Stereoisomers of the Bacterial Volatile Compound 2,3-Butanediol Differently Elicit Systemic Defense Responses of Pepper against} Multiple Viruses in the Field

C. M. RYU, University of Science and Technology, Daejeon, Korea, Republic of (South)

KRIBB, Daejeon, Korea, Republic of (South)

The volatile compound 2,3-butanediol, which is produced by certain strains of root associated bacteria, consists of three stereoisomers, namely, two enantiomers (2R,3R and 2S,3S-butanediol) and one meso compound (2R,3S-butanediol). The ability of 2,3-butanediol to induce plant resistance against pathogenic fungi and bacteria has been investigated; however, little is known about its effects on induced resistance against viruses in plants. To investigate the effects of 2,3-butanediol on plant systemic defense against viruses, we evaluated the disease control capacity of each of its three stereoisomers in pepper. Specifically, we investigated the optimal concentration of 2,3- butanediol to use for disease control against Cucumber mosaic virus and Tobacco mosaic virus in the greenhouse and examined the effects of drench application of these compounds in the field. In the field trial, treatment with $2 \mathrm{R}, 3 \mathrm{R}$-butanediol and $2 \mathrm{R}, 3 \mathrm{~S}$ butanediol significantly reduced the incidence of naturally occurring viruses compared with $2 \mathrm{~S}, 3 \mathrm{~S}$-butanediol and control treatments. In addition, 2R,3R-butanediol treatment induced the expression of plant defense marker genes in the salicylic acid, jasmonic acid, and ethylene signaling pathways to levels similar to those of the benzothiadiazole treated positive control. This study reports the first field trial showing that specific stereoisomers of 2,3-butanediol trigger plant immunity against multiple viruses.

Periarbuscular membrane signaling in plant host promoting arbuscular mycorrhizal fungal fitness H. MONTERO (1), P. Eastmond (2), U. Paszkowski (3), (1) University of Cambridge, Cambridge, U.K.; (2) Rothamsted Research, U.K.; (3) University of Cambridge, U.K.

The prevalent arbuscular association relies on mutual recognition between plant and fungal partners across consecutive symbiotic developmental stages. As symbiosis progresses, nutrient exchange dynamically alters the physiological status of the symbionts. Arbuscules are the main landmark for the association and upon their formation several proteins have been described to function, mainly in nutrient transport. Arbuscular 
Receptor-Like Kinase 1 (ARK1) was recently shown to be a rice periarbuscular membrane specific signaling component of the association. Mutation of this protein renders the symbiotic fungus unable to sustain effective spread, leading to an overall attenuation of the association although arbuscules developed normally. We have demonstrated that this defect can be recovered if mutant plants are grown alongside wild type plants suggesting that signals or nutrients are not properly delivered to the symbiotic fungus. We here document our efforts to estimate the contribution of lipid nourishment in the ARK1-mediated fungal fitness support role. To this end, lipid imaging has been implemented revealing alterations in lipid dynamics in the arkl mutant. This observation is reproduced in Brachypodium arkl mutant and in a rice mutant impaired in a close homolog of ARK1. Analytical efforts to complement these microscopy-based inspections and the contribution of other candidate arbuscular specific signaling components in supporting fungal fitness will also be discussed.

Detection and analysis of bidirectional cross-kingdom communication in a plant-endosymbiont system E. SECIC (1), K. H. Kogel (2), (1) Institute of Phytopathology, Justus-Liebig-Universität, Gießen, Germany; (2) Institute of Phytopathology, Justus-Liebig-Universität Gießen, Germany

Small RNAs (sRNAs) are potentially novel communication mediators between plants and microbes, thereby employing the RNA interference (RNAi) pathways. Such bidirectional cross-kingdom communication has been discovered in a few host-pathogens. Microbe-derived sRNAs are proposed to modulate the plant immune response, while plant-derived sRNAs regulate expression of microbial virulence and other essential genes. Of note, RNA-based communication in symbiotic interactions has not yet been shown, and thus it is an open question whether beneficial fungi use sRNA to successfully colonize their host plants. To answer this question and, if applicable, elucidate the underlying mechanisms of RNAi-mediated establishment and maintenance of mutualism, we investigated the interaction between the model grass Brachypodium distachyon and the mutualistic root endosymbiont Piriformospora indica, with a special focus on RNAi-related activities and identification of cross-kingdom (ck)RNAs. Based on deep sequencing of sRNA and mRNA data sets and application of an established bioinformatics pipeline, we gained first evidence for RNAi-mediated action of ckRNAs in the Brachypodium-Piriformospora symbiosis.

Identifying the genetic determinants of barley colonisation by the growth-promoting biocontrol agent Pseudomonas fluorescens A. PACHECO-MORENO (1,2), R. Goddard (1), S. de Vos (1), P. Nicholson (1), C. J. Ridout (1), J. G. Malone (1,2), (1) John Innes Centre (JIC), Norwich Research Park, Norwich, U.K.; (2) University of East Anglia, Norwich, U.K.

Plants can shape its rhizosphere microbiome through the secretion of root exudates whose composition depends on the host genotype, plant development and environmental characteristics. The ability of different microbes to degrade these exudates determines their success in the rhizosphere and results in a shift in the rhizobiome composition driven by the plant. At this stage, the genetic determinants of the plant-microbe recognition process in the rhizosphere are largely unknown. A better understanding of this interaction will provide the basis for selecting more efficient biocontrol strains, and breeding plants with an improved ability to recruit beneficial microbes. In my $\mathrm{PhD}, \mathrm{I}$ am examining the relationship between barley and the widespread PGPR P. fluorescens. I used carbon-metabolic regulatory biomarkers based on the strain SBW25 to examine how carbon utilisation relates to rhizosphere colonisation competence in different barley cultivars. The elite variety Tipple showed markedly compromised colonisation by our SBW25 simple-C source biomarker, suggesting a shift towards complex-C source exudation relative to the heritage variety Chevallier. This phenomenon was analysed by GC-MS analysis and corresponds to a shift in the Tipple Pseudomonas rhizosphere population towards complex-C utilisation. Specific metabolite biomarkers were then used to isolate individual exudate differences in planta, and ultimately, to screen a CxT mapping population for Pseudomonas colonisation loci.

Recognition of chitooctamers and lipochitooligosaccharides activate symbiosis signaling in plants J. SUN, F. Feng, G. Oldroyd, Sainsbury Laboratory Cambridge University, Cambridge, U.K.

AM fungi signal to the plant via diffusible lipochitooligosaccharides (LCOs) or chitooligosaccharides (COs) signaling molecules that can activate the common symbiosis signaling pathway with induction of calcium oscillations. Recently, we have studied how COs and LCOs produced by AM fungi, Sinorhizobium meliloti and Mezorhizobium loti can activate calcium spiking, a marker of symbiosis signaling, in barley, wheat and maize. We have found that LCOs can be perceived by cereals, but that this recogntion lacks the stringency of perception present in legumes. Whereas legumes show LCO perception early in development, cereals only percieve LCOs when they are showing signs of nutrient starvation. Both nitrogen and phosphate status contribute to the regulation of LCO perception in cereals.

Transcriptomic comparison of Medicago truncatula during symbiosis

T. LEE, K. Schiessl, G. Oldroyd, Sainsbury Laboratory Cambridge University, Cambridge, U.K.

Plants have evolved to form alliances with microorganisms in the rhizosphere to acquire nutrients that limit their growth. The two most well studied symbionts are arbuscular mycorrhizal fungi and rhizobial bacteria. The arbuscular mycorrhizal symbiosis appeared in the very early stages of plant evolution-about 450 mya-while evidence at the rhizobial symbiosis evolving from arbuscular mycorrhizal associations between 60-80 mya. The two share many biological aspects in common: capturing nutrients and trading them for carbon with the plant, secreting specific molecules to be recognized, and entering the plant root to directly associate with the plant cells. However, there are also key differences: the formation of nodules that form to harbor rhizobia, which are absent in arbuscular mycorrhizal associations. To further understand the differences and similarities, we have collected the transcriptomes during the processes of these two symbioses in Medicago truncatula. We have identified the expression levels of genes with RNA-seq for 3 time points after Rhizophagus irregularis infection and 15 time points after Sinorhizobium meliloti infection. By applying unsupervised fuzzy c-means clustering to the transcriptomes, we were able to unbiasedly classify the gene profiles during symbiosis. Looking more closely into these clusters, we can distinguish both commonalities and differences between these two associations. 
Use of blumenols as reliable shoot markers of arbuscular mycorrhizal colonisation in rice.

E. K. SERVANTE (1), J. Choi (1), R. Halitschke (2), M. Wang (2), E. Mindt (2), I. T. Baldwin (2), U. Paszkowski (3), (1) University of Cambridge, Cambridge, U.K.; (2) Max Planck Institute for Chemical Ecology, Jena, Germany; (3) University of Cambridge, U.K.

Arbuscular mycorrhizal fungi (AMF), Glomeromycotina, form intimate associations with $>80 \%$ land plants present today; colonising within their roots to form a mutually beneficial symbiosis. Recently, it was found that levels of blumenol-type metabolites, apocarotenoids, in leaves and roots correlated with AMF colonisation rate in multiple fungal, crop and model species including N.attenuata, S.lycopersicum and T.aestivum ${ }^{1}$. This project aimed to assess the use of the blumenol method of detection of AM colonisation in rice. A time course experiment using different phosphate conditions and mutants of AM symbiosis was used to give distinct AM colonisation phenotypes. By using calcium- and calmodulindependent protein kinase (ccamk) mutants the AMF-specific response could be determined. In addition, the use of different phosphate conditions allowed for variable developmental phenotypes that were then used to assess the extent of blumenol accumulation. Levels of AM colonisation detected by 'classical' methods of microscopy and AM marker gene expression were compared to the extent of blumenols measured in leaf tissue. This project was conducted as a pilot study with the intention of exploiting this high throughput method of detection for future GWAS investigating the genetic potential for AMF benefit in rice.

Genotype-Species Dependence of Tomato-Trichoderma Interaction Effects on Plant Sprouting and Growth I. VUKELIC (1), D. Radic (1), L. Prokic (2), L. Jovanovic (1), M. Bojovic (1), G. Racic (1), D. Pankovic (1), (1) Educons University, Sremska Kamenica, Serbia; (2) University of Belgrade, Belgrade, Serbia

The effect of five Trichoderma species on sprouting and plant growth of two tomato cultivars (Narvik and Gružanski zlatni), was examined in pots with sterile soil $(\mathrm{pH} 8)$ and semi-controlled conditions. Tomato seeds were treated with spore suspension $\left(10^{6} \mathrm{CFU} / \mathrm{ml}\right)$ of $T$. harzianum, T. brevicompactum, T. virens, T. longibrachiatum or T. citrinoviride, at sowing. The effects on plant sprouting of cultivar Narvik ranged from $13 \%$ inhibition with $T$. longibrachiatum, to $50 \%$ stimulation with $T$. citrinoviride, and for the cultivar Gružanski zlatni it ranged from $0 \%$ with $T$. brevicopmactum to $63 \%$ with T. longibrachiatum. Plant fresh weight was inhibited from $20 \%$ and $32 \%$ with T. citrinoviride, to $47 \%$ and $73 \%$ with $T$. virens of cultivars Narvik and Gružanski zlatni, respectively. The percentage of fresh weight inhibition of aerial plant parts and roots were similar in cultivar Narvik. However, the inhibition of aerial plant parts fresh weight was higher (50\%) in comparison to root in Gružanski zlatni. Dry matter production per plant was more inhibited by Trichoderma application in general, in cultivar Gružanski zlatni in comparison to Narvik. Non-destructive parameters of chlorophyll, anthocyanin and flavonol contents did not change significantly in examined conditions. In conclusion, Trichoderma application stimulated tomato seed germination in vitro, and sprouting in sterile soil, however, mainly led to plant growth inhibition depending on tomato-Trichoderma genotype-species combination.

\section{Comparative analysis of Medicago truncatula mutants deficient in NCR peptides essential for symbiotic nitrogen fixation P. KALO, Biological Research Center, Szeged, Hungary}

Medicago truncatula is able to establish one of the most effective nitrogen-fixing symbiotic interaction with the symbiotic partner Sinorhizobium spp. In the infected cells of the M. truncatula nodules, the rhizobia undergo morphological and metabolic transformation. This differentiation is irreversible in species belonging to the inverted repeat-lacking clade (IRLC) of legumes and this process is driven by the family of the nodulespecific cysteine rich (NCR) peptides produced by the plant. In the M. truncatula genome, approximately 700 genes encode NCR peptides. The high number of genes can imply that the function of NCR peptides may overlap or partially overlap and the NCRs act redundantly. Recent studies revealed that NCR169 and NCR211 peptides are essential for terminal bacteroid differentiation and hence for effective symbiotic nitrogen fixation (SNF). Recent genetic analyses identified two novel NCRs that are also required for SNF between M. truncatula and Sinorhizobium sp. In order to further dissect the bacteroid differentiation regulated by essential NCRs, we analyzed the bacterial morphology and the degree of the bacteroid elongation in the four M. truncatula mutant lines defective in production of NCR genes. To study the structural alterations in bacterial morphology and define what stage of the bacterial differentiation is blocked in the NCR deficient lines, mutant nodules were analyzed by laser scanning confocal and scanning electron microscopy.

Transcriptional profiling of arbuscular mycorrhiza symbiosis in the liverwort Marchantia paleacea.

M. SGROI (1), G. V. Radhakrishnan (2), G. Oldroyd (3), U. Paszkowski (4), (1) University of Cambridge, Cambridge, U.K.; (2) John Innes Centre, Norwich, U.K.; (3) Sainsbury Laboratory Cambridge University, Cambridge, U.K.; (4) University of Cambridge, U.K.

Arbuscular mycorrhizal symbiosis (AMS) arose in land plants more than 400 million years ago, perhaps acting as a major contributor to plant terrestrialization. Whilst the components of AMS are well characterized in higher plants, little is known about the molecular mechanisms that underpin AMS in extant bryophyte clades, such as hornworts and liverworts. Marchantia paleacea, a member of the most ancient extant land plants clade, engages in AMS with fungi of the Glomeromycotina phylum. The genome of M. paleacea contains multiple homologues of symbiosis genes associated in higher plants with the establishment of AMS. In order to characterise this bryophyte-AMS system on a molecular level, we performed an RNAseq time-course to observe transcriptional changes in M. paleacea thalli in response to (1) germinated $R$. irregularis spore exudates, (2) intracellular colonization, (3) arbuscule formation and (4) late symbiotic stages of AMS. As part of this analysis, we focused on the expression pattern of known symbiosis gene orthologues in M. paleacea and on a comparative transcriptomic approach with similar RNAseq datasets obtained from higher plants. This analysis provides an evolutionary perspective of the arbuscular mycorrhiza symbiotic interaction, identifying components of the signaling machinery with a conserved pattern of expression across land plants, which were possibly involved in ancestral AMS associations.

Spatiotemporal dynamics of nutrient exchange at the arbuscule

J. MCGALEY (1), R. Roth (1), R. Morris (2), T. Ott (3), U. Paszkowski (1), (1) University of Cambridge, U.K.; (2) John Innes Centre, U.K.; (3) University of Freiburg, Germany 
Bidirectional nutrient exchange lies central to arbuscular mycorrhizal symbiosis, the intimate, mutually beneficial relationship between Glomeromycotina fungi and most land plant species. It involves plant-to-fungus transport of organic carbon and fungus-to-plant transport of mineral nutrients (e.g. phosphate and ammonium) at the highly specialised interface of the arbuscule. Despite its importance for the nutrition of both partners and suspected role in arbuscule branching and maintenance, little is known about the actual dynamics of symbiotic nutrient exchange at the arbuscule. Two approaches are taken to address this in an Oryza sativa- Rhizophagus irregularis system. To dissect the apparent link between nutrient transport dynamics and arbuscule development, a computational modelling approach is applied. To then resolve the underpinning spatiotemporal dynamics, multiple microscopic and time-lapse techniques are used to image fluorescently labelled transporter proteins that are involved in the symbiotic nutrient transport. This enables the detection of any correlation or disparity in dynamics between the reciprocal directions of nutrient exchange during arbuscular mycorrhizal symbiosis.

NODULE INCEPTION recruits the lateral root developmental program for symbiotic nodule organogenesis

K. SCHIESSL (1), J. Lilley (2), T. Lee (3), G. Oldroyd (3), (1) SLCU, Cambridge, U.K.; (2) previous address: John Innes Centre, Norwich, U.K.; (3) Sainsbury Laboratory Cambridge University, Cambridge, U.K.

Nodules are root organs that occur in legumes as a result of cytokinin signaling induced by perception of nitrogen-fixing bacteria. In contrast, lateral root development involves preinitiation of founder cells, whose further development is promoted by auxin, but blocked by cytokinin. Here we demonstrate that despite these different modes of induction, lateral roots and nodules converge on a common developmental program, involving the formation and interpretation of a local auxin maximum. This common program can be activated in the roots of Medicago trunctula by cytokinin treatment, in a manner dependent on the transcriptional regulator NIN. Our work implies that evolution of nodulation involved recruitment of a common root developmental program, whose induction by cytokinin, a unique feature of legumes, is explained by innovations in NIN.

Unraveling root-type specific phosphate uptake in rice plants under arbuscular mycorrhizal conditions D. ALBINSKY (1), R. Roth (1), S. Abbas (1), K. Cragg-Barber (1), I. Jakobsen (2), P. Flis (3), D. Salt (3), U. Paszkowski (1), (1) University of Cambridge, U.K.; (2) University of Copenhagen, Denmark; (3) University of Nottingham, U.K.

Phosphate is a major nutrient for plants, but it is only limited available to the plant root system due to its high retention in soil. Hence, the symbiotic relationship with mycorrhizal fungi of the subphylum Glomeromycotina in which the majority of all land plants engage in is an important way of acquiring the essential phosphate from the soil through the widespread fungal mycelium. The relative contribution of the mycorrhizal $\mathrm{P}$ uptake (indirect $\mathrm{P}$ uptake) to the total $\mathrm{P}$ nutrition of mycorrhizal plants can vary depending on the availability of $\mathrm{P}$. $\mathrm{P}$ uptake is mediated by transporters belonging to the PHT1 family. The rice (Oryza sativa) root system consists of crown roots, large lateral roots and fine lateral roots showing different symbiotic properties. Our previous molecular studies revealed a functional diversity among the individual root types (RTs) that changed upon mycorrhizal colonisation. These observations prompted us to propose that each RT contributes distinctly to plant P uptake in the presence and absence of AM fungi. Here, we present molecular and physiological data elucidating the P uptake properties of the different rice RTs under different P regimes, in asymbiosis and during symbiosis with Rhizophagus irregularis.

Zaxinone, a natural apocarotenoid, is involved in the establishment of the arbuscular mycorrhizal symbiosis V. FIORILLI (1), I. Haider (2), C. Votta (1), J. Y. Wang (2), M. Jamil (2), J. Mi (2), B. A. Kountche (2), K. P. Jia (2), A. Balakrishna (2), L. Lanfranco (1), P. Bonfante (1), S. Al Babili (2), (1) University of Torino, Torino, Italy; (2) King Abdullah University of Science and Technology, Saudi Arabia

Carotenoids are precursors of several plant hormones and signaling molecules, which are involved in the establishment and the maintenance of arbuscular mycorrhizal (AM) symbiosis. Recently, we have identified zaxinone, a product of the rice carotenoid cleavage dioxygenase (OsZAS), as a novel natural metabolite and carotenoid-derived signaling molecule that is required for normal rice growth and development, affects arbuscular mycorrhizal symbiosis, and down-regulates strigolactone (SL) biosynthesis (Wang et al. 2019). A loss-of function mutant (oszas) showed decreased zaxinone content in roots, decreased root biomass, higher SL release and decreased mycorrhization level. To gain further insights into the role of zaxinone in mycorrhization, we generated $O s Z A S$ over-expressing lines under the control of $35 S$-promoter. These lines exhibited an increased mycorrhizal colonization level compared to WT. In order to understand whether zaxinone has a direct impact on the fungus during its pre-symbiotic phase, we tested the effect of this compound on spore germination of Gigaspora margarita, in comparison with the synthetic SL analog, GR24. To unravel the role of zaxinone during the colonization process, we determined the content of this compound and of SLs in oszas mutant and WT plants during a time course experiment. In their whole, the results suggest that, zaxinone, plays a crucial role in the development and establishing AM symbiosis in rice.

NSP1 and NSP2 regulate a large group of genes associated with strigolactone biosynthesis of a symbiotic state X. LI (1), L. Luginbeuhl (2,3), G. Oldroyd (4), (1) Sainsbury Laboratory, University of Cambridge, CAMBRIDGE, U.K.; (2) Department of Plant Sciences, University of Cambridge, CAMBRIDGE, U.K.; (3) Department of Cell and Developmental Biology, John Innes Centre, Norwich, U.K.; (4) Sainsbury Laboratory Cambridge University, Cambridge, U.K.

NSP1 and NSP2 are essential for root nodule symbioses and contribute to colonisation by arbuscular mycorrhizal fungi in Medicago truncatula. We have previously shown that NSP1 and NSP2 form a DNA-associated protein complex (Hirsch et al 2009). While the root nodule phenotyes of these mutants are very clear, the mycorrhizal phenotypes are more subtle, with $n s p 1$ and $n s p 1 / n s p 2$ mutants showing delayed colonisation by Rhizophagus irregularis, while in contrast, we were unable to see any impact on $R$. irregularis colonisation in nsp 2 mutants across a time course of $R$. irregularis infection. NSP1 and NSP2 have been shown to control the expression of genes involved in strigolactone biosynthesis. Using a RNAseq approach we demonstrate that NSP1 is involved in the regulation of many genes involved in strigolactone biosynthesis, while we find less impact on this pathway in $n s p 2$ mutants. The impact on the regulation of strigolactone biosynthesis in $n s p 1$ occurs in the absence of $R$. 
irregularis colonisation. Our work supports the previous observed effects of NSP1 on R. irregularis clonisation and regulation of strigolactone biosynthesis, but our work does not support a role for NSP2 in these processes.

Processing of NODULE INCEPTION is essential for legume-rhizobial symbiosis J. FENG (1), G. Oldroyd (2), (1) Sainsbury Laboratory, University of Cambridge, Cambridge, U.K.; (2) Sainsbury Laboratory Cambridge University, Cambridge, U.K.

The transcription factor NODULE INCEPTION (NIN) is a key regulator of the symbiosis between legumes and rhizobia, that is known to coordinate both bacterial infection and nodule organogenesis. Here, we show that NIN is processed during nodulation, with at least three processed forms of NIN detectable by immunoblot analysis using multiple anti-NIN antibodies. When NIN is expressed transiently in leaves of $N$. benthamiana, we primarily observe a product consistent with the full-length NIN protein and larger forms, that are likely agglomerations of NIN. In contrast, we never see the full-length NIN product in M. truncatula, under normal symbiotic conditions, instead we see accumulation of much shorter products. NIN is specifically induced by rhizobial infection, but we have found no evidence for alternate splicing of the NIN transcript, suggesting that the NIN protein itself is processed to create the shorter forms. Processed NIN is still detectable after treatment of the protein synthesis inhibitor cycloheximide, supporting the proposition that NIN processing is a posttranslational event. This implies a protease that may be activated during nodulation. Further studies will be focused on the identification of the protease involved in NIN processing and functional analyses of the different NIN processed forms during the rhizobial symbiosis.

The impacts of Delftia sp. JD2 in the symbiosis among bradyrhizobia and soybean B. Riviezzi (1), C. Cagide (1), S. Castro-Sowinski (1,2), C. Olivaro (3), M. MOREL REVETRIA (1), (1) IIBCE, Montevideo, Uruguay; (2) Facultad de Ciencias, Udelar, Uruguay; (3) CUT, Udelar, Tacuarembo, Uruguay

Root exudates (RE) contain both high- and low-molecular weight compounds (LMWC) that function as signaling molecules in plant-microbe interactions. Among LMWC, plant fractions present high number of phenolic compounds, and flavonoids are some of the most common polyphenolics. They are especially relevant in Nod Factor synthesis in rhizobium-legume symbiosis. Substantial developments in research on plant products focus on the analysis of phenolics. However, existing methodologies are not always applicable to satisfy all systems. While complexes with proteins, carbohydrates or other elements hinder complete analysis of flavonoids, the high amount of phenolics make protein extraction and analysis also tricky. Delftia sp. JD2 is a plant-growth-promoting bacterium that produces auxins and increases soybean yield and nodulation rate when is co-inoculated with bradyrhizobia. In this work, we present a methodology to analyse the LMWC and proteins from RE of soybean co-inoculated with bradyrhizobia and JD2. A specific design of a hydroponic system was developed. Radicules of pre-germinated seeds were inoculated/co-inoculated with B. elkanii U1301+U1302 and Defltia sp. JD2. A series of filtrations allows recovering LMWC and macromolecules, separately, from hydroponic liquids. The profiles of flavonoids, aminoacids and auxins are analysed by UHPLC/MS and UHPLC-HRMS while proteins are extracted by a two-step of ultra-diafiltration and characterised by a "bottom up" proteomic approach.

Multi-level control of Pseudomonas fluorescens rhizosphere adaptation

L. Grenga (1), R. H. Little (2), S. D. Woodcock (2), G. Chandra (2), R. Morris (3), J. G. MALONE (2,4), (1) Laboratory 'Innovative technologies for Detection and Diagnostics' CEA-Marcoule, Bagnols-sur-Cèze, France; (2) John Innes Centre (JIC), Norwich Research Park, Norwich, U.K.; (3) John Innes Centre, U.K.; (4) University of East Anglia, Norwich, U.K.

The plant rhizosphere is a highly complex environment, containing thousands of competing and cooperating microorganisms and dynamic fluxes of nutrients, toxins and signaling molecules. Microbial colonisation of the rhizosphere is a correspondingly complex process that is controlled by environmental inputs at multiple levels, including gene transcription, translation and through modulation of protein activity. It is becoming increasingly apparent that key regulators of the colonisation process function in a non-linear, pleiotropic and multi-level manner, with interconnections to other signaling pathways and phenotypic outputs that vary profoundly in response to multiple environmental cues, both defined and undefined. As part of our ongoing research into the signaling network that controls rhizosphere colonisation by the commensal soil bacterium Pseudomonas fluorescens, we have identified the RimABK system that influences the transition between active and sessile lifestyles in both pathogenic and commensal microbes. Deletion of $\operatorname{rimK}$ affects phenotypes including root attachment, motility and cytotoxicity, compromising rhizosphere colonisation by $P$. fluorescens and infection by the pathogen $P$. syringae. Here we show that RimABK is a novel translational regulator that converts environmental signals into rapid changes in translational output and adaptive remodeling of the proteome through specific modification of bacterial ribosomes.

Reprogramming of GIPC glycosylation by GINT1 is critical for the persistence of perimicrobial membranes in Medicago truncatula W. MOORE (1), C. Chan (1), T. Ishikawa (2), E. Rennie (1), J. Mortimer (1), H. V. Scheller (1), (1) Joint BioEnergy Institute, Emeryville, CA, U.S.A.; (2) Saitama University, Saitama, Japan

Glycosyl inositol phosphorylceramides (GIPCs) are major components of the plant plasma membrane that have been implicated in membrane organization, signaling, and cell death. The carbohydrate moieties of GIPCs are both developmentally regulated and structurally diverse across plant species. Recently, the first glycosyltransferase enzymes involved in biosynthesis of GIPC glycan head groups have been identified. GIPC MANNOSYL TRANSFERASE (GMT1) and GIPC N-ACETYLGLUCOSAMINE TRANSFERASE (GINT1) produce distinct Hex and HexN(Ac)-GIPC species, respectively. Arabidopsis thaliana gmt1 mutants are severely dwarfed and Oryza sativa gint1 mutants are seedling lethal, indicating that glycosylation is important for GIPC function. In Medicago truncatula roots, GINT1 is highly upregulated during nodulation and arbuscular mycorrhizal symbiosis. The GINT1 promoter is active in the nodule apex and cortical root cells developing arbuscules, indicating that GINT1 is expressed in cells actively synthesizing perimicrobial membranes. RNAi-mediated silencing of GINT1 impairs nodule development, culminating in bacterial senescence. Similarly, GINT1-silenced roots have lower levels of AM colonization and contain senescent arbuscules and hyphae. Taken together, reprogramming of GIPC glycosylation by GINT1 is important for the persistence of Sinorhizobium meliloti and Rhizophagus irregularis in M. truncatula roots. 
Characterization of nitrogen uptake and transfer in plant-arbuscular mycorrhizae symbioses S. L. ROWE, Michigan State University, East Lansing, MI, U.S.A.

Approximately $80 \%$ of terrestrial plants form symbiotic associations with arbuscular mycorrhizal fungi (AMF). AMF are widely beneficial with their best-studied contribution to hosts being the uptake and transfer of soil phosphorus (P). In exchange, hosts provide AMF with carbon in the form of hexose sugars and fatty acids. AMF are also known to uptake and transfer inorganic nitrogen (N) from the soil environment to the host plant. Previous work suggests that soil $\mathrm{N}$ is assimilated by AMF and transported within AMF as arginine and released to the plant without carbon. However, the uptake and transfer of $\mathrm{N}$ by AMF required further characterization. In particular, the regulation of $\mathrm{N}$ fluxes through the symbiosis under different environmental conditions has not been quantitatively characterized. We have developed and tested compartmented microcosm systems that allow differential nutrient uptake and exchange by the roots and associated AMF of whole plants to be measured under a range of conditions. These microcosms have allowed us to demonstrate substantial growth benefits to host plants from $\mathrm{N}$ transfer by the fungus. We are pursuing high throughput isotopic labeling experiments to investigate the regulation of nutrient uptake and exchange among plants and their AMF.

Is auxin signaling part of the RAM1-regulated arbuscocyte developmental program?

F. DU, C. Gutjahr, Plant Genetics, TUM School of Life Sciences Weihenstephan, Freising, Germany

The exchange of nutrients in arbuscular mycorrhiza symbiosis between plants and glomeromycotan fungi is performed by fungal broccoli-shaped structures, called arbuscules. REDUCED ARBUSCULAR MYCORRHIZA1 (RAMI), encoding a GRAS transcription factor, was identified to be a core regulator of arbuscule development. The ram 1 mutant displays a stunted arbuscule phenotype. Arbuscule branching also requires auxin signaling. We wondered how auxin signaling is placed relative to RAM1 in a signaling network regulating arbuscule development. We found in L. japonicus that the auxin reporter DR5:GUS was active in arbuscocytes (arbuscule containing cells) in the wild type but not in ram 1, indicating that $R A M 1$ is required for DR5:GUS induction in these cells. Furthermore, ectopic expression of RAM1 induced DR5:GUS in a patchy pattern in absence of the fungus. In addition, some AM-induced auxin response genes were not induced in the ram 1 mutant. Taken together, this indicates that activation of auxin signaling or biosynthesis in arbuscocytes may involve RAM1.

Phosphoproteomics reveals the downstream phosphorylation signaling targets of the lectin receptor-like Kinase PtLecRLK1involved in plant / mycorrhizal symbiosis R. L. HETTICH, Oak Ridge National Laboratory, Oak Ridge, TN, U.S.A.

Previous QTL mapping revealed the G-type lectin receptor-like kinase (PtLecRLK1) as a plausible locus responsible for the mycorrhizal symbiosis of the Populus-Laccaria bicolor system. Transgenic expression of this gene in Arabidopsis thaliana promotes L. bicolor root colonization. To characterize the downstream phosphorylation signaling of this kinase, LC-MS/MS proteome data were collected from phosphoenriched samples of Arabidopsis wild type and transgenic lines (containing PtLecRLK1) with and without L. bicolor inoculation to identify differentially abundant phosphopeptides and proteins. In total, phosphopeptides were identified that mapped to 3,416 proteins, and there was a significant downregulation of phosphopeptides in inoculated PtLecRLK1 transgenic Arabidopsis. Proteins related to defense response, such as EMSY-LIKE 3 and tetraspanin-8, were substantially lower abundance in inoculated transgenic Arabidopsis, suggesting that PtLecRLK1 plays a role in the suppressing signaling of defense related genes during mycorrhizal symbiosis. A few phosphopeptides were upregulated in inoculated transgenic Arabidopsis and mapped to proteins such as phototropin-1 and photosystem II reaction center, suggesting that PtLecRLK1 also plays significant role in upregulating genes related to metabolic processing and cellular organization. These differentially abundant proteins and phosphoproteins provide a detailed glimpse into cellular responses during the plant / mycorrhizal symbiosis.

\section{Bradyrhizobium sp. LVM 105 forms nitrogen-fixing nodules with Glycine soja, but is impaired in its ability to form nodules with agronomically improved soybean cultivars H. B. KRISHNAN, USDA-ARS, Columbia, MO, U.S.A.}

Bradyrhizobium sp. LVM 105 is an alphaproteobacterium that was originally isolated from nodules of partridge pea (Chamaecrista fasciculata), a member of the subfamily Caesalpinioideae. The nodulation biology in Chamaecrista is believed to have evolved independently from that of subfamily Papilionoideae and represents around 60 million years of independent evolution. The genome sequence of Bradyrhizobium sp. LVM 105 revealed a total of 7,453 protein-coding sequences, 3 rRNA genes, and 51 tRNA genes. Bradyrhizobium sp. LVM105 is phylogenetically related to the soybean symbiont Bradyrhizobium diazoefficiens. This phylogenetic relationship promoted us to examine the nodulation compatibility of Bradyrhizobium sp. LVM105 with domesticated soybean (Glycine max) and with wild soybean (Glycine soja). Bradyrhizobium sp. LVM105 efficiently nodulated Glycine soja, however, it was impaired in its ability to form nodules on agronomically improved soybean cultivars. Interestingly, LVM105 was able to form a few nodules sporadically on the primitive soybean cultivar "Peking", and nodules were much larger than those formed by USDA110. In contrast, on the roots of agronomically improved cultivars such as McCall and Williams 82 , LVM105 elicited "popcorn" nodules. These observations suggest that breeding soybean for better agronomic performance (yield, disease resistance etc.) has inadvertently restricted the ability of LVM105 to efficiently nodulate soybean in modern American cultivars.

Rapid phosphorylation of soybean RIN4 upon rhizobial treatment K. TOTH, University of Missouri, Columbia, MO, U.S.A.

The signaling cascade, that leads to the formation of nitrogen-fixing symbiosis between legumes and rhizobia, is initiated by receptor-like kinases such Nod Factor Receptor1 and 5 triggering downstream phosphorylation. In order to better understand the biology of this mutualistic interaction, we conducted a phosphoproteomic study on soybean root and root hairs in response to the compatible symbiont Bradyrhizobium japonicum. 
RIN4 (Rpm1 Interacting 4), a protein well characterized in plant immune responses, was found to be phosphorylated within one-hour postinoculation in soybean root hairs in response to B. japonicum. RNAi-targeted gene silencing and CRISPR-Cas9 targeted knock-out of one of the homologs resulted in a significant reduction in nodule numbers. Intriguingly one of the identified phosphorylation sites is located within a 15 amino acid region, which appears to be present only in proteins derived from leguminous plants. When a phospho-minus version of this phosphorylation site was introduced into transgenic soybean roots, significantly fewer nodules were formed, suggesting that the site might be required for the symbiotic signaling. Interestingly, one of the receptor-like kinases involved in the early symbiotic signaling (Symbiosis Receptor Kinase), phosphorylates this abovementioned residue located within a legume-specific motif in vitro. Currently, efforts are made to confirm the phosphorylation in planta.

Role of metabolic genes during the infection process in Rhizobium - legume symbiosis R. LEDERMANN (1), R. Wheatley (2), V. K. Ramachandran (2), J. Terpolilli (3), P. S. Poole (2), (1) University of Oxford, Oxford, U.K.; (2) University of Oxford, U.K.; (3) Murdoch University, Australia

Rhizobia are a diverse group of Alpha- and Beta-proteobacteria that undergo a mutualistic relationship with legume plants. Intense chemical crosstalk between rhizobia and the plant host takes place during symbiotic establishment. One major bacterial signaling factor are secreted lipochito-oligosaccharides. These so-called Nod-factors trigger root hair curling in the host which entraps root hair-attached bacteria. From there rhizobia enter the root of host plants via infection threads. These are plant-derived structures which guide the bacteria into the root cortex where they are released into plant cells. Eventually, rhizobia will differentiate into nitrogen-fixing bacteroids and exchange the fixed nitrogen for reduced carbon sources and other nutrients. Within infection threads rhizobia are enclosed completely by plant tissue and hence, all nutrients needed for growth must be provided by the host. Likewise, rhizobia growing in the rhizosphere receive most of their nutrients from root exudates. So far, the microscopic nature of early infection stages has hampered biochemical experiments and the physico-chemical nature of these important structures remains unknown. A novel experiment using INseq has revealed genes of the pea symbiont Rhizobium leguminosarum bv. viciae 3841 which are essential or advantageous during the infection process. Here, we describe mutations in such metabolic genes and their effect on the symbiotic efficiency of $R$. leguminosarum.

\author{
Activation of the general stress response by stress-specific sensor histidine kinases is crucial for host infection by Bradyrhizobium \\ diazoefficiens \\ J. WÜLSER (1), C. Ernst (1), D. Vetsch (1), R. Ledermann (1), J. A. Vorholt (2), H. M. Fischer (1), (1) ETH Zurich, Zurich, Switzerland; (2) \\ ETH Zürich, Zürich, Switzerland
}

The general stress response (GSR) enables bacteria to respond to adverse, potentially lethal environmental conditions. Contrary to specific stress responses, the GSR is activated by a variety of different stress signals and provides cross-protection against stressors unrelated to the inducing conditions. In Alphaproteobacteria, the decisive step in GSR activation is phosphorylation of the hybrid response regulator PhyR by sensor histidine kinases harboring a characteristic HRXXN motif. The nitrogen-fixing bacterium Bradyrhizobium diazoefficiens requires PhyR-mediated GSR activation not only for tolerance to a multitude of stresses under free-living conditions (e.g. salts, hyperosmosis, alkaline $\mathrm{pH}$ ) but also for formation of efficient endosymbiosis with its host plant soybean. To date, stress conditions encountered by $B$. diazoefficiens during early stages of host infection are poorly defined, and little is known about the molecular key players acting at the GSR-sensing level. Here, we assessed the role of eleven candidate GSR-activating HRXXN kinases in the $B$. diazoefficiens-soybean symbiosis. Through phenotypic characterization of $B$. diazoefficiens mutants lacking all or subsets of the kinases, or complemented with distinct combinations thereof, we identified a minimal subset of two kinases that are involved in symbiosis. Their stress specificities and differences in signal perception pathways will be presented.

The role of lipid signaling in the recruitment and activation of the Nox complex in the fungal endophyte Epichloë festucae B. Hassing (1), C. Eaton (1), C. H. Mesarich (1), B. D. SCOTT (2), (1) Massey University, New Zealand; (2) Massey Univ, Palmerstown North, Manawatu, New Zealand

Epichloëfestucaeforms a mutualistic association with perennial ryegrass. This fungus colonises the intercellular spaces of aerial tissues of the host. In many plant-associated fungi signaling via reactive oxygen species (ROS) generated by the NADPH oxidase (Nox) complex has been shown to play a crucial role in establishment and maintenance of this association. Studies on the regulation of the Nox complex in plants and animals have shown that lipid signaling via phosphatidic acid (PA), which is generated by phospholipaseD, is required for the activation of the Nox complex. The E. festucaegenome contains two phospholipaseD genes, pldAand $p l d B$, coding for two structurally different proteins. Only deletion of pldBresulted in reduced colony growth and deformed hyphae that were unable to undergo hyphal fusion. A PA biosensor expressed in the wild type strain localized to the plasma membrane (PM) but in the pldBdeletion strain mislocalised to the cytoplasm of hyphal tips. The pldBdeletion additionally resulted in a change in localization of superoxide. Inoculation of the pldBmutant strains into ryegrass seedlings resulted in a defective symbiotic interaction phenotype with a dramatic increase of hyphal biomass and severe stunting of the host. These results show that $\mathrm{PldB}$ is required for $E$. festucaehyphal fusion and establishment of a mutualistic symbiotic interaction with ryegrass and indicate an important role PA signaling in this symbiotic interaction.

\author{
Improved Network Biology Approach Discovers High-confidence Candidate Host targets of Pathogen TAL Effector Target Genes in \\ Bacterial Leaf Streak of Rice \\ B. MISHRA (1), N. Kumar (1), A. J. Bogdanove (2), S. Mukhtar (1), (1) University of Alabama at Birmingham, Birmingham, AL, U.S.A.; (2) \\ Cornell University, Ithaca, NY, U.S.A.
}

Network biology has proven instrumental for the identification of key players in different biological systems including plant-pathogen interactions. Bacterial leaf streak (BLS) of rice, caused by Xanthomonas oryzae pv. oryzicola (Xoc), is an important disease that is becoming more prevalent with climate change. Xoc takes advantage of highly conserved transcription activator-like (TAL) effectors delivered into host cells to manipulate diverse biological processes including nutrient transport and defense response pathways by direct transcriptional activation of 
target genes. We applied network structural centralities and weighted $k$-shell decomposition methods to reveal the most influential players in a rice computational protein-protein interactome (RicePPInet). We found that highly connected nodes (hubs) as well as nodes that situate in the center of the network (internal layered proteins) are enriched for candidate Xoc TAL effector target genes reported earlier. Further, we constructed a large rice response-to-pathogen co-expression network and integrated with RicePPInet to generate a Rice Immune Protein-Protein Interaction Network (RIPIN). Inner layered proteins in RicePPInet are enriched in RIPIN, and involved in several important biological processes. In summary, the results of our multi-omics, integrative network systems biology approach reveal that network centrality and structural properties may be highly predictive of effector targets in different pathosystems.

Long-lasting priming by $\beta$-aminobutyric acid is marked by de novo DNA hypomethylation M. R. ROBERTS (1), D. Worrall (1), G. Holroyd (1), R. Alvarez-Venegas (2), E. Luna Diez (3), M. Catoni (3), J. Ton (4), (1) Lancaster University, Lancaster, U.K.; (2) CINVESTAV-Irapuato, Irapuato, Guanajuato, Mexico; (3) University of Birmingham, U.K.; (4) University of Sheffield, U.K.

Plants use a variety of constitutive and inducible defences to resist attack by herbivores and disease-causing microbes. In addition, plants can also enhance future defences based on past experiences to optimise the trade-off between costs and benefits of defence. This phenomenon, known as priming, represents a heightened state of alert in which defence responses can be triggered more rapidly and/or to a greater degree and is established following a prior stress or chemical priming treatment. We have previously shown that $\beta$-aminobutyric acid (BABA) root drenches of Arabidopsis and tomato seedlings can generate long-lasting priming responses. Here, we investigate the mechanisms of long-term BABA priming of tomato seedlings. Transcriptomic analysis of control and primed plants inoculated with Botrytis cinerea reveals a clear acceleration of inducible expression of defence genes and indicates increased expression levels of defence genes in primed plants. Whole genome bisulphite sequencing reveals genome-wide hypomethylation in BABA-treated plants in all three cytosine sequence contexts ( $\mathrm{mCG}, \mathrm{mCHG}, \mathrm{mCHH})$. Differentially methylated regions can be identified throughout the genome, but the most striking effect of BABA treatment is $\mathrm{CHH}$ hypomethylation in genic regions. Altogether, our results suggest that epigenetic changes at the level of DNA methylation can mark long-term BABA priming of gene expression.

Dual dissection of the molecular dialogue driving the bread wheat - Fusarium graminearum interaction reveals the co-regulation of candidate susceptibility factors and fungal effectors

A. Fabre (1), M. Vignassa (2), S. Urbach (3), T. Langin (1), L. BONHOMME (1), (1) UMR GDEC INRA/UCA, France; (2) CIRAD, France; (3) FPP IGF, France

Fungal plant diseases are controlled by a complex molecular dialogue that involves pathogen effectors able to manipulate plant susceptibility factors at the earliest stages of the interaction. By probing the wheat-Fusarium graminearum pathosystem, we profiled the co-regulations of the fungal and plant proteins shaping the molecular responses of a 96-hour-long infection's dynamics. While no symptoms were yet detectable, fungal biomass swiftly increased along with an extremely diverse set of secreted proteins and candidate effectors supposed to target key plant organelles. Some showed to be early accumulated during the interaction or already present in spores, otherwise stored in germinating spores and detectable in an in vitro $F$. graminearum exudate. Wheat responses were swiftly set up and were evidenced before any visible symptom. Significant wheat protein abundance changes co-occurred along with the accumulation of putative secreted fungal proteins and predicted effectors. Regulated wheat proteins were closely connected to basal cellular processes occurring during spikelet ontogeny and particular coregulation patterns were evidenced between chloroplast proteins and fungal proteins harboring a predicted chloroplast transit peptide. The plant and fungal coordinated responses provide a resourceful set of data and expand our understanding of the wheat-F. graminearum interaction.

Multiple regulatory hubs are involved in the RKS1-dependent gene network controlling quantitative disease resistance against Xanthomonas campestris

F. DELPLACE (1), C. Huard-Chauveau (1), F. Roux (2), R. Peyraud (3), D. Roby (1), (1) LIPM, INRA, CNRS, Université de Toulouse, Castanet-Tolosan, France; (2) LIPM, Université de Toulouse, INRA, CNRS, INPT, Castanet-Tolosan, France; (3) iMEAN-biotech, Toulouse, France

The importance of pathogen perception and signaling pathways in the regulation and execution of plant immune responses have become apparent during the last years. Notably, $R$ gene-mediated immunity has been shown to be the most efficient form of resistance in plants. Quantitative resistance that is considered a more durable type of resistance with partial effects on plant immunity, have gained increasing attention for breeding purposes. One of the most important disease of crucifers (including cabbage, broccoli and rapeseed), is the black rot, caused by the bacterial plant pathogen Xanthomonas campestris pv. campestris (Xcc). Based on results obtained by genome wide associating (GWA) mapping and functional approaches, we cloned Resistance related KinaSe $1(R K S 1)$ as the gene underlying a major QTL conferring approximately $50 \%$ resistance in Arabidopsis thaliana against the strain Xcc568 (Huard-Chauveau et al., 2013). Our main objective is now to decipher the RKS1 dependent regulatory pathway(s). We used comparative transcriptome analysis of mis-expressing RKS1 lines, and we then reconstructed the molecular RKSI dependent network using our dataset and published interactions. Multiple putative functional hubs were identified within this network, and mutant-based experiments were performed to functionally validate them. The results and perspectives of this work based on a combination of molecular biology, computational biology and genetics will be presented.

Modeling effector-host interactions in the context of the barley protein interactome

V. VELASQUEZ-ZAPATA (1), S. Banerjee (1), P. Surana (1), J. M. Elmore (2), R. Wise (2), (1) Iowa State University, Ames, IA, U.S.A.; (2) USDA-ARS/Iowa State University, Ames, IA, U.S.A.

Pathogen effectors are excellent tools to explore dynamic regulation of plant resistance and susceptibility. To discover novel mechanisms of effector action, we exploited the biotrophic powdery mildew fungus, Blumeria graminis f. sp. hordei (Bgh), and its host, barley (Hordeum vulgare). We used next-generation sequencing to identify interacting partners from high-throughput yeast two-hybrid assays, using $B g h$ effectors 
as baits, and as preys, a time-course cDNA library from infected barley and isogenic immune mutants. We evaluated selected vs. non-selected conditions for positive interactors using a robust informatics and statistics pipeline, including mapping reads to barley and $B g h$ genomes, reconstruction of prey fragments and fusions with GAL4-AD, and processing of count data. We used this information to develop a ranking system for the preys, comprising: 1) significant enrichment under selection for positive interactions, 2) in-frame with GAL4-AD, and 3) degree of enrichment in pairwise comparisons of baits under selection. Outputs from this pipeline facilitated sorting and validation by binary $\mathrm{Y} 2 \mathrm{H}$. We integrated the top ranked effector targets with a predicted barley protein-interactome to identify barley- $B g h$ interaction hubs. Additionally, we filtered the interactions based on co-expression to detect tightly regulated genes during the immune response. Immune modules exhibited enrichment for genes associated with transcription, phosphorylation and intracellular transport.

\section{The transcriptional landscape of plant pattern-triggered immunity}

M. BJORNSON (1,2), S. Ranf (3), T. P. Nuernberger (4), C. Zipfel (5,6), (1) Inst. of Plant and Microbial Biology and Zürich-Basel Plant Science Center, UZH, Zürich, Switzerland; (2) The Sainsbury Laboratory, University of East Anglia, Norwich Research Park, Norwich, U.K.; (3) Technical University of Munich, Freising, Germany; (4) Eberhard-Karls Univ Tubingen, Tuebingen, Germany; (5) The Sainsbury Laboratory, University of East Anglia, Norwich Research Park,, Norwich, U.K.; (6) Inst. of Plant and Microbial Biology, University of Zürich, Zurich, Switzerland

Plant immunity is initiated upon perception of molecular signatures of microbes or damage by cell-surface pattern recognition receptors (PRRs), which instigate signaling cascades resulting in a large-scale transcriptional reprogramming. A key question in this process is whether plant cells induce different immune responses depending on the origin or nature of elicitors perceived by distinct PRRs. To address this, we assayed transcriptional responses of Arabidopsis thaliana to an array of seven elicitors comprising a variety of source and PRR categories, across a six point time course focusing on early responses. We find a strong correlation among transcription patterns induced by all elicitors, suggesting that plant cells mostly perceive danger. Accordingly, this core response is shared among published responses to abiotic stresses, highlighting the importance of a plant general stress response (GSR). Genetic manipulation of the GSR affects elicitor-induced resistance against bacteria, and gene regulatory network analysis suggests novel regulators which may play a role in this response. Despite the preponderance of the GSR, a subset of genes represents a core immunity response, and ongoing work is investigating the role and regulation of these genes during immunity. Through combining a panel of elicitors with a rapid time series, we have defined the transcriptional landscape of surface immunity, and have identified potential novel regulators and executors of plant immunity.

\section{Spatiotemporal Analysis of Potato hypersensitive response-conferred Resistance to Potato Virus Y: RBOHD is required for successful} Virus arrest

K. GRUDEN (1), T. Lukan (2), M. Pompe-Novak (3), M. Tusek Znidaric (1), A. Kladnik (1), A. Blejec (1), M. Zagorscak (1), A. Coll (2), K. Morgiewicz (4), J. Hennig (5), S. Baebler (3), (1) National Institute of Biology, Slovenia; (2) National Institute of Biology, Ljubljana, Slovenia; (3) Natl Inst of Biology, Ljubljana, Slovenia; (4) Polish academy of sciences, Poland; (5) Institute of Biochemistry and Biophysics, PAS, Warsaw, Poland

Mechanisms of viral arrest in hypersensitive response (HR)-conferred resistance have remained elusive despite intensive ongoing research. We previously showed that in potato-PVY HR interaction it is not the cell death that prevents virus spread. Here we studied the molecular mechanisms of HR in a spatiotemporal manner. All analyses were performed in parallel in cv. Rywal and its SA-depleted transgenic counterpart, which cannot restrict viral spread. No particular ultrastructural features were detected distinguishing between both genotypes. We further selected 23 genes based on responsiveness in time-stamped whole leaf transcriptomic data. These included genes involved in ethylene, jasmonate and salicylate signaling, redox state related genes and a set of immune signaling actuator genes. Analysis of small sections surrounding the site of viral infection has shown that responses can be tightly spatiotemporally regulated. Interestingly, the response of redox state related genes was showing spatiotemporal response that differed between both genotypes. In particular, induction of RBOHD gene is focused on the border region of the lesion and overlap with the expression of TRX-H gene. We hypothesised that RBOHD is essential for signaling leading to successful arrest of the virus in HR-conferred resistance in potato. Indeed, in transgenic plants with suppressed RBOHD gene activity the virus can spread systemically, breaking the HR-conferred resistance, thus validating our hypothesis.

Molecular Cloning and Characterization of Capsicum spp. NBS-LRR Gene Related to Anthracnose Resistance

S. PARK (1), S. Kim (2), K. S. Lee (3), Y. H. Lee (3), E. J. Suh (3), J. Park (3), D. J. Hwang (4), J. Han (3), (1) National Institute of agricultural Sciences, Korea, Republic of (South); (2) National institute of Agricultural Sciences, Jeonju, Korea, Republic of (South); (3) National institute of Agricultural Sciences, Korea, Republic of (South); (4) Natl institute of Agricultural Sciences, jeonju, jeolabukdo, Korea, Republic of (South)

Capsicum spp. (sweet and chili pepper) is one of the most popular and widely horticultural crops cultivated worldwide. Biotic stresses represent one of the main challenges for Capsicum spp. production. One of them, the anthracnose disease caused by Colletotrichum spp. is an important fungal disease that caused significant yield losses during pre- and post-harvest. Typically, in plant disease resistance, the intracellular immune receptors are proteins having a central NB-ARC domain and a C-terminal leucine-rich repeat (LRR) domain terms as a nucleotide-binding domain and LRR (NLR) proteins, which recognize pathogen effector proteins. Therefore, to study the function of NLRs gene from pepper $C$. baccatum shown anthracnose disease resistance capsicum species, we isolated one NLR gene CbNLR1202 from C. baccatum and then overexpressed in $N$. benthamiana. This gene overexpressed tobaccum plants were shown more susceptible to anthracnose disease caused by $C$. acutatum than wild type. CbNLR1202 gene regulated several PR genes, NbPR1, NbPR2, NbPR4, and NbPR10. It may important role of regulator in anthracnose disease resistance in tobacco and pepper. This study was carried out with the support of 'Cooperative Research Program for Agricultural Science \& Technology Development (Project No. PJ01323102)’, Rural Development Administration, Republic of Korea.

Metabolic niches in the rhizosphere microbiome investigated using exometabolomics

R. P. JACOBY (1), S. Kopriva (2), (1) University of Cologne, Botanical Institute and CEPLAS, Cologne, Germany; (2) University of Cologne, Botanical Institute and CEPLAS, Germany 
The ability of microorganisms to use root-derived metabolites as growth substrates is a key trait for success in the rhizospheric niche. However, few studies describe which specific metabolites are consumed or to what degree microbial strains differ in their substrate consumption patterns. Here, we present results derived from an LC-MS exometabolomics workflow, developed to investigate the metabolic footprint of root-associated bacterial strains when cultivated using plant root extracts as the sole carbon source in the growth medium. Our initial work profiled strains isolated from the Arabidopsis root microbiota, revealing a surprising diversity in substrate consumption patterns between strains. An ongoing project studies a synthetic community of bacterial strains isolated from maize roots, defining the distinct metabolic niches occupied by specific strains. In both studies, we observe a large number of microbe-derived metabolites that are secreted into the growth medium. Our methodology delivers new mechanistic information about processes that shape the microbial ecology of the rhizosphere, such as: niche differentiation, competitive exclusion, cross-feeding, signaling and antibiosis.

Identification of new plant virus $3^{\prime}$-cap-independent translational enhancers M. Miras (1), M. A. Aranda (2), V. TRUNIGER (3), (1) CEBAS-CSIC, Spain; (2) CEBAS-CSIC, Murcia, Spain; (3) CEBAS-CSIC, Murcia, ME, Spain

While most plant-encoded mRNAs contain a $5^{\prime}$-cap and a poly(A)-tail that act synergistically to stimulate translation, $\sim 80 \%$ of known positivestrand RNA plant viruses lack one or both of these features in their genomic and subgenomic RNAs. Thus, translation of some of the viral genomes lacking both features has been shown to be controlled in cis by RNA elements present in their 3'-UTR able to enhance cap-independent translation (3'-CITEs). Our group has identified three different active 3'-CITEs in Melon necrotic spot virus (MNSV, family Tombusviridae, genus Carmovirus) and has shown that at least one of these has been acquired by recombination with an Asiatic isolate of Cucurbit aphid-borne yellows virus (CABYV; family Luteoviridae, genus Polerovirus), suggesting that 3'-CITEs are modular, interchangeable structural elements). Here we show that CABYV cap-independent translation is controlled by two $3^{\prime}$-CITEs, differing in sequence and structure between viruses belonging to the Asiatic and European groups. Their translation enhancer activity depends on the presence of the genomic $5^{\prime}$-end in cis, and their modes of action seem to differ, depending on different eukaryotic translation initiation factors (eIFs): The 3'-CITE of most MNSV isolates depends on eIF4E while our recent experiments seem to indicate that the CABYV 3'-CITEs require eIF4G but not eIF4E for activity.

Exploring the landscape of existing motifs in networks of Arabidopsis thaliana and Solanum tuberosum under biotic stress Ž. RAMŠAK, A. Coll, T. Lukan, K. Gruden, National Institute of Biology, Ljubljana, Slovenia

The need to better understand stress-mitigating mechanisms in crop plants is increasing rapidly. Most often discussed biological systems are networks of genes or proteins. In our previous work, we have demonstrated the power of network generation and analysis approaches for generation of novel hypotheses. Large-scale analyses of these networks can be complemented with network decomposition approaches, that divide the network into smaller units, that can be analysed independently. Motifs are structurally conserved simple building blocks found in analyses of real-world networks, implicating their importance in gene functional regulation. Pathogen infection of a plant triggers a complex interaction between both players involved, resulting in changes of the complex signaling network, such as changes in gene activity or reprogramming of the cell metabolism. In order to understand the mechanisms and dynamics, we generated environment specific networks of Arabidopsis thaliana under biotic stress by Botrytis cinerea and Pieris rapae and Solanum tuberosum under biotic stress by Potato virus Y. For each system, two separate networks were generated (with and without the stressor(s)), and analysed for the presence of network motifs unique or used by both. This in combination with experimental transcript expression data enabled us to form novel hypotheses on the condition specific responses of a model and crop plant to environmental cues, appropriate for testing in the laboratory.

EvoMPMI: Genetic signatures for phenylpropanoid biosynthesis in streptophyte algae

J. DE VRIES (1,2), S. de Vries (1), J. M. Archibald (1), (1) Dalhousie University, Halifax, NS, Canada; (2) Technische Universitaet

Braunschweig, Braunschweig, Germany

During their earliest steps on land, plants were subjected to various challenges. These included abiotic stressors but also biotic ones: on land, plants will have experienced a different microbial load and composition as compared to those that their algal progenitors-the streptophyte algae - have experienced in the aquatic environment. The algal progenitors of land plants likely had a fortuitous combination of features that eased their passage from an aquatic to a terrestrial environment. Extant land plants produce various phenylpropanoid-derived metabolites that aid them in warding off microbes; these are currently considered metabolites that are specific to land plants. Yet, making use of the recent rise in sequencing data on streptophyte algae we have uncovered that streptophyte algae have orthologous genes for almost all phenylpropanoid biosynthesis-associated genes known from land plants. This includes candidates for the key entry point into the phenylpropanoid pathway, the phenylalanine ammonia lyase (PAL). In retrospect, our data provide an explanation for decade-old descriptions of lignin-like metabolites in various streptophyte algae. We infer that the earliest land plants had the genes to utilize various routes of land plant phenylpropanoid biosynthesis, equipping them with broad-spectrum response metabolites for biotic and abiotic cues. Biochemical characterization of this pathway in streptophyte algae will usher in an understanding of its functional evolution.

Elucidating the transcriptional networks underlying the salicylic acid response in Arabidopsis R. HICKMAN (1), M. Pereira Mendes (1), M. van Verk (1), K. J. Denby (2), C. M. J. Pieterse (1), S. C. Van Wees (1), (1) Plant-Microbe Interactions, Department of Biology, Utrecht University, Utrecht, Netherlands; (2) University of York, York, U.K.

The phytohormone, salicylic acid (SA) is a central regulator of plant immunity that controls a large and diverse set of defense response genes. To obtain a detailed understanding of the dynamics and architecture of the transcriptional regulatory networks that underlie the SA-response we have used two different, yet complementary, genomics approaches in the model plant Arabidopsis thaliana. First, we performed a high-resolution RNA-seq time series analysis of SA-treated leaves to obtain a detailed temporal map of transcriptional reprogramming induced by SA over a 16-h 
time course. Analysis of this large-scale, information-rich data set allowed us to build dynamic regulatory maps of the SA response, identify gene regulatory modules, and infer novel defense regulators; we predicted and validated a role for two homologous NAC transcription factors (TFs) in SA-mediated immunity. In the second approach we profiled the Arabidopsis chromatin accessibility landscape by sequencing ATAC-seq libraries prepared from seedlings treated with SA or a mock control. These data allowed us to delineate regions of accessible chromatin on a genome-wide scale and link these with SA-mediated changes in gene expression. Digital genomic footprinting analysis with these ATAC-seq data provided information on TF occupancy within the regulatory DNA of target genes and provided a direct and empirical approach for reconstructing TF networks that may regulate the SA response.

Capsid dynamics of tripartite brome mosaic virus assembled independently in vivo A. Chakravarthy, A. RAO, University of California, Riverside, CA, U.S.A.

The packaging of the viral progeny by structural protein components leading to the assembly of infectious virions is an essential step in the life cycle of RNA viruses. In BMV, the genome is divided among three nucleic acid species and is packaged into three separate virus particles. The three virus particles are indistinguishable either by electron microscope (TEM) or any known separation techniques (e.g. density gradient centrifugation). Therefore, the segregation of independent virions still remains a formidable task due to particle homogeneity. We have developed a novel and robust Agrobacterium-mediated transient expression system amenable for coupling replication and packaging that can independently assemble each of the three BMV virion types in planta. Our results indicate that all three virions of BMV are identical in morphology, size and surface charge conformation. However, trypsin digestion followed by MALDI-TOF analysis revealed that three virions are divided into two classes: Virions packaging RNA 1 and RNA2 are grouped into Class 1 while those co-packaging RNA3 and 4 are grouped into Class 2 . We also observed that the trypsin digestion profiles of Class 1 type of virions are distinct from those of Class 2 . This novel in vivo virion assembly approach can be used to assemble pure virions of multipartite plant RNA viruses like Cucumber mosaic, Barley stripe mosaic and Alfalfa mosaic viruses.

A genetic screen for important elements in bacterial pathogenesis

J. WU, X. Mei, S. Wan, X. Xin, Institute of Plant Physiology \& Ecology, Chinese Academy of Sciences, Shanghai, China

Plant disease is a major threat to global plant yield. Previous work showed that, in addition to suppressing plant immunity, pathogens utilize type III effectors to drive the formation of an aqueous apoplast (water soaking) to multiply aggressively in plant leaves. AtMIN7 is important for the induction of water soaking upon Pseudomonas syringae infection. The min $7 / f l s 2 /$ fr $1 /$ cerkl mutant plant, in which pattern-triggered immunity and AtMIN7 pathways are disabled, displayed significantly enhanced susceptibility to $P$. syringae infection. Here, we started an EMS mutagenesis using the $\min 7 / f l s 2 /$ efr $1 /$ cerklmutant as parents and screen to identify1) other elements in bacterial pathogenesis; 2) unknown components, in addition to MIN7, involved in water soaking pathway. Two rounds screening test were performed. Bacterial spray assay was performed in the $1^{\text {st }}$ round screening and over 300 candidates which show stronger disease symptoms were uncovered. In the $2^{\text {nd }}$-round screening, bacterial infiltration assay was performed, and a few lines with enhanced susceptibility or stronger "water soaking" phenotype with pathogen infection or "diseaselike" symptoms without pathogen infection were identified. Mapping-by-sequencing approach will be used to identify the causal mutations in the mutants and further functional study will be performed.

High Resolution Transcriptomics Reveals the Significance of Cell Identity in Directing Cell Type-Specific Immunity Networks C. Rich (1), R. Eichmann (2), S. Ott (1), P. SCHÄFER (3), (1) University of Warwick, U.K.; (2) The University of Warwick, U.K.; (3) University of Warwick, Coventry, U.K.

Plant roots represent a complex organ consisting of dozens of different cell types. Cell type-specific transcriptomics have significantly advanced our knowledge of the individuality of cell type function and revealed the importance of a coordinated regulation of cell type-specific gene networks to secure overall root functionality under abiotic stress. While root diseases pose a major threat in crop production, the function of root cell types in regulating root immunity remains elusive. In an effort to decipher principles in the regulation of root immunity, we conducted cell type-specific transcriptomics of Arabidopsis roots treated with two immunity elicitors - bacterial flagellin or plant-derived Pep1. Our analyses indicated that both elicitors activate different immunity networks in each cell type and uncovered the contribution of each cell type in mounting an effective immune response. By developing a novel promoter paired motif analysis tool, we were able to predict and validate in planta key transcription factor (TF) pairs involved in the regulation of cell-type-specific immunity networks. Moreover, we found that TF pairing is a regulatory principle employed by cell identity networks to direct cell type-specific immunity according to cell type functionality. Our analyses provide a regulatory landscape of innate immunity in roots that we now use to model immunity vs. abiotic stress signaling with the ultimate aim of generating crops improved in root stress adaptation.

Using X-ray Computed Tomography to phenotype the fungal pathogen Zymoseptoria tritici C. LAWLESS (1), J. I. Burke (2), S. Tracy (3), A. M. M. Tiley (1), P. Pilo (1), A. Feechan (4), (1) UCD; School of Agriculture and Food Science, UCD Earth institute, Ireland; (2) University College Dublin; School of Agriculture and Food Science, Ireland; (3) UCD School of Agriculture and Food Science, Ireland; (4) UCD, School of Agriculture and Food Science, UCD Earth institute, Dublin, Ireland

The fungal pathogen Zymoseptoria tritici is the causative agent of Septoria tritici Blotch (STB) which is one of the most devastating diseases of wheat (Triticum aestivum L.), particularly in Northern Western Europe. The effectiveness of commercial fungicides as a control mechanism against STB is decreasing, therefore there is a need to identify novel methods to control STB. X-ray Computed Tomography (CT) is a nondestructive imaging technique, which enables $Z$. tritici to be visualised in 3-dimensions in planta during infection. Our aim is to combine molecular techniques with X-ray CT scanning to investigate $Z$. tritici infection in the host and in the non-host grass Brachypodium distachyon. Preliminary data shows pycnidia can be visualised using X-ray CT imaging. From this, fundamental new knowledge will be gained on the life cycle and structure of $Z$. tritici and enable phenotyping of the disease. Understanding a non-host interaction will inform future resistance 
strategies to Z. tritici. This phenotyping method of visualising fungal structures can be used for comparing the disease progression in wheat and in non-host grass systems.

Phytohormone network-mapping reveals a function for TOPLESS in Salicylic Acid-mediated defense signaling P. A. RODRIGUEZ (1), M. Altmann (1), S. Altmann (1), N. Marin de la Rosa (1), A. Garcia i Molina (2), P. Falter-Braun (3), (1) Helmholtz Zentrum Muenchen, Germany; (2) LMU München, Germany; (3) Helmholtz-Zentrum München, Germany

Phytohormones play an essential role in mediating integrative responses in plants towards environmental cues like drought or pathogens and during developmental programs as growth, cell division and differentiation. We generated a systematic experimental map of the phytohormone network to identify mediators of crosstalk and to obtain a systems level perspective onto this central plant signaling network. Our local network map further suggests that the co-repressor protein topless (TPL), previously implicated in auxin (IAA), jasmonic acid (JA) and ethylene signaling pathways, also mediates SA-dependent immune responses. From this local SA network, the hypothesis of TPL indirectly interacting with the SA receptor NPR1, in a regulatory ternary complex that includes NIM1-INTERACTING (NIMIN) protein family members, emerges. As TPL has also a regulatory role in circadian transcription, we profiled the transcriptional diurnal oscillations along $24 \mathrm{hr}$ of the genes involved in our local SA network on a wild-type background compared to a $t p l$ mutant background. These preliminary data suggest that TPL disrupts the diurnal oscillations patterns of certain clock genes, like TOC1 and PRR5; of NPR4, another SA receptor; and of NIMIN1 and NIMIN3, and importantly of defense genes, like PR-1, PR-2 and PR-5. Thus, we present an initial validation of a SA signaling module in our phytohormone network map and demonstrate a regulatory role of TPL during a circadian clock-mediated SA defense signaling.

Temporal regulation of maize genes expression during sugarcane mosaic virus infection

K. Du, J. Xie, T. Jiang, Z. Fan, T. ZHOU, Department of Plant Pathology, China Agricultural University, Beijing, China

Sugarcane mosaic virus (SCMV) causes maize dwarf mosaic disease worldwide. We use maize-SCMV as a pathosystem to study molecular mechanisms for virus infection and symptom expression. To understand the altered host genes expression patterns during virus infection, we conducted a comparative transcriptomics analysis with maize shoots at early systemic infection (3 days postinoculation, dpi) and steady infection ( 9 dpi) stages. Similar number of differentially expressed genes (DEG) at 3 dpi and 9 dpi were obtained that were 8,893 and 10,134, respectively. To separately investigate differences of gene expression caused by SCMV and seedling development, total DEGs were categorized into DEG1 (DEGs only at $3 \mathrm{dpi}$ ), DEG2 (only at 9 dpi) and DEG3 (both at 3 and 9 dpi) by a generalized linear model. GO and KEGG pathways analysis showed that the pathways of DEG1 included protein processing in endoplasmic reticulum and fatty acid biosynthesis, which might indicate the regulated genes were targeted for virus replication and protein translation. Interestingly, DEG2 formed clearly significant GO enrichment in plastid and thylakoid related cellular components, which indicated altered photosynthesis activity. Pathways of DEG3 were mostly involved in plant hormone signal transduction, circadian rhythm, phagosome, ubiquitin mediated proteolysis, plant-pathogen interaction and photosynthesis.

Transcriptomic analysis unveils gene networks associated with the Fusarium head blight resistance transferred from Triticum turgidum ssp. carthlicum into durum wheat

E. SARI (1), A. L. Cabral (1), B. Polley1 (1), Y. Tan (1), D. J. Konkin (1), E. Hsueh (1), R. E. Knox (2), Y. Ruan (2), P. R. Fobert (3), (1)

Aquatic and Crop Resource Development, National Research Council Canada, Saskatoon, SK, Canada; (2) Swift Current research and Development Centre, Agriculture and Agri-Food Canada, Swift Current, SK, Canada; (3) Aquatic and Crop Resource Development, National Research Council Canada, Ottawa, ON, Canada

Fusarium head blight (FHB) resistance in the durum wheat gene pool is poor. Triticum turgidum ssp. carthlicum line Blackbird is a relative of durum wheat that offers partial FHB resistance. The objective of this study was to identify candidate regulatory resistance genes in the defense mechanisms underlying the resistance of Blackbird. RNA-seq analysis was conducted to identify differences in the transcriptome of Blackbird, durum cv. Strongfield and two progenies of the Strongfield/Blackbird population with extreme disease rating. Weighted Gene Correlation Network Analysis identified five gene-networks significantly $(P<0.05)$ associated with the resistance to FHB spread, two of which show moderate to high correlation with the plant height and one with relative maturity. Two gene networks showed subtle difference between Fusarium graminearum inoculated and mock inoculated plants in all the genotypes, supporting their involvement in constitutive defense. The candidate regulatory genes encode proteins involved in pathogen recognition, signaling pathways (e.g. abscisic acid and mitogen activated protein kinase signaling), transcriptional regulation of both defense and development, cell death regulation and cell wall reinforcement. Hub genes within the five significant modules co-located with seven previously reported FHB resistance QTL. SNPs associated within the hub genes are available for future high-resolution mapping studies.

Quantitative disease resistance to Phytophthora sojae and species of Pythium: It's complicated A. DORRANCE (1), C. Million (2), W. Rolling (3), S. Karhoff (3), K. Scott (2), A. Stasko (2), L. McHale (4), (1) The Ohio State Univ, Wooster, OH, U.S.A.; (2) The Ohio State University, Wooster, OH, U.S.A.; (3) The Ohio State University, Translational Plant Sciences Program, Columbus, OH, U.S.A.; (4) The Ohio State University, Dept. of Horticulture and Crop Sciences, Columbus, OH, U.S.A.

Watermolds consistently have a negative impact on soybean stand and yield in the North Central Region of the United States. Numerous sources of host resistance have been identified to several watermolds. In total, we have mapped more than 30 quantitative disease resistance loci (QDRL) to one or more of the following: Phytophthora sojae, Pythium irregulare, Py. ultimum var ultimum, Py. ultimum var sporangiiferum. The major finding is that each resistant cultivar has different loci mapped for resistance to each of these watermolds, indicating varied control of disease progression in the necrotrophic phases of these pathogens. High levels of quantitative disease resistance (QDR) to $P$. sojae have proven durable, but the mechanisms of QDR remain elusive. Through high density mapping of several bi-parental populations, eQTL analysis of a Conrad $\mathrm{x}$ Sloan mapping population, and GWA analyses of more than 900 plant introductions, two patterns have emerged. The first is that auxin plays a role in susceptibility which suggests that the plant response to the hormone can dictate the final disease severity. Second, the resistance is 
complex, and multiple loci and pathways are coordinated to respond to and limit pathogen growth in seedling roots. These results will guide breeding as resistance to watermolds is introgressed into high yielding adapted cultivars.

Indolic glucosinolate metabolon formation and PEN2-mediated entry control of non-adapted powdery mildews in Arabidopsis L. WAGENKNECHT, H. Ghareeb, E. K. Petutschnig, V. Lipka, Georg-August-University, Goettingen, Germany

The Arabidopsis myrosinase PEN2 is required for broad-spectrum invasion resistance to filamentous plant pathogens, including non-adapted barley powdery mildews. We previously showed that PEN2 is a tail-anchored protein with dual-membrane targeting to peroxisomes and mitochondria and that PEN2 has the capacity to form homo-oligomer complexes (Fuchs et al., Plant Cell 2016). Moreover, we observed pathogen-induced recruitment and immobilization of mitochondrial subpopulations at sites of attempted fungal invasion and showed that mitochondrial arrest is accompanied by peripheral accumulation of GFP-tagged PEN2. Simultaneously, PEN2 substrate production by the cytochrome P450 monooxygenase CYP81F2 is coordinated on the surface of the Endoplasmic Reticulum, which becomes structurally reorganized in immediate proximity to the immobilized mitochondria. Exclusive targeting of PEN2 to the outer membrane of mitochondria complements the pen 2 mutant phenotype corroborating the functional importance of the mitochondrial PEN2 protein subpool for controlled local production of PEN2 hydrolysis products at subcellular plant-microbe interaction domains. Currently, we analyze the role of MAMP-dependent signaling for CYP81F2 expression, ER rearrangement, mitochondrial arrest and PEN2 aggregate formation. At the meeting, we will report on the current state of our findings.

An ancient and conserved regulation of lipid production allows carbon exchange in arbuscular mycorrhiza symbiosis M. K. RICH (1), N. Vigneron (1), G. V. Radhakrishnan (2), T. Vernié (1), J. Keller (1), P. M. Delaux (1), (1) LRSV UMR5546 CNRS/Université de Toulouse III, Castanet-Tolosan, France; (2) John Innes Centre, Norwich, U.K.

Arbuscular mycorrhiza symbiosis (AMS) is found in most extant land plant families and allows the exchange of nutrient between Glomeromycota fungi and plants. Recent findings show the dependence of arbuscular mycorrhiza fungi to lipids delivered by their host plants. It was shown that WRINKLED (WRI) family transcription factors are essential for AMS in Medicago and Lotus. Those transcription factors promote the expression of lipid biosynthesis genes, including the glycerol phosphate acyltransferase Required for Arbuscular Mycorrhiza 2 (RAM2), in the arbuscular cells. Using phylogenomics and functional studies in the bryophyte Marchantia paleacea, we are investigating the origin and evolution of the AMS lipid pathway. We found that MpaWRI and MpaRAM2 are expressed in arbuscule containing cells and that expression of a dominant repressor form of MpaWRI leads to defect in the establishment of AMS. In addition, overexpression of MpaWRI induces expression of lipid biosynthesis genes and we find direct binding of MpaWRI on the promoter of MpaRAM2. Finally, transient expression of MpaWRI induces accumulation of triacylglycerol in Nicotiana benthamiana leaves. Our results show that the regulation of lipid production by WRINKLED during AMS is conserved between bryophytes and angiosperms, suggesting that this mechanism is ancient and could trace back to the first mycorrhizal symbiosis more than 450 million years ago.

Re-shaping of effector repertoires in different pathotypes of Magnaporthe oryzae

K. T. KIM (1), J. Ko (1), H. Song (2), G. Choi (2), J. Jeon (2), K. Cheong (2), Y. H. Lee (1,3,4,5), (1) Department of Agricultural Biotechnology, Seoul National University, Seoul, Korea, Republic of (South); (2) Interdisciplinary Program in Agricultural Genomics, Seoul National University, Seoul, Korea, Republic of (South); (3) Center for Fungal Genetic Resources, Seoul National University, Seoul, Korea, Republic of (South); (4) Plant Immunity Research Center, Seoul National University, Seoul, Korea, Republic of (South); (5) Research Institute of Agriculture and Life Sciences, Seoul National University, Seoul, Korea, Republic of (South)

Magnaporthe oryzae is a pathogenic fungus, which can infect mainly rice and wheat, and other Poaceae grasses including Setaria, Elusine, and Lolium. This fungus secretes protein effectors to manipulate host physiology and immune system to promote its viability. These effector proteincoding genes are known to rapidly evolve in pathogenic fungi due to their interactions with host plants. We tracked the homologous effector genes in M. oryzae isolates with different pathotypes in order to reveal how different hosts change the effector repertoires. The effector genes were mined by in silico secretome analysis from the reference genome and the homologous effectors in the other pathotypes were identified by homology search. Then, the genomic variations and the selection pressures exerted by different hosts were measured. We found that the different hosts changed the effector repertoires of each $M$. oryzae isolate as a various degree of sequence polymorphism was observed among them. This sequence variance was related with selection pressures exerted by different hosts. The genomic contexts of effector genes in each isolate were also re-organized and this was induced by the repetitive sequences near the effector genes. Taken together, we revealed that the host range expansion of M. oryzae has led the adaption of effector repertoires to different hosts. This study would enable us to better understand the evolution of effectors interacting with host plants.

\section{Identifying the receptor of Phytophthora Pep-13/25 in Solanum species}

Y. TORRES ASCURRA (1), X. Lin (2), H. Fillianti (1), R. G. F. Visser (1), T. Nuernberger (3), V. G. A. A. Vleeshouwers (1), (1) Plant Breeding, Wageningen University and Research, Wageningen, Netherlands; (2) The Sainsbury Laboratory, University of East Anglia, Norwich Research Park, Norwich, U.K.; (3) Centre for Plant Molecular Biology, Eberhard Karls University of Tübingen, Tübingen, Germany

The late blight disease caused by the oomycete pathogen Phytophthora infestans is the major threat for potato production. Breeding for resistance to late blight has been focused in the introgression of cytoplasmic resistance $(R)$ genes into potato cultivars. However, these $R$ genes have failed to provide durable resistance to the disease. An alternative source of immunity relies on pattern recognition receptors (PRR) that are located on the cell surface and recognize apoplastic effectors or microbe-associated molecular patterns (MAMPs). One well characterized oomycete MAMP is Pep-13/25, a conserved peptide motif derived from a transglutaminase that is present in all Phytophthora species, including P. infestans. Infiltrations with Pep-13/25 lead to HR-like cell death responses in various wild Solanum species and cultivated potatoes. To identify the PRR of Pep13/25, a genetic mapping approach was conducted and combined with BSA-RNA seq analyses. This has resulted so far in an interval of 330 $\mathrm{kb}$ based on the DM 1-3 516 R44 (DM) potato reference genome. Physical maps have been reconstructed to a region and various receptor-like 
kinases (RLK) candidate genes have been obtained. Overall this work aims to identify novel surface immune receptors in potato that will contribute to understand cell surface-triggered immunity. Exploiting the PRR and stacking them with $R$ genes can provide a more profound strategy to achieve a more durable resistance to late blight in potato.

Phenotyping wheat mutants with enhanced disease resistance to Puccinia striiformis f. sp. tritici A. DEATKER (1,2), J. Hegarty (3), D. Raats (4), C. Schudoma (4), F. L. Stefanato (2), A. Heckmann (2), M. Watkins (5), J. Dubcovsky (3,6), K. V. Krasileva (1,2,7), (1) University of California Berkeley, Berkeley, CA, U.S.A.; (2) The Sainsbury Laboratory, Norwich, U.K.; (3) University of California, Davis, CA, U.S.A.; (4) Earlham Institute, Norwich, U.K.; (5) University of East Anglia, Norwich, U.K.; (6) Howard Hughes Medical Institute, Chevy Chase, MD, U.S.A.; (7) Earlham Institute, U.K.

We conducted a forward genetics screen on 2,000 mutagenized lines from the mutant population of Triticum turgidum cv Kronos grown in the field and uncovered enhanced disease resistance to the yellow (stripe) rust pathogen ( $P$. striiformis f. sp. tritici). We identified 35 enhanced disease resistance lines from our primary screens in Davis, California. Further field experiments allowed us to test our candidate mutants across environmental conditions in U.S.A. and U.K. over five years. We confirmed that resistance in several mutants was heritable over the years and was effective in both U.S.A. and U.K. In order to identify the mutations, we have backcrossed mutant lines to the wild type progenitor, constructed mapping populations and screened F3 families. In addition, we started to characterise these mutants on the microscopic level through pathogen staining to identify at what stage of infection yellow rust is arrested in resistant mutants. We also noticed that a subset of mutant lines displayed a 'necrotic lesions' phenotype. We are testing if these phenotypes result from upregulated immunity and could potentially confer broad range resistance. Rapid identification of new sources of resistance is key to keep one step ahead of rapidly evolving pathogens. Mutations identified from mutagenized populations can be deployed immediately in breeding programmes as well as be part of the toolkit for modern genome editing approaches to improve crops.

Symbiotic relationship between tomato and Pseudomonas in phytoremediation Z. VERSHININA, IBG UFRC RAS, UFA, Russian Federation

Phytoremediation is the elimination, neutralization or conversion of pollutants to a less toxic form with the help of plants. This method is often used for heavy metal (HM) contaminated soils. On the one hand, it is important to select suitable plants-hyperaccumulators of HM to restore the biological productivity of ecosystems. On the other hand, the search for soil bacteria that increase the availability of HM for plants is important for effective phytoremediation. Bacteria of the genus Pseudomonas are the most promising in this regard. Tomato is widely cultivated in agriculture. In recent years, studies on the accumulation of HM in tomato plants and the potential use of tomatoes for phytoremediation have become popular. The strain $P$ s. sp. 102 can significantly increase growth parameters and biomass of tomato plants, including under the toxic effects of cadmium. The greatest positive effect was observed in plants transformed with the bacterial adhesin gene rapA1, the product of which is important for colonization of plant roots by bacteria. It was also shown that shoots of transgenic tomato plants accumulated the greatest amount of cadmium during inoculation of $P s$. sp. 102. The ability to extract high concentrations of cadmium and accumulate a large biomass under stress opens up prospects for the further use of associative interactions between tomato and Pseudomonas for phytoremediation. This research was supported by the RFBR (18-34-20004 mol_a_ved and 34-00033 mol_a).

Characterising Solanum dulcamara as a reservoir host of Ralstonia solanacearum

P. SEBASTIÀ MIRAVET, Center for Research in Agricultural Genomics, Barcelona, Spain

Ralstonia solanacearum is a gram-negative bacterium that causes bacterial wilt in wide range of plant species, with high aggressiveness, broad geographical distribution and lack of effective control measures in the field. Previous research has focused on how R. solanacearum infects different susceptible hosts (e.g. potato, tomato). However, there is scarce knowledge on the behaviour of this bacterium in other ecological niches: water, soil, reservoir hosts. Studying the interaction between $R$. solanacearum and its reservoir hosts might give us new insights to unravel how this bacterium overwinters and also the reservoir hosts could provide a suitable environment for horizontal gene transfer between different microorganisms. Here, we characterise two reservoir hosts (Solanum nigrum and Solanum dulcamara) of R. solanacearum to understand the symptomatology of the disease in these plants and also if they provide any defence strategy to protect themselves against this pathogen. Our results show that even though both $S$. nigrum and $S$. dulcamara are able to withstand better $R$. solanacearum than a susceptible host (e.g. potato, tomato), $S$. dulcamara presented a more resistant phenotype. Moreover, we were able to see how the latter reservoir host restricted the spread of $R$. solanacearum by using different bacterial reporter strains. These early results provide a basis to understand the interaction between $R$. solanacearum and one of its main reservoir hosts S. dulcamara.

An RxLR effector from Phytophthora infestans interacts with a lipid binding protein to regulate plant susceptibility H. ZHAO (1,2), H. McLellan (2), Q. He (2), P. Boevink (3), P. R. J. Birch (2,3), (1) State Key Laboratory ofCropStressBiology for AridAreas, Northwest A\&F University, Yangling, China; (2) Division of Plant Science, James Hutton Institute, University of Dundee, Dundee, U.K.; (3) Cell and Molecular Sciences, James Hutton Institute, Dundee, U.K.

Phytophthora infestans is an important oomycete pathogen and poses a serious threat to potato production. It secretes a range of RxLR effectors which are translocated inside plant cells and attenuate plant immunity by manipulating host target proteins. Therefore, we screened key effectors, important for pathogenicity, to identify their virulence targets in the host in order to help create new sustainable disease control strategies. The effector Pi00366 strongly suppressed the HR induced by INF1 and promoted the infection of $P$. infestans. Pi00366 interacts with the phosphoinositide binding protein Phox1 in both Y2H and Co-IP assays. Over expression of Phoxl also suppressed INF1 cell death and significantly promoted $P$. infestans infection. In contrast, on Nicotiana benthamiana silenced for Phoxl the HR induced by INF1 emerged earlier, and plants were more resistant to P. infestans than the GFP control. When co-expressing Pi00366 with Infl on Phox-VIGS plants, the ability of Pi00366 to suppress INF1 induced HR was attenuated, which indicates that this virulence function of Pi00366 is probably dependent on Phox1. Additionally, Phox1 localized to the endosome, and Pi00366 partially colocalized with Phox1 at endosomes upon co-expression. All results 
above indicate that Pi00366 promotes $P$. infestans colonization and suppresses PTI through interaction with Phox1 at endosomes, and Phox1 acts as a susceptibility factor to help $P$. infestans attenuate plant immunity.

Biochemical characterization of MLO2 in Arabidopsis

F. LEISSING (1), N. Huck (1), L. Huang (2), R. Panstruga (1), U. Conrath (3), G. J. M. Beckers (1), (1) RWTH Aachen University, Aachen, Germany; (2) University of California Irvine, U.S.A.; (3) RWTH Aachen University, Plant Physiology Department, Aachen, Germany

Mildew Resistance Locus O (MLO) proteins are plant-specific seven transmembrane domain proteins which are conserved among monocots and dicots. Loss of function of Mlo in Hordeum vulgare or loss of function of MLO2 in Arabidopsis thaliana provides durable resistance against powdery mildew fungi. Until now the biochemical function(s) of MLO proteins remain(s) unknown. To gain more insight into its biochemical function(s), we used a novel XL-MS strategy to identify protein interaction partners of MLO2 in Arabidopsis thaliana. Putative MLO2 complexes were in vivo crosslinked by formaldehyde. Subsequently, these complexes were purified by tandem affinity chromatography followed by mass spectrometry. In addition to a known interaction partner of MLO2, calmodulin, different putative in vivo protein interaction partners with a link to plant defense responses were identified. Moreover, phosphopeptides of MLO2 have also been recorded by mass spectrometry. Therefore, the phosphorylation of MLO2 by different pathogen-induced kinases, e.g. mitogen-activated protein kinases (MPKs) and calciumdependent protein kinases (CPKs), was confirmed by a non-radioactive in vitro kinase assay. Additionally, the phosphorylated residues of MLO2 were determined by the in vitro kinase assay. Taken together, we could identify new putative in vivo protein interaction partners of MLO2 and we confirmed MLO2 as an in vitro substrate of different pathogen-induced kinases.

Site specific proteolytic processing - An irreversible contribution to plant immune signaling?

A. PERRAR (1), F. Demir (1), P. F. Huesgen (1,2), (1) Forschungszentrum Jülich, ZEA-3 Analytics, Jülich, Germany; (2) University of Cologne, Medical Faculty and University Hospital, Cologne, Germany

Proteolysis plays a major role for the outcome of plant-pathogen interactions, by regulating protein abundance and as an irreversible posttranslational protein modification that creates new proteoforms with altered functions, interactions and subcellular localization. To identify proteins affected by proteolysis and proteolytic processing in bacteria-infected plants, we profiled changes in the Arabidopsis thaliana (At) leaf proteome and N-terminome on a global scale during the early stage of infection with Pseudomonas syringae pv. tomato DC3000 (Pst). Changes in protein abundance were measured by quantitative proteomics and differential proteolytic processing was investigated by High-efficiency Undecanal-based $\mathrm{N}$ termini enrichment (HUNTER). To differentiate between endogenous protease activity contributing to plant immune signaling and the action of bacterial effector proteases we compared distinct $A t$ infection scenarios using virulent (WT) and avirulent (delta hrcC) Pst strains. We found specific changes in protein abundance already at early stages of both infections compared to control, followed by stronger changes in proteome composition in the later stages. This reflects the early degradation of target proteins and proteome changes due to altered transcription. Proteolytic processing as assessed by N-terminome analysis shows a greater dynamic at the later stages of infection. Overall, differential infections led to distinct changes in proteome and $\mathrm{N}$-terminome.

EXORIBONUCLEASE 4 regulates MLA10 expression at post-transcriptional level to ensure proper immune responses in plants C. Zhang, Z. Chen, L. ZHAO, Q. H. Shen, INSTITUTE OF GENETICS AND DEVELOPMENTAL BIOLOGY, CHINESE ACADEMY OF SCIENCES, BEIJING, China

Barley Nucleotide-binding domain and Leucine-rich Repeat containing receptor MLAs recognize isolate-specific effectors from powdery mildew fungus and initiate immune responses. Previous studies have revealed that a miRNA family and an E3 ligase are involved in down-regulating MLA mRNA accumulation and protein stability, respectively. In order to identify more components that are required for maintaining proper $M L A$ transcript abundance, we employed a forward genetic approach to isolate suppressors of a transgenic Arabidopsis line expressing $M L A 10(F 99 E)$ autoactive variant driven by an estradiol-inducible promoter. Our results showed that a mutation in EXORIBONUCLEASE 4 (XRN4) could reduce the expression of $M L A 10$ at post-transcriptional level. We hypothesize that XRN4 degrades the MLA10 transcripts to an appropriate level to avoid the post-transcriptional gene silencing of this gene. Virus induced gene silencing of $H v X R N 4-1$ and $H v X R N 4-2$, encoding a cytoplasmic and a nuclear localized exoribonuclease, respectively in barley, resulted in the decreased accumulation of $M L A 10$ mRNA and compromised disease resistance to powdery mildew fungus, further confirming that XRN4 is a positive regulator of $M L A 10$ expression. Our study suggests that the expression and activity of MLAs are tightly regulated in barley to maintain an ideal balance between immunity and optimum growth and development.

RNA 2 of Cucumber mosaic virus mediates aphid deterrence in tomato.

W. ARINAITWE, A. Guyon, T. Tungadi, S. J. Rhee, A. E. Pate, J. P. Carr, University of Cambridge, Cambridge, U.K.

Cucumber mosaic virus (CMV) is a positive-sense single stranded-RNA virus comprising three genomic segments, RNAs 1, 2 and 3, which encode five proteins. CMV infects over 1000 plant species, including tomato (Solanum lycopersicum). It is non-persistently transmitted by more than 80 aphid species. CMV causes biochemical changes that enhance or diminish host preference by aphids. Using pseudorecombinants (exchanges of RNA segments between different viral strains) specific CMV RNA segments have been identified that influence aphid settling behaviour in certain hosts. For instance, in tobacco (Nicotiana tabacum), RNA 1 of Fny-CMV (severe CMV strain) stimulates strong resistance to Myzus persicae (Tungadi et al. unpublished). A recent mathematical model by Donnelly et al. 2019, Ecology in press) postulates that such virus-induced aphid behaviour may have distinct and substantial epidemiological effects. We investigated the effects of CMV on tomato-aphid interactions. We assessed settling preference of two aphid species, Myzus persicae, a generalist and Macrosiphum euphorbia, a specialist on tomato infected with different CMV pseudorecombinants. We made infectious CMV pseudorecombinants by systematic exchange of RNA segments of LS-CMV, a mild strain with those of Fny-CMV. Our findings suggest that the Fny-CMV $2 \mathrm{a}$ and or $2 \mathrm{~b}$ viral protein, both encoded by RNA 2 mediate aphid resistance in tomato. We also found that the virus is affecting more visual cues than volatile cues. 
Provenance, Transmission and Dynamics of Bacteriomes and Mycobiomes in Arabidopsis, Brachypodium, Maize, Wheat, Rice, Tomato, Soy, Cassava and 9 Other Important Species of Angiosperm Plant

D. JOHNSTON-MONJE (1,2), J. Guittierrez (2), T. Ovalle (2), A. Gkanogiannis (2), L. A. Becerra Lopez-Lavalle (2), (1) Universidad del Valle, Cali, Colombia; (2) International Center for Tropical Agriculture, Cali, Colombia

Plant microbiomes play an important role in sustaining all human life, but there is much we still have to learn about where these microbes come from, how they are selected for by the host and how they are transmitted between generations. To help understand the provenance, transmission and dynamics of microbiomes in important angiosperm plants, we carried out a comparative study of the microbes found in seeds and seedlings of the monocot crops maize (B73), rice (Nipponbare), switchgrass (Alamo), Brachiaria (Basilisk), wheat, sugarcane, barley and sorghum; the dicot crops tomato (Heinz 1706), coffee (Geisha), bean (G19833), cassava, soybean, pea and sunflower; and the model plants Arabidopsis (Columbia-0) and Brachypodium (Bd21). Seeds were planted in either sterile sand or field soil inside hermetically sealed jars and after reaching the $2^{\text {nd }}$ or $3^{\text {rd }}$ leaf stage, DNA was extracted to allow for next generation sequencing of the bacterial and fungal populations that developed in rhizospheres, endospheres, phyllospheres, spermospheres and substrate. Preliminary analysis supports our hypotheses that there is a core microbiome of seed transmitted bacteria found in all angiosperm plants, that there has been co-evolution of plant genomes with endosphere bacteriomes, and that unlike bacteria, the majority of fungal endophytes are derived from the soil.

Back to the Roots: Deciphering the taxonomic and functional diversity of the root microbiome of wild and modern tomato in the Ecuadorian Andes

P. VAN 'T HOF, Universidad San Francisco de Quito - USFQ, Quito, Ecuador

Ancient societies first attempted to domesticate wild plants for food production, which gave rise to present-day agriculture. Currently, more than a third of agricultural production is lost due to abiotic or biotic stress and future increases in crop yields should be achieved with fewer input of fertilizers and pesticides. These challenges have increased awareness of the importance of the plant root microbiome.

Plants are colonized by a surprising number of microorganisms, revealing in most cases an intimate symbiotic relationship, but the impact of plant domestication on the functional diversity and beneficial activities of this root microbiome is still largely unknown. Recent studies showed taxonomic differences in the root microbiome between wild relatives and modern cultivars, mainly in root architecture and root exudation. This leads to the hypothesis that present-day cultivars might have lost traits to recruit and activate host-specific beneficial root microbiota.

Our project investigates tomato species and native soils in the Andes to explore the taxonomic and functional diversity of their root microbiomes. Next generation sequencing and 'omics technologies, combined with classic microbiological techniques are being used to obtain insight in the diversity of root-associated microbial communities of tomatoes. We hypothesize that wild tomatoes grown in their native soils harbor unique and higher frequencies of beneficial root microbiota, compared to modern cultivars.

Development of a TYLCV resistance breeding system in tomato utilizing gene-specific molecular markers and infectivity assays J. H. Lee (1), D. J. Chung (2), J. M. Lee (2), I. YEAM (1), (1) Andong National University, Korea, Republic of (South); (2) Kyungpook National University, Korea, Republic of (South)

Tomato Yellow Leaf Curl Virus (TYLCV) is one of the most threatening diseases in tomato production worldwide. Among several TYLCV resistance genes known to date, $T y 1$ resistance has been most intensively investigated and utilized in the TYLCV resistance breeding programs. However, exploration of novel resistance sources and resistance gene pyramiding is still to achieve durable and stable resistance against TYLCV. TYLCV resistance genes reported to date include $T y 1 / 3, T y 2, T y 4, t y 5$ and $T y 6$ derived from wild-type tomatoes. Sequence information of each TYLCV resistance genes were analyzed and molecular markers were developed and applied. Using these markers, we are in the process of combine these resistances in multiple combinations. Quantitative infectivity assays using infectious TYLCV clones are optimized and performed to precisely address the resistance levels of each resistances and combination of these resistances.

Plant immune components controlling growth and structure of the bacterial leaf microbiota

S. PFEILMEIER, C. Field, M. Bortfeld-Miller, J. A. Vorholt, ETH Zürich, Zürich, Switzerland

The plant microbiota consists of a variety of microorganisms, including bacteria, fungi and oomycetes, which form stable communities and play critical roles in the survival and adaption of the plant to its environment. Various plant-associated bacteria promote plant growth and protect against pathogens. However, the fundamental mechanisms of host-microbe interactions that act in situ and underlie community establishment are currently unclear. While the immune system efficiently protects the plant against most pathogens, it is unknown to what extent it affects the commensal microbiota. We describe a reverse-genetic screen of Arabidopsis thaliana mutants defective in different parts of the immune system. We identified candidate genes that alter the composition of a bacterial synthetic community derived from the $A$. thaliana phyllosphere. Current work aims at disentangling how these plant mutant lines impact growth and niche occupation of immune-sensitive bacteria individually and in a community context. Overall, this study will contribute to our understanding of the plant immune system and its role for the leaf microbiota.

Small RNAs regulate barley and powdery mildew interaction

H. PEI, A. Ahmad, D. Liu, X. Liu, Y. Liu, L. Zhao, Q. H. Shen, INSTITUTE OF GENETICS AND DEVELOPMENTAL BIOLOGY, CHINESE ACADEMY OF SCIENCES, BEIJING, China

Small RNAs (sRNAs) and RNAi pathways play an important role in regulating plant immunity. Many trans-kingdom sRNAs have been shown to function in a number of pathosystems. To identify miRNAs in barley $(\mathrm{Hvu})$ and powdery mildew $(B g h)$ interaction, we constructed 10 small RNA libraries obtained from healthy or infected barley leaves, as well as $B g h$ conidiospores and epiphytic hyphae. We identified $B g h$-derived sRNAs ( $B g h$-sRNAs) and barley derived sRNAs ( $H v u$-sRNAs) by high-throughput sequencing and bioinformatical analysis. Among $314 \mathrm{Hvu}$ miRNAs identified, 107 of them were predicted to have target genes in $B g h$, with 3 of them to have solely predicted targets in $B g h$. Among 21 
$B g h$ milRNAs, 14 of them were predicted to have target genes, with 6 of them to have solely predicted targets in barley. Interestingly, we identified two $B g h$-sRNAs, $B g h$-sRNA10T and $B g h$-sRNA23T, that may target barley $M L A$ immune receptor genes. Co-expression of $B g h$ sRNA10T or $B g h$-sRNA23T with barley $M L A$ genes in Nicotiana benthamiana suggests that both $B g h$-sRNAs negatively regulate the expression of different subsets of $M L A$ genes. Further qRT-PCR at 16h, 24h, $48 \mathrm{~h}$ and $72 \mathrm{hpi}$ showed inverse correlation between $B g h$-sRNAs and $M L A$ target gene expression. These results indicate that cross-kingdom sRNAs exist and may function to regulate barley immunity and $B g h$ virulence during barley and powdery mildew interaction.

Population genetics of Hyaloperonospora arabidopsidis (Hpa), a natural oomycete pathogen of Arabidopsis thaliana F. PAUL, G. Shirsekar, R. Schwab, C. Tahtsidou, M. Lucke, P. Consortium, D. Weigel, Max Planck Institute for Developmental Biology, Tuebingen, Germany

Many plant pathogens cause devastating diseases, continually threatening food security worldwide. Due to the antagonistic nature of hostpathogen interactions, the interaction partners pose high selection pressures on one another. In agricultural pathosystems, which are less genetically diverse compared to wild plant pathosystems, such interactions have been well-studied, but little is known about how they play out in natural plant-pathogen systems. In 2018, we sampled wild populations of the annual plant Arabidopsis thaliana infected with a natural obligate biotrophic oomycete pathogen Hyaloperonospora arabidopsidis ( $\mathrm{Hpa}$ ) throughout a large part of their natural range in Eurasia. After propagation on immune-compromised host plants in the lab, we whole-genome sequenced $137 \mathrm{Hpa}$ isolates as well as host plants from 76 populations. We expect tight co-evolutionary dynamics between both partners because of the pathogen's host-specificity and its dependency on host resources to complete its lifecycle. We will present first insights from studying genetic diversity and population structure of Hpa. By looking closer at genomic variation within and among pathogen isolates, we aim to understand how the pathogen adapts to its host. Additionally, combining knowledge about the pathogen with information about the host, we will be able to investigate co-evolutionary dynamics in this pathosystem to deepen our understanding of natural plant-pathogen interactions.

Fungicide Sensitivity for a diverse Set of Fusarium Species causing the FHB Complex of Wheat M. JENNIFER (1), S. Bieri (2), S. Helge (2), R. Odile (3), (1) Syngenta Crop Protection AG, Stein, U.S.A.; (2) Syngenta Crop Protection AG, Stein, Switzerland; (3) Syngenta Crop Protection AG, Basel, Switzerland

Fusarium head blight (FHB) is a severe fungal disease affecting the ear of cereals such as wheat, barley and maize, caused by multiple species from the genus Fusarium spp. While visual lesions and losses of grain yield may be limited, the production of mycotoxins in the grain is of concern. FHB control by fungicides relies mainly on triazole fungicides. Syngenta Crop Protection is developing ADEPIDYN ${ }^{\mathrm{TM}}$ (abbreviation: APN; compound name: pydiflumetofen), a broad-spectrum Succinate Dehydrogenase Inhibitor fungicide (SDHI fungicide) with high control level of FHB. Aim of the work is to understand if the use of APN would significantly change the Fusarium population composition, in terms of species frequency or mycotoxin content capacity. We have tested the effect of APN and five other Fusarium active fungicides on 13 species and more than 500 Fusarium isolates using in-vitro assays. Moreover, sequence information was generated covering a large amplicon of the ITS and three relevant SDH enzyme subunits sdhB, sdhC and sdhD. Results indicate a superior in-vitro efficacy of ADEPIDYN ${ }^{\mathrm{TM}}$ compared to all tested fungicides. The advantage is shown for all species and all mycotoxin chemotypes. We will also discuss the sequence diversity found for the SDH subunit genes in relation to the species, the mycotoxin chemotype and in-vitro activity of SDHI fungicides. Results suggest no major shift is expected in the population composition for Fusarium species within the FHB complex of wheat.

DNA glycosylase MBD4L modulates chromatin structure in Arabidopsis infected tissues

J. R. Torres (1), S. Arias (2), F. Nota (2), C. I. Lescano (2), N. M. Cecchini (3), M. E. ALVAREZ (2), (1) CIQUIBIC-CONICET- Universidad Nacional de Cordoba, Cordoba, ARGENTINA; (2) CIQUIBIC-CONICET, Universidad Nacional de Córdoba, Cordoba, Argentina; (3) CIQUIBIC-CONICET- Universidad Nacional de Cordoba, Cordoba, Argentina

Chromatin structure controls gene expression, transposon silencing and DNA repair, among many other nuclear processes. Under certain stress conditions, plants exhibit chromatin remodeling apparently due to changes in the epigenetic marks of the genome. We previously described that Arabidopsis tissues infected with Pseudomonas syringae pv tomato (Pst) trigger decondensation of chromocenters, demethylation of DNA, and transient activation of pericentromeric transposons. In addition, other authors showed that the host genome is damaged under such condition, and that plant defense mechanisms help to maintain DNA integrity. We wondered if DNA glycosylases help repair DNA and/or remodel chromatin in Pstinfected tissues. To evaluate this we used comet assays to monitor DNA strand brakes in wild type and mutant plants. Mutants impaired in the DNA glycosylase MBD4L (Methyl Binding Domain 4-Like) had lower DNA damage than wild type plants. By confocal microscopy we performed a time course analysis of Pst-induced heterochromatin relaxation and found that this response is reduced in $m b d 4 l$ plants. Consistently, MBD4L maintained a nuclear localization in both basal and infection conditions, and its two splicing variants reached different subnuclear domains under different stress conditions. Thus, our results suggest that MBD4L mediates genome repair and chromatin remodeling under biotic stress conditions.

\section{The development of a transgenic sweet potato (variety Brondal) that is putatively resistant to Sweet potato feathery mottle virus (SPFMV) using the Coat protein method and the RNA interference method}

R. T. Masekesa (1), A. I. Robertson (2), E. Ngadze (1), A. Matikiti (1) and I. Sithole-Niang (1), (1) University of Zimbabwe, Harare, Zimbabwe; (2) Agri-Biotech (Pvt Ltd), Harare, Zimbabwe

Most Zimbabweans grow sweet potato Ipomoea batatas for household food security but weevil infestation/viral infections often reduce yields by up to $90 \%$. Breeding to counter these pests is hampered by the hexaploid genome nature of the crop. Molecular methods can be used but these require reproducible means of regenerating shoots in vitro and transformation protocols for adding relevant traits. This study aimed to develop regeneration and transformation protocols for s.potato genetic improvement by adding either the coat protein gene or a customized miRNA gene of Sweet potato feathery mottle virus. For leaf regeneration, ratios of $2.4 \mathrm{D}$ to kinetin were evaluated. Shoot organogenesis was stimulated by 0.05 $\mathrm{mg} / 12.4 \mathrm{D}+0.5 \mathrm{mg} / 1$ kinetin while somatic embryogenesis was stimulated by $0.5 \mathrm{mg} / 12.4 \mathrm{D}+0.1 \mathrm{mg} / 1$ kinetin. For meristem regeneration BAP, NAA and $\mathrm{GA}_{3}$ were evaluated. Treatment $1 \mathrm{mg} / \mathrm{l} \mathrm{BAP}+20 \mathrm{mg} / 1 \mathrm{GA}_{3}$ had a significantly $(\mathrm{p}<0.01)$ higher plant regeneration efficiency of $66 \%$. In the transformation experiments, 221 plants were regenerated after co-cultivation with Agrobacterium harboring either pCambia1305.1-CP or pCambia1305.1-RNAi. Of these, seven proved to be transgenic by PCR analysis, GUS histochemical test and dot blot hybridization. The coat protein approach and novel interfering RNA method have shown stable transgene integration into the s.potato genome. In conclusion, two reproducible s.potato (c.v Brondal) regeneration and two transformation procedures were developed. 